\title{
(1)
}

:

(\#)

(.).

-

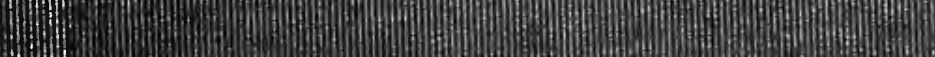
thitio

H. 


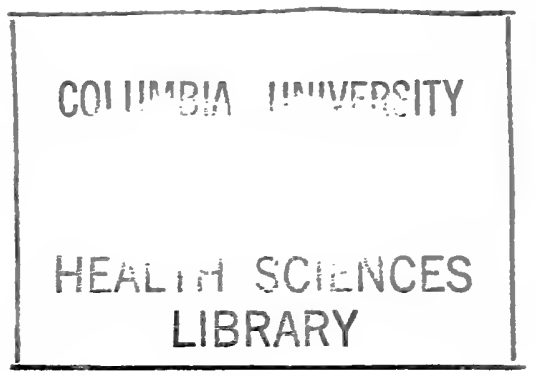



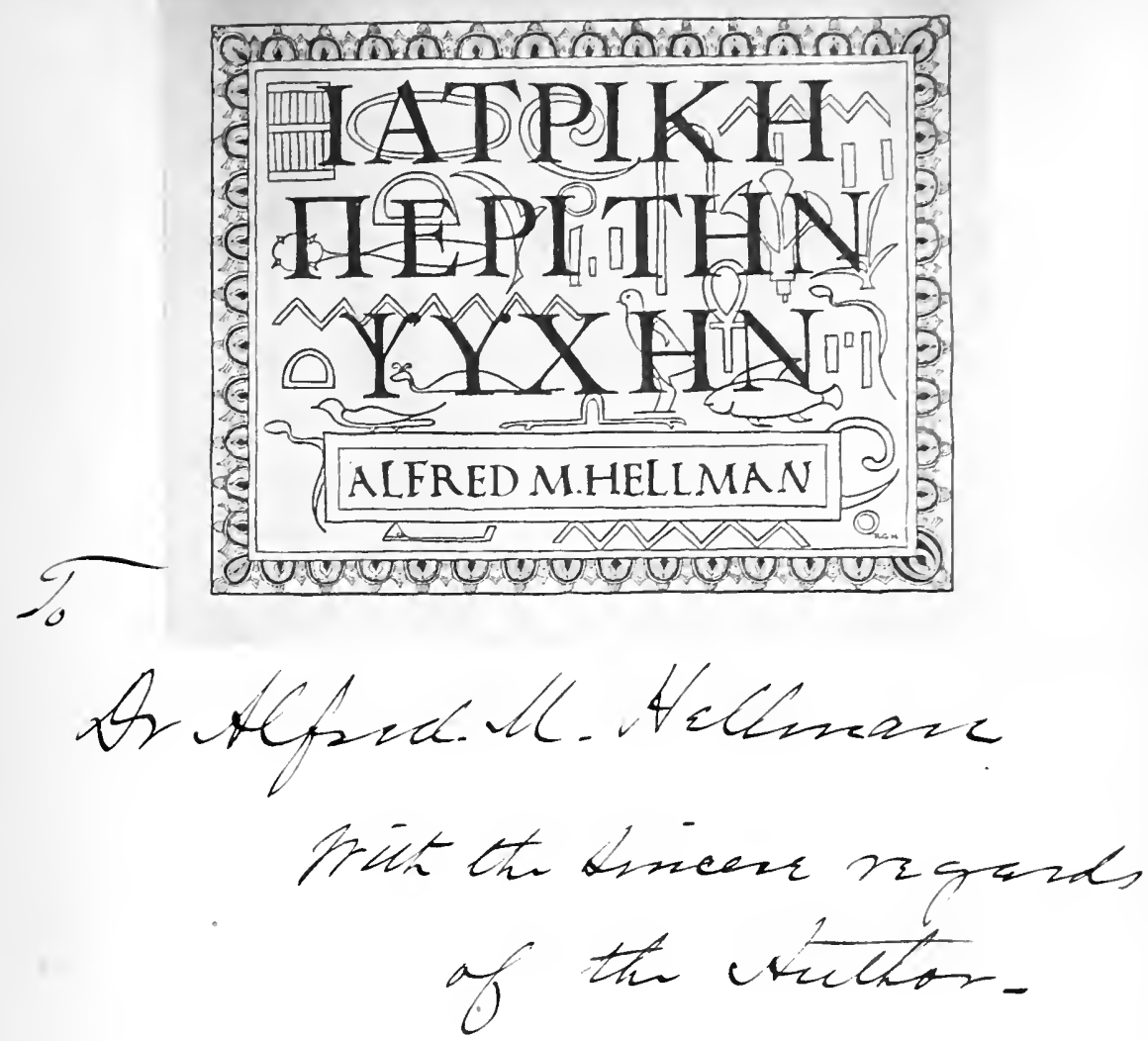



\section{ABDOMINAL HERNIA}

ITS

DIAGNOSIS and TREATMENT

BY

$$
\text { W. B. DE GARMO, M.D., }
$$

NEW YORK.

Professor Special Surgery (Hernia), New York Post-Graduate Medical School and Hospital;

Fellow New York Academy of Medicine; Member American Medical Association, New York State and County Medical Societies, Honorary

Member of the Medical Society of Virginia.

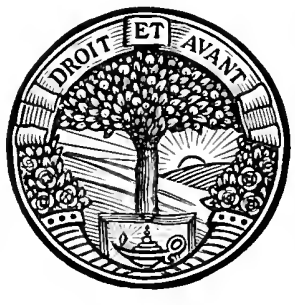

PHILADELPHIA \& LONDON

J. B. LIPPINCOTT COMPANY 


\section{Copyright, I907}

By J. B. Lippincott Company

Electrotyped and Printed by J. B. Lippincott Company" The Washington Square Press, Philadelphia, U.S.A. 


\section{DEDICATION}

70 the many practitioners of medicine

1 and surgery who, during the past twenty years at the New York Post-Graduate Medical School and Hospital, have patienty followed the Author's attempts to simplify the teaching of hernia, this work is dedicated. 
Digitized by the Internet Archive in 2010 with funding from Open Knowledge Commons 


\section{PREFACE.}

The first appeal for aid by those afflicted with hernia, is ustrally made to the family physician.

While the surgeon has plenty of literature to guide him in his part of the work, the physician, tpon whom many times the full responsibility of the case must rest, finds little practical aid from that source in any language. It is to the physician that this book is especially addressed, and if it fails to furnish him useful suggestions regarding the diagnosis and treatment of adominal hernia, then certainly it will have fallen far short of its intent.

The author's severest critic will scarcely accuse him either of writing a book too early in his professional career, or of compiling one from the experience of others. Many authors have been consulted, and quoted with credit, but the fundamental part of the work is based upon personal experience. and the illustrations are largely from photographs of patients under treatment.

The author realizes his liability to criticism for omitting mention of other forms of operation, popular, perhaps, in the country of their origin; but he justifies himself in the fact that they have not shown the success, that has followed the use of those here given. Furthermore he has tried to make clear the principles involved in curative measures rather than to suggest any special method.

Acknowledgment of obligations to many friends is freely given, especially the following who willingly granted the use of original drawings which are a valuable addition to the text: Dr. W. S. Halsted, Baltimore: Dr. WV. J. Mayo, Rochester, Minn.; Dr. William S. Bainbridge, New York: Dr. Charles N. Dowd, New York; Dr. A. E. Halstead, Chicago; Dr. 
W. Jay Seaver, New Haven; Dr. Samuel W. Kelly, of Cleveland, and to Professor Julius A. Becker for special dissections from which Mr. K. K. Bosse has drawn some of the anatomical plates, and to Dr. Alfred M. Hellman for compiling the index.

Manufacturers of appliances for the relief of hernia have allowed the publishers to draw freely upon them for electrotypes, with which to illustrate different forms of trusses, and the author wishes to thank in this public manner, Wm. H. Horn \& Bro., The J. Ellwood Lee Co., Chesterman \& Streeter, and his brother Mr. G. J. De Garmo. 


\section{CON'TEN'TS}

CHAPTER PAGE

I. Surgical Anatomy of the Inguinal Region . . . . I7

II. Descent of the Testicle. . . . . . . . . . . . 34

III. Cause of Inguinal Hernia . . . . . . . . . . 44

IV. Types and Conditions of Inguinal Hernia . . . 54

V. The Hernial Sac .............. $7 \mathrm{r}$

VI. Symptons and Diagnosis of Inguinal Hernia . . . . $\mathrm{S}_{2}$

VII. Mechanical Treatment of Inguinal Hernia . . . 1 I4

VIII. Truss Fitting . . . . . . . . . . . . . . . . I52

IX. Mechanical Treatient of Inguinal Hernia in InFANCY AND ChILDHOOD. . . . . . . . . . . 189

X. Treatment of Inguinal Hernia by Gyinastics . . . 204

XI. Surgical Cure of Inguinal Hernia . . . . . . . 214

XiI. Conplications in the Surgical Cure of Inguinal HERNIA . . . . . . . . . . . . . . . . 239

XIII. Sigmoid, Cafcal and Bladder Hernia . . . . . 277

XiV. Surgical Cure of Inguinal Hernia in the Female . 292

XV. Femoral Hernia . . . . . . . . . . . 297

XVI. Mechanical Treatment of Femoral Hernia. . . . 317

XViI. Surgical Cure of Femoral Hernia . . . . . . 33 I

XVIII. Umbilical Hernia . . . . . . . . . . . . . . . 344

XiX. Mechanical Treatment of Umbilical Hernia . . 355

XX. Surgical Cure of Umbilical Hernia . . . . . . . . 372

XXi. Ventral Hernia . . . . . . . . . . . . . 383

XXII. Rare Formis of Hernia . . . . . . . . . . . . $39 \mathrm{~S}$

XXili. Contra-Indications to the Surgical Cure of Abdominal Hernia . . . . . . . . . . . . . . . 409

XXiV. Strangulated Inguinal Hernia . . . . . . . . . . 413

XXV. Medical Treatment of Strangulated Hernia . . . 427

XXVI. Surgical Treatment of Strangulated Inguinal Hernia 432

XXVII. Str.angulated Femoral Hernia . . . . . . . . 439

XXViII. Strangulated Umbilical Hernia . . . . . . . . 443 



\section{ILLUSTRATIONS}

FIGURE

PAGE

I. (Original drawing.) Showing position of superficial vessels.. 2I

2. (Original drawing.) Aponeurosis of external oblique muscle and

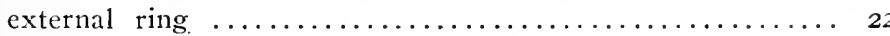

3. (Original drawing.) Aponetrosis opened to show deep part of

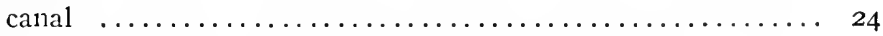

4. (Cooper.) Anatomy of inguinal and femoral region........ 25

5. (Original drawing.) Proximity of deep epigastric and iliac arteries to ingtinal canal....................... 30

6. (LEIDY.) The greater omentum................... 32

7. (Godard from Eccles.) Partial descent of testicle........ 35

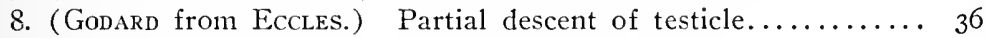

9. (Eccles.) Testicle in Scarpa's triangle.................. 37

Io. (Original photo.) Right testicle in canal, left at internal ring. . 38

I I. (Original drawing.) Multiple cysts of cord............ 40

12. (Original photo.) Hydrocele of cord simulating irreducible

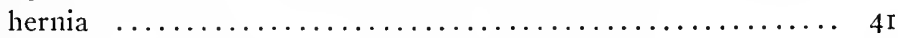

I3. (Macready.) Side view of abdomen of old man.......... 46

I4. (MACREAdy.) Early inguinal hernia................. 48

15. (Original drawing.) A form of physical culture that produces

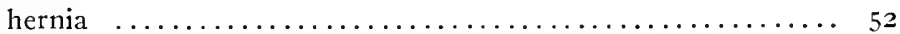

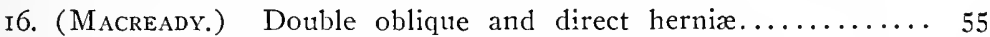

17. (Eccles.) Incomplete inguinal and direct herniæ........ 56

I8. (Original photo.) Right complete inguinal and enormous left

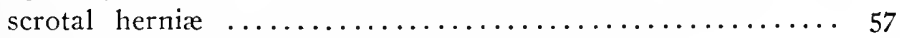

I9. (Original photo.) Scrotal hernia of enormous size......... 58

20. (Original photo.) Labial hernia in woman............ 59

2I. (Macready.) Double direct hernia................... 60

22. (Original photo.) Sigmoid hernia.................. $6 \mathbf{r}$

23. (Original photo.) Right direct and left sigmoid herniæ...... 62

24. (Original photo.) Left interstitial hernia, right testicle in canal. 63

25. (ECCLES.) Right interstitial hernia with retained testicle..... 64

26. (ECCles.) Right testis in cruro-scrotal pouch with hernia.... 65

27. (MACREADY.) Right interstitial hernia with retained testis.... 66

28. (MACready.) Left interstitial hernia simulating femoral hernia. 67

29. (Original drawing.) Showing formation of interstitial sac... 68

30. (Original drawing.) Three fibrous rings in acquired sac..... 72

31. (Original drawing.) Two fibrous rings with strangulation in

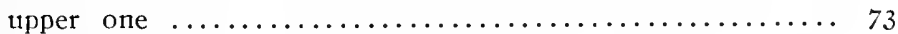

32. (Original drawing.) Omentum incarcerated in ring in Con-

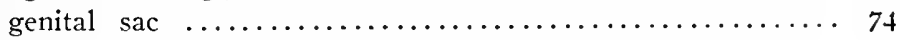

33. (MACREADY.) Hernia in funicular portion of tunica vaginalis.. 75 
FIGLRE

34. (Original drawing.) Hernia into funicular portion of tunica vaginalis with cyst below..................... 76

35. (Original drawing.) Infantile sac with closed tunica vaginalis

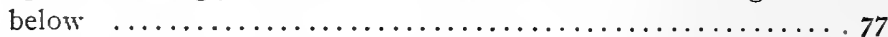

36. (Original drawing.) Sac of peculiar shape............ 78

37. (Original drawing.) Interstitial sac................ 79

38. (Original drawing.) Relative thickness of tissues covering sac. 80

39. (Original photo.) Irreducible omental scrotal hernia........ 83

40. (Original photo.) Large reducible scrotal hernia......... 84

4I. (Original drawing.) Improper method of examining for

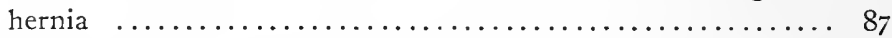

42. (Original photo.) Enormous irreducible left scrotal hernia... 89

43. (Original photo.) Large scrotal hernia with true peritoneal

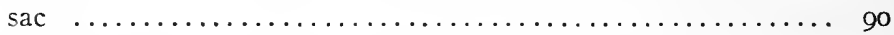

44. (Original photo.) Typical congenital hernia in adult....... 92

45. (Original photo.) Double congenital hernia in boy......... 93

46. (Original photo.) Double congenital hernia mistaken for hydro-

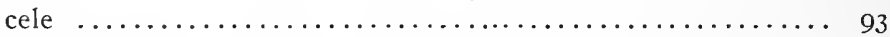

47. (Original photo.) Double congenital hernia retained by truss. 94

48. (Original photo.) Double congenital hernia two years after

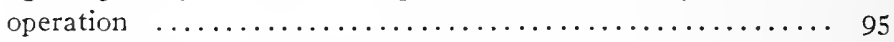

49. (Original photo.) Typical scrotal hernia of acquired type.... 96

50. (Original photo.) Right labial hernia ................ 97

5I. (Original photo.) Right labial hernia, four weeks after opera-

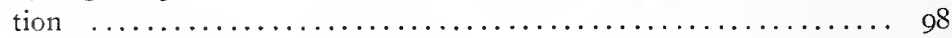

52. (Original photo.) Sigmoid hernia $\ldots \ldots \ldots \ldots \ldots \ldots \ldots \ldots$

53. (Original photo.) Crecal hernia ................... I00

54. (Original photo.) Sigmoid and Cæcal hernia in same patient.. Ior

55. (Original photo.) Cæcal hernia in woman.............. Ior

56. (ECCLES.) Left varicocele and femoral varix............. I03

57. (Original photo.) Varicocele mistaken for hernia.......... I05

58. (Le Progre's Med., redrawn.) Statuette showing truss. 900 B. C. II5

59. (Original drawing.) Showing shape of pelvis........... I22

6o. (Original photo.) Illustration of bad truss-fitting......... I27

61. Original photo.) Properly applied truss............... I28

62. (Original photo.) Usual manner of applying German truss... I29

63. (Original drawing.) Position in which a truss spring should

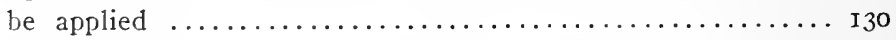

64. (Original photo.) De Garmo-Hood truss applied............ I40

65. (Original photo.) De Garmo-Hood truss applied, back view.. I4 $\mathrm{I}$

66. Group of variously shaped pads (thirteen figures)......... I49

67. A standard of sizes for truss pads (four figures)......... 150

68. (MAcready.) Measuring for inguinal truss............. I 53

69. (Original drawing.) Methods of taking diagram with lead tape 154

7o. (Original drawing.) Method of taking diagram, second position I 55

7r. (Original drawing.) Pelvic diagrams contrasting shape..... I56 
IIGURE

PAGE

72. (Original drawing.) Pelvic diagrams contrasting shape..... I.57

73. Shaping truss springs (seven figures)...............

74. (HoRn.) Hard-rubler cross-body truss applierl............ I6I

75. (Original photo.) Large labial hernia.............. 62

76. (Original photo.) Labial hernia retained by cross-borly truss.. I6.3

77. (HoRn.) Hard-rubber Hood truss applied............... I64

78. (Original photo.) Recurrent hernia in woman............ 164

79. (Original photo.) De Garmo-Hood truss applied to recurrent

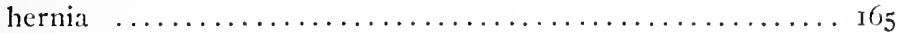

8. (Original photo.) Large double hernia ............... I66

81. (Original photo.) Large double hernia retained by Hood truss. I67

82. (Horn.) Combination of radical-cure truss with ordinary dou-

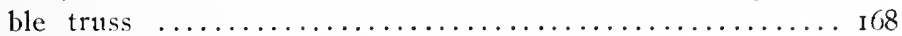

83. (Macrendy.) English "Rat-tail" truss, applied............ I70

84. (Eccles.) English double truss, applied............... I7 I

85. (HoRn.) Hard-rubber French truss, applied............. 172

86. (Original photo.) Donble retained testes and hernia; truss

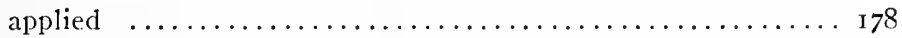

87. (Original photo.) Double retained testes and hernia....... I79

88. (Macready.) Truss for reducible interstitial hernia........ I 8 I

89. (Original photo.) Large irreducible scrotal hernia......... I84

90. (M.acready.) Hinged-cup truss for irreducible hernia....... I 85

91. (Original photo.) Enormous irreducible left scrotal hernia.... I85

92. (Original drawing.) Method of supporting large inreducible

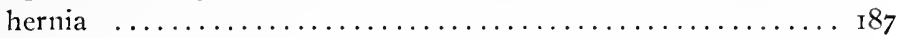

93. (Original drawing.) Five-weeks-old boy with cross-body truss

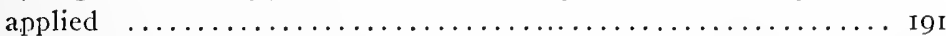

94. (Original drawing.) Hood's truss applied to girl six months old 192

95. (Original photo.) Cross-body hard-rubber truss........... I 93

96. (Eccles.) Hank of worsted truss.................. 194

97. (Original photo.) Cross-body hard-rubber truss with perineal

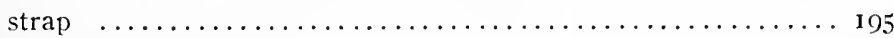

98. (Original photo.) De Garmo-Hood truss on girl.......... 196

99. (Original photo.) De Garmo-Hood truss on boy.......... I97

10o. (Original photo.) German scrotal hernia truss on boy....... I98

IOI. (Seaver.) Gymnastic exercise no. I ................... 205

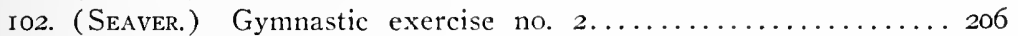

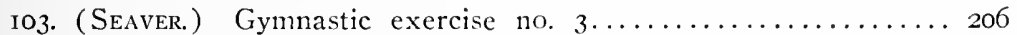

I04. (Seaver.) Gymnastic exercise no. $4 \ldots \ldots \ldots \ldots \ldots \ldots \ldots \ldots \ldots . \ldots \ldots$

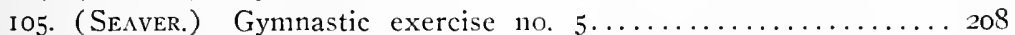

I06. (Seaver.) Gymnastic exercise no. $6 \ldots \ldots \ldots \ldots \ldots \ldots \ldots \ldots \ldots$

107. (Senver.) Gymnastic exercise no. $7 \ldots \ldots \ldots \ldots \ldots \ldots . \ldots . \ldots 209$

I08. (Seaver.) Gymnastic exercise no. $8 \ldots \ldots \ldots \ldots \ldots \ldots \ldots \ldots$ г

I09. (Original drawing.) Sac separated from cord and anatomy of

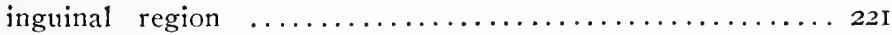

I Io. (Original drawing.) Curved blunt needle .............. 223 
FIGLRE

PAGE

III. (Original drawing.) Internal oblique stitched to Poupart's

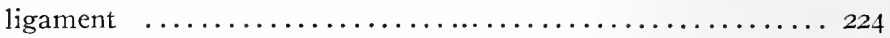

I12. (Original drawing.) Aponeurosis closed by continuous suture. 225

II3. (Halsted.) Relative position of sac, cord and vas deferens... 229

I14. (Halsted.) Cremaster fastened under internal oblique......230

II5. (Halsted.) Internal oblique fastened to Poupart's ligament... 23I

II6. (Halsted.) Overlapping of aponeurosis (first step) ........ 232

I17. (Halsted.) Overlapping of aponeurosis (second step) ...... 233

i I8. (Halsted.) Sectional view of fascial layers.............. 234

119. (Wullstein, redrawn.) Transplanting of cord (first step)...2 235

I20. (Wullstein, redrawn.) Transplanting of cord (second step).. 236

I2I. (Halsted.) Halsted's method of titilizing split sheath of

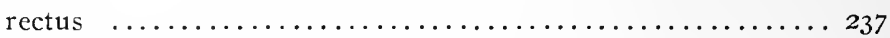

122. (Original drawing.) Right retained testicle in boy of to years. 240

123. (Original photo.) Right retained testicle in boy of 9 years... 24I

124. (Original photo.) Double retained testes in boy of $\mathrm{I} 3$ years.. 24I

125. (Original drawing.) Delayed testicle surrounded by sac.... 242

126. (Original photo.) Double retained testes two years after opera-

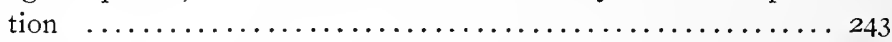

127. (Original photo.) Retained left testicle and interstitial hernia. 244

128. (Original photo.) Sac of interstitial hernia before opening... 245

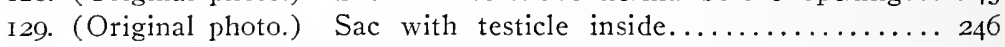

I30. (Original drawing.) Retained testicle, sac opened......... 247

I3I. (Original drawing.) Purse-string suture in sac...........247

I32. (Original drawing.) Sac tied by purse-string suture.......248

I33. (Original drawing.) Lifting muscles to place testicle beneath

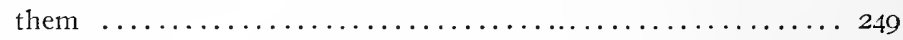

134. (Original photo.) Interstitial hernia and retained testicle....250

135. (Original photo.) Interstitial hernia three weeks after opera-

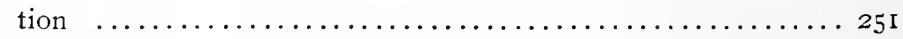

136. (Original photo.) Double hernia in child of 7 years supposed to

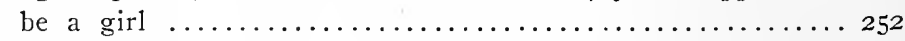

I37. (Original photo.) Same as preceding, with hernia reduced... 253

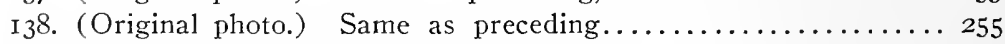

I39. (Original drawing.) Appendix adherent to anterior wall of

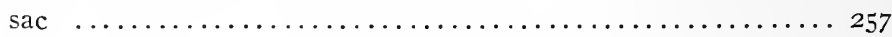

I40. (Original drawing.) Appendix adherent to posterior wall of

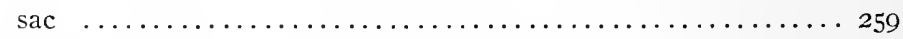

I4I. (Original drawing.) Appendix incarcerated in fibrous ring... 260

142. (Original photo.) Sac and omentum removed together....... 262

I43. (Original photo.) Omentum spread out for ligating..........263

I44. (Original photo.) Omentum irreducible from shape...... 264

I45. (Original photo.) Omentum, hypertrophied $\ldots \ldots \ldots \ldots \ldots 265$

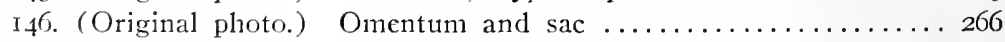

I47. (Original photo.) Scrotal hernia to within two inches of knee.. 268

I48. (Original photo.) Side view of preceding case.......... 269 
FIGIRE

149. (Original photo.) Preceding case seven weeks after operation, 270

50. (Original photo.) Large serotal hernia containing bladder... 2,

15I. (Original photo.) Preceding calse six weeks after operation.. 272

152. (Original photo.) Preceding case five years later with hernia

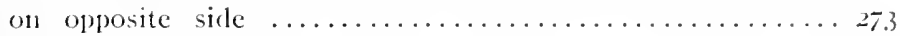

153. (Original photo.) Enormous scrotal hernia in man of go years $27 . t$

154. (Original photo.) Irreducible scrotal hernia, intestine only... 275

155. (Original drawing.) Peculiar mesentery found in preceding

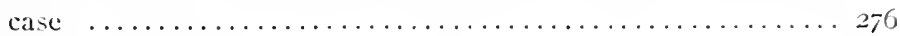

I56. (Original drawing.) Hernial sac containing free cecum and loops of intestine..........................

157. (Original drawing.) Hernial sac in front of cacum...... 279

I58. (Original drawing.) Form of purse-string suture for sigmoid

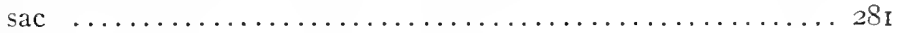

I59. (Original drawing.) Lifting internal oblique to bury stump of

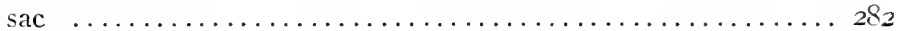

I6o. (Original drawing.) Protrusion consisting of hernial sac and bladder ..................................

I6I. (Original drawing.) Hernial sac and bladder protruding sep-

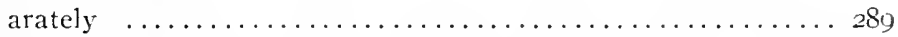

162. (Original drawing.) Closure of canal in female by single suture 294

163. (Original drawing.) Anatomy of femoral region...........298

164. (Original drawing.) Transverse section of femoral region... 299

165. (Redrawn from GRAY.) Showing relative position of femoral hernia and large vessels....................... 301

I66. (Original photo.) Double femoral hernia in man of 50 years. 302

167. (Original photo.) Reducible femoral hernia of enormous size. 303

168. (Original photo.) Irreducible femoral hernia............. 305

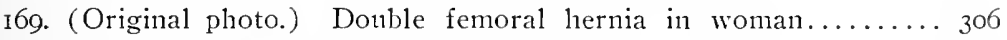

I70. (Original drawing.) Cystic femoral sac .............. 30 ;

I7I. (Original photo.) Femoral hernia of peculiar shape, in woman. 308

I72. (Eccles.) Left varicose saphena rein.............. 312

173. (Original photo.) Femoral and labial varix in pregnant woman

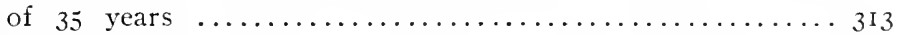

I74. (Original photo.) Lipoma simulating femoral hernia..... 315

I75. (Original photo.) Femoral hernia in woman, cross-body truss

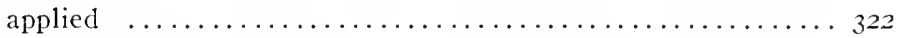

176. (Original drawing from photo.) Cross-body hard-rubber truss

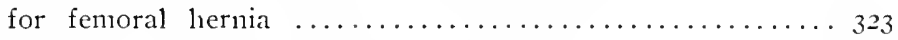

177. (Redrawn.) German femoral truss applied................ 325

I78. (Redrawn from MACrfanr.) English femoral truss applied... 325

179. (Original drawing from photo.) Adjustable truss applied to

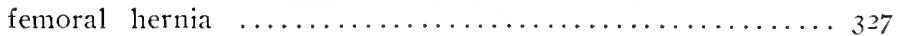

I8o. (Original drawing from photo.) De Garmo femoral truss ap-

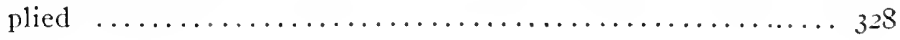


FIGURE

I8I. (Original photo.) Inguinal and femoral hernia in woman, truss

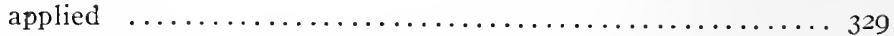

I82. (Original drawing.) Location of incision for femoral hernia... 334 183. (Original drawing.) Showing femoral protrusion under Poupart's ligament .............................. 335

I84. (Original drawing.) Femoral operation; sac drawn down... 336 I85. (Original drawing.) Femoral hernia operation, sutures in place 337 I86. (Original drawing.) Femoral hernia operation sutures tied.. 338 I87. (Original drawing.) Femoral hernia operation; blunt needle.. 339 I88. (Original photo.) Double reducible femoral hernia, woman of

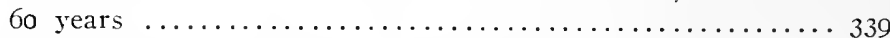

I89. (Original photo.) Same case, side view............... 340 190. (Original photo.) Same case six weeks after operation..... 34I 191. (Original photo.) Femoral hernia of unusual type......... $34^{2}$ 192. (Original photo.) Umbilical hernia in child of 3 years.... 347 193. (Original photo.) Enormous irreducible umbilical hernia.... 348 194. (Original photo.) Same case, side view............... 349 195. (Original photo.) Enormous umbilical hernia; man of 45 years 350 196. (Original photo.) Reducible umbilical hernia in 200-pound

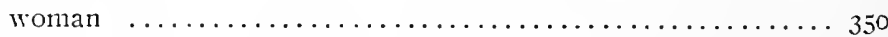

197. (Original photo.) Irreducible umbilical hernia in woman.... 35 I 198. (Original photo.) Irreducible umbilical hernia in man..... 35I

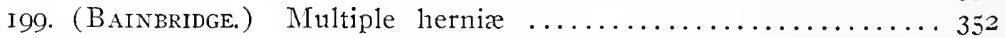
200. (BAinbridge.) Same case showing 5 herniæ in median line... 353 201. (DR. S. IV. Kelly.) Plate and plaster for umbilical hernia... 356 202. (Dr. S. W. Kelly.) Plate and plaster applied............ 357 203. (Original photo.) Truss applied for umbilical hernia, child of

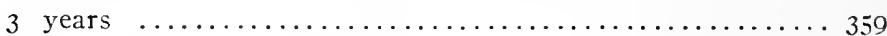

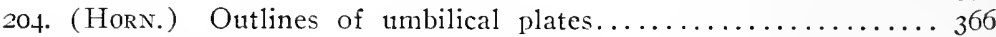

(Continued.).Size of umbilical plates................ 367

205. (Eccles.) An English umbilical truss.................. 368

206. (Horn.) Diagram for abdominal belt measure........... 369

207. (Eccles.) English rim-plate concave umbilical truss........ 370

208. (Original drawing.) Vertical overlapping of abdominal wall. 376

209. (MAYo.) Transverse elliptical incision ................ 378

2го. (MAYo.) Transverse overlapping sutures in place........... 379

2II. (MAro.) Transierse overlapping, complete .............. 380

212. (Original photo.) Post-operative ventral hernia........... 387

213. (Original photo.) Deposit of fat simulating ventral hernia... 388

2I 4. (Original photo.) Ventral hernia following gun-shot wound.. 389

2I5. (Original ploto.) Same, front view...................... 390

216. (Original photo.) Bilateral post-operative ventral hernia... 39 39

217. (Original photo.) Ventral hernia after appendix operation... 393

218. (Macready.) Spontaneous double lumbar hernia........... 399

219. (MACReAdr.) Truss for right lumbar hernia............ 400

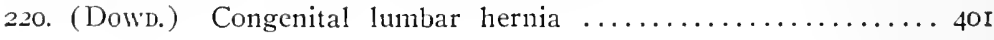


FIGURE

221. (Dow.) Anatomy of congenital lumbar hernia.......... 402

222. (Dowd.) Operation for lumbar hernia sutures placed....... 403

223. (Dowd.) Operation for lumbar hernia sutures tied......... 404

224. (Original photo.) Exstroply of bladder and vaginal hernia.. 406

225. (Original photo.) Sane case with appliance.............407

226. (Horn.) Appliance for perineal hernia ................. 408

\section{Group of:}

\section{Classification of Trusses by Groups.}

Springless trusses (seven figures)................

French, German and English trusses (eight figures)........ 125-125

Cross-body type of trusses (eight figures)............. $131-132$

Chase type of trusses (eight figures).................

Hood type of trusses (Twelve figures)............. 37 -1 39

Unclassified trusses (four figures) .................... $43^{-1} 44$

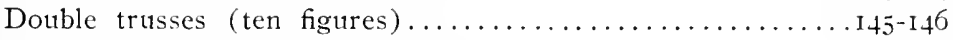

Trusses for femoral hernia (sixteen figures) ..............

Infant umbilical trusses (nine figures) $\ldots \ldots \ldots \ldots \ldots \ldots \ldots \ldots . \ldots \ldots$

Adult umbilical trusses (eighteen figures).............. $36 \mathrm{I}-365$

Trusses for ventral hernia (six figures.................. 394-395

Diagrams (original drawing) showing formation of femoral hernia,

extra-peritoneal fat and lipoma..................... 3 co 



\section{ABDOMINAL HERNIA}

ITS

\section{DIAGNOSIS AND TREATMENT.}

\section{CHAPTER I.}

INTRODUCTION.

Definition.-Hernia is the protrusion from a cavity, of any of its natural contents; as hernia of the brain from the cranial cavity, or hernia of the lung from the cavity of the chest. Abdominal hernia is, therefore, the protrusion through the retaining wall of any of the enclosed viscera. This generally occurs at some point in the muscular wall that is weakened by the transmission of nerves and blood vessels, at points congenitally defective, or through muscular parts that have been previously lacerated or incised.

The word "Rupture," so commonly used to denote a condition of hernia, will be, as far as possible, avoided in this work, as it leads to an erroneous impression of what actually occurs. In the early ages this term was applied under the supposition that there was actual rupture of the peritoneum. It is now well known that there is rarely laceration of tissue. Hernia results, in almost every instance, from the gradual stretching of tissue and escape of the abdominal contents, either into a preformed (congenital) sac, or by the formation of a sac (acquired) from the peritoneal lining of the abdomen.

Abdominal hernize derive their names from the part of the abdominal wall through which they pass. The terms inguinal, fomoral, or umbilical, denote at once their place of 
escape, the exception to this being ventral hernia, which may occur at any point in the anterior abdominal wall other than in the regions named. As ventral hernia occurs at points in the muscular wall so strong as ordinarily to resist hernial protrusion, it follows that when it does occur, it is either due to some congenital defect or is the result of some injury, such as a stab wound or a cutting operation. Extreme distension of the abdomen may also result in such separation of its aponeurotic fibres as to allow of protrusion.

A little more than 73 per cent. of all hernia are of the inguinal type. Next in frequency is femoral hernia, with 18 per cent., and third, umbilical hernia with about $8 \mathrm{r} / 2$ per cent. This leaves about I per cent. for all of the rarer forms.

The individual may have a single hernia or multiple herniæ. It is not uncommon to find inguinal and umbilical, or inguinal and femoral herniæ in the same subject.

The type, or form, of hernia is notably influenced by sex, as shown by the following comparison:

Male: Inguinal, 96.33 per cent.; femoral, 2.53 per cent.; umbilical, I.I4 per cent.

Female: Inguinal, 50 per cent.; femoral, 33.I 5 per cent.; umbilical, I 5.9 per cent.

That age has a decided influence on the occurrence of hernia is shown by the exhaustive studies of Paul Berger. His tables show 19.6 cases to $\mathrm{I}$, OOO individials in the first year of life, and drops to 4.2 per $\mathrm{I}, \mathrm{OOO}$ in the second year; then there is a gradual decline up to the twentieth year when only 0.88 is found. From this time on to the seventy-fifth year the increase of proportion is constant, reaching at this age its highest point, 24.20 per $\mathrm{r}, \mathrm{OOO}$ individuals.

Hernia consists of the sac and its contents; the sac being formed from peritoneum, the lining membrane of the abdominal cavity. It may be formed at the time of the first protrusion and is then termed an acquircd sac. As will be demonstrated later, a congenital sac may have existed long before the protrusion of the hernia, by the persistence of a pouch of 
peritoneum (Tunica Vaginalis) which normally should have been obliterated at, or shortly after, birth.

The sac consists of its body, or the expanded portion, which contains the bulk of the protrusion; the neck, which is the constricted part running through the muscular wall; and its mouth, the aperture of communication with the peritoneal cavity.

The acquired sac on first protruding, may be free from adhesions, and reducible, but readily becomes attached to surrounding tissues and from that time is irreducible. It then furnishes a permanent, moist, serous lining to the canal through which it protrudes.

Hernia of the bladder, of the cxecum and sigmoid flexure, may occur without a true hernial sac. The anterior bladder wall is not covered by peritoneum, and it may form the actual protrusion in inguinal hernia. If the protrusion is of fairly large size, it will also drag that part of the organ into the canal that is covered by peritoneum, when both bladder and abdominal contents will be found. This same condition exists in sigmoid and cacal hernia, except that in these the peritoneum covers the anterior wall of the gut and the posterior wall is dragged down without this covering.

Following previous operations where the peritoneum has for some reason failed to unite, there may be protrusion immediately beneath the skin without sac formation. This is most frequently met with in ventral hernia following laparotomy, but I have found in one instance this condition existing in a recurrent inguinal hernia. It was quite evident that the previous operator had either failed to ligate the neck of the sac properly, or what is more likely, the ligature had slipped off, and both omentum and intestine were in contact with scrotal tissue.

The contents of a sac may be either omentum, intestine, or in fact any of the movable contents of the abdomen. In some rare cases even those organs that are not ordinarily considered movable, as the kidney or a part of the liver, have been 
found in the hernial sac. The contents of a sac may be freely reducible, or its reduction may be prevented by the great size of the mass and the smallness of the neck of the sac, or from adhesions of the protruding mass to the inner sides of the sac; also by the formation of fibrous bands which transverse the sac in different directions.

Omentum and small intestine are most frequently found in the hernial sac; next in frequency, in about the order named, will be found the sigmoid flexure, cæcum and transverse colon. The bladder may also protrude into an inguinal hernia sac, but is more frequently found without peritoneal covering.

The term enterocele refers to hernia, the contents of which is exclusively intestine, epiplocele to one containing omentum, and the use of the words combined, as entero-epiploccle, to one containing both intestine and omentum. While these are in some instances convenient terms, they will be avoided in this work on the ground that multiplicity of names adds to the confusion of the subject.

\section{SURGICAL ANATOMY OF THE INGUINAL REGION.}

The lower lateral third of the abdominal wall, known as the inguinal region, is an irregularly shaped triangle. Roughly, its outer boundary is Poupart's ligament, its inner boundary the median line of the body, and its upper boundary an inaginary transverse line from the crest of the ilium to the median line. The anatomy of this triangle, although the region is comparatively small and easy of access, has proven one of the most difficult to comprehend and teach of any connected with the muscular system.

The author assumes that his reader has already acquired an anatomical education from works upon the subject and from practical demonstration upon the cadaver, therefore, the anatomy here given is merely to refresh his mental picture of the parts. If the picture is presented from a different view to 
that which he is accustomed, and minus some of its technical details, it may be even clearer in outline, in which case the object sought will have been fully accomplished.

Immediately beneath the skin of this region we come upon the two layers of superficial fascia. These layers contain a deposit of fat of variable thickness according to the condition

FIG. I.

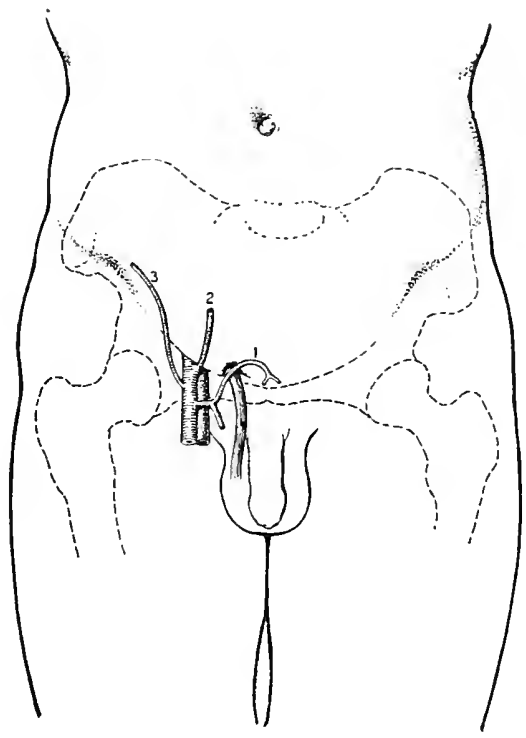

Showing position of superficial vessels (size exaggerated). I, Superficial branch of external pudic artery. 2, Superficial epigastric artery. Both are in deep layer of superficial fascia and are divided in hernia operations. 3, Circumflex iliac artery. Not usually divided.

of the patient. In operating it will happen many times that the dividing line between the two layers is not discovered, but occasionally it is so well defined as to mislead the operator into the belief that he has already reached the aponeurosis of the external oblique muscle. The only surgical importance connected with this fascia is that the deep layer contains two sets of vessels that are usually cut in the first incision in hernia operations (fig. I). 
These arteries both come from the femoral space and are first, the supcrficial cpigastric, crossing Poupart's ligament at its middle third and passing on upwards over the internal ring towards the umbilicus. Second, a superficial branch of the crternal pudic, leaving the femoral space and passing up directly over the external abdominal ring, and arching over to

FIG. 2.

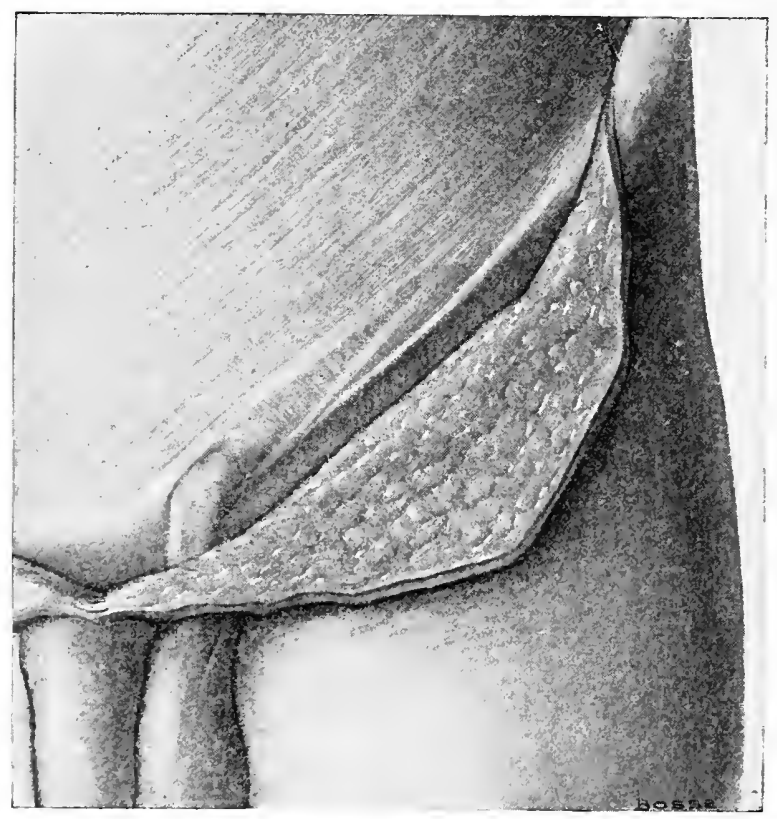

Aponeurosis of external oblique muscle, in which is shown the external ring covered by the intercolumnar fascia.

the root of the penis. These vessels are not important in size lut may require ligation at time of operation.

Beneath this fascia we find the aponeurosis of the external oblique muscle which is easily distinguished by its glistening surface and from the fact that its fibres run obliquely downward towards the public bone (fig. 2). In operations for hernia the fleshy part of the external oblique muscle is seldom 
seen. The fibres of the aponeurosis are bound together by the overlying intcrcolumnar fascia, which is tendonous in character and furnishes strong protection to the upper angle of the external abclominal ring, by arching across from one pillar to another. In opening down to the external abdominal ring, it frequently obscures the upper angle of that aperture and prevents the easy passage of the director under the aponeurosis, until it has been scraped away with some blunt instrument. The cord receives this fascia as its last covering as it comes out between the pillars of the external ring.

The aponeurosis of the external oblique muscle is a thin, but very tough, inelastic tenclon, which splits easily in the direction of the fibres, especially after the intercolummar fascia is cut, but furnishes one of the most important parts of the retaining wall of the abdomen. At its lower border it is reflected back under the abdominal wall forming Poupart's ligamont. This lower portion of the aponeurosis is attached to the anterior superior iliac spine above, and to the spine of the pulses below. Just above the pubic attachment there is a split in the fibres for the transmission of the spermatic cord in the male and round ligament in the female. This aperture is called the cxtcrnal abdominal ring. The term "ring," an unfortunate one, conveys the iclea of a circular opening, when in reality it is triangular in shape. This name, however, has from long use become so firmly fixed in the medical mind that it would probably result in even more confusion to adopt any other. The base of this triangular opening is formed by the crest of the pubic bone, and its upper angle is prevented from splitting still higher in the aponeurosis by the intercolumnar fascia. The sides of the triangle are formed by the free split borders of the aponeurosis and are called the pillars of the ring. The c.rtcrnal, lower pillar, curves around in such a manner as to form a groove upon which the cord rests. The internal, superior pillar, passes over the cord to the crest of the pubic bone, to interlace in the median line with its fellow of the opposite side. On account of the cord being larger than the round liga- 
ment, and to the fact that the testicle passes down through this opening, the external abdominal ring is considerably larger in the male than in the female.

Poupart's ligament, extending from the iliac spine to the spine of the pubes, is also attached to the pubic bone at the pectineal line for about one inch, forming Gimbernat's liga-

FIG. 3.

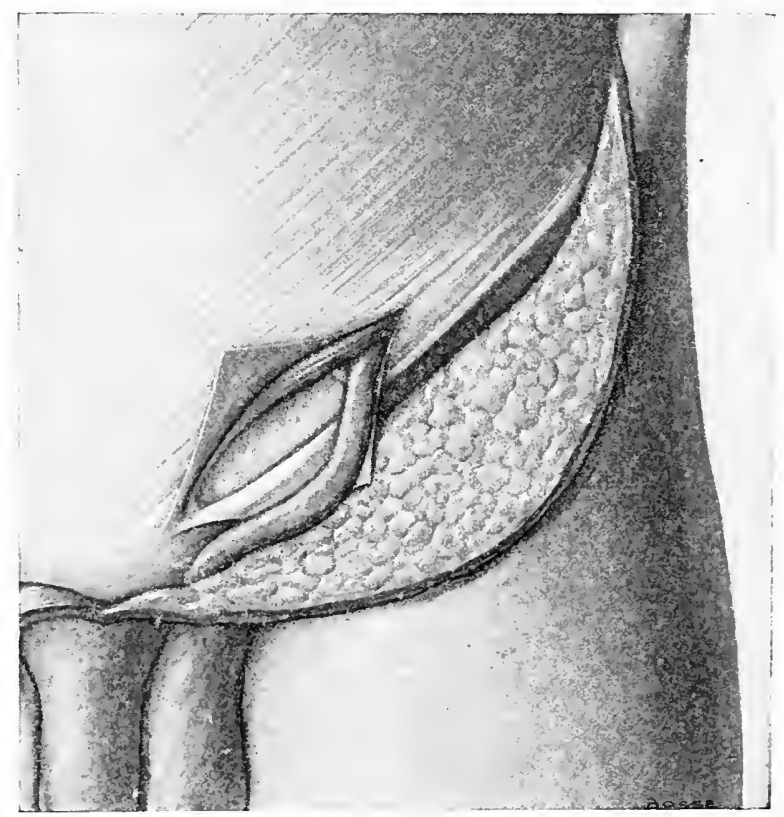

Aponeurosis opened to internal ring, showing lower border of internal oblique muscle; transversalis fascia in deep wall of canal.

- ment. The crural arch, beneath which emerges the femoral vessels, is formed by Poupart's ligament, internal oblique and transicersalis muscles (figs. 3 and 4 ). All works on anatomy show these muscles, as shown in the accompanying cuts, nicely dissecter one from the other. No such picture is presented on the operating table and for this reason the two muscles will be spoken of together. The lower border of the internal oblique 
is attached to the outer half of Poupart's ligament, and the transversalis is attacherl to the same ligament immerliately beneath it, but only to the outer third. The filses of both muscles are fleshy in character and arch up orer the cord, the

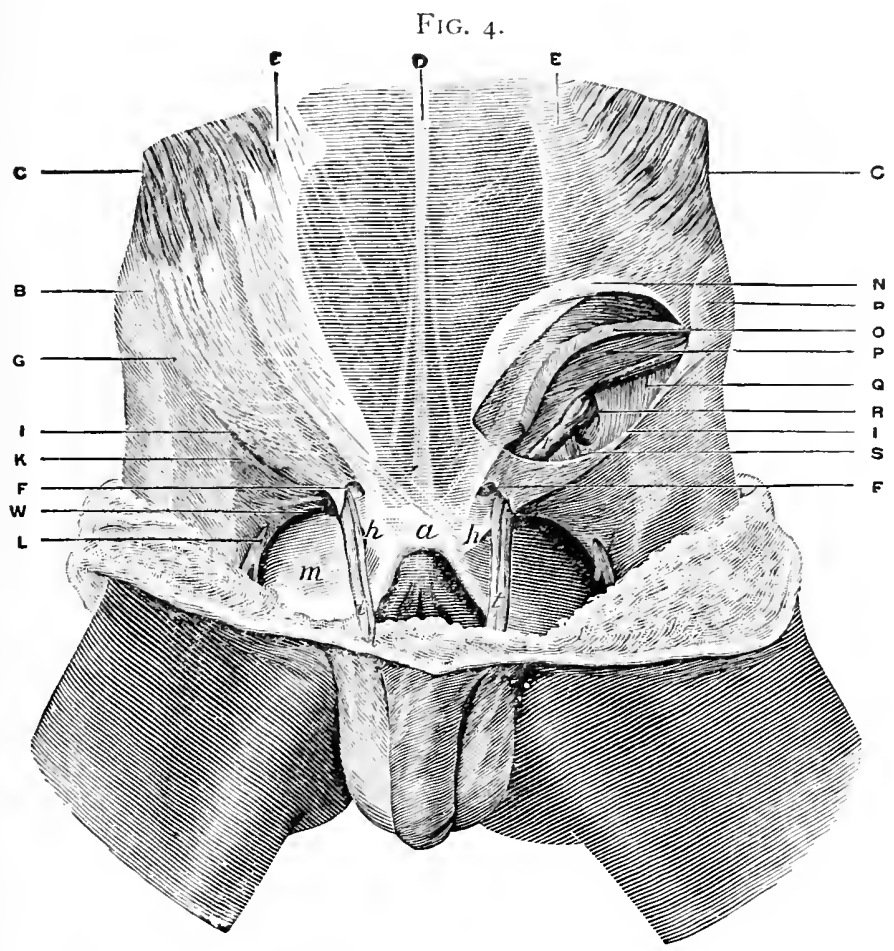

$A$, Symphysis pubis. $B$. Anterior superior spinous process of crest of ilium. $C$, Muscular part of external oblique muscle. $D$, Linea aiba. $F, F$, External abdominal rings. $I, I$, Poupart's ligament. $S$, Aponeurosis of external oblique muscle cut open to show deeper parts. $O$. Internal oblique muscle. This is turned up at lower edge to show ( $P$ ) Transversalis muscle. ( 1 operative work these muscles are seen as one.) $Q$, Transversalis fascia. $R$, Internal abdominal ring. $S$, Epigastric artery, $t, t, t$, Spermatic cord. Oblique inguinal hernia leaves the abdomen at $R$, (Internal ring), and follows the cord to $F$ (External ring). Direct hernia protrudes directly through the wall at $F$. Femoral hernia protrudes at $l l$. (From Sir Astley Cooper on Hernia.)

transversalis even higher than the internal oblique, and then the two muscles becoming blended into an aponeurotic structure, the conjoined tondon, pass down back of the cord and find insertion in the pectineal line of the os pubis. Here conjoined 
tendon is exactly back of the external abdominal ring, and should furnish the most important barrier against the occurrence of direct inguinal hernia. Some of the fibres pass towards the median line where it joins its fellow of the opposite side.

The lower edges of these muscles are indistinguishable on the operating table, and are treated as one structure. Normally, the fibres of these muscles start from Poupart's ligament in front of the cord, and arching over close to it, descend back of the cord to the pubic bone. In many people this arch is abnormally high, and the insertion of the conjoined tendon is well towards the median line, leaving the muscular wall back of the cord very deficient throughout the whole length of the inguinal canal.

At the time of descent of the testicle, through the canal, there is a covering taken from the lower edge of the internal oblique muscle which develops some muscular fibres, and is afterwards known as the cremaster muscle. This fascia, or muscle, frequently forms one of the coverings of a hernial sac. The muscle receives its blood supply from the cremasteric artery, a branch of the epigastric, and its nerve supply from the genital branch of the genito-crural nerve. Neither of these are ordinarily seen in operations for hernia.

When the aponeurosis of the external oblique muscle is first split and retracted, so that the canal is freely exposed, the ilio-inguinal nerve will usually be seen following closely the lower border of the internal oblique muscle. The ilio-hypogastric nerve will also frequently be seen a little higher on the surface of the same muscle.

The rectus muscle should, perhaps, be mentioned here on account of its relation to the aponeuroses of the muscles just considered. The aponeuroses of all of the abdominal muscles below the umbilicus pass in front of the recti, leaving the posterior surface of the latter in contact with the transversalis fascia. The muscle is attacher below to the pubic crest as far out as the pubic spine and doubtless affords some protection to the external abdominal ring. 
TRANSVERSALIS FASCIA OR EXTRA PERITONEAL SIIEATII.

This is inmediately beneath the transwersalis muscle and while in the upper part of the abdomen it is thin, in the inguinal region it is thicker and stronger. It gives the cord its first covering, the infundibuliform fuscia. It also forms one of the coverings of oblique inguinal hernia.

In the transversalis fascia is situated the internal abdominal ring. This is a purely arbitrary term given to the beginning of the inguinal canal, as there is neither a ring nor an opening into the abdominal cavity proper. Immediately beneath this sheath, or fascia, crossing the canal at right angles just below the internal abdominal ring. is the decp cpigastric artery. In operations for hernia this important ressel is frequently hidden by the transversalis fascia and its location must be kept constantly in mind in order to aroid injury to it. This vessel and its accompanying two veins are embedded in the subperitoncal arcolar tissuc. This sheet of fat, between the transversalis fascia and the peritoneum, is of variable thickness in different individuals, and in the same individual at different times. It is very abundant in the vicinity of the cord, and is frequently an important factor in the production of hernia : ( I) In its liability to the formation of lipomatous tumors which descend through the canal, dragging a process of peritoneum after them. (2) During violent muscular effort this fat may be forced into the canal, the point of least resistance, where it acts as a dilating wedge, stretching the tissues so that hernial protrusion readily follows. Beneath this subperitoneal fat we have the peritoneum, the lining membrane of the carity of the abdomen. Further attention will be given this important structure when we have finished our consideration of the abdominal wall.

Haring reviewed briefly the structure of that part of the abdominal wall involved in iguinal hernia, let us clearly understand the inguinal canal through which most hernire of this region descend. We have seen that the transversalis fascia 
(or extra peritoneal sheath), where it envelops the cord as the infundibuliform fascia, is really the internal abdominal ring and the beginning of the canal. From this point, deep in the abdominal wall, the canal runs parallel with Poupart's ligament obliquely towards the surface, coming out beneath the skin through the split in the external oblique known as the external abdominal ring. At birth these two rings are almost directly opposite each other, but on reaching adult life they have become separated by a distance of about one inch and a half. This change takes place rapidly in the early life of the child.

The exact boundaries of the canal are somewhat difficult to understand, as in some instances the same structures form its outer boundary high up, its roof lower down, and its posterior border still lower. This remark applies to the lower border of the internal oblique and transversalis muscles. Its boundaries are perhaps better unclerstood by following the steps of the operator rather than by following the classical methods of the dissector, even though not quite as accurate.

After division of the skin and superficial fascia, and splitting the aponeurosis of the external oblique muscle over the whole length of the canal, we find that this tendon has served as the principal part of the anterior wall.

Arching over the cord, in front of the internal ring, will be found muscular fibres belonging to the internal oblique and transversalis muscles. For a distance of about three-quarters of an inch, from the internal ring down, these fleshy fibres form the anterior wall of the canal, the remaincler of the distance being formed by the aponeurosis of the external oblique. The roof of the canal is principally formed by the lower border of the internal oblique and transversalis muscles; its floor by Poupart's ligament.

Its posterior boundary, in its upper two-thirds, is formed by transversalis fascia, and the lower third by the conjoined tendon. My personal experience is, that in by far the greater number of cases operated upon for hernia, nothing is found back of the cord but transversalis fascia throughout the entire 
length of the canal. Beneath this is the sulb-peritoneal fat and the peritoneum. Embeded in the stils-peritoneal fat are the epigastric vessels, crossing the posterior wall of the canal at right angles about half an inch below the internal ring. The inguinal canal is occupied, normally, by the spermatic cord in the male and the round liganent in the female. In addition to these we frequently find the ilio-inguinal nerve, either following the surface of the cord or along the lower border of the internal oblique muscle.

The spermatic cord, as it passes through the inguinal canal, receives the following coverings: ( I) The infundibuliform from the transversalis fascia. (2) The cremasteric fascia (or muscle) from the internal oblique. (3) The intercolumnar fascia from the external oblique, as it passes between the pillars of the external abdominal ring.

\section{DEEP EPIGASTRIC ARTERY.}

The relation of the deep epigastric artery to the inguinal canal is important. By the present methods of operating the artery is frequently exposed to accident, eren though not always seen. It lies immediately back of the spermatic cord between the transversalis fascia and the peritoneum, and crosses the cord at an oblique angle just at the inner and lower border of the internal abdominal ring. It passes from the external iliac, its origin, just inside of Poupart's ligament, obliquely upwards to the sheath of the rectus muscle. It is usually accompanied by two veins. The close relation of the cxtcrnal iliac artcry to the parts under consideration must always be borne in mind (see fig. 5). Although it is within the abdominal cavity, it is frequently in contact with the deep parts of the canal, and several accidents to this ressel during operations are known to me, and one has been published by an eminent operator. The other blood vessels of this region, outside of those forming the spermatic cord, are unimportant from a surgical standpoint. 
The nowe supply to this region is by the ilio-hypogastric and ilio-inguinal from the upper lumbar nerves, and is purely sensory in character. These nerves are frequently seen when operating, just below the aponeurosis of the external oblique,

FIG. 5.

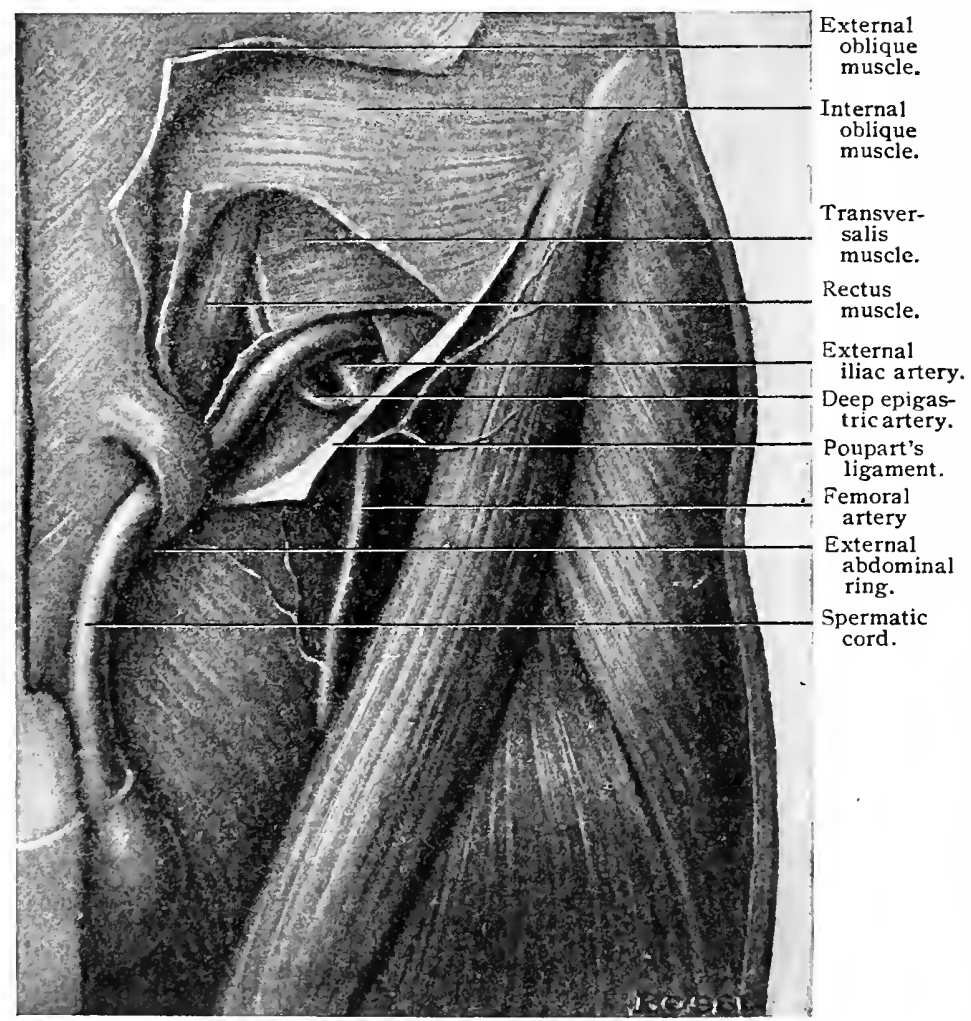

To illustrate proximity of deep epigastric and iliac arteries to inguinal canal.

and with a little care can be avoided, but their division is only attended by temporary loss of sensation over a small area of surface.

Peritoncum.- We have constantly to deal with this structure in hernia operations, as all hernial sacs are formed from 
it, and it rarely happens that abdominal hernia occurs without peritoneal covering which becones its sac.

This serous membrane not only completely lines the abdomen, but entirely or partially envelopes every hollow organ contained in this cavity. Its surface is normally moist and shiny. It wraps itself completely about the small intestine and passes backwards to the spine, where it is attacherl as the mesentery. The mesentery gives to the intestinc at least partial support; in hernial protrusions, however, it may become not only enormously elongated, but so changed in character as seriously to interfere with operative procedures. The right segment of the mesentery is longer than the left, allowing of greater pressure from the intestine in the right hypogastric fosse. This may, in a measure, explain the greater number of hernice on the right side. The external inguinal fossa is at the outer sicle of the epigastric artery and immediately back of the internal ring. This is the deepest of the fosse, and that on the right is deeper than the one on the left, another reason for the more frequent occurrence of inguinal hernia on the right side. Hernixe entering the external inguinal fossa and passing into the canal become oblique inguinal hernia.

The fossa towards the median line from the epigastric artery, is the internal hypogastric fossa, and it is in this pocket that direct inguinal hernia originates. In the relation of the protrusion of hernia to the epigastric artery originated the terms, cxtcrnal and internal inguinal homia, the protrusion into the external fossa (oblique) being termed external, and that into the internal fossa (direct) internal hernia. The author has for many years avoided using these terms in his teaching, as they are confusing and misleading. Internal herniac are believed to be such as occur within the body, as hernia through the diaphragm into the chest cavity, or hernia through the foramen of Winslow.

While any organ within the abdominal cavity may become involved in hernia, there is only one other structure that we shall consider in this part of the work, and that is the omentum. 
This forms a rather constant factor in the diagnosis and treatment of hernia (fig. 6).

The omentum hangs as a great, fatty apron between the abdominal contents and anterior muscular wall, doubtless forming an important means of protection to the bowels from sudden changes of temperature, and against injury from blows

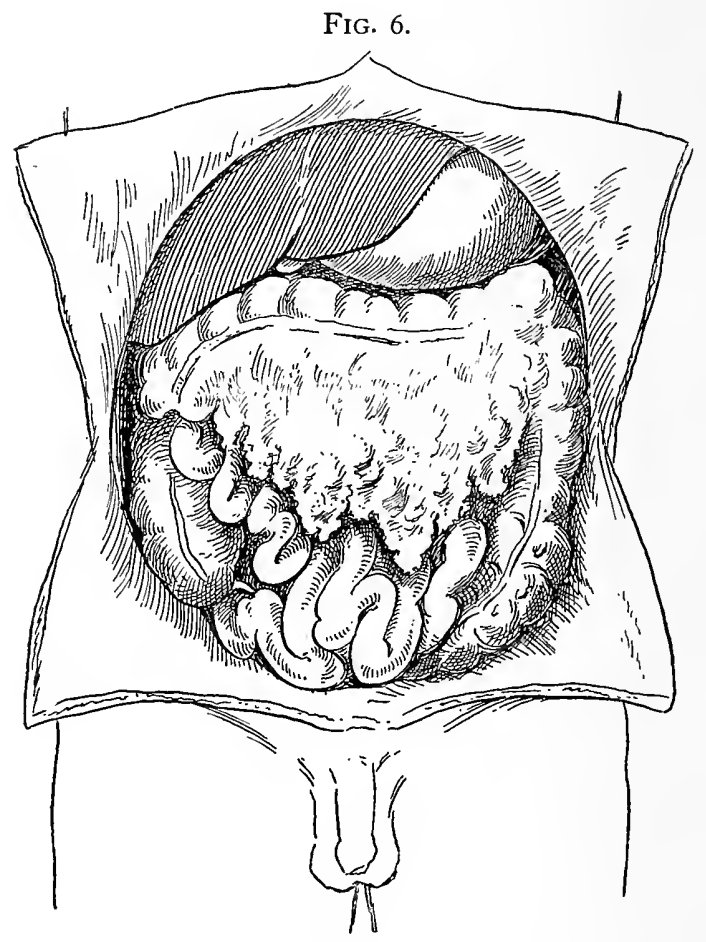

The greater omentum as seen from the front.

upon the abdominal surface. To those suffering from hernia, it is the source of much trouble, and necessarily much will be said about it in the pages to follow.

It hangs in two sheets, or layers, each covered by peritoneum, from the lower convex border of the stomach and from the transverse colon. Perhaps it would convey a clearer idea to say that it passes down to the pelvis from its attach- 
ment to the stomach, is folded upon itself, and returns wo the transverse colon. The space between is normally a shut sac, but frequently, owing to the delicate structure of the onentum, openings into it will be found. Intestinal obstruction is sometimes clue to constriction of a loop of bowel in such an opening. The two layers camnot always be distinguished; in fact, they selclom are when dealing with its lower part, but it must be remembered that they exist, as it not only happens that cysts may form between these layers, but intestine has slipped in between them, and in this position has been ligated with the omentum and actually cut away.

The nerve supply to the onentum is poor, but its blood vessels are large and numerous. These vessels latve very delicate walls, and as they are not surrounded by muscular fibre, there is no tendency to contract. A blcoding woin that perhaps would be of no importance elsewhere, in this structure might easily cause fatal hecmorrhage.

When omentum first protrucles in hernia it has all of its normal characteristics, but if allowed to protrucle frequently, or remain in the hernial sac, it becomes hypertrophied, indurated, nodular, and may then at times act as a foreign body if returned to the abdominal cavity. It no longer belongs within that cavity. Omentum allowed to lie in the hernial sac soon becomes adherent to its sides and forms the most common type of irreducible hernia. 


\section{CHAPTER II.}

\section{DESCENT OF THE TESTICLE.}

A knowledge of the formation and descent of the testicle affords an insight into the cause and origin of many herniæ, especially in those that occur in early life, and it has direct bearing upon some of the complications which may arise during treatment. It is important, therefore, that we give this process careful consideration.

The genital mass which is primarily formed just below the kidney and behind the peritonetm, begins its transit towards the pelvis, before it can be definitely stated whether it is to become ovary or testis. In the male this transition is not complete until it has passed through the abdominal wall to its natural abiding place in the scrotum. In the female the descent of the genital body is normally to the pelvic cavity, where it remains as the ovary. The analogy between the sexes is sometimes carried farther by the persistent effort of an ovary to descend into the labium majora.

It has occurred several times in my experience that the ovary in its descent has followed the ordinary course of the testicle, and has been found outside the external ring, in which position it is, in some instances, irreducible. Another noticeable fact is, that when the ovary has once entered the canal, it is almost as persistent in its effort to descend through it, as the testicle is under the same circumstances. Like the testicle, the ovary may become arlherent, or, from other causes, lodge at any point in the canal and not pass entirely outside the abrominal wall. It may then lead to difficult and painful menstruation, to the formation of cysts, or, as found in one case, such degeneration of its structure as to require removal.

In the elephant and some other animals, the testicles remain permanently in the abdomen, while the stallion, in some instances. has then under voluntary control, so that they may 
be found either within the abdomen or in the scroturn. In some other animals, they descend at the "rutting" season only. Instances where they are wholly, or partially, retained within the abdomen in the human subject are not uncommon. Where, however, they descend late in life or at any time after birth, they are quite certain to leave the inguinal canal in such a weakened state, that hernial protrusions are liable to follow. The testicle, in passing through the muscular wall of the abdomen, carries with it the various layers of fascia which

FIG. 7.

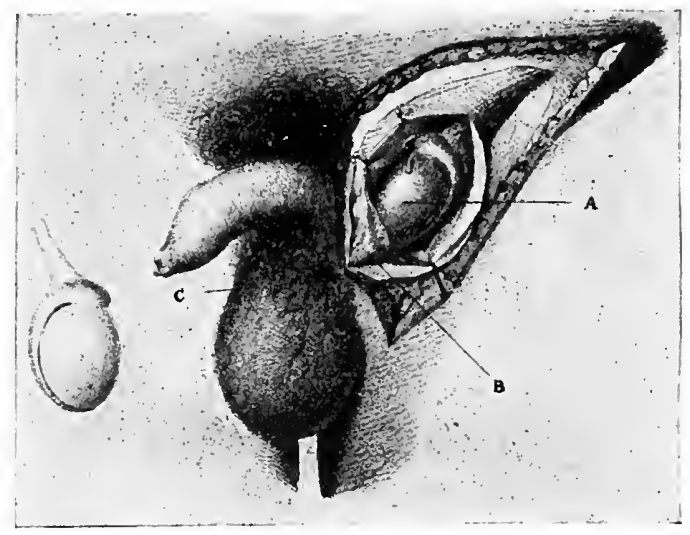

A left testis retained in the cruro-scrotal fold.-A. Testis. B, Fasciculus of gubernaculum attached to scrotal tissue at C. (Godard, from Eccles' "Imperfectly Descended Testicle.")

form the sheath of the cord; these fascial layers are only demonstrated by the most minute dissection, and are unimportant in the present consideration of the subject.

The gubernaculum testis is a bundle of fascia containing muscular fibres, which are attached to the testicle at one end. and in the scrotum at the other. At its lower end a few fibres pass off towards the thigh and some go to the perineum. It is these fibres outside the scrotum that are believed to be active in guiding the testicle into abnormal positions in some instances. The action of the gubernaculum testis is not perfecly uncier- 
stood, and it is not well known whether it aids in the descent of the testicle by a contractile force, or merely serves as a guide in a process that is carried out by some other method of development.

The accompanying illustrations (Eccles) clearly illustrate two distinct types; one (fig. 7 ) showing the testicle just

FIG. 8.

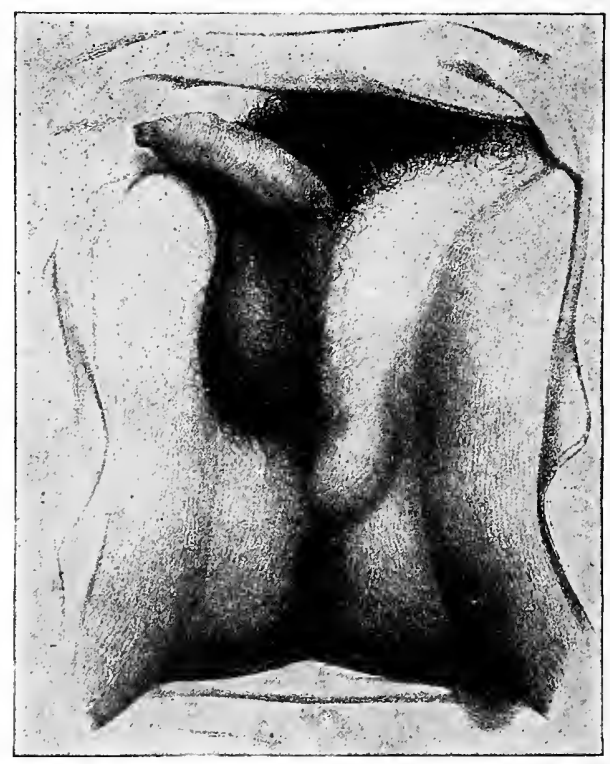

A left testis lying in the perineum.-The testis was the size of an almond, and its component parts could be readily made out through the skin. The left half of the scrotum was undeveloped. The right testis was of normal size in the right half of the scrotum. (Godard from Eccles' “ Imperfectly Descended Testicle.")

outside of the abdominal wall with the overlying tissues turned back. It also graphically shows the action of the gubernaculum testis upon the scrotal wall. The other illustration (fig. 8) shows a small testicle lodged in the perineum, and the scrotum on the left side undeveloped. Dr. W. B. Coley has called the hernia that sometimes develops with this form of mal-descent "inguino-perincal hernia." Still another posi- 
tion is shown in fig. 9, where the testicle has descender into the tissues of the thigh, presenting in Scarpa's triangle the appearance of femoral hernia. Fig. Io shows a testicle lodged just outside the external abdominal ring, with hernia in the inguinal canal. ${ }^{1}$

FIG. 9.

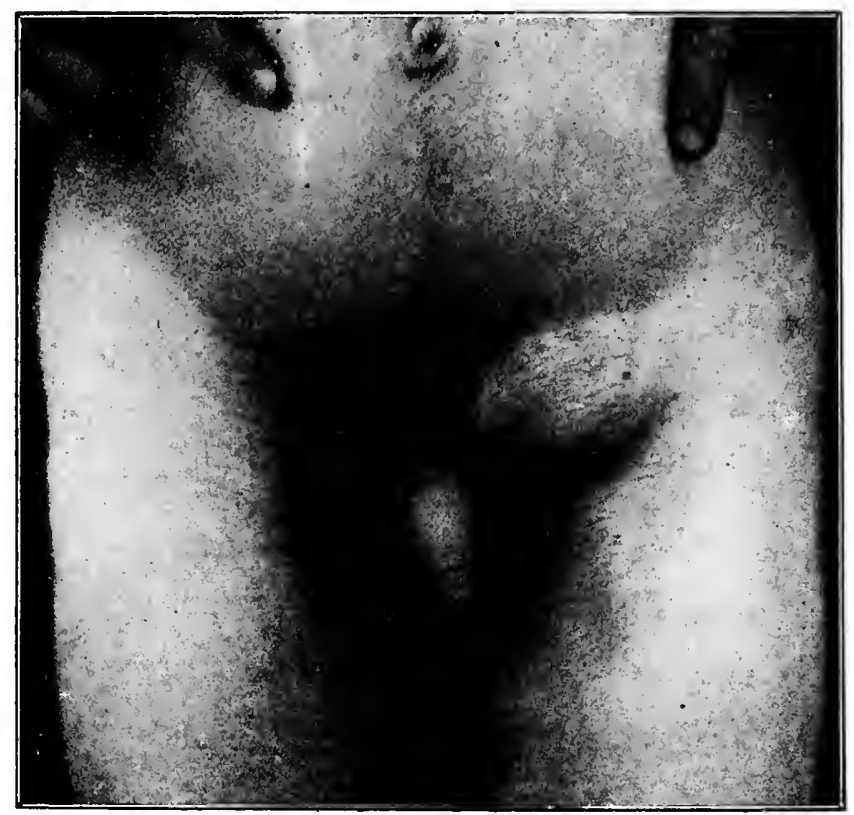

A left testis in Scarpa's triangle. Its cord could be traced through the superficial abdominal ring. (Eccles.)

The descent of the testicle may be arrested at any point within the abdomen, or it may pass into the iliac fossa and remain there. It may lodge at any point in the inguinal canal between the internal and external rings, or it may pass on to a wholly abnormal position outside the scrotum and be found in the perinemm, at the root of the penis, or into the subcutaneous

${ }^{1}$ Dr. Albert E. Halstead of Chicago reports a case where both testicles were found on one side of the scrotum. Ectopia Testis Transversa, Surgery Gynecology and Obstetrics, p. 129. February, 1907. 
tissues of the upper part of the thigh. At times, it comes out of the external ring, but turns upwards upon the surface of the aponeurosis of the external oblique muscle towards the crest of the ilium. Its normal descent may be prevented by a deficiency in the attachment, or formation, of the gubernaculum testis, by achesions, or by lack of development of the inguinal canal. The external abdominal ring may be too small to

FIG. Io.

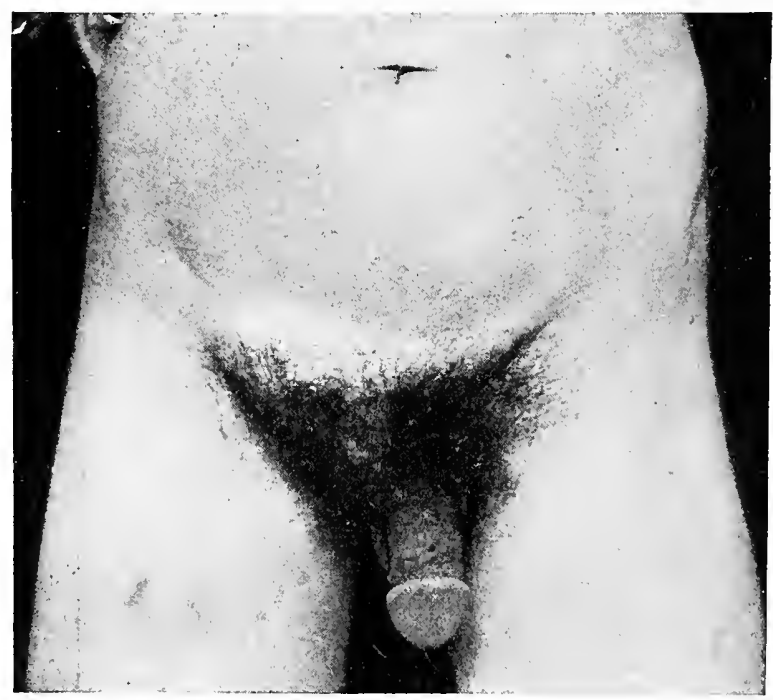

Right testicle in canal at external ring. Complete oblique inguinal hernia above it. Left testicle at internal ring. Scrotum rudimentary.

allow it to pass, or the scrotum may be so undeveloped as to prevent its entrance. It may even be retarded and its descent prevented by a truss improperly applied for associated hernia.

There is considerable difference of opinion as to whether a testicle which has been prevented from reaching its normal position, is functionless, and the preponderance of evidence would seem to inclicate that in a large number of cases it is. This has lerl eminent anthorities to arlvise their removal at the 
time of operation. Recent studies prove beyond question, however, that there is an internal secretion from these functionless testicles, which is markedly beneficial to the proper development of the individual, especially at the period of change from youth to adult life. It is, therefore, very desirable that they should be preserved in every instance, unless their diseased condition should prove a menace to the health of their possessor.

In view of the considerations just stated and the mental effects of castration, an operation will be detailed uncler its proper heading. which enables us to leave these testicles under the muscular wall of the abdomen. in cases where the cord is so short as to make it impossible to place them in the scrotum.

\section{TUNICA VAGINALIS.}

Previous to the descent of the testicle, and apparently not dependent upon the transit of that organ, a pouch of peritoneun, subsequently known as the tunica araginalis, descends into the scrotum. The neck of this pouch, which communicates with the cavity of the abdomen, should be obliterated at birth, leaving that part which is anterior to the testicle a closed sac, the cavity of the tunica vaginalis. Failure to complete the obliteration of the neck of communication between these cavities, leads to the formation of certain types of hernix, and produces several forms of complications.

This process of peritoneum should be obliterated at birth. or in early infancy, but for unexplained reasons it sometimes continues patulous until adult life. It is the remaining open, or the dilating of the partially closed neck of this pouch, that allows congenital hernia to pass down in front of the testicle. without true peritoneal covering. The normal obliteration of the neck of this process begins by three points of closure. These, it is well to remember, have special bearing upon cystic formations, which occur between them before complete obliteration has taken place. They are at the deep abdominal open- 
ing, at the superficial abdominal opening, and immediately above the testicle. That portion which lies immediately in front of the testicle forms the carity of the tunica vaginalis, where hydrocele of this membrane may occur.

The word "congenital," as applied to that type of hernia which protrudes into this cavity, has been and is confusing. It places, by inference, the date of origin of the hernia at the birth of a child, whereas, as a matter of fact, congenital hernia may

FIG. II.

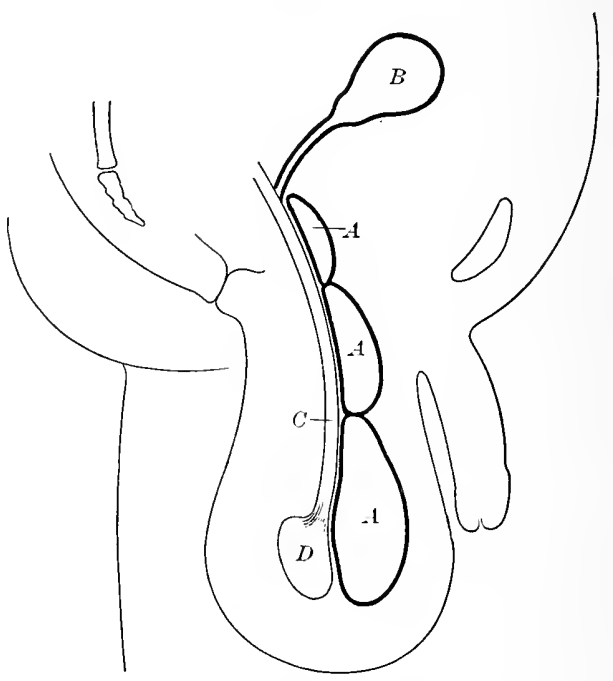

$A, A, A$, Cysts in front of cord. $B, C y$ st hanging within abdomen by pedicle three inches long. $C$, Cord. $D$, Testicle.

occur at almost any period of life, in subjects where the process of obliteration of the neck of this cavity has never taken place. It is only in recent years that anatomists have ascertained that in many subjects, in whom hernia has never occurred, there remains a small opening between the cavity of the abclomen and the cavity of the tunica vaginalis throughout life. The term congenital, therefore, refers to a defect which was present at birth, and which predisposes to a certain type of hernia. 
The drawing shown in fig. I thas been mate from one of a number of similar cases met with in operative work. It is introduced to illustrate how cysts, forming in the partially. obliterated tunica vaginalis, may prove confusing in diagnosis and troublesome during operation. A young man, of $2+$

FIG. I2.

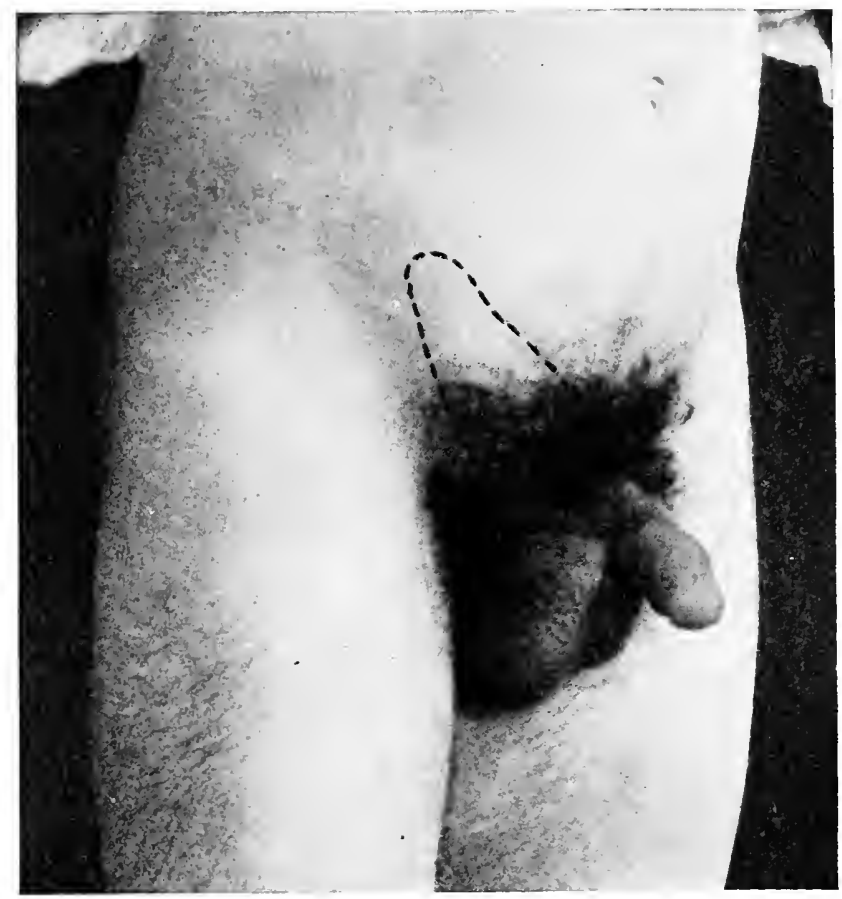

Hydrocele of cord simulating irreducible hernia. Dotted line shows tumor extending to the internal ring. Notice separation between tumor and testicle, showing that tunica vaginalis proper is not involved. Entire cyst removed by operation.

years, had experienced much trouble in wearing a truss. and upon examination it was decided that a part of his hernia was irreducible. Upon operating. a sac about three inches long was found, and back of this were three cysts, shown in the sketch, closely associated with the cord. Within the neck of the sac was noticed a cord-like attachment which, when drawn 
upon, brought from the abdominal cavity a cyst the size and shape of a small pear, hanging in the abdomen by a pedicle fully three inches long.

There is little doubt that all of these cysts formed in unobliterated portions of the tunica vaginalis, and at the upper portion of the canal, had bulged forward into the forming hernial sac, and finally dropped back into the peritoneal cavity. This occurrence was undoubtedly aicled by the truss which had been worn over the canal lower down.

Fig. I 2 shows a young man with hydrocele of the cord, extending from the top of the testicle to the internal ring. He was subsequently operated upon, the entire cyst removed, and the canal closed by the Bassini method.

\section{CANAL OF NUCK.}

The process of peritoneum under consideration, enters the canal of the female fetus the same as in the male, and while ordinarily obliterated at birth, it may persist, producing similar conditions and complications.

Nuck, in the seventeenth century, recognized this condition, and when present it bears his name.

When it remains patulous, it allows of the occurrence of congenital hernia in the female, or of congenital (reducible) hydrocele. Its presence also accounts for encysted hydrocele in the canal of the female, not uncommonly met with in operative work. It may be coincident with hernia, or exist alone, and I have long felt that it accounts for some failures to cure the female infant by truss treatment.

\section{SPERMATIC CORD.}

The cord extends from the internal abdominal ring through the canal to the testicle, and is composed of the vas deferens, three arteries with their return veins, lymphatics, and nerves. These vessels are held together and the cord formed by its sheath, which is composerl of three layers of fascia given 
off fron the exlges of the abdominal muscles as the cord follows the testicle in its descent. These are from the deepest towards the surface: (I) Infundibuliform fascia. (2) Cremasteric fascia. (3) Intercolumnar (or spermatic) fascia. The cremasteric fascia, or muscle, as it is frequently callerl, contains muscular fibre as well as fibrous tissue, and aicls in the support of the testicle. In some children and young persons, this muscle acts so violently at times, as to draw the testicle against the external abdominal ring with such force as to cause considerable pain. This action will be referred to again in connection with diagnosis, as "Retraction of the Testicle." Except the cremaster these fascize are seldom demonstrable at the time of operation.

The nerve supply to the cord is from the renal plexus and by the genital branch of the genito-crural. To the latter is clue the "cremasteric reflex" noticeable in many boys on touching suddenly the inner surface of the thigh, that surface being supplied by another branch of the same nerve.

The ras deferens, or excretory duct, lies at the inner side of the cord, especially at the upper part of the canal, and turns sharply at the internal ring to go to the base of the bladder, while the ressels continue on their course. This separation accounts for the fact, that in the protrusion of hernia the vas deferens may be on one sicle of the sac, while the ressels are on the other. In hernia the usual position of the cord is back of the sac. but it is frequently found in front of, or at either sicle of it. 


\section{CAUSE OF INGUINAL HERNIA.}

Inguinal hernia forms about three-fourths of all herniæ, and should therefore have most careful consideration. Its causes naturally arrange themselves into two groups; those that are predisposing, and those that are immediate, or direct.

\section{PREDISPOSING CAUSES.}

The following list of predisposing causes of inguinal hernia has been compiled for use in this work: (I) Heredity; (2) Age; (3) Sex; (4) Descent of processus vaginalis (male and female) ; (5) Descent of testicle; (6) Anatomical defects; (7) Fat, excess, or sudden increase of.

I. Heredity.-The result of the study of inheritance as a predisposing cause of hernia has not been very satisfactory, as it has proven little; but it has been strongly urged by some authors and doubted by others. The subject is attended by too many uncertainties and too many statistics to receive extended consideration in this work. While in my own experience I have had some striking indications that the tendency to hernia is occasionally transmitted from one generation to another, I have arrived at no conclusion as to what that tendency may consist of.

Sir Astley Cooper attempted over a century ago to show that it was due to the shape of the bony pelvis, and others have attributed it to an inherited laxness of muscle, which proves inefficient in the retention of the abdominal contents. The theory of an inherited weakness of the abdominal muscles appeals to me, and appears to be sustained by experience. In one family, the members of which have been under my care for twenty-five years, three different generations have been cared for, and the three principal forms of abdominal hernia (in- 
guinal, femoral, and umbilical) have been represented in their ordinary proportion. The cases are largely among the female members of the family, who have every appearance of good health and strong muscles. No member of this family would consent to operation, so that opportunity has not been afforded to confirm the belief that muscular deficiency is the cause of the inheritance.

2. Age.-The extremes of life are undoubtedly more productive of hernia than the intervening period, and for discernible reasons. In early infancy we have not only the short canal, the internal ring being directly back of the external ring at birth, but we have the defects due to mal-descent of the testicle and lack of obliteration of the tunica vaginalis.

Lockwood $^{1}$ has also demonstrated that in early life the mesentery is longer than in the years of maturity, being onefifth the length of the body. At forty years of age it is only one-ninth the length of the body. In old age we have the degeneration of tissue so common to advancing years, and the change in form well shown in fig. $1_{3}$. when the stooping forward and relaxation of the abdominal muscles places them at great disadvantage. It has been stated that this form of abdomen is the result of large herniæ, but to my mind it is more frequently a cause of hernia.

3. Sex.-The influence of sex upon the incidence of inguinal hernia, is clearly shown by the large preponderance of this type of hernia among males. This difference is probably due to the large size of the spermatic cord as compared with the round ligament in the female, to the descent of the testicle through the inguinal canal, and its lodgements and detentions. The descent of the tunica vaginalis also plays an important part in the increase of hernize among males. It is true that this same process occupies the canal of the female, and, as the canal of Nuck, aids in the production of hernia, but not to the same extent as in the male.

\footnotetext{
${ }^{1}$ Pathology and Treatment of Hernia. C. B. Lockwood, F.R.C.S., London, 1889 .
} 
4. Descent of Tunica Vaginalis.-The failure of complete obliteration of the connecting neck, between the cavity of the tunica vaginalis and the cavity of the abdomen, beyond doubt leads to the formation of many inguinal hernix. This opening may exist for years without the protrusion of hernia, but usually, where it is present, it only needs unusual strain to fill the already formed sac.

FIG. I.3.

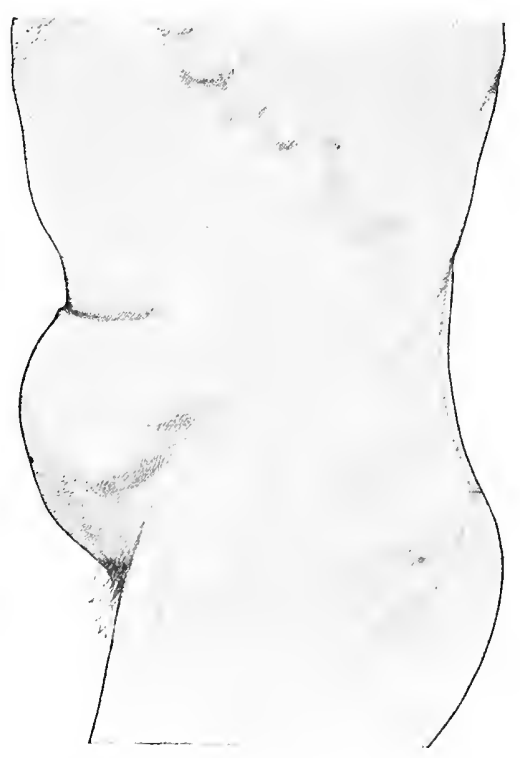

Side view of the abdomen of an old man with scrotal hernia. To show the flattening above the umbilicus. (Macready.)

5. Descent of the Testicle.-The formation and descent of the testicle, through the ablominal wall, is believed to have such an important place in the production of inguinal hernia, in the male, that this transit has alrearly been carefully consiclered in the chapter levoterl to this subject.

6. Anatomical Defects.-There are certain anatomical clefects in the muscular formation, about the inguinal canal, which 
unquestionably lead to a very large number of hernice, yet they have not attracted specific attention until recent years, when frequent operations have demonstrated them to every extensive operator upon hernia.

The lower borders of the internal oblique and transversalis muscles, passing over the cord as the latter enters the canal at the internal ring, run parallel with it and find insertion into the pubic bone. In front, the canal is protecterl by a dense, fibrous layer, the aponeurosis of the external oblique muscle, but back of the cord there is little protection against protrusion, except that afforded by the peritonem and the fascia transversalis. This muscular defect allows of deep pocketing from the peritoneal surface into the hypogastric fossa, and constitutes a strong predisposing cause of hernia. In the olcler operations this weakness was not considered, and all repair work was done at the external ring, leaving the original defect to invite a recurrence of hernia.

Another anatomical defect which is a frequent cause of hernia, especially in early life, is the nonobliteration of the communicating neck, between the cavity of the tunica vaginalis and the cavity of the abdomen. That portion of the pouch of peritoneum which descends in front of the testicle, becoming the tunica vaginalis, should be closed off from the abdomen by the obliteration of its neck, which passes through the inguinal canal. This stage of development should have been reached at, or before, birth, but unfortunately it fails to occur in a large number of persons, and the peritoneal surface remains continuous down into this pouch in the scrotum, inviting a protrusion through the moist, tube-like opening.

Probably numerous persons have this condition throughout life without developing hernia, but in many others it is an important predisposing cause. When a hermia occurs in this pouch, it is known as congenital hernia: the term not meaning that hernia was necessarily present at birth, but that the defect was, which led to its occurrence. IVe see numerous cases of congenital hernia occurring at all ages up to adult life, 
showing that this tubular neck of communication, between the two cavities, has persisted during all of the previous years that the patient has lived, and needed only some additional cause to produce the hernia.

The two anatomical peculiarities named are considered by far the strongest predisposing causes, the first existing to a

FIG. I4.

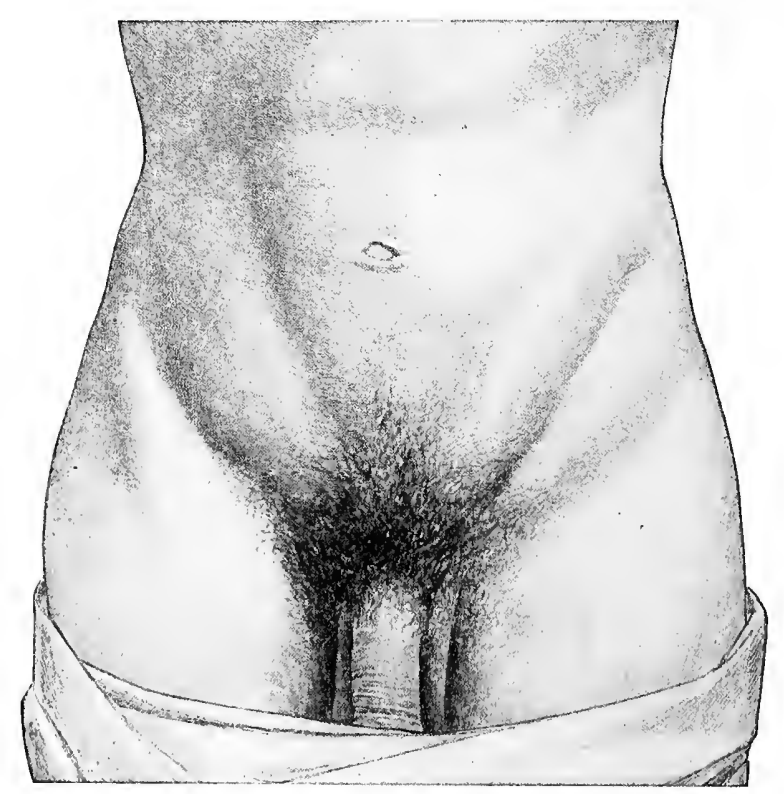

Abdomen of a male, age 39 , with early inguinal hernia. To show the lateral bulgings often present in hernial subjects. (Macready.)

greater or lesser degree throughout life in nearly all people, and the second being particularly active before puberty.

There is a form of abdomen which some authors think is strongly predisposed to hernia, which was first described by Malgaigne as "Triple Bulging;" and by Velpeau it was classed as ventral hernia. It is fairly well illustrated in fig. I4. The median bulge is due apparently to the strong contraction of 
the recti muscles, diminishing the antero-posterior dianeter of the abdomen, and causing the inguinal region to bulge on either side. Some of these cases seem due to excessive strength in the recti, rather than to any special weakness in the side muscles of the abdomen. I have watched some of them for years, where the condition was marked, without seeing hernia (levelop.

7. Fat.-That fat, either in excess, or suddenly accuirerl, is productive of inguinal hemia, is abundantly proven. It acts in several ways: (a) By increasing intra-abdominal pressure: (b) by slipping into the canal, the point of least resistance under violent muscular exertion; $(c)$ by the formation of subperitoneal lipona, which may descend through the canal, dragging with it a process of peritonem which then becomes hernial sac; $(d)$ fat accuired by excessive beer drinking, has been found particularly productive of hernia, by a two-fold action. First, in the accumulation of fat: second, in causing fatty clegeneration of muscular tissue. This condition makes these patients poor subjects for the curative operation, increasing the danger and diminishing the chances of permanent cure.

Abnormally weak and premature children are especially prone to the occurrence of hernia in the early months of life, and debility from old age, or other causes, frequently leads to it. Those suffering from pulmonary tuberculosis are particularly susceptible, as they not only have the relaxation of tissue, but also have the cough as a direct cause.

The theories suggested in the early studies of the causation of hernia seem rather unimportant in view of recent experience. Most writers have sided with either those who have assigned the predisposing cause of hernia to purely mechanical reasons, or with those who clamed that it was due to pathological changes. Those holding to the mechanical causation, adhere to the belief that relaxation of the abdominal muscles is the primary cause, while the "pathologists" believe that in the abnormal lengthening of the mesentery we may find the first change which results in abdominal hernia. 
Anatomists have given the normal length of the mesentery, from its vertebral attachment to its intestinal border, as from six to nine inches. It is quite evident, therefore, that in many of the hernize of large size, the elongation of this attachment must be considerable. Experience during the past decade, with an operation that corrects a mechanical defect in the abdominal wall, and cures permanently a large percentage of these hernix, of enormous size with lengthened mesenteries. seems to prove conclusively that the primary cause it not in the length of the mesentery.

The problem of curing the defect having been solved, it is not in accord with the purposes of this work to further consider theoretical causes.

\section{IMMEDIATE OR DIRECT CAUSES.}

The immediate causes of inguinal hernia are too numerous to allow of naming each one. Any violent muscular compression of the abdominal contents may act as a direct cause of hernia.

The following is a partial list and will, perhaps, be suggestive of many others not enumerated: ( 1 ) Constipation ; (2) Vomiting; (3) Cough; (4) Lifting; (5) Shouting; (6) Posture: $(7)$ Obstructions to urination; (8) Crying in children : (9) Acites.

r. Constipation.-After many years of observation I do not hesitate to place constipation at the hear of the list. The history of an attack of obstinate constipation immediately precerling the discovery of a swelling in the groin is surprisingly common, and at once suggests an important part of the subsequent treatment of the case. In chronic constipation the cause is more effective in that there is longer continued repetition of the straining at stool and greater distention of the bowel.

2. Vomiting.-Straining during this act is frequently an inmerliate cause in an individual otherwise predisposed to hernia. 
3. Cough.-Violent and persistent coughing, as in whonping cough, chronic bronchitis, and phthisis, are actire causes, as may also be an elongated uvula with resultant violent cough.

4. Lifting.-This is a common direct cause of inguinal hernia, but no more so to the laborer than to the merchant or editor. The muscles of the working man are trained to their work and when he makes even a heary lift, they protect him. It is when he is caught suddenly, unprepared, that harm comes. The merchant or clerk is maccustomed to heary work, but upon occasion takes hold "with the boys" and his muscles not trained to withstand the strain, the effort frequently results in hernia.

A former patient was in charge of the gymnasium in one of the larger colleges and was accustomed to lead in all of the exercises which he taught, and felt that he could exceerl, in strength tests, any of the younger men. He gave this occupation up to become the editor of a daily paper, and in this position was closely confined to his desk. It was a mystery to him why, after he had abandoned very active and extreme exercise, that he should develop hernia. The mystery was explainerl when it was discovered that he had in his home a pair of extra heavy dumb-bells, his pets of former days, with which he exercised at rare intervals.

5. Shouting.-The vigorous calling out of wares in the open streets, as clone by hucksters, pellers, and street fakirs, is quite liable to bring on hernia, and it is very hard to treat them successfully by mechanical means or cure them by operation as long as they continue their occupation.

6. Posture.-There are certain positions of the borly which favor the occurrence of hernia, i.c., any muscular effort. while the abdominal muscles are relaxed, as in stooping forward, or while the arms are extended over the head. Golf has furnished a fairly large number of hernixe in men past midcle life. The schools that teach so-called "Physical Culture by Correspondence" aid many midclle-agerl men in prodlucing hernia. The reason that I name particularly this class of 
schools, is to point out the fact that the fault lies more in the orer-ambition of the pupil, than in the lack of knowledge on the part of the teacher. I feel warranted in illustrating here an exercise that has brought under my notice from 6 to Io cases of hernia a year (fig. I 5). This consists in throwing the body back as far as possible, with the hands above the head,

FIG. I5.

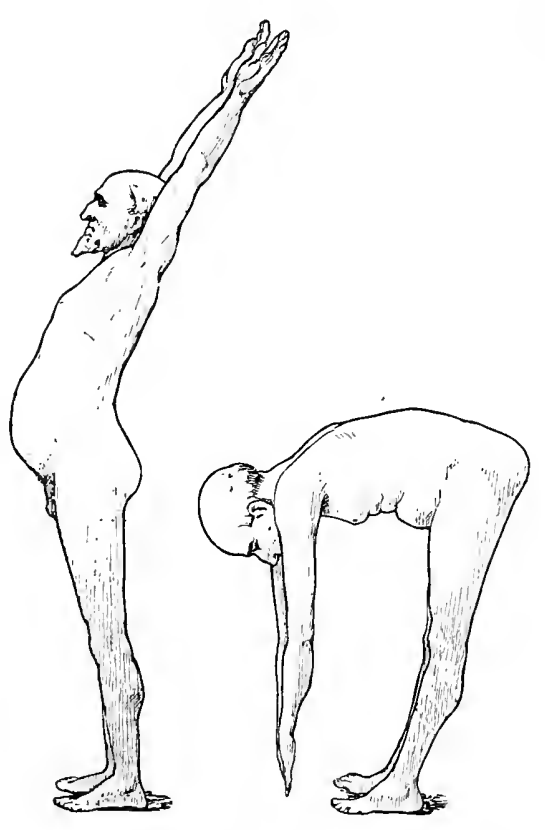

$A$ form of "Physical Culture" that produces many herniæ in men past middle age.

and then stooping forward until the finger tips rest upon the floor. This one exercise had apparently been the cause in these cases; at least this is the only one form that all had taken. I am not a disbeliever in physical exercise, even for those who have hernia as I shall demonstrate later, but I do think that those past the muscle building age should not resort to it except under strict personal supervision of an inst ructor. 
7. Urinary Obstructions.-These urinary disturbances, either as stricture, calculi, or prostatic enlargenent, may easily lead to the development of hernia. Men who have prostatic trouble are especially liable to it, both on account of the straining in attenpted urination, and to the fact that this trouble usually occurs at a time of life when the tissues are not in condition to withstand this frequently repeated strain.

8. Crying.-In infancy and early childhood there is no doubt that crying has an inportant place. The infant is strongly predisposed to hernia by its short canal and developmental imperfections; ackl to these constipation and crying, and we have two very active direct causes.

One other cause that I clo not see as frequently as in my earlier years of experience, is the belly-band that "grandmother" said "must be very tight or the baby could not be expected to thrive." By having the belly-band tight the intestines are driven into the lower abdomen and extreme pressure made upon the delicate tissues, especially when the child cries.

Crying may not only act as an immediate cause, but it is very sure to aid in increasing its size rapidly, when hernia is once formed. When the infant cries, and the bowel is forced into the canal, causing pain, this in turn causes the child to cry more.

9. Acites.-An effusion of fluid into the abclominal cavity is sometimes the origin of inguinal hernia, but for obvious reasons such cases are not common.

In adclition to the above enumerated causes, phimosis has been mentioned by eminent writers as a direct cause of hernia, otherwise it would not be referred to here. If the foreskin is so long or the opening in it so small as to cause the child to strain when he urinates, then we have a reasonable cause of hemia, otherwise not. Very rarely has such a case been seen; furthermore, in the Jewish orphan asylums where all of the male children have been circumcised in early infancy the usual proportion of hernia exists. 
CHAPTER IV.

\section{TYPES AND CONDITIONS OF INGUINAL HERNIA.}

In early writings upon the subject, and even in many of more recent date, the divisions and subdivisions of inguinal hernia have been so numerous and its nomenclature so prolific, that much unnecessary confusion has been added to an already complex subject. Accepting risk of criticism, I have endeavored to simplify this division to the extreme limit, and to use only those terms, so far as possible, which carry with them their own meaning.

The necessary divisions for the proper consideration of inguinal hernia are: Oblique, direct, sigmoid or cacal, and intcrstitial hernia. Fig. I6 shows two oblique and one direct hernia in the same patient.

The oblique type may either be of the congenital, or of the acquircd form. In dcgree of development the congenital is nearly always a scrotal (or labial) hernia, as when it passes the inguinal canal it drops into a preformed sac. Acquired hernia, on the contrary, may be, according to its degree of development, either incomplete, complete, or scrotal (or labial) in character. Direct inguinal hernia seldom becomes scrotal in character even though it attains large proportions.

Sigmoid or Cæcal hernia belongs to the direct hernia type, but on account of its peculiar anatomical characteristics must be considered under a special heading. This statement holds equally true of interstitial hernia, except that it may belong either to the acquired or congenital type, but very rarely to direct hernia.

Oblique inguinal hernia is so called because the protrusion enters the inguinal canal at the internal abdominal ring and passes obliquely through the abdominal wall. If it protrucles into the canal only, it is then called incomplete in- 
guinal hernia (see fig. 17). If, however, it passes out of the inguinal canal through the external abdominal ring, then it becomes complete oblicute inguinal hernia (see fig. I 8 , right side). If these small herniae are neglected or unskillfully cared for, they become by progressive development scrotal hernia in the male (figs. 18 and 19), or labial hernia in the female

FIG. 16.

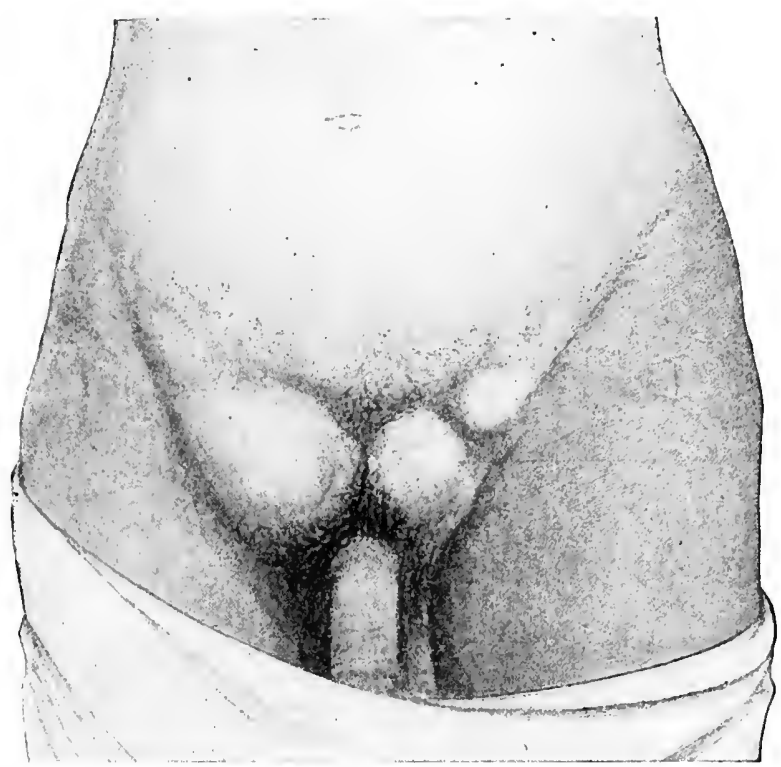

A male, age 79, having an oblique inguinal hernia in an early stage, and a direct hernia on the left side, and on the right side an oblique hernia more advanced than that on the left. (Macready.)

(fig. 20). The terms " scrotal " and "labial " indicate a stage of growth and should not be used to designate types of hernia. Some truss manufacturers make what they call a "scrotalhernia truss," which is based upon the idea that it is for a special type of hernia, and it is only mentioned here because the author knows that some misapprehension exists in the minds of many practitioners. 
Oblique inguinal hernia may protrude into a preformed sac, present at birth (from lack of obliteration of the tunica vaginalis), in which case it forms a congenital hernia. This protrusion may occur after the person has reached adult life, even though the defect has existed during all of the preceding years, but by far the greater number of congenital herniæ do occur in infancy or early childhood. When we speak of con-

FIG. I7.

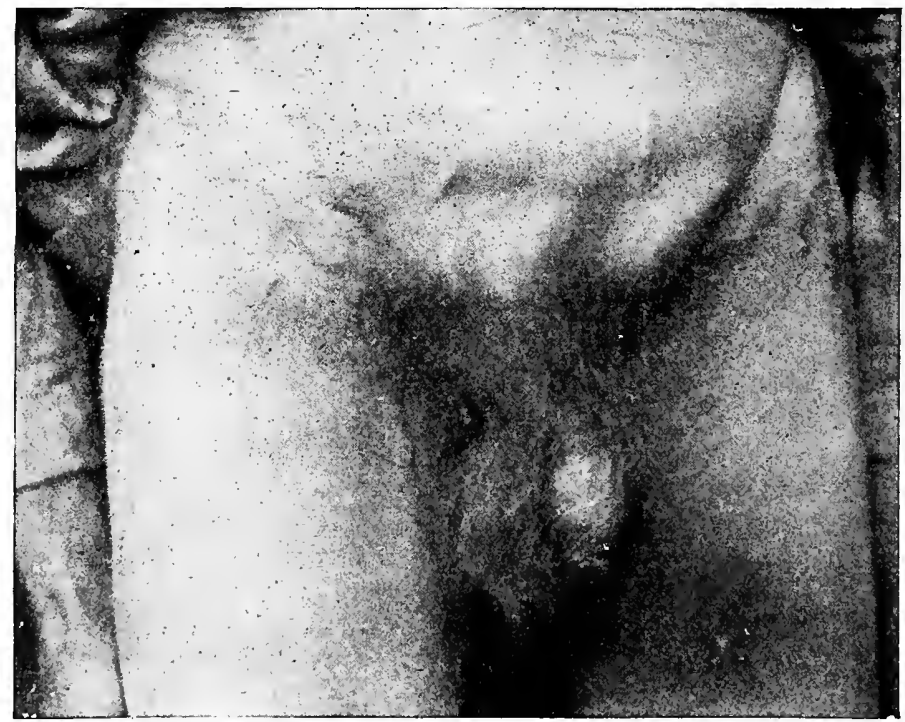

incomplete inguinai nernia on left. Direct inguinal hernia on right, poorly shown. (Eccles.)

genital hernia, therefore, it does not indicate at what age such hernia may have developed, but does clearly mean that the hernia has come down into a sac already formed.

Acquired inguinal hernia refers to a condition wherein the protruding mass carries with it, as a sac covering, the peritoneal lining of the abdomen. This peritoneal pouch is known as the hernial sac and, in the course of time, loses some of its characteristics as peritoneum by becoming thickened and otherwise changed in structure. It continues, however, as a moist, 
serous mebrane, and as such farors the protrusion of the abdominal contents.

Direct inguinal hernia does not enter the upper part of the inguinal canal at the internal ring, but protrucles directly through the external abdominal ring and presents a tumor

FIG. I8.

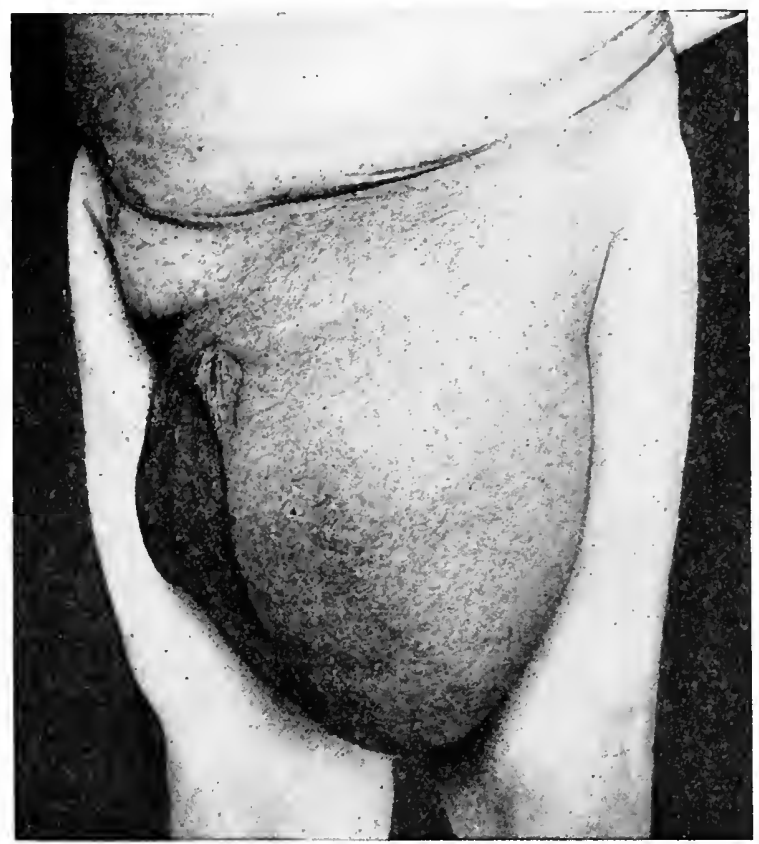

Showing on the right side a complete oblique inguinal hernia. On the left an enormous oblique inguinal (scrotal) hernia. Circumference, 32 inches; within two inches of knee-joint.

circular in form that, even though it is large, has little tendency to descend into the scrotum (fig. 2I). A tumor as large as a cocoanut has been seen standing out from the body over the pubic bone. Tumors of this type should be approached by the operator with care, as they are liable to contain the fundus of the bladder, or they may prove to be sigmoid hernia as shown 
in fig. 22. The deep epigastric artery passes across the inguinal canal just below the internal abclominal ring, and oblique hernia passes over it in going down the canal. Direct inguinal hernia, on the contrary, passes through the abdominal wall inside of the artery, towards the median line. This is usually

FIG. I9.

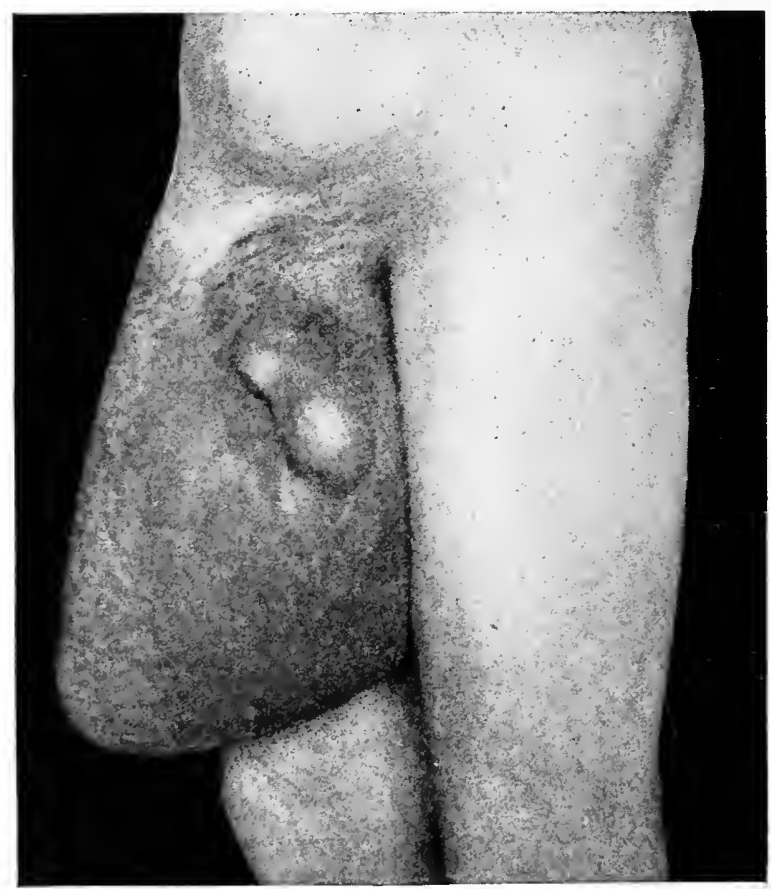

Showing the enormous size which scrotal herniæ may attain by neglect. Right oblique inguinal hernia (scrotal) in a man over 70 years of age.

so, but in some persons with abnormally short canals the artery runs lower down, and while actually the protrusion is oblique, it has every appearance of a direct hernia. It must be remembered therefore in operating, that the deep epigastric artery may be found at either sicle of the neck of the hernial sac. 
Herniae of the direct type are not met with as frequently in women as in men, and the reasons for this are shown in the recent studies of Marie Donati (Archicio per le Slicnac Mcdischc, No. 3, I905; Abstract Mcdical Record. July 8. 1905). He believes that "the smaller number of the clirect inguinal type that we find in women, is due to the different formation of the inguinal canal in the two sexes, and to estab-

FIG. 20.

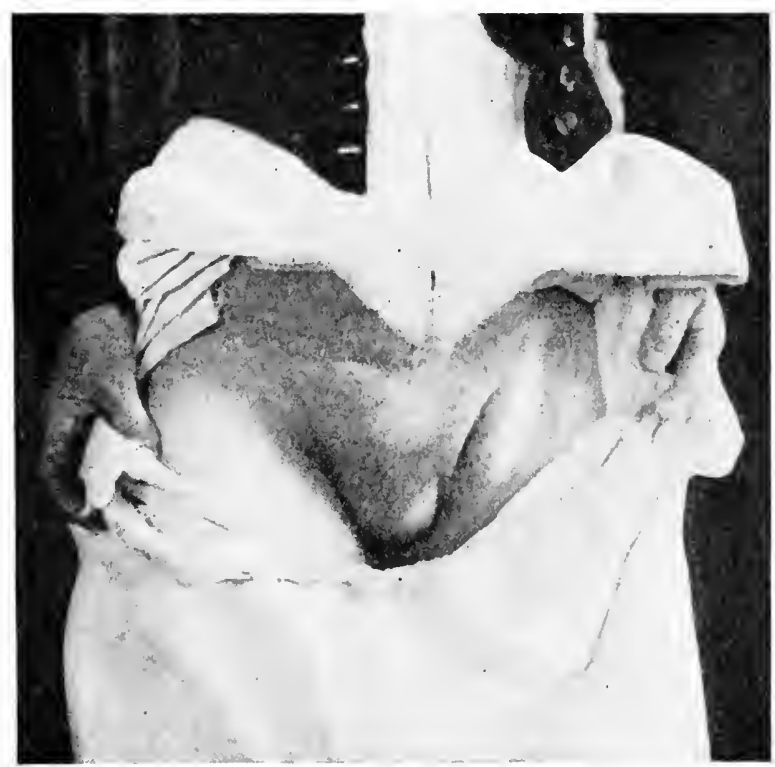

Showing oblique inguinal (labial) hernia in a woman of 45 years.

lish this conclusion he has dissected 52 carlavers of both sexes, $3 \mathrm{I}$ women, $2 \mathrm{I}$ men, and has found marked differences in them. The aponeurosis of the insertion of the great oblique muscles has linear interstices, which are much larger in the male than in the female. The pillars of the internal oblique are stronger in front of the canal in women. The arciform fibres (inter-crural) are in women often mingled with cross fibres which strengthen the ring, which are absent in men. The 
orifice of the external inguinal ring is generally smaller in females than in males. It is generally situated higher and a little more external in men than in women, contrary to what has been supposed to be the case. The aponeurosis of the large oblique, which forms the anterior wall of the canal, is much stronger in the female sex than in the male. The lower margin of the ring in women is more horizontal, that in the

FIG. 2I.

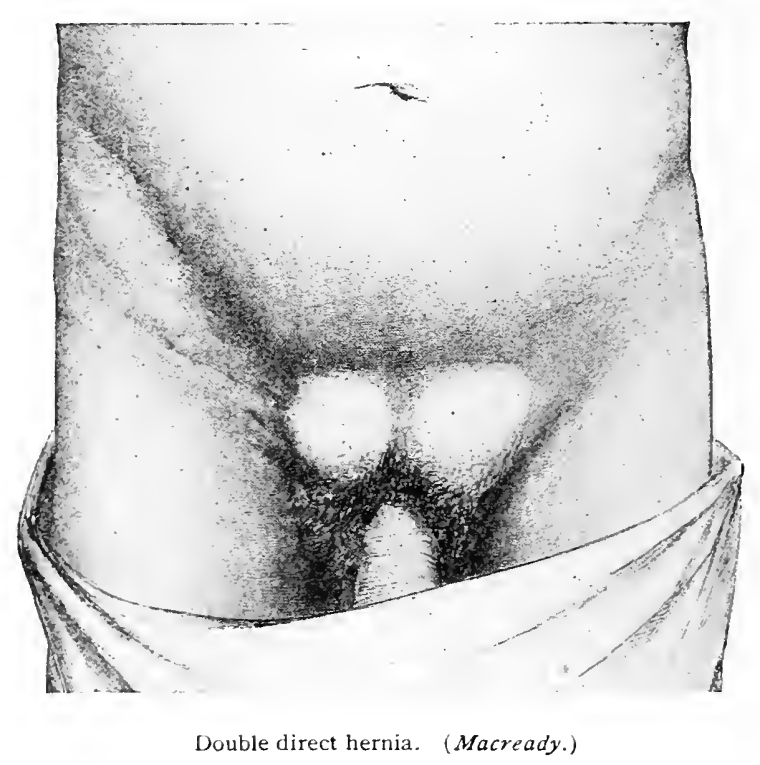

male more oblique. Hence this point, which is often weak in the male, is in the female most resistant."

Sigmoid hernia, the beginning of which is usually that of a rlirect inguinal hernial protrusion, has certain peculiarities in its development which make it essential that it should have a rlistinctive classification, and none of the terms applied to it seems so rlescriptive as the one here userl.

It will be remembered that the peritoneum not only lines the cavity of the abdomen, but envelops most of the hollow 
organs, forming back of them the mesentery which gires them partial support. In the case of the sigmoirl flexure, the caecum, and the bladcler, this covering is only partial. The sigmoirl flexure is covered in front and on the sides, but a portion of a back part of the bowel is in clirect contact with the posterior wall of the pelvis (iliac fossa). When hernia occurs to this

FIG. 22.

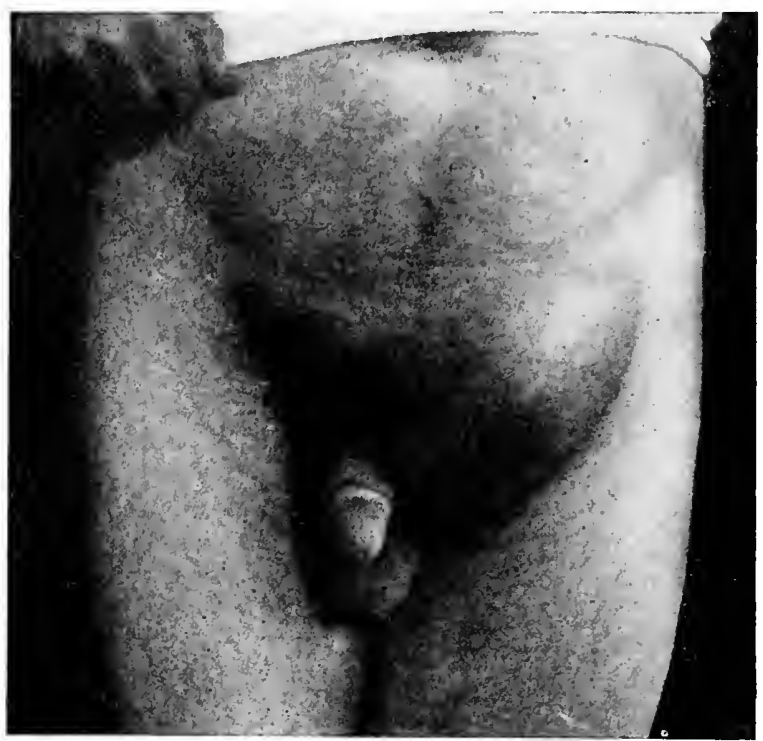

Sigmoid hernia. Peritoneal covering in front of intestine, none at its back. Loops of small intestine protruding in front of sigmoid flexure.

part of the large bowel it pushes through the abdominal wall. carrying the portion of peritoneum that is in front as a peritoneal pocket into which other parts of intestine may protrucle. The posterior intestinal wall, as it protrudes, usually precedes the part covered by peritoneum, forming the most prominent part of the tumor, and is entirely devoid of peritoneal covering. If the operator does not anticipate this condition, and opens low down on the tumor he will find that he has made an 
incision directly into the large bowel. If, however, he be so fortunate as to open high up on its anterior surface, he will then have opened into the sac which is in communication with the peritoneal carity, recognize the true condition, and thus prevent an embarrassing situation.

FIG. 23.

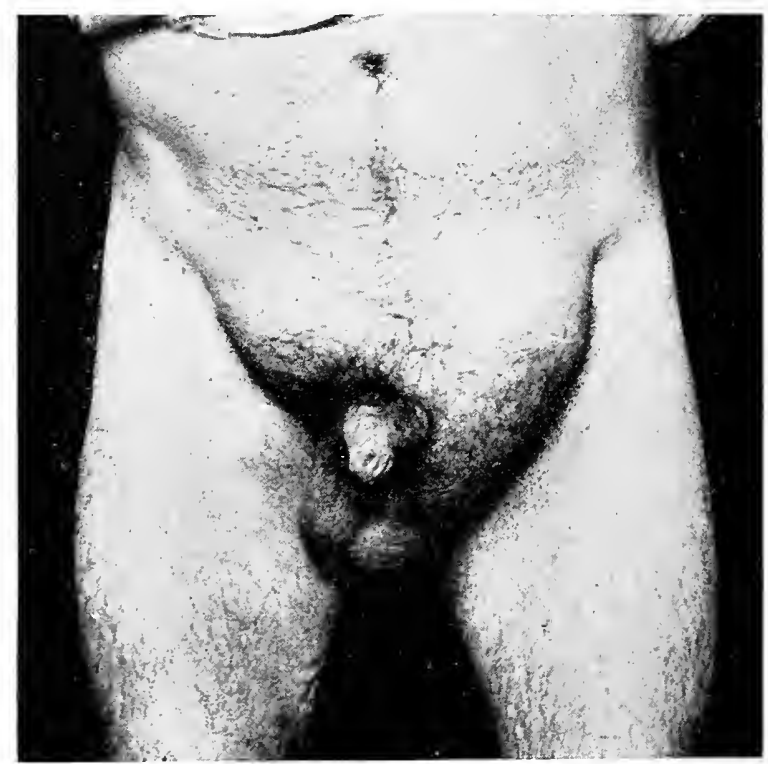

Direct inguinal hernia on the right; sigmoid hernia on the left. The illustration is typical of the peculiar shape of sigmoid protrusions.

In treating of diagnosis, suggestions will be made that may airl in recognizing this condition before operation, but this can not always be clone. It is fortunate that I am able to present the photograph of such a typical case as that shown in fig 23. On the right sicle an ordinary direct inguinal hernia appears; on the left sicle can be seen what prover at operation to be a hernia of the sigmoid flexure. Its size has pushed it down to the top of the scrotum, but it is not a true scrotal hernia. 
Cæcal (inguinal) hernia does not assume the imprortance of the sigmoicl type. both on account of its greater rarity and from the fact that the conditions presented by it are not as liable to learl to disastrous results. The free ciecum and the appendix vermiformis are not uncommon occupants of a true hernial sac, but these are not termed caecal hernia. It is only

FIG. 24.

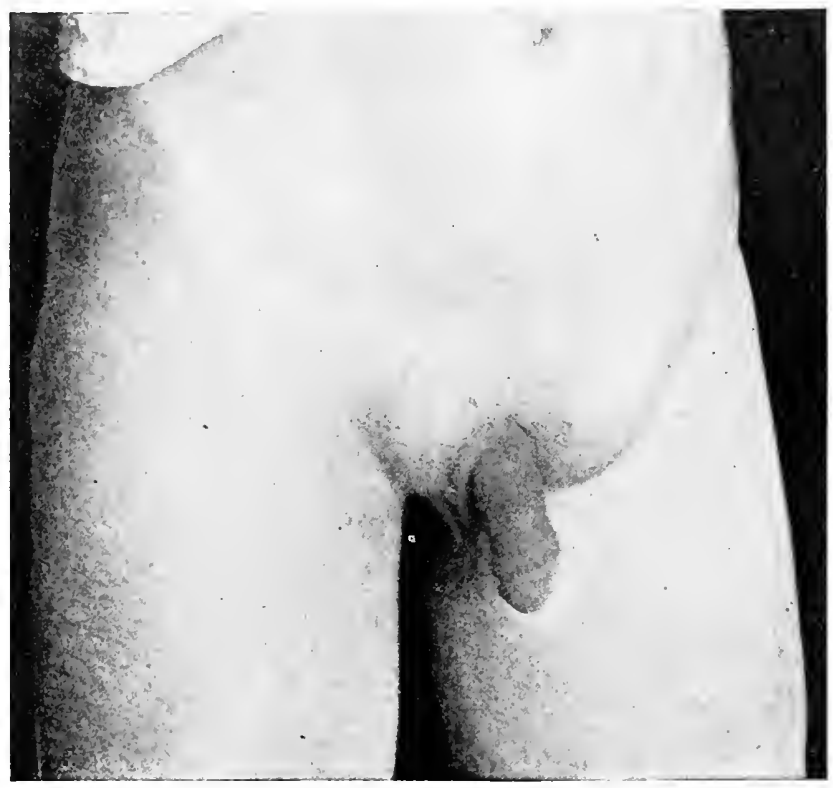

Left interstitial hernia caused by delayed descent of testicle. Right testicle is inside of external abdominal ring.

when the colon slicles down so that the portion not covered by peritoneum forms a part of the protrusion that it is given this title.

Interstitial inguinal hernia. Included under this title will be consiclered all of those hernize that have been variously termed "properitoneal." " interparietal," " bubonocele rara." "superficial inguinal," and "interstitial." Any hemia that 
protrucles between the layers of muscle, or tissue, which constitute the abdominal wall at this point, will be termed interstitial hernia. Such hernire usually begin as oblique inguinal hernia and, in their passage down the canal, meet with some obstruction which turns them to some point of less resistance. Ordinarily these diverticula of the hernial sac are small, but

FIG. 25.

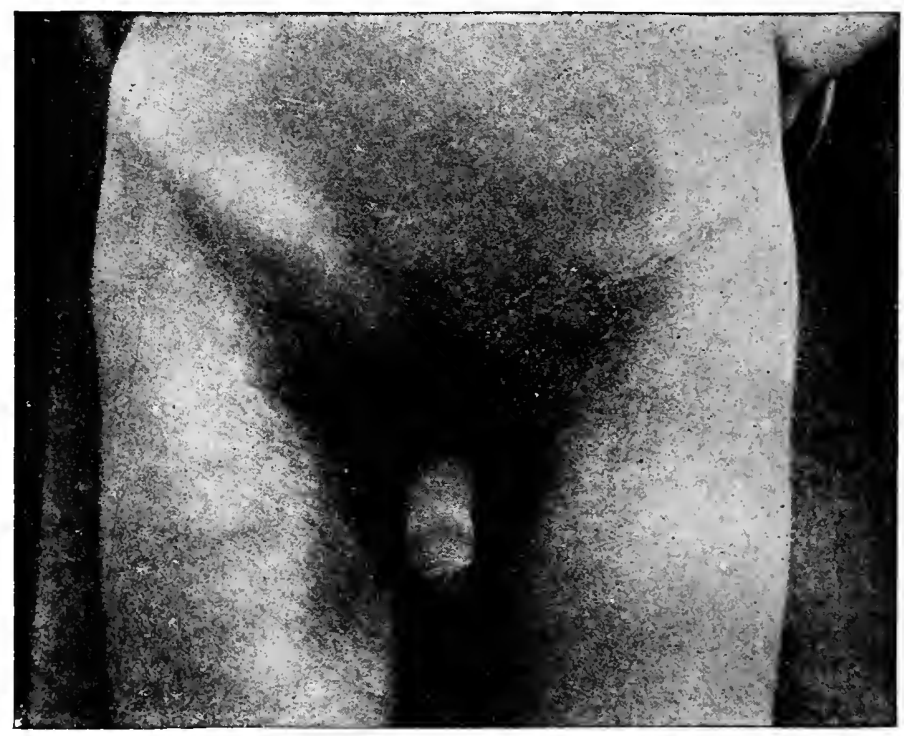

A right interstitial hernia, associated with the right testis in the canal. (Eccles.)

sometimes they reach proportions quite in excess of the size of the true sac.

Interstitial hernia, in the male, is very frequently associaterl with the imperfect descent of the testicle as shown in fig. 24, but a similar condition is not infrequently met with in the female. I have seen two such hernize in the female that were associaterl with an ovary contained in the same sac, showing a probalble formation identical with the imperfectly descended testicle in the male. The ovary had first entered the 
canal followed by the hernia, which, being obstructed in its downward course, crowded itself between the abdominal layers. These protrusions may be between any of the layers of the abdominal wall, but will be found most frequently in the order named: ( I) Beneath the aponeurosis of the external oblique, and between this structure and the internal oblique. Such a

FIG. 26.

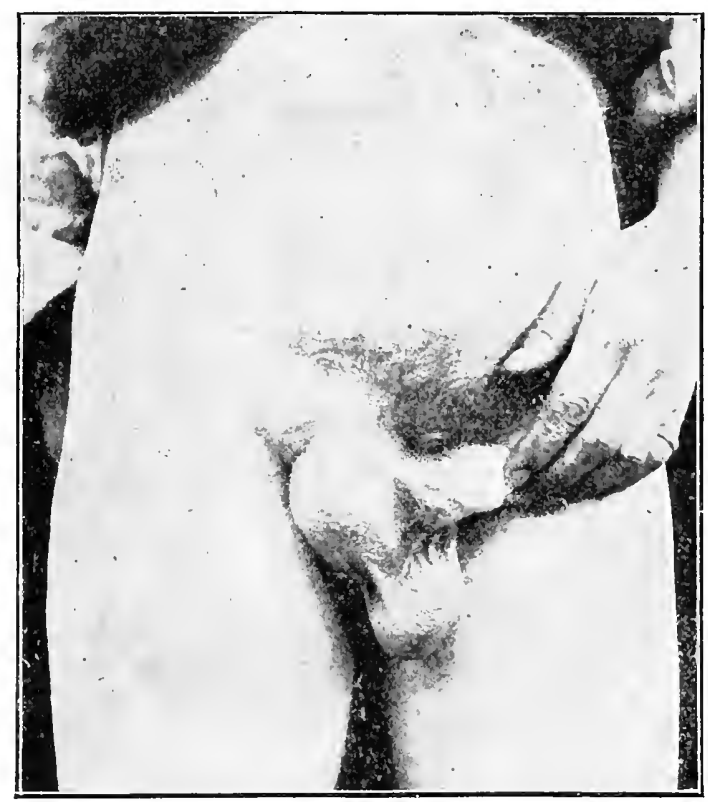

The right testis in a cruro-scrotal pouch, accompanied by a hernia. (Eccles.)

formation is shown in fig. 25 , as well as in the one just referred to. (2) Into the subperitoneal fat between the peritoneum and the transversalis fascia. (3) Between the skin and external oblique muscle, or into the tissues of the thigh as shown in fig. 26.

In formation the first type most frequently develops towards the iliac crest. The form that passes under the fascia transversalis more frequently forms a pocket towards the 
median line, while that forming immediately beneath the skin may go in almost any direction. A hernia of the latter type is shown in fig. 27, which has dissected up the subcutaneous tissues of the thigh and simulates femoral hernia (fig. 28). Not only may these herniæ develop, from obstructions within the canal, but some that I have operated upon have been the

FIG. 27.

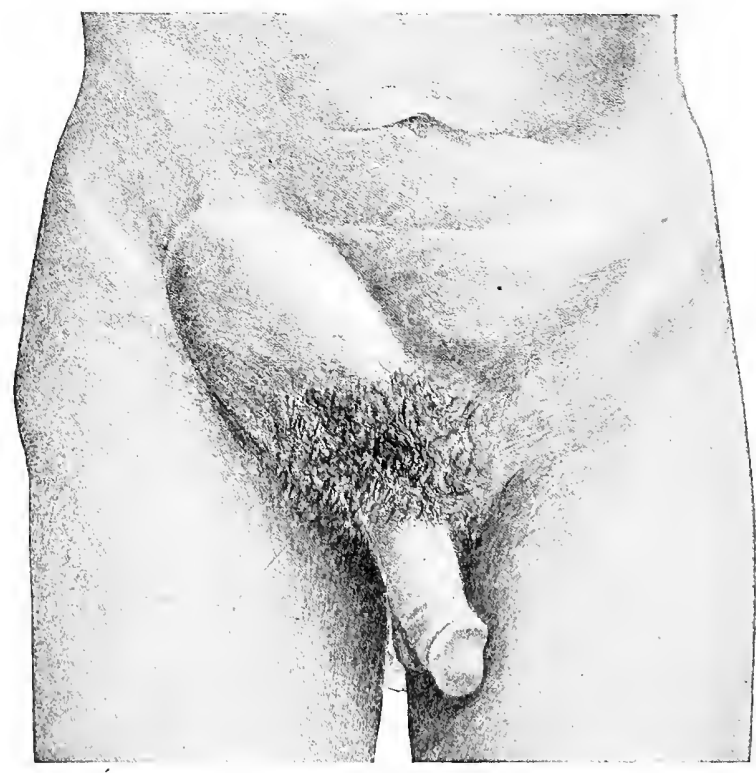

Interstitial hernia. The right half of the scrotum is absent and the testis, which is very small, lies within the canal near the external ring. The external oblique is in front of the hernia. From a man, age 5o. (Macready.)

result of trusses improperly applied. The truss pad had obstructed the passage of the hernia through the external abdoninal ring, but had not kept it out of the canal. The sac had, therefore, enlarged laterally where there was less resistance.

Interstitial hernia that forms between the peritoneum and transversalis fascia is believed to be the most dangerous type of 
this class, owing to its greater liability to become strangulated and to the obscure location of the exact place of strangulation. This was strongly impressed upon me several years ago while operating upon a woman seventy-five years old, for a strangulated inguinal hernia. On opening into an ordinary inguinal

FIG. 28.

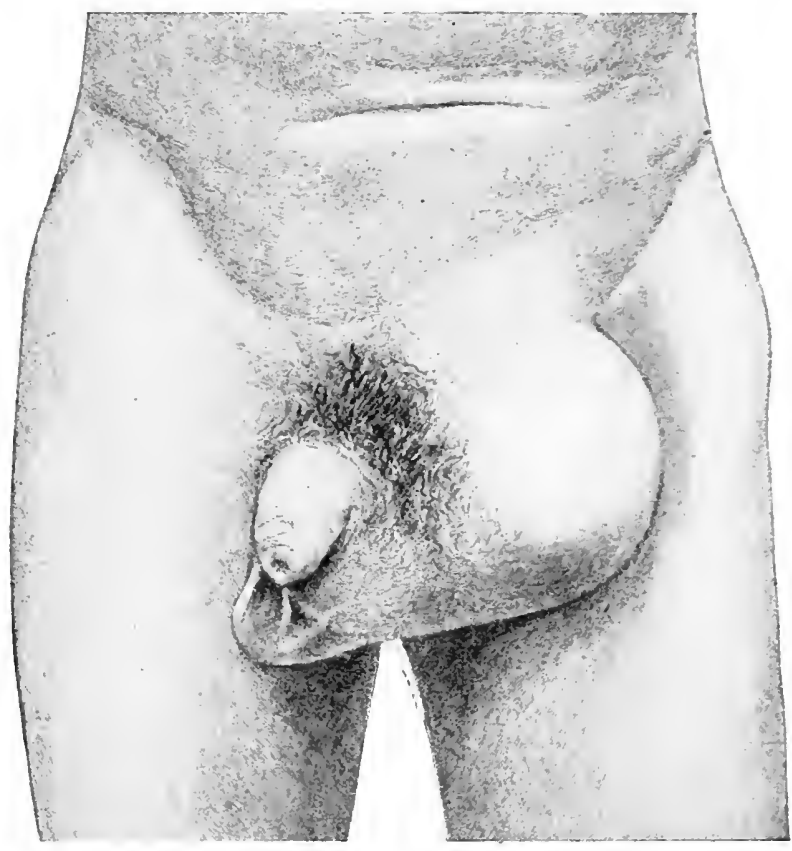

Interstitial hernia, falling over the thigh and simulating femoral hernia; in a man, age 56. (Macready.)

sac it was found to contain small intestine. in fairly normal condition, which was reduced without much difficulty. A tumor was then discovered nearer the median line, which was found to be an interstitial sac just outsicle of the peritoneum. and it was in the neck of this sac that strangulation existed. The form of the sac is roughly shown in the following illustration (fig. 29). 
Conditions. - The conditions in which inguinal hernia may be found, at the first examination, are as follows: Reducible, irreducible, incarcerated, inflancd, or strangulated.

Reduciblc inguinal hernia is where, irrespective of its size, its contents are wholly reducible to the cavity of the abdomen. Such hernixe are uncomplicated and can usually be retained within the abdomen by truss pressure. In infants this method of treatment may result in a permanent cure, but in adult life this fortunate result is rarely obtained. The truss ordi-

FIG. 29.

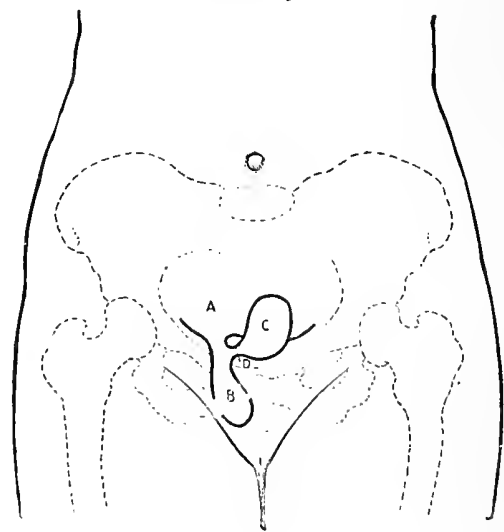

1. Axlominal cavity. B, Sac in unguinal canal. C, Interstitial sac. D, Neck of interstitial sac, where strangulation existed.

narily guards against strangulated hernia, providing its design and fitting are suited to the requirements of the case.

frreducible hernia may occur from one of many causes. (a) I very large mass of the abdominal viscera, either intestine or omentum, may protrude gradually through a small neck into the sac, and, when attempts at reduction are made, give the casce the appearance of an irreducible hernia. Such a case, by perfect rest in bed and repeated gentle taxis, would be readily converted into a reducible hernia and could then be treated as strch. (b) Umentum that is allower to remain in a hernial sac, is under pressure at its neck, and this partial obstruction to 
the return flow of blood may cause an enlargement or nypertrophy of the omental mass. The omentum becomes hard and nodular and without undue violence it cannot be forced back through the small neck of the sac. Even when this can be clone, it is attended by much danger, the hypertrophied omentum acting as a foreign body within the abdominal cavity.

In my early experience with hernia, when operations were attended by more risk and the ultimate result far from satisfactory, while I had no actual mortality, I was more than once concerned regarding the welfare of my patient, upon whom I had succeeded in reducing what appeared to be an irreducible hernia. In the present day, work of this type is seldom justifiable, as the skillful surgical care of the case is probably attended by much less risk and by an incomparably greater degree of success. (c) The reduction of hernia may be prevented by adhesions of the protruding parts to the sides of the hernial sac. These acthesions most commonly occur between omentum and the sac wall. Adhesions between protruding intestine and the sac wall are more rare than those involving the omentum, because of the peristaltic action of the bowel. Intestine, even when packed into a sac in large quantities, must continue this action in order to carry forward its contents, and this constant motion undoubtedly frequently prevents adhesions. Bowel in this abnormal position, however, becomes constantly less active and is still further crippled by adhesion to the sac wall. If only a small area is adherent the bowel still performs its function, but as a greater amount becomes disabled obstinate constipation is followed by intestinal obstruction, and then, even the art and science of surgery is powerless to afford more than temporary relief. In such cases, when operation for strangulated hernia is resorted to, the patient for a few days may appear to have been rescued from death, but symptoms of intestinal obstruction gradually reappear and death follows. The operation releases the bowel from imprisonment, and at that time it may appear to be in fairly good condition, but its paralysis has been complete and there is no hope of recovery. 
Incarcorated hernia is a form which, though ordinarily reducible, has for some cause become temporarily irreducible. There is no strangulation of tissue, however, and no symptoms of intestinal obstruction, hence the term as here used is not intended to convey the idea of strangulation. (These two conditions are frequently confused in the medical mind.) If the protruding contents of the sac are intestine, the bowel may have become twisted in such a manner that for the time being its return is prevented, but the pressure upon the bowel is not sufficient to produce stasis, either of its blood supply or its contents. When the protruding contents are mostly, or wholly, omentum, the cause of irreducibility may be identical with that just named. An incarcerated hernia is not a condition of immediate danger, but may become so in two different ways. If intestine is involved, acute intestinal obstruction may result at any moment, while if omentum forms the bulk of the tumor. it may become inflamed, adding to the danger.

Inflamed hernia is a term that should be limited to those cases of omental protrusion where this structure has become inflamed, which condition is most frequently brought on by over-violent attempts, either by the patient or his medical attendant, to reduce a mass of protruding omentum. The bowel, if also protruding, usually comes down into the sac back of the omentum and returns to the abdominal cavity upon the patient assuming a recumbent position. If the bowel is prevented from returning, the case is quite sure to result in strangulated hernia.

Strangulatcd hernia is a type in which the intestine is usually the part involved, although we may meet with cases where it is the omentum, or, as most commonly occurs, both intestine and omentum are present. As this accident will be fully considered under a special heading, it will not be necessary to treat of it here further than to say that it usually presents a picture of acute intestinal obstruction with its accompanying intense physical suffering. 


\section{THE HERNIAL SAC.}

Formation.-In order to comprehend more clearly the conditions in which we may find hernia, some consideration must be given to the formation and development of hernial sac. It usually begins by the bulging forward of the peritoneum into one of the hypogastric fossæ. If the hernia is to be of the direct type, it is into the fossa between the epigastric artery and the median line; if oblique, into the external hypogastric fossa outside of the epigastric artery. This bulging may occur under some unusual strain, and the elasticity of the peritoneum is stuch that it will, perhaps many times, recover its nomal smooth surface, but when this undue stretching is repeated too frequently, or too violently, a pocket is formed in the canal lined with peritoneum. From that moment the peritoneal lining of this pocket becomes the hernial sac, and it is with its increase in size and change in character that we have to deal.

It is highly probable that a sac in the early period of its formation may be reducible, as well as its contents, but as a matter of fact we do not find it so upon the operating table, even though the case be one of very recent origin. Doubtless very shortly after its protrusion, it forms firm adhesion to the surrounding tissues. Its subsequent development, dependent somewhat upon the treatment of the hernia, is one of growth both in size and thickness of the tissue of which it is composed. An old and large hernial sac has lost entirely the characteristics of peritoneum. The thickening of its structure may be uniform or it may become thickened in some parts while it remains thin in others, but it is no longer peritonemm, nor is it necessary to treat it as such. Not only has it ceased to belong to the abdominal cavity, but it has become a foreign body in the canal, and a cure can seldom be effected until it is completely eradicated. 
There are certain changes in structure that $I$ have met with frequently in operating, that have been little spoken of in works on hernia, and still they seem to me important because they frequently are the immediate cause of strangulated hernia. I refer to fibrous, tough, inelastic rings, that form not only in many old sacs, but in some of rather recent origin. These rings are fairly well shown in fig. 30 taken from a man of thirty-five years, who had a right scrotal hernia for ten years.

FIG. 30.

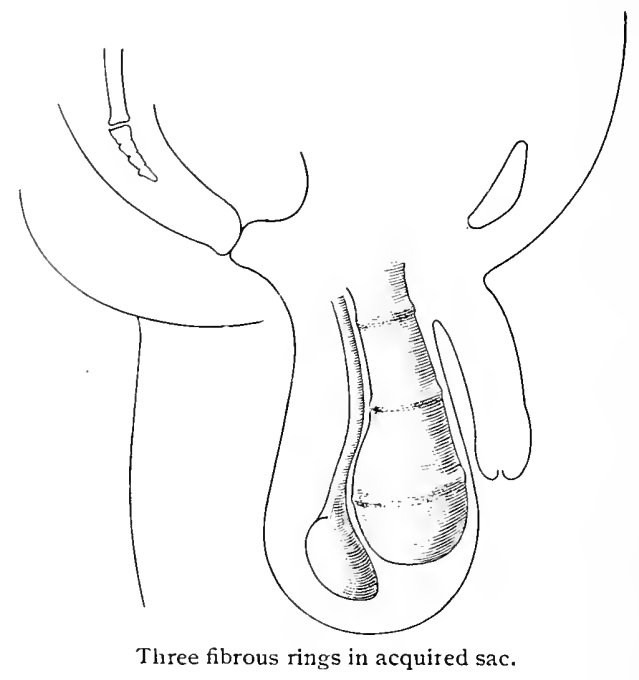

When I first began to meet with these rings I supposed that they were confined to congenital sacs and represented the original points of closure, or obliteration, of the tunica vaginalis, but further observation has convinced me that they are more frequent in sacs of the acquired form. These rings are so tough that if a loop of bowel is forcibly driven into one of them, strangulation is quite sure to result. They usually surround the entire sac, but sometimes only a portion of it, the balance of the sac being thin. From one to four may be found in the same sac, located at any point from the vicinity of the 
testicle to the internal ring. In some instances they have narrowed the sac to a complete closure as shown at the lower ring in fig. 3I. In this case intestine and bowel were found strangulated in the upper ring while the pocket below the lower ring was filled with fluid. The sac was unquestionably of the acquired type and was removed entire from the scrotum. There was no communication between the upper and lower cavities.

FIG. 3I.

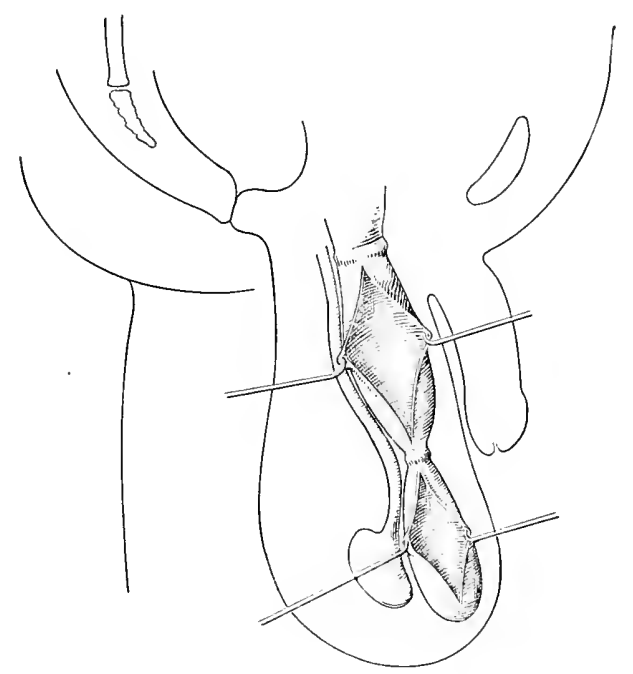

Showing two fibrous rings in sac. Strangulation of intestine and omentum was present in upper one. Lower cavity contained fluid only.

Fig. 32 shows a similar ring, in a congenital sac, in which was incarcerated (not strangulated) an omental protrusion. This was in a boy, ten years of age, who had been under treatment for some time and who was wearing a truss. Considerable thickening was felt in the scrotum, but the neck of omentum, connecting with the abdomen, was small, and the true condition was not recognized until the operation. In this work will be found an illustration of an appendix, the end of which was incarcerated in a ring near the bottom of the scrotum. 
Congenital Sac.-It has been already stated that the congenital sac is present at birth because nature, for some unknown reason, has failed to carry to completion one of the processes of development. This applies as well to the inguinal canal of the female, but the condition with her is unquestionably more rare. It is not intended to repeat here what has been said in connection with the descent of the testicle and the formation of the tunica vaginalis, but to call attention to a form of sac where

FIG. 32.

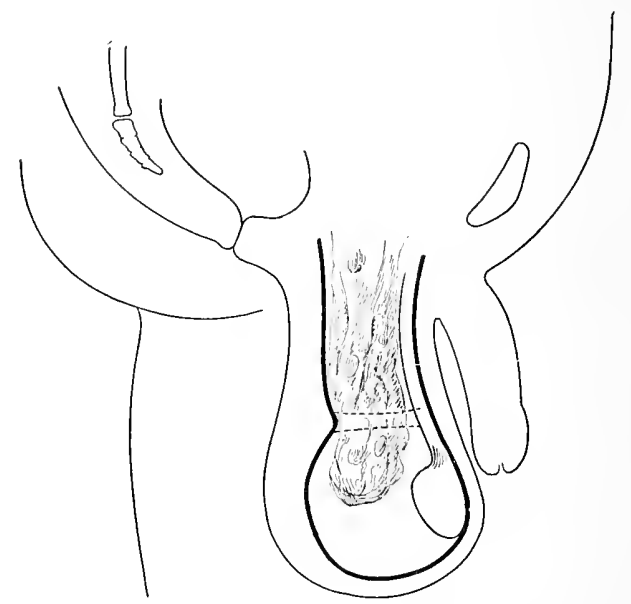

Boy, to years of age, with omentum incarcerated by ring in congenital sac.

the hernia is in the funicular portion of the unobliterated tunica vaginalis (fig. 33 ). In these cases is found what appears to be a true hernial sac, and beneath this may be found an empty serous-lined cavity, or a cyst filled with fluid as shown in fig. 34 . This sketch was made from a case as found in a young man, about twenty years old, who gave a history of hernia existing only one or two years. The tninica raginalis had completely closed, above the testicle, and probably had partially closed at the internal ring. When hernia occurred the upper closure was dilated and the hernia then dropped at once to the top of 
the testicle. Shortly after this the cyst cleveloperl in front of the testicle. The closure of the tunica raginalis may be complete at the internal ring, but nowhere else, leaving a capacious, serous-lined sac in front of the cord and testicle. Behind this may descend a new sac forming what has been termed infuntilc hernia ( fig. 35).

FIG. 33 .

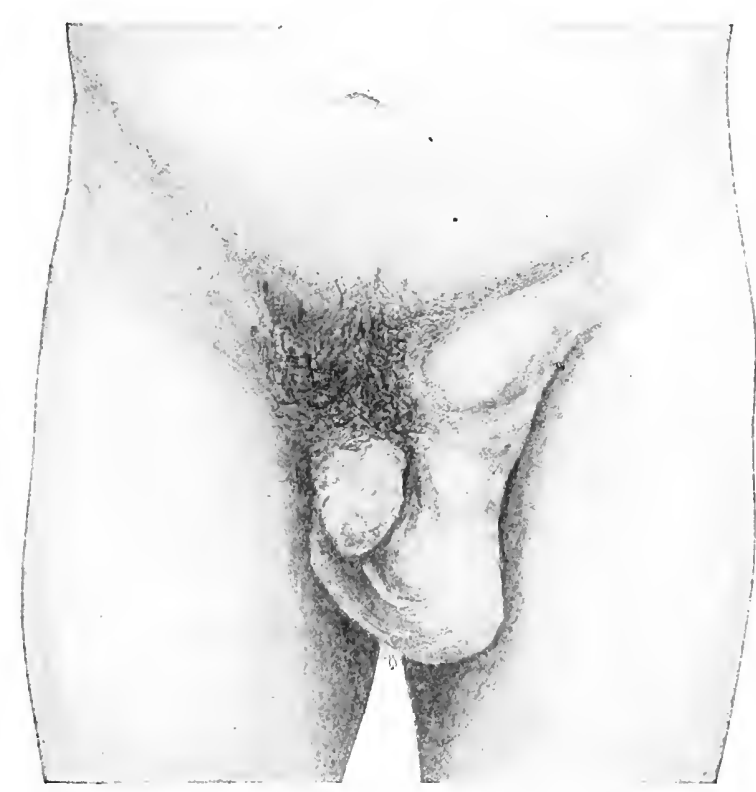

Hernia in the funicular portion of the tunica vaginalis. (Macready.)

It is especially important to keep this complication in mind as, on opening the tunica vaginalis, it might easily be mistaken for the hernial sac and the latter overlooked entirely. One should always assure himself that the serous sac, into which he has openerl, has communication with the abdominal cavity.

These serous-lined cavities are sometimes very perplexing, when found in unusual situations, and are not easily accounted for. In one of my own cases, a man 40 years old. I found such a cavity entirely disconnected, with the cord or true hernial sac 
at the inner side of the cord and above the pubic bone, in a position that at first led me to believe I had opened the bladder wall. The most careful investigation failed to demonstrate communication with any other cavity. It was a serous-lined cavity capable of containing about one ounce of fluid, empty, and was removerl from the subperitoneal fat in which it seemed imberlded. The testicle was in its normal position in the scrotum. It is quite possible that these cysts may sometimes

\section{FIG. 34.}

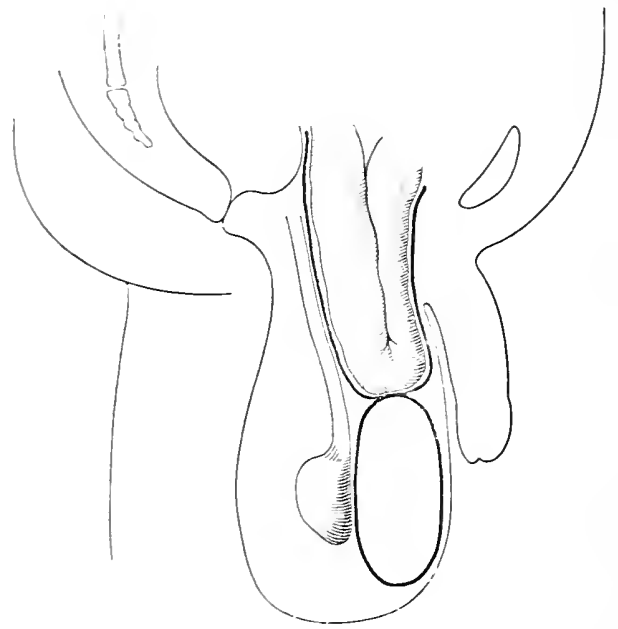

Hernia into the funicular portion of the tunica vaginalis, with cyst below. Age, 20 years.

arise from a hernial sac that has become occluded at its neck from truss pressure or other causes. I have found similar conditions in at least two femoral hernire.

In the case shown in fig. 36 the shape of the sac resembled that of a large mitten, and the diverticula corresponding with the thumb was full of small intestine. A sac may vary in size from that of a hickrory-nut to an enormous pouch, reaching to the knee-joint. I have removed a number of sacs large enough to have slipped completely over the patient's hearl. A sac may form in a lateral rlirection if its rlescent is olstructed, or, if 
already formed and its contents are prevented from entering it freely, it may expand between the fascial layers of the aldominal wall, forming interstitial hernia (fig. 37). Several illustrations of this have been seen, where the condition was considered due to the wearing of strong truss pressure upon the pubic bone, instead of retaining the hernia within the internal ring.

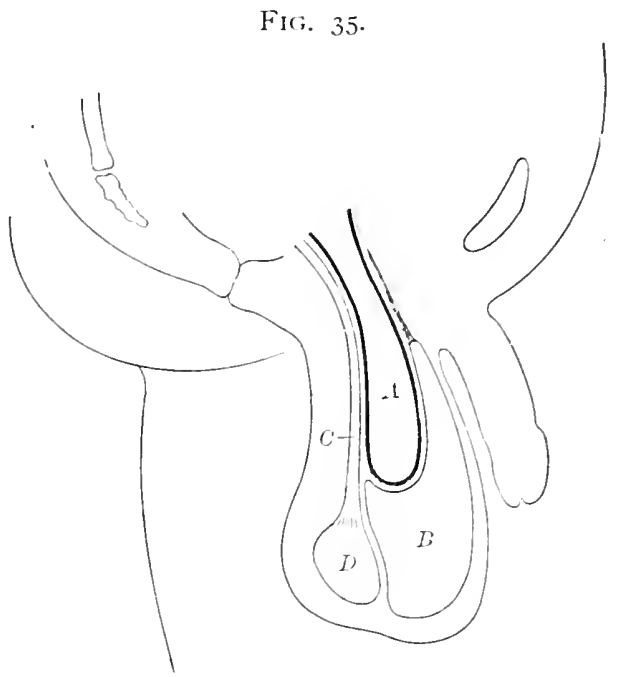

Infantile sac. A, True hernial sac. 1, Tunica raginalis, closed sac. C, Cord. D, Testicle.

Coverings of the Sac.-A classical description of the formation and coverings might be as follows: First, the pocketing into the upper part of the canal of the peritoneum. which eventually becomes the sac: second, as this pushes forward it is covered by the transversalis fascia; third, as it passes down through the canal, the cremaster muscle; fourth, as it protrudes from the external abdoninal ring it takes with it the intercolumnar fascia; fifth, the superficial fascia : and sixth, the skin. The minute clescription and enumeration of the corerings of the hernial sac have led to much timidity on the part of the occasional operator, or with the physician who is forced into 
an operation as an urgent life-saring measure. If he refers to his works on anatomy, his confusion becomes worse by the very exact and exhaustive accounts, properly given, of these unimportant fascial layers.

Again let us follow the operator's knife and see these tissues as we see them at the operating table. If the hernia is direct, or complete oblique, and we cut down upon the sac, we divide the skin and its underlying fatty tissue (superficial

FiG. 36.

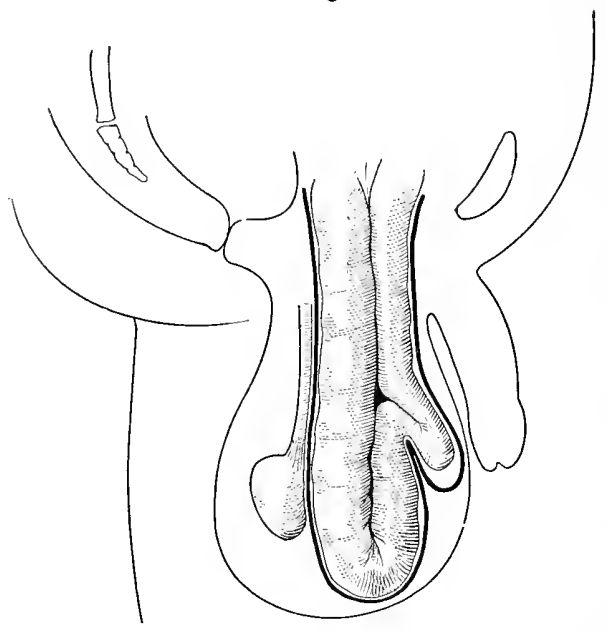

Showing sac shaped like a large mitten.

fascia), and at once see the sac bulging up into the wound. It is true that this sac is covered by a layer of fascia, containing more or less muscular fibre and loose cellular tissue, so blended together as to make individual identification impossible. It is easily cleared from the sac surface by blunt dissection. Frequently it is so thin as to allow the sac to show plainly through it. It is composed of the transversalis fascia and the cremaster muscle, and possibly the intercolumnar fascia. If we are dealing with an incomplete oblique inguinal hernia, we will not see the sac until we have split the aponeurosis of the external 
oblicute. In the accompanying diagrammatic sketch (fig. 38 ) I have attempted to show the relative thickness of the coverings of the hernial sac, and a glance at that will inclicate at once what a small part these two or three layers of fascia form.

In the shape, formation, and direction in which the hernia may develop, these fasciæ not infrequently play an important part, but I am now speaking from a purely operative standpoint and the exaggerated idea of their importance in the mind of the young man, and the medical man who at times must, in justice to his patient, do surgical work and do it promptly.

FIG. 37 .

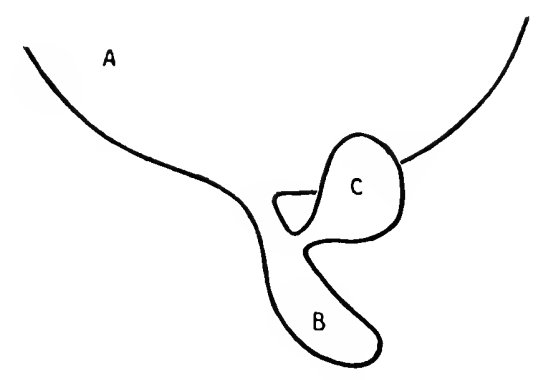

Formation of an interstitial sac. A, Peritoneal cavity. B, True hernial sac. C, Interstitial sac.

Only those who have taught hernia work to the practitioner can fully realize how often competent men hesitate to do the right thing at the right moment, because of their confused ideas of the anatomy of the parts, and consequently their excessive dread of approaching them surgically. It is to such men that I am trying to make this subject more clear; not encouraging incompetent men to do surgical work; far from it, but trying to lighten the burden of the conscientious practitioner who is striving to do his best as a conservator of human life.

Contents of Sac.-As this work is not historical it cannot enter very fully into an account of all the many unexpected things that have been found in the hernial sac. As has been 
previously stated all of the movable organs of the interior of the abdomen are found in it. On account of its longer mesentery the small intestine is most frequently met with. Next in frequency, if not fully equalling it, is the omentum. The large intestine may have a long enough mesentery to allow it to freely occupy the hernial sac. The appendix and crecum are many times found in right side hernix, and while it has not occurred in my own experience, my associate, Dr. George E. Doty, has found an appendix in a left-side inguinal hernia, showing the extreme lengthening of the mesentery in some

FIG. 38.

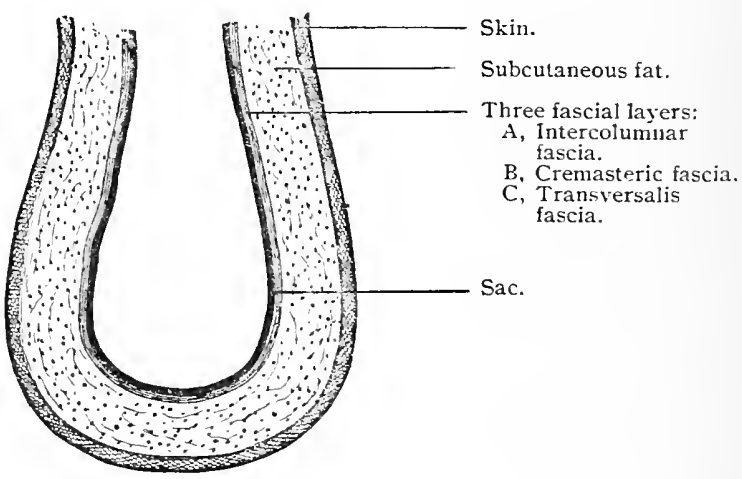

Showing relative thickness of tissues covering complete inguinal sac.

people. The ovary and tubes are often found, and the uterus, pregnant or otherwise, has been recorded as found in a number of cases. Is early as i6ıo, Trautmann did a Cresarean section upon an incarcerated uterts and succeeded in saving both the mother and child.

The stomach, liver, gall-bladler, spleen, pancreas, and kidney, have all been reported as found in inguinal sacs, as well as concretions of various shapes and sizes. In the case shown in the photograph, fig. Is, I found at the time of the operation a perfectly round ball the size of a ping-pong ball, free in the sac. It was white and had a smooth exterior. It could be 
easily cut, however, and doubtless originated from a picce of omentum which had been separated from the larger mass, or from a hypertrophied appendix epiploica, which had finally cast loose from the intestine and dropped to the bottom of the sac. The patient had himself been aware of its presence for several years.

The contents of a sac in an ordinary hernia, however, is almost always either intestine, omentum, or broth together. Large sacs almost always contain a certain amount of fluid also. The fluid found in a reducible hernia is usually light, straw-colored, and clear. That found in the sac of a strangulated hernia may vary in color from a light coffee-color to that of a clark, heary chocolate, according to the length of time the strangulation has existed and the intensity of the constriction. The bladder has been mentioned as having been found in a hernial sac, and while protrusion of this organ, through the internal inguinal ring, is not uncommon. it is more frequently found by the side of the hernial sac, uncorered by peritoneum, than in its interior. 


\section{CHAPTER VI}

\section{SYMPTOMS AND DIAGNOSIS OF INGUINAL HERNIA.}

\section{REDUCIBILITY OF NON-STRANGULATED INGUINAL HERNIA.}

Hernia is seldon irreducible from its inception, but almost always becomes so as the result of neglect, producing one of the following conditions: (a) Gradual protrusion through a small canal until the bulk of the mass prevents its reduction. Adhesions of protruding contents to sac walls, or bands within sac. (c) Hypertrophy of protruding omentum.

(a) Hernia named under the first heading are not necessarily irreducible, as by confinement to bed and repeated manipulation they frequently can be returned to the abdomen. This was illustrated in a series of cases, reported before the New York Acarlemy of Medicine, by the author a number of years since. The subsequent histories of these very cases, however, proved conclusively that it was not an advisable procedure, as several of them furnished emergency operations by the recurrence of the protrusion (in some instances, several months afterwards) with symptoms of strangulation, notwithstanding the fact that careful truss-fitting had been carried out. It is best, therefore, to consider and treat these as cases of irreducible hernia.

(b) Adhesions of omentum to the sac wall are very common in inguinal hernia, but it is rather rare to find the bowel adherent. This is rloubtless due to the sluggish character of the former. and the peristaltic action of the latter. In very old and large hernixe, the normal motion of the bowel may be lost, and then aclhesions form. Such cases are subject to the most obstinate constipation, terminating in true intestinal obstructiron, ancl this is quite liable to cause rleath from paralysis of the bowel. even if operation is done. In these hernix, bands of 
connective tissue may also be found rumning in various directions through the salc, effectually preventing the return of its contents.

(c) A very common cause of the irreducibility of hernia is the hypertrophy and growth of protruding omentum. It

FIG. 39 .

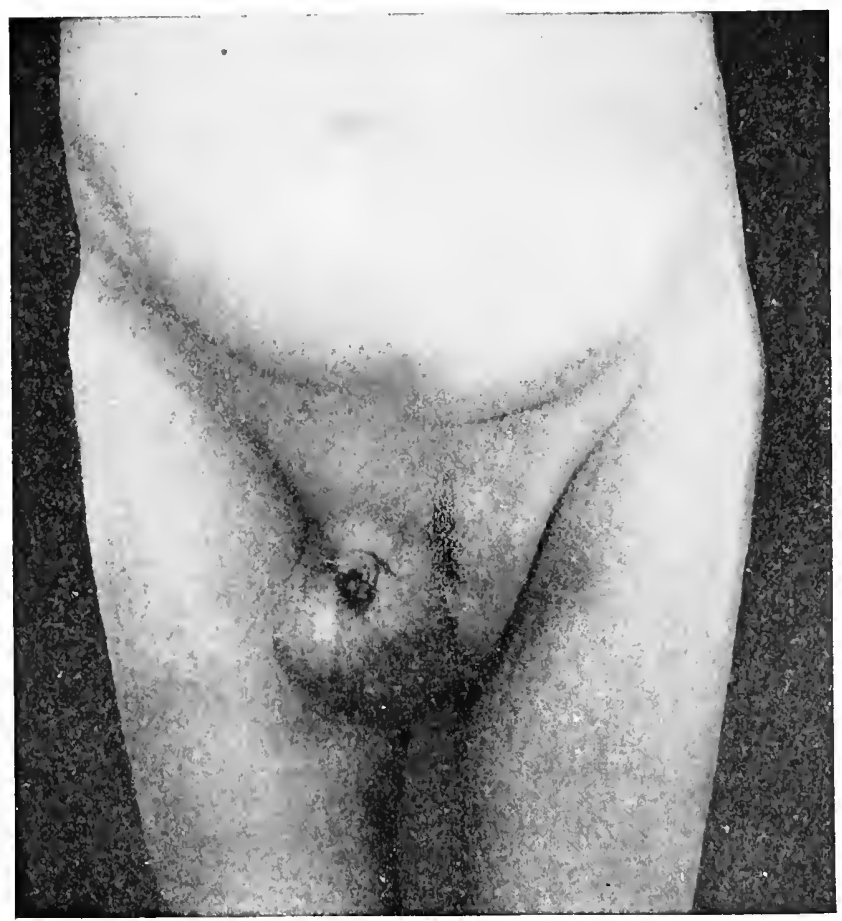

Left scrotal oblique inguinal hernia containing large mass of irreducible omentum.

becomes hard, nothlar, and of such size that it is impossible to pass it back through the canal even though no adhesions are present. It rarely happens that these masses, of protruding omentum, plug up the canal so that the intestine cannot protrucle. On the contrary, such cases are always attended by increased dangers of strangulation. 
Fig. 39 shows an irreducible hernia where a large mass of hypertrophied omentum was found at time of operation. Fig. to shows an even larger hernia when the contents were mostly intestine and wholly reducible.

Methods of Examination.-Inspection should be with the patient standing, and if a truss or support is being worn, it

FIG. 40.

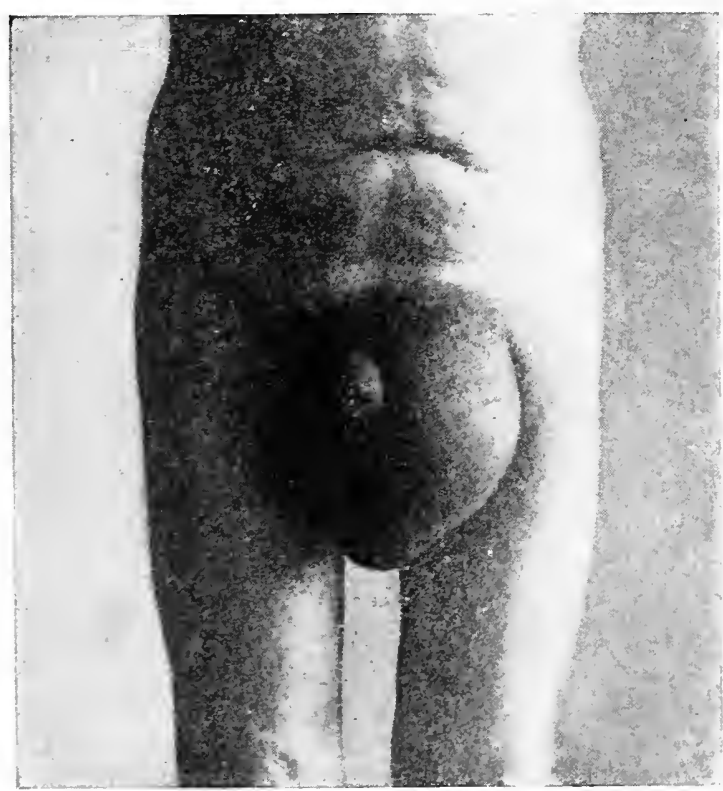

Enormous left scrota hernia in man of 60 years. Note testicle at bottom of tumor, showing that hernia is not of the congenital type.

should be removed. By this we will gain an accurate idea of the size and shape of the tumor, which will be a great aid in rliagnosis. If nothing is seen but a small bulging in the vicinity of the internal ring with no history of larger swelling, make suddcu pressure over the upper part of the canal with the encls of the fingers and if something is felt to slip back into the abrlomen, we may safely conclude that we have to deal with 
an incomplete inguinal hernia. If the swelling extends outside the external abdominal ring, and it is oblong in shape and reducible, it is probable that the case is one of complete oblique inguinal hernia; its reducibility, however, may not be ascertainable until the patient has been placed in the recumbent position. On inspection it may be seen that the swelling is circular in form and stands out from the body without much, if any, tendency to follow the cord, and this would indicate a direct inguinal hernia. If, on the contrary, it has already lropped into the scrotum, we may be certain it is an oblique inguinal hernia, and is either congenital or acquired. If congenital, and the hernia is protruding, the location of the testicle is obscured. If the swelling is reducible, there will be found an amount of thickening of the tissues, about the cord, that does not exist normally. If the photographs are carefully examined it will be readily seen that all congenital herniæ present a swelling, uniform in shape, and usually with a comparatively small neck, while in those with the acquired sac, no matter how large they may be, the location of the testicle is plainly visible. This absence of testicle is also noticeable in Sigmoid and Cacal hernia, but it will be seen that these herniæ are frequently quite different from any of the other forms.

Palpation.-The feeling of a swelling carries with it many valuable suggestions as to whether it is hard, nodular, and perhaps irreducible, indicating adherent and hypertrophied omentum, or smooth in outline, fluctuating and elastic, as in a fluid cyst. The pressure of the fingers on the tumor may produce the "gurgling" of gas in the intestine, so characteristic of bowel protrusion.

Under this heading may be noticed, also, the character of impulse on having the patient cough, to note whether it gives the impression of merely pushing the tumor forward without expansion. It must not be forgotten that in an abclominal varix, or large varicocele, there is upon coughing a thrill in the enlarged ressel that might easily be mistaken for impulsc. This holds equally true of a partially filled fluid cyst. It is also true 
that many people who have no hernia and perhaps little tenclency to it, have a decided impulse on coughing; it is, however, projectile and not expansible impulse. In view of all these facts as an indication of hernia, impulse is not considered of great importance even though it is given a prominent place by almost every writer on hernia. In my own work, both public and private, I have placed little reliance on it.

I desire to suggest here an excellent diagnostic test which I have used and taught for many years. While the patient is standing and with the tumor at its largest size, the fingers of one hand are held firmly over the inguinal canal, maintaining firm pressure while he lies down. Gentle pressure may now be made on the tumor by the unoccupied hand. It is usually not difficult to distinguish the character of the contents of the tumor as they pass under the fingers which still compress the canal. The rush of fluid, the nodular, irregular feeling of omentum, or the "gurgling" of gas in the bowel, all tell their own story.

The tumor having been entirely reduced, reverse this test by supporting the canal while the patient gets on his feet. After standing for a time, fluid will gradually pass the supporting fingers, but omentum or bowel will be retained. This is a diagnostic test, and indicates just what will occur in the mechanical treatment of these cases, i.c., that fluid cannot be retained by external support.

Pcrcussion aids materially in deciding between the solid character of omentum with its flat note, and the resonant note given off by gas imprisoned in protruding bowel.

Ina'agination of scrotal tissue, upon the finger (fig. 4I), is a method that needs more condemnation than praise, and I have repeatedly cautioned my classes against its indiscriminate use. It is useful and allowable in large herniae in order to decide whether it is perfectly reducible, but its use on a person suffering from a small complete or an incomplete hernia in this way is an injustice and of little benefit to the examiner. By forcing the finger up into the external abrominal ring, that 
aperture is certainly enlarged, and but little real information is obtained. It is no uncommon thing to find people with very large external abdominal rings, who have no hernia, and some of those who have it of morlerate size have comparatively small rings. In my clinic at the New York Post-Graduate Medical School I have had, within the past ten years, three men tell me

FIG. 4I.

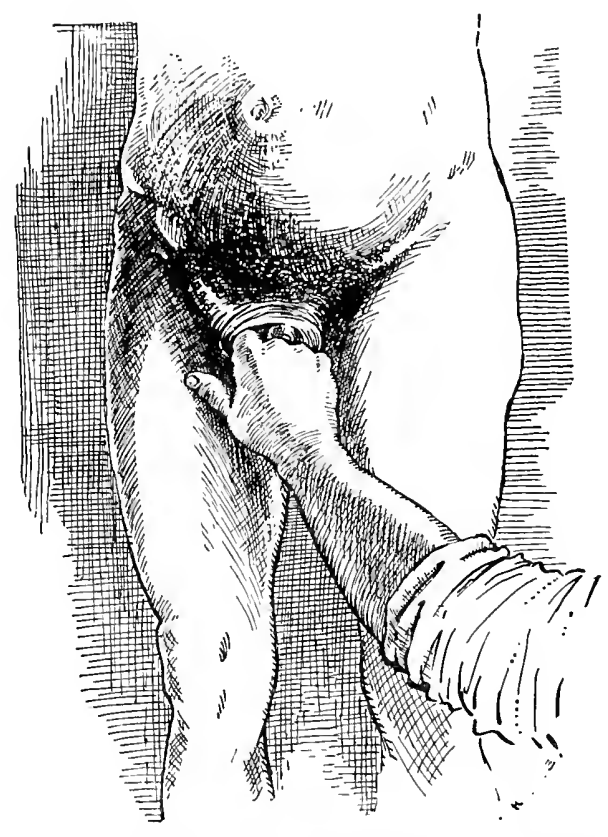

The method of examining hernia here shown-passing the finger into the canal by invaginating the scrotal tissues-camnot be too strongly condenined.

that they had hernia produced in just this way, in order to aroid military service in Germany. Two of these men had employed a woman who seemed to be an adept at it. The usual time required to produce a noticeable hernia was three days, and it was accomplished by dilating the inguinal canal with the finger invaginated in the scrotal tissues, following the cord as a guide. 
Indigestion, constipation, and prostration are results, and therefore symptoms, of hernia more frequently than generally supposed. It has been no uncommon experience to see cases that persistently resisted drug treatment, entirely cured of obscure intestinal troubles by the perfect retention of hernia. While constipation is undoubtedly more frequently a cause, it is, in large and uncontrollable hernix, a most common and dangerous result. It indicates a paralysis of the protruding bowel which, if not heeded, will result in intestinal obstruction that has hitherto proven beyond medical or surgical skill to relieve. There is also an amount of prostration present in many of these cases that is wholly disproportionate to the degree of departure from a normal condition. The effect of an unretained hernia upon men has been compared with that produced upon women by some uterine derangement that may in itself be trifling. The mental effect is sometimes very serious and should be promptly counteracted by the physician. Three cases of suicicle, the impulse having its direct origin in existing hernia, are within the personal knowledge of the author.

Pain.-In by far the greater number of cases, the first inclication of hernia is in the presence of a swelling. This may be discovered by the patient when it is only a slight bulging, but it is no uncommon thing to have him come with swelling as large as an egg or orange, which unquestionably has been of slow growth and still has not been noticed. Naturally he assumes that it has just occurred and gives a history that is misleading. It proves conclusively, however, that hernia may reach considerable proportions without sufficient pain to call the patient's attention to the parts.

If the development of hernia is rapid and from violence, it may be attended by a certain amount of pain, but under orclinary conditions this is unusual. There is sometimes a smarting or burning sensation in the inguinal region, and this is very liable to be present in omental protrusion. It is believed that those patients who complain of extreme sensitiveness and pain, should be looked upon as suspicious and doubtful cases, so far 
as diagnosis of hernia is concerned. If hernia really exists, other troubles are also liable to be found; as an inflaned cyst on the cord, an inflammation of the cord itself, or possibly an acute adlenitis of the inguinal glands. Sometimes a burning sensation is complained of as occurring late in the day and disappearing during the night. It is more common, however, for

FIG. 42.

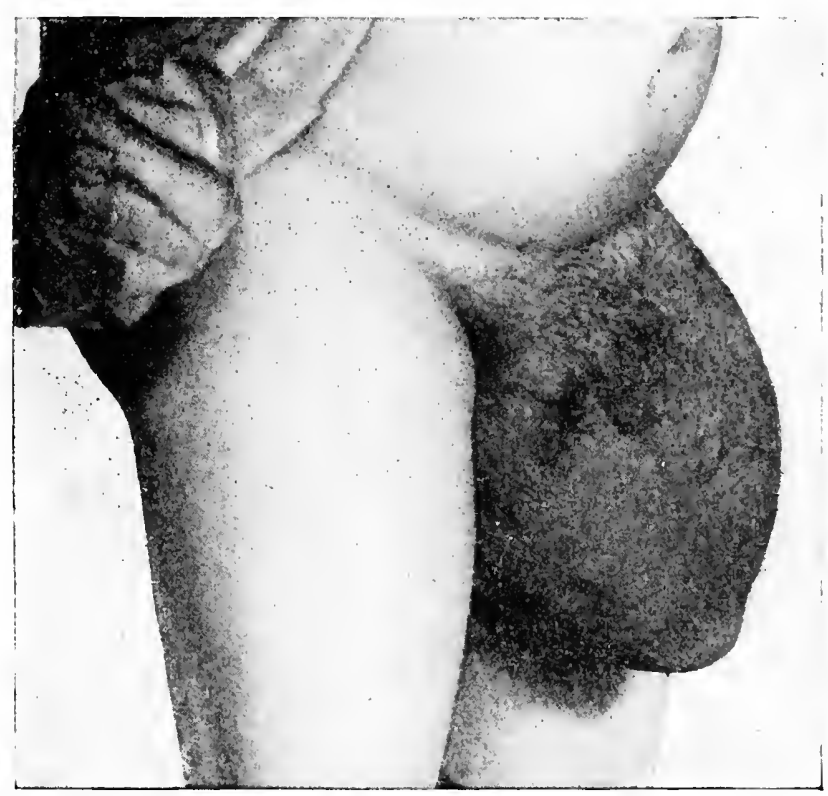

Enormous irreducible left scrotal hernia, 20 years' duration, no treatment. Age, 45 years. Notice testicle at bottom of scrotum, showing that it is an acquired sac.

the patient to complain of weakness, or dragging pain, in the lower part of the abdomen, worse at night and better in the morning, which is increased while straining at stool. sneezing, or coughing. Almost all writers on the subject agree that it seldom happens that hernia of goodly proportions develops suddenly, except in the congenital type where the sac is already formed. In the latter cases, when the neck of the sac 
is sufficiently dilated, a good-sized hernia may drop at once into the pouch that awaits it.

Swelling.-The swelling, which to the patient may be the first indication of hernia, usually disappears when he is in the recumbent position at night, and may not return the next day until he has been on his feet several hours, or it may return

FIG. 43.

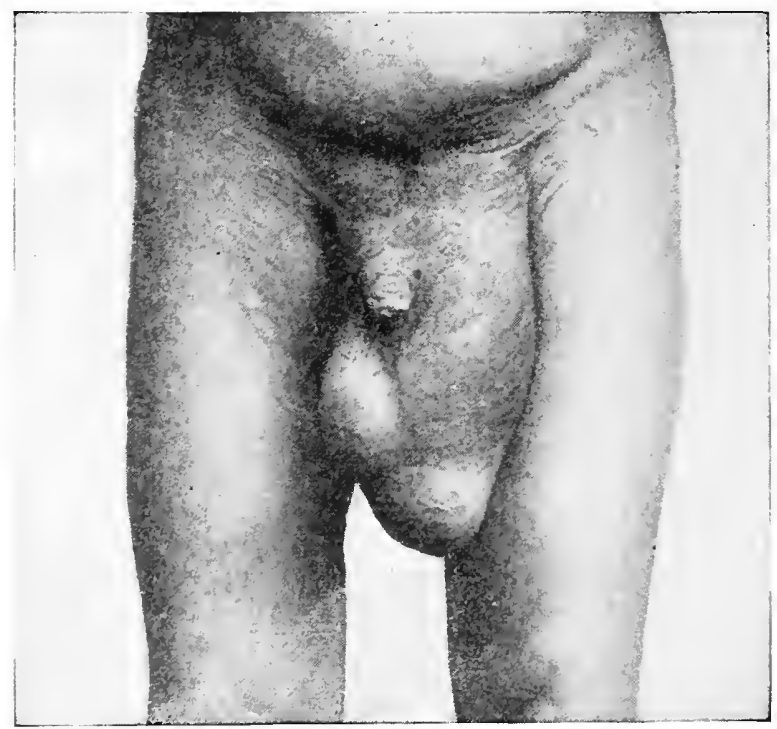

Showing large scrotal hernia with true peritoneal sac; outlines of testicle well defined on the same side.

as soon as he gets out of bed. When it is well cleveloped it is quite certain to drop into its sac as soon as he is in an upright position. Such a swelling is probably a reducible hernia. If neglecterl. the neck of the sac and the tissues surrounding it become more and more dilated, allowing the hernia to increase in size until it attains proportions that are truly enormous, and the patient becomes a burden to himself and friends (see figs. I8. $19,+2$ and 43 ). 
At any stage of the development of hernia there are complications, that may occur, that materially change the character of the case. The first and most dangerous is, that such an amount of protruding viscera may be forced into the narrow neck of the sac that strangulation of the parts occurs. Not only are the contents of the intestine prevented from passing through the protruding loop, but the circulation of blood is at first retarded, then checked entirely, producing ultimately gangrene of the parts. The second change that may alter the swelling in character is in the contents of the sac becoming adherent to the sac wall, and it is then an irreducible hernia. Irreducible hernia is one step farther away from the normal condition and increases its dangers to the patient as well as adding materially to the difficulties of treatment.

\section{DIFFERENTIAL DIAGNOSIS.}

The differential diagnosis between types of inguinal hernixe is usually not attended by much uncertainty in uncomplicated cases. The manner of formation, size, and shape, all aid in deciding whether we have to deal with an incomplete, complete, or scrotal hernia, of the oblique ingtinal type; with a sigmoid, crecal, or ordinary direct hernia.

Congenital Hernia.-Perhaps more frequently than any other type, we are called upon to differentiate between the congenital and acquired forms of inguinal hernia, and if we are dealing with infants and children, and especially if we hope to cure them without operation, it is quite important that we should recognize the difference. Congenital hernia is always of the complete oblique form, as after passing through the canal and out at the external abdominal ring, it drops immediately into the scrotum in front of the testicle. If the congenital sac is in the female, it comes well down into the labitum majora. Its history is usually of sudden formation and in the male is first seen in the scrotum. The mother of the child will tell you that she saw the swelling in the scrotum first, which means that in all probability it is a congenital hernia. If she gives a history 
of seeing the swelling at the external ring for some days before it descended lower, it indicates that the hernia is of acquired type. In the swelling produced by congenital hernia the location of the testicle is always obscure, and if search is made for it, usually it will be found at the back part of the scrotum. The swelling which includes the testicle is smooth in its outlines as

FIG. 44 .

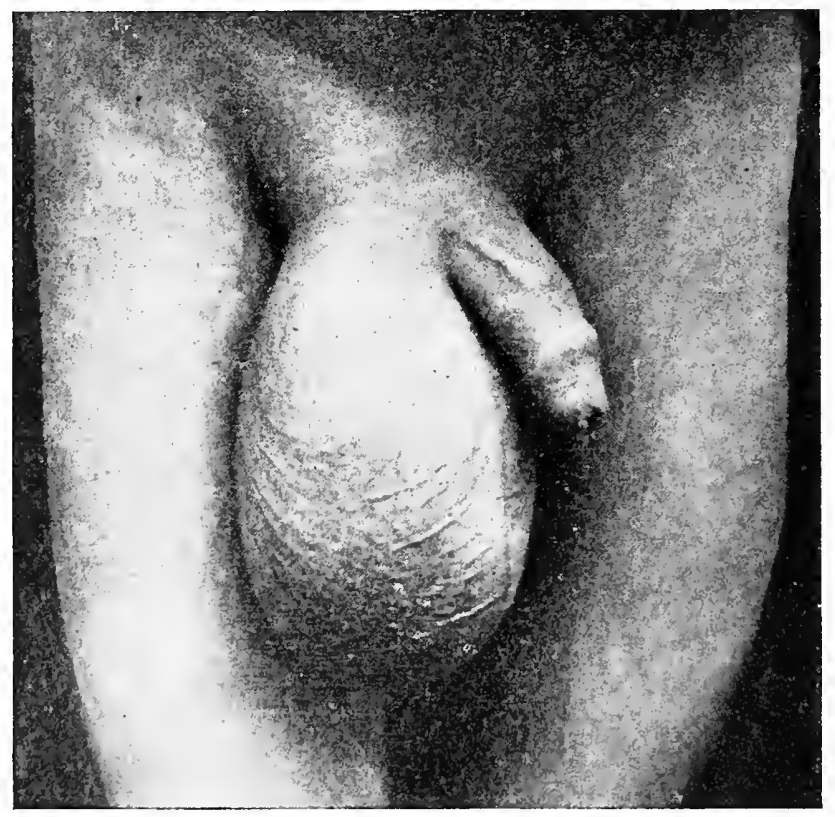

Typical congenital protrusion in adult. Note that location of testicle cannut be sten.

shown in figs. 44 and 45 . By comparing these with the photographs of the other large hernice it will be seen that, in the latter, the testicle can always be located. In the case shown in fig. 46 a mistake in diagnosis was made by the family physician, who supposed it to be an ordinary hydrocele, and, not having a trocar at hand, incised both sides with a scalpel. It is rather remarkable that, after cutting into one, he should 
then have opened the opposite sicle. In the latter instance. protrudling intestine convinced him of the true condition, and he

FIG. 45 .

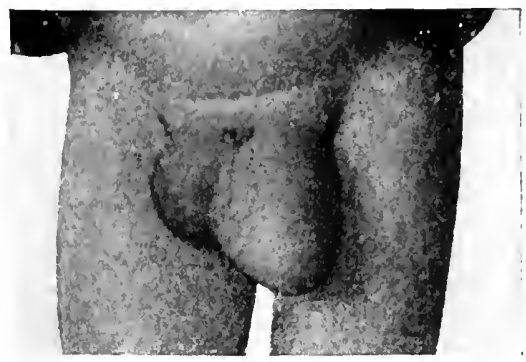

loy 6!/2 years old. Double congenital hernia. Delayed testicle on right. Both testicles placed in scrotum and hernix cured by operation.

FIG. 46.

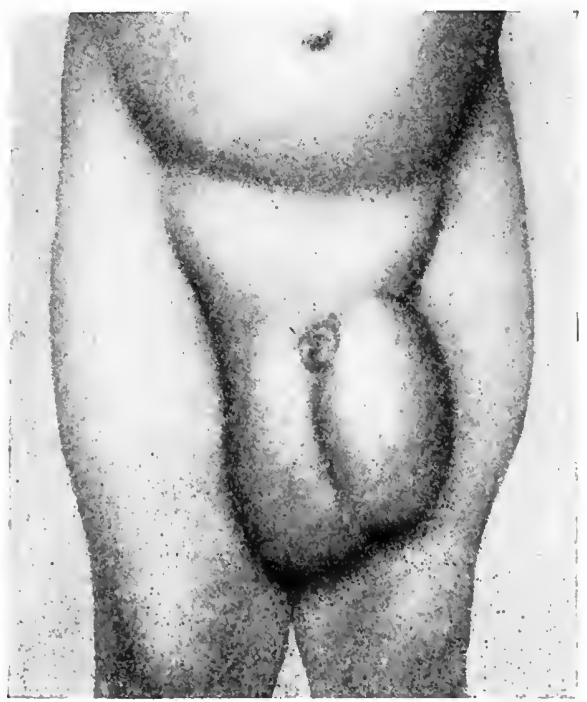

Donble congenital hernia mistaken and incised for hydrocele by fanty physician.

closed the wounds with plaster. The child was quite sick following this, but recovered. It was several weeks later that 
I saw the case, but the scars were distinctly visible and they show faintly in the photograph. As the child's hernize were reducible, a truss (fig. 47) was applied and worn for one year, at which time he was cured by operation. Fig. 48 shows the same boy two years after the operation.

The shape of a congenital hernia should orclinarily suggest its diagnosis, its size usually being very large in proportion

\section{FIG. 47 .}

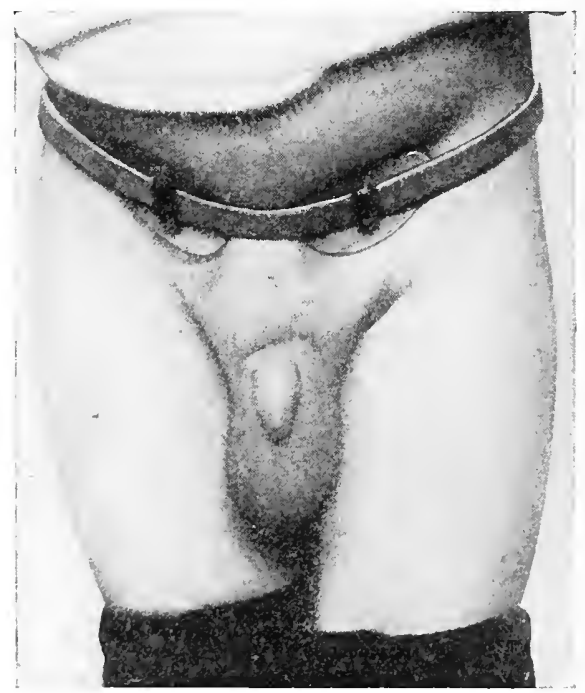

Same case as Fig. 46, showing herniæ retained by truss, which was worn one year before operation for cure.

to the size of the neck of the tumor. If these hernice have become rery large and are of long duration, this does not always hold true. Fig. 44 shows typical congenital hernia as seen in the adult. Compare with fig. 49, which shows a typical scrotal hernia of the acquired form; fig. 5o shows a typical lalial hernia in the female.

Oblique Inguinal Hernia, Acquired.-While congenital hernia will ahways present itself as a scrotal protrusion, the 
acquired form may be found as a small bulging into the upper part of the canal, or from this to any degree of development up to the enormous protrusions already shown. Even in the latter it will be noticed that the testicle can almost always lue located by inspection. The shape of ollique hernia that has not passed into the scrotum, is oblong, and its presence in the canal can usually be detected.

FIG. 48 .

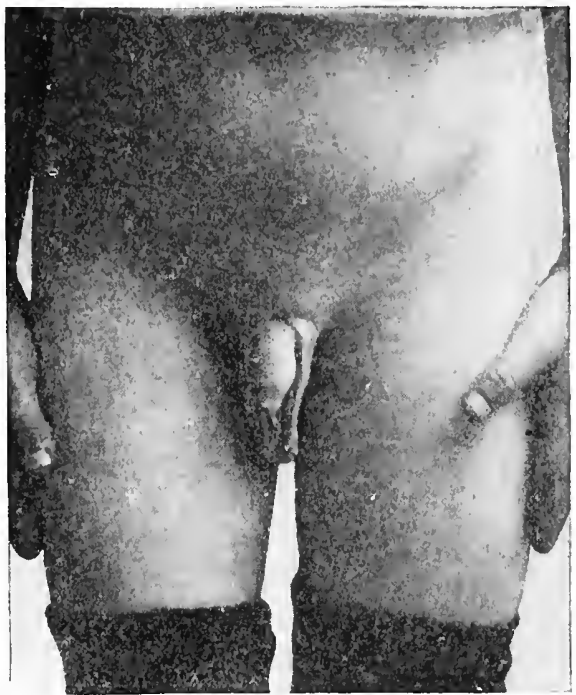

Case shown in figs. 46 and 47 , two years after operation for cure.

Cases are frequently seen where there is a decided bulging over the canal which comes from the pushing forward of the whole wall of the abdomen in this region, but where there is no actual hernia. Such cases may require light support for a time, but frequently eren this is unnecessary. Certainly they are not in need of operation.

It is in the very earliest stages that diagnosis is difficult, and it is here that the following test has often been of great service to the author and his students. This test is especially applicable. 
in cases where there is doubt in the mind of the examiner whether hernia actually exists. Stand by the side of the patient, who shall also be standing, place the fingers gently orer the canal. Hare the patient cough, and notice first, whether there is a feeling of expansion in the bulging part: then, without changing the position of the fingers, make sudden, sharp

\section{FIG. 49.}

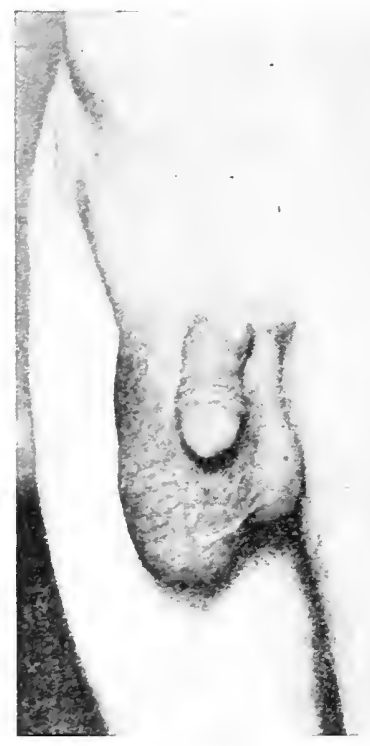

Front.

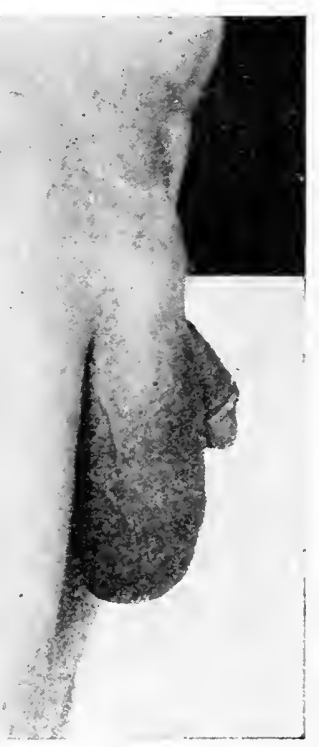

Sitle.

Typical right scrotal hernia of acquired type. Note outlines of testicle at bottom of scrotum in front view.

pressure over the canal, and note whether you feel anything slip from beneath them. This pressure nutst be quick and positive to be effective. but must not be roughly executer. It may be repeated any number of times, liaving the patient cough before each time. to assure one's self the slipping is, or is not, present. If present, the dliagnosis of hernia is guite certain. Its absence is not quite. but nearly, as positive evidence that it does not 
exist. In the work of a large clinic which must be done rapidly and still as accurately as possible, this test has proven itself of the greatest value.

Direct Inguinal Hernia presents a round tumor having the appearance of being nearer to the median line than the oblique form, and even when quite large, stands abruptly out from the

FIG. 50.

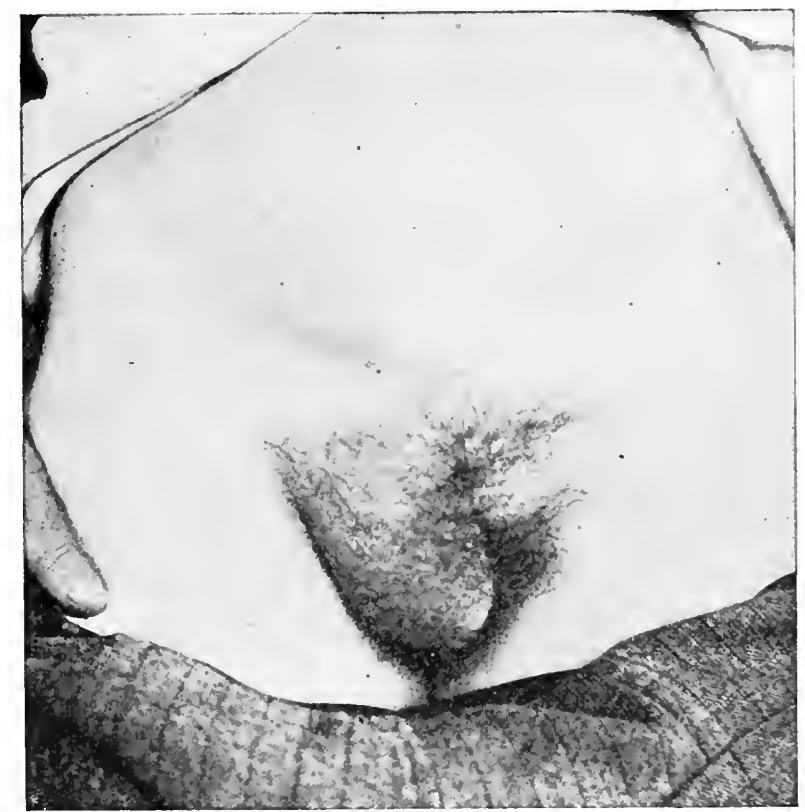

Right labial hernia (complete oblique inguinal), similar to scrotal hernia in the male.

body without much tendency to follow the cord (figs. 2 I and 22): Coming out to the inner side of the deep epigastric artery. it protrudes directly through the external abdominal ring. The cord is at its outer side from the median line. and its covering of intercolumnar fascia prevents its descent into the scrotum. When small, it can usually be easily reduced by the pressure of the hand with the patient standing. Its diagnosis is attended 
by less difficulty than in the oblique, but the difficulties of operative work upon it are greater. The rather common presence of the bladder, either in association with a hernial sac, or alone as a direct protrusion, makes special caution necessary.

In operating, after the sac is removed, a much more difficult opening to close is presented than in oblique hernia, and there is a greater liability to acciclental injury to important blood ressels on account of anatomical changes in the parts.

FIG. 5r.

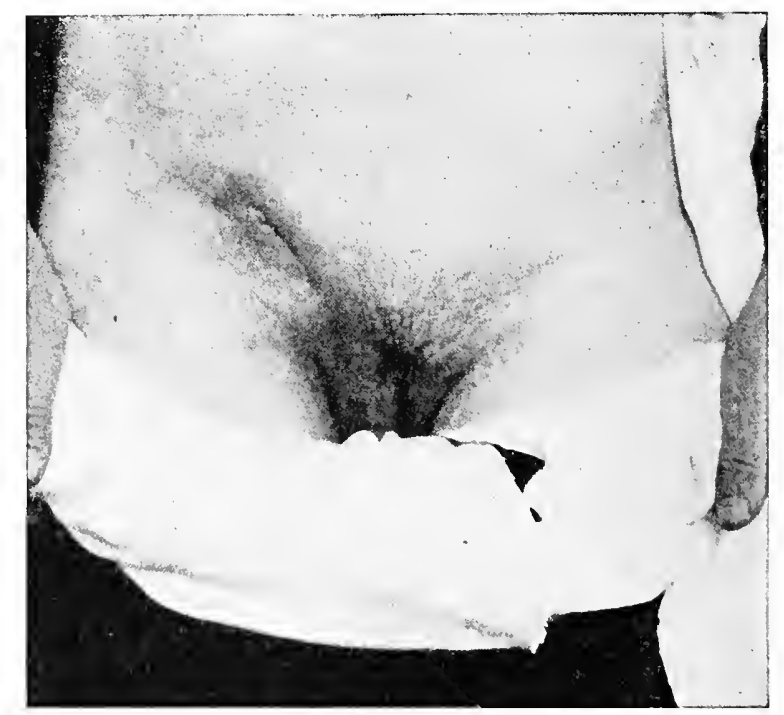

Same as Fig. 5o. Four weeks after operation for right labial, inguinal hernia.

Sigmoid and Cæcal Hernia can properly be considered under one heading owing to their similarity in anatomical conditions and in general appearance. Their diagnosis is particularly important owing to the difficulties in successfully and safely operating upon them, and I consider it fortunate that I can present several excellent photographs of cases where the diagnosis was confirmed at operation. It is not always possible to cliagnose this condition before operating, as in cases of 
blaclder protrusion, but we usually have a peculiar type of swelling, and careful study of the photographs presented will make this more clear than words could (figs. 23, 52, 53. 54). Fig 54 shows both caeal and sigmoid hernia in the same patient, a condition rarely met with. It will be seen that no matter what size the tumor may be, its neck is enormonsly

FIG. 52.

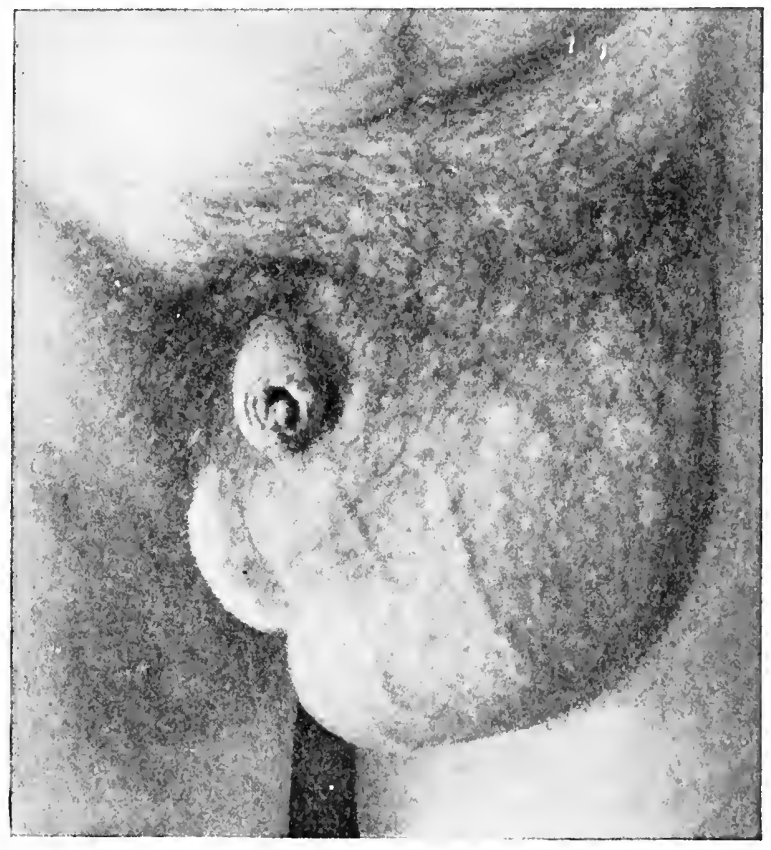

Sigmoid hernia.

large. These, as well as hernia of the bladder, are most frequently direct protrusions, but the latter never reaches the enormous proportions obtained by the sigmoid-cacal type. If examined early, they present the general appearance of an ordinary direct hernia. The slowness of reduction may lead even in their early growth, to suspect something out of the usual order. They do not, like oblique inguinal and many 
cases of ordinary direct hernia, slip back suddenly and with a "gurgle." In truth their reduction is never perfect, as the posterior wall of the bowel is usually anchored ouside of the abdominal cavity, but this fact cannot often be ascertained by the feeling communicated through the covering tissues. The reduction is accomplished slowly and with considerable pain to the patient. That is, there is a degree of sensitiveness that

FIG. 53.

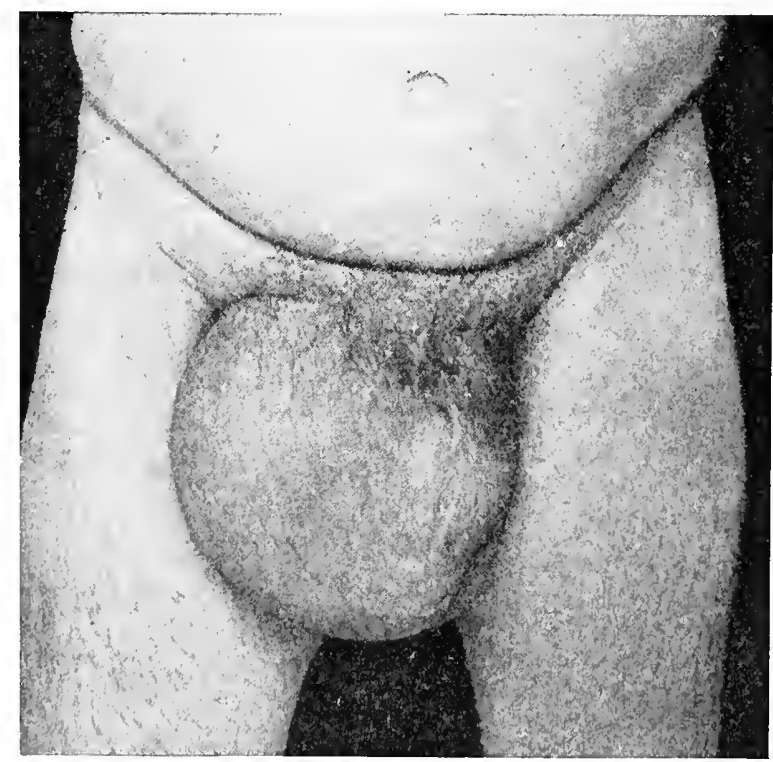

Cæcal hernia.

seldom exists in other forms of hernia. It is impossible many times to decide between a very much thickened sac and a hernia that is only partially reducible.

There is one other important indication that may tell us hefore operation what we are likely to find, and that is, that patients having this form of hernia can seldom tolerate the pressure of a truss. They frequently abandon treatment as they are far more comfortable without support, and seek 


\section{FIC. 54.}

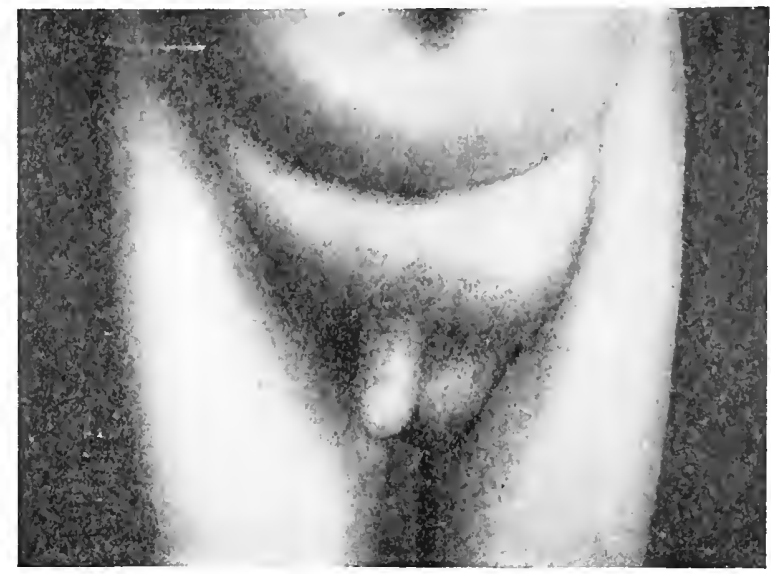

Sigmoid and cecal hernia in the same patient.

FIG. 55.

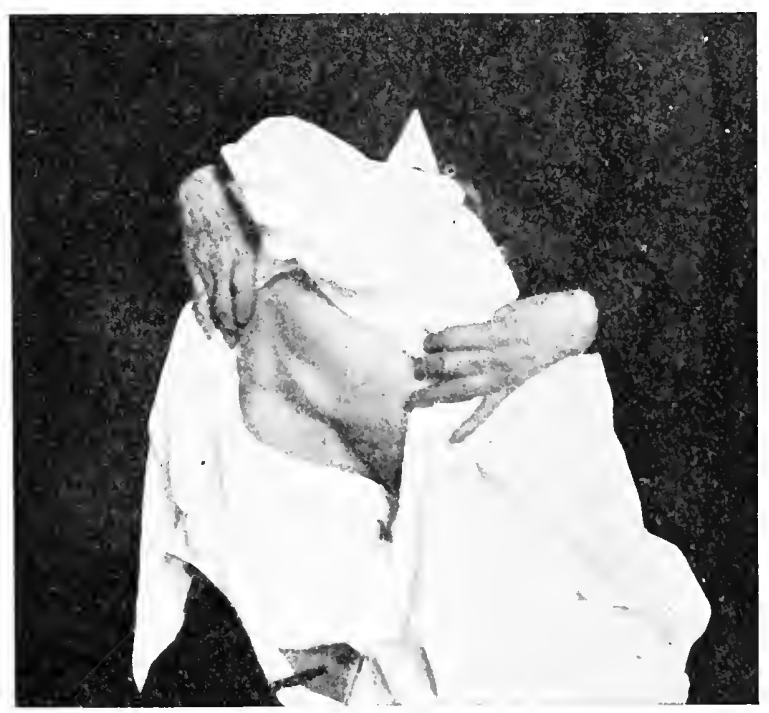

Woman 75 years of age with cæcal hernia. Much larger and strangulated when first seen. 
surgical relief only when the tumor becomes an intolerable burden. On account of the large size of the neck of these tumors they are not especially liable to strangulation, but gas is frequently present in the bowel in large quantities, as may be easily demonstrated by percussion.

\section{DIAGNOSIS OF OBLIQUE INGUINAL HERNIA FROM OTHER CONDITIONS.}

In the consideration of this branch of the subject it is well to divide tumors, which are liable to be mistaken for hernia. into the reducible and those that are not reducible:

REDUCIBLE TUMORS.

Hernia.

Congenital Hydrocele.

Varicocele and Varix.

Partially descended testicle.

Lipoma.
IRREDUCIBLE TUMORS.

\section{Hernia.}

Hydrocele of cord.

Hydrocele of tunica vaginalis.

Partially descended testicle.

Hæmatocele.

Lipoma.

Inguinal Adenitis.

Impulse upon coughing, which is always referred to in text-books, is in many instances deceptive, as a large varicocele, or a partially filled fluid cyst, may give the same sensation. Furthermore, it will be found that many persons who are not afflicted with hernia produce a strong impulse in the inguinal region upon coughing. It is not the impulse, but expansion of the tumor under strain of coughing, which furnishes a safer guide. When the hand is placed orer the swelling, the latter will be felt to expand, fill up, and increase in size, with a subsequent contraction to its previous condition.

In the acquired variety of inguinal hernia, when the protrusion has a true peritoneal covering, the swelling is usually first noticed at the external ring, and may vary in size from that of a chestnut to a hen's egg. It is usually soft, easily compressed, and disappears on lying down.

A small tumor found in the inguinal region, presenting these characteristics, is pretty sure to be hernia. It must be 
borne in mind, however, that a large Vari.r, as sometimes seen in pregnant women, or a hydrocele of the cord where the fluirl is partially reducible into the abdomen or into the tissues, will closely resemble hernia. In the case of varix, it can wisually be differentiated by the fact of a general varicose conclition of the labia or, perhaps, of the entire leg of that side. An excellent diagnostic test in all reducil)le tumors is as follows:

FIG. 56.

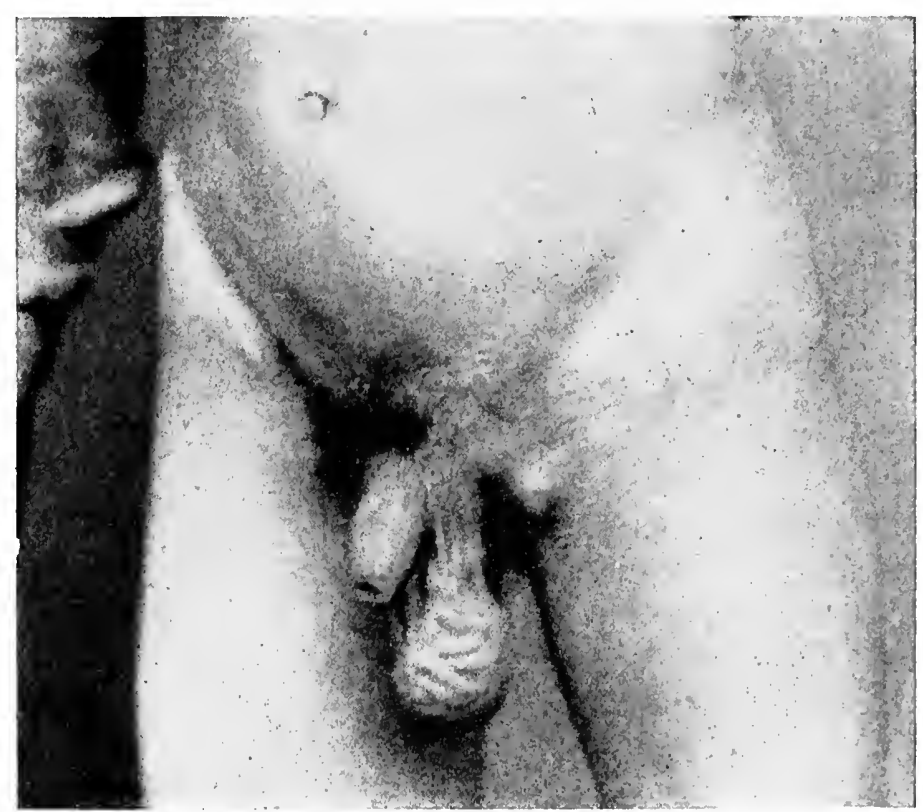

A left varicocele and femoral varix on same side. (Eccles.)

While the patient is standing and the tumor largest in size, the fingers of one hand are held firmly orer the inguinal canal, maintaining firm pressure while the recumbent position is assumed. Gentle pressure may now be marle on the tumor by the unoccupied hand. It is usually not difficult to distinguish the character of the contents of the tumor as they pass under the fingers which still compress the canal. The rush of fluid, 
the nodular, irregular feeling of omentum, or the "gurgling" of gas in the bowel all tell their own story. The tumor having been entirely reduced, reverse this test by supporting the canal while the patient gets on his feet. The fluid will then gradually pass the supporting fingers, but omentum or bowel will be retaineil. This is not only a diagnostic test, but indicates just what will occur in the mechanical treatment of these cases, i.c., that fluid cannot be retained by external support.

Congenital Hydrocele is reducible and forms the most common fluid tumor; it may be accompanied by a true hernial protrusion, making diagnosis even more obscure. In many of these cases, diagnosis can be made only by the action of the supposed hernia under mechanical treatment and inability to retain its fluirl part. Translucency and the smallness of the neck of the tumor must be borne in mind. It usually reduces slowly and the fluid can be felt rushing into the abdomen by holding a finger over the canal, while pressure is made from below by the other hand. It occurs almost exclusively in very early life. If mistaken for hernia and treated as such, it will lue a fortunate mistake for the patient, as it will be quite sure is cure a condition that later might develop into hernia. As a truss will not retain the fluid, it is important to diagnose the condition and enlighten the parents as to what they may expect. In some instances of protrusion into a congenital sac of thin omentum. the tumor is quite elongaterl and has the feeling of fluid contents.

Varicocele and Varix (figs. 56 and 57 ), it would seen, shrould be recognized at sight, but the fact that patients have been sent hundrerls of miles for the treatment of hernia when the latter dirl not exist. proves that it is not always so plain to the arerage examiner. The test given for fluid tumors, carefully executer?, should give conclusive information.

The crmmon saying that a varicocele feels like a bunch of earthworms is not in itself a safe guirle. as a mass of thin omentum may convey very much the same sensation and present a similar apjearance. It seems hardly pardonable, however, that 
such a case as that shown in fig. 57 , where the dilated surface veins at once suggested its character, should loe mistaken for hernia. In some men, however, not only does the scrotal surface present a perfectly normal condition, but the cord is so enlarged in the canal that it has the feeling of hernia. Here again, if the hand is placed over the canal and the patient made to assume a lying-down position, the tumor will rlisappear without any part of it having been felt to pass under the fingers.

Fig. 57.

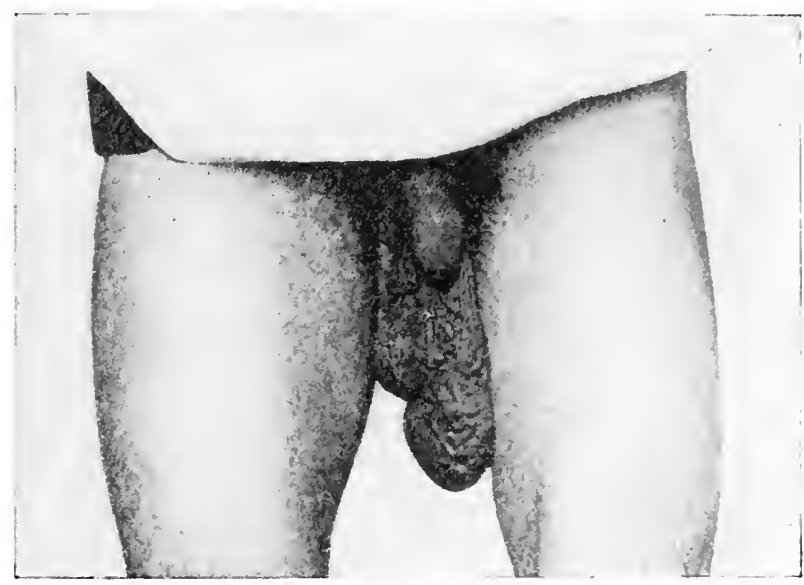

Extreme case of varicocele that had been mistaken for hernia.

In a case of clouble abdominal varix which had been mistaken for hernia, sent to me from a distant state, the superficial epigastric vein, on either side, was so enomonsly dilater as to simulate fairly well double inguinal hernia. The tumors were easily compressible, which gave then the appearance of being reducible, and a truss had been worn over them. They gave the peculiar thrill of reducible fluid tumors when the patient coughed, and this had been mistaken for impulse. There were no other enlarged veins in the inmediate vicinity, but below the knee the varicose condition was quite marked. 
LABIAL VARIX may be even more misleading and is more frequently met with. The tumor disappears almost completely on lying down and appears promptly on standing up, but it can easily be demonstrated by the test for fluid tumors, already given, that it is not reducible to the abdomen. Labial varix occurs most frequently during pregnancy and usually disappears soon after confinement. These cases simulate labial hernia, which is not nearly so common in women as scrotal hernia in men. The following case was seen in consultation (June 27, I898) : Mrs. A., age thirty-five years, had been seen by several physicians for swelling in the left labium. Diagnosis of hernia had been made by all, and several different kinds of trusses had been applied with no effect except great discomfort. She was five months pregnant and in perfect general health. The swelling was first noticed two months since and gradually increased. It was not attended by actual pain, but smarting and burning, and she experienced a sense of weight that was very disagreeable. It disappeared almost wholly when lying down and returned promptly upon standing.

A soft, compressible tumor the size of a large goose-egg, or small orange, was found in the left labium majora and extended well up towards, but not to, the external ring on that side. It also extended up the left vaginal wall and connected with deeper parts by a cord nearly as large as one's thumb. Diagnosis._Varix of veins of labia. Treatment.-Expectant; recumbent position for relief when in pain. If trouble is experienced after confinement, obliteration of veins is advised. Letter from family physician (Jan. 2I, I 899) gives subsequent history as follows:

"Perhaps it might interest you to learn the history of Mrs. A. of this city, whom you saw with Dr. M. last summer, and who had a varicocele of the left labium major; she was also pregnant. We had supposed that she might have a hernia. As her pregnancy progressed the tumor became smaller, and I was able to feel the varicose veins. By the time labor came on the tumor diminished to one-half its former size, was no im- 
pediment, and, though forceps were used, she escaped without injury to perineum or labium. It is now four months since her baby was born, and she makes no complaint of her varicocele."

The above letter illustrates in a graphic manner how, when the pregnant uterus left the pelvis as pregnancy advanced, the vessels were released from pressure and practically resumed their normal size.

Undescended Testicle lying just outside of the external ring, easily reduced into the canal, but with a cord too short to allow it to go into the scrotum, may be misleading. The empty scrotum on that side should direct attention to the true condition. Furthermore, by deep pressure over the canal it will be seen that the reduction is not complete. Numerous illustrations of this condition, in various parts of this work, will convey not only a correct iclea of their appearance, but will also indicate their very common occurrence.

In cases where apparent reduction has been accomplished and there is still felt a thickening in the canal, while the patient is in a recumbent position, one of the following conditions should be suspected; adherent omentum, retained testicle, (ovary if female), or fluid encysted within the sheath of the cord. Liponna, or loose fat in canal, might also be mentioned.

An abscess, especially of the slow forming variety seen in cases of Pott's disease, with a long sinus, may prove very perplexing when it points in the inguinal region. Under moclerate pressure, with the patient on his back, it may be so far reducible as to give the appearance of hernia. The slowness of reduction, and "boggy" feeling should lead to suspicion, especially if Pott's clisease is known to exist. Iliac abscess may present a tumor in the inguinal region, but Psoas abscess will more likely point in the femoral space.

Lipoma.- In the sense of a true fatty tumor that forms in the subperitoneal fat, and from its own size and weight forces itself clown through the inguinal canal, it is not believed that lipoma is very common. Elongated pieces of fat occupying the canal and protrulling at the external ring with or without 
hernia, are extremely common, and perhaps the most convenient term is to call them lipoma. It is believed that they are formed by normal subperitoneal fat being forced into the canal under some unusual strain and acting as a dilating wedge; not only this, but it pulls down a pocket of peritonetm which becomes a true hernial sac. The diagnosis of such masses of fat from hernia is extremely difficult and often impossible. It will be found, however, that when a swelling of this character is reduced to the canal, it can still be felt under its fascial coverings. It does not drop back suddenly as would a piece of free omentum. Exact diagnosis is not, however, very important, as the treatment should in any case be the same as for hernia. If there is nothing but fat in the canal, the wearing of a truss for a year may destroy it, and it is in such cases that occasionally we have records of the remarkable cure of hernia, in the adult, by truss-wearing.

Irreducible Tumors are, as a rule, more easily distinguished from hernia. An acutely inflamed inguinal gland should never mislead an examiner, as its heat, tenderness, and closeness to the skin, are characteristics which are foreign to hernia. They are also unaccompanied by any of the general symptoms found in strangulated hernia.

Encysted hydrocele, and hydrocele of the cord, especially within the canal, make diagnosis uncertain in rare cases. If on the cord lower down, they are usually easily recognized by their round, smooth, elastic feel, and by their attachment to the corcl. Cysts just outsicle the ring, which may be reduced into the canal, are particularly liable to cause mistake. Under trusstreatment, Jowever, they usually demonstrate their character by the impossibility of their retention. Furthermore, when they are reclucerl, they lift the testicle high up in the scrotum. A gentleman came to me a few years since, who had visited most of the best-known truss makers in attempts to retain a small swelling which protruted at the external ring. Recognizing its true character, I drew off with a small aspirating needle about half an ounce of amber-colorerl fluid and injected 5 drops 
of 95 per cent, pure phenol. The cure was complete, as no hernia had ever been present.

Hydrocele of the tunica vaginalis should be diagnosed from incarcerated or irreducible hernia by the fact that it is first discovered in the scrotum and enlarges from the bottom upwards; that the cord is of normal size where it passes through the abdominal ring, and that the tumor is translucent, tense, and fluctuating, and the location of the testicle uncertain. In the beginning of hernia there is a history of a reducible tumor, while in hydrocele there is a history of a gradual growth without any period of reducibility. Hematocele lacks the diagnostic test of transmitted light, but has a history of traumatism which should guide us aright.

Lipoma that are only partially reducible may sometimes form in the canal and slip through the external ring. These tumors and intramuscular layers of loose fat, work their way into the canal, become elongaterl and protrude. It is, at times, impossible to recognize them until the patient reaches the operating table. As it is always considered advisable to operate, on account of the certainty with which they are followed by hernia, the uncertainty of diagnosis is umimportant.

The incomplete descent of the testicle forms a far more common complication than is generally supposed. If it is arrested by adhesions, or shortness of its cord, in the canal or near the external ring, it may make diagnosis uncertain and simulate irreducible hernia. Young men who have this defect are frequently very sensitive about it, feeling that they are physically imperfect, and add to the difficulties of the situation by trying to disguise the fact that the scrotum is empty. A case of this kind that had been previously examined by two physicians, was seen in consultation for supposed incarcerated hernia, but proved to be a post-gonorrhceal epididymitis with a testicle permanently located near the internal ring. The patient had deluded the attending physicians by arranging and persistently keeping his clothing so that the scrotum was not exposed. He also assured me that he had repeatedly been 
fitted by truss makers without ever allowing them to see the scrotum. Another case of this type was seen that had been diagnosed inflamed inguinal gland, and an incision was about to be performed when the true character of the case was discovered by finding one sirle of the scrotum empty.

In the female, an ovary lodged in the inguinal canal presents a form of complication resembling, in most features, that of delayed testicle. It is as persistent as is the testicle, in remaining outside the abdominal wall. It is not as sensitive to pressure from truss-wearing, nor does it seem so liable to inflammatory action. It can seldom be fully replaced within the abdomen by manipulation, and even when apparently this has been done, it readily slips out from under the retaining pad of the truss and may then become quite painful. Phey are almost always painful at the menstrual period. Ovaries found in this position are frequently cystic and, in rare instances, degeneration of structure has been so far advanced as to warrant their entire removal.

Cysts forming along the cord in the male, or in the canal of Nuck in the female, may complicate the mechanical treatment of hernia to a moderate extent, and may be very perplexing in making a correct diagnosis. Small cysts, when they come under truss pressure, usually disappear after a time; if larger, and in the way, they can be aspirated. It is when they are unrecognized and attempts are made to replace and retain them within the abdomen, that they cause the most trouble.

Inguinal Adenitis.-An inflamed inguinal gland may form one of the complications of hernia, making diagnosis obscure, as indicated by the case of a woman who gave a perfectly clear history of reducible inguinal hernia, retained by truss-wearing for several years. At the time of examination, she presented a hard. slightly painful tumor, in the inguinal region, which could not be reduced. It presented, in fact, every appearance of an incarceraterl omental protrusion, except that there was a suspicious amount of heat and tenderness. Attempts to reduce the protrusion failed, and upon the points named it was diag- 
nosed inguinal adenitis. This diagnosis was subsequently confirmed by the subsidence of the inflammation and the return of her hernia, which was easily reducible. During the active inflanmation of the gland, hernia was prevented fron entering the canal, even when the truss was temporarily abandoned.

I have seen a case of this kind that was even more obscure. A gentleman who has a large surgical practice in a neighboring city brought to me a healthy, red-cheeked young man of twenty-six years, six feet tall and weighing nearly two hundred pounds. Two weeks before he had felt some discomfort in the right inguinal region and shortly after discovered a small swelling. The discomfort had continuted, but it was not sufficient to keep him from his occupation. My diagnosis was an inflamed cyst in the canal or an incarcerated piece of omentum surrounded by fuid, which commonly occurs in the latter condition. Favoring especially the last-named diagnosis, an operation was advised, and the doctor invited me to do it. My surprise was very great when, on splitting the aponeurosis of the external oblique muscle, I found confined to the limits of the canal an abscess containing about half an ounce of pus. Careful examination convinced us that this did not conmmunicate either with the abdominal cavity or with the subcutaneous parts. The young man disclaimed any venereal disease, and how infection had taken place in this munusual location we were unable to decide. This was not an ingtinal adenitis, but an abscess within the inguinal canal.

Sarcoma, and even carcinoma, of the inguinal gland must be considered in making a differential diagnosis of incarcerated or irreducible hernia. One case sent to me as irreducible ingtinal hernia, was sarcoma, which later invaded the abdominal cavity and caused the death of the patient.

DIAGNOSIS OF FEMORAL FROM INGUINAL HERNIA.

Ordinarily this is not in the least difficult, but in some instances may be extremely so. If hernia is found in the scrotum or labium it is at once recognized as having an 
inguinal origin. Femoral hernia usually protrudes as a small, circular swelling, in the femoral space just below Poupart's ligament, but it sometimes comes in contact with resistent tissues that turn it sharply upwards, and it then occupies almost exactly the position of an incomplete inguinal hernia. Such a hernia is always reduced with difficulty, and this fact alone, occurring in a moderately small hernia, should lead us to suspect that it is of the femoral variety. In addition to this, howerer, is the fact that reduction in femoral hernia, especially of the kind named, will be clownwards and backwards, apparently directly into the thigh. As the hernia slips back the finger can usually follow it into the aperture through which it has returned. Pressure over the inguinal canal will also demonstrate the fact that this is empty. A test that $I$ have used for many years in cloubtful cases and that has proven valuable, is as follows:

Locate the spine of the pubes and place a finger upon it. Then, by the fingers of the other hand, locate the iliac spine upon the crest of the ilium and place a finger upon that. Now draw an imaginary line between the two, representing Poupart's ligament, and if the bulk of the tumor is below that line it is femoral: if above it is inguinal. In this connection it is also well to remember that the internal ring is just half-way between the spines upon which the fingers rest.

The pubic spine is an important landmark, complete inguinal hernia passing over the pubic bone between it and the median line. and femoral hernia always presenting outside and below this bony prominence. It is well to remember that femoral hernia is rery rare in youth and childhood, and that while it does occur in the male, it is far more common in the female.

PROGNOSIS.

A small reducible inguinal hernia is not in itself a serious matter. but the fact that it carries with it potentialities that may at any moment transform it into a highly dangerous condition, is sufficient to make it a matter worthy of the most 
earnest solicitude on the part of both doctor and patient. These dangers increase with the degree of development, and neglect merely invites disaster. It is a condition that demands immediate attention, as its history from earliest beginnings is one of constant increase in size and added complications, if allowed to go mattended. Sometimes, though rarely, physicians advise mothers to allow their children to outgrow hemia; this is wrong and due to utter ignorance of the subject.

Hernia has, until recently, been classed among the incurable surgical affections, but, fortunately for humanity, it has finally been permanently removed from that list. There remain, lowever, two forms of treatment, palliatiz' and curatio' ${ }^{\prime}$, and while the physician may advise, the patient must elect which form is to be used.

Palliatice treatment may, under certain conditions, be also curative, but in by far the greater number of cases it is only temporizing, and the patient is carrying with him a degree of dlanger that is greater than that involved in submitting to methorls designed to cure the affection. Palliative methorls consist in the adjustment of retaining apparatus, such as trusses, bandages, etc., that prevent the protrusion of the hemia, and, while they remain efficient, protect the patient from the danger of strangulated hernia. In very young children the wearing of such apparatus for one, two, or more years frequently produces a permanent cure, but in the adult this fortumate result is seldom obtained, and they are obliged to continue the use of artificial support throughout life.

Curatioc treatment consists in a surgical operation for the removal of abnormal conditions and restoration of the parts to their normal strength. For many centuries it was the opprobrium of surgery that attempts in this direction not only uniformly failed, but were attended by serious danger. One of the greatest achievements of modern surgery is that not only have the dangers been almost wholly eradicated, but that the degree of success attained is far beyond the expectations of the most hopeful. 


\section{MECHANICAL TREATMENT OF INGUINAL HERNIA.}

There can be no question respecting the rapid advance in the surgical treatment of hernia during the past ten years. Operations for the relief of this defect have not only become comparatively safe, but any impartial observer must admit that more cures are being obtained than were formerly thought possible. The increased safety in operation is unquestionably due to the wonderful advance made in all branches of surgery, while the increase in the percentage of cures results not only from improved methods, but from the fact that now many cases of small and recent hernia are being operated upon, whereas in former years only those cases considered desperate were subjected to surgical treatment.

No matter how much advance there may be in the art of surgery and in the cure of hernia by operative means, the mechanical treatment of this affection must always remain an important source of relief to a large number of sufferers. Patients requiring artificial support for retention of hernia form a great army of sufferers who look to the general practitioner for aid. He should not allow them to drift about and put up with the services of the inexperienced and non-medical truss seller, but take them under his own care and know that they are perfectly protected from the danger of strangulated hernia. The wearer of a truss is a chronic invalid, and he should submit himself to his physician, at regular intervals, for inspection and the correction of defects in the truss and in the manner of wearing. Some neglected and complicated hernix may demand the attention of those who have had large and special experience, but the prevention of this extreme condition rests with the general practitioner, and he can in all ordinary cases carry out the necessary details for securing the safety and comfort of the patient. 
Thirty years ago the practice of that special branch of surgery now known as orthopedic was almost wholly in the hands of instrument makers and " quacks." The crippled and deformed have been rescued from the hands of the ignorant,

FIG. 5S.

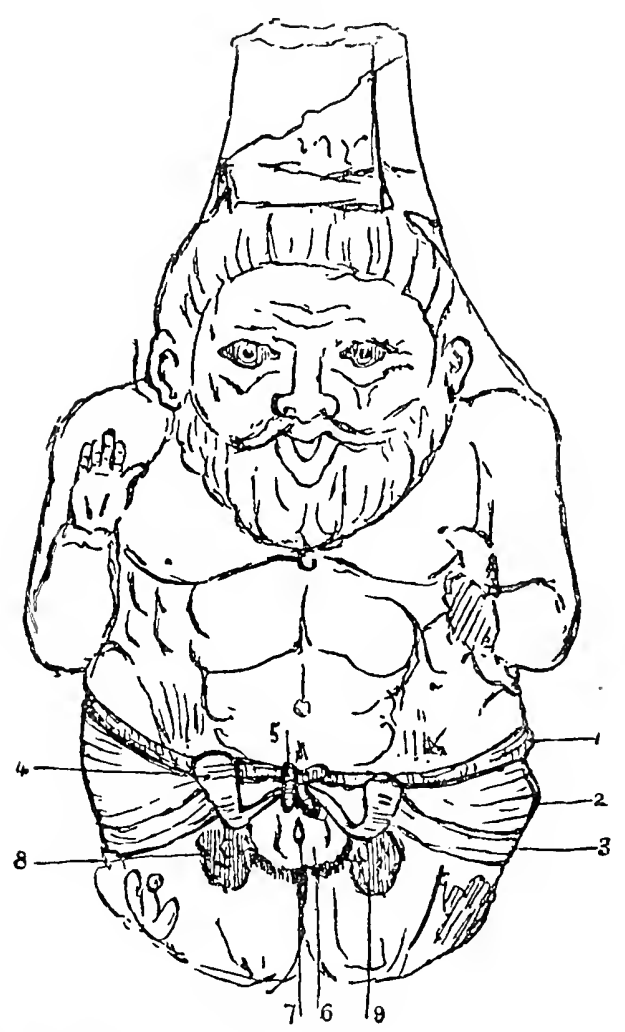

Statuette found at Susa by M. Fouvet while exploring an ancient Phcenician cemetery; pronounced by M. Maspero and other Egyptologists to be an example of the Egyptian deity Bés, or Bizou, and to date back to about the year goo B.C. The fortunate owner is Dr. F. Poncet, Cluny, France. It shows a truss applied to double inguinal hernia, and also presents double femoral hernia. (For full description see Le Progris Mídical, June r, 1.ion.)

and there is, perhaps, no more careful scientific surgical work than that done by the orthopedist. Quackery, undoubtedly, still exists in this line, but it is not supported by the wealthy and 
intelligent people as in the past. Instrument makers construct orthopedic apparatus, but they seldom either devise or apply then. They certainly do not treat the patient. For many years the author has hoped to rescue the mechanical treatment of hernia from the hands of those who know nothing of the anatomy, diagnosis, or pathology, and therefore little of the proper treatment of these important cases. It was hoped that orthopedic surgeons would introduce mechanical treatment of hernia into their special work. As one of the founders of the American Orthopedic Association, he endeavored to convert his friends to his views, but failed. No members of the profession are so well educated to do the mechanical work as they, but now, since the great advance in the surgical side of the subject, it is still farther away from them.

This is a misfortune to the large number of truss wearers that must always remain with us, no matter what advance is made in surgery, because it leaves them in the hands of those who must necessarily do poor work and make serious mistakes, no matter how honest their intentions may be. Druggists and instrument makers should continue to sell trusses, but the diagnosis of the case, selection and application of the truss, and subsequent care of the patient, should be in the hands of those who have been qualified by medical education to fulfil these duties. These remarks are not intended to reflect upon men who, while they have not had a medical education, have from many years' experience as truss fitters become expert in this line of work and fairly accurate even in diagnosis. Many of these men have taken the trouble to study the anatomy of the parts involved in hernia. The most serious charge that can be made against some of them is, that they frequently favor some special style of truss, and their expertness, as applied, lies more in their ability to fit the patient to this truss than in the selection of and fitting to him a truss best suited to his condition.

History and Construction of Trusses.-The wholesale manufacture of trusses has become an important business in this country, and correspondence with all of the larger firms 
enables me to state, that a conservative estimate of the number made every year would be fully three fuarters of a million. Some of the manufacturers gave a much higher estimate, ancl the one given is that of the most conservative. One maker alone claims to manufacture this number, but he not only has an American trade, but does an extensive export business. Truss manufacturers, many of whom have had large personal experience, have done what they could to aid their patrons, the retailers, in the art of truss-fitting. For this purpose, some instrument makers have found it advisable to select an employé who shall have acquired skill in the fitting-room, but to the average druggist truss-fitting is distasteful, and most frequently he sells the truss after securing a measure of his customer, usually taken over the clothing. On the other hand, physicians, as a rule, avoid truss-fitting because of their total lack of instruction during college days. They have been duly instructed regarding the alarming conditions found in cases of strangulated hernia, and the operation for its relief, but have not been taught how to protect their patient against this danger by the proper application of a truss. They have been especially well drilled in how to apply " Professor Somebody's pet splint," but of truss-application, which they will have ten opportunities to make for every fracture they see, nothing has been taught them. The patient is therefore in the unfortunate dilemma, between the truss seller and the doctor, of having to select and usually to apply his own truss.

This work of truss-fitting is even more foreign to the calling of the druggist than would be the prescribing of medicines. He has usually a very good knowledge of materia medica, but knows little anatomy, pathology, or surgery. The responsibility is therefore with the physician, and here it must rest. When he sees a case of hernia he should decide upon its needs, select the truss, apply it, and give his patient to understand-as long as he wears a truss he requires the doctor's care, and that he should report several times each year for inspection, even though everything seems, to him, in perfect condition. Some 
manufacturers devote a portion of their catalogues to instructions in truss-fitting, but one of them sums up the whole subject in the following laconic sentence: "The best way to learn truss-fitting is to do it." This is good advice to the physician, but in this work I hope to give a more serviceable guide than has hitherto been within the reach of the general practitioner; the surgical part of the subject has been skillfully dealt with by others, but the equally important mechanical part has been given only superficial attention.

Trusses are remedies for the treatment of hernia, and from the excellent variety made it is usually possible to select one suited to the requirements of a case in hand. He who expects to treat all types of hernia with a single form of truss is as far wrong as the doctor who is looking for one drug, or a combination of clrugs, that shall be suited to the cure of all his patients. For this reason patented trusses are unreliable, as a rule: in fact, I know of no patented trusses worthy of extensive use.

A considerable variety of good trusses are made by reputable manufacturers, who are rery willing to make any rariation that physicians may suggest in their construction. The difference between such trusses is largely in detail of construction, the types being essentially the same, and none will be mentioned in this work that cannot be obtained from any of the large producers. The United States leads the world in production; in fact, it is safe to say that more good trusses are made in this country than in all of the rest of the world put together.

\section{CLASSIFICATION OF TRUSSES.}

It has been a serious problem to present to my reader a large variety of good appliances, from which he may select intelligently, without transforming my work into a trussmaker's catalogue. It is essential, however, that the reader be shown what is believer to be the better types, as well as some of the lack ones, and told wherein the latter are defective, so that 
Group of Springless or Elastic Truss Type.

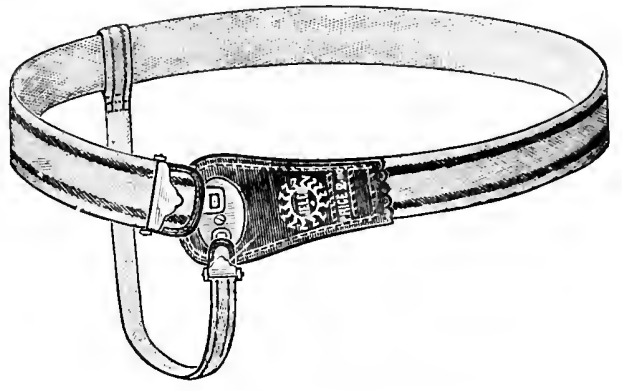

r. Ordinary single elastic truss.

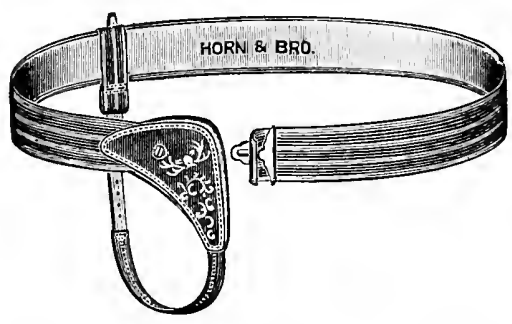

2. Elastic truss with special parl.

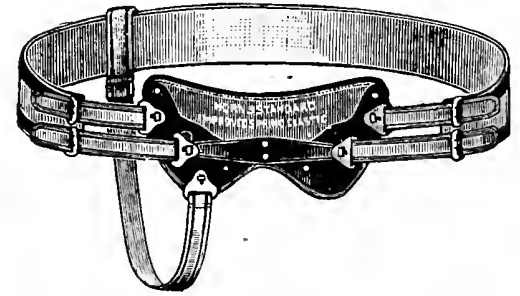

3. Double elastic truss with elliptic spring on front plate.

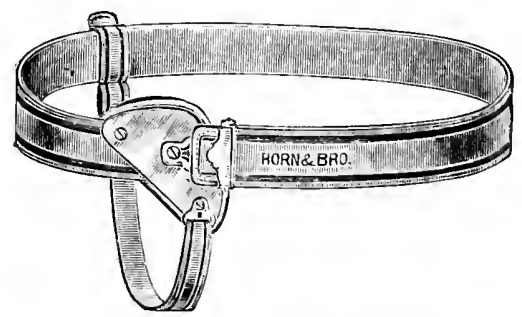

4. Elastic truss with German pad. 
Grovp of Springless or Elastic Truss Type (Continued).

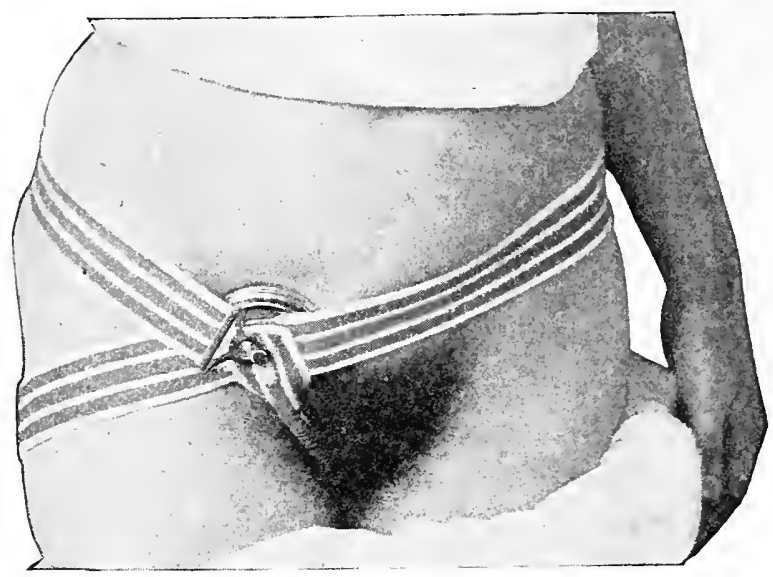

5. One piece elastic truss.

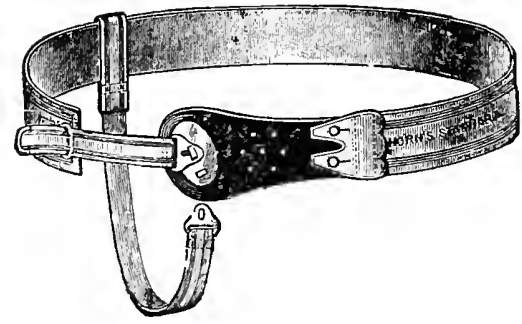

6. Elastic truss with hard rubber front plate.

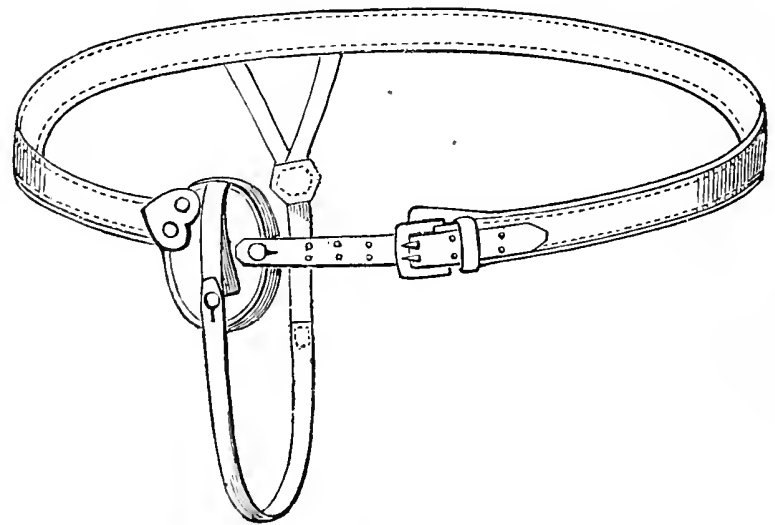

7. Moc-Main (English) truss, leather. 
he may discriminate against them. I have encleavored to solve this problem by grouping together those of a certain type and speaking of them in a general way, rather than incliviclually. All worthy of favorable or unfavoralble mention have been placed in a few groups, and the reader can readlily fix in mind the principles involved in each. This grouping is also in a measure historical, for, as a matter of fact, endless varieties have descended from a few types. These groups have been placed in the following historical order:

(I) Springless type. Earliest history (900 в.c.) to date.

(2) French-German type. I306.

(3) Cross-body type (English), Royal Pat., October 27, 1806.

(4) Chase type (American). 1837.

(5) Hood type (American). 1847.

(6) Unclassified.

Perhaps the remotest historical record of the truss is contained in the statnette, a cut of which is at the beginning of this chapter. There is little doubt that the earliest form consisted of a pelvic belt with a compress over the inguinal canal, held in place by a perineal strap. From this prehistoric truss used by many nations, civilized and uncivilized, has descended the group of springless trusses, a very few of which are shown. In this country they have been best known as the "elastic truss" because of their being made of heavy elastic web. In England this type is known as the MocMain, and consists of a strong pelvic belt, made of leather, to which is attached a good-sized parl held down by a perineal strap (no. 7 of group). It is believed to be in many respects better than our elastic truss. It is more durable, and, being nonelastic, is more reliable in the retention of the hernia. There is also upon the pad a small spring, to which the perineal strap buttons, that turns its lower end under, giving an, upward as well as a backward pressure.

I regret to state that, in this country, more elastic trusses are sold than any other form. That this is not because of their 
122

ABDOMINAL HERNIA.

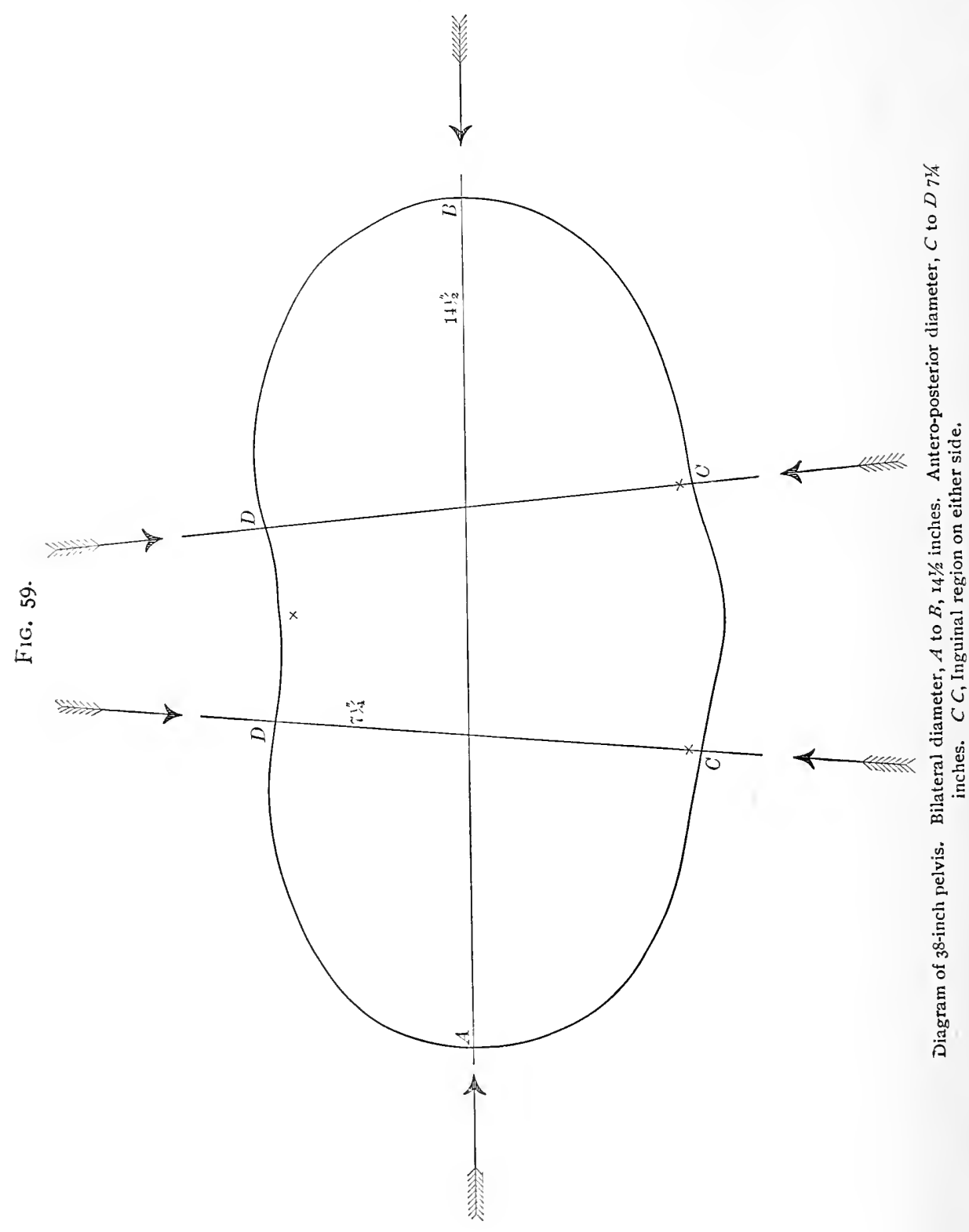


greater value is clemonstrated by the fact that some manufacturers who supply such a large demand seldom use them, in their own fitting-rooms, when the truss-selection is left to their best judgment. Their extensive sale is due largely to the fact that they require no fitting. Those suffering from hernia can buckle them on without difficulty. Furthermore, they wear out much sooner than other forms, the wearer frequently being obliged to renew his truss two or three times a year instead of wearing it several years. Springless trusses are, however, very defective in action, and their use in many instances results in serious trouble. Few people wear them for any length of time without finding their hernize worse than when they began. This occurs because the pad is drawn down against the pubic bone by the perineal strap, leaving the upper part of the canal unprotected and consequently occupied most of the time by a part of the hernia. A hernia held at the external ring only, is poorly held and sure to increase. If the pelvis were as round as a barrel, the springless truss would be more effective, but as its transverse diameter exceeds by fully one-third its anteroposterior diameter, it is thoroughly unscientific (fig. 59).

A flexible band surrounding the hips and drawn tightly, will produce far more pressure over each hip than over the inguinal region where it is needed. A band of this character will not maintain its position upon the body without the perineal strap, and this is, for reasons which will suggest themselves, an abomination, as well as positively injurious. The importance of retaining hernia within the intcrnal ring cannot be too strongly emphasized. In many instances injury results from placing the supporting pad over the pubic bone and external ring, allowing thereby the upper part of the canal to be constantly occupied by a loop of bowel or piece of omentum, and compressing the cord against the bone. producing atrophy of the testicle in some instances. Added to this is their lack of cleanliness, which alone is quite enough to condemn them for general use. They have one valuable use and that is, as a night truss. Ordinarily this is not needed 
by the adult, but if from the enormous size of the hernia or a persistent cough the protrusion takes place at this time, these springless trusses serve a good purpose. There is little doubt that most wearers would get a greater degree of improrement by the use of a night truss, but the one that is cractly suited for the day is cntircly unsuited for the night, and the reverse holds equally true.

French, German, and English Trusses.-In the second group is shown a type of truss that has been well known in this country, and in most foreign countries, for many years. There are an endless number of variations in the minor details of its construction, but the general type remains. Doubtless it represents the first form of metal spring, and it had its origin either in Italy or France, probably the latter. The first recorded use of metal for a truss spring was, according to Macready (A Treatise on Ruptures, Jonathan F. C. H. Macready, F. R. C. S., p. I95), the iron-band truss recommended by Gordon in I 306 . Steel was first used by Nicolas le Quin of Paris ("The Sign of the Golden Truss") in I628. It is possible to present only a few of the numerous descendants of this type. In this country they have been known to manufacturers as the French (nos. 6, 7 . and 8 of group) or German (nos. 3,4 , and 5 of group) truss, the only distinguishing feature between them being that the former are made lighter, and usually bear some decorations in the form of fancy stitching or embossed flowers upon the leather forming the outer surface of the pad.

This form of truss as made in England (nos. I and 2 of group) is superior in two important particulars to those made here, and whether their origin was the same is not known. In the French-German truss the spring passes around the back to a point three or four inches beyond the spine, terminating over the gluteal muscles. In the English form this spring continues far enough around to clasp the opposite hip, thereby holding itself securely in place. It also has the retaining pad placed in a line almost parallel with the spring instead of arching 
Group of Trisses, French, German, axi Exrilisil TrPes.

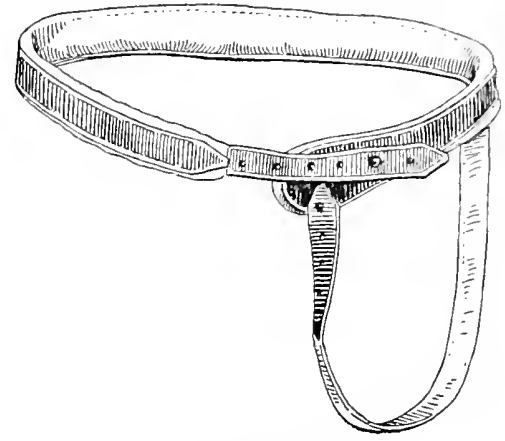

I. Single English type.

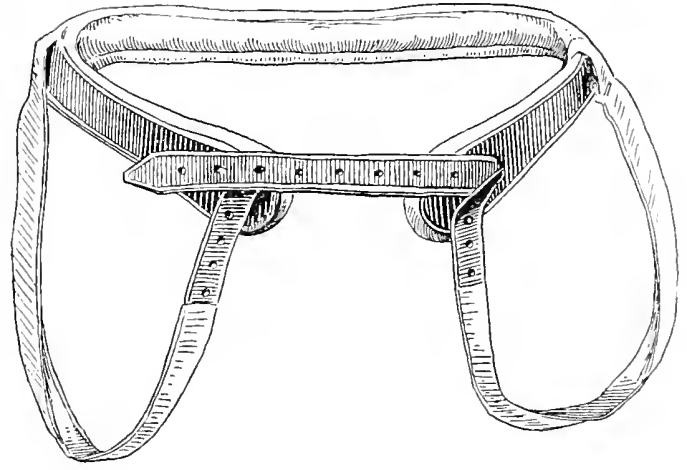

2. Double English type.

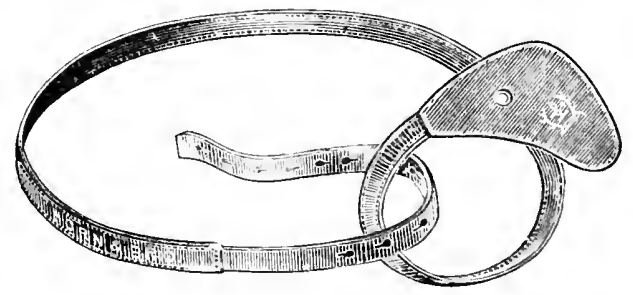

3. Single German type. 
Group of Trusses, French, German, and English Types (Continued).

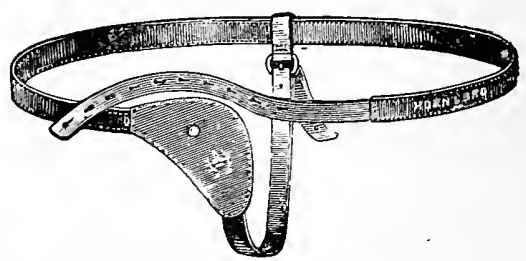

4. German type (so-called "scrotal-hernia truss").

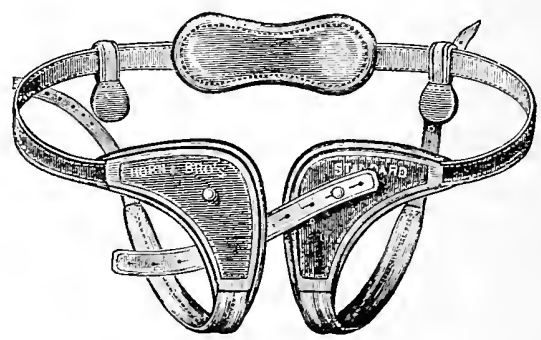

5. Double German truss.

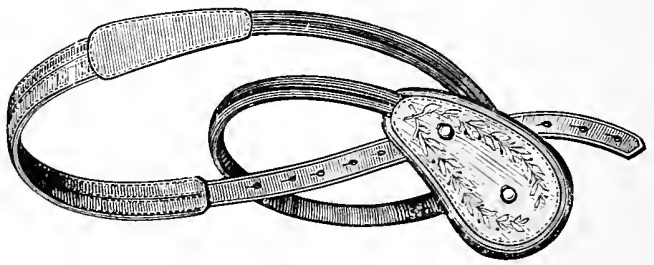

6. French truss.

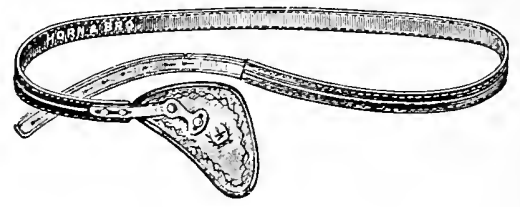

7. Adjustable French truss.

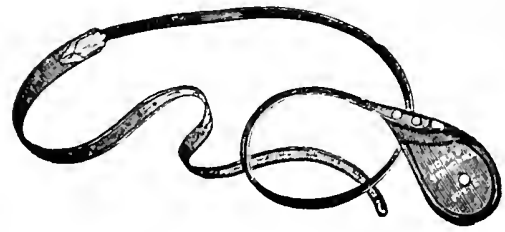

s. Hard-rubher or celluloid Frencl truss. 
abruptly down over the pubic bone as in the French-German truss. These are two serious lefects in most of the trusses made in the United States and Germany. Fortunately some of the larger manufacturers are recognizing this defect and encleavoring to correct it. The centre of the truss pad should be very nearly on a line with the centre of the spring, in order to have it effective and comfortable. When the pad is thrown

FIG. 60.

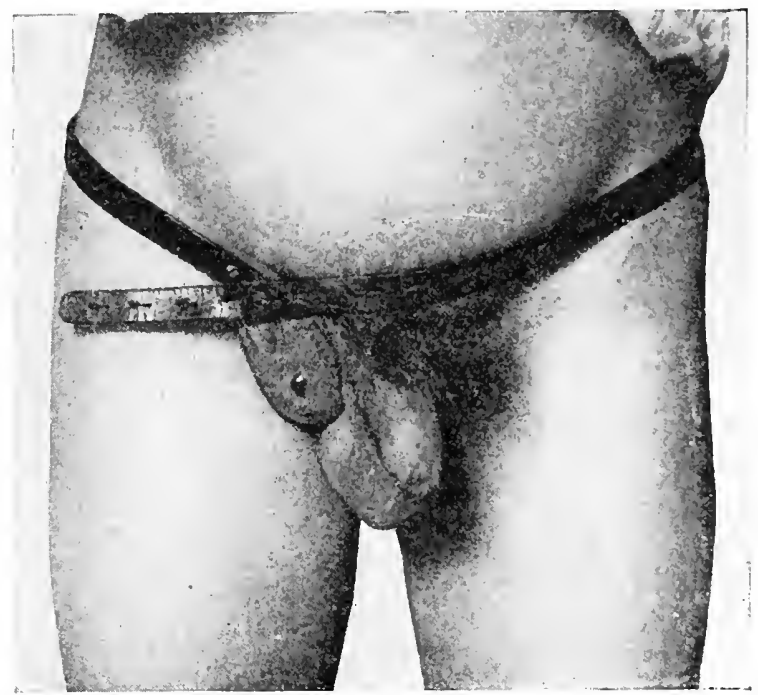

Typical illustration of bad truss fitting with a French-German truss, frequently seen. Note that the pad acts as a compress directly over the pubic bone, and that the hernia is in the canal above.

down so low that its lower edge rests upon the pubic bone it ceases to be a reliable support, acts as a compress over the bone, and may for a time keep the hernia out of the scrotum, but the canal is gradually being dilated to such an extent that the hernia eventually becomes almost, if not quite, uncontrollable. The accompanying illustrations (figs. 60, 61, and 62) show this serious defect in truss-making and truss-wearing. The first pictures the case of a young man who, uncler the use of this 
type, had gradually grown worse until it was almost impossible to retain his hernia, it is not uncommon to see these trusses worn in this way. The second photograph shows him with a Hood truss holding his hernia in proper position. As he was weak upon the left side, a thin pad for moderate support was placed over that region.

Perhaps the worst feature of this form of truss, as now made, is in the fact that even if the pad is properly placed over

FIG. 6I.

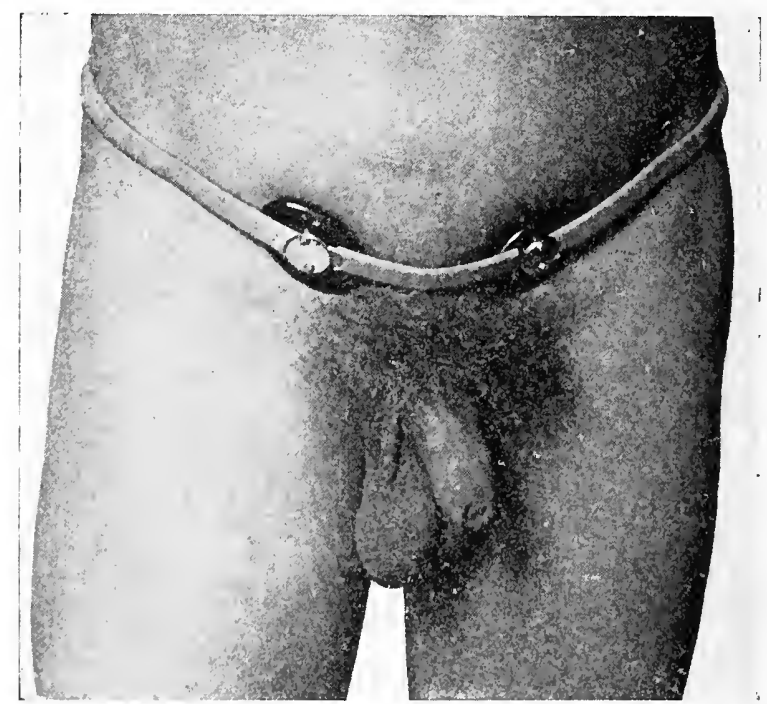

Same case as Fig. 60, with properly adjusted De Garmo-llood truss retaining hernia within abdomen and with thin pad for support of opposite side.

the ingtinal canal, it will in a short time clrop down over the pubic bone. The reason for this is that the spring naturally seeks the spot around the hips where it is least influenced by muscular action. This neutral point is midway between the crest of the ilium and the trochanter major (fig. 63), above the active muscles of the thigh and below those of the abdomen. In this position the front end of the spring terminates over 
the middle of the canal. If the retaining parl is two inches below the spring, it will be seen at once that it must rest upon the pubic bone when the spring rests in its normal position. This criticism applies with equal force to all of that vast variety of trusses of the type shown in the group under consideration and by the Chase type, to be spoken of shortly. The English

FIG. 62.

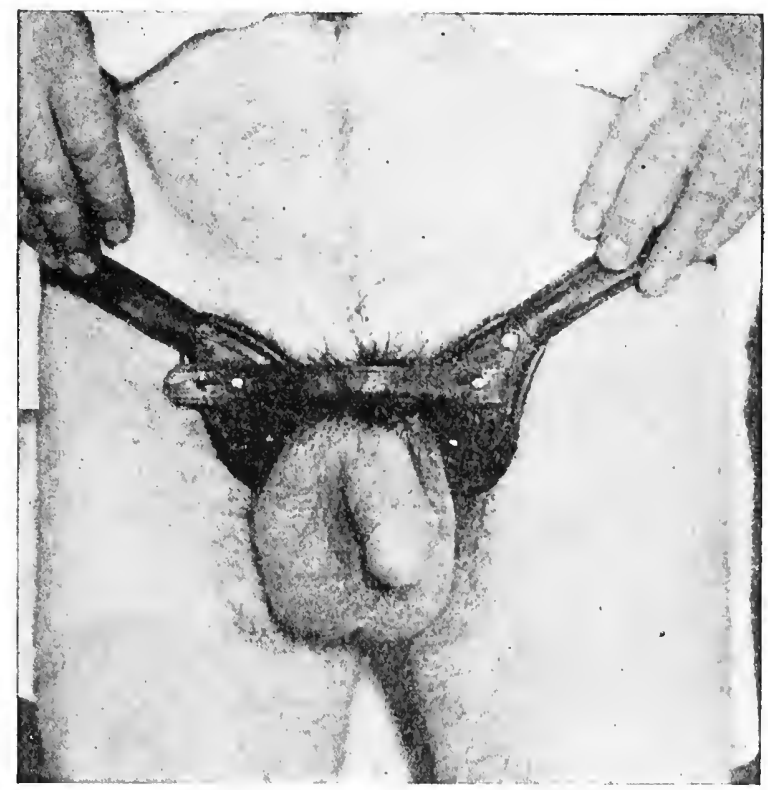

Usual manner of wearing the so-called German style of truss. The hernize on both sides are protruding into the scrotum. The canals are entirely unprotected.

type of this group is very good, and the farther away from this form the more imperfect is the truss of this pattern. While trusses of this group have an enormous sale few expert trussfitters use thenn; the German instrument and truss makers. however, use them almost to the exclusion of all others.

Cross-Body Type.-Towards the close of the eighteenth century, Salmon \& Ody, an English firm still in existence, made 
an important change in the form of truss springs, establishing one of the most valuable types of truss that we now have for the treatment of single inguinal and femoral hernia, known as the cross-body truss (no. I of group). The spring, instead of surrounding the hip on the side of the hernia, passes from the canal directly across the lower abdomen and around

FIG. 63.

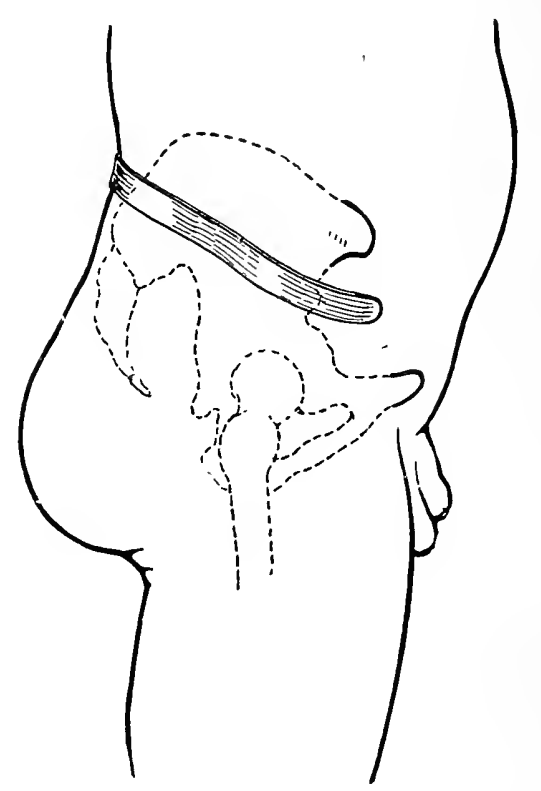

Position in which spring should rest about hip. Midway between crest of ilium and trochanter major, its end in front over internal ring. If it comes across abdomen it should term:nate at the same point.

the hip of the opposite side. As originally made this truss had a convex par over the inguinal region, held to the spring by a ball-and-socket attachment. The spring after passing across the abdomen and around the hip opposite the hernia, terminated over the spine, where there was another circular pad held by the same methorl of attachment.

This truss was later modified, in this country, by putting 
Group of Trusses of the Cross-Body Type.

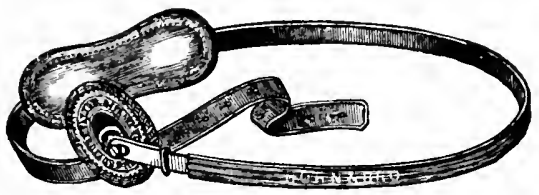

I. Cross-body truss, leather cover, ball-and-socket pad

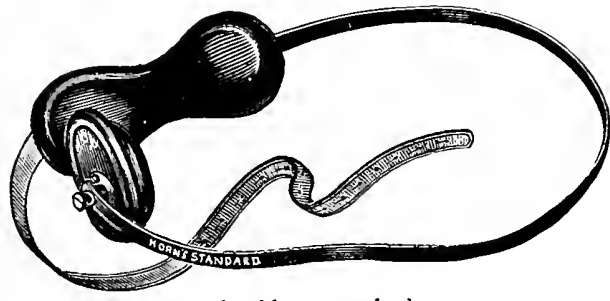

2. Hard-rubber cross-body truss.

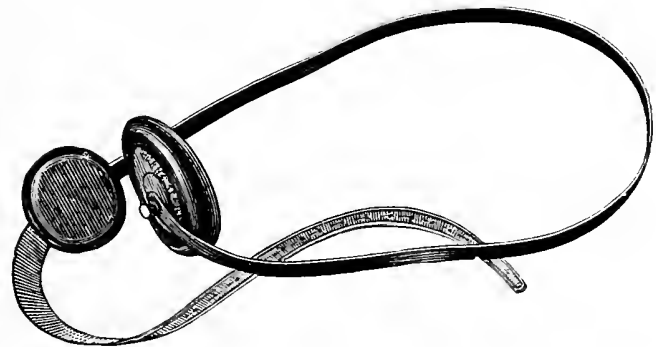

3. Hard-rubber, cross-body truss, small back pad. Continuous-spring.

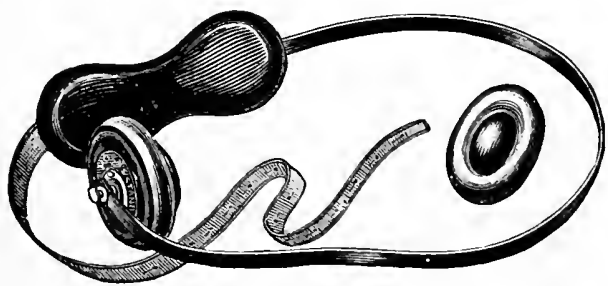

4. Hatd-rubber "radical-cure truss." 
Group of Trusses of the Cross-body Type (Continued).

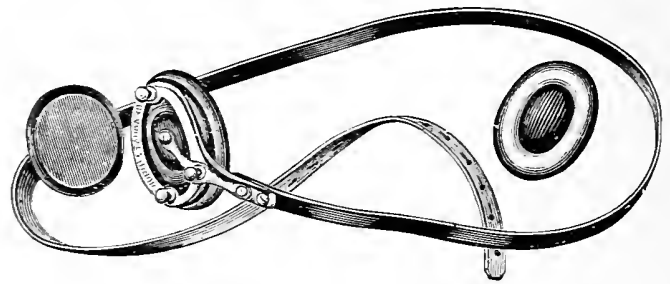

5. Radical-cure truss. Continuous-spring cross-body

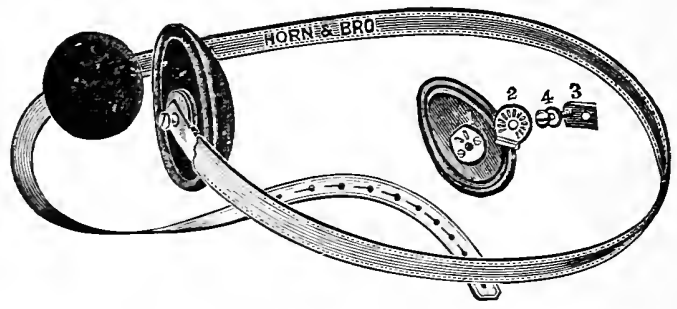

6. Leather covered continuous-spring cross-body truss.

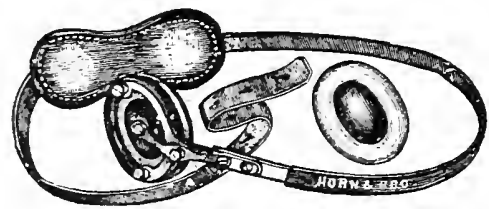

7. Radical-cure truss, large leather covered back pad.

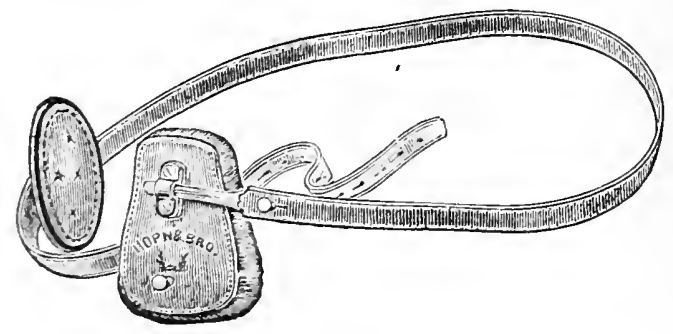

8. Army and Navy truss. 
Group of Trusses of the Chase Type.

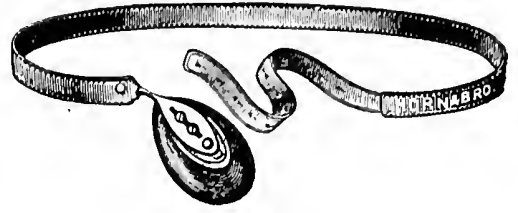

I. Chase truss.

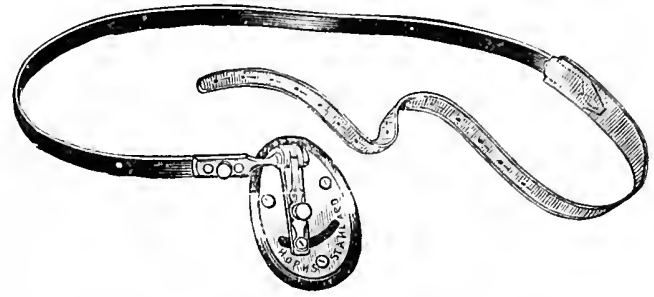

2. Foster ratchet modified Chase truss.

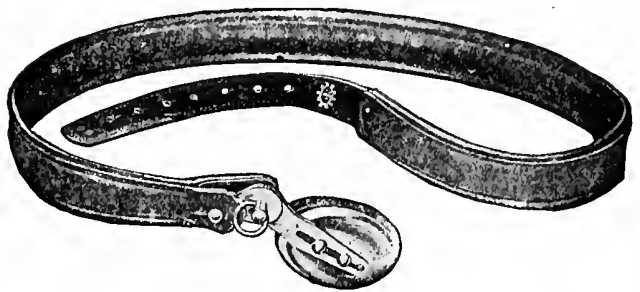

3. Adjustable pad Chase truss.

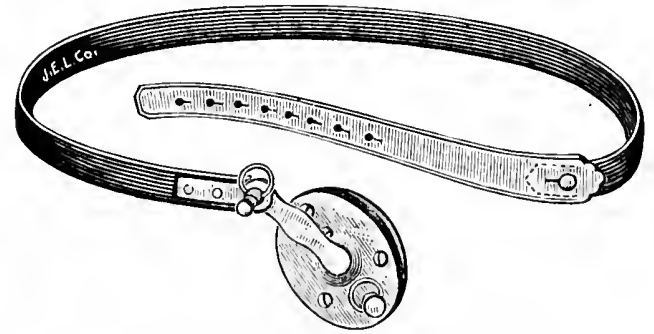

4. Adjustable ball-and-socket, with set screw. 
Group of Trusses of the Chase Type (Continued).

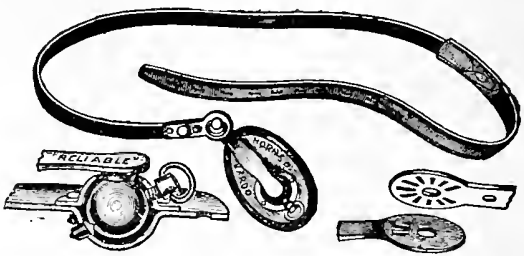

5. Modified set screw Chase truss.

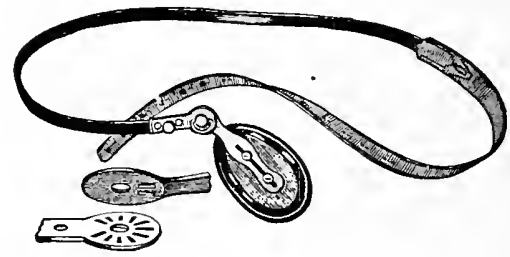

6. Modified hard-rubber Chase truss.

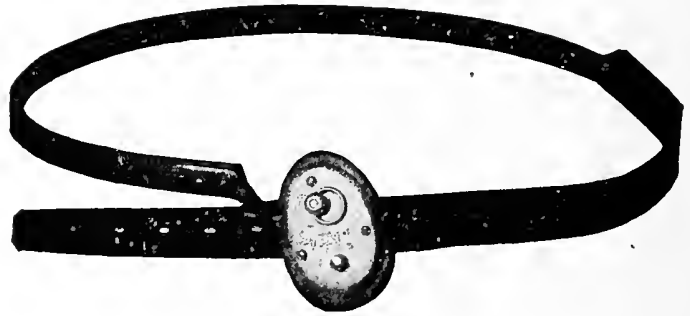

7. Curved neck, ratchet pad.

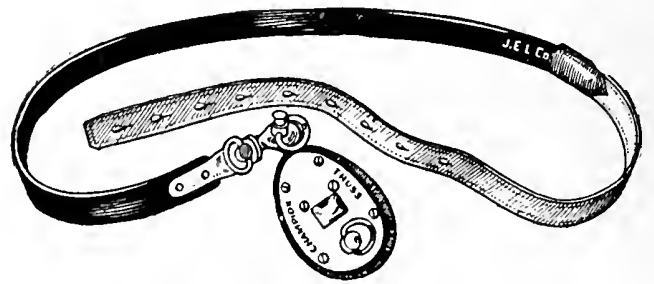

8. Adjustable pad and set screw. 
on an elongated back pad (nos. I, 2, and 4) that should press on either side of the spine, its centre being arched; and by making the spring longer (nos. 3, 5, and 6) so that it would terminate over the gluteal region, directly back of the hernia, either in a circular pad or continuous with the strap that completes the circumference. The special advantages of this spring are-that it surrounds fully three-fourths of the body and, when properly fitted will retain its position, even though no strap is used; it furnishes a longer, and therefore a more elastic, spring. The direction of the pressure is from the front pad to the centre of the back, and as it crosses the back at a slight elevation over the front, it has a slight upward pressure. It is convenient to the dealer because it can be quickly converted from a right- to a left-side truss. There are many modifications as to the form of retaining pad and its method of attachment to the spring, but, while convenient, they are not essential.

In this form, with such modifications as can be made to suit individual peculiarities, we have one of the most valuable appliances for the treatment of hernia that has ever been devised.

Chase Type.-This form began with the truss bearing the name of its inventor, Dr. Heber Chase, 1837 . It was issued during the war of the Rebellion (I86I-5) to soldiers who developerl hernia in the service. It consisted of a spring of the French type to which was attached in front a soft, malleable iron neck, curved downwards, holding a polished cedar pad. The pad was held by screws, passing through a slot in the iron neck, so that it could be raised or lowered and the neck easily bent into any desired position. The truss was considered quite an improvement on its prototype which undoubtedly was the French truss. It has many descendants, a few of which are shown in their group.

To a lesser degree they are all open to the objections that have been offered to the French type. The pad-centre being considerably below the spring-centre is an objection already mentioned. Also, the springs like all those that go on from the 
same side as the rupture, are dependent upon the strap for retention of position, and when this stretches, or is improperly adjusted, they are very liable to shift their position and allow the hernia to protrude. For perfect fitting, comfort, and security, they do not compare favorably with the cross-body or Hood type of truss.

Hood Type.-This is apparently an original type, purely American, the invention of Dr. J. W. Hood of Kentucky. In its existence of nearly seventy years, this truss has passed through many hands and has been the subject of many improvements, but its general type remains. Its spring is solid in front, surrounding both hips, and terminates within about two inches of the spine on either side. Usually it has circular pads attached to the spring ends in the back upon which the counter pressure is taken, but some makers put on instead a flat, oblong disk which broadens the spring at this point and distributes the pressure over a greater surface. The retention pads used in front are of various shapes and designis, and may be selected to suit individual requirements. The original Hood pad is, however, for general use, an excellent form. It is thick at its lower edge, thin at the top, and in action presents a moderately convex surface over the inguinal canal. The pubic portion of the Hood spring has, on either side, a slot which runs parallel with, and is clirectly over, the inguinal canal. Transversly to this slot is another in the pad, and between the two there is quite a wide range of movement for accurate adjustment. When the adjustment is complete the pad is soliclly fixed to the spring by set screws, which prevents motion between them while in use.

The action of this truss is peculiar in that it does not depend wholly upon compression or spring action. It has, in fact, been extensively and very successfully used in a metal that has scarcely any spring action. It acts as a resisting frame about the pelvis with the pad making firm pressure over the inguinal canal when the wearer is in an upright position. Should he cough or strain, the abdominal wall is thrown for- 
MECHANICAI, TREATMENT.

Group of Trusses of Hood Type.

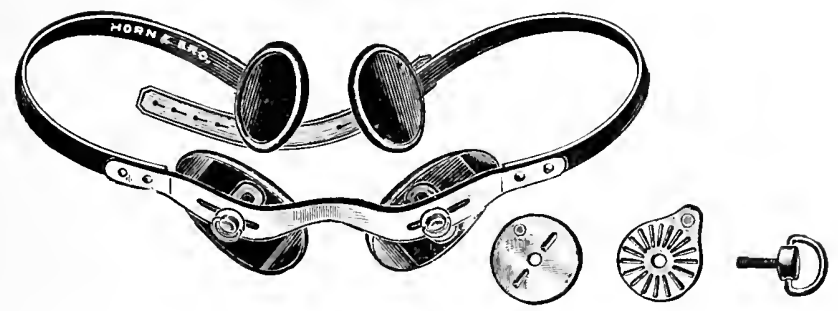

I.

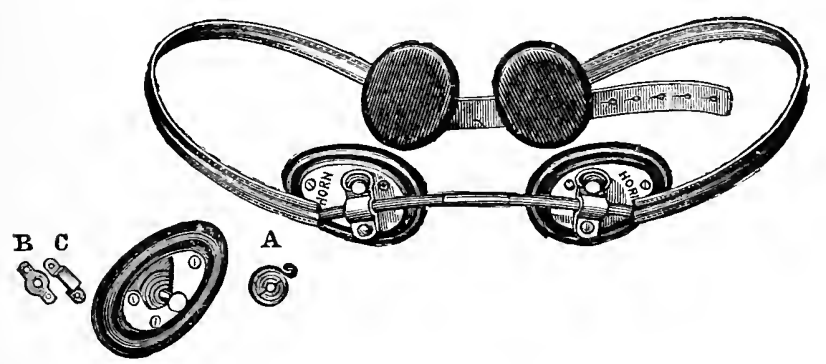

2.

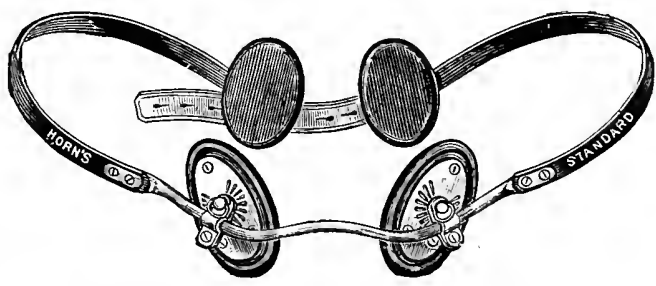

3.

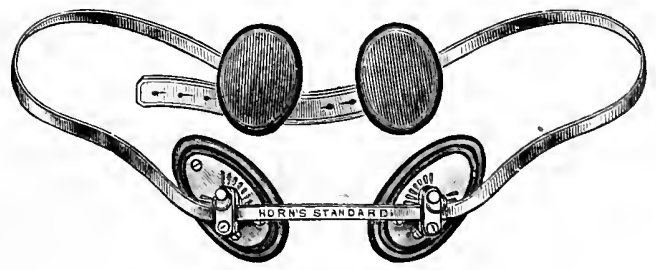

4 . 
Group of Trusses of Hood Type (Continued).
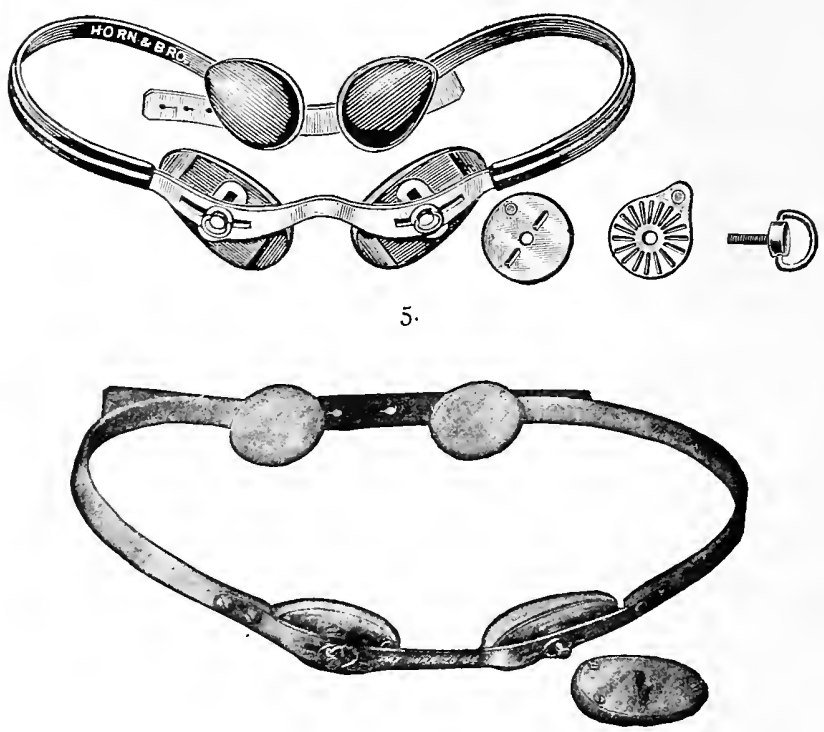

6.
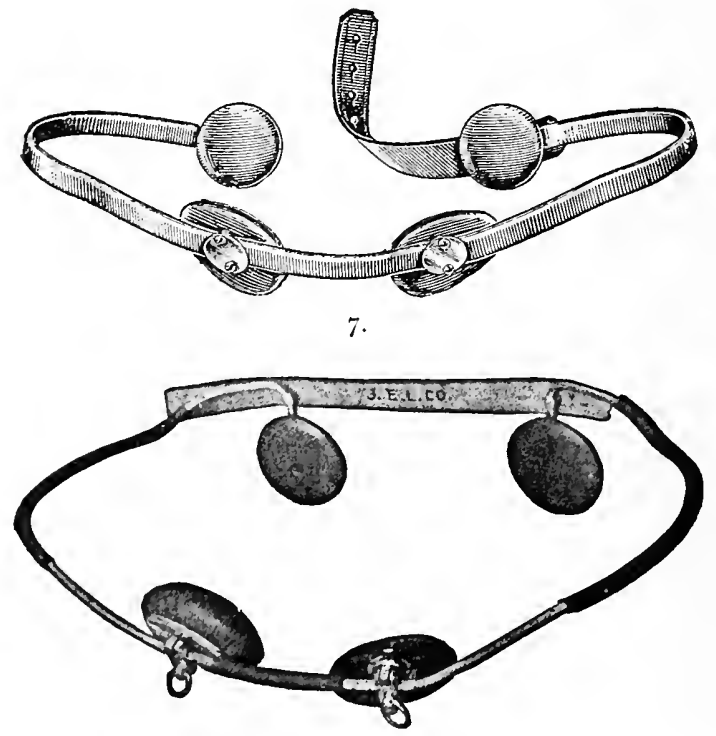

s. 
Grocp of Trusses of Hood Trpe (Continued).

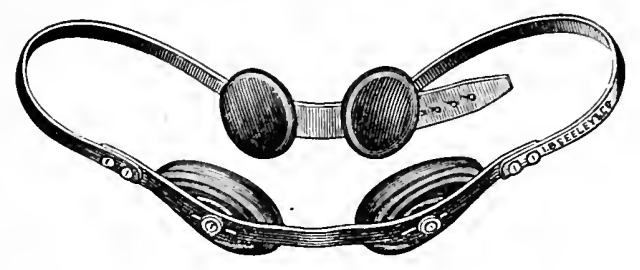

9.

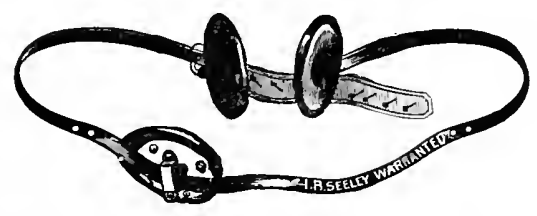

IO.

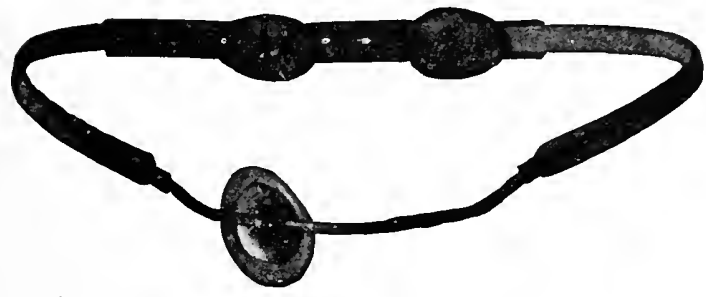

II.

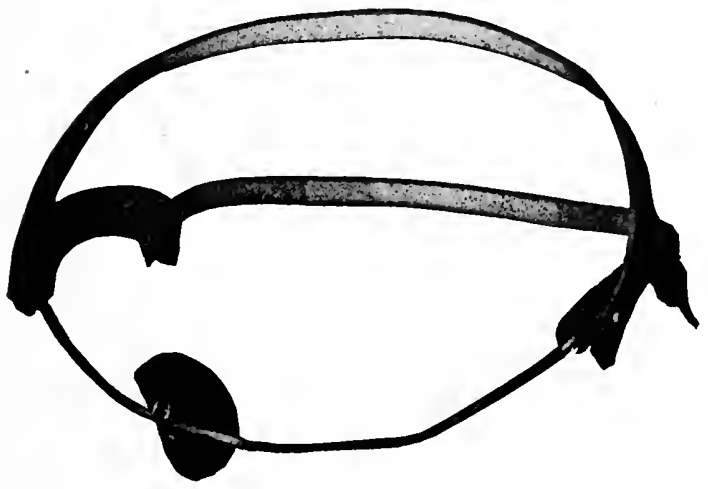

T2. 
ward, but firmly met and restrained by the pad fastened to the pelvic frame. If, however, he should lie down, the frame would not follow to any extent the receding surface of the abdominal wall. Many truss makers have not fully comprehended this action of the Hood truss and have made the

FIG. 64.

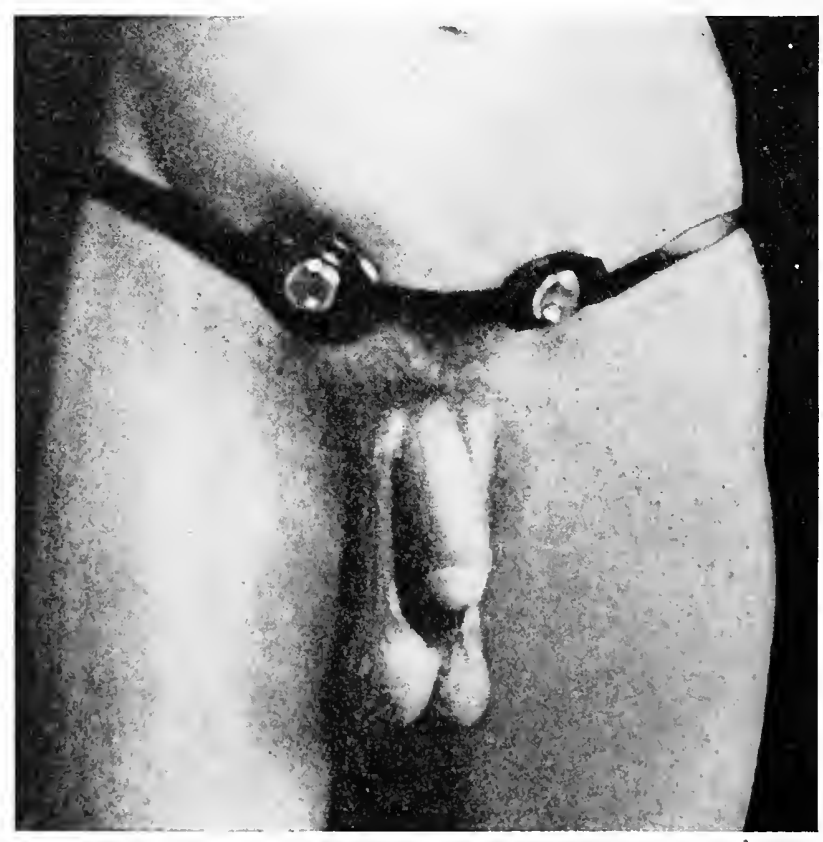

De Garmo-Hood truss applied. Right complete inguinal hernia. Left incipient hernia retained by thin "dummy" pad.

springs too heavy and with too much action, this error being fostered by the necessity of strong spring action in other forms of truss. The fact that in this truss a larger amount of compression can be clispensed with, makes it a much easier form for the patient to wear. When completely at rest, the wearer is in a measure reliever of pressure, which is a great comfort, fuite in contrast with the tireless and never ceasing pressure 
from a spring; the ends of which are endeavoring to come together.

The Hood spring should surround the pelvis very nearly in a straight line, and some manufacturers have ruined it by arching the sides too high. As soon as this is clone it loses its pelvic-frame action, the pads drop down over the pubic bone, and the truss then has many of the oljectionable features of the trusses which have the pad set on $a$.

FIG. 65 .

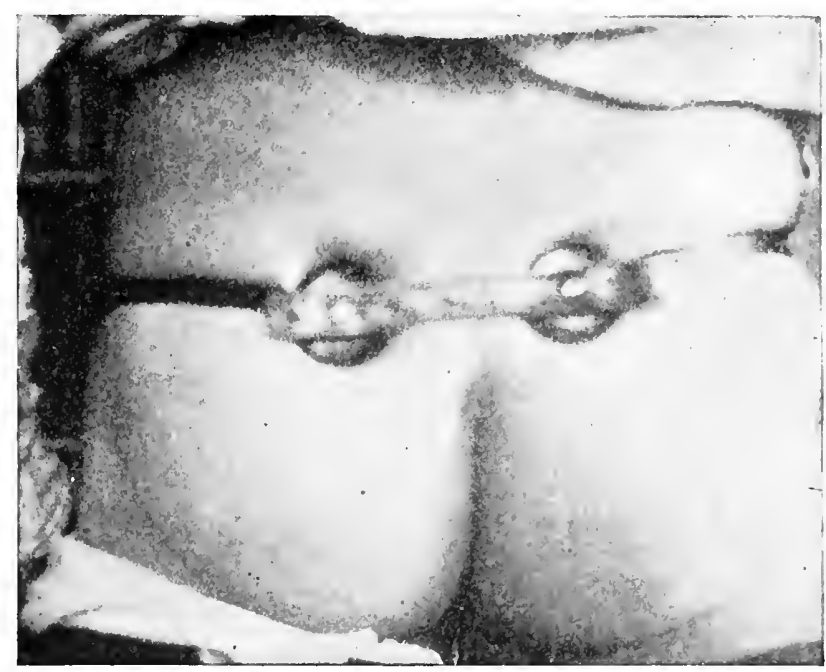

De Garmo-Hood truss applied. Back view.

descending arm. The shapes which are best for general use are shown in Nos. 6, 7, and 9 of the Hood type group. The Hood spring also has the advantage of carrying one or two pads, but it is always best with two. The one over the side not ruptured may be very thin and is called in the trade, a "dummy" (figs. 64 and 65 ).

A person with one hernia is quite liable to develop another on the other sicle, which is more likely to happen when a single truss is worn than when none is used, for the reason that 
the pressure against one side of the abdomen throws the intra-abdominal pressure towards the other side. Again, where double hernia exists, this solid-front spring is desirable because there can be no change in the relative position of the pads, by the stretching of a strap as in other forms of double truss, or by error of adjustment by the wearer. Increased weight and consequent change of size, is also better provided for by the adjustment of the strap in the back than in front. A person may gain or lose several inches in size without any relative change in the position of the two inguinal canals. When the wearer becomes tired and wishes to relax the constriction of a truss, it can be done if fastened in the back, without disturbing its position in front; on the contrary, if the pads are held in position by a strap in front, the moment this is loosened they are out of adjustment and the wearer is liable to accident.

The truss of this type that I have used for many years, more than any other, is shown in no. 7 of group, or fig. 64 and 65 , applied. It differs from the others principally in its lightness, simplicity of construction, and in the fact that the spring is made of hard-rolled German silver instead of steel. It is covered by hard rubber and the pads are attached by simple clasps that allow of arljustment.

There are few expert truss fitters in this country who have not used extensively the Hood type of truss, while some have used it almost to the exclusion of all other forms. It is easier to fit than almost any other, and certainly easier to wear. The special advantages of the Hood truss are believed to be:

(I) It passes around the pelvis at the most immovable part.

(2) Surrounding both hips gives it stability.

(3) It retains with relatively less pressure.

(4) It protects one or both canals.

(5) Counter pressure is on the gluteal region where best tolerated.

It has, however, the rlisadvantage of being somewhat more expensive to manufacture than other forms, and for this 
UNCLASSIFIED Group.

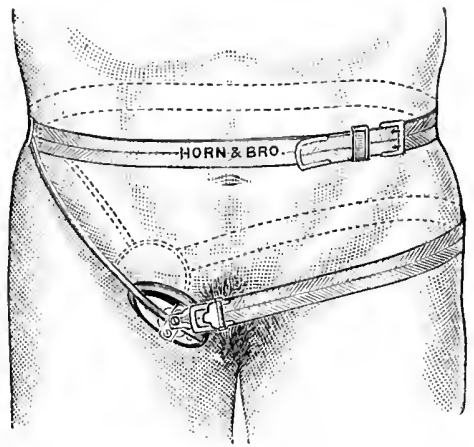

I. Wire-Spring Truss.

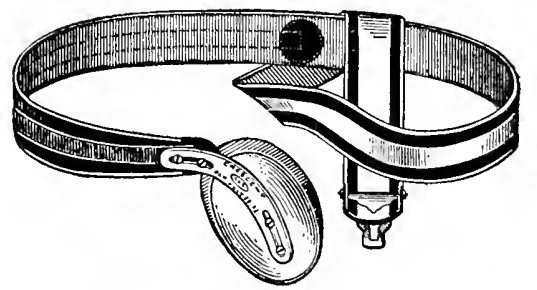

2. Spring and elastic web combined

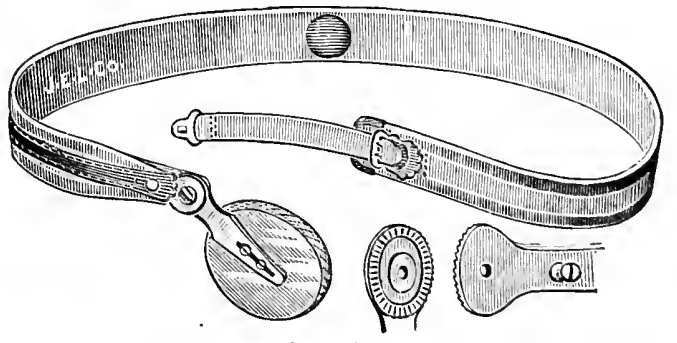

3. Spring and elastic web combined. 
Double Truss Group.

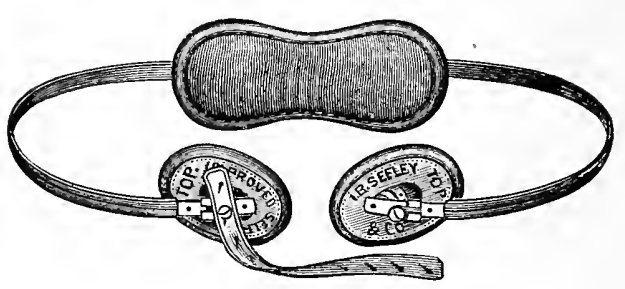

I. Double ball-and-socket truss.

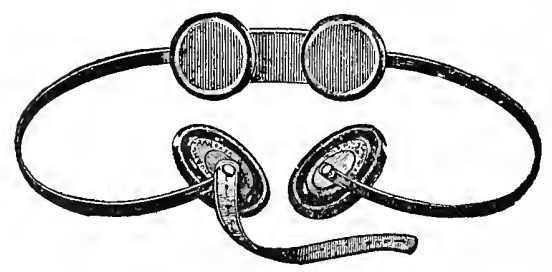

2. Double hard-rubber truss.

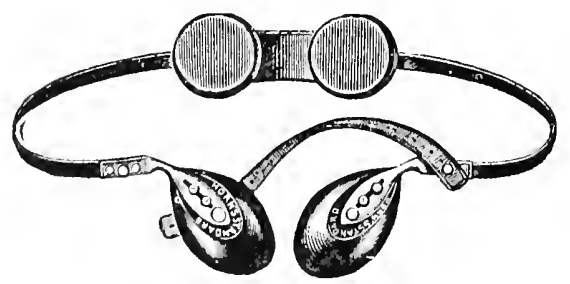

3. Modified Chase Truss. 
Double Truss Group (Contimed).

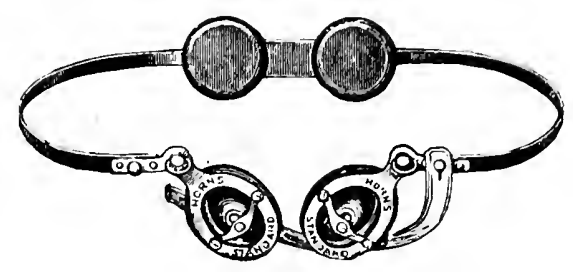

4. Modified radical-cure truss.
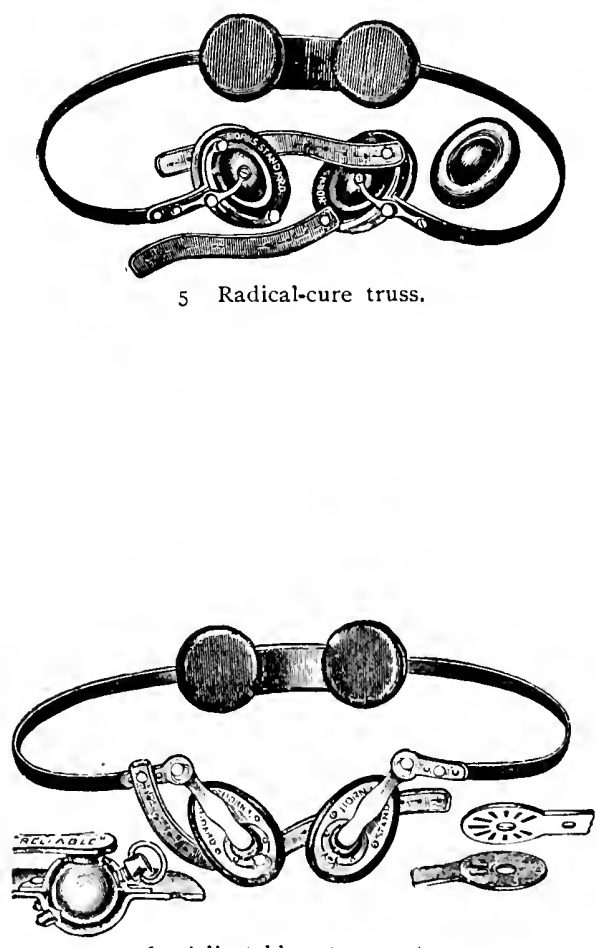

6. Adjustable set-screw truss. 
Dotble Truss Group (Continued).

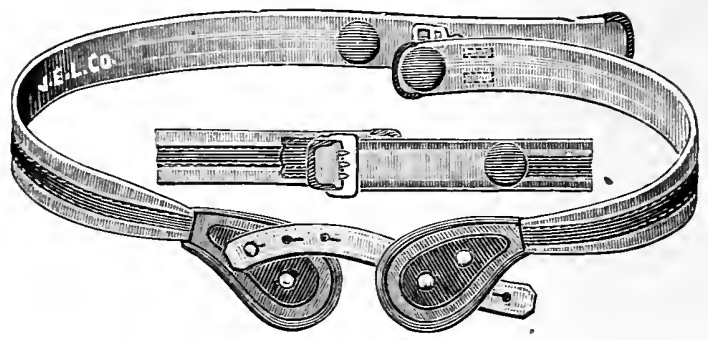

7. Elastic web and spring truss.

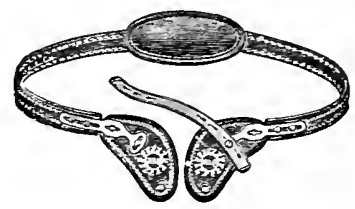

8. Adjustable French truss.

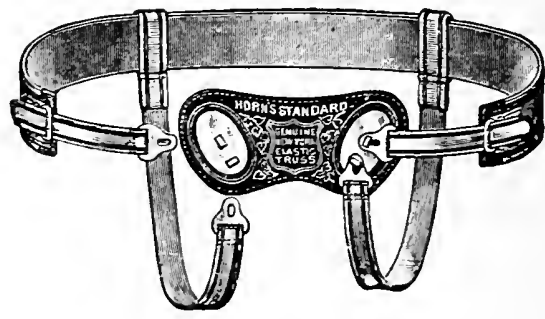

9. Double elastic truss.

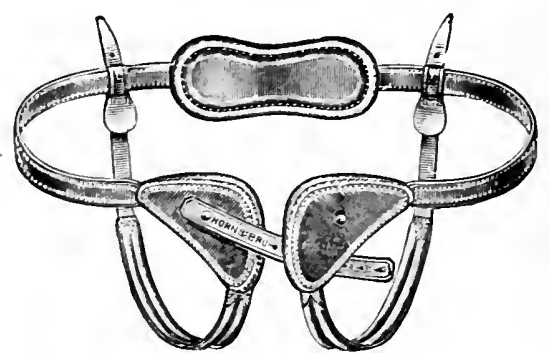

Io. Double German truss. 
reason alone, many truss sellers have declined to carry them in stock. The physician should impress upon his patient the truth, that to the ruptured man a good truss, well fitted, is more important to him than his clothes. Undue economy in this connection is poor policy.

Unclassified Group.-There is also a group of trusses that cannot be classified with any of those already mentioned. It contains one upon which I have never looked with much favor though I have known good truss fitters (no. I) unbiased by personal interest. who claim to have found it satisfactory. Large numbers have been sold to dealers throughout the country, and for that reason it seems best to mention it here. It has the appearance of an original type of truss, and I believe the makers considered it such, but as a matter of fact Dr. Tod of Lon(lon, England, patented (about ${ }_{1} 8_{5} 8$ ) a truss to which this is exactly similar except in details of construction, his being made of a steel spring occupying exactly the same position as this, held in place by a similar band, while this is made of spring brass, or other wire, so shaped that one piece forms not only the spring, but the frames for both the front and back pads. The advantages of this truss are in its extreme lightness and small cost of construction. Its disadvantages, as they present themselves to me, are its great liability to become displaced. lack of durability and general inefficiency. Its retention in place is absolutely dependent upon the web band which surrounds twothirds of the hip. Furthermore, the length of the spring, from the crest of the ilium to the hernial pad in front, is such, that in bending forward the pad is forced down over the pubic bone. Wearers have complained to me in reference to this as a great inconvenience, and it is certain that any truss pad that is so liable to displacement, must be looked upon as very dangerous.

Nos. 2 and 3 of this group represent a combination of a short steel spring with an elastic band. It is a modification of, and I should think something of an improvement upon, the elastic truss. 
Double Truss Group.-This group merely presents the same type of trusses already illustrated, as arranged for double instead of single hernia.

Retaining Pads.-Pads for the retention of the hernia are made of many shapes, and are usually interchangeable so that the fitter can make almost any combination of spring and pad that he may desire (fig. 66). In ordering pads it is only necessary to state the form of spring they are intended to be used on, and suitable screws for attachment will be placed on them. For general use the best form is a moderately convex, oblong pad such as shown in nos. 3 and 7 of the pads grouped on the accompanying plate. These oval pads are made in several sizes, as shown in the diagram of sizes (fig. 67); using an unnecessarily large pad is a mistake most frequently made. This is especially triue in the treatment of large sized hernize. The larger the pad, the stronger the spring pressure must be. With the smaller pad the pressure is concentrated immediately upon the spot, while if that same pressure is distributed over a large area, it ceases to be effective. A thin, flat pad answers well for a thin person, but upon a fat patient, a deeper pad, such as No. 8 of pad group, must be selected.

The pads are made either of soft material, felt, hair, or of hard material, such as wood, hard rubber, or celluloid. A soft pad that has proven useful is known as the water pad. This is made in every conceivable size and shape, and consists of a rubber bag filled with water or glycerine, sealed. Over this is a layer of felt and then a covering of silk, kid, or chamois. In some special cases this is a valuable pad, but its lack of durability is a serious defect. If the wearer is cautioned about its tendency to flatten out and leave him umprotected, it may save serious trouble. This change is so gradual that unless attention is drawn to it, it may not be noticed. For general use there is no parl made that equals in durability, cleanliness, and reliability, those marle of hard rubber or celluloid. The skin maintains a much healthier condition under the pressure of a highly polished, impervious surface, than any soft material 
MECHANICAI, TREATMENT

FIG. 66.

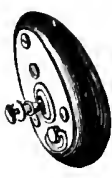

2.

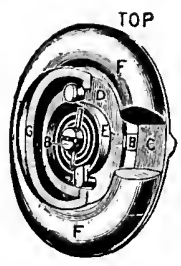

4.

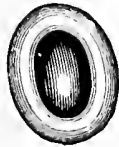

6.
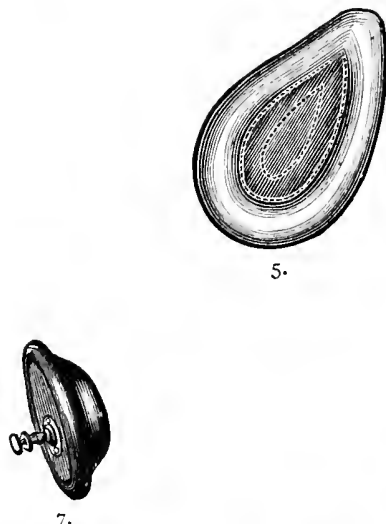

5 .

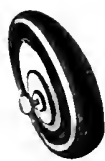

3.

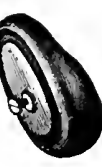

8.

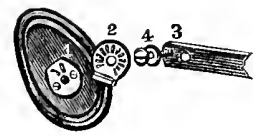

9

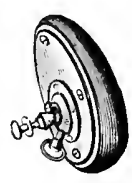

Io.

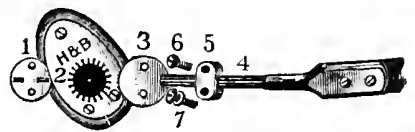

11.
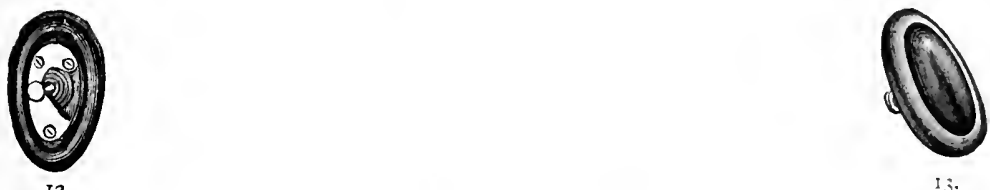

Group of Variously Shaped I'ads 
FIG. 67.
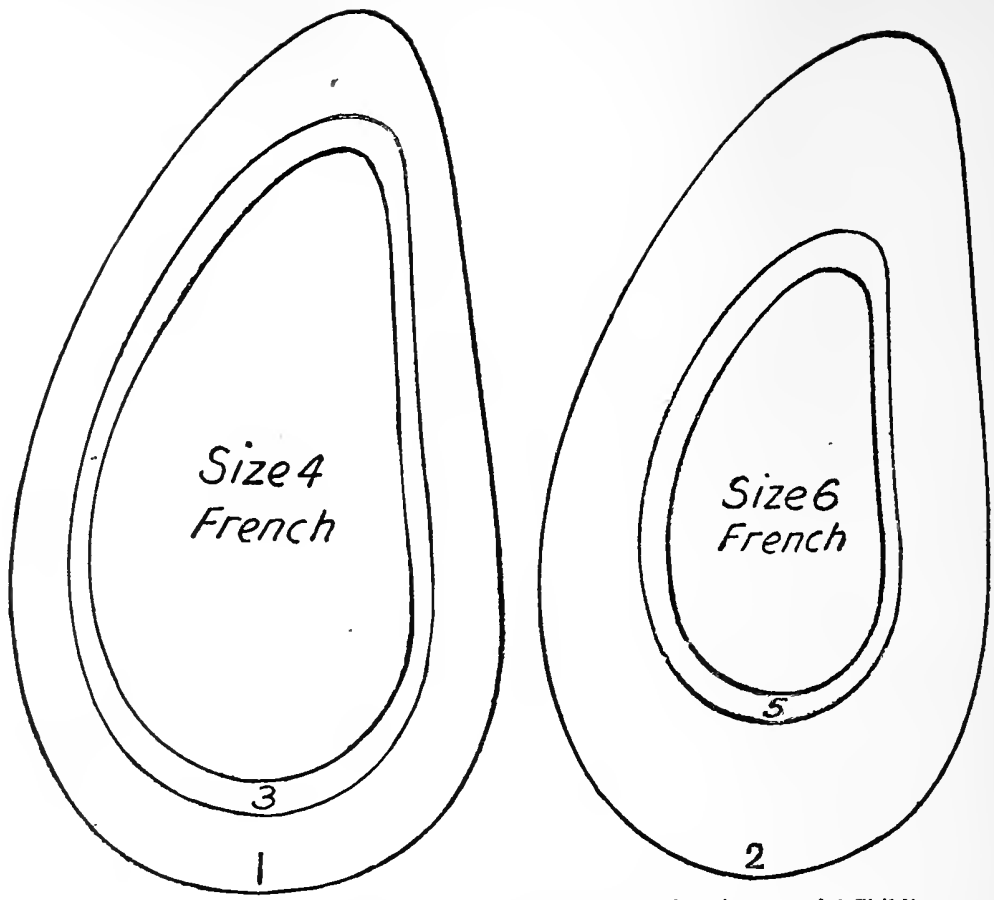

French pads. Sizes I and 2 Adult's; sizes 3 ancl 4 Youth's; sizes 5 and 6 Child's.
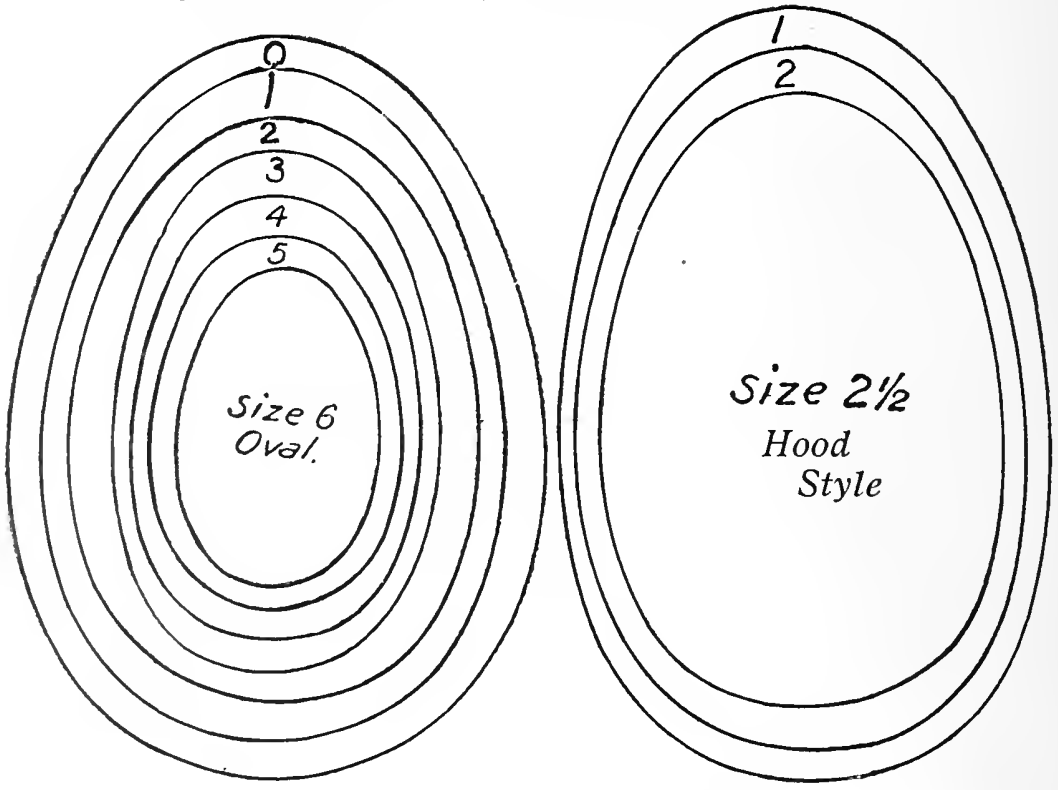

A Standard of Sizes for Truss Pads. (Jlorn.) 
that is constantly accumulating the excretions of the skin. Ordinarily the hard pad is efually comfortable to wear, if placed in proper position, and does not impinge upon the bone or other hard parts. The tissues back of the parl are soft and flexible, conforming readily to the shape of the parl, thus closing the upper part of the canal.

Truss Coverings.-The material with which a truss spring is covered has little to do with its efficiency, but may make much difference in comfort, cleanliness, and durability. Cleanliness of both person and appliance is the first essential of comfortable truss wearing, and it is for this reason that hard rubler and celluloid make the most desirable materials with which to cover truss springs and make the pads. In using these materials retaining pads can be made hollow, and therefore very light.

The use of hard rubber in truss-making was the invention of Dr. J. W. Riggs of New York City, about I 865 , and was one of the most valuable contributions ever made in the interest of the truss wearer. The names of Riggs, Chase, and Hlood, all reputable physicians, should long be remembered in connection with the great advance of this country over other nations in truss construction. Manufacturers are to be congratulated upon the excellence of their products, but we must still claim for the medical man the honor of having marle the most valuable suggestions. In individual cases, especially in aged, thin, and sensitive people, it may be very advisable to have trusses constructed of the softest possible material, but for the average wearer there is nothing equal to the hard rubber or celluloid, which insures cleanliness, as they will not absorb the excretions of the skin. They can be washed in water, or boiled if worn during contagious disease. Physicians frequently make the mistake of speaking of the "Har(1-Rulb)er Truss" or "Celluloid Truss" as though they were some definite type of truss. This is an error, as these names merely refer to the materials used in construction and are applied by makers to every known type of truss. 


\section{CHAPTER VIII.}

\section{TRUSS-FITTING.}

The fitting of trusses is an art that is difficult for a person to acquire who has no mechanical tastes or ability. Such a man would seldom become an expert, but if persistent, would, with practice, do the work fairly well. It requires in addition to some mechanical skill, patience unlinited, persistence until the ideal is attained, and tact in managing the patient, especially if he is an old truss wearer and has "ideas" regarding his needs. Unfortunately every beginner has had to acquire skill by personal experience, and when he has obtained this it has been considered shrewd business policy, by the non-professional expert, to impart as little of his knowledge as possible to others. There is no valuable guide to truss-fitting and largely because those who have written on hernia have had surgical experience only, while those who have had experience in truss-fitting have " bottled it up," fearing that their rivals might be benefited by it.

Truss-fitting consists of obtaining the measure and shape of the patient, selecting the truss suited to the case, shaping of the spring, and its application to the patient. The patient should also be instructed in the reduction of his own hernia, in the removal and readjustment of the truss, in the necessity of care and cleanliness of the skin, and last, but not least, in the importance of returning for refitting and inspection.

Taking Measure and Shape.-Every person is differently formed, even though the circumference be exactly the same, so that it is equally as important to consider the shape as the measure. Manufacturers can only follow one general shape for a certain size, therefore, if a patient buys a truss from stock that fits him, it is because he happens to fit the truss. Practically each truss should be shaped to the form of the person that is to wear it, and recognizing this fact, truss makers temper their 
springs so that they can, with care, be bent to the required form with little risk of breaking.

The measure for a truss (fig. 68) for inguinal hernia should be the entire circumference of the pelvis, about level with the internal ring, passing midway between the crest of the ilium and the trochanter major, and with the tape a little higher in the back than in front, corresponding with the pelvic obliquity.

FIG. 68.

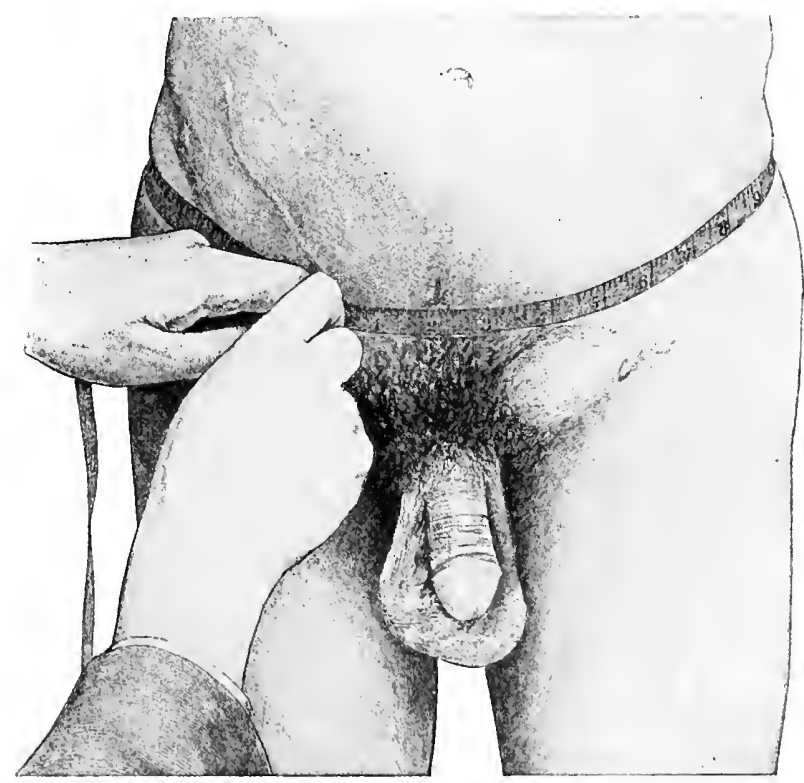

Showing location in which measure should be made for inguinal truss. Tape should pass, midway between trochanter major and crest of ilium. (Macready.)

This measure should be recorded in number of inches, and follows the line properly covered by the truss spring as shown in fig. 63. It is also well to record the measure from one inguinal canal to the other in double hernia in order to locate the pads at a proper distance apart, remembering that in direct hernia the pads must be nearer together than in the oblique form. 
Diagram.-Placing a diagram, on paper, of the patient's pelyis will materially aid even an expert fitter, and it puts trussfitting within the possibilities of the inexperienced. The

FIG. 69.

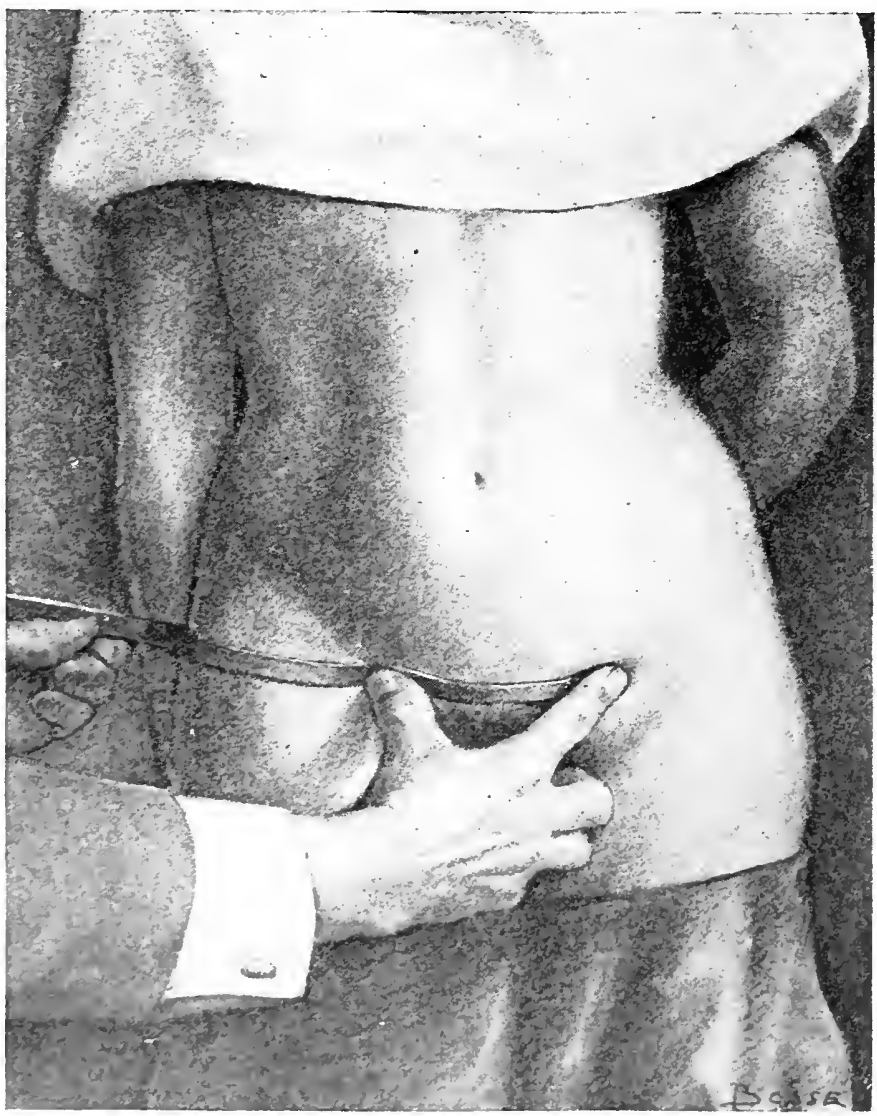

Showing method of taking diagram with lead tape.

"Ifearl-Tape Method" suggesterl by the author many years ago, has proven a very easy and valuable way of roing this, but has not been as extensively known as it should have been. This cliagram is obtained by the use of a strip of sheet lead, half 
an inch wide, one-sixteenth of an inch thick, and for use on adults, about twenty inches long. It can be cut from the sheet by any plumber. The end of this lead tape is placed over

FIG. 70.

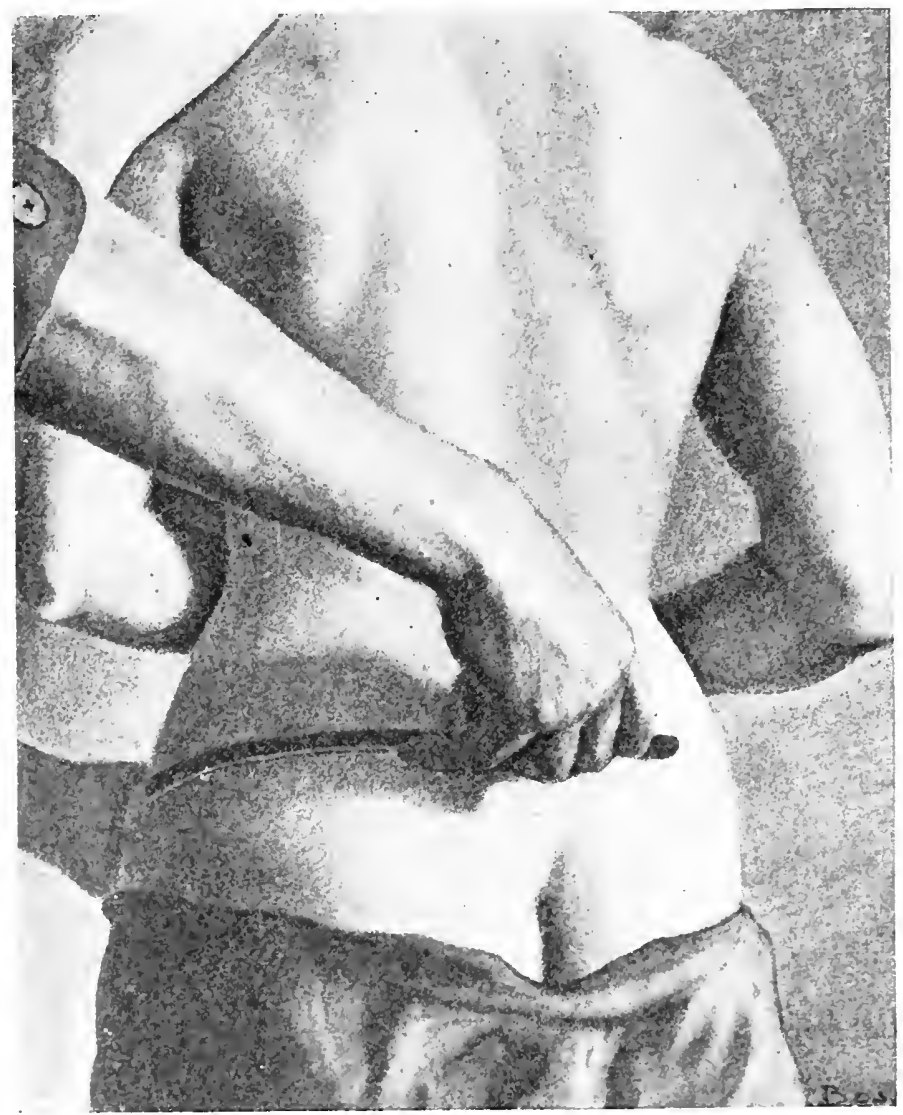

Showing method of taking diagram; second position.

the hernia (fig. 69), extending from this point across the front of the abdomen and around the hip on the opposite sicle. thence across the back (fig. 70 ). The lead is gently pressed to the form of the body, carefully removed and placed 
edgewise upon a sheet of paper, or case book, of suitable size. A tracing is now made of its inner surface with a lead pencil. This will represent about two-thirds of the circumference of the pelvis and when transferred to paper the diagram may be completed by turning the lead over and completing the tracing, or

FIG. $7 \mathrm{I}$.
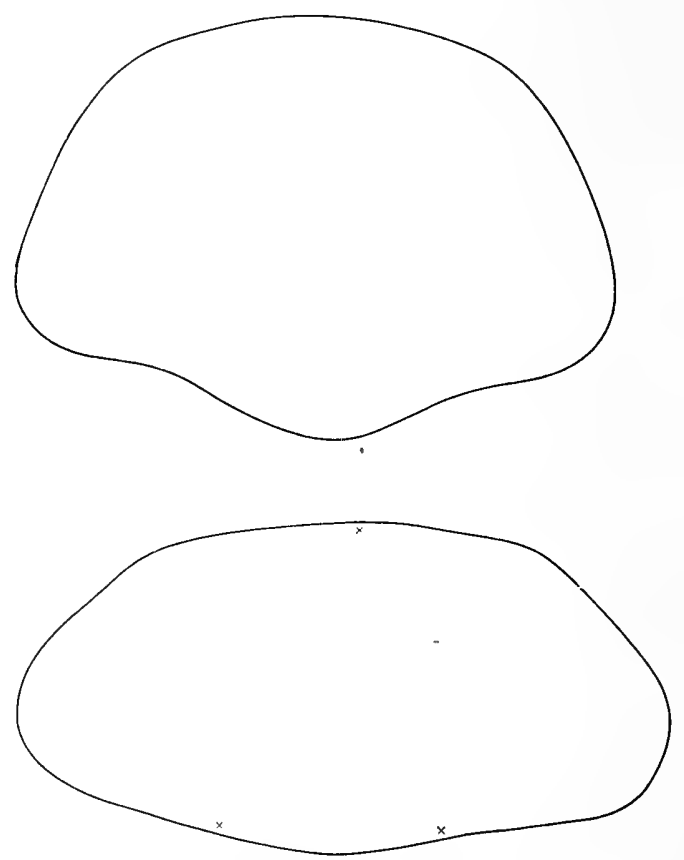

Diagrams of two persons of $3^{2-i n c h ~ m e a s u r e . ~}$

if preferred by repeating the process for the other side. This diagram gives the shape of a section of the hips taken on a line covered by the truss spring.

The shaping of a truss spring by this diagram is much easier than shaping it to the patient's body. Time is saved to the fitter, and the embarrassment to both the patient and the physician of repeated trials upon the borly is saved. Usually if the spring is carefully shaped to the diagram, very few, if any, alterations will be required when it is put upon the person 
of the wearer. This method so far simplifies truss-fitting as to place it within the reach of every practitioner who is willing to devote the time necessary. The patient should be, and usually is, willing to pay for this time in order to be relieved of a very dangerous condition. The two diagrams shown (figs. 71, 72) are reproduced from those of two taken from my case book of

FIG. 72.
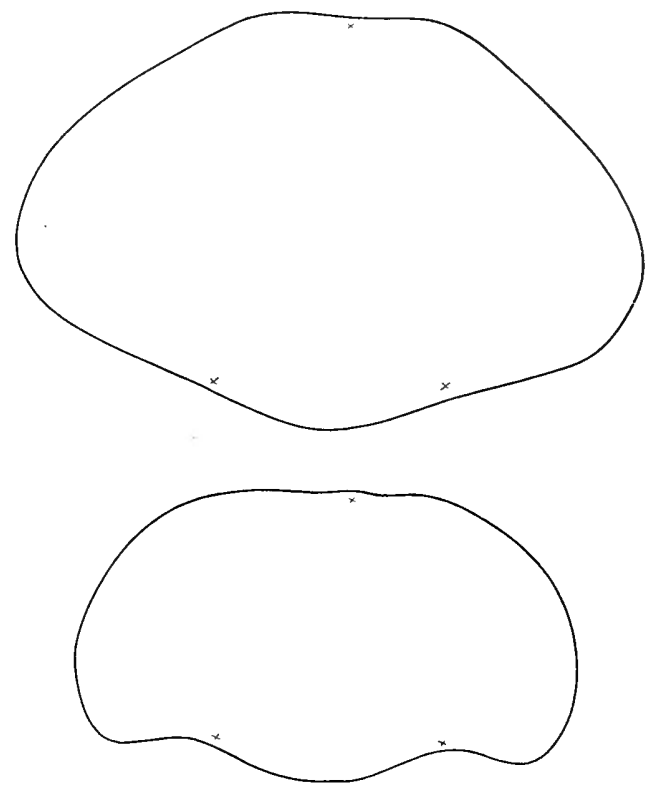

Diagrans of two persons whose circumference is identical.

two persons of exactly the same measure, and illustrate at once how impossible it would be for one to wear with comfort a spring shaped for the other.

Shaping.-In truss-fitting one should have a pair of strong pliers, a screw-driver, and a pair of good lands, the latter being the most important part of the outfit, as nearly all of the actual bending of the spring should be clone by them. The bending into the required shape of a tempered spring must be done carefully and nct by a sudden, jerky force. Grasp the 
spring firmly in the hands as shown in no. 3, fig. 73 , and by a steady, firm pressure gradually bend it to the point required. In shaping by the diagram, begin at the point over the hernia and shape first across the front of the abdomen, if a cross-body truss, and then around the hip and across the back. If the spring crosses the back it must not be allowed to press upon the spine, but the pressure should be taken up by the heavy side muscles. If the lower edge of the spring needs twisting farther in or farther out to change the bearing of the pad or improve the fitting of the truss, this should be done by the pliers. It must be remembered in fitting a spring to the diagram, that allowance must be made for pressure. The spring must be forcibly held out to the shape of the diagram.

All springs corered with hard rubber must be thoroughly warmed before attempting to bend them, otherwise the rubber covering will crack, damaging seriously the durability of the truss. The warming is done by passing the spring rapidly through a gas flame (no. I, fig. 73), or through the flame of an ordinary spirit lamp. The latter method is the best, as the gas flame smokes it and unless constantly wiped will soil the hands or patient's body. The skill necessary for this warming process is quickly acquired, and all that is necessary to prevent burning, is to keep the spring constantly moving. An equally good way, when convenient, and one free from danger of burning, is to dip the spring in boiling water for about one minute. Celluloid springs do not need warming before bending, except to see that they are not extremely cold. They must not, under any circumstances, be placed in a flame. They are readily distinguished from hard rubber, which is always black, by their being pink or white. Ordinarily this material is sufficiently flexible to stand any necessary shaping. It is only when the springs have been kept long in stock that they become somewhat brittle, and then the pouring upon them of boiling water will prevent cracking.

Every time a truss spring is bent its pressure is somewhat reducerl, and for this reason it is best to start with a spring that 
TRUSS-FITTING.

159

FIG. 73.

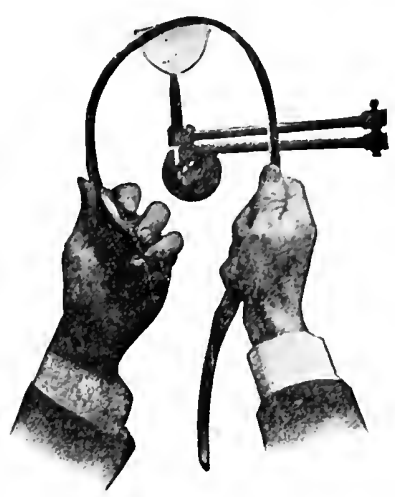

I.

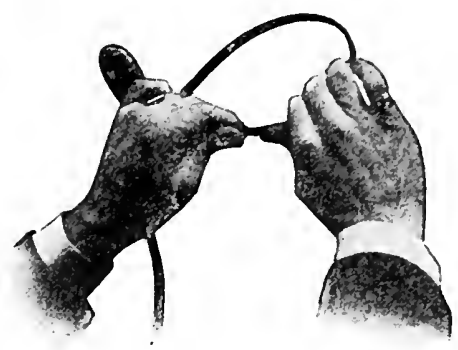

2.

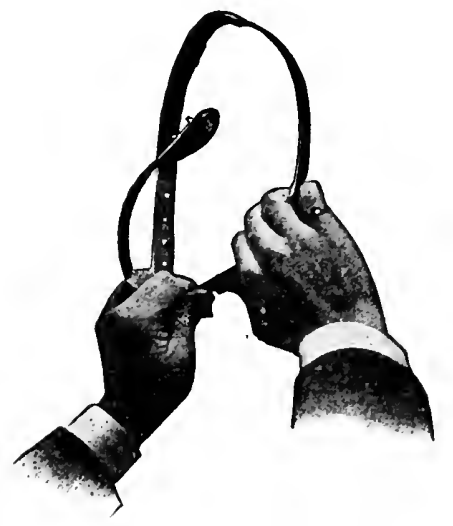

3.

Shaping truss springs. 
Shaping truss springs (Continued).

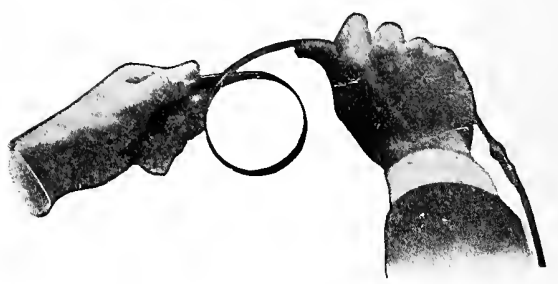

4.

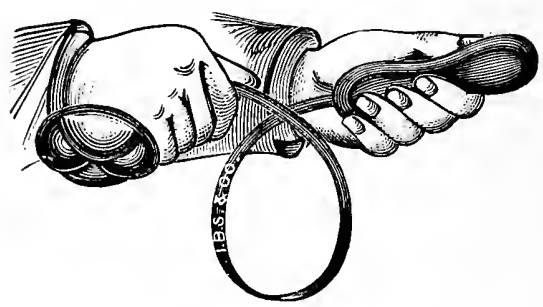

5 .

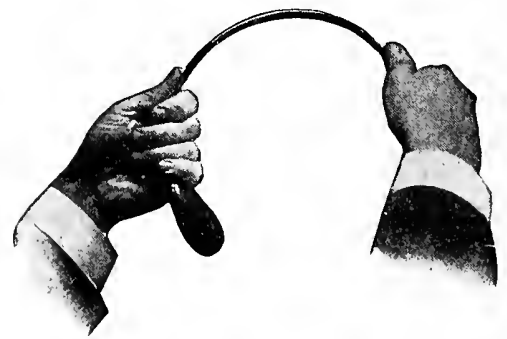

6.

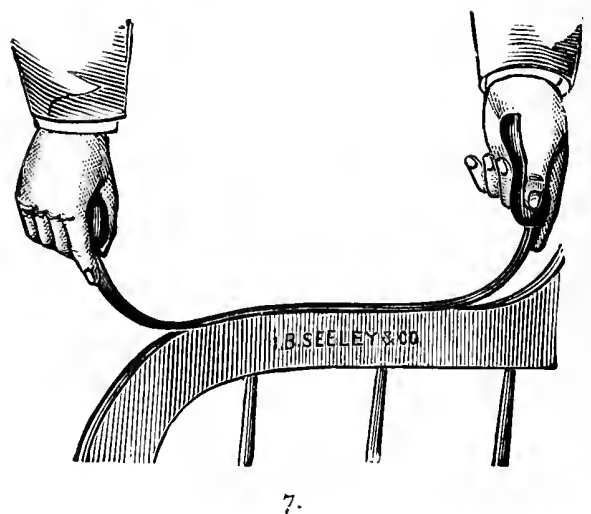


is somewhat stronger than needed. Its pressure can be rerluced by taking it firmly in hand and stretching out (nos. 6 and 7 , fig. 73), or bending it over the arm of a chair. Caution is necessary not to reduce its strength too much, as it is casier to decrease than increase it. Its pressure may be increased, however, by adding to its curves, by short, firm bends between the hands, or curling up the spring as shown in nos. 4 and 5 , fig. 73. If increase of pressure obtained this way is considerable, it will be to quite an extent transient, and it is usually better to exchange for a stronger spring. It is to be remembered that all hard-rubber covered springs must be warmed before bending, but that this is not necessary in shaping celluloid or leather covered springs.

FIG. 7 .

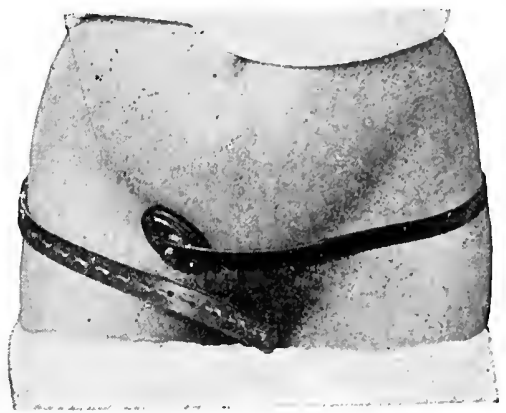

Hard-rubber cross-body truss applied to complete oblique hernia. In small hernia the pad may be higher.

SELECTING TRUSS.

Oblique Inguinal Hernia.-An incomplete or a small complete oblique inguinal hernia is usually retained by a very moderate pressure and a small pad, which should be either directly over the internal ring or upon the canal immediately beneath that point. It is more difficult to select a truss exactly suited to the treatment of a small hernia, from the stock of the average dealer, than it is for a large hernia. This is because many dealers will have nothing to do with a light truss spring. believing that its virtue is clepenclent upon its strength. Of the 
trusses carried in stock by the dealers, few will be found better for a small and recent hernia than a light spring cross-body, preferably with a hard-rubber or celluloid covering (nos. 2, 3, and 6 , cross-body group). If the spring seems too strong, reduce its pressure in the manner already described, while shaping it to the diagram of the patient. When applied it should occupy the position shown in fig. 74, or still better,

FIG. 75 .

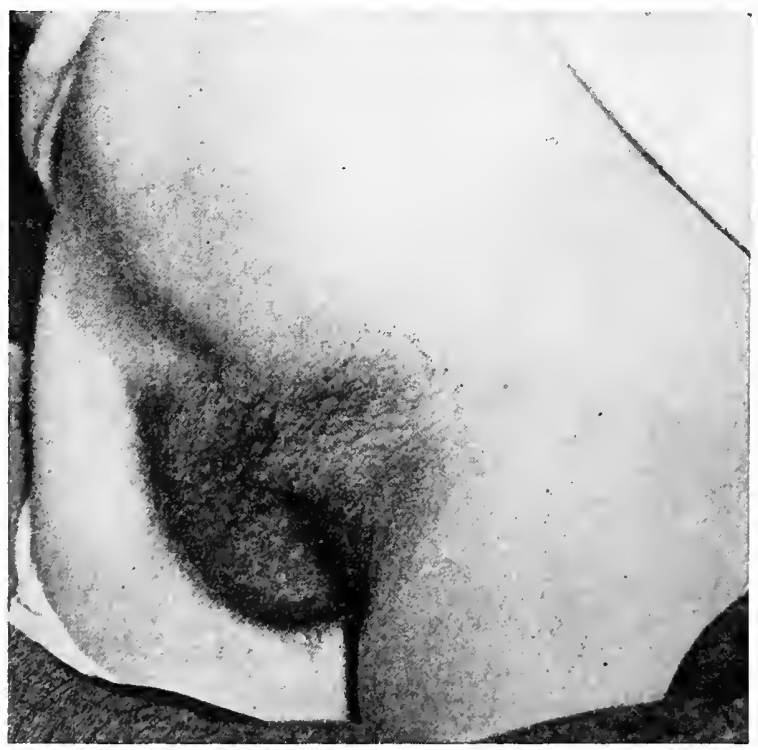

Woman aged 40 years, with right labial hernia of seventeen years' duration.

with the pad a little higher. The pad as there shown, compresses the entire canal, while in a small oblique hernia it is only necessary to compress its upper part. The higher the pad, with retention of the hernia, the greater the comfort of the wearer, and the greater will be the improvement obtained from its use. Fig. 75 shows a large labial hernia retained, in fig. 76 , by a hard-rubber, cross-body truss. The pad should be about no. 3 or 4 oval (fig. 67 ) and moderately convex. Some 
manufacturers make what they call a narrow-spring cross-body, which is particularly well suited to light cases.

The next choice in such a case would be a light Hood truss, and if very light, is to be preferred even to the crossbody (fig. 77). If the meager stock of the dealer makes it necessary to select a Chase type of truss, select a size smaller

Fig. 76 .

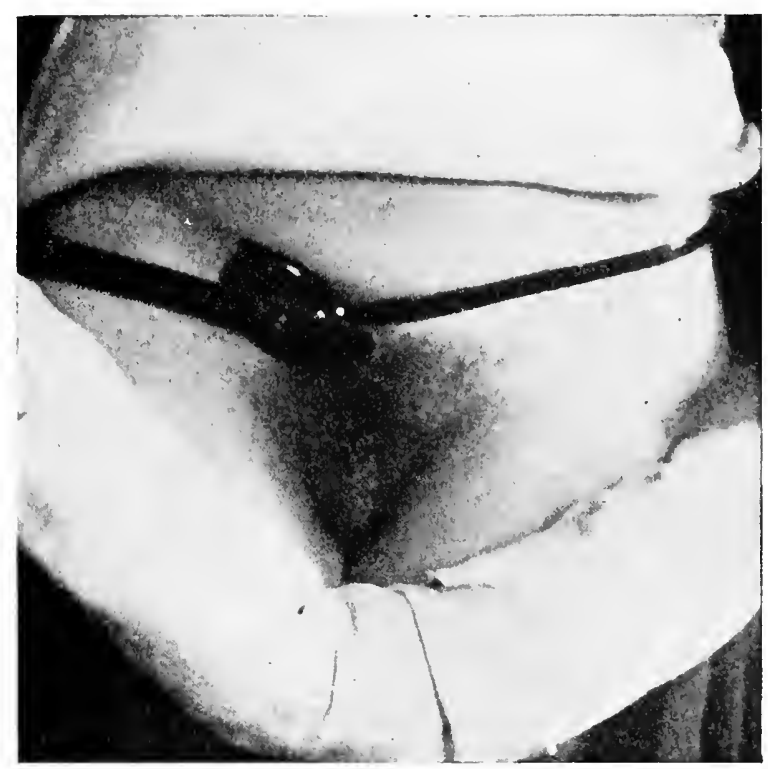

Right labial hernia retained by hard-rubber cross-body truss.

than called for by the measure, and straighten out its neck nearly parallel with the spring (no. 3, Chase group), and shape by diagram. The smaller size is suggested because in turning the neck nearly parallel with the spring, the latter is thereby lengthened. The most objectionable feature of this type of truss is in the length of its neck, and low bearing of the pad. If driven to the necessity of putting on a truss of the French or elastic type, let it be for temporary use until a better form can 


\section{FIG. 77.}

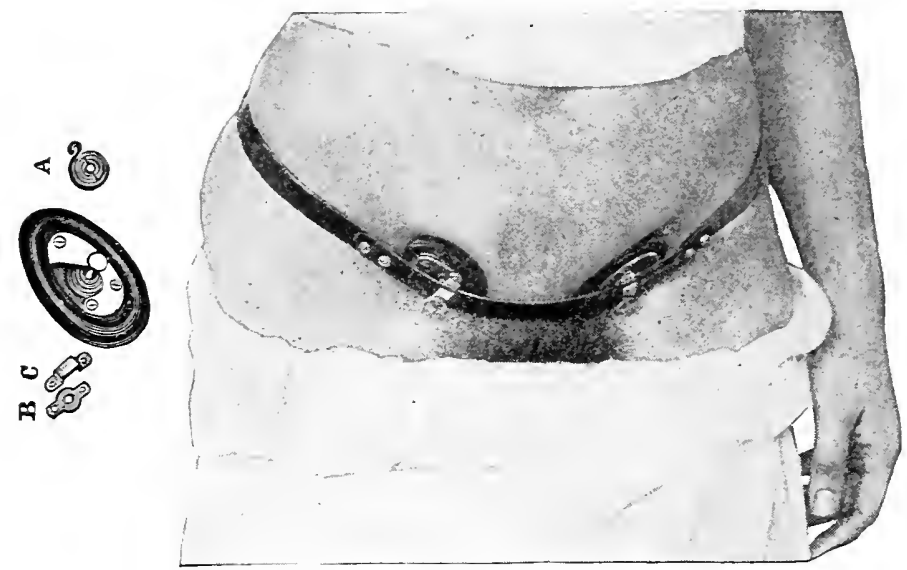

Hard-rubber steel-spring Hood truss with pad on spiral spring. For double or single hernia. This atrangement of pad is better suited to fat than to thin people and where strong pressure is required.

FIG. 78 .

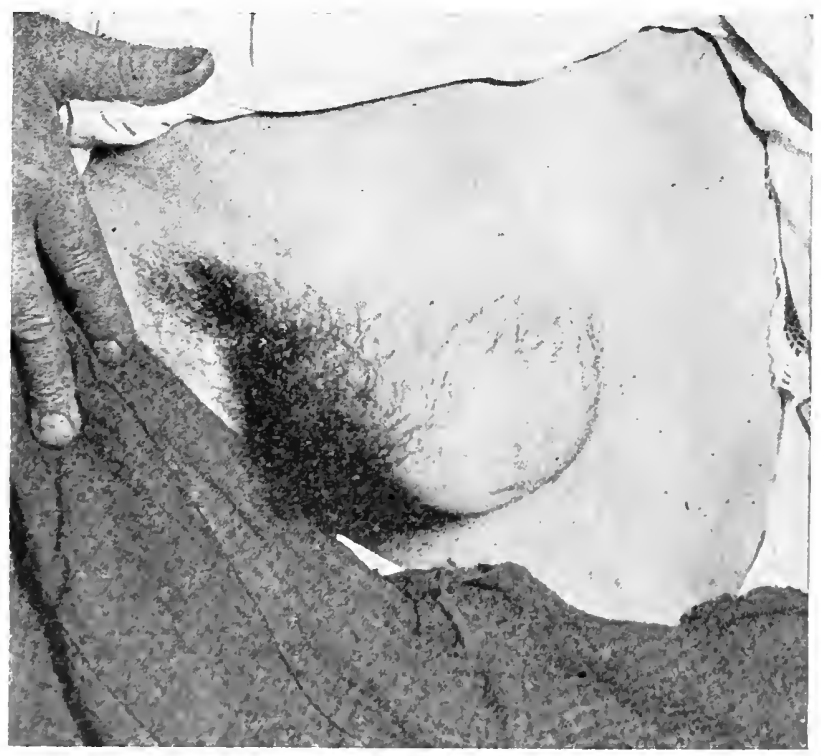

Complete oblique inguinal hernia. Recurtent after operation by MacEwen method. 
be obtained, for under the permanent use of an inferior truss, small hernire are yuite sure to grow worse. Fig. 78 shows an inguinal hernia in a woman of middle life that would require a cross-body spring of more pressure, or what was lelieved better in this case, a Hood truss, as shown in fig. 79. This is a very light form of the Hood truss and, in some cases, might

FiG. 79 .

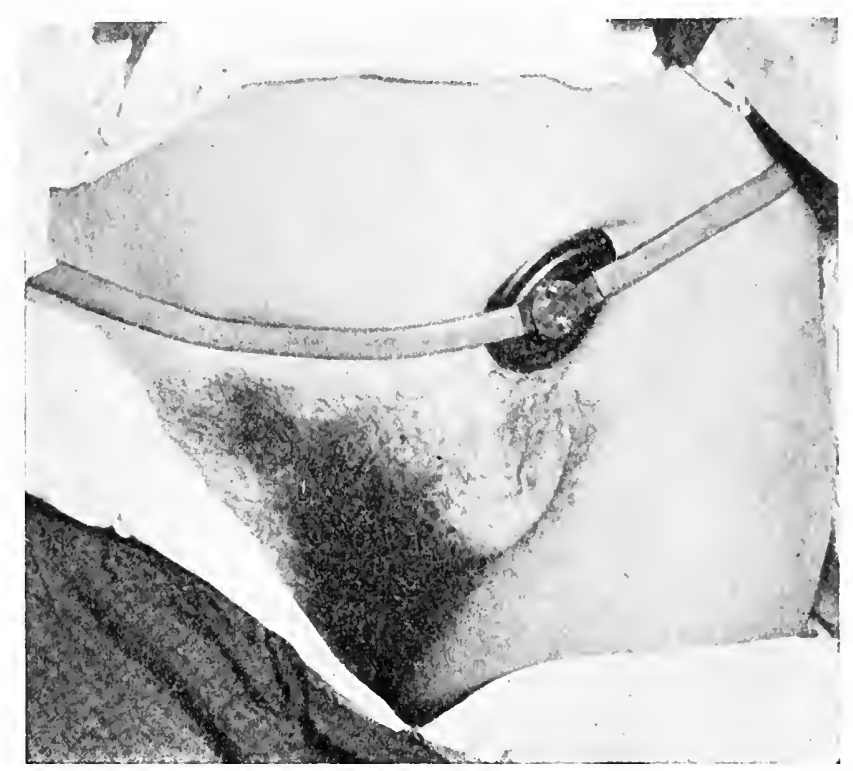

Same as Fig. 78 , with De Garmo-Hood truss applied. Hernia is perfectly held within the abdomen. Bulging over pubic bone is from a thickened sac and loose skill.

not be sufficiently strong to retain the hernia perfectly, and it would be well to use the regular Hood form with steel springs, as shown in fig. 77 .

As oblique inguinal hernia increases in size there is a corresponding shortening of the canal, by the dragging down of the internal ring, until it is nearly opposite the external, thereby increasing the difficulties of treatment. Instead of having a canal an inch and a half long to act upon, we then have a large hole beneath the skin leading directly into the abdominal cavity. 
In addition to this direct opening, there is usually a thickened sac, in these neglected cases, that also prevents in a measure the efficient action of a truss. Then again the pad must rest nearer the pubic bone, and this, by pressing the cord or other tissues against the bone. adds seriously to the discomfort of the patient. Even in these extreme cases the Hood truss arranged with deep

Fig. So.

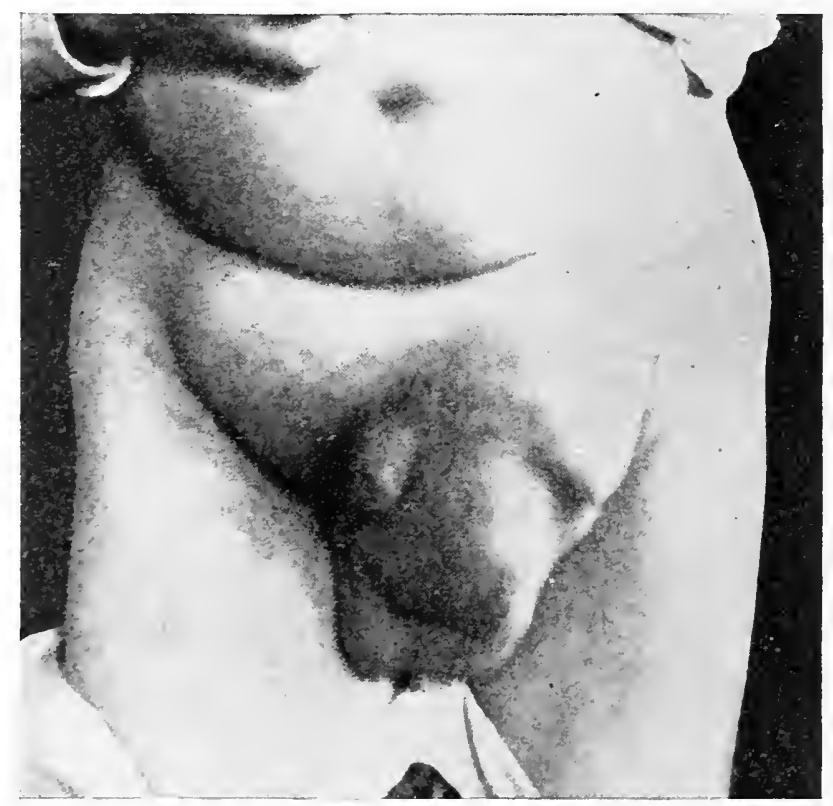

Large double hernia. Protrusion on the left not complete when photograph was taken. Size of patient's head when fully out.

pads (fig. 77 ) is an excellent truss. Fig. So is a photograph of enormous ciouble scrotal hernia, which was retained by a Hood truss of the ordinary type, as shown in fig. 8I.

In order to clearly comprehend proper and inferior forms of truss. it is advisable to compare this with what the Cermans call a "Scrotal-Hemia Truss.' In the latter there is an enormous compress over the pubic bone held in place by a 
strap between the legs. The amount of pressure necessary to retain the hernia, by such a large surface, must necessarily be great. In these enormons hernixe I have for many years held as a last resort, the truss known as the "Radical-Cure Truss" (nos. 4, 5, and 7 of cross-body group). This will many times retain a hernia upon which every other form of truss

FIG. 8I.

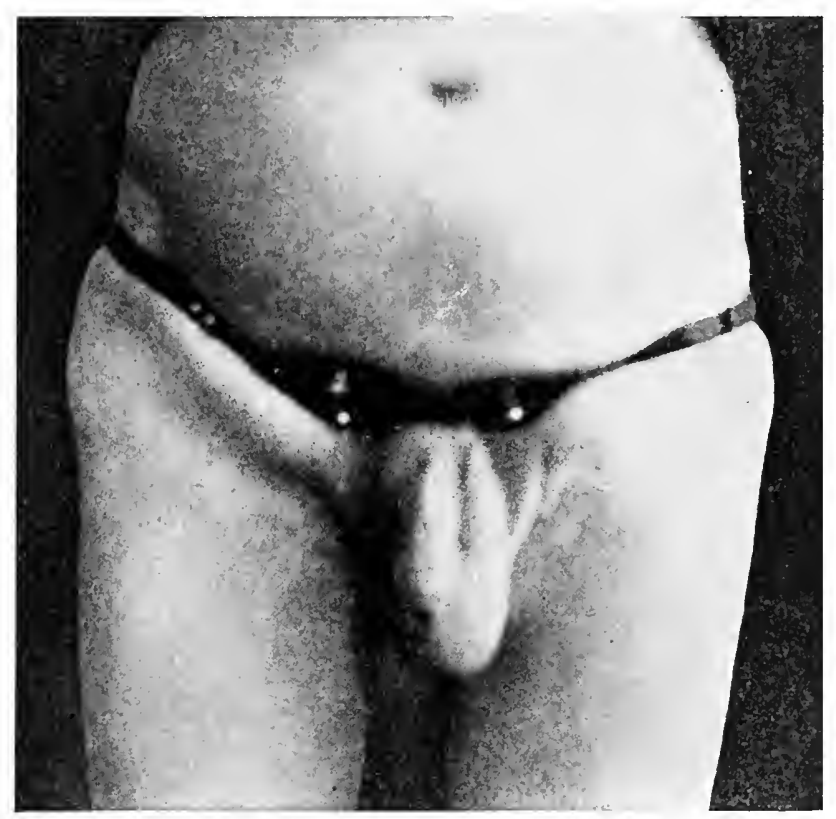

Same as Fig. 8o. Hernia retained $b y$ double steel-spring hard-rubber Hood truss.

has failed, but it should be used only as an extreme measure, as it is most uncomfortable. Its peculiarity is in the construction of the retaining pad, which has a small, hard, oblong centre, surrounded by a soft-rubber or kid-leather ring. In action the greater amount of pressure is concentrated upon the small central pad, and to this, and the fact that they are usually made with an especially strong spring, is to be attributed their greater retaining power. The name had its origin in the 
fact that it was advertised for many years by its originator uncler this title. That it possessed any special virtues, implied by its name, is not believed, except that it retained hernia securely and was skillfully applied by its inventor. Fig. 82 shows half of one of the double Radical-Cure trusses applied in combination with half of an ordinary double hard-rubber truss. The single truss should always be of the cross-body type. Some makers have attached this pad to a French spring, but this combination is practically worthless.

FIG. S2.

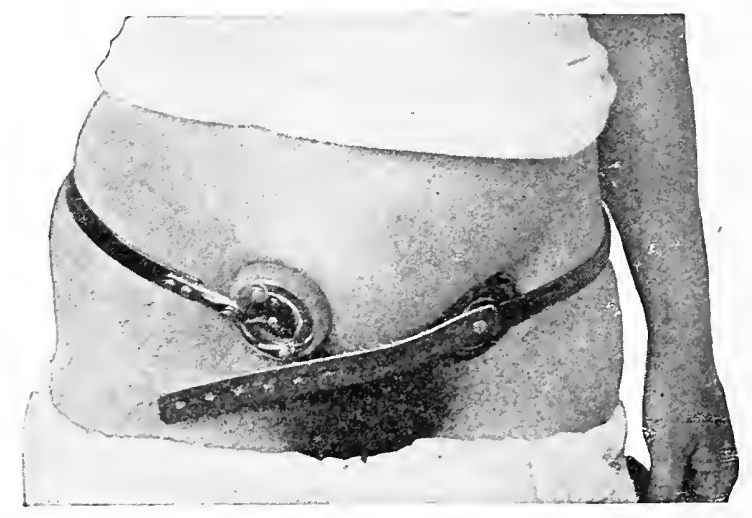

Hard-rubber radical-cure truss on right side, combined with ordinary double hard-rubber truss on left.

In fitting these large and neglected cases, it must be remembered that the canal is destroyed, and that the point of greatest pressure must be very nearly over the external ring and consequently nearer the centre of the abdomen than it would be applied in snall oblique hernia. In many of these large hernize the use of the water pad, which can be combined with any form of spring. will be found to retain better than the smonth, hard pad. When the water pad is used, it should, if possible, be changed to the hard pad as soon as sufficient improvement warrants it. for, while valuable, they are not very durable and therefore need to be watched. Under proper 
treatment, that is, perfect retention and gradual reduction of pressure, large oblique hernix improve in almost every instance. This improvement is sometimes so great, that if the patient allows a protrusion of the hernia, he may be unable to reduce it, and serious results ensue. Patients under treatment for large hernice must be particularly cautioned regarding this danger, and advised never to stand without a truss on.

In the Author's experience there have been very few reducible herniæ, even of enormous proportions, that could not be controlled by a suitable truss properly adjusted, and he has relied largely upon either the Hood form or cross-body spring, with such variations of pad as seemed to be required by the peculiarities of the case. The retention of these hernize must be undertaken seriously, and not considered as trifling cases to which a truss can be applied and the patient sent away. It has frequently happened that patients, coming from a distance, have been sent away unattended until such time as they could be given the uninterrupted attention necessary to successful treatment. This course might entail a delay of from five to ten days, or longer, with the patient in bed part of the time, if retention could only be accomplished in the recumbent position. Cases of this type that had previously met with repeated failure, managed in this way, have had the most gratifying results. It is true, now that the surgical treatment of these cases is so successful, that there is not the incentive to this persistent and hard work as in former years: still, there may be good reasons why an operation is not advisable, and they should under no circumstances be abandoned as hopeless.

When these enormous hernixe are reduced and retained, in the male sex, there remains a large mass of thickened sac and fascia as well as elongated cords and scrotum. It aids greatly in the retention of the hernia and adds much to the comfort of the patient, to adjust a firm and tight-fitting suspensory bandage. In people who have large, pendulous abdomens and flabby muscular walls, a light, but strong, abdominal belt will aid. Such belts are usually kept in stock by dealers, but it is 
far better, when possible, to have them made to order, as they usually contain rubber, which material rapidly deteriorates when lying unused. A group of these belts, and directions for measuring, will be found in the chapter on the mechanical treatment of umbilical hernia.

The English use, for cases of very large herniæ, what they term the "Rat-Tail Truss" (fig. 83). It will be noted

FIG. 83.

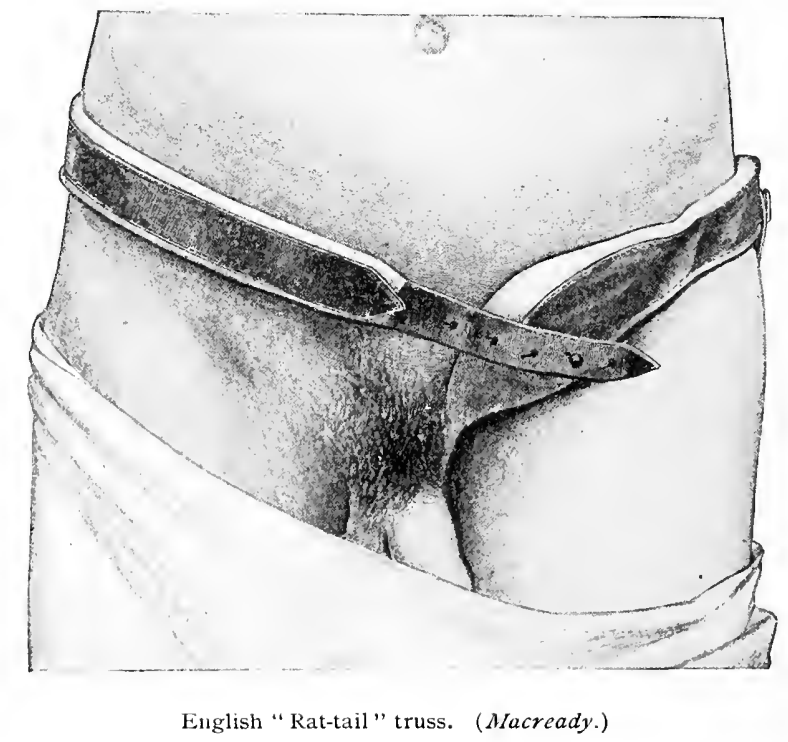

that this truss is quite similar to the French type, except that it is vastly superior in that the direction of the pad is nearly continuous with the spring, bringing it directly over the canal, instead of over the pubic bone as in the German truss. The spring in this truss is also long enough to clasp the opposite hip, which greatly aids in holding it firmly in place. It is to be hoped that, eventually, our truss makers will abandon the patterns now used for making the French-German type and adopt those more nearly approaching the English design. The 
advantages of the English truss are well shown in fig. 84, as applied to double inguinal hernia; we believe, however, that with accurate fitting of the spring, the understraps, which are always objectionable, could usually be abandoned, and we can speak in praise of the position of the parls only. In contrast to this, attention is called to a truss of the same type (fig. 85 ),

FIG. 84.

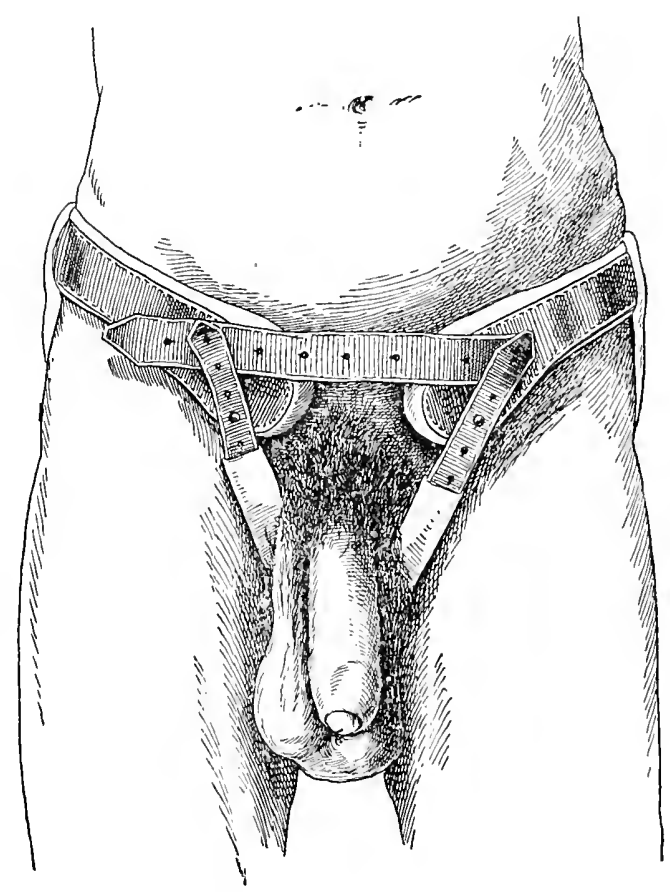

An English type of double truss, applied. (Eccles.)

very extensively sold in this country, which, while excellent in construction, has the serious defect of having the centre of the pad too far below the spring. This truss, as shown applied, is in very good position, except that the spring is too high. When the wearer stoops, the abdomen strikes the upper edge of the spring; and forces the pad down orer the pubic bone. 
Direct Inguinal Hernia.-The mechanical treatment of direct hernia is, in most respects, similar to that of the oblique variety, except that the difficulties are somewhat increased on account of its close proximity to the pubic bone. The contents of a direct hernia may sometimes account for truss-wearing being more painful. In direct hernia we may have the bladder, cæcum, or sigmoid flexure, forming part or all of the protrusion. In such a case pain is caused by direct trusspressure upon the bladder or bowel. Several cases of sigmoid

\section{F1G. 85 .}

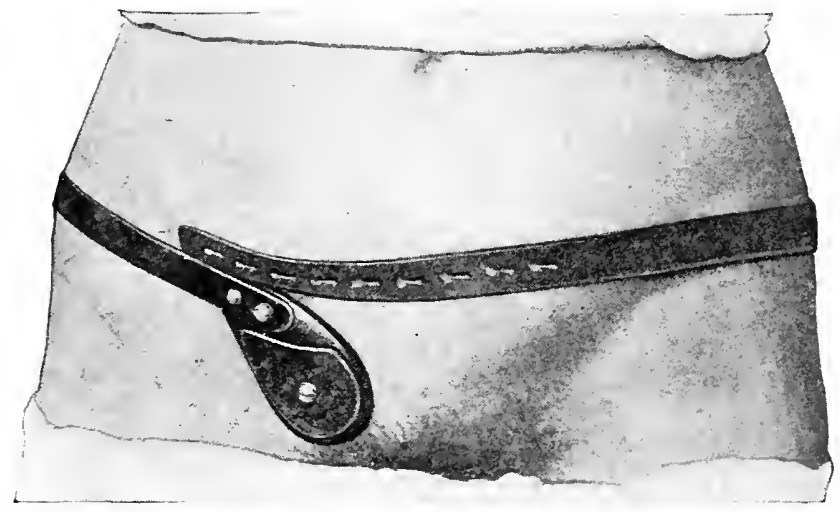

Hard-rubber French type of truss applied. The strap may be buttoned on stud-head at end of spring or on another below centre of pad. The latter is preferred. The tendency of this truss is to slip down over the pubic bone.

and crecal hernia have been seen, that could not tolerate any form of truss pressure that was sufficient to retain the protrusion. In direct hernia a pad that is nearly circular in shape is frequently better than the oblong form. The water pad is particularly good, for, even if some pressure is made against the pubic bone, it does not cause the pain that a hard pad would. Thatever pad is used it must be placed nearer the median line, and lower, than for oblique hernia. Little or no improvement must be expected in direct hernia from truss-wearing. If the case is prevented from increasing in size and the patient is made fairly comfortable, it must be looked upon as successful. 


\section{COMPLICATIONS.}

There are certain complications met with in the mechanical treatment of hernia that require special consideration, and notably are the following:

(I) Cysts, within, or just outside the canal.

(2) Elongated sub-peritoneal fat.

(3) Reducible hydrocele or other fluid in sac.

(4) Varicocele.

(5) Delayed descent of testicle.

(6) Interstitial hernia.

(7) Pregnancy.

(8) Adhesions of hernial contents.

Cysts.-It is not very uncommon, and may occur in either sex, that cysts form within the canal or in the vicinity of the external ring, where they interfere seriously with truss-wearing and lead to doubts as to diagnosis. Those that form lower down on the cord, or in front of the testicle, are not now under consideration, as they are sufficiently far away from the canal to escape truss pressure. These cysts most commonly form in that part of the tunica vaginalis which occupies the canal and which has not been completely obliterated. They may be closely associated with the cord (or round ligament), or hang by a pedicle, so as to slip out of the canal and have the appearance of being reducible to the abdominal cavity. It is the latter type that prove the most troublesome and misleading in the mechanical treatment of hernia. They may be associated with hernia, or occur alone and be mistaken for the latter condition. Their recognition is usually not difficult, if their possible occurrence is kept in mind. If a round, somewhat elastic tumor repeatedly slips under the truss pad, it is in all probability either one of these cysts, a piece of hypertrophied omentum, or subperitoneal fat. While the cysts present a round, elastic surface, that of omentum or sub-peritoneal fat is usually nodular, and its connecting neck is larger than the pedicle of a cyst. Furthermore, if the patient is lying down, omentum is fully reducible 
to the abdomen, and the cysts will appear to be reduced, yet deep pressure over the canal will reveal their presence.

The treatment of such cases must depend largely upon the position of the cysts. If in the way of the truss pad, it is best to draw the fluid off. Frequently this can be done by a hypodermic needle, but if they are large, an aspirating needle will be better. It has been my habit to make no further attempt to cure them on the first tapping, as this alone is sufficient in many instances. If they reform, they are, upon the occasion of the second tapping, injected, through the same needle that has been used for drawing off the fluid, with from five to ten drops of 95 per cent. pure phenol. On the day following this injection, there will be a recurrence of the fluid, almost, if not quite, equal to the original quantity. By the fifth day their absorption will be noticeable, and by the tenth to fourteenth day it will usually be complete, and the cure of the cysts permanent. If these cysts drop so far below the truss pad as to cause no discomfort, they may be entirely ignored, as they seldom acquire sufficient size to be inconvenient; but if within the canal, they are, by their distention of the tissues, a positive injury, besides causing the patient a great amount of discomfort.

Sub-peritoneal fat is often a forerunner of, or associated with, inguinal hernia. During muscular action it is pressed into the canal from in front of the peritoneum, and in this position it becomes elongated, so that it may protrude at the external ring. It is entirely outside of the hernial sac, if one exist, and to the average observer it cannot be distinguished from protruding omentum. Its action under truss-pressure is very similar to that of a fluid cyst; it will persistently slip from under the truss-pad and lead the patient, and probably also the doctor, to believe that it is the hernia that protrudes. Its feeling and shape are quite different from that of a cyst. It remains in the canal when the patient is lying down, and careful examination will usually reveal this condition. It cannot ordinarily be differentiated from omentum adherent within the 
sac. The truss pad can be worn over it without harm, and in many instances this pressure will result in its absorption. If recognized, it is best to assure the patient that no harm will come from it, even though it does protrude from beneath the truss. It is, of course, better that it should be held within the canal in the hope that it will be destroyed, but this cannot always be accomplished. The absorption and disappearance of this fat, under truss pressure, would doubtless account for some of the apparently remarkable cures of hernia in the adult by truss-wearing. In such cases the fat is present, but there is no hernial sac. In other words, no hernia exists.

Reducible Hydrocele or other fluid in the sac. The condition of reducible or congenital hydrocele is not a very common one, and, as might be expected, is seldom found except in children. Its recognition is the most important part, as very little modification will be necessary in the mechanical treatment. Where free fluid exists in the hernial sac no amount of truss pressure will retain it within the cavity of the abdomen. Fortunately, this condition is usually temporary, and if the truss pressure is maintained in order to retain the hernia, it will usually right itself. It is quite inadvisable to resort to any method but that of an operation for the cure of existing hernia and the destruction of the secreting membrane. It is seldom advisable to tap the hydrocele, and, when this is done, it must be remembered that in reality the cavity of the abdomen is being entered, and every precaution as to asepsis should guard the patient against infection.

Free fluid in the cavity of the abdomen (ascites) from heart, kidney, liver or other lesions, is a very troublesome complication in the mechanical treatment of inguinal hernia. and frequently causes the patient greater mental concern than the possibly fatal malady which is its cause. It is entirely futile to attempt the retention of such fluid by truss pressure. If it is not large, truss-wearing should be continued in order to prevent the protrusion of bowel or omentum, and the patient should be assured that no harm will come from the fluid in the 
sac. If the abdominal distention is great, it is really unimportant whether the patient wears a truss or not, as the bowel and omentum both float at the upper abdominal cavity on the fluid, and there is no tendency for either to protrude through the inguinal canal. A large and strong suspensory bag affords about the only approach to comfort that can be afforded. Ascites, following scarlet fever or from other causes, should be managed in the same general way, except that it is more important, in these cases, to continue the use of the truss even though a large quantity of fluid is present. When this fluid is reabsorbed, it may require temporarily increased truss pressure on account of the damage done the abdominal wall by overdistention, but as the case improves the pressure should be again diminished.

Varicocele.-The small or moderate size varicocele demands little attention in the mechanical treatment of inguinal hernia, except to keep in mind the fact that compression of the cord, against the pubic bone, will retard the return flow of venous blood from the testicle much more than it will obstruct the arterial supply, so that poor truss-fitting tends decidedly to increase the condition. A large varicocele, combined with hernia, requires the use of a snug-fitting suspensory in addition to the truss. It will also make necessary the resorting to stronger spring pressure, as the cord is almost always much larger than normal. Various special pads have been designed, so made as to press on either side of the canal and avoid extreme pressure on the cord, but they are of little real service. A small pad placed high on the canal will protect the corl better than any other way known to the Author.

Delayed Descent of Testicle.-This condition complicates, perhaps, more frequently than any other, the mechanical treatment of hernia. It is not uncommon in children that the absence of the testicle, from the scrotum, remains undiscovered by parents until bulging in the inguinal region is noticed. This bulging indicates the attempts of the testicle to get into its normal position, and it is safe to state that it is always accom- 
panied by hernia, and, that the hernia occupies a congenital sac. In view of these facts, it is a condition worthy of the most careful attention at whatever period in life it is discovered.

It is obviously the duty of the physician to so treat the case, if possible, as to aid the attempts of nature to place the testicle in the scrotum, and to this end the truss should be so adjusted as to retain the hernia without preventing the descent of the testicle. If the patient is very young, the bulging slight, and the testicle not outside the canal, it is considered good practice to keep the case under observation, but to apply no truss. As the hernia increases in size, both it and the testicle will protrude at the external ring, and they may then be separated; the former retained by a small pad over the upper part of the canal, while the testicle is kept from re-entering the canal, from below, by the same pad. The preferred spring, for these cases, is of the cross-body type, (no. 3, cross-body group), as light as possible, and to this should be attached a small, prominent pad the size of no. 3 or 4 of the oval pad diagram (fig. 67).

If a truss of this type can be worn with comfort, it is advisable to continue its use for two or more years, in children under five years of age. If, however, it causes any amount of pain, especially colicky pain in the abdomen, this indicates that the intestine is not fully reducible and the use of the truss should be discontinued. Such cases are unsuitable for mechanical treatment and operation is advisable at the earliest date convenient. Even where reduction and retention are complete, cases of hernia, associated with delayed descent of the testicle, are very seldom cured by truss treatment, as the congenital sac in communication with the testicle persists, and it is believed that they are properly operative cases. The only reasonable excuse for delay is, perhaps, the early age or bad general condition of the patient.

Where the testicle is retained high in the canal, associated with troublesome hernia of good size, some relief and protection against strangulation of the intestine may be obtained by 
using, after reducing the protrusion, a soft water pad directly over the testicle. A concave pad and one made in horseshoe shape have been used with at least partial success. The wearing of any pad that presses directly upon the testicle is, however, attended by more or less discomfort and aids in the destruction of the already impaired organ. That it leads to

FIG. 86.

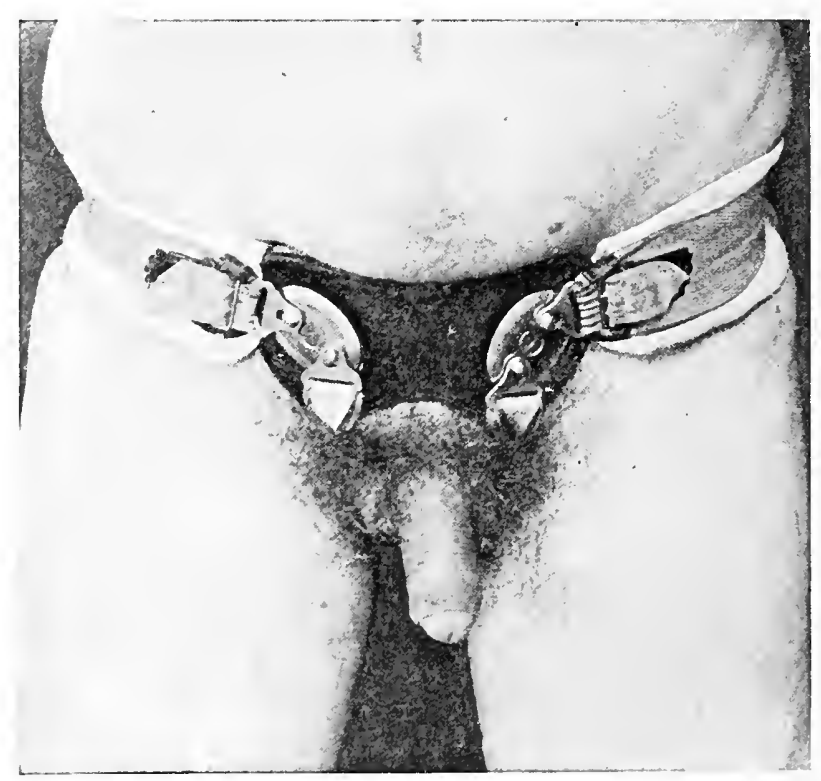

Double retained testes associated with double congenital hernia.

cancer of the testicle, as stated by some writers, has not been borne out by the author's experience. A testicle may be permanently retained within the canal by truss pressure, but if there is sufficient length of cord to allow of it, slipping beneath the truss parl is pretty sure to occur, and at such times is liable to prorluce the most excruciating pain.

In the case illustrated in fig. 86 a young married man of twenty-eight years of age had enjoyed perfect health in every 
respect except the defect under consideration. Both testicles were retained within the canals. At times one or buth would slip outside the external ring and he would then suffer extreme torture until he was able to reluce them to the canal. On the other hand, if he abandoned truss-wearing, he was subject to symptoms of strangulated hernia. Between these two

FIG. 87 .

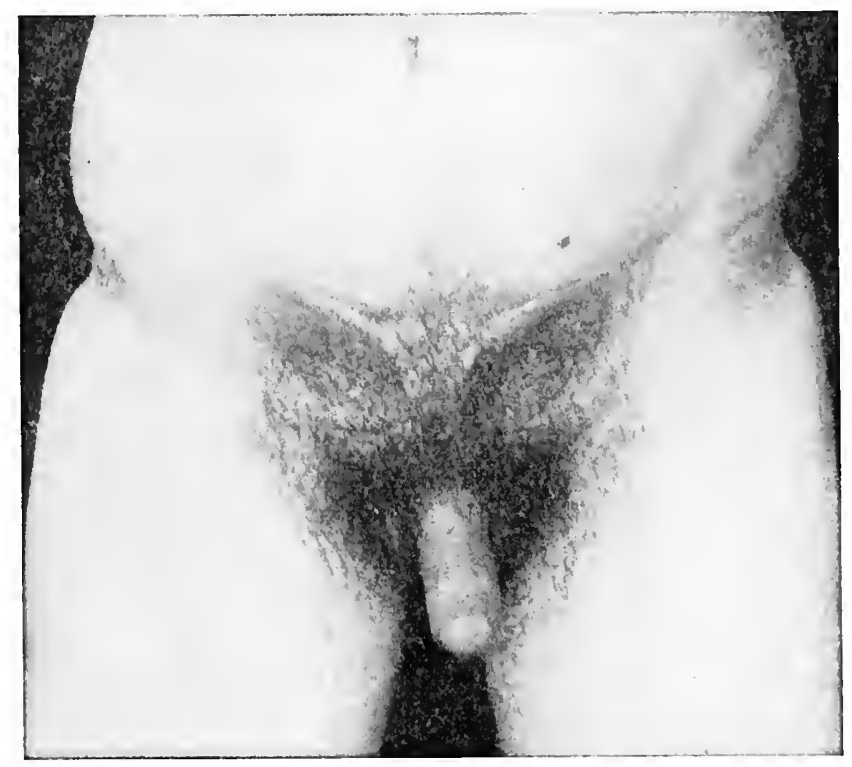

Double retained testes. Same as Fig. S6 with truss removed. Showing effects of extreme pressure.

dilemmas he found truss-wearing the safer, and the truss shown on him seemed to be the best for his use. Examination of the photograph, with the truss removed (fig. 87), shows the result of the extreme pressure necessary to retain the testicles within the canals. This case was sulssequently operated upon. Delayed testes may, in some few instances, be retained in the canal under truss pressure for years with comparatively little trouble. and then become so irritable as to necessitate the abandonment 
of the support. One patient, sixty years of age, had throughout his life worn a truss that retained the testicle in the canal, but the organ finally persisted in slipping under the pad and eventually descended into the top of the scrotum at that late period in life. A small, deep truss pad was adjusted so as to compress the upper part of the canal, retaining the hernia and thereby securing safety and comfort.

Descent of the ovary into or through the inguinal canal complicates, more rarely, the mechanical treatment of inguinal hernia. It carries with it the same form of congenital sac and the same difficulties of treatment. It forms a mass in the canal which is hard to diagnose from adherent omentum. If the ovary is present the mass will usually at each period of menstruation become larger and more sensitive to pressure.

As regards pain, truss pressure is better tolerated in these cases than where the testicle is similarly placed, and in several instances a small truss pad has been worn over the upper part of the canal, retaining the hernia while the ovary has been protruding at the external ring. In two or three cases a concave pad has been used directly over the ovary, retaining the hernia without any amount of discomfort. One patient, under observation for twenty years, has been obliged to remove her truss at each menstrual period, but suffers no discomfort at other times, and has declined operative relief. An ovary that has once entered the canal is seldom again fully reducible to the abdomen, and it is quite likely to become diseased in this abnormal position, especially when under truss pressure, and for this reason it is consiclered best to look upon them as operative cases unless especially contra-indicated by some other more serious condition.

Interstitial hernia instead of following the canal and descending into the scrotum or labium, forms a sac for itself between the layers of the abdomen, and while it usually presents a tumor covering a large area, its contents, if reducible, are returnerl to the abclominal cavity through a comparatively small opening at the internal ring. Personal experience leads 
to the belief that almost any of the trusses used for oblinue inguinal hernia will be equally successful in this condition.

The English prefer in these cases a larger parl such as shown in fig. 88, lut the water pad of medium size on either the cross-borly or Hood spring has been found less cumbersome, and is believerl to be more effective. Owing to the peculiar formation of the sac in interstitial hernia there is greater

\section{FiG. S8.}

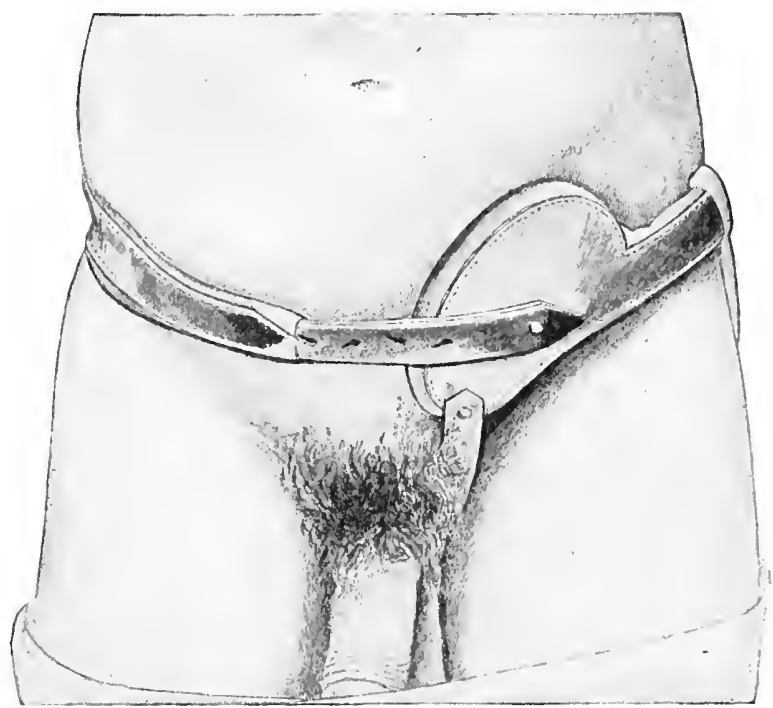

Truss for reducible interstitial hernia. (Macready.)

liability to the occurrence of strangulation, and unless the retention by truss pressure is complete at all times, early operation for cure should be strongly advised.

Pregnancy presents conplications in the mechanical treatment of hernia that should be considered. In the earlier months difficulty in retaining the hernia is increased by intra-abdominal pressure, and it is frequently necessary to add spring pressure. Later, this pressure should be removed and the size of truss increased. 
As pregnancy advances and the uterus rises up out of the pelyis, it carries the intestines and the omentum higher in the abdominal carity and away from the inguinal region. It therefore commonly happens that a woman could with safety abandon her truss entirely during the last six weeks of gestation without suffering any protrusion of her hernia. It is not considered best that this should be done, as a very light truss can be worn with safety. The disappearance of the hernia during the last months of pregnancy has frequently led both the patient and the doctor to believe that it had been cured, and consequently into the mistake of allowing the woman to get out of bed during her convalescence without having her truss on. This is franght with special danger at this time, when the muscles are weak and flabby from recent distention. The truss should be very carefully readjusted before the woman leaves the bed.

Adhesions of Hernial Contents to Sac Wall.-These cases will be more fully considered under the heading of irreducible hernia, and reference here is to those cases only where the bulk of the protrusion is reducible, but a small part, usually omentum, is held in the hernial sac by adhesion.

This condition is not always recognized by examining the hernia, but may be first suspected by the extreme difficulty experienced in retaining it by the truss applied. In most cases where a hernia of moderate size cannot be retained by means of a well-fitting truss, there probably is a small piece of actherent omentum within the canal that is not reclucible. After the truss is applied this omentum acts as a guide or actually drags down the bulk of the hernia. If the adherent part is omentum, no harm will come of wearing strong pressure across it, and eventually this will destroy it and the hernia become easily manageable by a truss of ordinary pressure. It is best, therefore, to start with as strong a pressure as can be tolerated, and the patient should be instructerl to reduce the hernia as soon as he feels that it has protrucled under the truss pad. The so-called "Radical-Cure Truss" has been found most frequently suc- 
cessful (cross-body group, fig. no. 4), and a night truss is desirable. For the latter purpose nothing has been found better than the elastic truss shown in the group of springless trusses. The day truss should be applied before the patient leaves the bed in the morning and after careful recluction of the hernia. The clange to the night truss should be after the patient is in bed. In using extreme truss pressure the skin must be thoroughly cleansed twice daily and kept dry with some good antiseptic or talcum toilet powder. If bowel is adherent to the sac wall it will be found that strong truss pressure cannot be tolerated. Colicky pains will be experienced similar to those in threatened strangulation, more frequently in the vicinity of the navel than under the truss pad, and truss-wearing should be at once abandoned and operative relief afforded.

\section{MECHANICAL TREATMENT OF IRREDUCIBLE INGUINAL HERNIA.}

It is not necessary to devote much space to this branch of the subject, as the proper treatment of irreducible hernia is by surgical, instead of mechanical means. There are cases, however, where from one cause or another operation is inadvisable and then the question of palliative treatment must be met. The older works on hernia taught that it was dangerous to wear a truss pad over protruding omentum. Experience has so often disproven the correctness of this statement that it can be flatly contradicted. The protruding contents of an irreducible inguinal hernia are almost always omentum and intestine; the latter being reducible, and the former adherent to the sides or bottom of the hernial sac. Such a case is shown in the photograph, fig. 89. Where the intestine is reduced it can, in many instances, be retained by strong truss pressure across the neck of omentum, where it passes through the canal. Such pressure may protect the patient against strangulated bowel. The inconvenience that he will suffer will usually be that attendant 
upon the wearing of an unusually strong truss. It seldom happens that pressure upon the neck of omentum does any serious harm, and in exceptional cases it has caused its absorption. In rare instances an inflammatory action has been set up, necessitating confinement to bed and application of an icebag for twenty-four or forty-eight hours, but nothing more

Fig. 89.

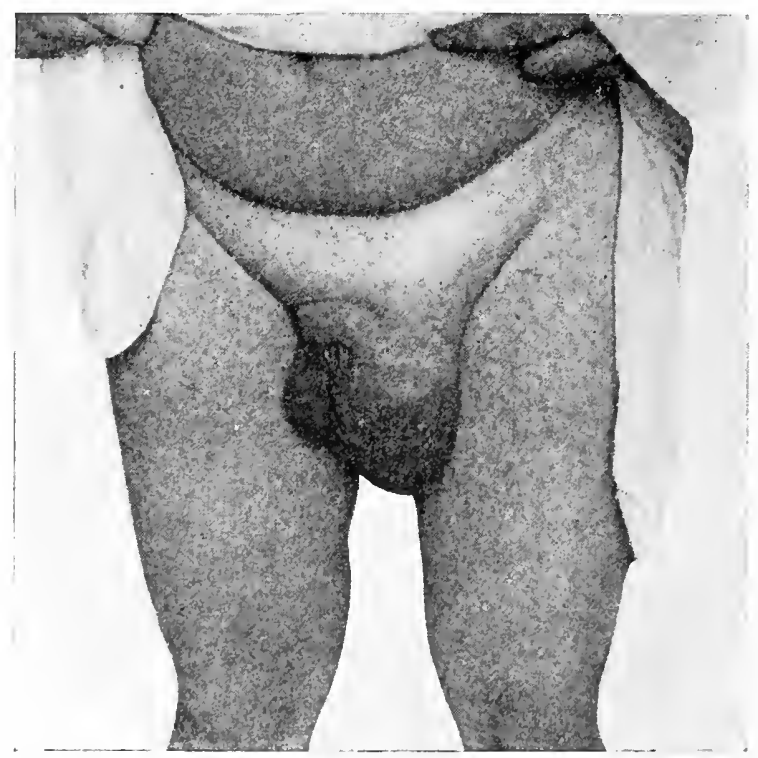

Large irreducible scrotal hernia. Intestine wholly reducible and could be retained by truss pressure. Subsequently cured by operation; large mass of hypertrophied omentum amputated.

seriouls has been seen. The case is certain to grow worse, even though protected against the dangers of strangulated hernia. The protruding omentum becomes hardened and takes on a condition of hypertrophy which may lead to its revelopment to an enormous size.

In selecting a truss for these cases a strong spring must l,e userl, usually of the cross-body variety of the ordinary type, or, still better, the radical-cure form. The water pad, aside from 
the fact that it is not durable, is a very good one. Nany times, however, a deep hard-rubber pad, of good size, may be worn with equal comfort and prove far more serviceable. If the irreducibility of the hernia is caused by adherent omentum in the canal, that does not extend down to the scrotum and is small in quantity, then a concave pad will prove useful. In these smaller cases it is not uncommon for the adhesions to

FIG. 90.

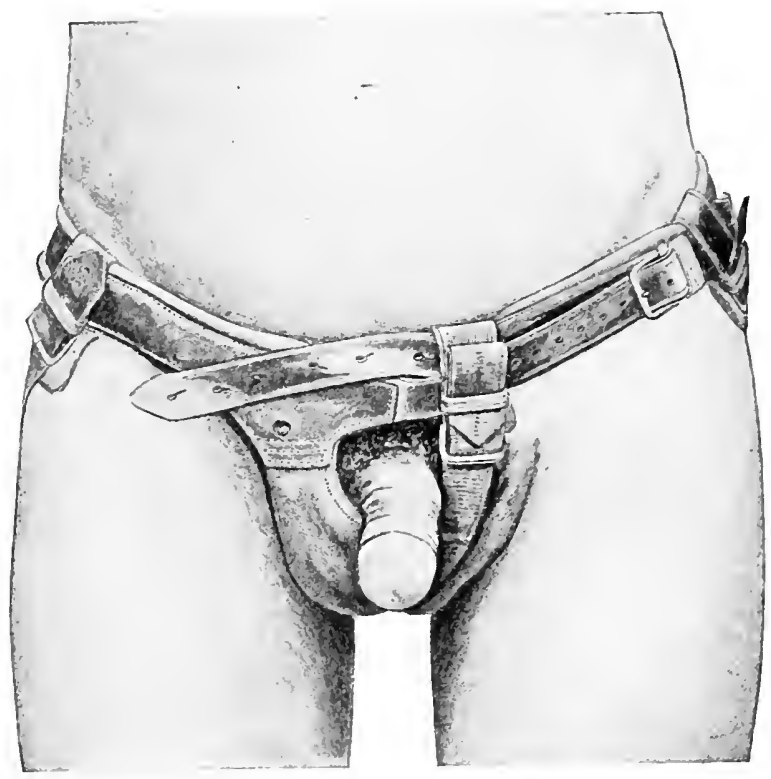

The hinged-cup truss for irreducible hernia. (Macrady.)

yield under the pressure of the truss and the hernia to become a reducible one. In such a case, where a concave pad has been used, it should promptly be changed to a convex one, in order to secure more perfect retention. The convex pad should be applied in every case where the protrusion can be reduced into the canal and is of small size.

Where a mass of omentum is adherent in the scrotum, and a truss pad has been applied across its neck, a good-fitting, 
strong suspensory bandage should also be constantly worn. The English use what they term a "Hinged-Cup Truss" (fig. $90)$, which appears to combine the pressure of a regular truss pad with a concave cup over the protruding omentum. Macready says of it:

FIG. oI.

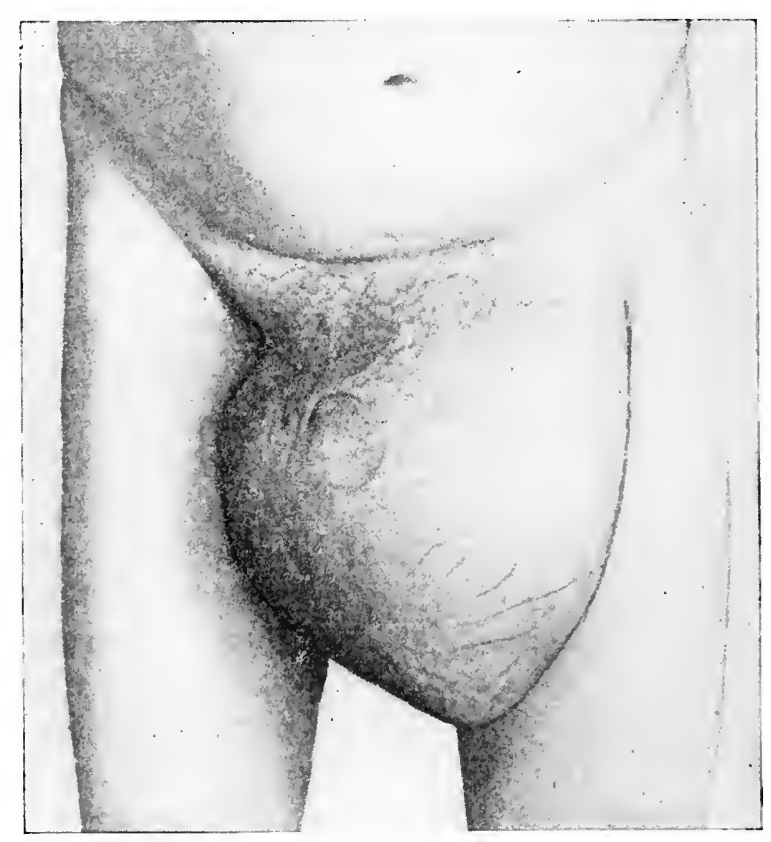

Enormous irreducible left scrotal inguinal hernia. Both intestine and omentum irreducible.

"It is seen to consist of two parts, of which one occupies very nearly the position of the pad of an ordinary truss and is not concave, and the other forms a scrotal portion, which is uniter to the former by a transverse hinge. The scrotal part is a three-sided frame of metal, covered in with chamois leather, curverl to adapt itself to the distended scrotum. The apex of the triangle is downwards towards the perineum, and to it are attached the understraps, which are fastened to the side of the 
truss just behind the shoulder, as usual. Every pull on the understrap presses the cup against the scrotum, whilst by means of the hinge, the movement is hindered from being convered to the pad." The author has had $n$ personal experience with it.

$$
\text { FIG } 92 \text {. }
$$
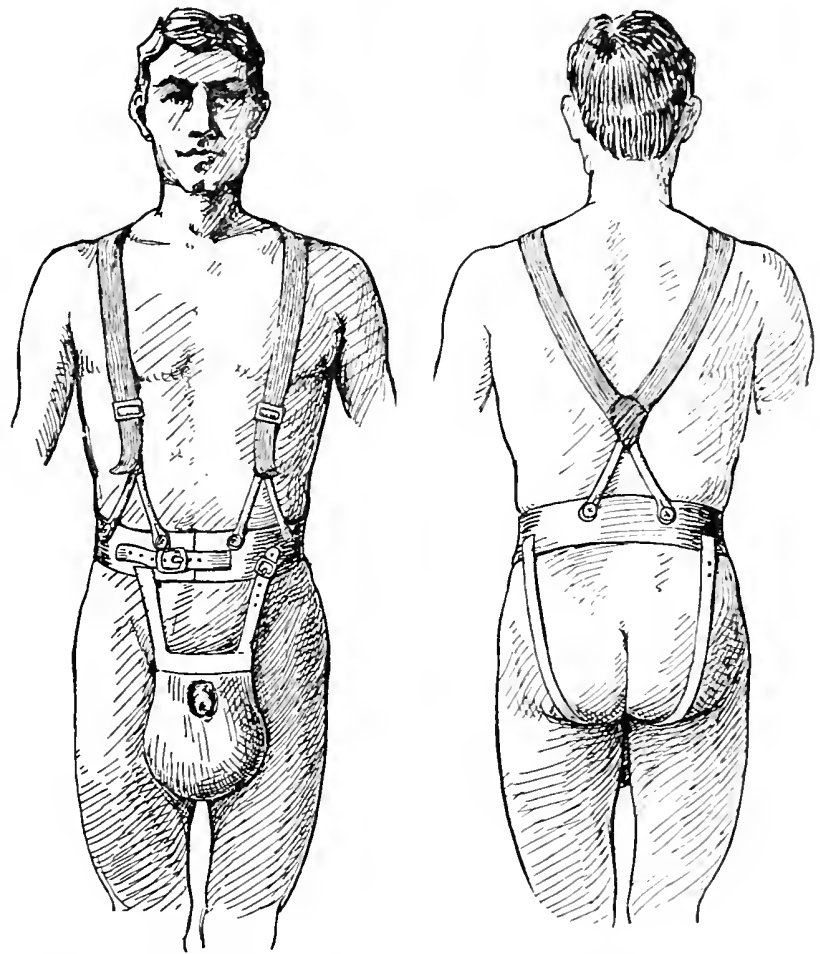

Showing method of supporting enormous irreducible scrotal hernia, when no form of truss can be worn. The weight is transferred to the shoulders by a pair of suspenders.

Irreducible scrotal hernia of enormous proportions, such as is shown in fig. 9I, in which truss-wearing is impossible, and when operation is inadvisable, should be protected by especially constructed supporting bags (fig. 92). The bag should be made from measures carefully taken, while the patient is recumbent and with the tumor at its smallest. There should be a strong band about the pelris to which can be buttoned an 
ordinary pair of non-elastic suspenders passing over the shoulders. A support of this kind will, in a measure, prevent increase in size besides adding materially to the comfort of the sufferer. Such hernize are pretty sure to cause the death of the patient eventually, and their operative relief should be seriously and immediately considered. It is true that danger attends such operations, but it must also be taken into account that the patient may be incurring a greater danger by declining it, besides almost complete disability. 
CHAPTER IX.

\section{MECHANICAL TREATMENT OF INGUINAL HERNIA IN INFANCY AND CHILDHOOD.}

One-half of all abdominal hernix occur during the first five years of life, and therefore come within the consideration of this branch of the subject. It may also be said that as regards treatment they form the most important, but by no means the most difficult cases. Important, because it is during this period that the defect must be cured, if this is ever to be accomplished without an operation. It is also important in the interest of the infant's good health, as it unquestionably affects the health and strength of the infant to a much greater extent than it does an adult.

The mechanical treatment of inguinal hernia is not difficult, and there are very few, if any, surgical affections that should be so thoroughly under the control of the family physician. Infants are easier to fit than the adult, and they will be brought to the physician with greater regularity; there is freedom from many complications which arise later in life, and they prove of more interest because they are cured if properly managed.

Unfortunately, however, it is a fact that children are growing up to manhood and womanhood, carrying with them the hernize of infancy because of lack of proper attention on the part of the family physician. The case has been brought to the doctor, and he has "prescribed" a truss, and recommended a druggist or instrument maker who deals in these articles. Having done this, he feels that he has discharged his whole duty to his patient, and gives the case no further thought. The dealer sells to the patient whatever truss he happens to be interested in, or has in stock, the case itself having little or no influence in deciding what form of appliance is to be worn; nor in the majority of instances is the instrument selected ever 
shaped to the form of the infant who is to wear it. In fact, the baby is fitted to the truss and not the truss to the baby. The sale of the truss having been made, no one but the parents feel any further responsibility in the case, and this rests lightly with them in the belief that just the right thing has been done. If, by the merest chance, the truss was approximately correct at the time applied, this does not hold true a few weeks later, as the child is rapidly changing in shape and size. The parents know nothing of the advisability of frequently refitting the truss, the dealer's interest and responsibility terminated with its sale, the doctor is out of the case altogether by having referred to the dealer, and the result is the child goes without a cure. This is all wrong, and the wrong begins with the family physician who first sees the case and passes it into unprofessional hands. $\mathrm{He}$ should become at once as responsible for its cure as he would for a fractured femur or a dislocated hip-joint. If he cannot bring to bear upon it better knowledge than his own, then it is his absolute duty to do the best he can himself.

In a few of the larger cities truss makers have, by practice, acquired considerable skill in fitting, and when they have taken the trouble to familiarize themselves with the anatomy of the parts, and have no special hobby or patent of their own to exploit, they do good work; but the physician, already possessing the knowledge of anatomy and methods of diagnosis, will soon obtain by experience the necessary mechanical skill, and carry out treatment in a far more scientific manner than possible for an unprofessional man, no matter how honest the intentions of the latter may be. $\mathrm{He}$ would also find that not only would the parents fully appreciate his efforts in behalf of their child, but by the cures which he would surely obtain his reputation would be materially enlanced.

Fully 95 per cent. of the inguinal hernix of infancy can be cured by careful mechanical treatment, but this must not be construed to mean the application of a truss, no matter how skillfully done, and the discharge of the case. It means taking the case under care and observation for not less than one year, 
or until a cure is effected. It is important to discover, when possible, the cause of the hernia in the infant. Of course, if due to congenital defect, time alone can remove it, but it will surprise a good many physicians to know how often the cause can be ascertained and removed. In this connection, I will again call attention to the frequency of hernia resulting from constipation, whooping-cough, tight belly-bands, and longcontinued crying. Attention to, and, so far as possible, removal

\section{FIG. 93 .}

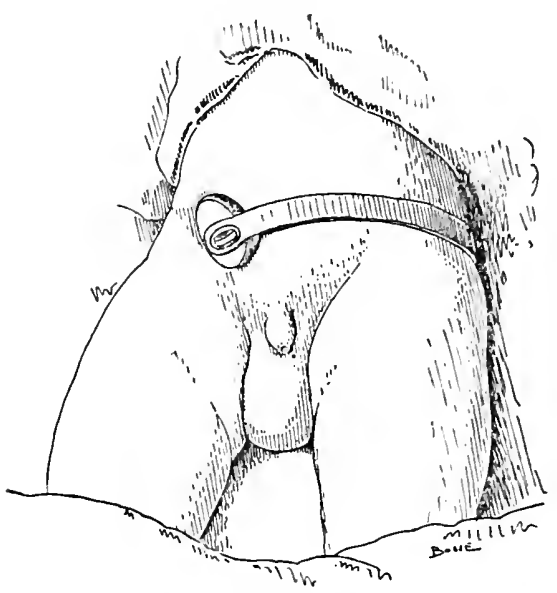

Five weeks old boy with right complete inguinal hernia and hydrocele of tunica vaginalis on the same side. Hard-rubber cross-body truss applied.

of, these causes is the first essential of successful treatment. This branch of the subject has already been considered under the canse and diagnosis of hernia.

The mechanical treatment of hernia in infancy is beliered to be the better method, for the reason that many can carry it out who are not qualified to do a surgical operation, and that the final result is equally good. The general practitioner can, if willing to derote the necessary time, secure good results without the use of the knife. There are very few ruptured infants that cannot be cured by the family plysician. State- 
ments that operations are advisable on children because trusses cannot be worn, are born of absolute ignorance of the mechanical treatment of hernia; as a matter of fact, infants tolerate truss pressure better, if that pressure is intelligently applied, than do adults. It is unfortunate that almost all infant trusses are made entirely too strong, and if applied as sent out

FIG. 94.

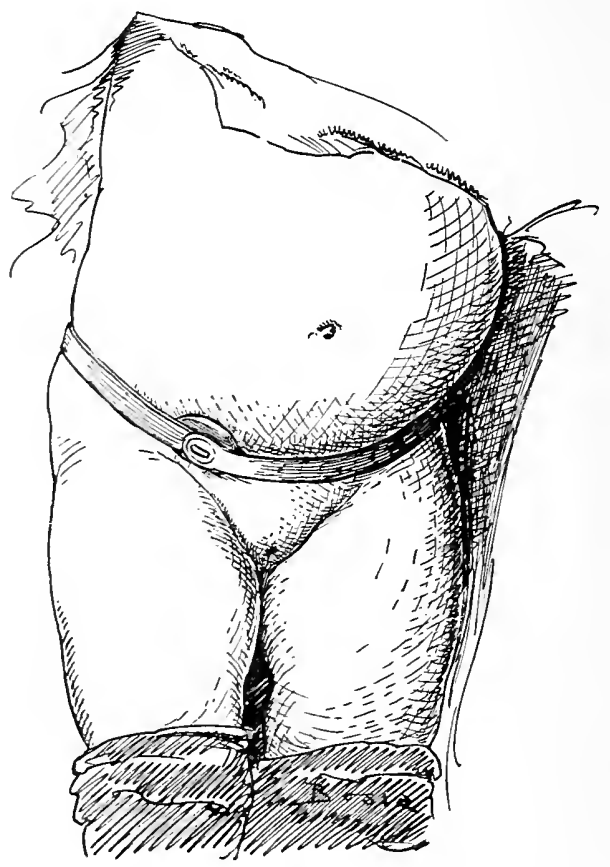

Hood truss applied to child six months old.

from the factory, must cause pain, if not actual injury, to the delicate tissues. It is just here that the physician's knowledge and supervision is essential. Nor is it a fact that hernia cured by truss is more liable to recur than when the same result has been brought about by an operation. It has been a matter of surprise that so few of the many children who have been under personal care and cured during the past twenty-five years have 
had a return of their ruptures. The recurrences have been wholly due to some violence, as whooping-cough, bronchitis, or some other cause, which would have been quite as likely to have produced hernia in a child who had never had it.

The age at which mechanical treatment may be begun is a question which many physicians are in doubt about, and one often asked in the lecture-room; the answer being that an infant old enough to be the possessor of a hernia is quite old enough to have the hernia treated (figs. 93. 94, and 95).

FIG. 95.

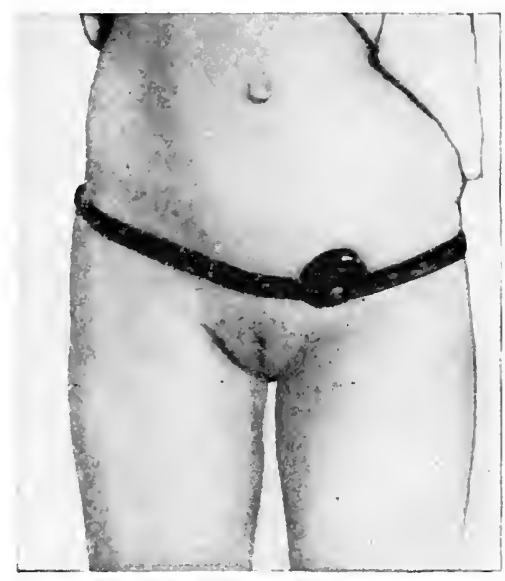

Cross-body hard-rubber truss as usually applied without perineal under-strap.

Trusses have repeatedly been put on babies ten days and two weeks old, and there has been no occasion to regret beginning treatment at this early date. It is an erroneous idea, and unfortunately a rather prevalent one, even with physicians, that a baby will " outgrow" this defect, or that it is " better to delay treatment until the child is olcler." It being conceded that it is advisable to begin treatment as soon after the development of the hernia as possible, the next question is as to the manner of supporting the protruding viscera. There is no bandage or makeshift of any description that will take the place, either for 
comfort, cleanliness, or efficiency, of a carefully applied truss containing a metallic spring, and covered by material impervious to moisture, such as hard rubber or celluloid. Elastic bands, so-called elastic trusses, and bandages such as a "hank" of worsted (fig. 96) have been recommended at various times; but they are a delusion and a snare, and usually an abonination.

There is no lack of good trusses in this country. The druggists of every town and hamlet in the United States are annually visited by representatives of manufacturers, whose

Fig 96 .

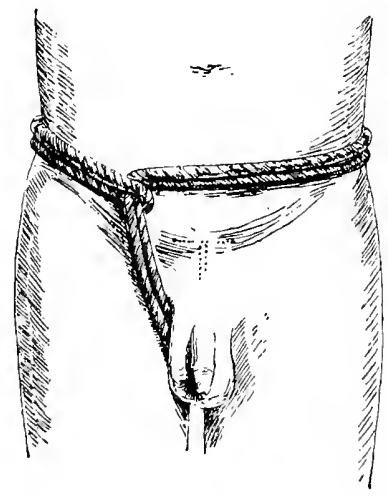

Application of a skein-of-wool truss. (Eccles) This is considered a very poor makeshift.

products are not equalled in any other part of the world, and still the number that are really suited for use on infants is very small, owing either to defects in the original design of the truss, or because of their being entirely too strong, even where the form of the truss is correct. All infant trusses which are macle to apply from the side of the rupture, all of those where the pad is placed upon a descending arm at a level lower than the pelvic spring, and all trusses cushioned or padded with soff material, are condenned. The so-called French and German style of trusses, which comprise the bulk of stock of many druggists, should have become obsolete a half-century ago. 
In truss selection the following prints should be remembered: (1) The spring should be so tempered, if steel, that it may be readily bent to the shape of the child, and its pressure adcled to or diminished by increasing or removing the anount of curve which it possesses. (2) The entire truss should be water-proof, that it may be frequently washed and not damaged by urine. Any material which absorbs, and holds in contact with the child's skin, its excretions, will cause scalding and

FIG. 97.

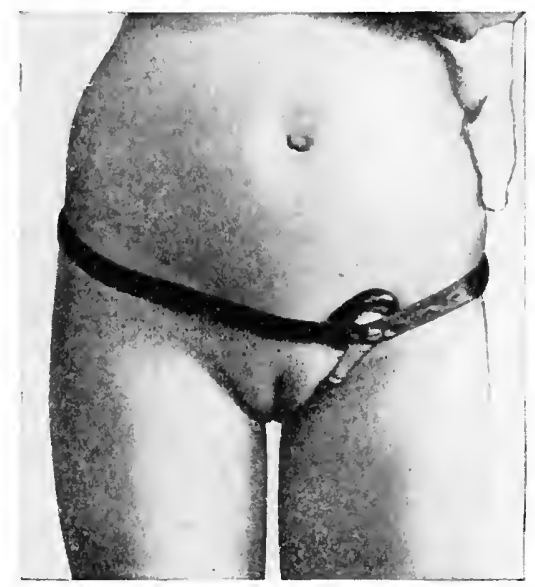

Left complete inguinal hernia in child 8 years old, retained by hard-rubber cross-body truss with perineal under-strap. The latter is seldom required.

excoriation, besides being actually filthy. (3) The truss shotid be simple and clurable. The more simple in design the better is the truss, as a rule. Pads with ball-and-socket selfadjusting action, so-called, or with complicated set screws for adjustment, are entirely unnecessary and soon become useless.

For the treatment of single inguinal hernia, in the infant, the cross-body spring, which, from the pad, crosses the front of the abdomen, passes around the hip of the opposite side, and across the back, is one of the most valuable appliances that can be used (figs. 93. 97). This truss can be obtained of 
almost every druggist in the country, the spring is covered either with hard rubber or celluloid and known in the trade as the "cross-body" truss. A spring of this kind will surround about two-thirds of the pelvis, and while it is supplied with a strap to complete the circumference, it readily holds itself in place whether the strap is used or not. Its pressure can readily be adjusted to the requirements of the case by increasing or diminishing the curve of the spring. Those covered with celluloid have the advantage of being readily shaped to the

Fig. 98.

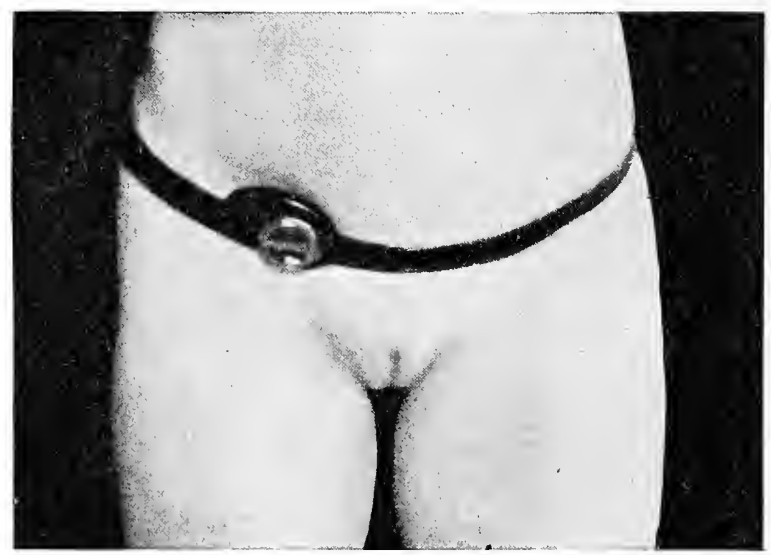

De Garmo-Hood truss, German silver spring, hard-rubber cover. Applied to small right oblique inguinal hernia.

form without heating (which is necessary in shaping the hardrubber springs), but have the disadvantage of being not quite so durable as those with the hard-rubber covering.

The "Hood" truss, covered by celluloid or hard rubber, is made in infant sizes and kept by most clealers, and forms an extremely desirable truss for children whose pelvic measure exceeds sixteen inches, but is not desirable in those of smaller size.

The De Garmo-Hood truss (fig. 98, 99) differs from the others in having a spring of German silver instead of steel, the 
covering being of hard rubber. This material for springs in this form of truss las proven very valuable, owing to its being readily shaped to form without liability of breaking, and because of its not having the action of compression found in the steel spring. This truss has been extensively used in my private work. Manufacturers have told me that they could not sell it to the dealers because it was considered too light, and this clearly indicates how little the clealers comprehend the requirements of a proper truss for infants.

FIG. 99 .

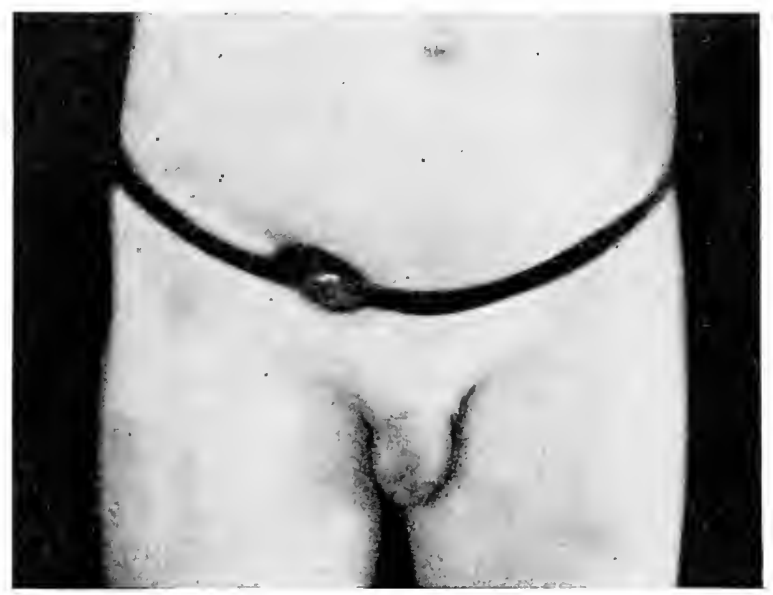

Right complete oblique hernia in boy of 8 years. Retained by De Garmo-Hood truss.

The measure for selecting the size of the truss should begin just above where the hernia is seen; that is, at the internal abdominal ring, passing around the hips midway between the crest of the ilium and the trochanter najor. This, in number of inches, will indicate the size of the truss required. In the shaping of the spring, the diagram method, previously described, will be found of the greatest service. The diagram is used instead of the child. If a spring covered with hard rubber is used, it should be passed through the flame of a spirit lamp until it is quite warm, and it can then be bent to the exact shape 
required. As before stated, and it cannot be repeated too often, most infant trusses, as sent out from the shops, are too strong in pressure, and this should be carefully guarded against. Only a light pressure is required if the location of the pad is at the right spot. A very common, almost universal, error, in applying trusses, especially to infants, is in putting the pad too low (fig. IOO). If the pad rests on the pubic bone its efficiency is at once destroyed and the discomfort of the child is assured.

FIG. I00.

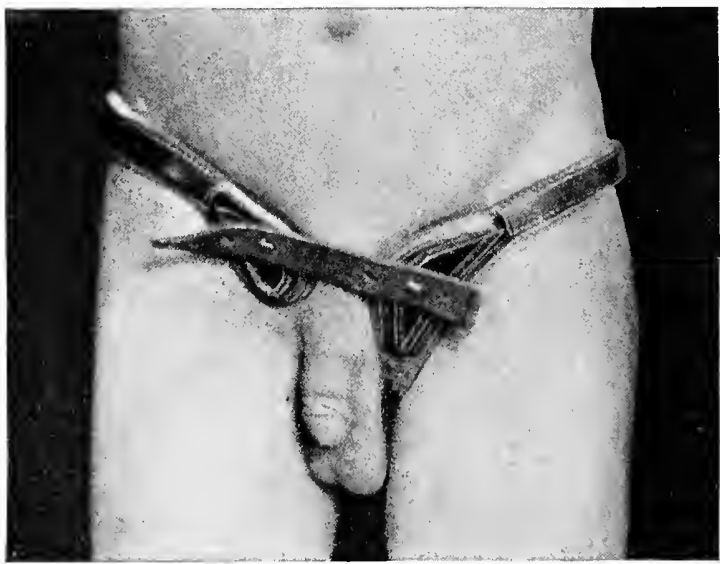

Boy of 12 years wearing the German type truss. Fitting on right side good, on left bad, owing to the use of what the truss makers call a scrotal hernia pad. In this case, omentum was adherent in scrotum.

It should be borne in mind that the design of truss-wearing is to keep the bowel entirely within the abdomen, and in order to accomplish this perfectly, the supporting pressure must be very nearly over the intornal ring. The descent of the hernia, stopped at the external ring, may in this way be kept out of sight, but still occupies the upper part of the canal, and a cure will never result. A truss pad that rests against the bone cannot thoroughly protect the upper part of the canal. It is held away from it, and the child is made uncomfortable. 
When the truss is fitted high the parts lack of the pad are soft and yielding, and discomfort is not caused.

Having fitted the truss, the care of the case has only just begun. The case must be kept under observation and the truss changed in shape and size as the child grows. This change and growth is very rapid, and the child should at first be seen at least once a week, and not allowed to pass entirely from care until it is curcd. In case of whooping-cough or severe bronchitis supervening, it is advisable to increase the truss pressure temporarily, but otherwise, after the first three months, it is well, if the hernia does not protrude, to begin to reduce the pressure. One year is the shortest period that a truss should be worn, and it should never be removed by the mother except for purposes of cleanliness, and this should be while the child is in a quiet and recumbent position. Absolute cleanliness must be insisted upon, and if the skin is kept clean and dry, it will tolerate strong truss pressure without abrasion. The free use of a good talcum toilet-powder is quite essential to the comfort of the infant truss wearer. After the careful cleaning and drying of the parts, it should be freely applied to the skin before placing the pad. Several good powders are on the market, or the formula given under general instructions, which was devised for this purpose many years ago and which has stood the test of time, may be used. Where an abrasion has once occurred and is slow to heal, on account of constant wetting by urine and the irritation of the truss, I have found nothing better than balsam of Peru.

As previously stated, the truss should be kept on for a period of one year; if, however, the case has been one of congenital hernia, it is best to prolong the wearing for two years. The truss pressure should be gradually lightened, until, during the last six months, it serves merely as a protective support against the recurrence of the hernia. If a strong truss were worn during the same length of time and then entirely removed, there would be a far greater liability of the return of the trouble. Attention to the child's general condition should 
not be overlooked. Constipation must be prevented and the digestive apparatus looked after. It adds greatly to the difficulties of controlling hernia if the intra-abdominal pressure is increased by flatulent distention of the intestines.

Among the complications mentioned, fluid in the tunica vaginalis is, perhaps, the most common and certainly the most perplexing. This fluid, which is usually reducible to the abdominal cavity through the neck of the tunica, may be present when the case first comes under observation, but more frequently forms during treatment. It occurs in many cases of congenital hernia, usually from one to two months after treatment has begun. The parent will bring the child back and tell you that the rupture is not as completely held by the truss as it formerly was, and that it is down almost all the time. The means of distinguishing this from the hernia have already been mentioned.

As regards treatment, it is best to let the fluid alone except in some rare instances where its quantity is so great as to inconvenience the child. If it ceases to return to the abdomen, indicating that the communicating neck has been obliterated. forming true hydrocele, it is well enough to tap with a small trocar, and this is usually sufficient to produce a complete cure. If from any cause the child has an effusion of fluid within the abclominal cavity and hernia, the fluid will also fill the hernial sac. In cases of this character truss pressure should be continued in order to protect the tissues about the canal. The fluid cannot be retained by any form of truss.

Non-descent of the testicle, associated, as it usually is, with hernia, requires careful consideration. We should never fail to examine carefully the scrotum of the ruptured child. It is not uncommon to see boys of eight and ten years old in whom it has never been discovered that only one testicle was present in the scrotum. In infancy this defect is likely to be overlonkerl, or. what is worse. if the testicle lies just outsicle the external ring it is mistaken for hernia, rednced, and kept back by a truss. When the testicle is in the canal, treatment will, 
in many instances, have to be delayed until it passes the external ring; then a small pad may be applied over the upper part of the canal.

In giving mechanical means the first place in the treatment of hernia in infancy, I do not wish to be understood as disapproving surgical measures. On the contrary, I believe that it is as justifiable to operate for the cure of hernia in a child as it is for the cure of clubfoot or other malformations. If by mechanical means we cannot correct either, it is our duty to operate. In clubfoot there are cases which an experienced orthopedic surgeon could confidently state would never be cured by mechanical appliances, but this is scarcely ever true of hernia, some of the most extreme cases in infancy yielding promptly and a permanent cure resulting without the use of the knife. This being true, beyond all question it is our duty to try the mild means first. If this is faithfully carried out, it will be found that few cases remain requiring operation.

\section{CARE OF SKIN AND GENERAL INSTRUCTIONS TO TRUSS WEARER.}

It is a matter of the utmost importance that the trusswearer be instructed how to care for the surface of the skin under the bearing of the truss pad, as it will save him much discomfort. He must also be taught how to put on and take off his truss and cautioned regarding the danger incurred by going about without it, even while in his own room. It is best that he should also know how to reduce his own hernia and how to act in case he finds this impossible. The fatality attending strangulated hernia is in many instances due to the ignorance of the patient regarding its dangers. The first essential to comfortable truss-wearing is that the skin pressed upon by the pads shall be kept strictly clean and dry. Not only must the skin be kept clean, but the truss itself must be frequently washed. Besides the keeping of the parts clean, it has been found desirable to bathe the skin frequently with equal parts of 
alcohol and fluidextract of hamamelis leaves; following this, after drying, by the application of a good, mildly antiseptic powder. The author has found nothing superior in the way of a powder to that published by him many years ago, which was designed for this special use, and the formula of which is here given :

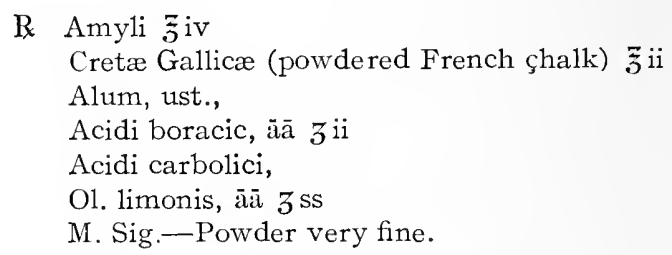

It should be made into a very fine powder and used by dusting the parts freely beneath the truss pad. As a toilet powder, for general use on infants, this will also be found superior to those commonly on the market.

If the skin has been broken, or suppuration has occurred beneath the truss pad, as it sometimes will, balsam of Peru has been found useful. In slight excoriations the use of benzoated oxide of zinc ointment heals the surface quickly. Its action is especially good, on infants, where the skin lesion is started by urine getting beneath the truss pad. If there is suppuration, the pus should be washed off thoroughly with a solution of hydrogen dioxide and the Peruvian balsam freely applied. Healing may frequently be accomplished in this way without confinement to bed or the discontinuance of the use of the truss.

There should be no compromise with a patient who suffers from a well-developed hernia, on the necessity of continuous truss-wearing. If for any reason he is obliged to discontinue its use temporarily, safety demands that he should maintain the recumbent position until such time as he can resume his truss. The man who has retained his hernia by a truss, for a time, and then discontinues its use, is in greater danger of strangulated hernia than he who has never worn one. 
Trusses worn by adults should be removed at night after the patient is in bed, being reapplied in the morning before getting up. If the patient has a persistent cough, or the hernia is so large that protrusion occurs while lying down, then a special night truss should be provided. The elastic truss is best suited for this use. He should understand that the truss suitable for night use is worthless, even dangerous, for day use, and that a truss suited for day is unfit for use at night. Also that, in a measure, he is disabled for life, or until cured, and that he must remain under the observation of his plysician. A properly selected and good-fitting truss restores him, for the time being, to normal condition. Owing, however, to changes in his shape, changes in the truss, or bad habits formed in the wearing of a good truss, he should, for safety, submit himself to frequent inspection.

A convenient way to instruct my patients has been to hand them a printed slip containing the following:

\section{INSTRUCTIONS TO TRUSS-IVEARERS.}

Apply your truss before rising in the morning.

Before applying, be sure that none of the hernia is protruding. Remove the truss after getting in bed at night; if correctly fitted, it will need no attending during the day.

In extreme cases a night truss may be needed. The one provided for day wear is unfit for such use.

Infants and young children should wear the truss both night and day.

Never go about your room without truss on.

In taking shower bath keep truss on.

Wash the truss--water will not harm it.

Extreme cleanliness will add to comfort.

Bathing the skin at night with equal parts of alcohol and Pond's extract will reduce irritation.

The free use of a good talcum powder in the morning is advised.

It is not safe to wear the truss over your underwear.

Any unusual abdominal pain or discomfort should lead you to examine your hernia. If protruding, it should be at once replaced and the truss readjusted. This should be done while lying down if possible. If replacement of hernia is impossible and pain is severe, apply an ice bag and send for physician. Delay is clangerous. 


\section{CHAPTER $\mathrm{X}$.}

\section{TREATMENT OF INGUINAL HERNIA BY GYMNASTICS.}

Works relating to abdominal hernia seldom mention gymnastics as an aid to palliative or curative treatment. It is, however, deemed quite worthy of consideration, and there is evidence both for and against it.

On one hand, a large number of patients have been seen who have developed hernia by misguided physical exercise or who, already having hernia, have forced it down to such an extent that strangulation or incarceration has occurred. On the other hand, many cases have been seen who have received decicled improvement from its use. I cannot say, however, that of my own personal knowledge I know of any herniæ permanently cured. I do not wish to intimate that I doubt the statement of scientific and careful observers who make contrary statements. Naturally those cases only who have hernia apply for relief, and those who have been cured have no occasion to. There is no doubt that the development of the muscles of the lower abdomen aids materially in the retention of hernia, even to the extent of its complete retention for a time in some instances, and I have for many years advised parents to allow their sons to go into almost any physical sport that they choose football excepted, provided they were wearing a truss that retained their hernix. In very early youth $\mathrm{I}$ am convinced that increased muscular tone aids in obtaining a cure without operation.

Furthermore, we must not pass by without due consideration the experience of such an able scientific observer as Jay IV. Seaver, A.M., M.D., who for many years has had charge of the physical education of the students at Yale College. He has tabulated the physical examinations of over 35,000 college 
students. Among this number he found nearly 3 per cent. of the young men had inguinal hernia (The Treatment of Inguinal Hernia in the Young, Yale Medical Journal, Feb., I900, and Feb., I904). Believing that those young men who had hernia, represented a type that especially needed physical development, he made it a rule not to excuse them from gymnastic exercises, unless especially requested to do so by the family physician. He directs that a suitable truss be applied and after the patient has become accustomed to it, the following exercises be taken twice daily, the severity of the movements being gradually increased:

FIG. IOI.

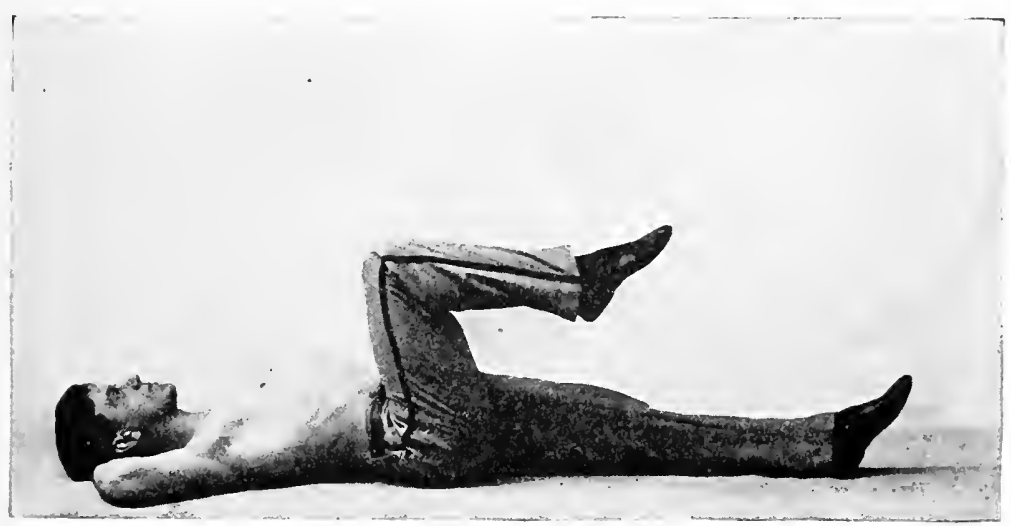

"( I) Lying on the back with thin cushion under head, raise the right knee, drawing it as close to the chest as possible, making special effort in the last part of the movement. Then extend the leg and perform the same movement with the left. Repeat the movement five times with each leg, then raise both knees toward the chest, doubling far enough to raise the pelvis from the floor, placing the hands, palms down, just under the hips; repeat five to ten times. It will be found much easier to take these exercises lying on a rug than on a bed, as a solid support gives the necessary resistance for the movement (figs. IOI, IO2). 
" (2) Lying on back with thin pillow under head and hands under back of neck, draw feet up to buttocks, then raise

FIG. IO2

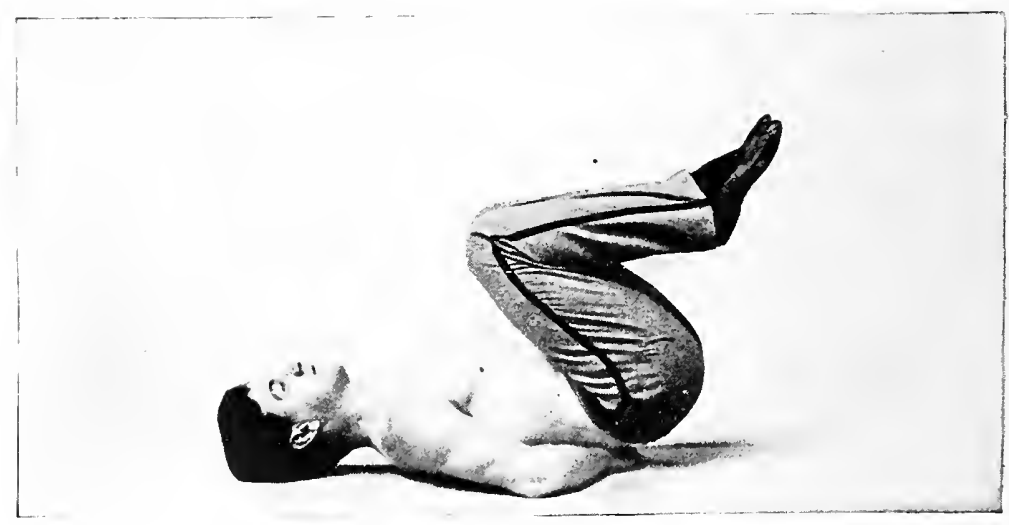

hips from floor as far as possible bearing the weight on the feet and shoulders. Repeat this exercise ten times. This movement will tend to strengthen the muscles of the abdomen and loins,

FIG. IO3.

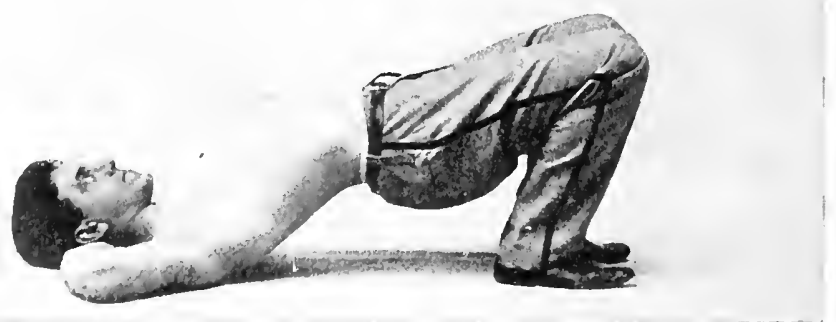

and is not a specially severe movement. This movement will be of increaser value if the person will inhale deeply while hold- 
ing the pelvis from the floor and exhale as the body is lowered (fig. 103 ).

* (3) From horizontal position on back, raise right leg to perpendicular, keeping the knee as straight as possible, and keeping left leg straight on floor, and hands with palms down, just under the hips. Repeat five times. Take the same exercise with left leg; then raise both legs to the perpendicular position an equal number of times, remembering to keep the leg straight at the knee. The movement may also be taken with fingers back of neck, but is more difficult. This is a

FIG. IO4.

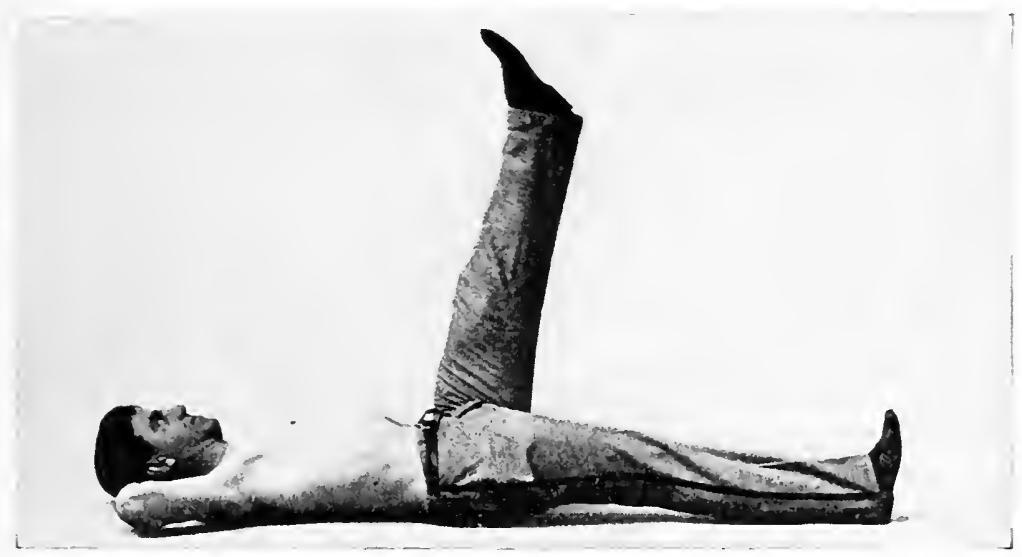

strong movement and must not be repeated so many times as to produce serious soreness of the abdominal muscles. By increasing at the rate of one movement erery second dar, a healthy person may expect to accomplish twenty repetitions without discomfort (fig. IO- ).

"(4) Lying on back, spread the legs so that the feet are three feet apart, then roll on to left shoulder, touching the floor with the right fingers as far as possible beyond the left shoulder. keeping the hips squarely upon the floor during the entire movement. Repeat fire times and then take the same exercise, twisting the trunk to the right (figs. I05 and I06). 
" (5) Lying horizontal on back, with fingers back of neck, raise right leg and touch on floor as near the left shoulder as

FIG. 105.

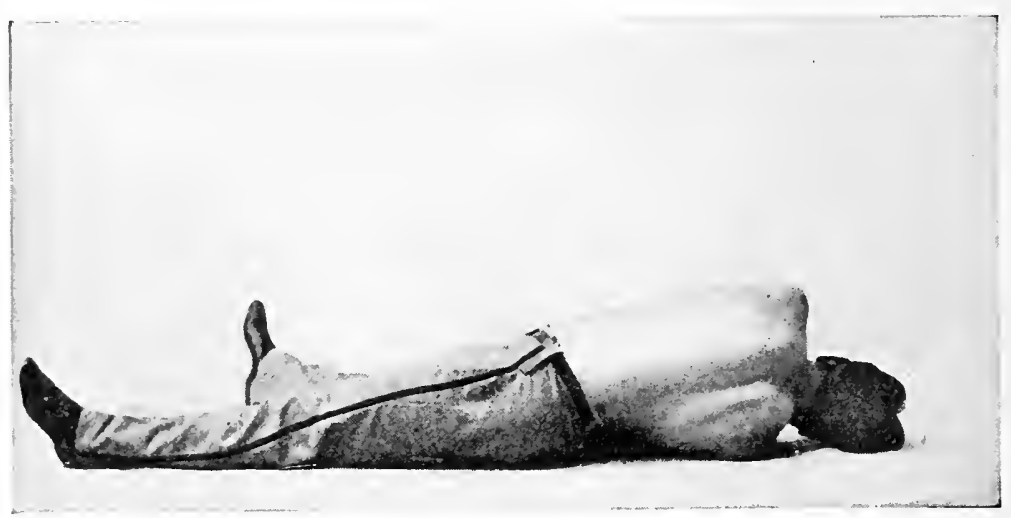

possible, keeping the shoulders square upon the floor. Repeat five times and take the same exercise with the left leg. This

FIG. I06.

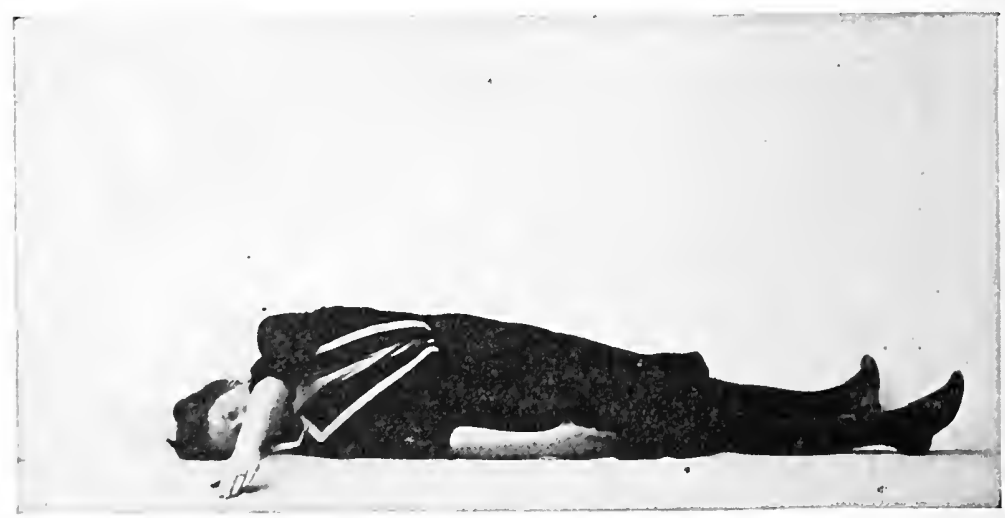

movement is of special value in strengthening the oblique muscles of the abdomen (fig. IO 7 ). 
“(6) Standing in erect position, with the head and hips well back, inhale deeply, and, at the same time, llaw in the lower abdomen as much as possible. Then relax the abdominal wall and exhale. Repeat from five to ten times, always trying to contract the lower part of the abdomen and, so far as possible, push out the upper part near the waist line. Then reverse this movement by contracting the waist line vigorously and cause, as nearly as possible, the wave of contraction to pass down over the abdominal wall.

FIG. I07.

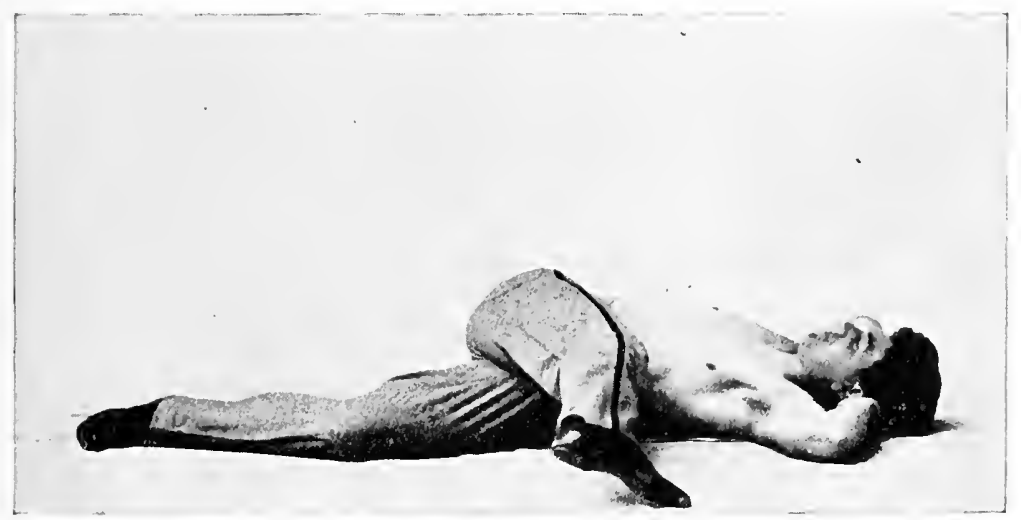

“ (7) Standing in position, with weight on the balls of the feet, with head and hips well back, raise the arms forward and upward, keeping the fingers well extended and palms parallel until, at the perpendicular position, they are the same distance apart as the breadth of shoulders. Inhale as the arms move upward, and use at least ten seconds in this inhalation. Then allow the arms to sweep downward to the original position, and the air to escape by the nose. Repeat six to ten times.

" ( 8 ) Standing erect, with head well back, raise the hands sideward and upward to the horizontal position. Then bend the arms at the elbows until the finger tips touch those of the opposite hand on the back of the neck. Then inhale deeply, 
pushing the elbows upward and backward as far as possible, keeping wrists firm, and then exhale as the elbows are lowered, move slightly forward, but retain the finger tips in position on the back of the neck, and the neck well back during both inspiration and expiration. Repeat fire to ten times. The abdominal wall must be made to move freely in this exercise. and it will be found that no free movement of respiration can be accomplished in this position. except by a use of the diaphragm and general abdominal wall.

FIG. IOS.

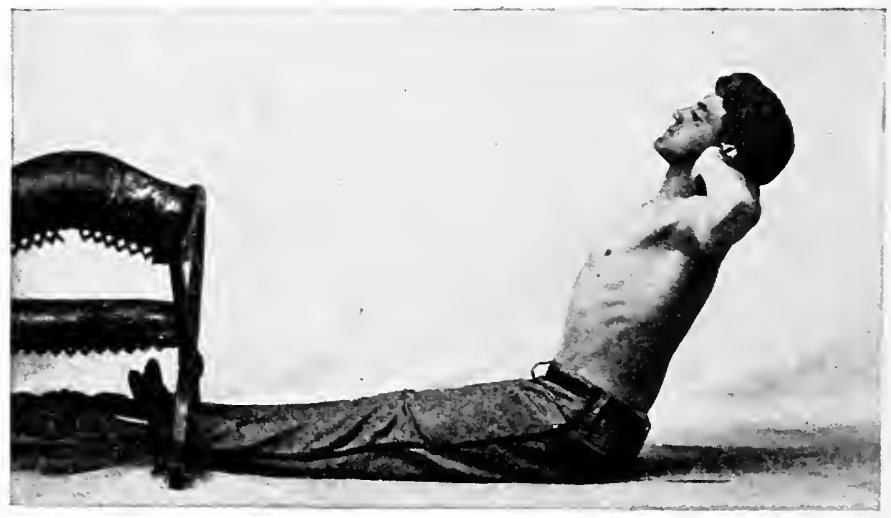

“ (9) Lying in a horizontal position with the toes under a sofa or any suitable piece of furniture, raise to a sitting posture, keeping the neck well back during the entire morement. There will be a decided tendency while taking this exercise to throw the head forward and bow the back. This must not be done. This exercise may be repeated from three to ten times according to the strength of the individual (fig. IOS).

"( IO) Lying on the stomach. extend the toes on the floor and the arms in the opposite direction so as to secure the greatest length possible from tip of toes to tip of fingers. Then, with feet raised as far from the floor as possible. inhale deeply 
for at least ten seconds. Then exhale slowly, using about onehalf this time in returning to the quiescent condition. Repeat from three to five times, kecping the muscles of the limbs tense as in stretching during inhalation."

Dr. Seaver advocates the use of the flattest truss pad that will retain the hernia and the gradual removal of pressure as the case improves. He believes that 75 per cent. of hernixe occurring in young men under twenty-five years of age, could be cured by the means suggesterl, and concludes as follows:

"First, I would recommend a surgical operation as the quickest and more efficient treatment for severe or long-continuted cases of inguinal hernia, where the matter of expense need not be consiclered, and where there is no serious mental antipathy to an operation. Second, I would recommend what may be properly called the gymnastic treatment of such cases as are recent in development, and where the inguinal ring is not unduly dilated, although the case may be of fairly long standing; also in all cases, however severe, where surgical operation is not possible. Third, I consider the mere application of a truss, however good it may be and whatever the price paid for it, as simply palliative treatment."

Geo. H. Taylor, M.D., publisherl a work in i 885 (John B. Alden, New York) on "Pelvic and Hernial Therapeutics," including "Process for Self-Cure." While this work contains many valuable suggestions, it has been productive of much harm in that it leaves the patient with the impression that he is fully qualified to carry out his own cure. I have, in speaking of the causes of hernia, already stated my experience with the "Correspondence Schools of Plysical Culture," and must again condemn any form of gymnastic exercise, for ruptured people, that is not personally superrised by an experienced instructor who appreciates its dlangers as well as its good parts. A small, but more recent work by Bernard MacFadden ("Natural Cure for Rupture," Physical Culture Publishing Co., New York, I902), gives a series of exercises which he terms first. second, and third system, showing a gradual but an extremely. 
trying exercise for the abdominal muscles, which should never be used except under personal supervision.

It is believed, as claimed by Dr. Seaver, that, for young people, systematic and carefully supervised gymnastic exercise is an important aicl in the non-operative cure of inguinal hernia, but it is also considered a dangerous method for people nearing, or past, middle life.

\section{CURE OF HERNIA BY INJECTION.}

This method of treatment would receive no mention in this work if it had not been so extensively brought before the profession and so strongly enclorsed (principally by those having a monetary interest in it) as to deceive those not familiar with the subject. From the fact that its use is almost always temporarily beneficial, and that it is curative in a few (very few) cases, it is not surprising that many physicians should be misled into recommending it. The opinions here expressed are based not upon prejudice, but actual trial in private practice and in a large clinic, with every facility for following the subsequent history of the cases. This trial was made before present successful surgical methods were known. It was then, perhaps, excusable to test a method which now seems so crude and unscientific, as we were seeking a cure for hernia. The ultimate history of the cases injected was, as a rule, that of recurrence, and the method has been entirely abandoned for several years. Its promoters recommend it as a perfectly safe method. Two deaths are known to have occurred from its use within the past few years, and one case was operated upon in order to save the life of a woman who had been treated by a notorious (advertising) adrocate of the injection method. In this case, his needle had punctured the wall of the intestine which was in the canal. Inflammation and strangulation rapidly supervened, and the woman was in a condition of collapse when first seen. An immediate operation, with the administration of oxygen and stimulants, barely saved her life. 
It is fully fifty years since attempts were first made by Velpean of Paris, Pancoast of Philadelphia, and others, to cure hernia by the injection of an irritating substance either into the sac or the tissues of the canal around the neck of the sac. Heaton of Boston, a few years later, devised a formula which he used secretly for thirty years and then publisherl. ${ }^{1}$ The early experimenters used for injection tincture of iodine, tincture of cantharides, and the essential oils. Heaton used a combination of the solid and fluid extracts of white-oak bark, which was probably better than the formula more recently used, consisting of chloride of zinc, carbolic acid, cocaine, etc. The Heaton method was deceptive, as a certain amount of improvement was noticeable in every case. A few appeared cured for from three to six months, but unfortunately with almost the entire number hernia eventually recurred. From our present point of view, the method cannot be too strongly condemned.

${ }^{1}$ The Cure of Rupture, Geo. Heaton, M.D., Boston, I877. 


\section{CHAPTER XI.}

\section{SURGICAL CURE OF INGUINAL HERNIA.}

In the great advance made in surgical work during the past few years the special surgery relating to the treatment and cure of abdominal hernia has occupied a foremost place. Perhaps, aside from pelvic surgery, no other branch has made an equal advance. The "radical cure of hernia" has been spoken of for many years, but the methods were not radical and seldom cured. There were operations for the radical cure, trusses for the radical cure, and still the fact remained that very few herniæ in the adult were ever cured, and many surgeons had given up hope of seeing this very common affliction placed on the list of curable diseases.

There is little time in this age of rapid advancement to discuss past failures, except, perhaps, to ask why they failed. Recent, and very large, experience has answered the question in a perfectly satisfactory manner, so that now it seems strange that light did not come to us before. It is a repetition of the old story of Columbus and the egg-after he had shown them how, they could all make the egg stand on end. The Columbus in the present instance was unquestionably Bassini, of Padua, Italy. ${ }^{1}$ At least two of our own countrymen had devised operations which would eventually have led to success, but the fact that Bassini published an operation, complete in its technique, and supported by a first report of over 200 cases, some operated on several years previously, makes it only fair that we should give to Italy an honor which we should have been only too glad to claim for America.

In operating to cure hernia at the present day the proposition is not " to restore the canal to its normal condition," which attempt failed through many centuries, but to construct a canal better than it ever was before. 
The great success of the present operation lies in the thoroughness with which all abnormal tissues are removed from the inguinal canal and the subsequent correction of an anatomical defect which exists in the larger part of the human family. The failure to do these very things, and the general incompleteness of all previous operations, are the very good reasons for their almost uniform failure to produce permanent cure. For many years the imperfect operations were persisted in, because of the ever-present fear of doing anything surgically that would approach or disturb the peritoneal membrane or cavity. Furthermore, it was long believed that the persistence of the hernial sac was the chief cause of the continuance of the hernia, and if this could be destroyed, without injury to the patient, a permanent cure would result. The attempts to cure hernia in the past have been made at or near the external ring, while now the corrections begin at the peritoneal surface and continue with each layer until the skin is closed. This is truly a "radical" operation for the cure of inguinal hernia.

The preparation of the patient for an operation for hernia should be made with the same care in every detail that would be exercised in any other abdominal operation. There is plenty of evidence at hand to indicate that discredit will be reflected upon the modern operation for the cure of hernia by those undertaking its performance who would not think of doing any other abdominal work. This is sure to reflect against the operation in two ways. It will cause a mortality that is entirely unjustifiable, and it will show a larger percentage of failures than should be presented. In most instances the applicant for an operation for the cure of hernia is in fairly good general health, and long preparation is unnecessary. It is important that the condition of the heart, lungs, and kidneys should be ascertained in order to intelligently decide upon the form of anzesthesia best suited for the case under consideration, and this should be done before the patient is prepared for operation. 
When possible, the patient should be under the control of a trained nurse, or in the hospital for about twenty-four hours before the operation is performed, to insure careful preparation and to regulate the food supply. A cathartic should be given and the parts prepared the night before the operation. If the cathartic is not effective, an enema should follow in the morning. The preparation of the field of operation on the preceding night consists of a complete shaving of the entire region, the cleansing and application of a liberal green-soap poultice, held in place by a suitable bandage, usually the Spica. In the morning this dressing is removed, the parts thoroughly cleansed with sterilized water, followed by 90 per cent. alcohol. Gauze moistened in a one to 2,000 solution of bichloride of mercury is then applied under a bandage and left in place until the patient is on the operating table. Here the field of operation is again scrubbed with liquid soap and a sterilized brush, and washed off with sterilized water, followed by a one to 2,000 solution of bichloride of mercury. Sterilized towels should now be spread so as to cover the entire patient, except the immediate field of operation, and all chemical solutions thrown away. No fluids, except sterilized water, or normal saline solution, should be used during the operation.

Every person connected with the operation in any way should prepare in the most careful manner. Many methods have been tried, but none has been found better than first scrubbing the fingers, hands, and arms to the elbows with soap in water that has been boiled, then washing in alcohol, followed by a thorough washing in a one to 2,000 solution of bichloride of mercury. The finger nails are doubtless the most frequent carriers of disease germs, and they should be given the utmost care. They should be cut and filed down as near the "quick" as possible, and then in cleansing should receive special care and scrubbing. Sterilized rubber gloves should be worn. but the use of these should be preceded by the same careful scrubbing and chemical sterilization as though they were not to be worn. 
Bassini Operation.-In the surgical cure of inguinal hernia, the work of the leading operators has crystallized into the following essentials, and while the operation is clone under various names, it appears to the author that Bassini was the first to demonstrate their importance.

( 1$)$ The complete removal from the inguinal canal of all foreign tissue (sac, fat, abnormal vessels). (2) Utilizing the fascial layers about the canal to so fortify it as to prevent recurrence. (3) The execution of the work with extreme asepsis to ensure safety and prompt healing.

Almost all operators select some form of suture material not quickly absorbed, but few in this country now follow Bassini in the use of silk. Every operator of experience will modify the operation to meet the special indications of the case in hand. Briefly, the Bassini operation is as follows:

( I) An incision through the skin, fatty tissue, and aponeurosis of external oblique muscle, which exposes the canal in its entire length.

(2) The sac is opened, its contents reduced and, after double ligation of its neck flush with the peritoneum, it is cut away. Any other tissue foreign to the canal is removed.

(3) The cord is held aside and the lower border of the internal oblique and transverse muscles attached to Poupart's ligament by interrupted sutures (silk according to Bassini, personally a continuous suture of kangaroo tendon is preferred).

(4) The cord is placed upon the muscular wall and the aponeurosis and skin closed over it in separate layers.

To Dr. Henry O. Marcy of Boston, a pioneer in modern methods for the cure of hernia, we are under obligations for showing us the value of kangaroo tendon. It is an almost ideal suture material for the deep buried sutures of hernia operations. We believe also that he was the first to use the subcutaneous stitch for the closure of the skin; two things which add materially to the success of this operation. My own modification of the Bassini operation has been principally in the use of the kangaroo tendon, and in using a continuous 
instead of an interrupted suture. In individual cases, it would seem that I had made almost every possible modification to meet the exigencies of the case in hand. Details, of the operation used in over 1,200 cases of inguinal hernia, follow.

\section{OPERATION.}

Oblique Inguinal Hernia.-In planning the incision, certain anatomical landmarks must be observed, and the first of these is to locate accurately the external ring. In thin subjects this is not difficult, as it is readily felt through the skin and subcutaneous fat, but where there is a thick deposit of adipose tissue it is not such an easy matter. It has been found that in a majority of cases, with male patients, the most satisfactory way is to roll the spermatic cord between the fingers and the pubic bone, following it up to its point of entrance into the abdominal wall, this giving accurately the external ring. The same test answers equally well in the female in most instances, as the thickened sac and its covering of fascia have very much the feeling of the cord in the male. Locate Poupart's ligament by placing a finger upon the anterior superior crest of the ilium and another upon the spine of the pubes. An imaginary line slightly curved downwards between these two points represents the ligament. In the adult the incision should be parallel with and about one inch towards the median line from Poupart's ligament, and from three to four inches long, according to the patient. In very fat patients, a much longer incision will be required through which to do good work than in those who are thin. The operation has been done frequently on adults by the author through an incision one and a half inches long, but this should be attempted by those only who are thoroughly familiar with the work.

The incision passes through the skin and the superficial and deep layers of fascia to the aponeurosis of the external oblique muscle. No attempt need be made to recognize the two layers of fascia, as in many instances they cannot be distinguished. In others, however, the dividing line is so clearly 
marked as to lead the operator to believe that he has already reacherl the aponeurosis. The latter should be recognized by its white, glistening surface and the direction of its fibres. The lower end of the incision should terminate at the upper exlge of the pubic bone, and its upper end should be well above the internal ring. Even in very large scrotal hernice, it is neither necessary nor is it advisable to extend the incision into the scrotal tissues, as the whole scrotal tumor and sac can be turned out through the inguinal incision. If this incision is of full length, both the superficial epigastric and superficial pubic vessels will be cut and require clamping. In many instances if the clamps remain on during the following steps of the operation up to the closure of the skin, ligature of the vessels will not be required. If the patient is fat, the clamps will obstruct the operative field, and it is better to tie the vessels at once or close them by torsion. There should be no uncertainty as to their complete closure, as subsequent oozing would almost certainly preclude primary union.

At this stage of the operation, the beginner frequently has trouble in locating the external ring, and this is because it is partially covered by the intercolumnar fascia. This fascia can readily be scraped away from above clownwards by the handle of the scalpel or the grooved director. The director can then be slipped through the external ring up under the aponeurosis of the external oblique muscle, and the latter is split in the direction of its fibres to a point a little above the internal ring. The inner split edge is taken by a clamp and the aponeurosis is stripped away from the internal oblique muscle towards the median line by slipping the fingers between the two. The outer split edge is clamped and all fascia removed from inner surface down to Poupart's ligament. The clamps should be allowed to remain on the flaps as a guide: The whole extent of the canal is now wide open, and still, in many instances, the sac is not visible and the beginner is perplexed as to its whereabouts, doing actual harm in some instances by cutting muscular fibre that should never be divided. In fact, no muscular fibre should 
be cut in this operation. Nuscle may be split and it will reunite without harm to its function; but once cut across its fibre, it is doubtful whether it will ever return to its former usefulness and strength. The ilio inguinal nerve will usually be seen passing down orer the sac and should be lifted up and put outside of one of the clamps holding the aponeurosis, in order to protect it from injury.

In finding the sac, pick up with thumb forceps the tissue presenting most prominently at the lower deep part of the wound. and almost invariably it will lead to the sac. The sac and the cord should now be lifted out of the canal together. The sac is corered by fascia that it has picked up from the edge of the muscles in its progress down the canal, and this can be readily divided by any blunt instrument. The cord should now be separated from the sac, and this is often attended by difficulty. It should be done by blunt dissection, and better by the fingers than by instruments. It is accomplished by carefully stripping one from the other, and the first point of separation should be as near the internal ring as possible. Many have failed in this part of the operation by trying to separate them at a point lower down. This is especiaily true in congenital hernia. The separation, to be accomplished at all, must begin as near the peritoneal surface as possible. At this point it can be done in almost erery case, and it is one important element in the success of the operation.

Especial caution is necessary in separating the vessels of the cord from the sac not to tear them, and it has been found most convenient and satisfactory to do this by clearing all tissue from the sac at one point and then to roll the sac in one direction over the finger end while all tissues and vessels are cleared off by and held in the fingers of the other hand. The vas rleferens, next to the artery of supply, is the most important ressel of the cord and the most likely to be orerlooked, as in color it is white like the sac. Its location can always be ascertained by rolling the tissues between the thumb and fingers uncler considerable pressure. Its hard, wiry feeling is char- 
acteristic. Communicating vessels broken while stripping away the sac, if at all important and on the cord, should be tied at

FIG, IO9.

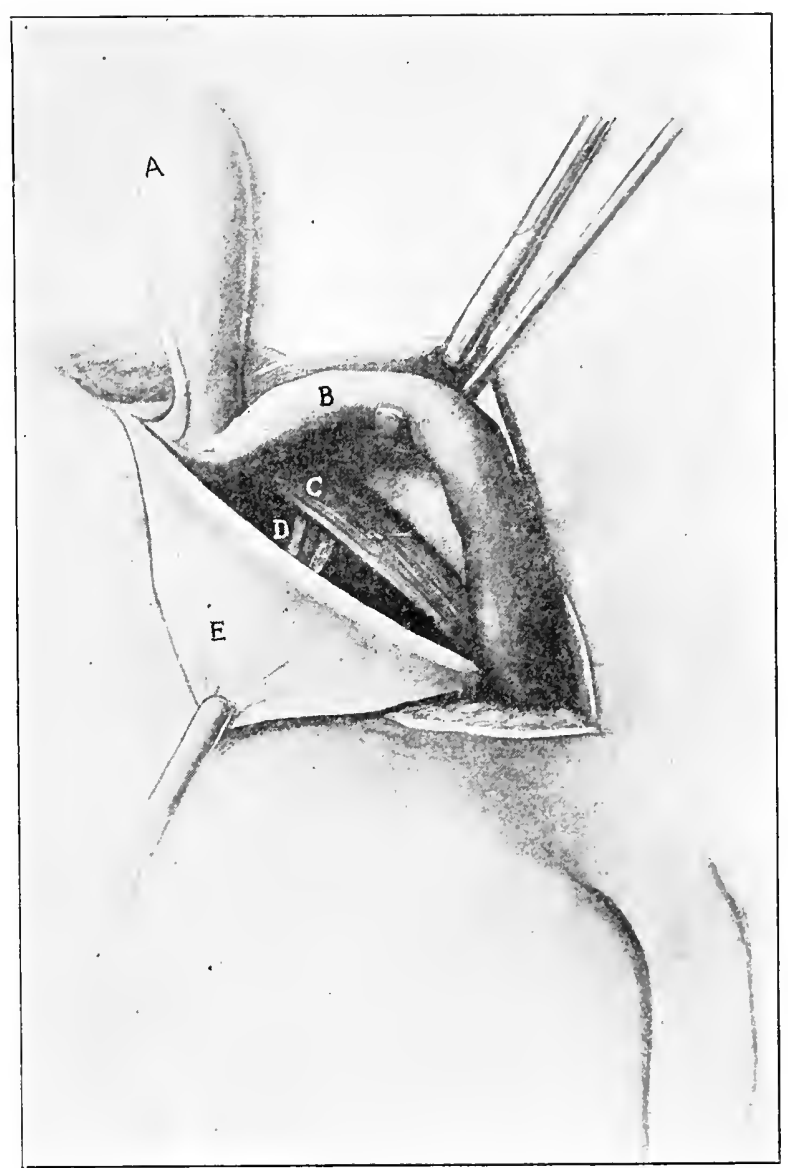

Showing, $\Lambda$, sac separated from cord; $B$, cord held up by biunt hook; $C$, lower border of internal oblique and trausversalis muscles; D, epigastric vessels, crossing wound transversely: beneath internal oblique; $E$, split aponeurosis of external oblique held back by clamps on either side, showing Poupart's ligament at extreme inner border.

once, as, if lost to view, they are hard to find again. Bleeding vessels on the sac surface are unimportant, as the entire sac is to be removed after ligation (fig. Io9). 
The sac should in every instance be opened and, if found empty, its neck ligated after clearing it of all ressels and fat. Where the size of the sac will permit, it is considered best that the operator hold the finger of one hand well within its neck, while the assistant ties down on the end of the finger. As the pressure of the ligature is felt around the end of the finger, the latter is withdrawn. By this method there is little danger of including a loop of bowel or piece of omentum within the ligature. After the first ligature has been completed, the sac should be perforated just outside of it, surrounded and ligatured again by the same strong catgut. In this manner two complete ligatures with perforation between them prevent the slipping off of either. The sac should be cut away before cutting the ligature, as by the latter the stump can be controlled and it can be seen that there is no bleeding. Ordinarily there are no important vessels in the sac wall, but there are very important exceptions to this rule, and a case is known where a young operator cut the sac without tying, expecting to close it as in an ordinary cœliotony. Hæmorrhage resulted, which could not be, or at least was not, found, and which proved fatal to the patient. Another case was seen in consultation where the operator had failed to find the bleeding ressel. While the sac is being ligated, traction should be made upon it so as to draw it well up into the wound. In this manner the ligature is placed flush with the peritoneal surface, and when the sac is cut away it leaves no pocketing of peritoneum from withinthe intra-abdominal surface is left practically smooth at this point.

An accident that must be guarded against, at this stage of the operation, is the looping up of the spermatic ressels on the neck of the sac and their subsequent ligation. As the sac is tied, the operator should make traction on it so as to draw its neck well up into the wound. When it is tied and cut, it will retract and the peritoneal surface will become smooth upon its inner surface at the site of the internal ring. The canal should be cleared of all loose fat or other abnormal tissue, and the 
operator is then ready to begin its closure, and upon the thoroughness and care with which this is done will depenrl to a very great extent the permanence of the cure. With the canal wide open and freed from the sac, it will be scen that there is a triangular space back of the cord, which is entirely unprotected by muscular or tendinous structure. In fact, there is usually nothing but peritoneum and its overlying fascia. This triangle, with its apex directed towards the iliac crest, is formed above by the lower border of the internal oblique muscle, below by Poupart's ligament, and the pubic bone forms its base. This muscular defect is overcome (in this operation) by freeing the lower part of the internal oblique and transversalis muscles from their connections above and below, slipping them down and stitching their lower border to Poupart's ligament.

FIG. IIO.

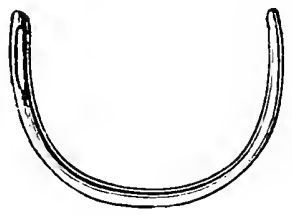

Blunt needle (actual size) used in placing kangaroo tendon sutures in the deep muscular tissue and the aponeurosis of external oblique.

The connective tissue between the aponeurosis of the external oblique and the internal oblique has been separated in the earlier part of operation. The internal oblique and transversalis should now be freed from their deep connections by gently passing the finger along under their lower border. When the deep muscles are thus freed above and below, their lower border can be brought into contact with Poupart's ligament without undue tension. With a strong, blunt, curved needle (fig. IIo) threaded with heary kangaroo tendon, the lower borders of the deep muscles are perforated well back from their edges, and at a point over the internal ring. The cord is held up by a blunt hook, or by the finger of an assistant, 
the needle passed beneath it and through Poupart's ligament, high up. When the suture is tied, it will be found that the

FIG. III.

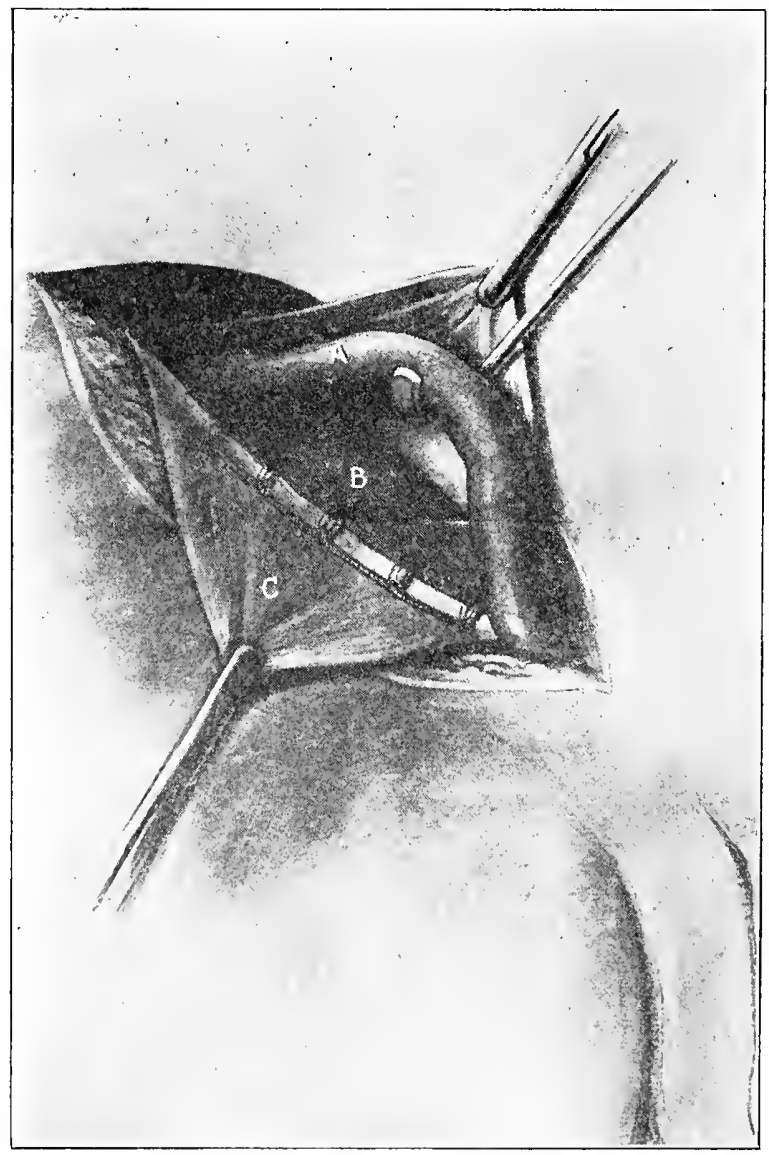

$A$, cord held up by blunt hook; $B$, internal oblique stitched to Poupart's ligament by kangaroo tendon: continuous suture made by passing suture over point of needle each time after puncture of tissue, and before tightening the thread; $\mathrm{C}$, aponeurosis held by clamps on both sides.

lower border of the internal oblicune surrounds the cord closely. The muscle is now stitched to the ligament throughout the 
length of the canal from the internal ring to the pubic bone (fig. III). In interrupted suture can be used if preferred, but the continuous is believed better, as it does away with the

FIG. 112.

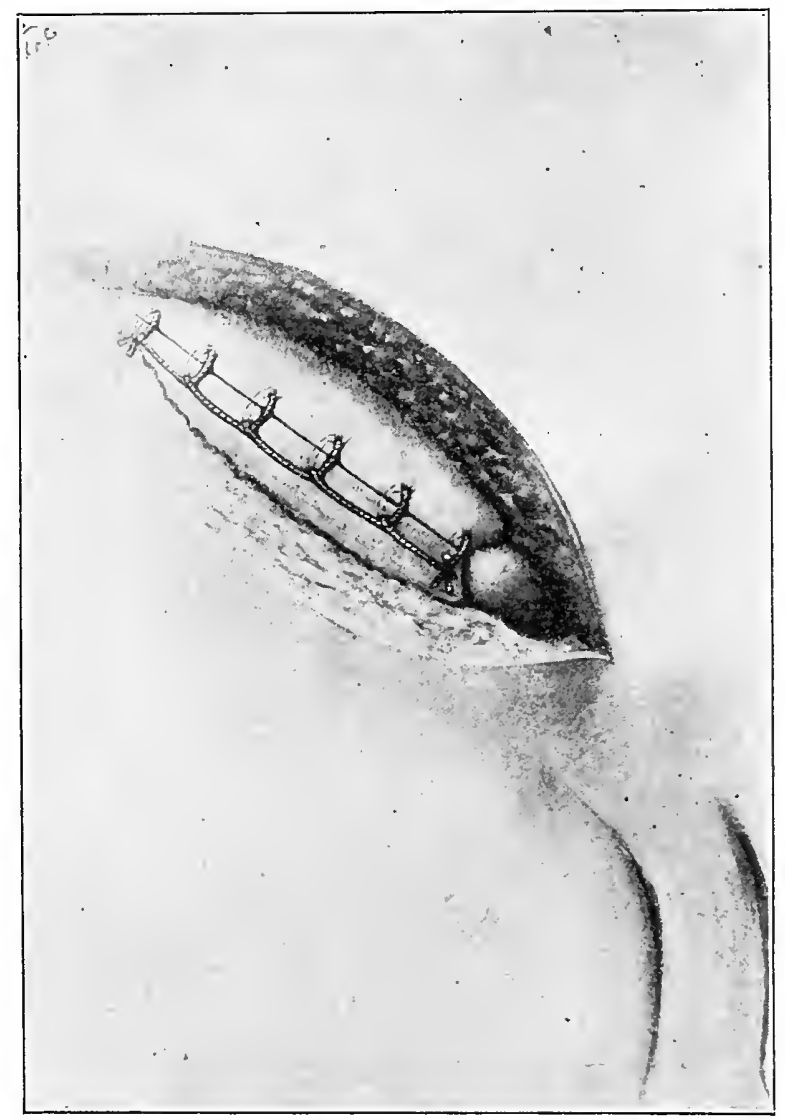

Aponeurosis of external oblique closed over cord by continuous suture of kangaroo tendon.

numerous knots and can be put in more rapidly. An additional suture placed above the cord has been suggested by Dr. IV. B. Coley. I have occasionally used this extra suture above the cord, but have ustually preferred to crowd the cord up into the 
extreme upper angle of the wound, making the canal as long as possible. The cord is placed on this muscular wall, which has been constructed beneath it, and the split aponeurosis closed over it by kangaroo tendon with the same stitch (fig. II2).

The split made in the aponeurosis is quite likely to extend up under the intercolumnar fascia, and its appearance is therefore deceptive. The first suture should be taken an inch, if possible, above the apparent split, otherwise a weak place is left, inviting recurrent hernia. It is neither necessary nor advisable that the aponeurosis be brought together edge to edge. It is better that the needle punctures be well back from the edge and not all in the same line. The closing of the skin by the subcutaneous (buried) suture and the sealing of the wound by collodion, complete the operation. A compress of folded, sterilized gauze, held in place by adhesive strips across the hips, with a liberal spica bandage, form the dressings.

Drainage need not be used except in enormous scrotal herniæ, where the stripping out of the sac is liable to leave considerable oozing. In these cases a rubber drainage tube is carried through the bottom of the scrotum and left in for twenty-four hours. During this time its outer end is carefully protected by moist bichloride gauze. The upper wound is completely closed and sealed in the manner described. The bandage and dressings on the hernial wound are not removed for ten days unless the elevation of temperature or pain demands it. An elevation of temperature on the day following the operation is not consiclered as indicating the removal of the dressings, but when it occurs about the fifth day it is suspicious, pointing to wound infection. Uncler proper aseptic work, deep infection is never seen, and that which does occur is superficial, coming largely from the skin. The skin in old truss wearers is frequently in particularly bad condition, and no amount of cleaning will, with certainty, sterilize it. When such infection does occur, the dressings should be removed and the wotnd cleansed daily with some good antiseptic. I have found nothing better than borolyptol for this purpose. Delayed 
healing does not, as has been clamed, prevent a permanent cure of the case. It is certainly undesirable, but not a serious complication.

There are certain peculiarities of sac formation that demand slight modification in the operation. In congenital hernia, it is not essential that the entire tunica raginalis should be stripped away from the cord and front of the testicle. That portion which occupies the canal, from the peritoneal surface to nearly the top of the testicle, should be separated from the cord and excised with ligatures applied at both the upper and lower points. There are instances also where it may not be advisable to remove the fundus of a very large acquired sac from the scrotum, and the communicating vessels are quite large enough to prevent its sloughing after the excision of the neck. This leaves quite a mass of thickened tissue in the scrotum and the possibility of hydrocele occurring within the sac, so that unless there is some good reason for not doing so it is better practice to remove it. In old and feeble men, it is much more important to terminate the operation with the greatest possible speed, than it is to be certain that no particle of thickened tissue shall remain in the scrotum.

It is the opinion of the author that the Bassini method as described, meets in an ideal manner the indications for the operative cure of ordinary cases of oblique inguinal hernia. It is desirable, however, that every operator should be familiar. with certain important modifications that may be necessary in cases where closure is extremely difficult.

In I895, Dr. Edward Wyllys Andrews of Chicago ("Imbrication or Lap-Joint Method," "A Plastic Operation for Hernia," Chicago Medical Recordcr. Ang.. I895) suggested that in certain cases where there was great deficiency of the internal oblique and conjoined tendon, increased strength could be obtained by "Imbrication" or overlapping of the fascial layers (see also Surgery, Gy'necology', and Obstctrics, vol. ii, p. S9. Jantuary, I906). This he accomplished in the following manner: 
The opening of the canal and treatment of the sac were in every particular similar to the Bassini operation. In closing, however, the split aponeurosis, and whatever of the deficient internal oblique could be obtained, were brought down together and stitched to Poupart's ligament under the cord. The cord was then laid upon this wall, and the remaining outer flap of the split aponeurosis brought over it and edges stitched down to aponeurosis.

This method places back of the cord the aponeurosis of the external and internal oblique muscles, and in front of it the additional overlapping layer of the aponeurosis of the external oblique. This would seem to utilize to the fullest extent the defective material that we may sometimes have to work with and the method has been used by the author on several cases with good results. Dr. Andrews says in his first paper, "I make use of it to supplement and reinforce existing methods without losing sight of their good qualities," but in his more recent article he declares that he has adopted it as a routine method. A somewhat similar operation has been elaborated at the Johns Hopkins Hospital by Dr. Halsted and Dr. Bloodgood " "The Cure of the More Difficult as Well as the Simpler Inguinal Ruptures," W. S. Halsted, M.D., Johns Hopkins Hospital Bullctin, Aug., I903).

In presenting this operation, I certainly cannot do better than to give it in Dr. Halsted's own words, and by the reproduction of the beautiful plates by Brödel:

\section{The Operation.}

"(I) The aponeurosis of the external muscle is divided and the two flaps reflected as in the Bassini-Halsted operation.

"(2) The cremaster muscle and fascia are split, not directly orer the cord, but a little above it.

“(3) The internal oblique muscle is made as free as possible. A little artifaction is here often necessary. If the muscle cannot be drawn, without tension, down to Poupart's ligament, it helps, I think, to make a relaxation cut or two in 
the anterior sheath of the rectus muscle under the aponeurosis of the external oblique muscle. This sheath being in part the aponeurosis of the internal oblique muscle, one can readily comprehend that incisions into it, if properly made, might be of service. It is well, however, to postpone making such incisions until the sewing of the internal oblique muscle to Poupart's ligament is begum, for then the amount of tension can be nicely gauged and the number, length, and precise position of the

\section{FIG. II3.}

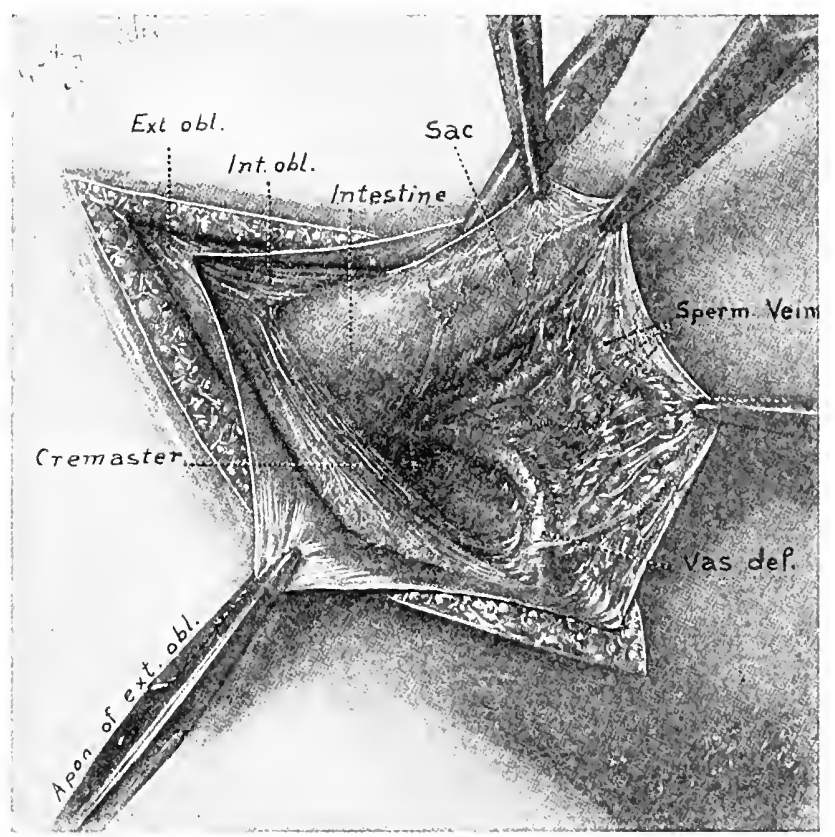

Showing relative position of sac, cord and vas deferens; method of handling. (Halstid )

relaxation cuts determined. A second reason for postponing relaxation incisions into the anterior sheath of the rectus muscle is that we sometimes use this portion of the rectus sheath to close the lower part of the inguinal canal, as already stated. "(4) When the veins are large, and this is usually the 
case, they should be excised with very great care to avoid even the slightest extravasation of blood into the tissues about the smaller veins and about the vas deferens which they accompany. And the vas deferens, as first emphasized by Bloodgood, should not be raised from its bed or handled or even touched, lest thrombosis of its veins occur (fig. II3). The

FIG. II4.

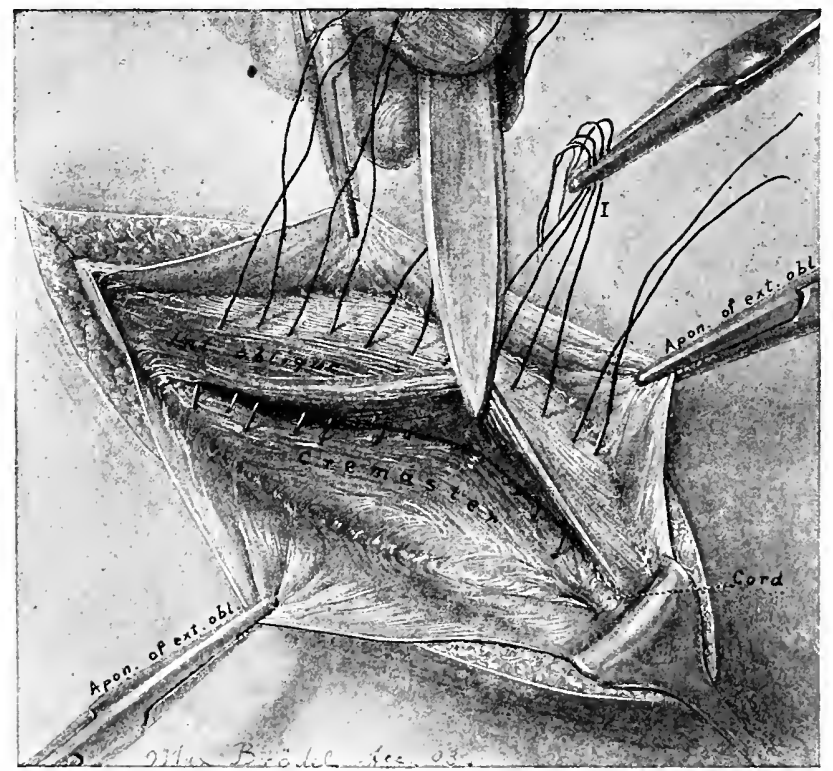

Showing the cremaster being fastened under the internal oblique muscle by fine silk sutures. (Halsted.)

veins should be ligated as high up in the abdomen as possible, being pulled down quite firmly just before the ligature (in a needle with blunt end first) is passed between them. As a precaution against slipping, we apply two ligatures of fine silk. both for the abrlominal stump and for the testicle stump of the veins. The farther from the testicle the veins are dividerl, the better, providerl, of course, that their stump is external to the external abdominal ring. 
" (5) Ligation of the sac by transfixion or by purse-string suture at the highest possible point. Both ends of this suture, after tying, are threaded on long curved needles, then carried far out under the internal oblique muscles from behind forwards, and, passing through the muscle, about $5 \mathrm{~mm}$. apart, are tied. The idea was suggested to the author by Kocher's operation, the principle being essentially the same.

FIG. II5.

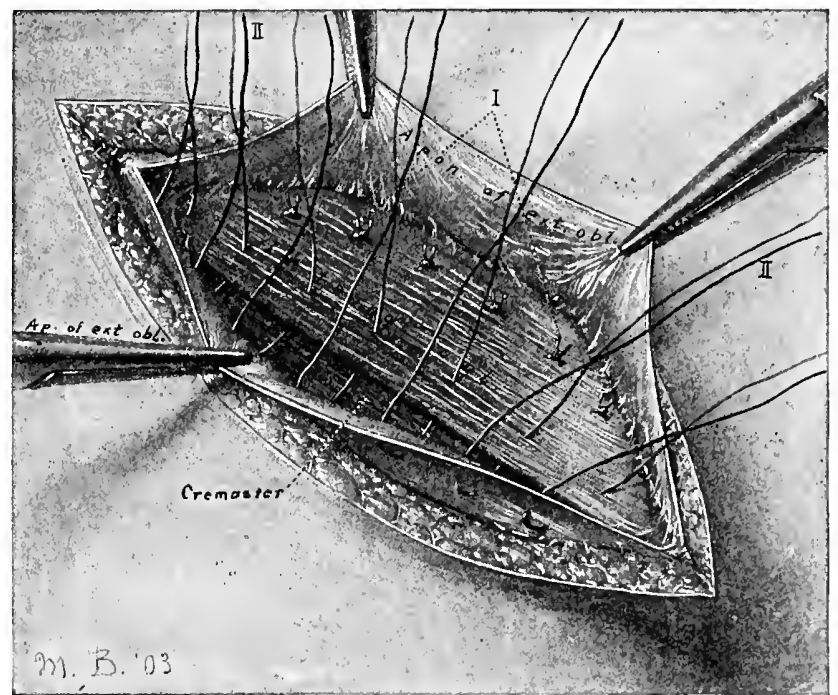

Internal oblique sutured to Poupart's ligament by catgut. (Halsted.)

"(6) The lower flap of the cremaster muscle and its fascia is drawn up under the mobilized internal oblique muscle and held in this position by very fine silk stitches, which, having engaged firmly a few bundles of the cremaster, perforate the internal oblique, preferably where it is becoming aponeurotic, and are tied on the external surface of the latter (fig. I I 4 ).

" ( 7 ) The internal oblique muscle, mobilized, and possibly further released by incising the anterior sheath of the rectus muscle, is stitched (the conjoined tendon also) to Poupart's 
ligament in the Bassini-Halsted manner (fig. I I5). Catgut is usually employed for this suture. The drawing was made from an unusually muscular subject and possibly exaggerated the size and extent of the internal oblique muscle, as well as of the cremaster, although the artist endeavored to record accurately what he saw.

"(8) The aponeurosis of the external oblique muscle is overlapped, as shown in figs. I 6 and I 7 . This is known as Andrews' method (The Chicago Medical Recorder, Aug.,

FIG. II6.

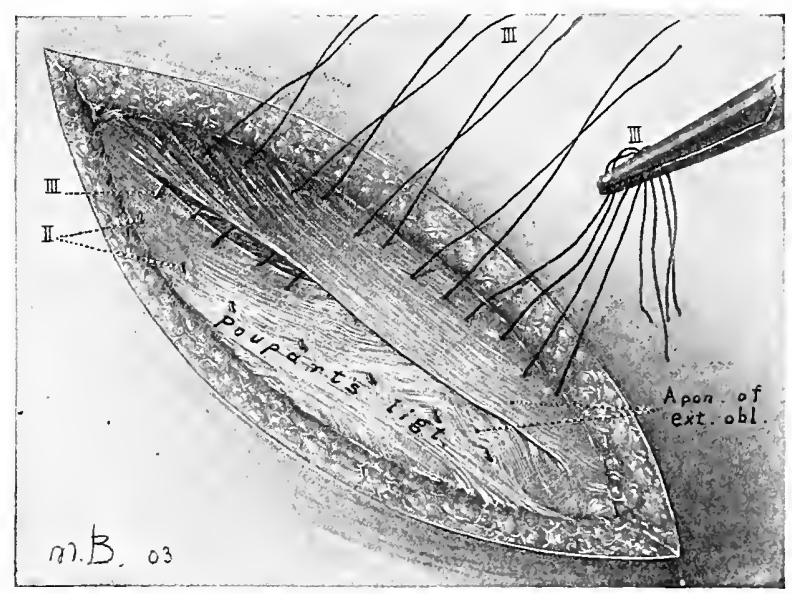

Overlapping of the aponeurosis of the external oblique, first step. (Halsted.)

I 895, vol. ix, p. 67), although devised independently by us.*

"(9) The skin is closed with a buried continuous silver suture, and the incision covered with five or six layers of silver foil. It is umnecessary to dress or examine a wound closed in this manner for two weeks, when the wire may be withdrawn.

* April I3, I4, 1906, Dr. Bloodgood demonstrated before the Society of Clinical Surgery (Surgery-Gynecology and Obstetrics, June, I906) his present method of operating upon inguinal hernia without transplanting the Corl, using the internal oblique fascia instead of the rectus for closing large hernial openings. 
Patients are kept in bed for a period of eighteen to twentyone days."

This operation differs from the Bassini method in the following particulars. First, that the essential part of the cord, the vas deferens and its associated vessels, is not lifted from its normal position. Second, in the ligation of a part of the vessels of the cord in most instances; and, third, in the closure of the fascial layers by overlapping. While I have great confi-

FIG. II7.

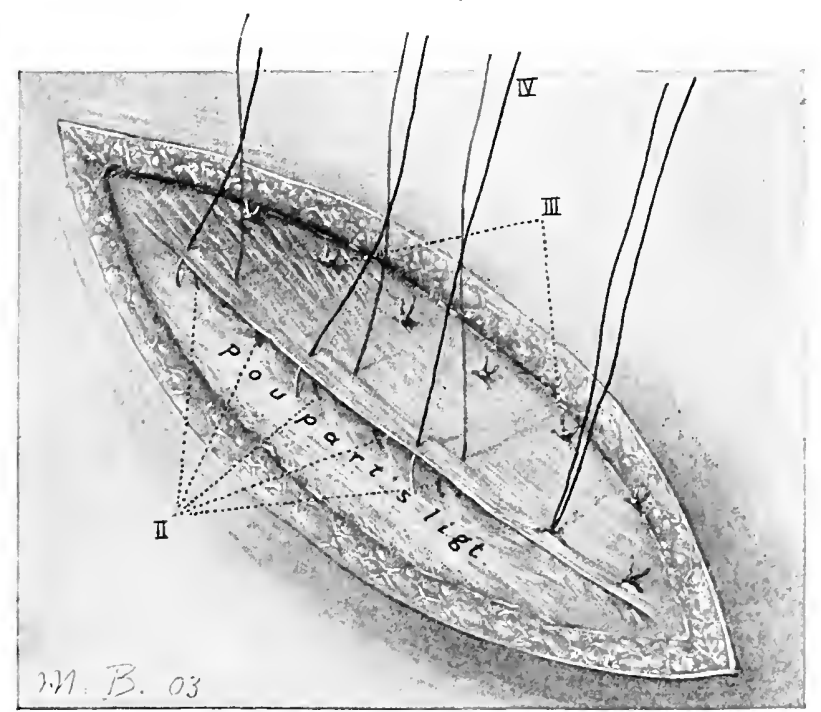

Overlapping of the aponeurosis of the external oblique, second step. (Hatsted.)

dence in this operation, and its use in the hands of its author and others has proven its value, it has not been adopted by me for the following reasons: In getting 99 per cent. permanent cures by the Bassini operation, I lave felt that more could hardly be expected from any method. I have also been tinirl. perhaps unnecessarily so, about closing the canal without transfer of the cord, as it did not seem possible to me to get as strong a closure. Excision of the reins I luave long used in exceptional cases where the cord was uncommonly large. In many 
of the cases a regular varicocele operation has been done through the inguinal incision.

In the displacement of the cord several modifications of the Bassini method have been suggested, one of the most recent being by Wullstein of Halle (Contralblatt fur Chirurgic, no. 38, 1906, Beilage). His incision starts at the pubic spine and makes a bow-shaped curve upward and outward to the neighborhood of the internal ring, one or two finger-breadths above Poupart's ligament, and the skin flap is reflected downward to it. The aponeurosis is split as in the Bassini operation, and the sac removed. The cremaster fibres are separated

FIG. IIS.

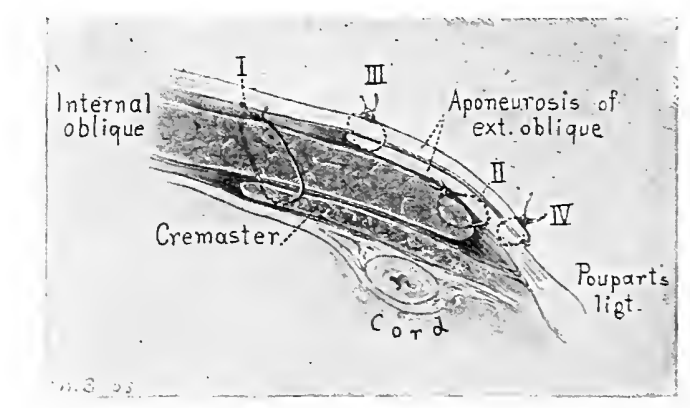

Sectional view of fascial layers in completed operation. (Halsted.)

from the cord, but not otherwise disturbed. The transversalis fascia is now split, being careful not to injure the epigastric ressels, and the cord displaced backward upon the extraperitoneal fat. The external and internal oblique muscles, transversalis muscle and fascia, are then all stitched to Poupart's ligament throughout the canal to near the external ring. as shown in fig. I I9. A flap is then made from the outer two-thirds of the anterior rectus sheath as shown in the cut, and the cord is transferred from the external ring to the upper angle of this incision. The fibres of the rectus muscle are then dissected and the end of the flap stitched beneath them and the corl as shown in fig. 120 . When the sutures are tied the 
aponeurotic flap is pulled behind the rectus muscle, and the cord assumes a course running well behind the same muscle, curving ontward and downward to reach the scrotum. The gap in the rectus muscle is repaired by stitching its edges together.

In a series of I,500 children under fourteen years of age, operated upon at the Hospital for Ruptured and Crippled, New York, on I25 the cord was not transplanted, and there were 5

FIG. II9.

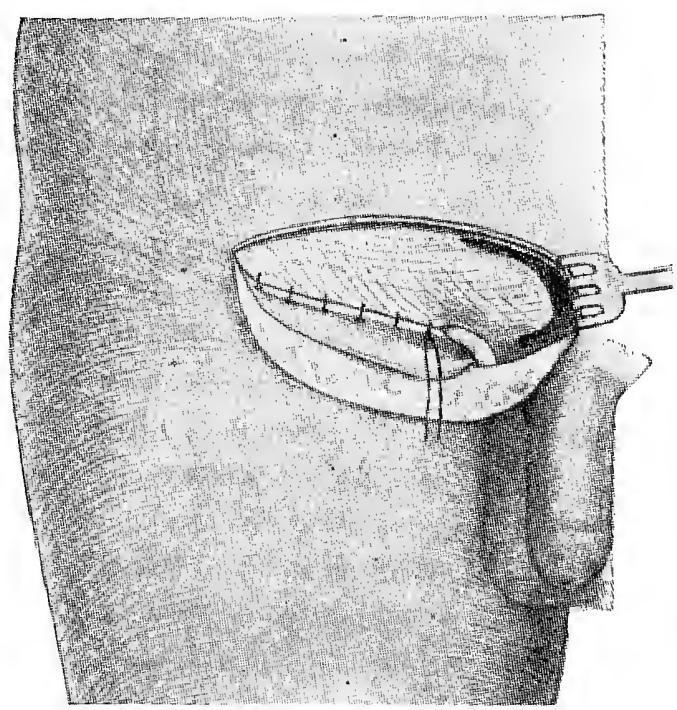

Cord placed next the peritoneum, tissues stitched over it to Poupart'sligament; flap cut in aponeurosis, into upper angle of which cord is to be transferred.

relapses, while in I,o76 Bassini operations there were only 6 recurrences ("Results of $\mathrm{I}, 500$ Operations for the Radical Cure of Hernia in Children," Wm. T. Bull and IVm. B. Coley, N. Y. Mcd. Record, March I8, I905). These results confirm my long established belief that the transfer of the cord adds to the permanence of the cure.

Direct Inguinal Hernia.-It is in direct hernia that we find, in many instances, a deficiency of tissue with which to make a closure that will permanently cure the case. It has been my 
custom in operating upon direct hernia to carry out the various steps of the Bassini operation the same as done in the oblique variety. The sac protrudes inside of the cord (i.c., towards the median line) and does not occupy the whole canal, but the latter has been opened in its entire length the same as in oblique hernia, the cord has been lifted from its groore, and the internal oblique closed beneath it. It has seemed to me that in

FIG. I20.

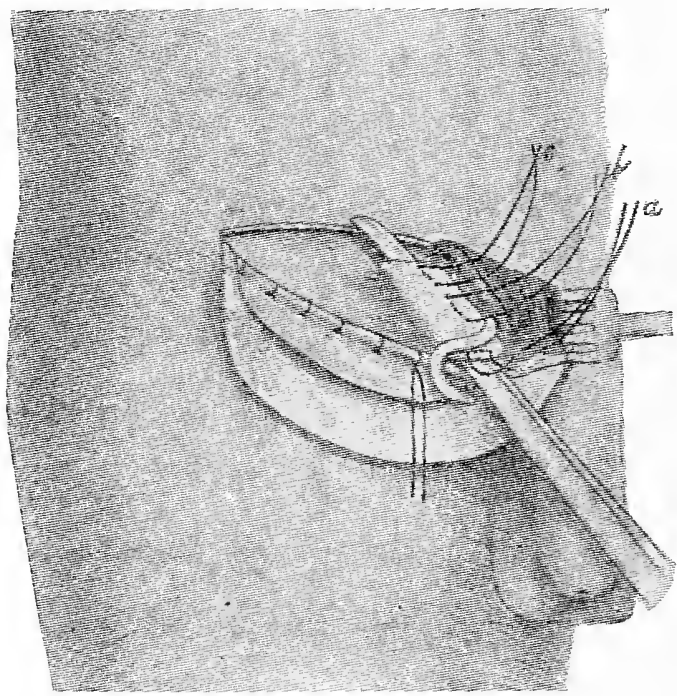

Cord transferred to upper angle of flap; the latter passing beneath it is stitched to the rectus muscle.

this way, especially if the internal oblique has been carefully freed from its attachments, a better closure may be made than possible without transfer of the cord. Cases are not uncommonly met with, however, where the muscular structure of the internal oblique and the conjoined tendon are so deficient that it is not possible to make a strong barrier against the return of the hernia with them alone.

We then have a choice of splitting its sheath and using the rectus muscle (suggested by Wöfler in I892. Beiträge z. Fest- 
schrift f. Th. Billroth, and in 1898 in a better form by Bloodgood), or what would usually seem better, using the sheath itself to close with as suggested by Halsted. In some instances it will be found that the muscle can be brought down without undue tension and the closure made strong. If any amount of tension exists, however, the sutures are sure to cut through its

FIG. I2I.

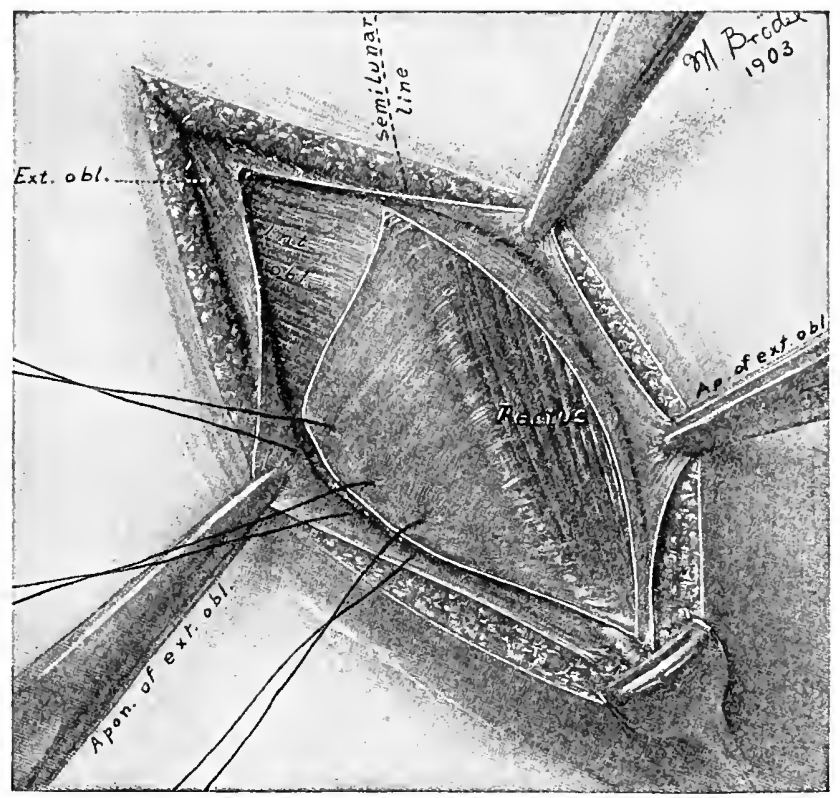

Halsted's method of utilizing the split sheath of the rectus in closing the canal where there is deficiency of conjoined tendon.

tibres and the muscle will resume its normal position. On the other hand, by cutting a flap from the sheath of the rectus it can be turned over and the weak triangle permanently fortified.

This is beautifully illustrated by the plate made by Brödel and reproduced from Halsted's article already quoted (fig. I2I). I have resorted to its use with success where I am confident failure would have resulted from the ordinary operation. This has been done in addition to bringing down all of 
the internal oblique that could be obtained. This method of interlapping of the fascial layers is one that has probably been utilized by almost every extensive operator for hernia, both before and since the publication of Dr. Halsted's article, but the details probably have not been as carefully elaborated anywhere as they have at the Johns Hopkins Hospital.

The sac of a direct hernia is almost always broad at its base or neck, and usually cannot be tied off with a circular ligature, but must be closed as a laparotomy wound is, by stitching its edges together. In these cases it must be constantly borne in mind that the work is being done in the immecliate proximity of important blood ressels and of the bladder. Two unpublished deaths are known to the author, resulting from hæmorrhage into the abdominal cavity from vessels injured by a needle used in making this form of closure. The parts had in both instances been closed, and there was no external eridence of the hæmorrhage. Another case, seen in consultation. where fortunately the hæmorrhage was external, the patient was sared by reopening the wound at once and tying the deep epigastric artery which had been perforated. The bladder has also been punctured in the same manner, leading to subsequent extravasation of urine.

In uncomplicated cases of inguinal hernia, if primary union has been obtained, the patient is allowed to sit up on the tenth day, and leave the hospital on the fourteenth day after operation: the bandage is continued for four weeks longer and then all support abandoned. 


\section{CHAPTER XII.}

\section{COMPLICATIONS IN THE SURGICAL CURE OF INGUINAL HERNIA.}

Oblique.-In the canal may be found fat, abnormal ressels, delayed testes, ovary, appendix, adhesions, cacum, sigmoid flexure, and bladder. In operating for the cure of inguinal hernia there are complications that cannot be anticipated, but there are others frequently seen where the operator should be prepared to instantly modify the operation to meet the demands of the case. The disposition of surplus fat in the canal has already been spoken of. No loose fat should be allowed to remain, either in or near the canal, and safety is always on the side of ligating it before removal, care being taken not to make such traction upon that layer of fat just outside of the peritoneum, as to draw that membrane or a fold of the bladder within the ligature. Remember that fat protruding into the canal, near the pubic bone, may be that covering the anterior bladder wall, of which more will be said later.

Enlarged or varicose a'eins will be found not only in the canal of the male, but (rarely) in the canal of the female, and these should be carefully ligated and excised. In the male it has been pretty well established that a fairly large number of the vessels of the cord can be cut away without harming the testicle, several writers claiming that if the vas deferens is protected and everything else divided, the testicle will still maintain its vitality and function. I must confess that I have not complete faith in the power of the very small vessels, that accompany the vas, to perform the full work of the testicle. This feeling remains, even though in the one instance where I cut away the entire cord except the vas and its closely associated vessels, no atrophy occurred.

Delayed Testes.-The imperfect descent of the testicle forms one of the rather common complications in the surgical 
cure of hernia. In $\mathrm{I}_{2}, 2 \mathrm{O}_{5}$ operations for the cure of inguinal hernia, I found 72 cases where this condition was associated. These patients have been largely boys between eight and sixteen years of age (figs. I22, I23, and I24), the youngest patient being five and the oldest thirty-five years old. The operation has not been encouraged in very young children who were having no trouble from the condition, under the belief that

FIG. 122.

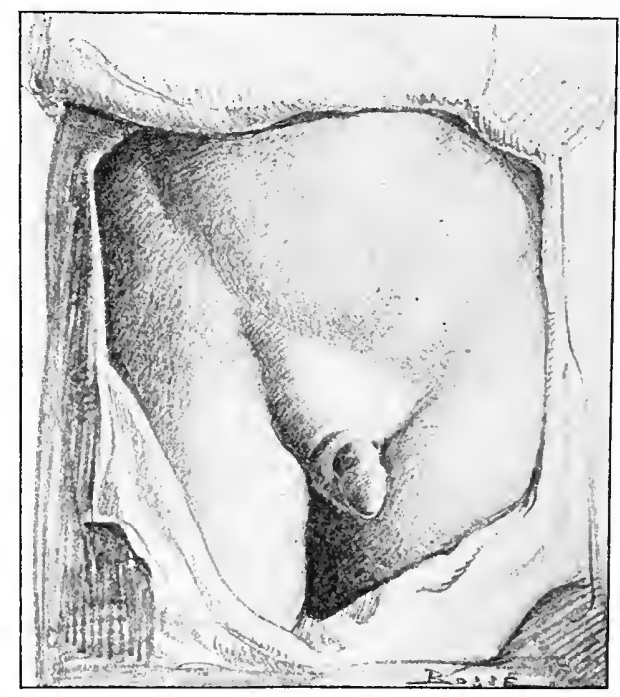

Right retained testicle and complete inguinal hernia in a boy to years old.

about the tenth year was the most favorable time. It is considered perfectly justifiable, however, and to be advised any time after the serenth or eighth year, or any time earlier when demanded by the discomfort of, or danger to, the patient. In 65 of the 72 cases it has been possible to place the testicle outside of the abdominal wall, and usually well down in the scrotum. Not in a single instance has there been retraction of the testicle into the canal subsequent to operation, so frequently complainerl of by the earlier writers. This fact is considered due 
to the extreme thoroughness exercised in freeing the cord from all tissues that would restrict its descent. Not in any instance

FiG. I23.

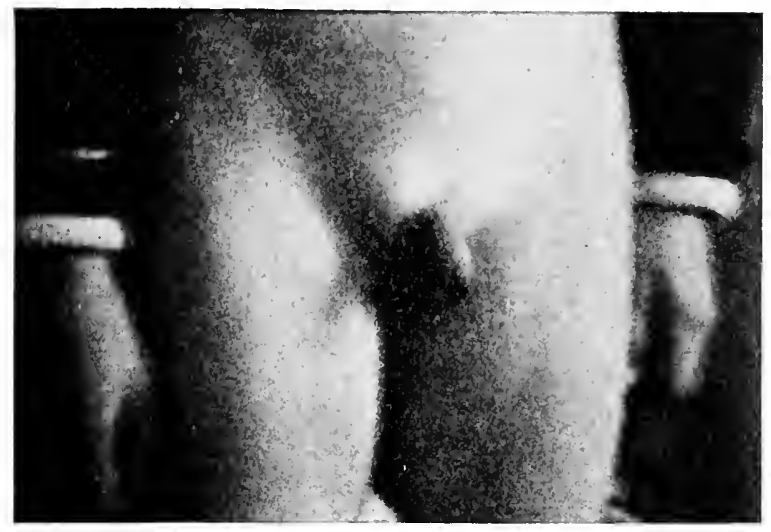

Boy 9 years old. Right testicle retained at external ring.

has the testicle been anchored to the scrotum or other tissue. Care has been taken to close the external abdominal ring so

FIG, I 24.

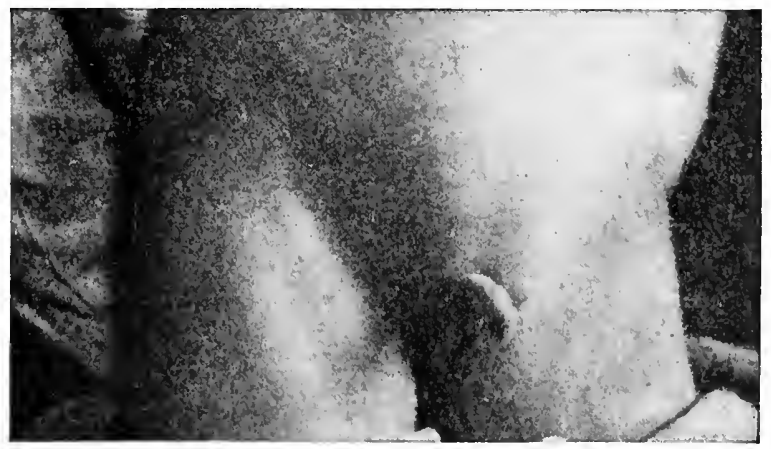

Double retained testes; age, 13 years. Right at internal ring, left at external ring. Subsequent operation put both in top of scrotum.

closely around the cord that the testicle conld not slip back into the canal. In 7 of these cases (all achults) the cord was found 
permanently so shortened as to preclude the placing of the testicle in a comfortable position outside of the canal. That is, if placed outside of the external ring, it would have been so tightly held against the pubic bone as to cause discomfort and danger of injury.

In delayed descent of the testes, even though hernia is not actually protruding, there is usually an accompanying sac suit-

FIG. I25.

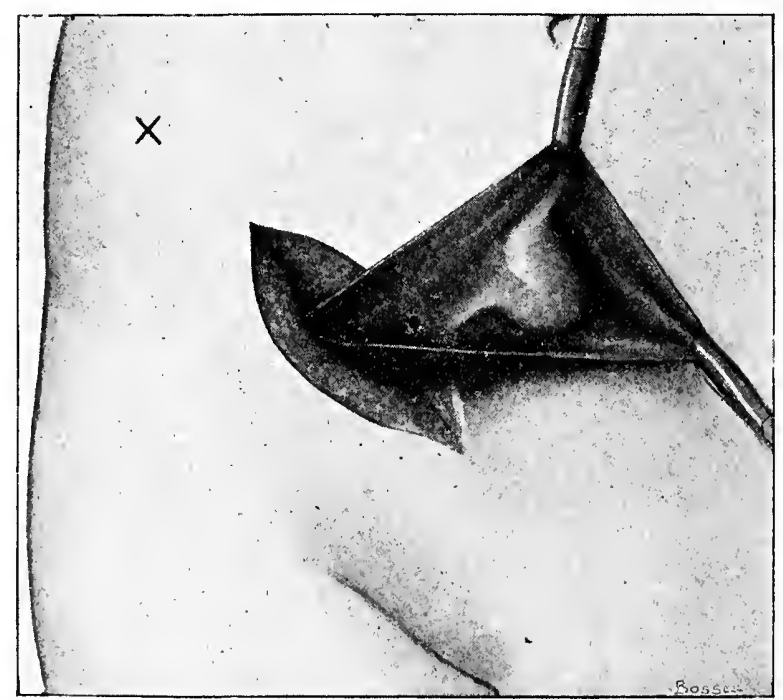

Showing sac around delayed testicle, the latter appearing to be inside of it. Sac held open by clamps.

able for its reception. The interior of such a sac is illustrated in fig. 125, and, as there shown, the testicle has the appearance of being inside the peritoneum. This in reality is not true in any case. The testicle may be completely enveloped by peritoneum and hang free in the abdomen, the peritoneum actually forming a mesentery by which it is supported and which, on the other hand, prevents its being placed in its normal receptacle. By careful manipulation the sac can usually be separated from 
the cord at a point near the internal ring, and when it and all fascia have been cut through, the vessels of the cord can be further stripped away from their attachment outside of the peritoneum, and thereby considerably elongated. Freeing the cord of everything but its essential elements is absolutely necessary to insure success. The neck of the sac should be closed either by ligature completely surrounding it, or by a pursestring suture applied from its inside. The cord should be free

FIG. I26.

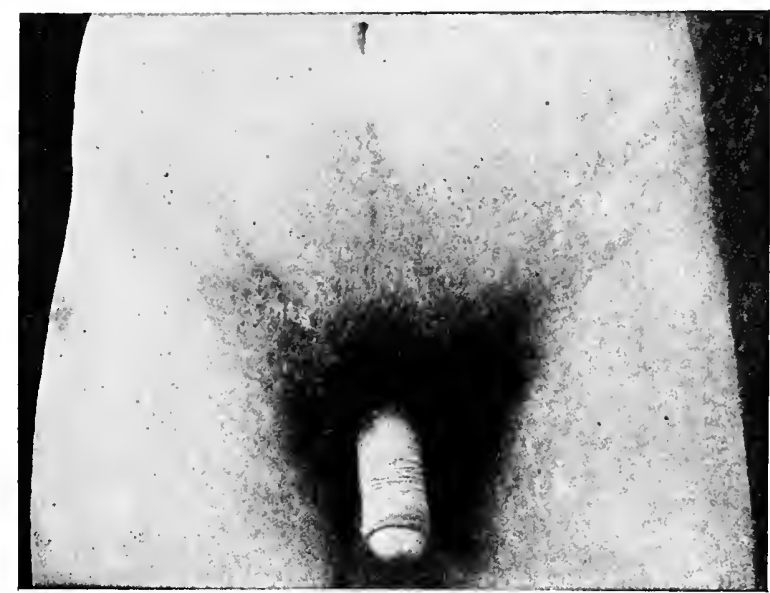

Double retained testicles, two years after operation, placing them under muscular wall and obliterating inguinal canals. For photographs of this case before operation, see figs. 86 and 87 .

from every form of adhesion, so that its vessels can be straightened out to their greatest length. When this is done, it will almost always be found that the testicle can be placed in the scrotum.

Before this can be done, however, a suitable pocket for its reception must be formed by running the finger down into the scrotum and forcibly dilating it. When this has been accomplished, the testicle should be placed in this pocket and the operation may be completed by the Bassini method. I have 
found no child in which this procedure could not be carried out, but in the adult the problem is quite a different one. In 7 of my adult cases there appeared to be no way of sufficiently lengthening the cord so that the testicle could be placed in its normal receptacle. Some writers glibly tell us to remove these testicles, that they are worthless and liable to cancerous or

FIG. I27.

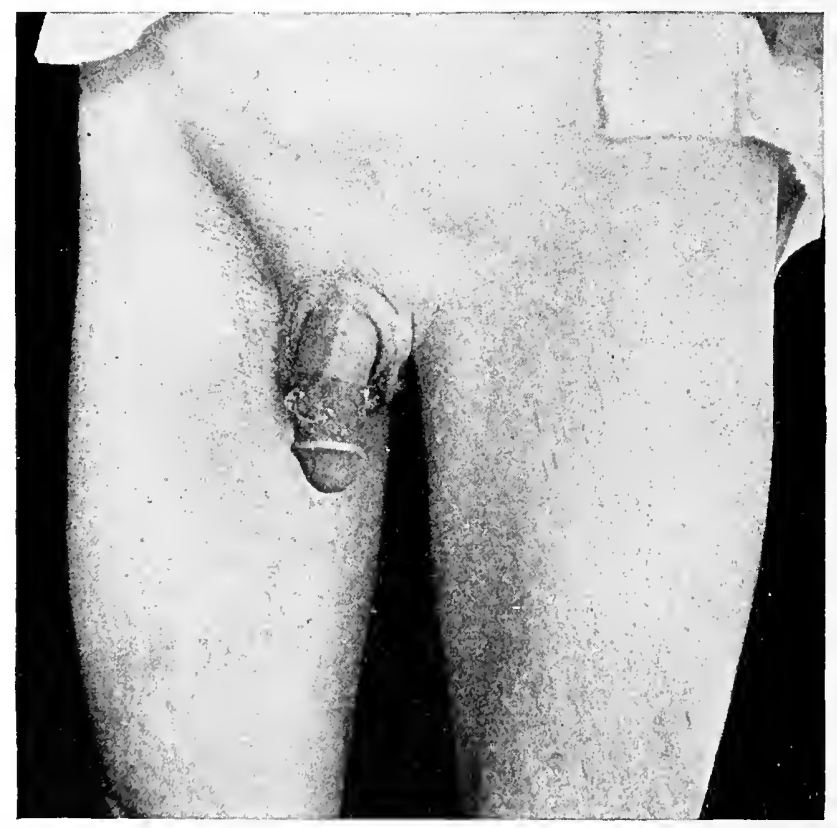

Retained left testicle and irreducible inguinal (interstitial) hernia; age, 24 years. A large, deep-seated tumor (shown in fig. 128), in left inguinal region, does not show.

tubercular affections. Recent studies, however, show conclusively, it would seem, that the testicle has a function aside from its procreative power, and that this function is beneficial to the growing child or young man. The well known mental effect following their removal is also to be taken into account before sacrificing one or both of these organs. Furthermore, it is yet to be proven that testicles lodged in the abdominal wall are any 
more liable to malignant disease than when in their normal position.

The necessity of having some form of operation that would provide for the saving of these testicles, where the corrl is too short to allow of their being put in the scrotum, was forcibly brought home to me in the case shown in fig. r26. two years after operation. This young man demanded that unless I could otherwise afford him relief, that both testicles should be

FIG. 128 .

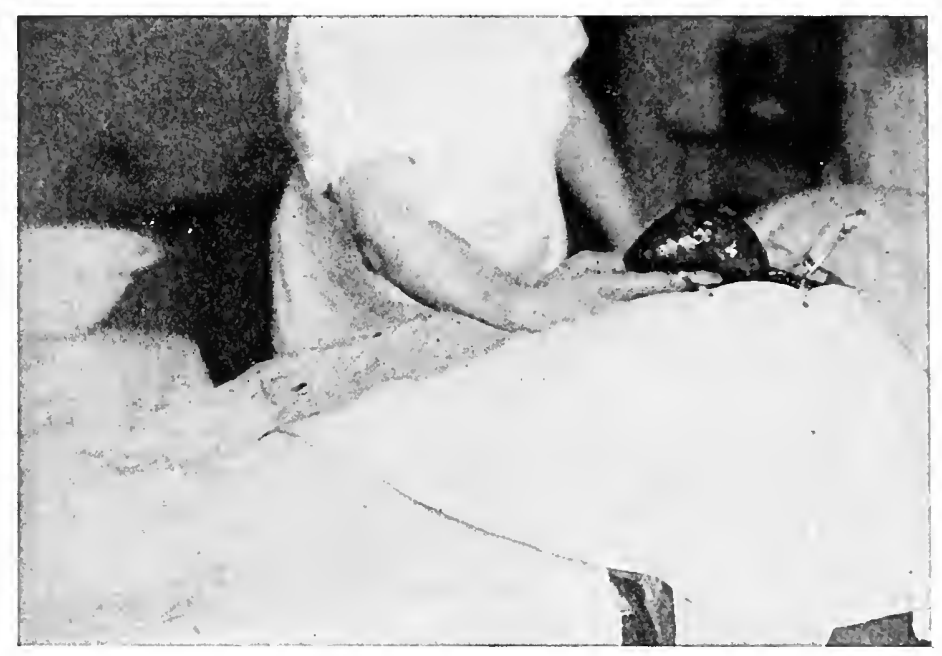

Case of retained testicle shown in fig. 127. Whole sac and contents before opening.

removed. He was 25 years old, had been happily married for two or three years, and was thoroughly competent to discharge his matrimonial obligations. He had suffered extremely, both from the slipping of the testicles under the truss pads, and from attacks of strangulated hernia whenever the truss was left off. The amount of constriction by the truss. which was found necessary in order to retain both the hernixe and the testicles, is shown in the photograph (fig. 87 ) which was taken upon the removal of the truss. In entering upon 
this operation, I had no very definite idea just what I should do, and when I had finished thought that I had devised a new operation for just such cases, but found shortly afterwards that my friend, Prof. Dawbarn, had recommended practically the same procedure. His operation had not especially contemplated the relief of cases of delayed testes, but the cure of ordinary inguinal hernia. ${ }^{1}$

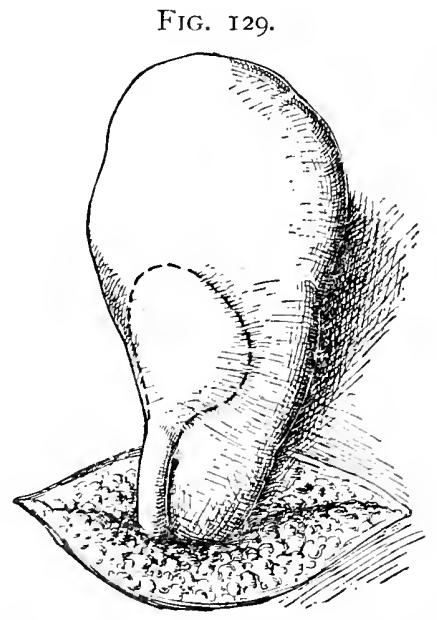

Sac with testicle inside, shown by dotted line. The location of the cord is not so distinct as here indicated.

Just how much I may have been influenced by this article, or whether I had even read it, is now impossible for me to say, but of this I am quite certain that I did not have it in mind at the time the operation was performed. The procedure is as follows:

The incision is made in every respect as in the Bassini operation, the aponeurosis of the external oblique being split to, or above, the internal ring. The sac is taken out completely (as shown in figs. I28, 129), before opening. In some cases it has

${ }^{1}$ Trancplanting Testicles for the Cure of Hernia, Robert H. M. Dawbarn, M.D., Wood's Reference Handbook, Vol. ix, p. 4I5. 
also been possible to separate the vessels of the cord and ras from the neck of the sac high up before opening, and this, when

\section{FIG. I3O.}

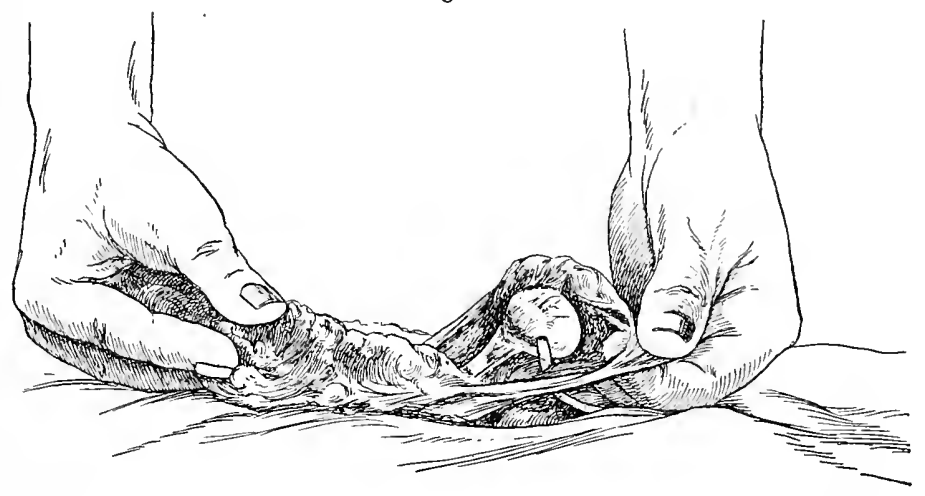

Retained testicle, figs. 127 and 128 ; sac opened. Omentum in right hand, testicle within sac in left hand.

it can be accomplished, is desirable. (In the case shown in figs. I 27 and I28, the sac was full of omentum, which was amputated and the stump reduced, leaving the testicle showing from

FIG. I3I.

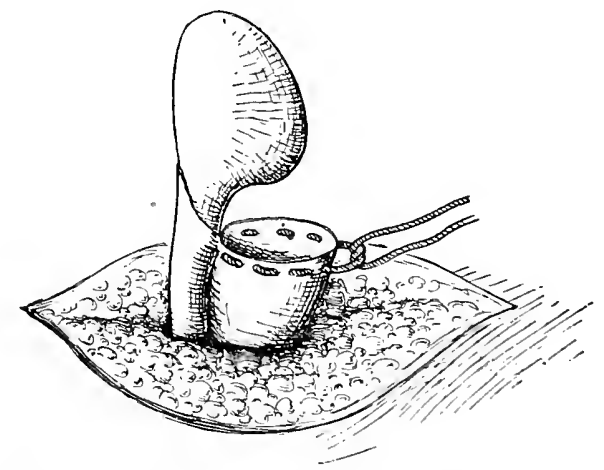

Sac cut away from testicle, ready to tie with purse-string suture. Should be closer to peritoneal surface than here shown.

the inside of the sac as seen in fig. I 30 .) The neck of sac (really the tunica vaginalis) must be gradually worked away 
from the cord just where the vessels leave it to dip down into the pelvic carity, and a ligature is either passed around it, or a purse-string suture is placed from its inside, and it is cut away from the cord and testicle (figs. I 3 I, I32). If it is now decided that the cord is too short to allow of the testicle being placed in the top of the scrotum, the fingers should be run under the internal oblique and transversalis muscles towards the median line, forming a pocket between these structures and the peritoneum (fig. I33). In this pocket the testicle is then placed and the canal entirely obliterated by closing it according to

FIG. I32.

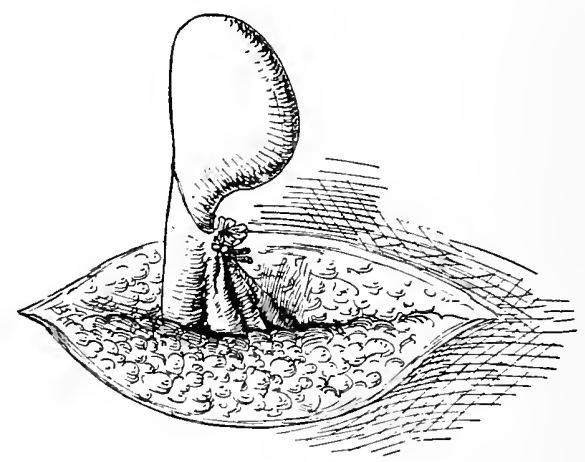

Sac tied by purse-string suture.

the Bassini method, except that there is no cord to provide for. The closure can be completed, as it is in most cases, in the female.

In the $\tau$ cases where I have placed the testicle in this position, the patients have, in every instance, experienced the most complete comfort. In one doubtful case on the left side, I found that the testicle would come well outside of abdominal wall, but not fully into the scrotum, and I made the mistake of leaving it in this position instead of placing it under the muscles as on the other side. It has since been a source of more or less discomfort to the patient. In young children, the subsequent and further descent of the testicle can 
COMPLICATIONS IN SURGICAL, CURE. 249

FIG. I33.

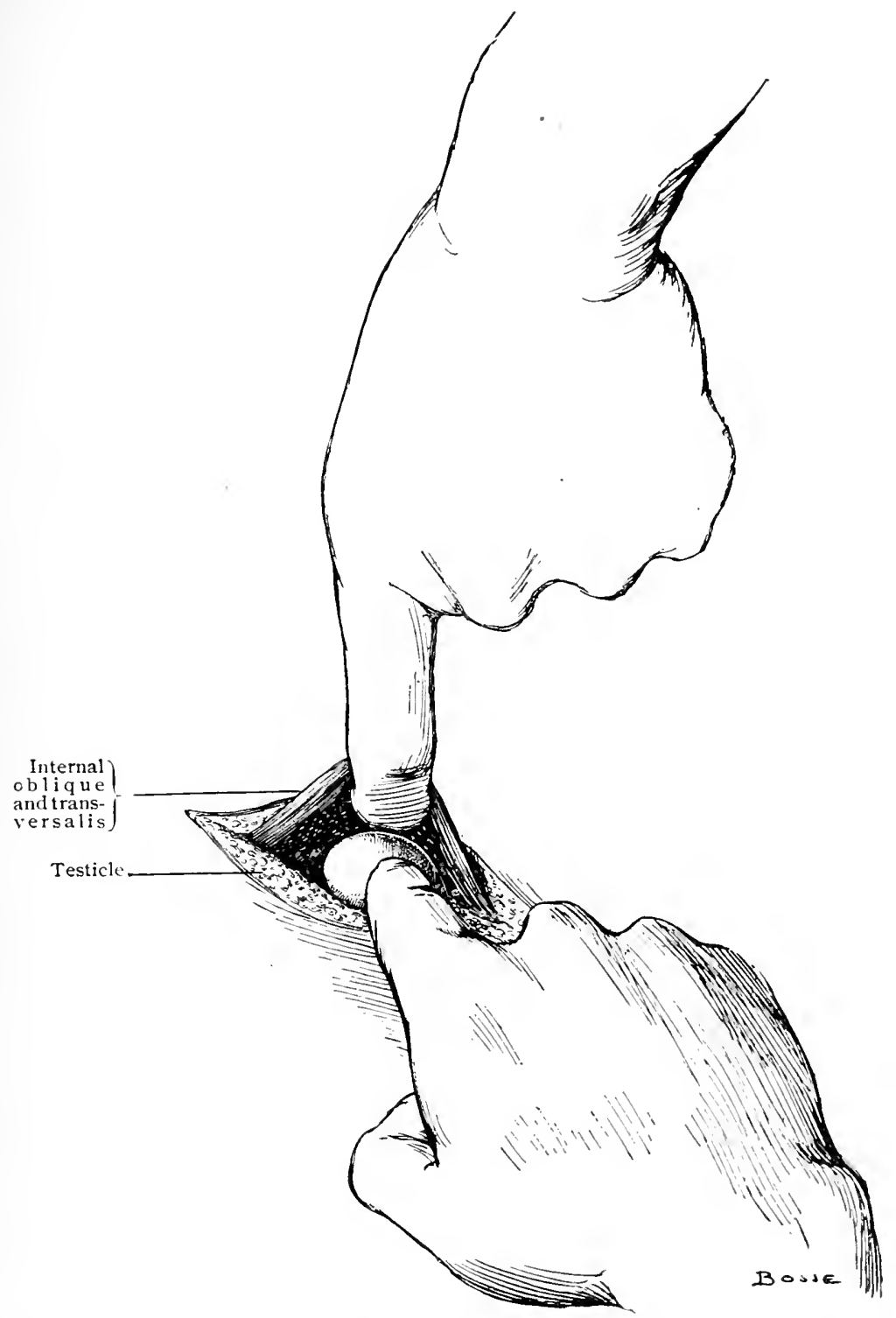

Lifting up internal oblique and transversalis muscles, to slip testicle into preformed pocket in front of peritoneum. 
be counted on, but not so in the adult. A photograph of the first case that I operated upon by this method, two years afterwards, is shown in fig. I26. This operation was clone ten years since, and the relief and satisfaction of the patient has been most gratifying. This is equally true with every patient upon whom the method has been used.

FIG. I34.

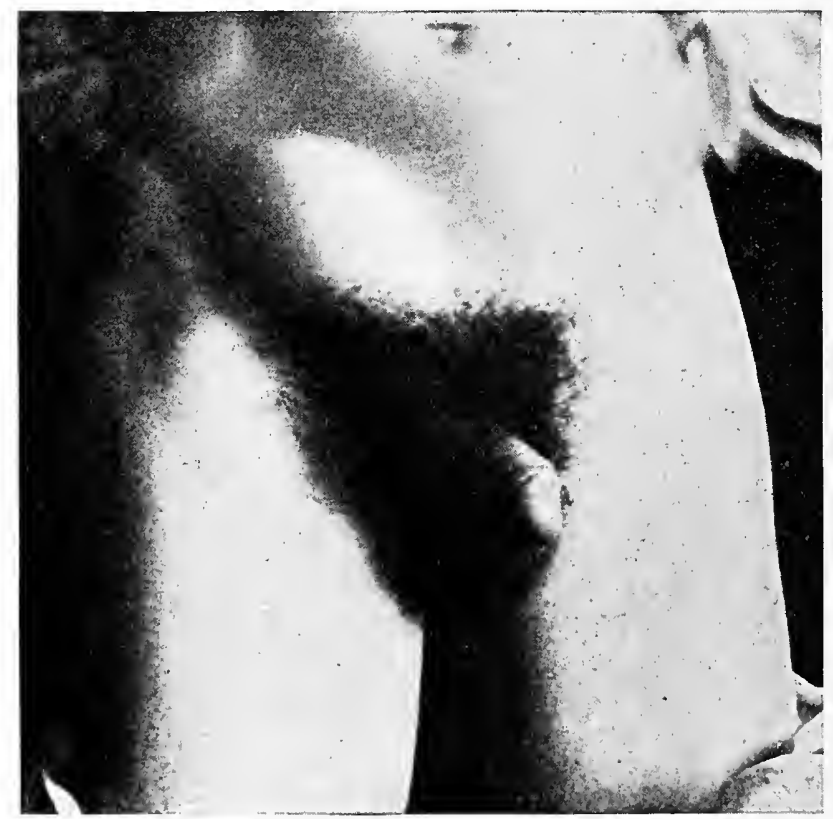

Interstitial hernia and retained testicle on right side in a man of 25 years. Right side of scrotum rudimentary. Hernial sac found beneath external oblique muscle. Testicle inside of external ring.

I wish it clearly unclerstood that I have not done, nor found it necessary to do, this operation of placing the testicle between the peritoneum and the muscular wall of the abdomen, on any young child, but I am convinced that it is far better than castration, in aclult cases. If the testicle can be brought down as low as in the case shown in figs. I34 and I35, it is 
better to put them in the scrotum. It is believer that any time after the patient has passed eight or ten years of age, these cases of delayed testes should be looked upon as belonging to the surgeon. They always carry with them an element of danger in the ever-present sac which frequently communicates with the abdomen by a narrow neck, making strangulation

FIG. I35.

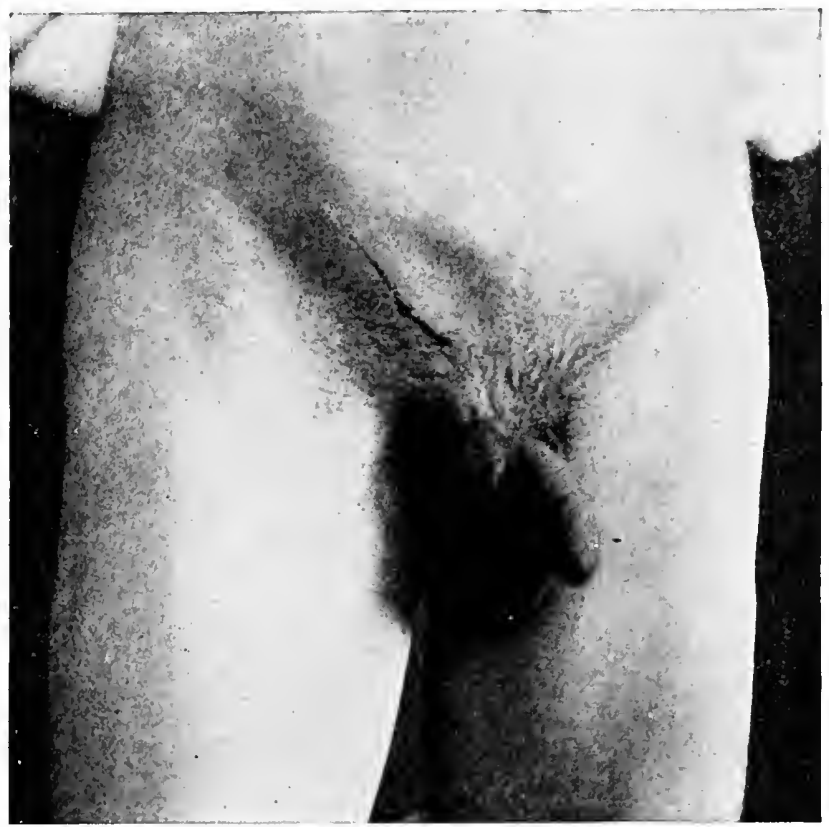

Same as preceding, three weeks after operation. Testicle in top of scrotum.

especially liable and dangerous. These sacs may exist for years without actual protrusion of intestine, and when it does occur, a small kntuckle of gut, strangulated beneath the thick muscular covering, is quite liable to be overlooked upon examination. The many individual peculiarities that have been met with in connection with this class of cases. indicate their exceptional danger in case of strangulation: as adherent omentum, 
adherent intestine, and, in one case, an adherent vermiform appendix.

Various other operations have been suggested for the relief of this condition, as suture of the testicle to the bottom of the scrotum, or carrying the suture through the bottom of the scrotum and fastening it to a wire frame, of attaching the

FIG. I36

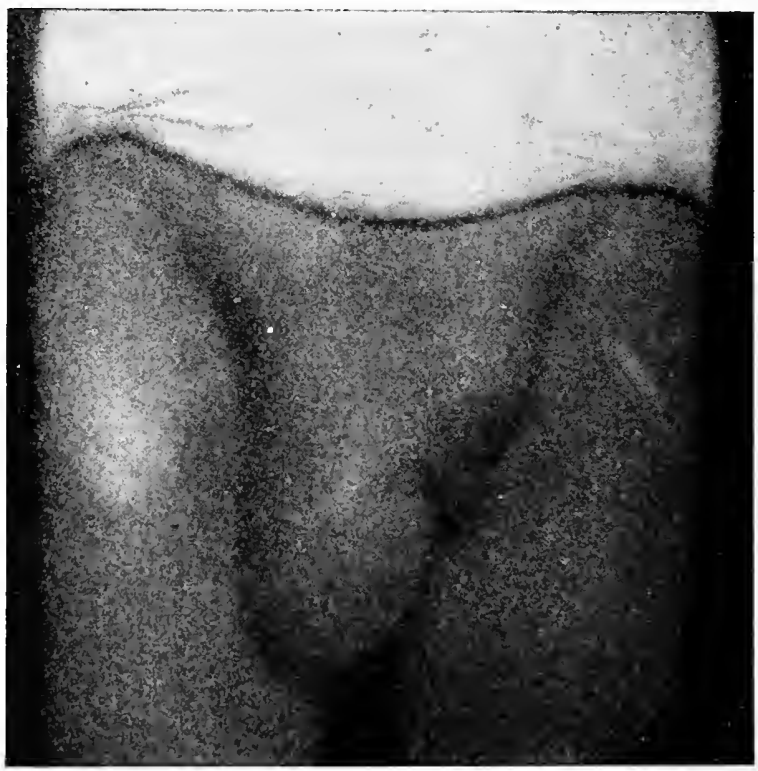

Sylvia L., aged 7 years. Double inguinal hernia. Supposed to be a giri and was so dressed. Operation demonstrated double retained testes.

testicle to a flap from the tissues of the thigh, or even attaching it to its fellow of the opposite sicle. None of these methods are given in 'detail, as they have not proven successful, but the one describerl has. One of my cases of this type is worthy of mention somewhat in cletail. as showing how analogous the physical indications of sex may become by certain tricks of development. 
Sylvia L., aged seven years, was apparently (without anything in a general way to inclicate that she was not just what she was named and (lressed for) a girl, of rather large size for the age. Until recently the mother had never doubted the sex of her child, but on consulting a physician, he found the large swelling shown in the photograph (fig. I36), and detected two small bodies within these swellings that he believed to be testicles. When the hernice were reduced (fig.

FIG. I37.

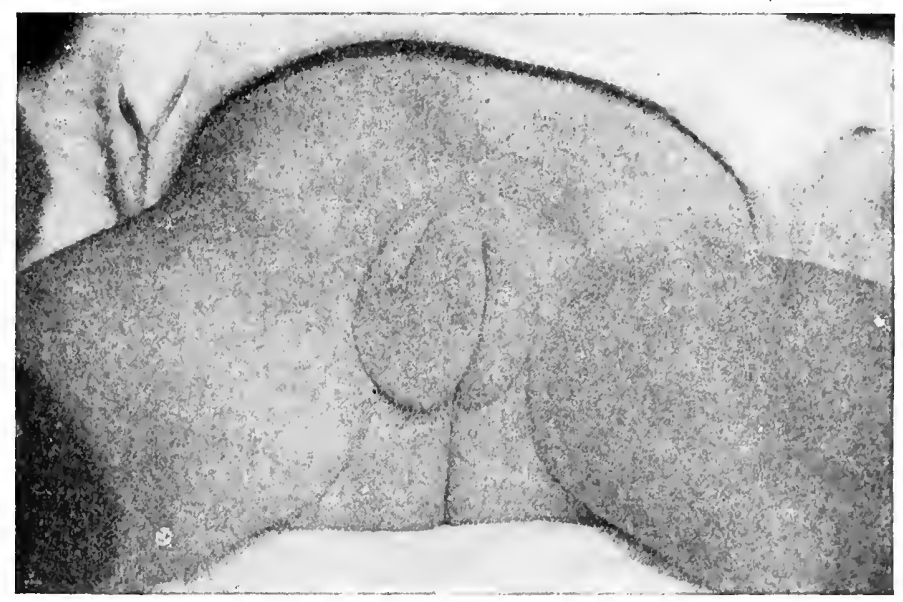

Sylvia L. Hernix reduced.

I37), the external parts presented what appeared to be the normal vulva of a female child, except that the labia were somewhat larger than usual. Upon separating the labia (fig. I3 8 ) the parts seemed to indicate an abnormally large clitoris and the entrance to the vagina.

I operated upon this child at the Post-Graduate Hospital, curing its hernia, and at the same time carefully examined the pelvic cavity, but, as expected, no trace of uterine appendages were found. The urethra opened just below the penis and the latter, when liberated, would have been nearly of normal size. 
It had been intended that considerable should be done in restoring him to the sex that nature had intended him to represent, by various plastic operations, and with this in view, the testicles were left down in what was afterwards to become the scrotum. The penis was to have been liberated and the urethra brought out through it, and then the divided scrotum was to have been united. The child had Potts' disease of the spine, and upon his recovery from the hernia operations, it was deemed advisable to delay further surgery for at least one year, but before this time arrived, he developed pulmonary tuberculosis and died.

In I,4 I I hernia operations done by the author, $\mathrm{I}, 205$ have been of inguinal type, and the testicle has acted as a complication as follows:

Removed for disease: sarcoma 2, degeneration I......... 3

Removed to save time in operating.................. 2

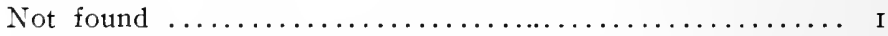

Found within abdomen (brought out I, left in I) ........ 2

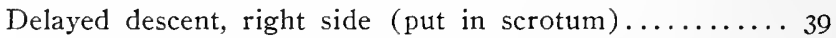

Delayed descent, left side (put in scrotum).......... 33. 72

( 8 were both sides)

Buried beneath abdominal muscles (3 sing., 2 dble.)...... 7

87

Ovary in Canal.-It is not very uncommon, in operating for inguinal hernia on the female, to find an ovary in the canal. As stated elsewhere, when the ovary drops into this position it is usually difficult to reduce without operation, and it is as persistent in attempts to slip under the truss pad, if one is being worn, as would be a delayer testicle in the same position. In operating, an ovary found in the canal, if not diseased, should be returned to the cavity of the abdomen after it has been entirely freed of achesions. In two instances I have been olliged to remove them on account of disease.

No case of doulble descent of the ovaries has come under my personal observation, but a number have been recorded by 
other operators. One such, that is of special interest on account of family history, is reported by Dr. William P. Matthews of Richmond, Va. ("Hernia of the Ovary Inguinal," N.Y. Medical Record, Nor. 30, Igor ). A girl of seventeen, who had double inguinal hernia present since birth, had never menstruated. Upon operation the ovaries were found in the canals, and it was ascertained that she had no uterus. Her great grandmother, twice married, bore children FIG. I38.

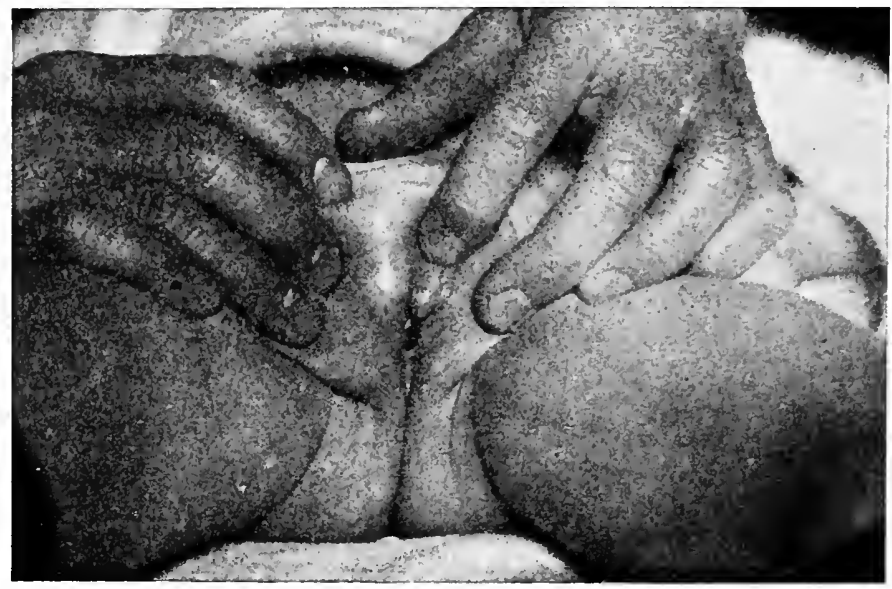

Sylvia L. Supposed labia held apart, showing what was thought to be the clitoris but which was really a small penis. The urethra was immediately beneath the penis, forming what appeared to be the vagina.

by each union. Eight female descendants of the first marriage were never unwell; one of the two daughters by the second marriage was never unwell. Two aunts of the patient have never menstruated and one has double reducible inguinal hernia. Five first cousins have never menstruated and two have double hernia. The patient's sister has never menstruated and has double hernia.

I had a woman of superb physique under my care for many years, for double inguinal hernia, who had never menstruated and whose uterus and ovaries were undiscoverable and prob- 
ably absent. She was twice married, but never bore children. Dr. R. Ferguson of London, Ontario ("Inguinal Hernia of an Imperfectly Developed Uterus and Appendages," American Medicinc, Sept. 26, 1903), found upon operation an incompletely developed uterus and both ovaries in a left inguinal hernia in a woman thirty-two years old. He ligated and removed them completely, dropping the stump back into the abdomen, and closed the canal by the Bassini method. Recovery was prompt and relief complete.

Dr. Frank T. Andrews of Chicago ("Hernia of the Tube Without the Ovary," Joumal of American Medical Association, Nov. 25, 1905), has in an exhaustive study of recorded cases of the involvement of the female pelvic organs with hernia, given the following division of cases. He has tabulated 366 cases, which are divided as indicated in the following table:

"Hernia of Tube without Ovary ............, 46 cases.

Hernia of Ovary and Tube ................. 80 "

Hernia of Ovary without Tube ............... I67 “

Hernia of Non-Gravid Uterus ................ 43 "

Hernia of Pregnant Uterus ................ 30 ."

Appendix Vermiformis in Canal.-In many instances the appendix is held in a position so remote as to cause no complication no matter how large the hernia may be, but in others it seems ever ready to clrop into the canal and become adherent or otherwise involved. The question of the removal of the appendix when found with other reducible parts of the hernia must be decided by the operator, but it has been the author's rule to remove them if unusually long; if nodular masses could be felt in its lumen; if it showed the least indication of former inflammatory conclitions.

In a case recently operated upon there were found adhesions of the omentum, in the vicinity of the internal ring, of an inflammatory character, that could lardly be accounted for by the presence of the hernia. Exploration through the internal 
ring with the finger brought to the surface a very much inflamed appendix, which clearly showerl a beginning or a subsiding appendicitis. It is quite probable that it was the pain of

FIG. I39.

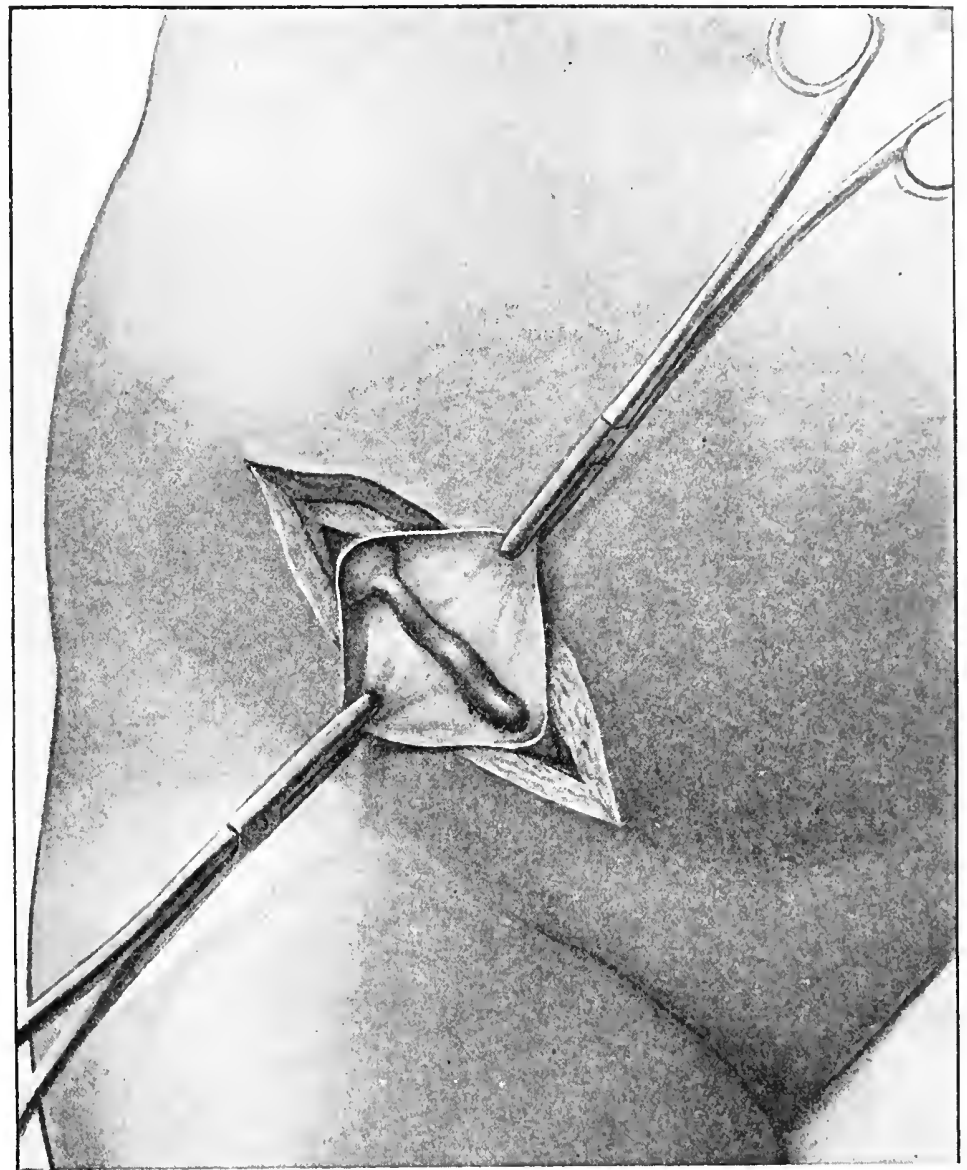

Appendix adherent to posterior wall of hernial sac, the latter held open by clamps.

this, which was attributed by the patient to his hernia, that brought the case to the operating table, and still it might easily have been overlooked. In a number of cases, where there has 
been a history of a previous attack of appendicitis, the appendix has been brought out and removed through the same incision while operating for hernia. This opening is, of course, not as convenient as one macle especially for the purpose, but I have failed only once to get it out through the internal ring when it was wanted, and, in this instance, it was obtained by splitting the internal oblique muscle in the direction of its fibre, just above and to the inner side of the internal ring.

The method of removing the appendix will usually be governed by the education and preference of the operator, as several excellent methods are taught. The author has for several years followed the one of ligating and burying the stump. The steps of the proceeding are:

(I) Ligating the vessels of the mesentery.

(2) Division of peritoneal covering only, usually by scissors.

(3) Tying in groove thus formed, with chromic gut.

(4) Placing the burying suture of fine silk with round needle through peritoneal coat of bowel, an inch away from and around insertion of appendix into cacum.

(5) Cutting off appendix and touching end of stump with pure carbolic acid ( wipe afterwards with alcohol on Sponge).

(6) Depression of stump and tying of burying suture.

The drawing shown in fig. I39 represents the condition found in a woman thirty-two years of age. She had noticed a sivelling for the past year on the right side, and it was found that considerable thickening remained after the reduction of the bulk of the tumor. At the first examination she was advised to have an operation, as it was believed that either the tube or ovary was involved. This conclusion was reached because of the fact that at each menstrual period she had increased pain. A truss was tried but proved uncomfortable, and operative relief was sought. On opening the sac the condition shown in the drawing was found. That is, the appendix was found lying along and firmly adherent to the posterior wall of the hernial sac, and the head of the crecum was firmly held in the internal abdominal ring, the vessels of the mesentery lying 
between the appendix and the sac surface. Little troulsle was experienced in ligating them, freeing the head of the ccecum,

Fir. I 40.

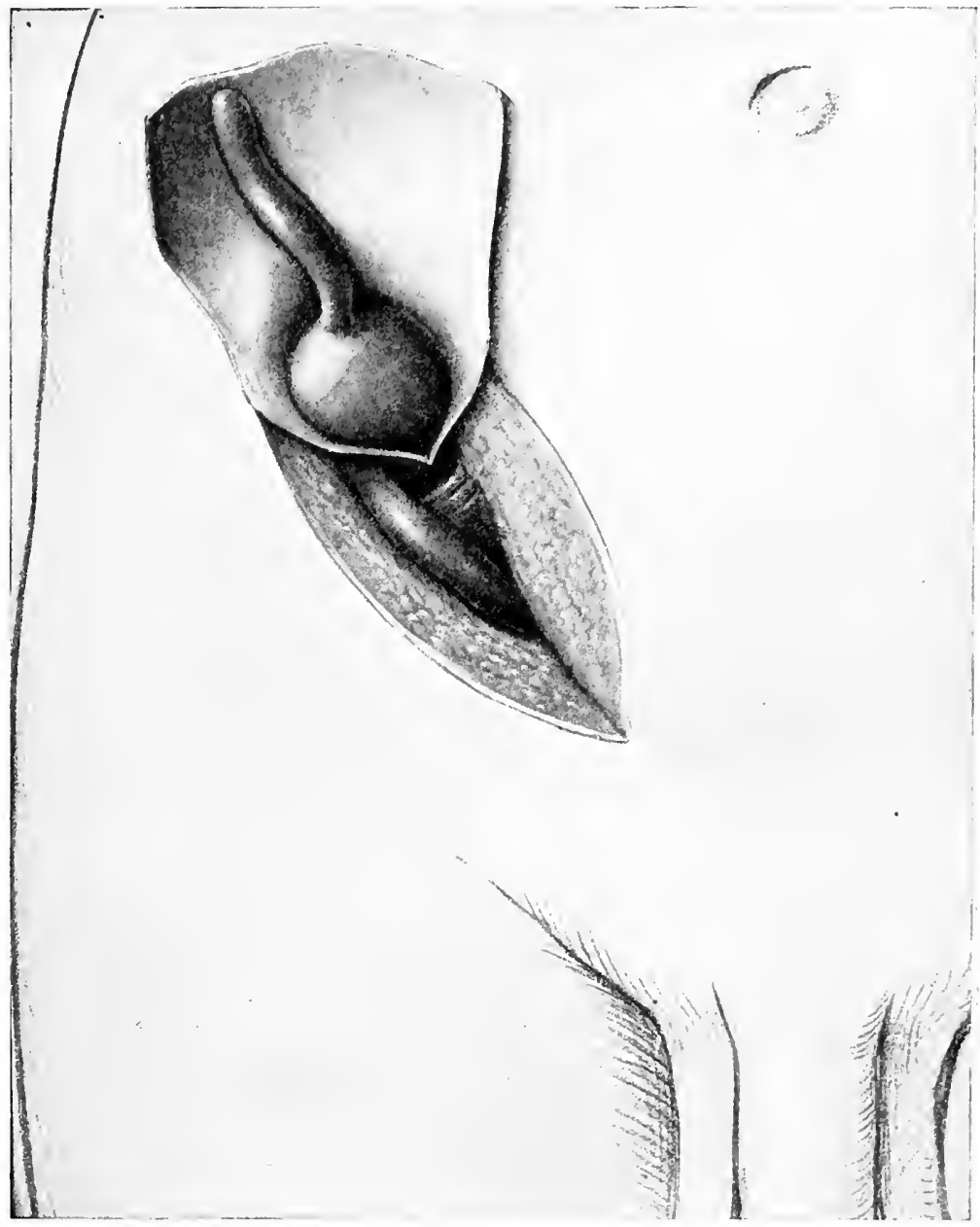

Appendix adherent to anterior wall of hernial sac, holding excum in internal ring Truss worn over appendix for many years.

and reducing it to the cavity of the abdomen after tying off the appendix. The neck of the sac was then ligated and cut away. 
Another quite similar case is shown in fig. I40. This man had been under my care for many years, and while he was obliged to wear an unusually strong truss, he suffered little inconvenience and was only brought to the operation through the influence of friends who had been cured of hernia. He not only followed his profession as a lawyer, but was an all around athlete and a long distance bicycle rider. It was therefore quite a surprise to find, on operating, an appendix

FIG. I4I.

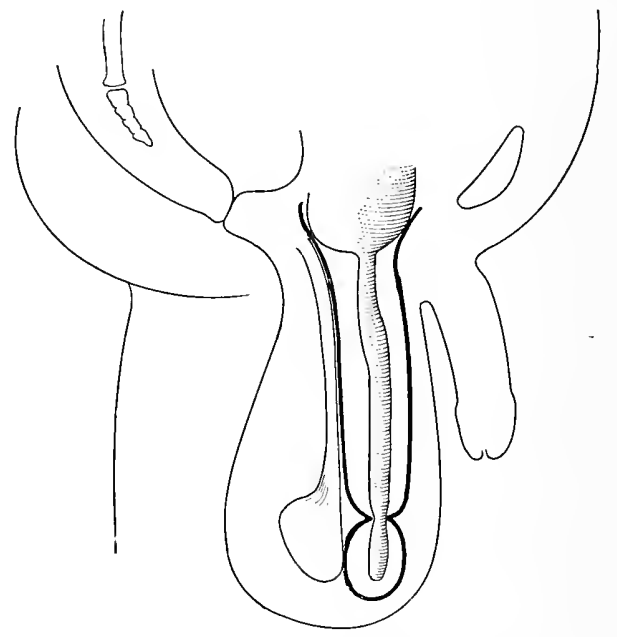

Top of appendix incarcerated in fibrous ring in sac.

adherent to the anterior wall of the hernial sac in such a position that his truss pad pressed directly across it near its junction with the crecum, and still it showed no evidence of inflammation or disturbance. The adhesions were of a character to clearly indicate that they had been there for many years.

Earlier in this work reference has been made to the white, fibrous rings that form in hernial sacs and the liability of strangulation to take place in them. A peculiar illustration of this is given in the case portrayed in fig. I4r. This was a 
boy about eight years old, under care at the clinic for the treatment of hernia at the New York Post-Graduate Medical School and Hospital, but who had not done very well under truss treatment, and as cure was not anticipated by this means, he was placed in the hospital for operative cure.

The end of the appendix was so firmly held within one of the rings above spoken of, that it could not be withdrawn, and still actual strangulation did not exist. It had the appearance. however, of having been there for a long time. It was an uncommonly long appendix, probably fully seven inches; the tip was down as low as the bottom of the "testicle, and the crecum was in the upper part of the canal. The crecum was elongaterl and merged so gradually into the appendix that it was difficult to lecide just where the latter began. It is not within my own experience, but my associate, Dr. George E. Doty, as well as several other operators, have fotnd the appendix in left inguinal hernia.

Adhesions Within the Sac are most commonly of omentum at its sides or bottom, but it occasionally happens that the intestine is adherent, and it then requires considerable care and skill to avoid serious injury. Attempts to tear such adhesions apart, unless they are of the most trifling character, should never be made. A large patch of the sac may, however, be cut ont and left adhering to the bowel without fear of harm resulting. Torn surfaces upon the bowel, even though they may not endanger its integrity, cause great delay and inconvenience from the liability of free oozing of blood. Adhesion of omentum, if small in area, can usually be easily broken up, but it is better to tie them by catgut rather than run any risk of stibsecfuent bleeding.

Amputation of Omentum is worthy of especial discussion. both on account of its frecuently being required, and on account of the dangers attending its removal if not carefully done. It is much better to remove large masses of omentum that have been outsicle the abdomen for some time, than to attempt its replacement, for two reasons : 
(I) It has become shaped to the canal and to the sac that contains it, and if crowded back into the abdominal cavity, FIG. I42.

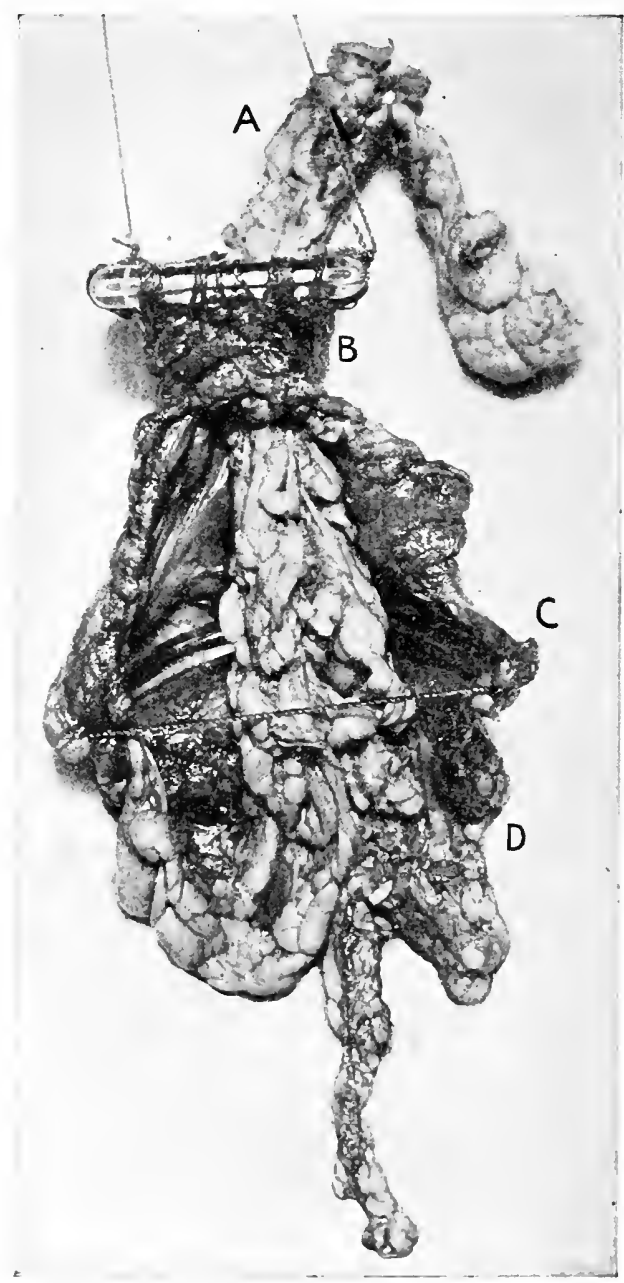

Omentum irreducible because of adhesions. A, Omentum. B, Neck of sac. C, Sac cut open D, Fundus of sac with omentum firmly adherent.

remains in a large mass at the internal ring ready to again dilate the canal and cause recurrence of the hernia. 
(2) Onentum long resident outsicle of the abdomen becomes hypertrophied and so changerl in character that it may act as a foreign body when returned within the peritoneal cavity.

Formerly it was considered goorl surgery to take a mass of omentum, such as shown protruding through the neck of the

FIG. I 43 .

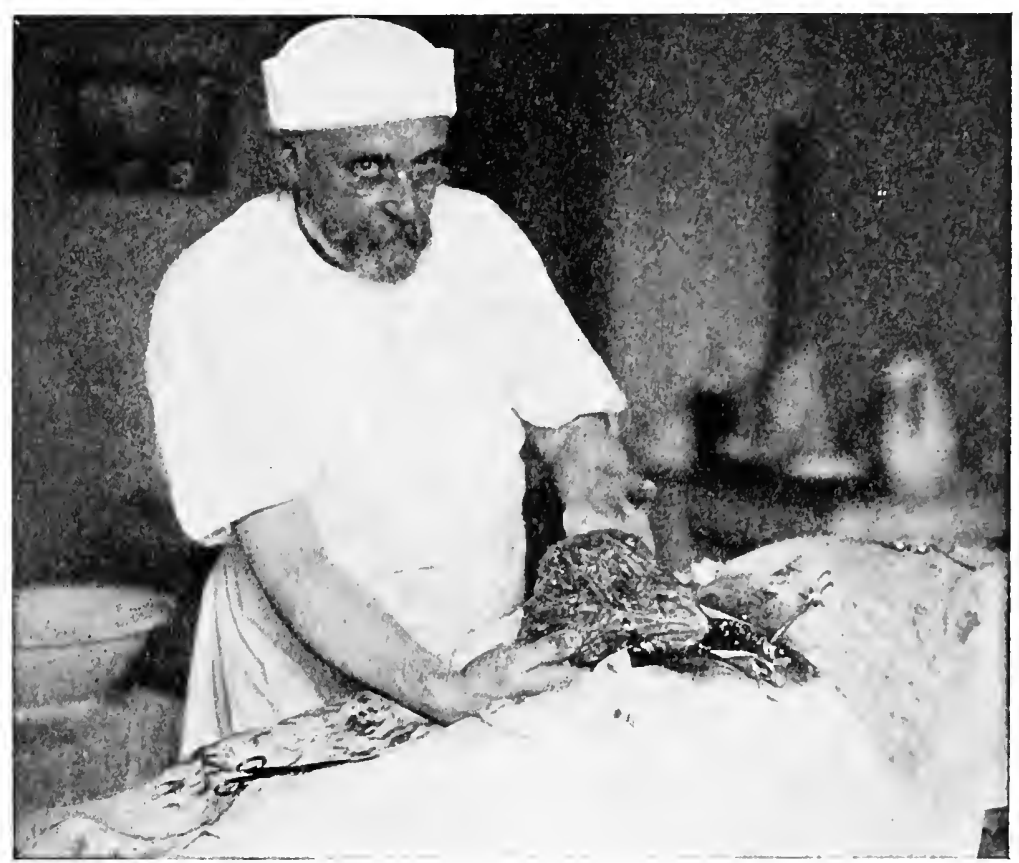

Onentum spread out before ligating to isolate vessles and prevent risk of injuring intestine.

sac in fig. I42, pass a ligature around it and cut it off. Work of this type was followed by fatal accidents from secondary hamorrhage, and in more than one instance, from injury to bowel that was lying unseen within the omentum.

Many years since I adrocated a methor of ligation that has become almost universally used by careful surgeons. It 
consists in drawing down the protruding mass until normal omentum is brought outside of the canal, being careful not to make undue traction. It is then spread out by the hands (fig.

FIG. I44.

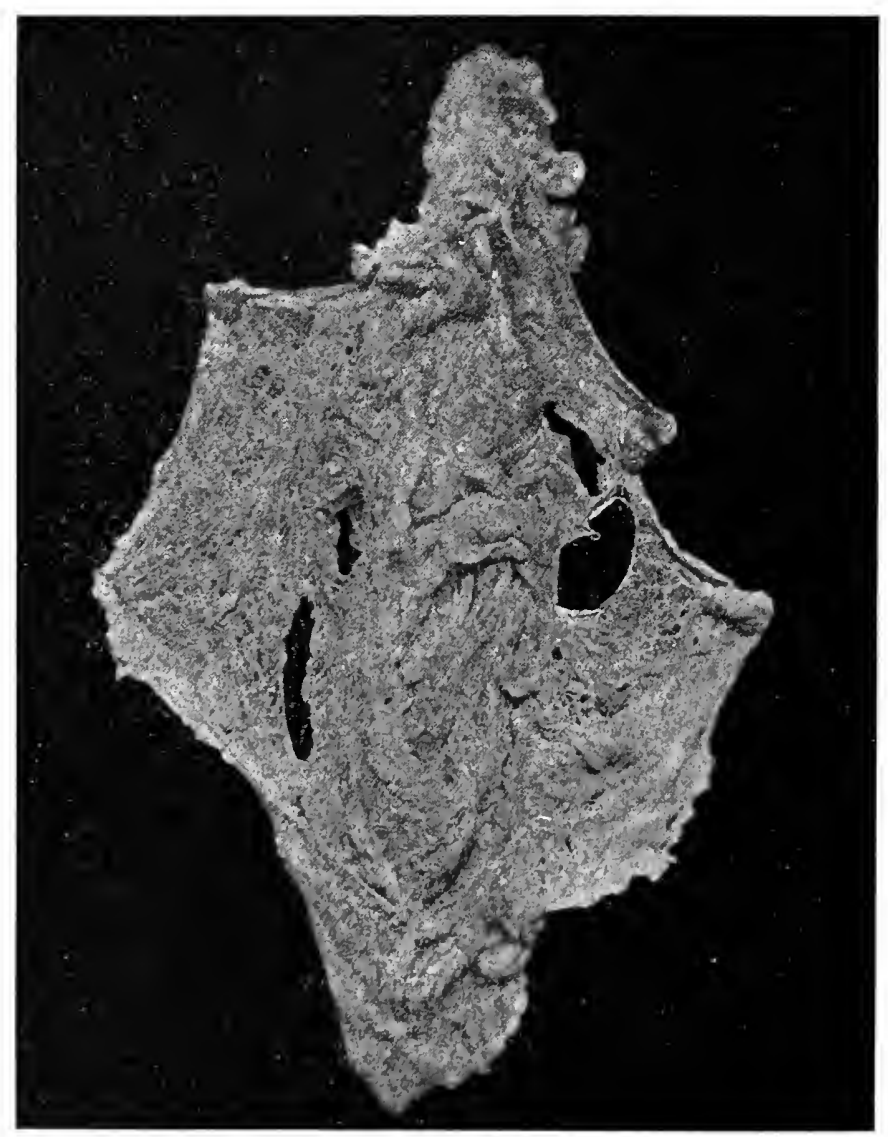

Mass of omentum irreducible from quantity and shape. Narrow part at top was in the canal.

I 43) so that every blood ressel of any consiclerable size can be easily seen. Ligation then legins at one sicle of this sheet of omentum and progresses carefully across to the other. Fat 
with very small ressels is tied in fairly good quantity. but each large vessel is carefully cleared of its surrounding fat and tied off separately. No. 2 chromic catgut has been most frequently used as suture material. As many as 25 ligatures have been applied in removing a piece of omentum. After the ligatures are in place and the omentum is cut away, the stump, which is usually left about half an inch long beyond the ligature, is examined for any bleeding points, and these are also carefully tied.

FIG. I45.

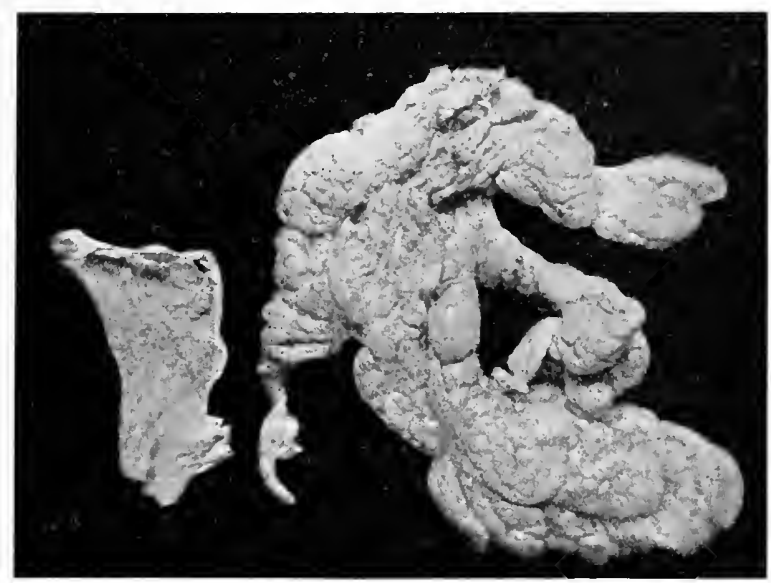

Omentum. Sac which contained it shown at left.

The freshly cut stump of omentum is dusted with aristol and returned to the abdomen. The use of the aristol may be entirely unnecessary, but it is believed to prevent adhesion of intestine to the cut surface and it has been used by the author for many years.

Herniæ of Unusual Size.-The large size of hernia does not in itself preclude its surgical cure. Unfortunately these extreme cases occur most frequently in the aged and infirm. and for these reasons operation may be quite inadvisable. If the general condition of the patient will allow of it, the surgeon 
should feel it his duty to give these sufferers not only relief, but freedom from the danger that is constantly with them of acute strangulated hernia; or what is eren more fatal, a gradual paralysis of the protruding bowel and eventual intestinal obstruction.

FIG. I46.

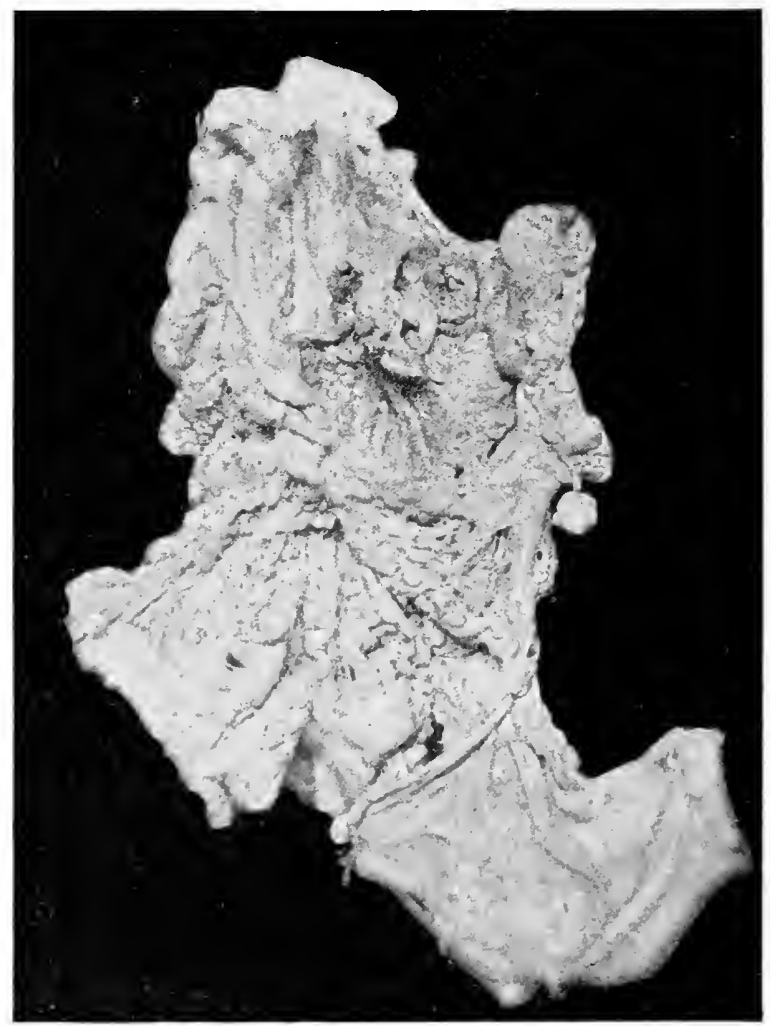

Omentum. At lower part is the sac which contained it.

It has been in my experience a noticeable fact, that many very large hernice are particularly easy to operate upon, and, as a rule. the difficulties that will be encountered can be fairly well estimated in adrance. if the following points are carefully considererl. 
(I) Is the patient's condition such as to stand an anesthetic and an operation of some magnitude?

(2) Is the hernia reducible and is the cavity of the abdomen large enough to receive it?

(3) If not reducible, is it omentum or intestine that cannot be reduced?

The patient's actual age is not nearly as important as his condition; in fact, people of advanced age usually stand the operation well. If any large portion is reducible, it is well to keep the patient in bed for some days before the operation, and keep as much of the hernia as possible within the abdomen. There is little doubt that some of these cases have had a fatal termination, due to extreme intra-abdominal pressure resulting from the return of enormous masses of intestine and omentum that have long been outside of the abdominal cavity. For this reason it is also advisable to remove as much of the protruding omentum as possible rather than to replace it. I have on several occasions removed what appeared to be nearly the entire omentum with no ill effects following. If, however, upon examination it is decided that the greater part of a large irreducible tumor is intestine, and this can usually be ascertained by. percussion and general feeling of the contents, then great caution should be observed in advising the operation. If the intestine is extensively adherent, the handling necessary to free it and return it to the abdomen may, in itself. cause collapse of the patient.

Patients who have become completely disabled by such enormous hernice are sometimes willing to assume unusual risks with the hope of obtaining relief. Such a case is shown in figs. I 47 and I48. This man was serenty-four years old, had a pulse rate never exceeding 50 , and had albumen in his urine. He was told that the risk of not surviving operation was believed to be fully one out of five, and he at once assured me that he had decided to have the operation if I thought he had even chances of living or dying. The operation was done and he completely recovered, and lived four years more in complete 
comfort, so far as his hernia was concerned. Fig. I49 shows him seren weeks after the operation, after which time he wore no bandlage or other support. One peculiar circumstance con-

FIG. I47.

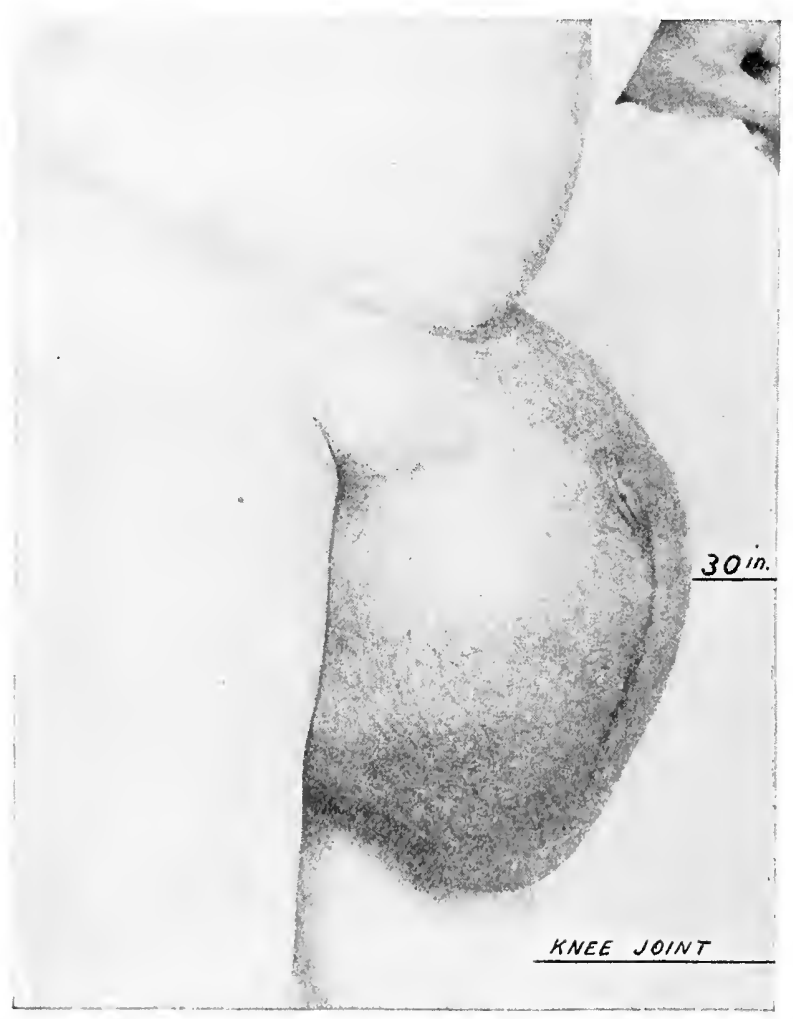

S. L. P. Age, 74 years. Operated upon Oct. 12, 1899. Duration of hernia, 20 years. No truss ever worn. No recurrence to 1903 , when he died of an affection of heart, present when olerated upon. No support worn. Note that the testicle is clearly outlined at bottom of scrotum.

necterl with this case was that the pulse, which we had been unable to fincl above 50 for two weeks previous to the operation, was 70 on the following morning, and did not drop materially 
below this during the subsequent eight or ten weeks that he was uncler observation.

In this case, in order to aid in rapid work, the testicle and sac were removed together after ligating the cord separately

FIG. I48.

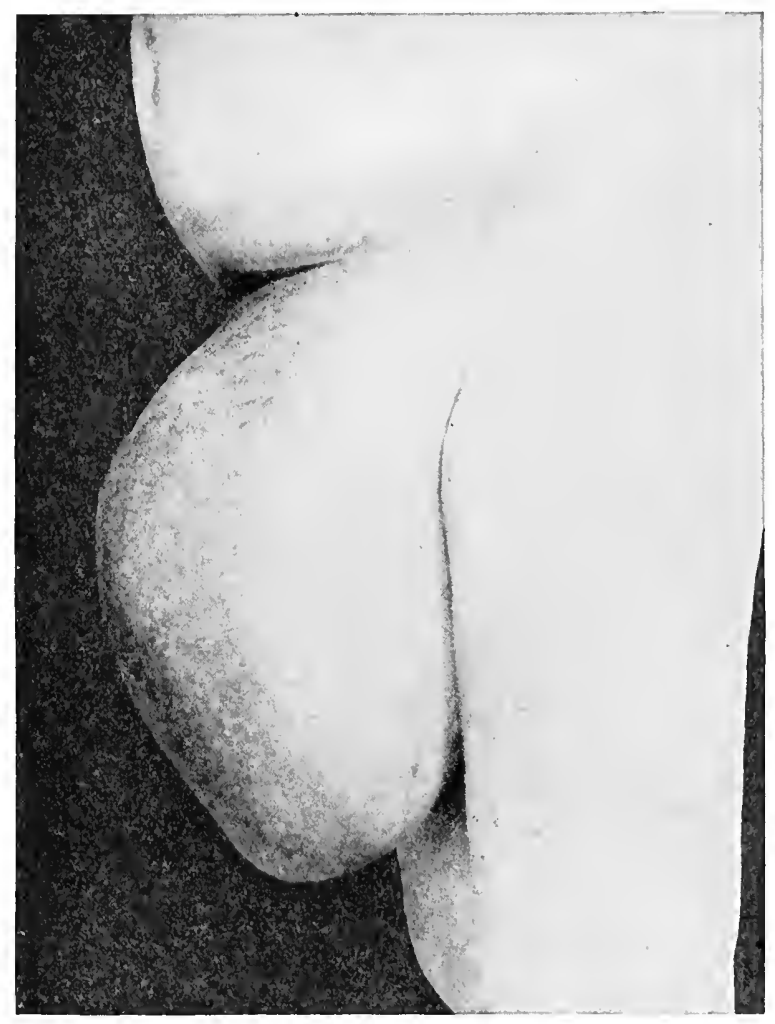

S. L. B. Side view of case shown in fig. 147.

at the neck of the sac. The sac was removed through the inguinal incision, the scrotal tissues not being touched. Nor was drainage of any kind used, the wound being closed conpletely and primary union obtained. If, however, there is considerable oozing following the stripping out of such an 
enormous sac, it is then advisable to put a good-sized drainage tube, preferably rubber with numerous fenestra cut out, down through the bottom of the scrotum. The protruding end of this tube should be very carefully guarded, by gauze moistened in I/ 2000 corrosive mercuric chloride, and the tube should be

FIG. I49.

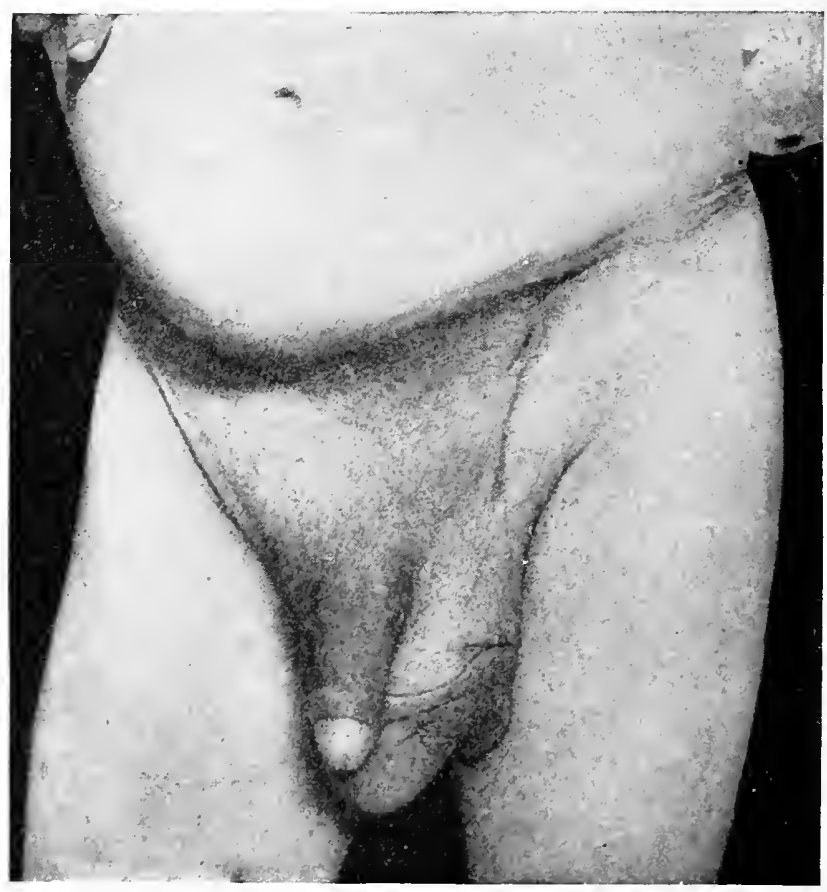

S. L. B, seven wceks after operation.

removed in twenty-four, or, at the outside, thirty-six hours. It is only by extreme care that infection can be avoided where a lrainage tulue is left in, and it has usually been my preference to take the risks of a good-sized hematoma rather than to subject the patient to the liability of infection. The blood clot will. as a rule, be absorber in the course of three weeks and does no harm. In only one instance have I seen such a clot 
become infected and require evacuation, and this case was unattended by symptoms of any importance.

The enormous hernix now uncler consideration do not seem so liable to recurrence as would naturally be expecterl. The case shown in figs. I 5O, I 5 I, and $5_{52}$ is an illustration of

\section{FIG. I50.}

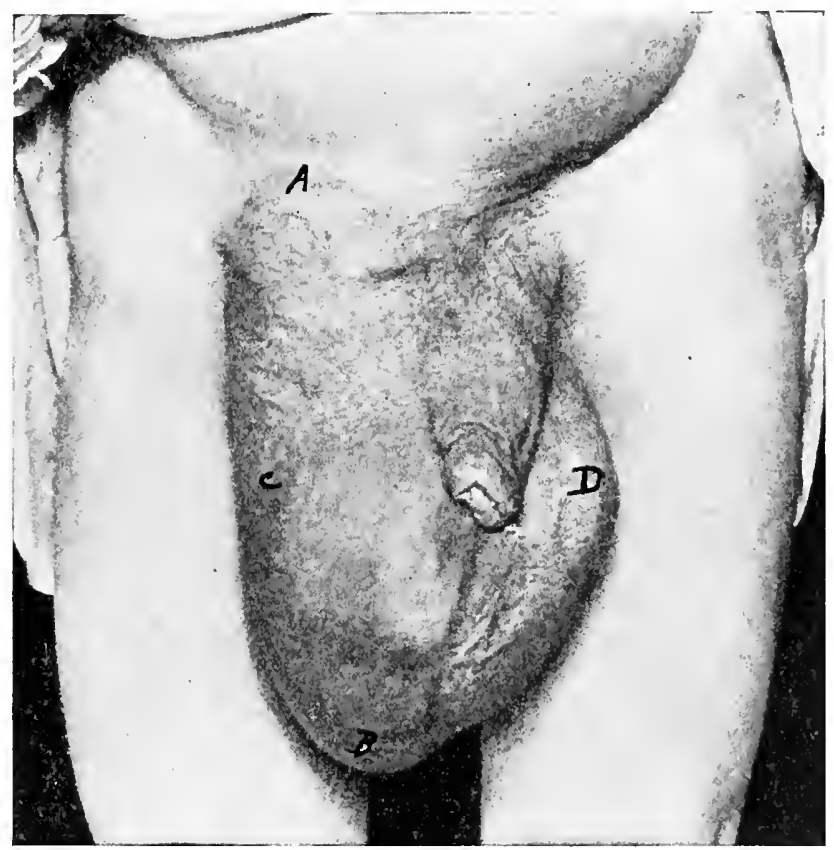

T.S., 50 years old. Riglit ingninal (scrotal) hernia for 20 years; never retained. Measures. $A$ to $B, I_{4}$ inches; circumference, $C$ to $D, 2$ feet, 2 inches. Operation Dec. 4,1895 ; no recurrence to 1906 . No truss worn. Contents, large and small intestines, omentum, bladder iuside of sac, and free fluid. Note right testicle at $B$, left at $D$. See figs. 15I, 152.

this fact. The man there shown was operated upon for an enormous uncontrollable scrotal hernia and made prompt recovery, healing by primary union. At that time there was no hernia on the left side. Five years later swelling was noticed on the left side (fig: I 52 ), and I found him with complete oblique hernia the size of a hen's egg, which was operated 
upon and cured. Many experiences of this kind convince me that in these very large hernize there is little trouble in effecting a permanent cure.

In the case shown in fig. I 53 the man was orer seventy years of age and had a hernia reaching nearly to his knee, of many years' duration, and wholly uncontrollable. The hernia,

FIG.I5I.

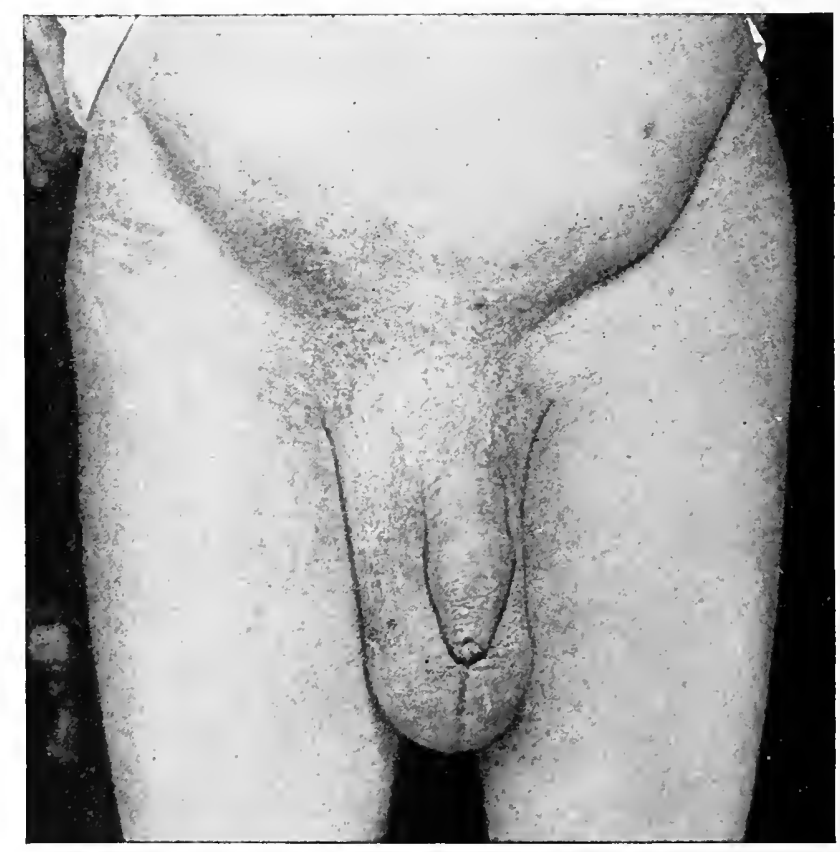

T. S., six weeks after operation for enormous right scrotal hernia. See figs. 150, and 152 .

which proved upon operation to contain mostly intestine, both large and small, could be reduced to the abdomen. He was kept in berl for fully one week before operating, and the hernia was kept reduced most of that time to accustom the abdominal cavity to its presence. The operation was not particularly difficult, and plenty of good muscle was found with which to close the very large opening. 
Fig. $15+$ is a case of very different type and one, if recognized, where an operation should not be attempterl. In this case it was easily ascertained that a large amount of intestine was present, a small amount of it reducible, and it was believed that a large mass of hypertrophier omentum was also in the sac. This unfortunately did not prove true. The man had

\section{FIG. 152 .}

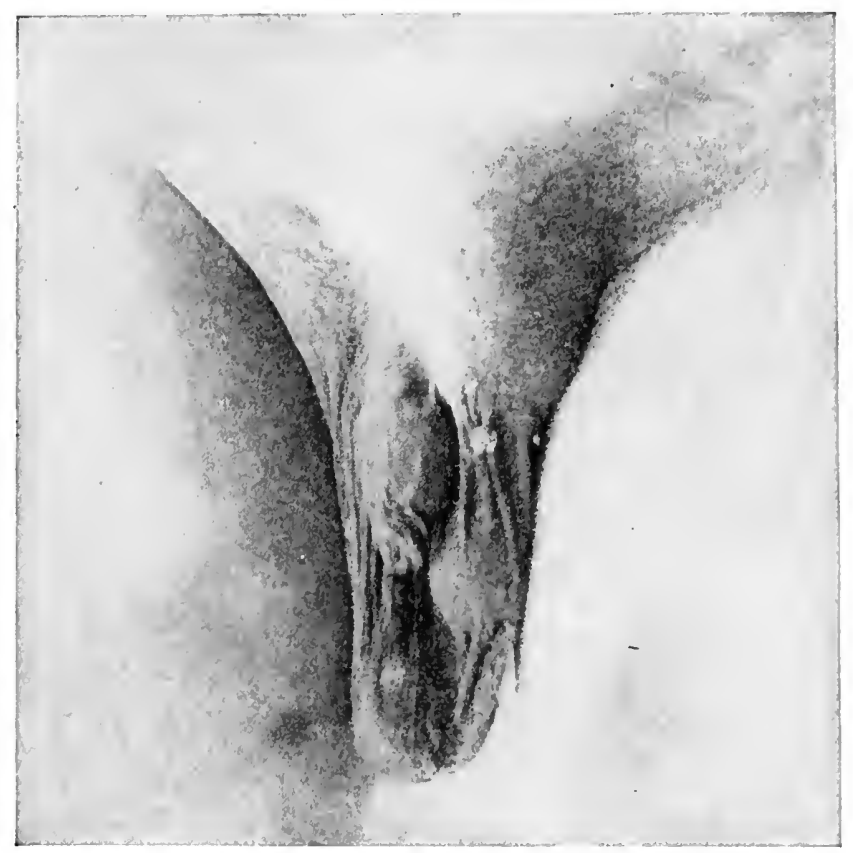

T. S. Recent left inguinal hernia; operation upon enormous right scrotal hernia five years previously. See figs. I50 and I5I.

suffered several attacks of partial intestinal obstruction unaccompanied by very acute symptoms of strangulation, but increasing in frequency, and the case had every indication that surgical relief was required. The condition observed upon operating was apparently a most unusual one. 
On opening the sac no omentum was found, on the contrary. it was packed with convolutions of bowel held on a mesentery of very unusual character (fig. I 55). This mesentery was a fan-shaped, board-like mass, fully an inch thick,

\section{FIG. I 53}

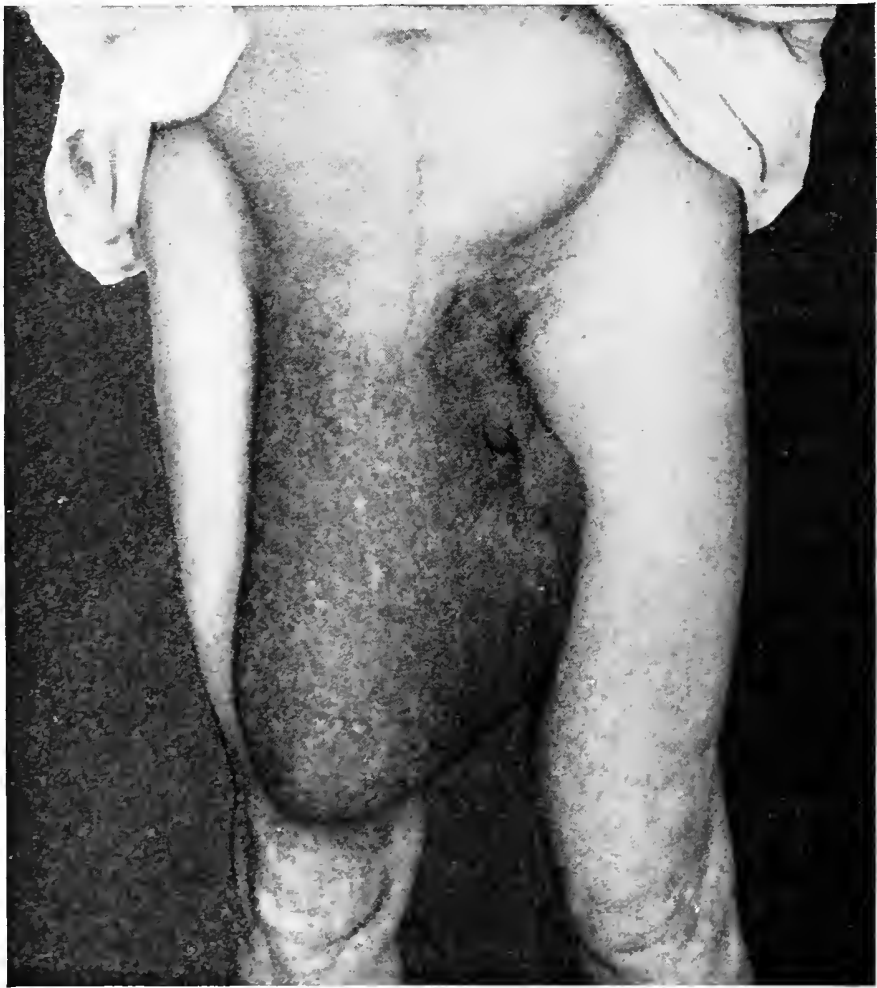

Enormous right scrotal hernia in a man over yo years old. Wholly reducible but not retainable by any truss that could be tolerated. Contents, large and small intestine and small amount of omentum.

having a feeling somewhat like that of a dense, broad tenclon. At the point where it entered the alydomen, it was bunched together intro a mass as large as an adult forearm, just below the ellow. and apparently almost as inflexible. 
Those who have operated upon large hernice will fully realize the almost insurmountable difficulties of the situation. The entire mass was finally returned to the abdominal cavity and the wound closed, but the patient did not long survive the operation. The feeling of the tumor, which led to the belief that it contained a large mass of omentum, was really produced

FIG. I54.

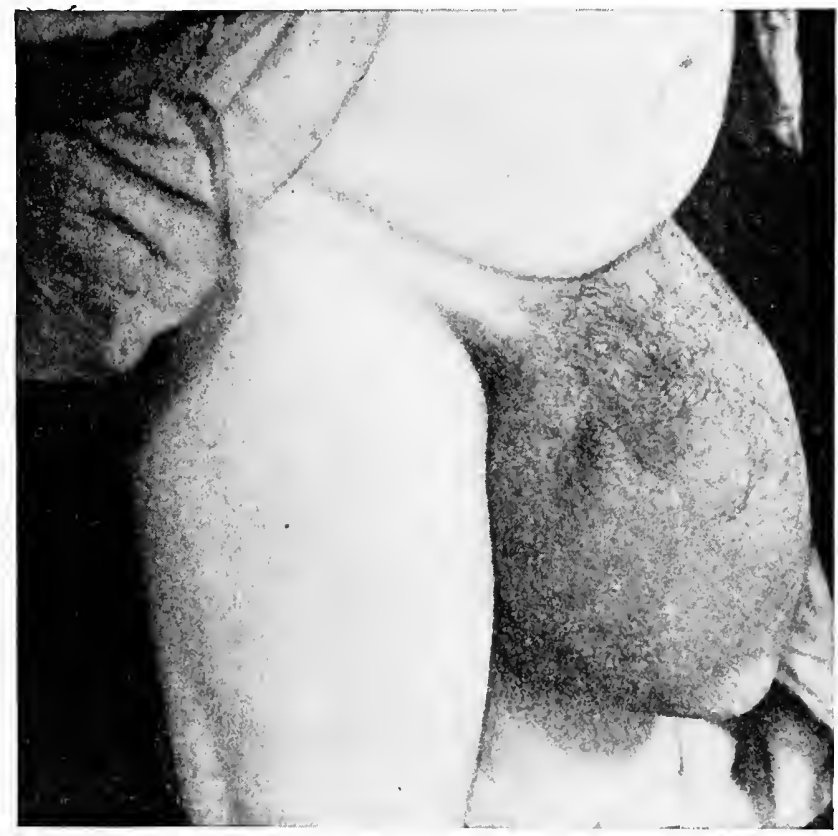

M. G., 40 years of age. Irreducible left scrotal hernia packed with intestine on peculiar hypertrophied mesentery. 1)uration, 2o years. Never retained.

by this peculiar hypertrophied (if it may be so called) mesentery. The ultimate termination of this case, if it had not been operated upon, would in all probability have been by intestinal obstruction produced by gradual loss of peristalsis in the protruding bowel. I have seen only one other case at all similar, nor do I recall having seen mention of any like it by other operators. 
My other case was in a woman with enormous femoral hernia shown in the photograph, fig. I69, and even this did not

FIG. I55.

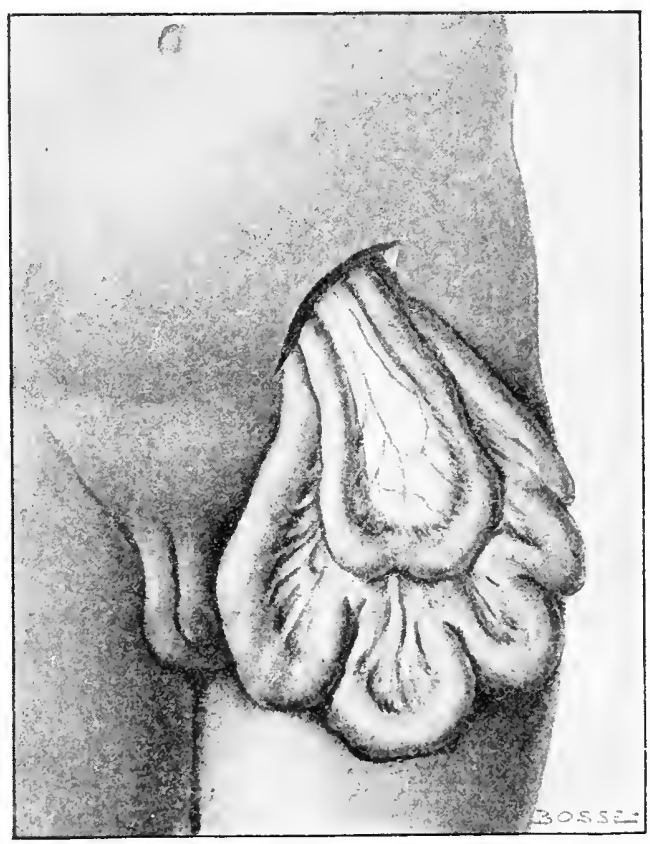

Intestine which was irreducible on account of hypertrophy and hardening of its mesentery.

present the extreme difficulties met with in the case under consideration. 


\section{CHAPTER XIII.}

\section{SIGMOID, CAECAL, AND BLADDER HERNIA.}

Hernix of the sigmoid, cæcum, and bladder, belong to the direct type of inguinal hernia and are classed together because of similar anatomical peculiarities. In the acciclents which may occur during the operation for their cure they are also similar.

In the chapter on diagnosis will be found suggestions which may, in some instances at least, lead to the recognition of sigmoid and cæcal hernia before the operation; in many others, however, there are no distinguishing signs previous to the opening of the parts, and even then serious mistakes may be made. It is perhaps wise to look upon all direct hernire as extra-hazardous and to use extraordinary care in operating upon them. Petit (Rechorches sur les causes des hernies) has truly said that hernial sacs are full of deception.

My own experience leads me to feel that sigmoid and crecal hernia can usually be recognized, or at least suspected, before operation by their peculiar form (see photographs of cases), their slowness of reduction, especially of the last part of the tumor, and by the fact that not only are they frequently uncontrollable by truss pressure, but the wearing of any mechanical support is intolerable because of the pain produced.

The difficulties in operating upon hernia of the direct type, especially the three forms now under consideration, are largely due to the distribution of the peritoneum. The protrusion takes place at a point where the peritoneum leaves the abdominal wall and is reflected over the pelvic organs. IThen it is carried through the abdominal wall it drags, by its attachments to them, one of these organs with it. This is especially true of the large bowel on either side and the bladder, on account of their being freely movable and conveniently 
placed. While the peritoneum completely surrounds the small and in some parts the large intestine it covers only the anterior surface of both the sigmoid flexure and cæcum. It is for this reason that a portion of the large bowel on either side may protrude without a hernial sac, and if not recognized by the operator may be opened by mistake. Tuffier (Etude sur le cacum et les hernies, Arch Gen. de Med., 7th ser., vol. xix, p. 642 ) found in the examination of Ioo bodies that 9 per cent. had the posterior surface of the cæcum uncorered by peri-

FIG. 156.

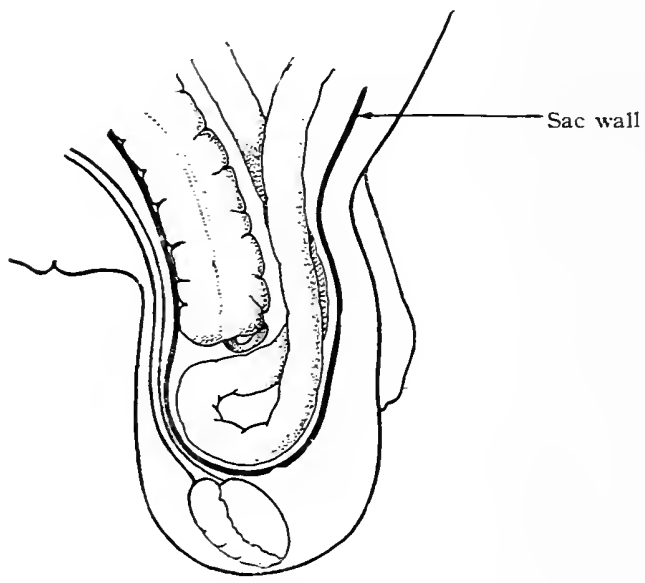

Hernial sac containing ftee cæcum and loops of small intestine.

toneum. The bladder is covered by peritoneum upon its summit and a small portion of its posterior wall, but not its anterior wall.

When this distribution of peritoneum is considered it will be readily understood how any one of these organs may protrucle in either one of three conditions: (I) It may become a part of the hernial contents, in a preformed sac-intra-peritoneal (fig. I 56). (2) It may protrude inclependently. without any serous covering-extra-peritoneal (fig. I 57). (3) It may be dragged down in the formation of the hernial sac and 
have a partial peritoneal covering, and become both intra- and extra-peritoneal.

As previously stated, protrusions into a preformerl sac have not been considered by the author to constitute either sigmoid, caecal, or bladder hernia, because they are easily reduced with the other hernial contents, quickly recognized, and not liable to accidental injury.

Sigmoid and Cæcal Hernia.-In these herniæe the bowel may be dragged down by a sac of peritoneum previously

FIG. I 57 .

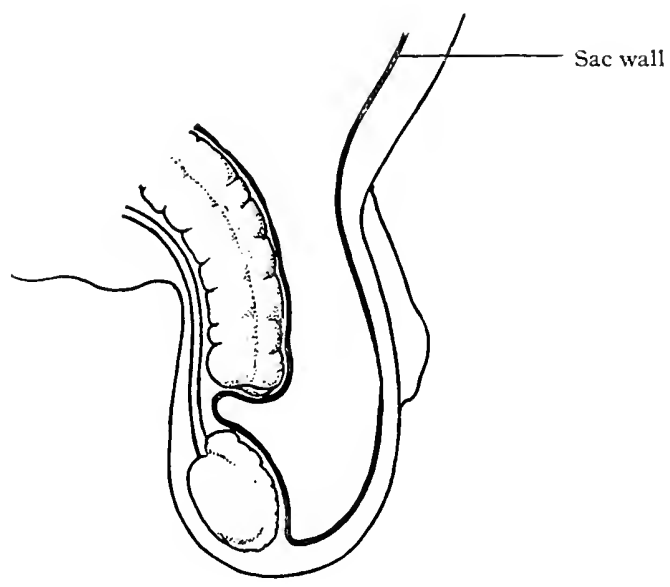

Hernial sac in front of cæcum. No peritoneal covering on posterior wall of bowel.

formed and containing other folds of the large, or many loops of small intestine and omentum. The posterior sac wall is then the normal peritoneal covering of the anterior surface of either the crecum or sigmoid, as the case may be. If the operator, fortunately, opens into the sac high up, near the internal ring, he will usually discover the true state of affairs and aroid injury to the bowel, either by tying off a portion of it with his sac ligature, or by rudely tearing it away from its deep attachments and perhaps lacerating the intestinal wall in a manner that would be difficult to repair. If, on the contrary, he opens 
into the fundus or lower portion of such a protrusion he may discover too late that he has opened directly into the bowel.

In operating upon these extremely difficult cases unusual caution is necessary throughout the entire operation: (I) In the opening of the sac. (2) In separating the fleshy attachments of the bowel from the deep parts. (3) In the closure of the sac. (4) In the closure of the enormously large opening which is left in the muscular wall of the abdomen after the reduction of the bowel.

In opening down upon any sac of the direct type, with broad base, it should be the invariable rule to separate the extra-peritoneal fascia well up towards the internal ring, pick up the peritoneum where it is thin and free, and open it carefully between anatomical forceps. If this one precaution were strictly adhered to it would prevent most of the accidental openings of the bladder and large bowel. Had I adhered to this point, which I have carefully taught my classes at the New York Post-Graduate Medical School and Hospital for many years, I would have avoided my only, but nevertheless mortifying, mistake of opening into the bladder. In this case I was so certain that I was dealing with a true hernial sac that I opened it even while speaking of the liability of bladder accidents in hernia operations.

When the peritoneal cavity has been entered near the internal ring, the finger can be passed down into the fundus of the sac, if present, or it can be ascertained that the protrusion is actually extra-peritoneal, and it can be dealt with accordingly. If the sac cannot be readily lifted from its posterior attachments it should be suspected at once that it is either sigmoid, cæcal, or bladder hernia. When the finger is within the peritoneum, the anterior sac wall may then be carefully cut upon it with blunt scissors, care being exercised to avoid intestinal adhesions that may be present, and remembering the immediate proximity of the epigastric artery. The contents of the sac may then be freely examined and so far as possible reducerl. 
The sccond step in the operation is to separate the posterior wall of the bowel from its attachment. This may be done by gently pushing the fingers upwarls between the bowel and the deeper parts, exerting as little force upon the intestine as possible, and watching closely for broken vessels, which should be tied at once eren though small. The point of separa-

FIG. 158 .

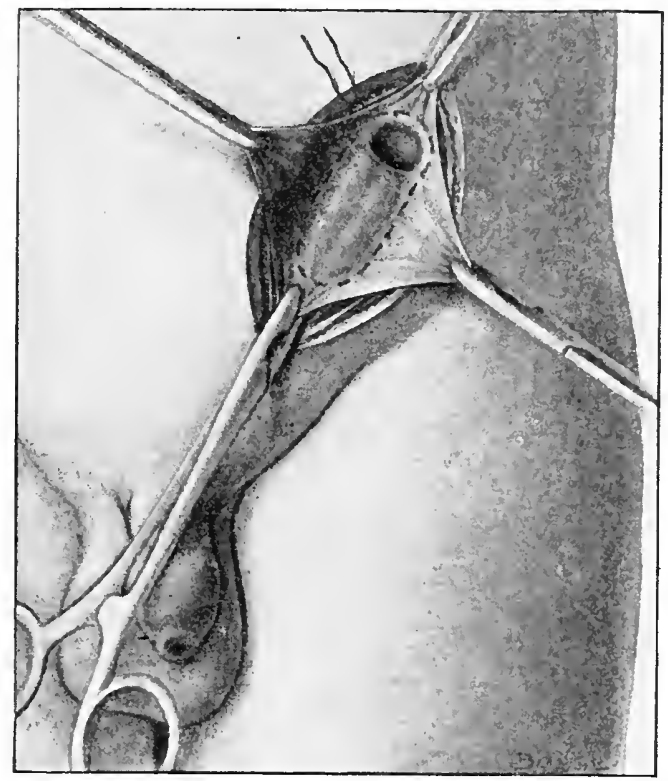

Form of purse-string suture for sigmoid sac.

tion between the structures should be carried well up into the abdomen so that the bowel can be reduced with perfect freedom. It is necessary to exercise caution in pushing the bowel back not to telescope it into itself, thereby producing an intussusception.

Closure of the Sac (fig. I 58 ). - It is quite obvious that a sac of this type cannot be closed by an ordinary ligature. as its posterior wall is formed of bowel. It is best done by a 
purse-string suture applied from the inside of the sac, going well up to the internal ring, or peritoneal surface on its anterior wall, and approaching with caution that part attached to the bowel. When this suture (usually no. 2 plain catgut used double) is tied, the sac is perforated, and any surplus of sac wall that exists is drawn up and ligated outside the pursestring suture and cut away. The ends of the sac ligature with

FIG. I 59 .

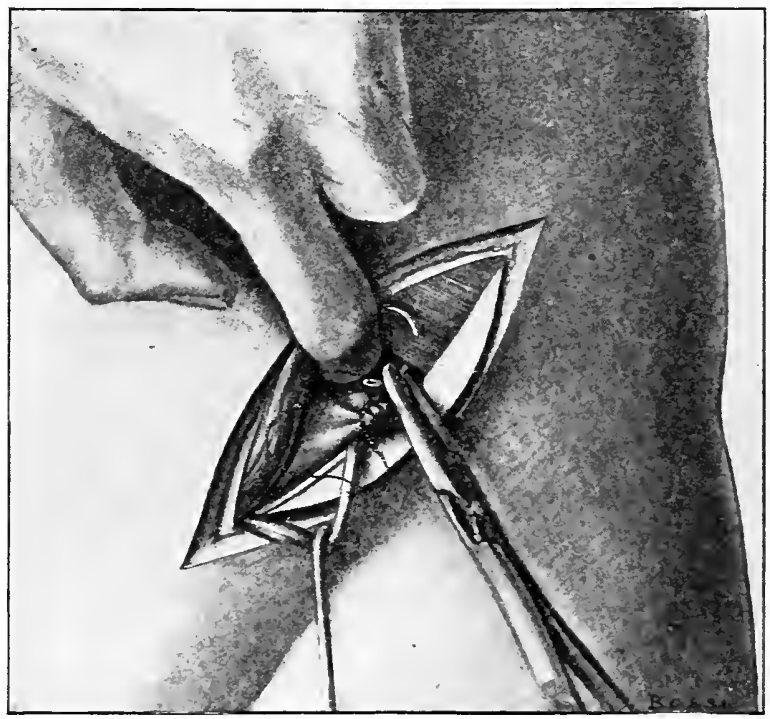

Lifting internal oblique muscle to bury stump of sac beneath it.

needle attached are used to anchor the stump of the sac, with attached bowel, in the following manner:

The internal oblique and transversalis muscles are separated from the peritoneum by slipping the ends of the fingers between them and the peritoneum, above the internal ring and towards the median line (fig. I59). The needle on the sac stump ligature is passed through these muscles from within outwards, and then back at another point. When it is tied 
with the free end of the stump ligature it will invert the sac stump and carry the attached bowel well away from the inguinal canal. I am convinced that the anchoring of this elongated loop of bowel well away from the internal ring is a great aid in the prevention of recurrence.

The details of the closure of these large openings in the abdominal wall must depend upon the condition of the surrounding muscular tissue and the preference of the operator. The first essential of success is thorough and careful work whaterer technique is adopted. If there is an abundance of good muscular tissue it has been my habit to close exactly as in oblique inguinal hernia. Even though the neck of the sac is at the external ring and the upper canal unoccupied, it is important that the cord should be displaced at its junction with the peritoneum, the lower edges of the internal oblique and transversalis muscles wrapped closely around it, and stitched to Poupart's ligament throughout the length of the canal. The continuous suture of the peculiar type already shown (figs. III, II2), is believed to make a more accurate closure than is possible with the interrupted. Especial care is taken in these cases to see that the closure is made as perfect as possible in the aponeurotic, as well as in the muscular structures.

In some of these cases the method of overlapping, already mentioned, has been used with advantage, and in one the rectus was split and turned out in order to get sufficient tissue to make a firm closure. Even where every precaution is taken there is little doubt that sigmoid and crecal hernia will furnish a larger percentage of recurrences than hernixe of the oblique inguinal type. In view of this fact it is advisable to keep them uncler closer scrutiny after operation, and, if any sign of weakness of the abdominal wall is discovered, to place a support upon them before actual protrusion occurs. At this time a good abdominal belt, that gives support over the whole lower part of the abdomen, is usually better than a regular truss, and should be worn with less discomfort. 
Bladder Hernia.-Like the hernix just discussed, the bladder may protrude through the abdominal wall, either inside of a true hernial sac without any peritoneal covering, or with both conditions present. Little attention has been given to those cases where the bladder protrudes within the sac covered by peritoneum, as there is no liability of accidental injury, and it is easily reduced with the rest of the hernial contents.

In I895 Dr. B. Farquhar Curtis made a most valuable contribution to this subject ("Wounds of the Bladder in Operations for Hernia," Annals of Surgcry', June, I895). In this study of the subject he collected $4 \mathrm{I}$ cases in which the bladder was wounded during operation, and $\mathrm{I} 7$ in which it was recognized and returned without injury. Only two years later Dr. C. L. Gibson ("Personal Experience in Hernia of the Bladder," Med. Record, March 20, I897) collected and added 45 cases to the list of Curtis, making a total of ro 3 cases. The report of Curtis's 58 cases covered the dates from 1575 to I895 (300 years), while Gibson's 45 cases, with three exceptions, included only those reported during the two subsequent years. In those two years there had been nearly as many reported as had appeared in all previous literature.

In the combined cases of Curtis and Gibson there were 76 males and 23 females; 70 males and 7 females had inguinal hernia: i 6 males and i 6 females had femoral hernia. More than half of the cases ( 52 ) were over fifty years of age, the youngest being two and one-half years. The bladder was intra-peritoneal in 7 , and extra-peritoneal in 73 instances; 18 were both intra- and extra-peritoneal.

Undoubtedly the most dangerous and deceptive, as well as the most common, variety is the extra-peritoneal, which comes through the abdominal wall uncorered by peritoneum. Frequently it constitutes the entire hermia and the surgeon is very apt to mistake it for the hernial sac. These bladder protrusions may vary in size from that of the end of one's finger to the entire bladder. The entire bladder and prostate gland have been found in a hernial protrusion of this type. The 
bladder protruding in this position has been mistaken for hernial sac, lipona, properitoneal fat, omentum, cyst, thickened patch in hernial sac, hydrocele of the cord, sacculation of the colon, or a second hernial sac. Plummer thinks that it would be particularly liable to be found in recurrent hernice, as the first operation would be apt to draw it toward this point.

Bladder wounds accidentally inflicted, during operations for the relief of hernia, have become too common to be longer consiclered surgical curiosities. This fact does not indicate increasing carelessness on the part of the surgeon, nor does it lessen the importance of the subject. It is unquestionably due to the greatly increased number of hernia operations being performed, and to the apparent impossibility of always being able to distinguish between the hernial sac and the bladder wall. It is to be hoped, and it is probable, that, as many operators are led to study it more closely, valuable knowledge will accumulate which will afford greater protection against this accident.

The diagnosis of bladder hernia is seldom made before operation, but as more attention is given to the subject there will be more cases that will be at least considered suspicious. In fact, the only safe method is to consider all cases of direct hernia, or hernia in very fat patients, as being a possible bladder hernia ; if on guard the operator will usually recognize the condition, if present. The indications may be divided into those to be looked for before, and those met with during. operation.

Before Operation.-The history of any blaclder symptom that can be obtained from the patient should be considered suspicious. In the case shown in the photographs (figs. I $5^{\circ}$ and $\mathrm{I}_{5} \mathrm{I}$ ) the patient informed me that while urinating he was usually obliged to compress the scrotal protrusion with both hands. At the operation a large protrusion of bladder wall was found (intra-peritoneal) protruding into the hernial sac. This was reduced as easily as the omentum and intestine that also occupied the sac in large quantities. After the operation, however, he was unable to void his urine, and it was then dis- 
covered that it was impossible to pass a catheter. Perineal section became necessary two days later. He obtained primary union in the hernia wound and made a rapid recovery.

Pressure upon the tumor may in some cases cause a desire to urinate. This was strikingly demonstrated in one of my cases where the bladder was found involved at the time of operation. Dr. J. F. Baldwin ("Hernia of the Bladder," Complete Inguinal Extra-peritoneal. Recovery. Am. Med. Jour., May I8, I90 I) reports a case, of eight years' duration, in a man of fifty-one years, where the bladder was protruding to the size of two fists. Ordinarily this swelling was reducible, but for forty-eight hours before operation it could not be rediced. The previous history showed that the patient was obliged to lift the tumor every time he urinated, and the protrusion had been uncontrollable by a truss.

The slowness of the reduction of these tumors, and in some its apparent incompleteness, should lead us to suspect that we have either large bowel or bladder to deal with. It has been suggested that there may also be felt a rush of fluid when quick and firm pressure is made. This, however, seems to the author a somewhat misleading suggestion, as many large hernial sacs contain fluid in appreciable quantities when no bladder protrusion is present. Furthermore, it is not in hernix of enomous size that we are liable to mistake, but rather in small or medium-sized herniæ of the direct type with no suspicious symptoms.

Dr. S. C. Plummer ("Inguinal Hernia of the Bladder," Jour. A. M. A., July 22, 1905) suggests that a test be made of having the patient retain his urine for a long time to see if it influences the tumor. The passing of a sound has, in some instances, verified the suspicion of bladder hernia, but in others it has failer. At the time of operation this has proved of great service to me, and has in several cases enabled me to decide that I was clealing with the blaclder wall.

Indications at Operation.-On opening into the canal a sac found protruding at the inner side of the cord, close to the 
pubic bone, should cause us to proceed with caution. This is especially true where it seems deeply inberlder in fat from which it is hard to separate it, and where it has a broad base.

FIG. 160 .

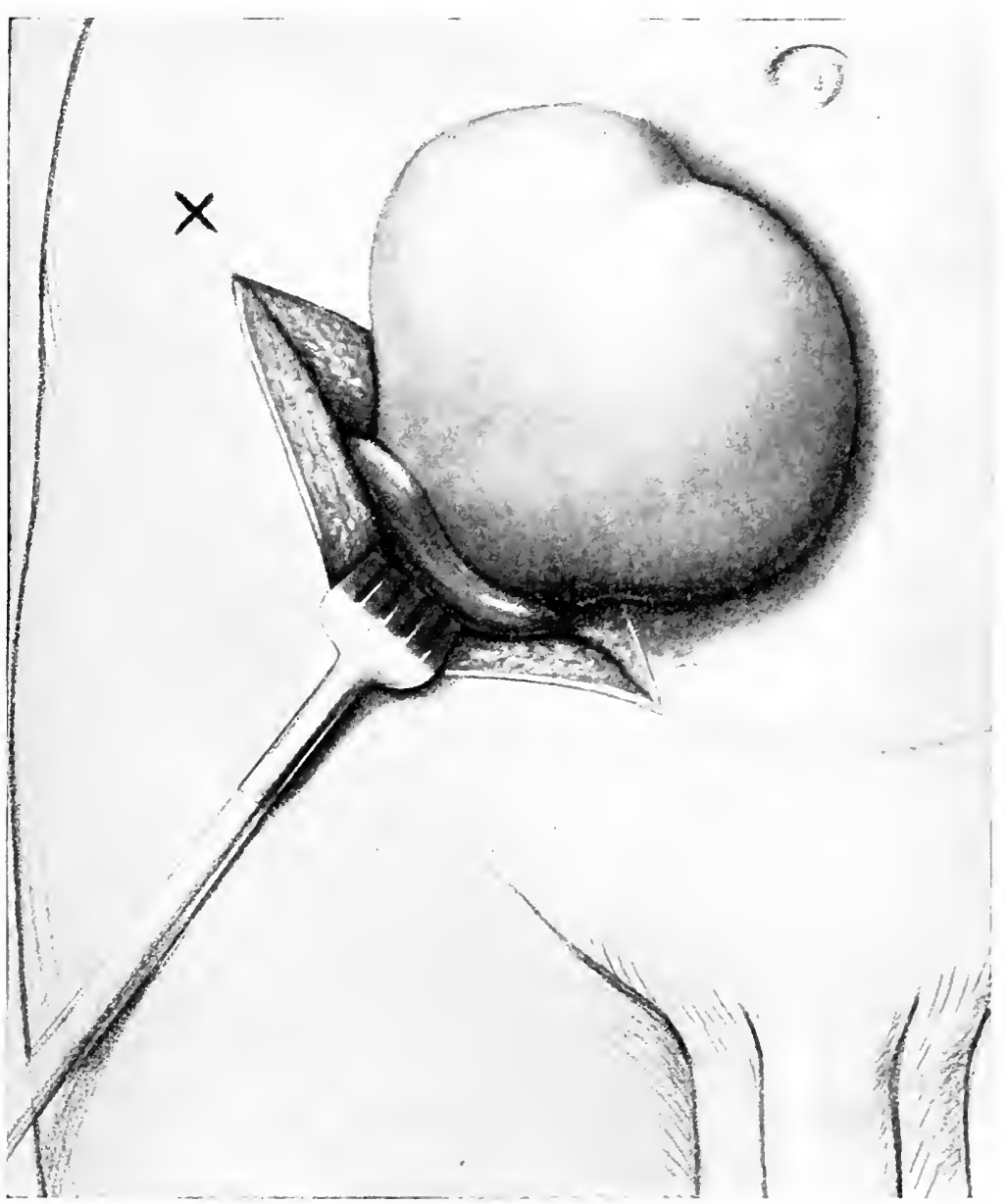

Showing a protrusion, the upper half of which was peritoneal sac containing intestine, the lower half, bladder. The dividing line has been intentionally exaggerated. In the patient it did not show so clearly.

One would think that the bladder wall could be recognized by its muscular covering, but this, in my experience, cannot 
be relied upon. This difficulty is well illustrated by the case reported by Dr. Rudolph IVinsboro ("Direct Inguinal Hernia," Injury to Bladder, Virginia Medical Semi-Monthly, Feb. IO, I904). He says: "To the inner side of the sac was a mass separated with great difficulty . . about the cord was a translucent area which evidently contained fluid." Thinking it an encysted hydrocele, he incised it and at once recognized urine by its odor. McLachlan states (Applicd Anatomy, rol. ii, p. 469) that "in sacculated bladder the walls of the sacculi contain no muscular fibres. The mucus membrane is forced through the bundles of muscular fibres, forming sacculi."

When, on pulling up an inguinal sac, there appears in its neck a mass of fat covered by peritoneum, it is highly important that it be neither ligated with the sac nor punctured with a needle, as it is in all probability bladder. This is most likely to occur in direct hernia, but $I$ have also seen it in the oblique variety. When two sacs are present in the canal it is safe to conclude that one is bladcler.

It has been stated that bladder wall is difficult to separate from the surrounding fat, and this, it is believed, is true. In my own case, when I committed the error of opening into the bladder, it was very readily lifted from the mass of fat which surrounded it and there was no true hernial sac. In the case shown in fig. I60, where the upper half of the tumor was hernial sac and its lower half bladder, the latter came out of the canal as easily as the former. The first appearance of the bladder part of the tumor was of loose fat in the canal. WVen this fat was lifted out, the bulging above, which proved to be the true sac, was brought to view. From the opening made in the latter it was easily demonstrated that the lower part was bladder. The sac was closed by purse-string suture, the bladder protrusion was inverted into itself and the muscular wall carefully closed. In the case shown in fig. If I the liability to mistake is in supposing that the lower protrusion is of an ordinary direct hernia. It is entirely possible to have oblique and direct 
hernia on the same side, and their true character can be fuickly ascertained by opening the upper sac and exploring the lower one through this opening.

Fig. 36 I.

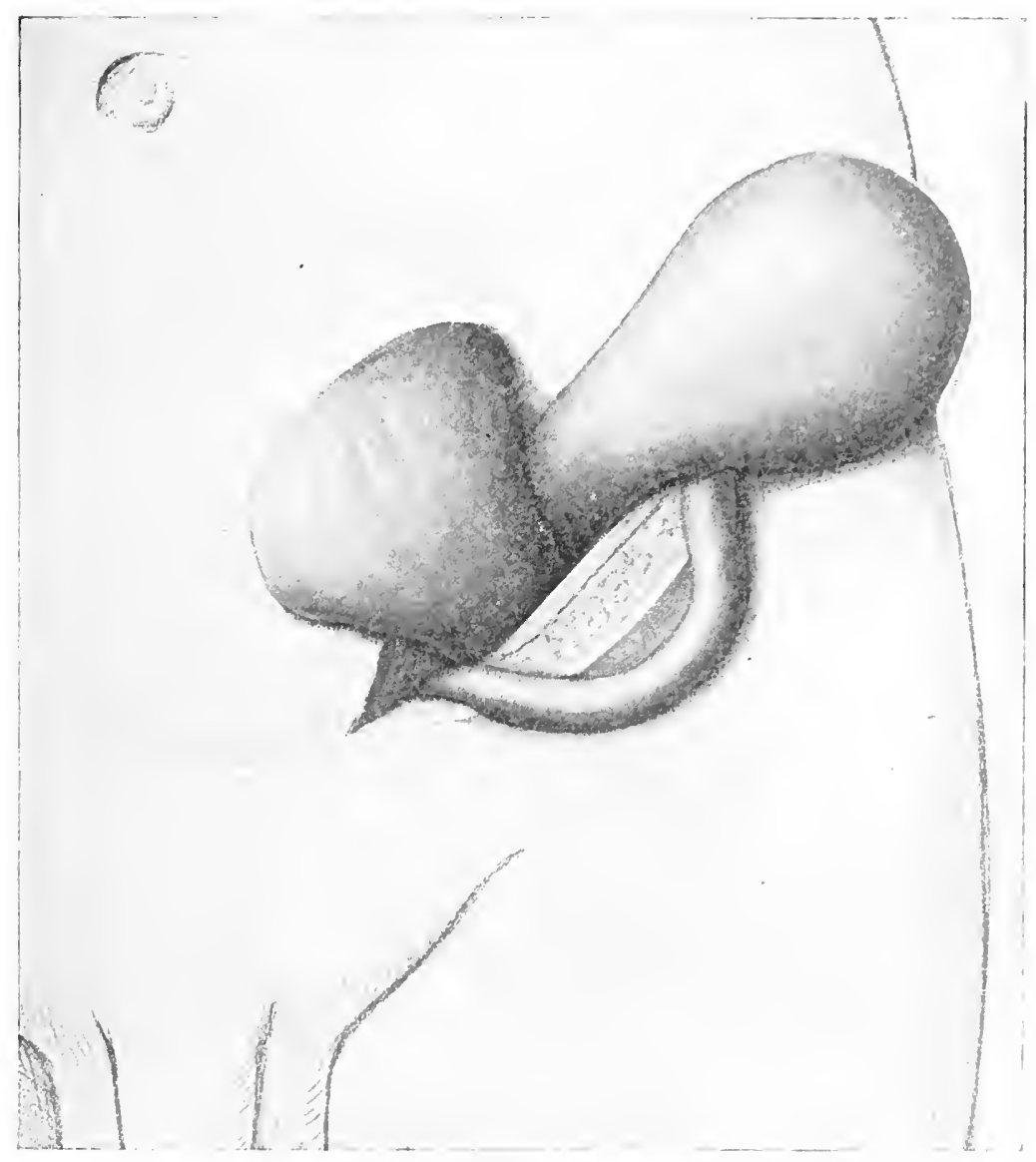

Hernial sae above, and bladder protrusion (extraperitoneal) below, in man 60 years of age.

In bladder as well as in sigmoid and cxeal hernia many. uncertainties in rliagnosis, and a great many operative difficulties, are avoided by opening into the sac or peritoneal cavity 
well up toward the internal ring. The bladder, the cæcum and the sigmoid are thus aroided, as well as the confusion that sometimes comes to an inexperienced operator by opening into an unobliterated tunica raginalis. It must be borne in mind that in double hernia the bladder may protrude on both sides. Gibson reports such a case and I have had one recently in a young man under thirty. When the bladder has been recognized before opening. it has been gently separated from its abdominal adhesions and pushed back into place, and the muscular"wall very accurately closed over it. In one instance I turned the sacculated part into the bladder by inversion, getting it entirely away from the canal.

Repair of Bladder Wounds.-An accident that has happened so many times and to so many different operators will certainly happen again and it is advisable, therefore, that those who contemplate doing the operation for the cure of hernia should have definite ideas as to what they will do when confronted by a bladder acciclentally opened.

On certain points in the repair of bladder wounds there are no differences of opinion, but on others, operators of experience differ widely. The merest tyro in medicine understands-for it is one of the earliest teachings in surgery-that in closing a bladder wound its walls must not be stitched through and through. Able operators also agree that such wounds are best closed by layers of sutures, some advising two, others three orerlying rows. Some advise that the mucous membrane lining the blaclder shall be first closed by small, plain catgut. and then the muscular and serous coats brought together above this by two more rows of silk or catgut; others prefer to put no sutures in the mucous membrane, relying entirely upon the muscular closure to prevent leakage. It seems rational that the use of small-size plain catgut to close accurately the mucosa ackls materially to the protection against leakage, anrl. owing to its early absorption, subjects the patient to little risk of having concretions form upon it within the bladrler. It is certain that silk should never be used for this layer. 
The tendency of late writers upon the subject is in favor of catgut throughout the operation. Curtis says on this point: "Whether silk or fine catgut is employed seems to be a matter of indifference, but the sutures should not penetrate the mucous membrane; they should be placed very close together, ten on twelve to the inch, and there should be at least two layers, and by preference three." Dr. Orville Horwitz (Annals of Surgcry, December, I905) expresses the following positive opinion on this subject: "It is generally conceded that the wound is best closed with fine silk, as the catgut sutures cannot be depended upon to endure for a sufficient length of time. Several cases have been reported in which this latter material was employed where a leakage occurred, owing to a too rapid absorption of the suture, death resulting from peritonitis." Horwitz recommends two layers of sutures, first, interrupted Lembert, second, mattress.

Personally I feel that in silk there is greater safety, and still, in view of the fact that in my own case a part of the silk was afterwards extruded into the bladder, became encrusted and had to be removed, I should hesitate to use it in an exactly similar case. If the wound is extra-peritoneal and there has been no opening into the peritoneal cavity, then one can take greater risks of leakage with little increase of danger.

The questions as to leaving provision for drainage in the wound and a permanent catheter in the bladder must be regulated by the judgment of the operator. If the operator has conficlence in the security of his closure it is far better to proceed with the operation for the cure of the hernia, as otherwise it will necessitate subjecting his patient to a second operation. The use of a catheter retained in the bladder four or five days would certainly seem indicated, but recent writers rather faror reliance upon either catheterization or voluntary urination every two hours. Again I say, if there is a possibility of leakage into the abdominal cavity the catheter should be retained for constant drainage: but if the danger is only of an extra-peritoneal vesical fistula I might rely upon frequent catheterization. 


\section{CHAPTER XIV.}

\section{SURGICAL CURE OF INGUINAL HERNIA IN THE FEMALE.}

The operations described in the preceding pages are ideal when applied to the inguinal canal of the female, where we have no cord to deal with. In many operations the round ligament has not been seen and the canal has been obliterated without any regard to its whereabouts. In a few cases, however, it will be found as large as a moderate-sized spermatic cord and should be treated exactly the same; that is, it should be lifted out of its bed and the internal oblique muscle closed beneath it, after the sac is tied off and cut away.

In some cases of congenital hernia in the female, where the process of peritoneum corresponding with the tunica vaginalis in the male was unobliterated, and so intimately blended with the round ligament as to make separation impossible, the entire mass was ligated and cut away. In these cases a purse-string suture is put around the inside of the sac first and then the ligature passed around ligament and sac. The stump has then been firmly stitched to Poupart's ligament and the internal oblique near the internal ring. In former times the Alexancler operation for shortening the round ligaments resulted in a large percentage of hernia, owing to the fact that the work was all done at the external ring. Gynecologists of the present day usually open the entire canal and close it by the Bassini method.

Dr. Charles P. Noble of Philadelphia, who has had a large experience, says (Reprint, Lackaramma Co. Mcdical Socicty, March 28, ro05): "If the ligament is pulled out through the external ring the process of peritoneum may be pullerl down and a hernia invited." Traction upon the ligament will always bring a peritoneal pouch into the upper part of the 
canal, and if this is left unobliterated it forms a convenient pocket into which intestine or omentum is almost sure to drop, eventually forming a good sized hernia. When the canal is open it is easy to push back and strip away this peritoneum before anchoring the ligament and closing the canal. In this connection S. Goldner has made a valuable study ("Does the Injury to the Round Ligament in Herniotomy Cause Retrodeviation of the Uterus?"-Z Zntralblatt für Gynäkologie, August I, 1903. In 50 women who underwent Bassinis radical operation he examined 28 in from three to six years after the operation. In I 3 cases the operation was bilateral and in I 5 unilateral. These patients belonged to the working class and went back to their labors undisturbed and free from pain. Of especial interest was the condition of $\mathrm{I} 2$ in whom the round ligament, on account of adhesions, was cut through and the stump of the hernial sac ligated with the round ligament; in 6 cases this was done on one side and in 3 cases on both sides, and in none of them was there any change in the position of the uterus. This condition led him to conclude that the severing of the round ligaments in the inguinal canal caused no disadvantageous results, and that the fact that even in those cases in which both ligaments were severed no retroflexion occurred, justified the use of the radical operation of Bassini.

My own experience has been that in I, 300 hernia operations, 306 were in the female; of these there were ${ }_{5} 56$ inguinal hernix, 25 being double. Five had inguinal hernia on one side and femoral on the other: I had inguinal on one side and double femoral; 2 had inguinal and femoral hernia on the same side; r had double inguinal and umbilical; I had inguinal and umbilical. When more than one hernia existed all were operated upon at a single anesthesia. Incidentally other surgical work was frequently done upon the patient where needed, as curetting, cervical, and perineal operations, for hremorrhoids, or the removal of small tumors. In none of these cases was there any untoward result, nor has there been a single recurrence so far as known. One of these women had experienced 5 
previous failures to cure a small inguinal hernia, and $I$ removed 5 or 6 large silver wire sutures that had been left in the canal. This case was particularly instructive in the fact that apparently in none of the previous operations had any attempt been made to bring the internal oblique muscle down to Poupart's ligament.

FIG. I 62 .

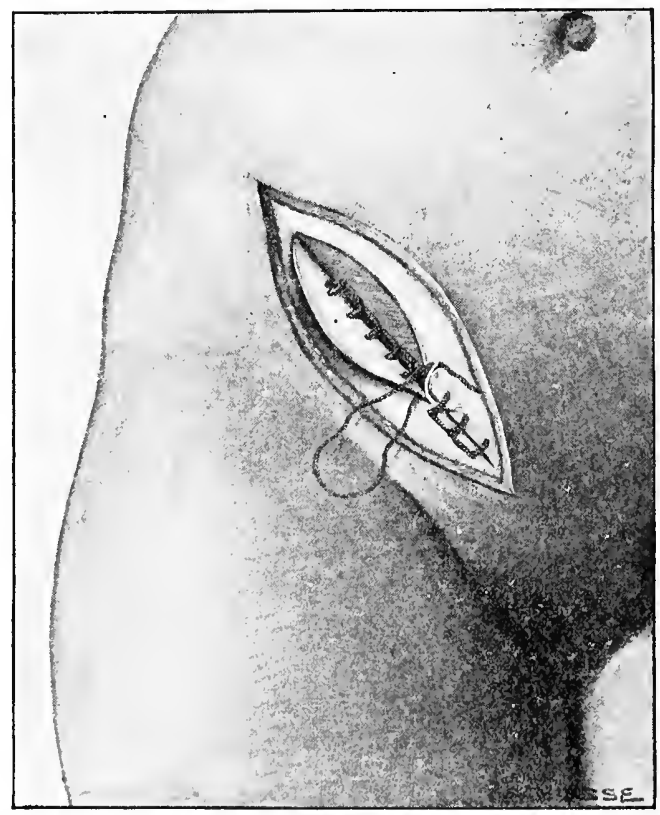

Continuous suture in muscular and aponeurotic layers as used in the female.

In the case of the double femoral hernia the woman was forty-three years of age, and the operation was for left strangulated femoral hernia caused by the incarceration of an epiploica appendix, which held a portion of the lumen of the intestine in the femoral ring. In the ingtuinal canal on the same side was found, firmly arlherent, the tube and ovary. The sac, includling ovary and tube, was tied off and cut away. The patient was out of bed on the tenth day and left the sanitarium 
on the fourteenth cured of her three hernixe and of much obscure abdominal pain from which she had suffered for many years.

The operation for cure is done in the female in every respect as in the male except that the canal is wholly obliterated. I have found it very convenient and effective to close the canal in the female by a single suture for both the deep muscles and the aponeurosis of the external oblique (fig. I62). The suture begins at the internal ring, bringing the lower edge of the internal oblique and transversalis in contact with Poupart's ligament all of the way down to the pubic bone. The blunt needle used, is then made to perforate the aponeurosis near its insertion into the pubic bone to the inner side. and then through the external pillar of the ring externally. When this is tightened it practically obliterates the external ring. The remainder of the continuous suture can be rapidly placed, the second and last knot being tied just above the upper angle of the split aponeurosis. The time required to operate on the female is less than on the male and the results are even more satisfactory, yielding in my own experience Ioo per cent. permanent cures.

The question of operating for the cure of hernia upon women who are pregnant is one that will come to most operators. In three instances I have operated upon women who were in the early months of pregnancy. In every instance they were less than four months adranced and, as would be expected, no umpleasant symptoms followed. It is inadvisable to do surgical work upon a pregnant woman unless especially called for, as in these cases.

I have recently been called upon to decide between two physicians as to whether or not an abortion was justifiable upon a woman three and a half months advanced in pregnancy because of her having an uncontrollable femora! hernia. It was not an unreasonable question in view of the fact that the woman had once required an operation for the strangulation of this same hernia. My decision was, as it probably would be in 
every other similar case, that such an act was entirely unjustifiable in view of the fact that women, even though suffering from extremely bad herniæ, seldom have any trouble with them during pregnancy or confinement. This is more especially true of inguinal or femoral hernia for the reason that as soon as the uterus begins to occupy the pelvis and lower abdomen, the intestines and omentum are lifted up and away from the hernial openings. It not uncommonly happens that in the last months of pregnancy a large hernia will disappear and not protrude even if no truss is worn. Women frequently are deceived by this into the belief that they are cured and make the mistake of getting up after confinement without applying a truss. 


\section{CHAPTER XV.}

\section{FEMORAL HERNIA.}

Eight per cent. of all hernize are of the femoral type, and it occurs more frequently in the female than in the male, as shown by the fact that of all women suffering from hernia, 38 per cent. have this variety. According to Nacready (Treatise on Ruptures) women during the child-bearing period have inguinal and femoral about equally, but after fifty years of age femoral hernia is slightly in excess. In men between twenty-one and sixty-five years of age, 3.9 per cent. have femoral hernia, but among bakers, as a separate class, it exists to the extent of 8.7 per cent. It is the most dangerous of all hernix, not only on account of its greater liability to strangulation, but owing to the fact that when strangulation does occur the destructive process is much more rapid than in any other form. This latter fact is undoubtedly due, first, to the inelastic structures which surround the femoral canal, and second, to the knife-like edges against which the intestine or other protruding abdominal viscera are violently pressed.

This variety of hernia is seldom encountered before early youth, and most frequently occurs in later life. The youngest case met with in the author's experience was in a boy four years of age who had double femoral hernia of nearly one year's duration. Another boy of eight years had right femoral, and the youngest girl, also eight years old, had right side hernia. That it seldom or never occurs in infancy is due to the anatomical fact that in early childhood the calibre of the femoral opening is very small, enlarging as adult life approaches.

This form of hernia may be single or double, and may be associated with inguinal hernia on either the opposite side or the same side. The author has seen one case of double inguinal 
and double femoral hernia in the same person. It may be reducible, irreducible, or strangulated. It is seldom irreducible without producing symptoms of strangulation, and in this it differs markedly from either inguinal or umbilical hernia, where we may find large masses of omentum or even intestine which do not produce any great amount of discomfort.

FIG. 163 .

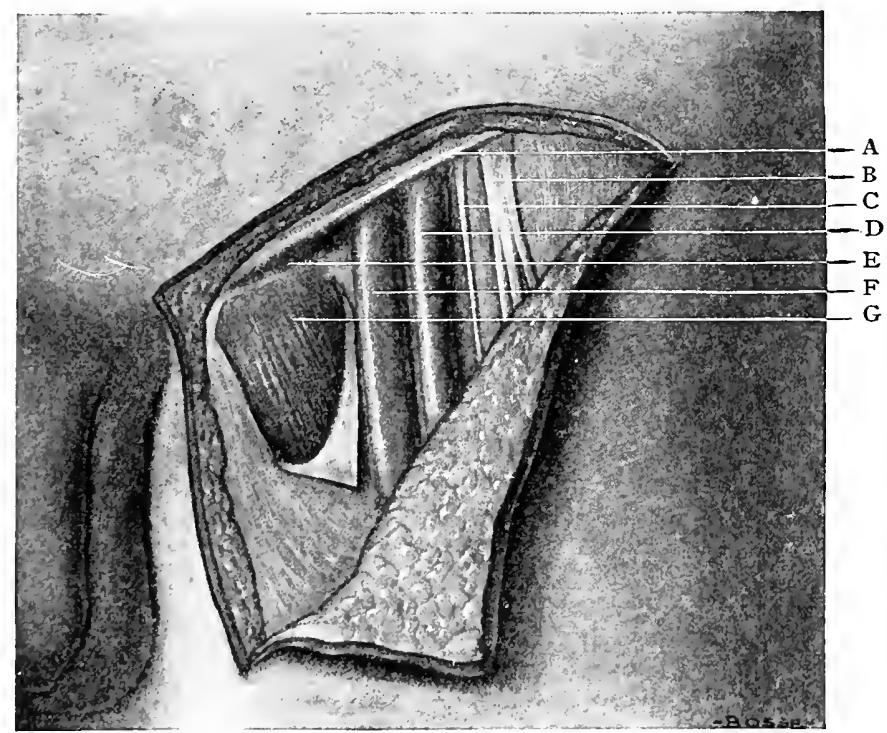

Femoral region. A, Poupart's ligament. B, Anterior crural nerve. C, Crural branch of genito-crural nerve. D, Femoral artery. E, Gimbernat's ligament. F, Femoral vein. $G$, Fascia lata cut away to show head of pectineus muscle.

ANATOMY.

Poupart's ligament is a strong, fibrous band extending from the anterior-superior spine of the crest of the ilium, to the spine of the pubes separating the abdomen from the thigh (fig. I 63 ). Peneath this structure (between it and the pubic bone) are the parts most concerned in the formation of femoral hernia. The transierse section of these parts is shown in the 
accompanying illustration, and a brief study of this will give a clearer idea of the relative position of the parts than can be given by mere description (fig. I64).

Gimbernat's Ligament (fig. I63) is attached to the ileopectineal line from the spine of the pubes outwards, and fills the triangle between this line and Poupart's ligament, its base being directed toward the femoral vein. Its total length is

FIG. I64.

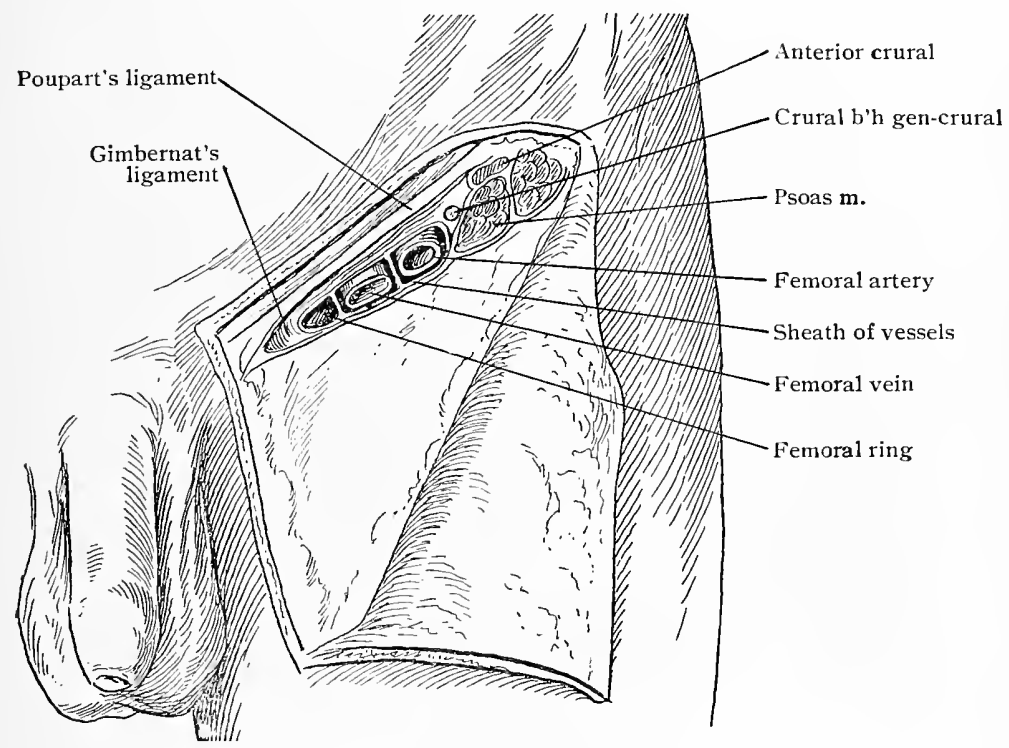

Transverse section showing relation of vessels and nerves to femoral canal.

from two-thirds to three-fourths of an inch. Immediately back of it is the conjoined tendon.

The Femoral Ring lies to the outside of the base of Gimbernat's ligament between that and the femoral vein, and is bounded above by Poupart's ligament and below by the ramus of the pubes. Hesselbach has given its measurement as Io $\mathrm{mm}$. in the female and $5 \mathrm{~mm}$. in the male. The femoral ring is ordinarily filled with subperitoneal fat and sometimes a small gland. When standing it is nearly horizontal. 
Diagrai Showing Formation and Covering of Femoral Hernia; Protusion of Extra Peritoneal fat and Lipona.

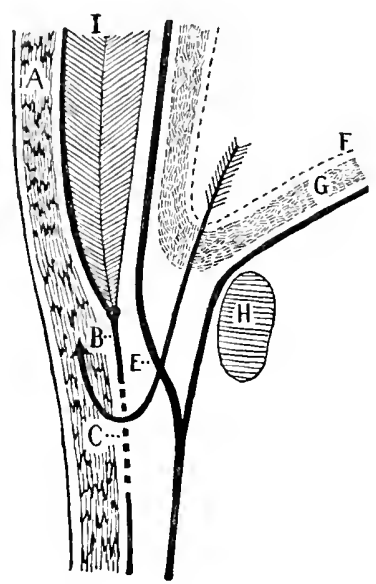

Diagram showing parts involved in femoral hernia. The arrow shows line of descent. A, Subcutaneous fat. I, Muscular wall. B, Fascia lata. C, Cribriform fascia. E, Femoral sheath (transversalis fascia near its junction with iliac fascia). F, Peritoneum. G, Extra-peritoneal fat. $\mathrm{H}$, Pubic bone.

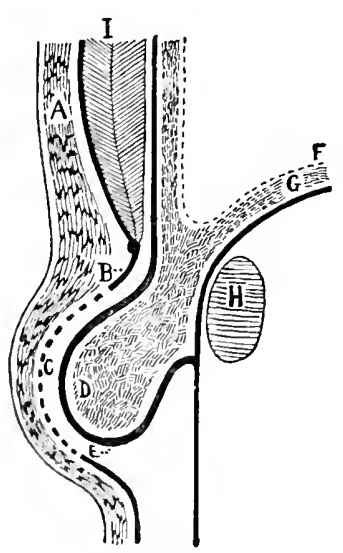

Fatty hernia through femoral canal. D, Extra-peritoneal fat.

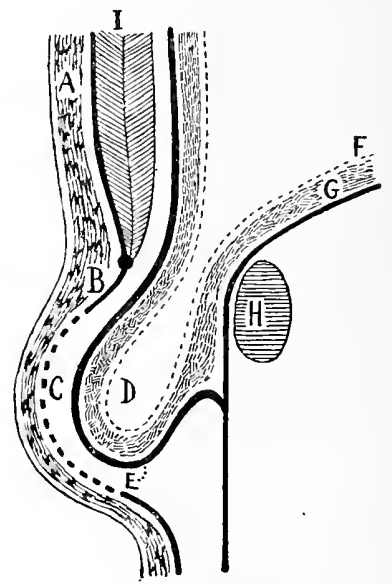

Femoral hernia. A, Subcutaneous fat. B, Fascia lata. C, Cribriform fascia. D, Hernial sac. E, Femoral sheath (transversalis fascia). F, Peritoneum. G, Extraperitoneal fat. $\mathrm{H}$, Pubic bone.

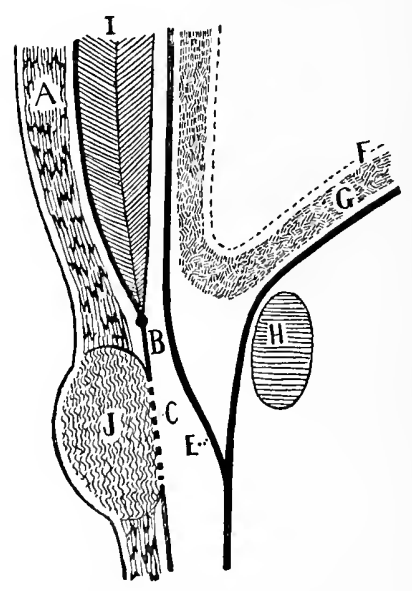

J, Fatty tumor. Not fixed below, but freely movable in subcutaneons tissue. 
Femoral Sheath.-The transversalis fascia, covering the anterior wall of the abdominal cavity, passes down under Poupart's ligament and forms the anterior layer of the femoral sheath, while the iliac fascia forms its posterior layer and is

FIG. 165 .

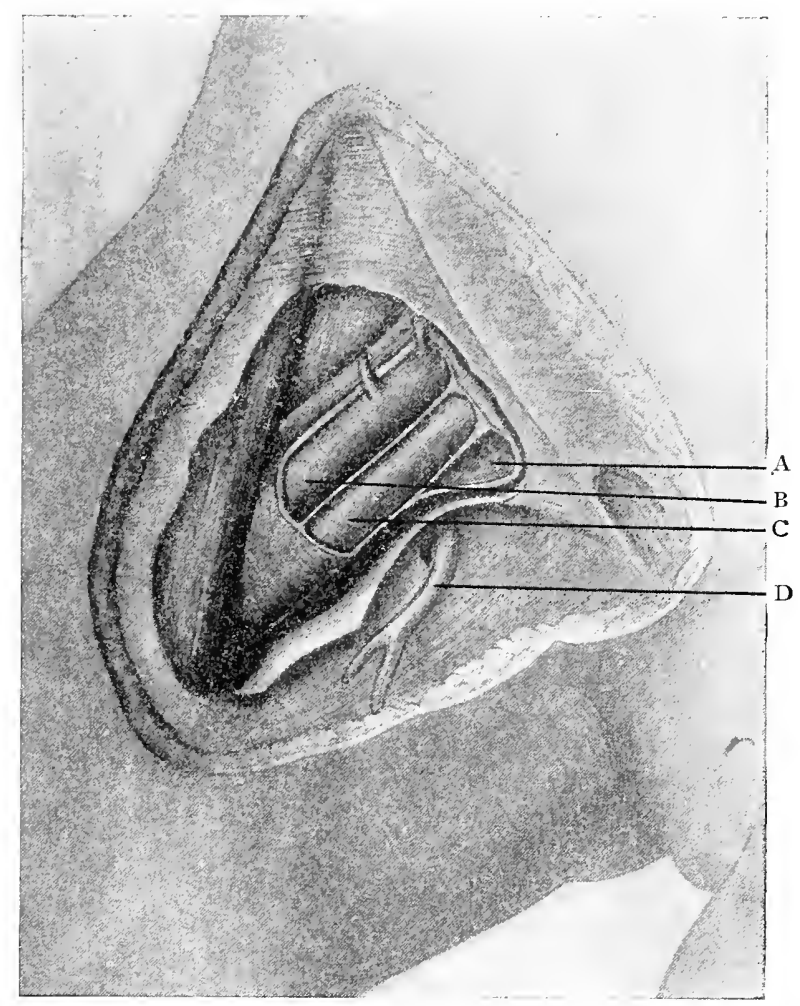

(Redrawn from Gray.) A, Showing small pocket by side of femoral vessels, where hernia usually protrudes, breaking down Gimbernat's ligament and forming an elongated, triangular opening. The top of this triangle is Poupart's ligament; the floor, the tissues covering the ramus of the pubes; its base, the femoral vessels; and its point, the spine of the pubes. $B$, Femoral artery. C, Femoral vein. D, Saphenous vein.

continuous with that on the pectineus muscle. The meeting and blending of these layers of fascia form a funnel-shaped space termed the femoral canal, which is about half an inch in length. Hernia protruding through this opening carries before 
it this fascia and subperitoneal fat. The fascia is sometimes so dense as to lead the operator to suppose that he is dealing with the hernial sac. At other times the tissues covering a femoral sac are so thin that it is only by extreme care that they can be incised without opening into the sac.

The most common point of protrusion of femoral hernia is within this sheath between the femoral vessels and the outer

FIG. I 66.

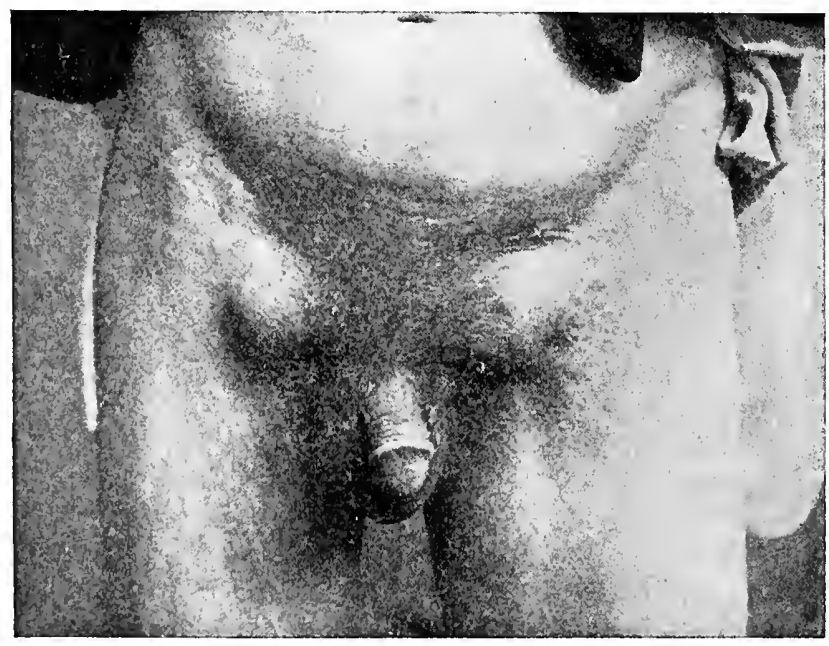

Double femoral hernia, in a man of 50 years. Typical illustration of fairly large femoral hernia. On the left the tumor has turned up partially over the external ring, but its centre is below the crease formed by the junction of the thigh and abdomen.

edge of Gimbernat's ligament. Below the sheath is the pectineus, and at the outer side the psoas muscle. To the outer side of the femoral sheath close to Poupart's ligament is the crural branch of the genito-crural nerve.

Femoral protrusions leave the abdominal cavity by escaping beneath Poupart's ligament, and passing to the inner side of the femoral vessels (fig. I65). Gimbernat's ligament is crowded towards the median line. It is this thin tendon which ordinarily produces the knife-edge cutting of the bowel that is 
so destructive in strangulated hernia of this type. These protrusions escape from beneath the fascia lata at the saphenous opening, penetrating or carrying before them the cribriform fascia, and form a tumor on the anterior and inner aspect of the thigh three-quarters of an inch to the outer side of, and the same distance below, the spine of the pubes. The long diameter of the femoral opening, after hernia has distended it, is trans-

FIG. I67.

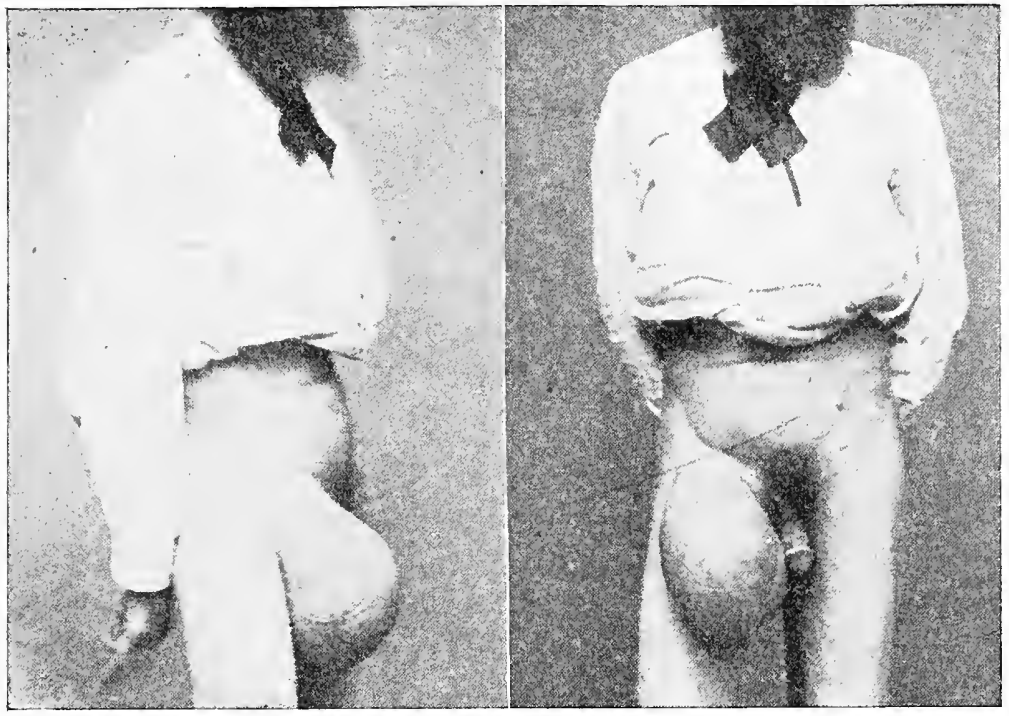

Side view.

Front view.

Reducible femoral hernia of enormons size. This hernia was reducible and could be retained by a truss.

verse, or parallel with the ramus of the pubes, and is triangular in shape, the term "Ring" being a misnomer.

Considering the parts from within, we have first, the peritoneum; second, the extra-peritoneal fat; third, the transversalis fascia, which forms the sheath of the femoral vessels; fourth, subcutaneous tissue; and fifth, skin. The vessels of the region are important and should be clearly fixed in mind, but their proximity need cause no timidity on the part of the 
careful operator. The saphenous vein passes over the falciform edge of the fascia lata to join the femoral vein, and here also are given off from the femoral artery the external pubic, the external epigastric and the circumflex iliac arteries.

Formation.-Femoral hernia usually develops by a slow process, frequently attended by some pain or a dull aching in that region and extending down the leg. This is often described as a burning sensation. In many instances, as in inguinal hernia, a swelling in the femoral space is the first inclication of anything wrong observed by the patient.

There is little doubt that the subperitoneal fat frequently acts as the entering wedge and is the forerunner of the hernia. Under some violent strain it is forced into the femoral sheath, and, slipping under Poupart's ligament, presents a small tumor at the saphenous opening. Shortly following this the peritoneum, dragged down by the attached fat, and pressed upon from within by the abdominal contents, is sure to be found protruding, and the hernia well established. Ordinarily femoral hernia does not attain a very great size, but presents a small, round tumor varying in size from a hickorynut to that of a good-sized hen's egg (fig. I66). As a rule femoral hernia remains small; as in other forms of hernia, however, there may be great variation in its size and shape. The enormous proportions that they may assume when neglected is well shown in the cases here illustrated.

Fig. I67 shows the case of a man 50 years of age, in good general health, who was being supported by a Hebrew charitable institution for complete disability. The hernia was consirlerably larger than the patient's head. Notwithstanding total lack of treatment for many years, the hernia could be completely reduced to the abdominal cavity and comfortably retained lyy truss pressure. The man was doing perfectly well uncler truss treatment until it occurred to him that he was losing his ronly possible claim upon the institution, after which nothing cruld be done with him, nor would he consent to operative cure, which cötld most certainly have been afforded 
him. The photograph shown in fig. $x 68$ is of a woman fifty-five years of age, who had an enormous irreducible femoral hernia. The full size of this hernia is not shown in the photograph, as it had not only formed down on the thigh, but had dissected up the skin from the fascia around toward the back part of the leg.

FIG. 168 .

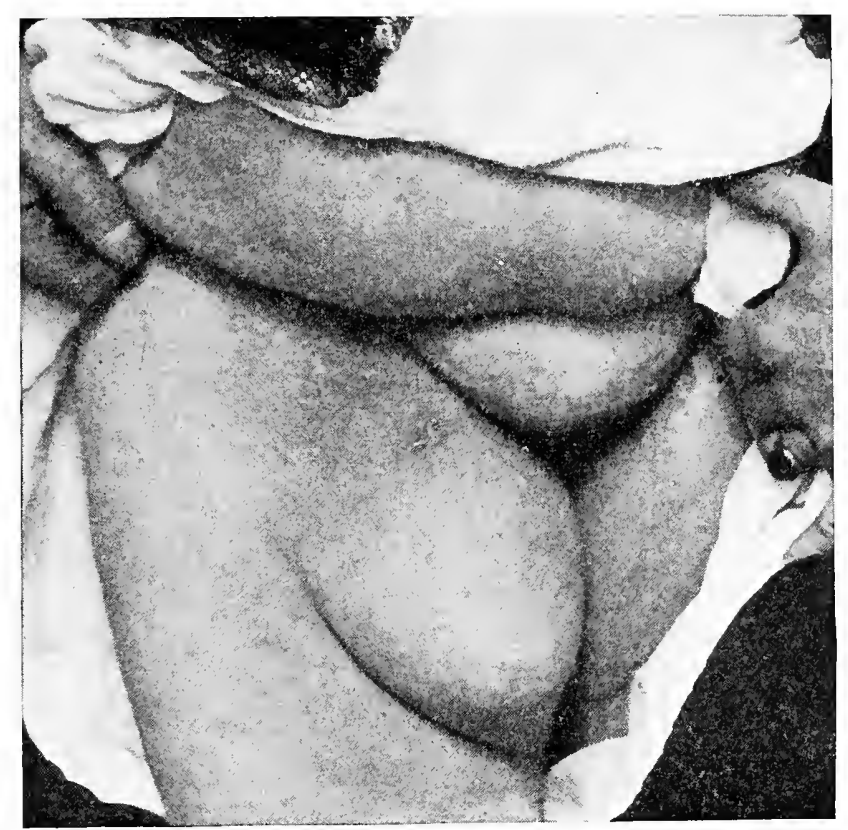

Woman 55 years of age. Irreducible femoral hernia. Extent of tumor not shown here, as it extended well around to back of thigh. Contents, intestine with a large mass of adherent omentum. Cured by operation.

Herniæ of this size are pretty sure to be made up largely of omentum, as in this case, but the author has seen one case fully as large where the protruding content was entirely intestine and irreducible. The case referred to is shown in fig. 169. This photograph fails to show the full proportions of the tumor, or another femoral hernia that existed on the opposite side. This tumor also extended around to the inner side of 
the thigh. Upon operating, the right-side hernia was found to consist of intestine held upon a mesentery so thick and inflexible that reduction appeared, at first, impossible. No adhesions existed, and no omentum was found protruding. Reduction was finally accomplished and the greatly distended femoral opening closed. The opposite side was also operated upon. The patient, more fortunate than my one other instance of

FIG. I 69 .

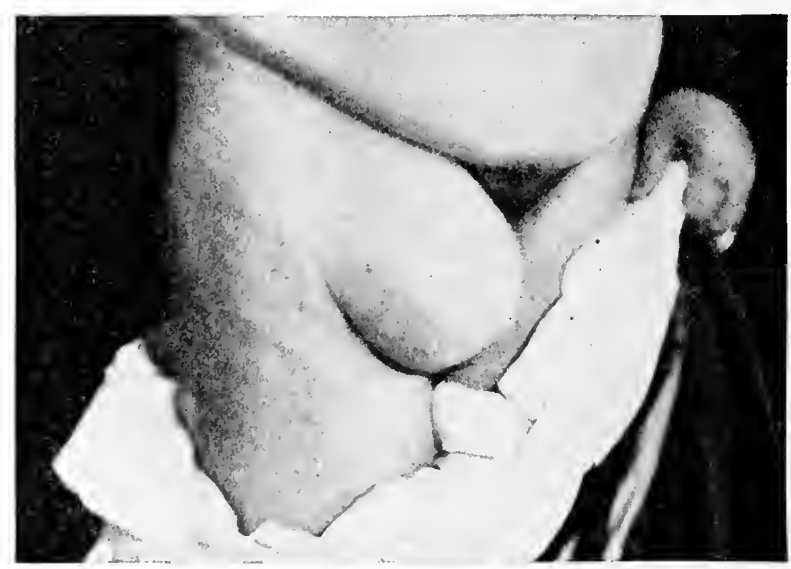

Double femoral hernia in woman aged 48 years. Right side only shown and not in its full size. Irreducible. Contents, intestine held down by hypertrophied mesentery. No adhesions. No omentum. Both herniæ cured by operation on the same day.

mesentery of this peculiar type, made a prompt recovery and remains sound four years later. No truss has been worn.

The dangers of acute strangulaton are not quite as great in hernix of such large size as in the smaller ones, inasmuch as they protrude through such enormously distended rings; they are (quite likely, however, to eventually reach a fatal termination where the bowel forms a large part of the protruling contents and is irreducible. This comes, first, with obstinate constipation, gradually increasing to intestinal obstruction and death. It is due to the fact that the bowel, in its abnormal position, under pressure at the abdominal orifice, gradually 
loses its peristaltic action and when obstruction occurs, an operation returns bowel which, while it may be otherwise in good condition, is paralyzed beyond recovery.

The sacs of femoral, like those of inguinal hernia, may also be modified in shape by bands of connective tissue forming in the interior. These bands become fibrous in character

FIG. I 70.

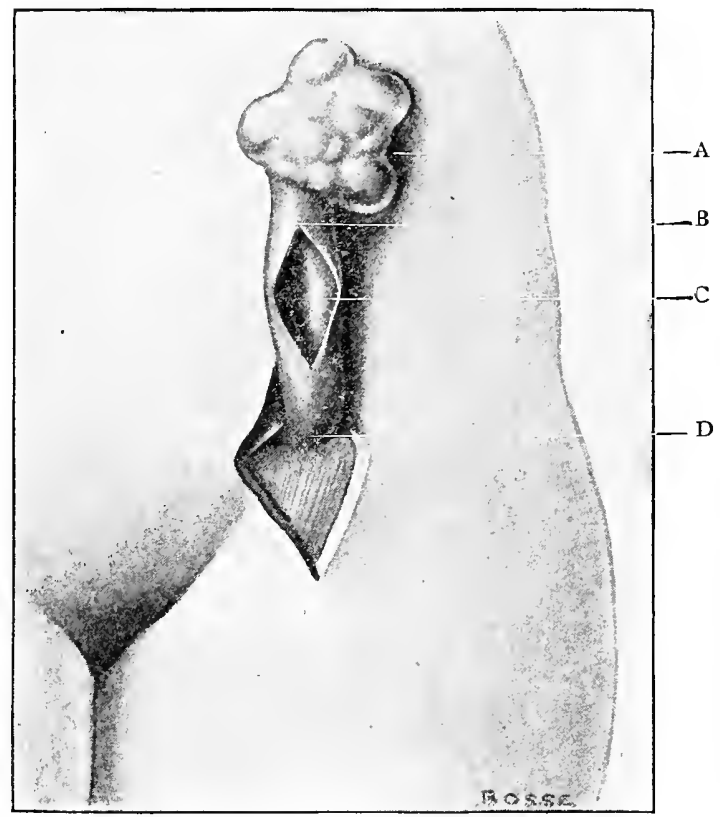

Cyst of old hernial sac. A, Cyst. B, Point of closure. C, Interior of sac. $D$, Femoral ring.

and add materially to the risk of strangulation. The neck of the femoral sac, where it passes under Poupart's ligament, is usually very small and this in itself becomes a source of danger to the protruding parts. The sac becomes tough and thickened at this point, and in some rare instances where a truss has been constantly worn, is closed off entirely. This closure is temporary in character and does not result in a cure. A sac 
that has been closed off in this manner is quite likely to take on a condition of hydrocele, and, as it occupies the exact site of the former hernia, is very likely to be mistaken for irreducible femoral hernia. Such a case is illustrated in a drawing (fig. I 70$)$. made from a sac removed from a patient supposed to have irreducible hernia. The hernia proper was in the upper part of the sac and easily reduced.

\section{FIG. I7I.}

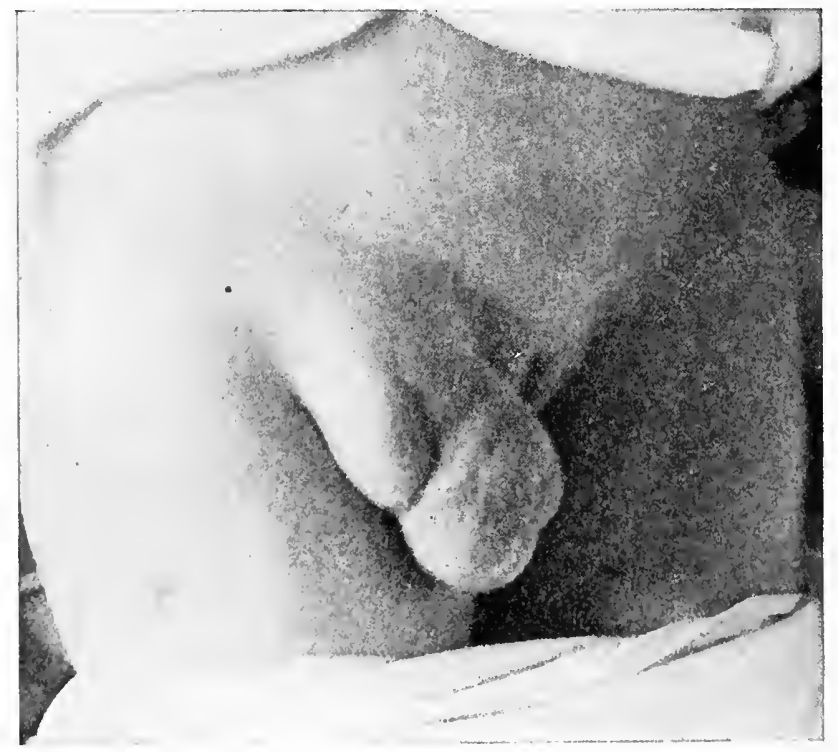

Irreducible femoral hernia of peculiar shape in a woman of $3^{8}$ years, due to resisting bands of fascia. Adherent intestine in sac. Cured by operation.

The shape of the sac, and even the exterior surface of the hernia, may be morlified by resisting vessels or fascia as it descends through the short canal or as it lifts up the cribriform fascia at the saphenous opening. A case with a lobulated sac from the latter cause is shown in fig. I7I. The patient was a woman thirty-eight years old and had the hernia for eight or ten years. Trusses trierl in this case could not be tolerated 
because, as was found on the operating table, she had bowel adherent in the sac. Both bowel and omentum were found, the latter being ligated and removed. It is due to these resisting bands that occasionally a femoral sac, instead of forming below the opening through which it protrudes, turns upwards over Poupart's ligament and simulates inguinal hernia.

Macready (Treatise on Ruptures, p. 6o) gives an illustration of a case where three femoral sacs were found upon the same side in one patient, and it is mentioned here to show the possibility of a sac protruding at unusual places. In Macready's case one protrusion was through Gimbernat's ligament close to the spine of the pubes, one at its usual place, and the third just to the outer side of the femoral vessels. The condition was not recognized during life.

The contents of a femoral sac may be almost any of the movable organs of the pelvic or abdominal cavity. Intestine is most commonly found, omentum next in frequency, but there are many recorded cases of tubes and ovaries, the appendix and the bladder, being found.

The subject of appendicular femoral hernia has been carefully studied by Dr. Alfred C. Wood of Philadelphia (Amnals of Surgery, May, 1906, p. 668). He has collected roo cases, from that of Garangeot, I 73 r, to his own 2 cases. He has collected only those cases where the appendix occupied exclusively the sac of a femoral hernia. Those where other portions of the intestine were present have been excluded. "Of the roo. cases of appendicular femoral hernia, $S_{I}$ were women, 7 men, I2 sex not given. Youngest 19 years, oldest 87 . More than half were over 50 years, and over 85 per cent. were past 40. Diagnosis previous to operation quite unusual."

\section{DIAGNOSIS OF FEMORAL HERNIA.}

In many instances it is more difficult to make a diagnosis of femoral hernia than of the inguinal variety, and not infrequently distinction between the two is attended by great uncertainty. The author has seen 2 patients who had been 
operated upon for inguinal hernia where the femoral type existed, and, of course, as soon as the patients were on their feet, the hernix protruded again. These occurred in the practice of men well known in connection with abdominal surgery. In one case the operator told of failing to find any sac, and later, when the femoral hernia appeared, it dawned upon him why he had failed. In both cases the patients refused to allow the original operator to operate again and were suspicious as to the true reason of failure.

Colicky abdominal pains are very likely to be present during the formation of femoral hernia if the intestine forms a portion of the protruding contents, but if it contains omentum alone, this may not cause the patient sufficient annoyance to attract attention. In a case recently seen of strangulated omentum in a left side femoral hernia, the patient complained of a dragging in the lower part of the abdomen on the right side. Even where the intestine is strangulated, the pain is far more likely to be abdominal than local.

If femoral hernia forms a small, round, typical reducible swelling in Scarpa's triangle, then the case is very clear, and the diagnosis easy; but in the descent it may come in contact with resisting tissues that turn it up over Poupart's ligament directly over the inguinal canal. In reducing such a tumor it must be followed in the line of least resistance, and then it will be traced to its true origin. If it is not reducible, by lifting it up and away from the abdominal wall, its neck may be surrounded and its point of exit located.

The anatomical points that must be constantly borne in mind in deciding between femoral and inguinal hernia are the anterior-superior spine of the crest of the ilium and the spine of the ptbes; an inaginary line drawn between the two with a slight downward curve representing Poupart's ligament. This line is roughly represented by the crease between the thigh and the abdomen, and is clearly shown in most people. The top of a femoral hernia just touches this crease, and an inguinal protrusion is above it. Furthermore, an inguinal hernia 
usually travels up the canal as reduced, while in femoral hernia reduction is directly back towards the thigh as it passes into the saphenous opening and slips under Poupart's ligament. In direct inguinal hernia the differential points may be more obscure, owing to its very close proximity to the femoral opening and to the fact that it reduces directly backwards. Here the spine of the pubes must be the guide, remembering that the femoral opening is three-quarters of an inch to the outer side and about the same distance below that point, and that the direct inguinal protrusion is almost immediately above the spine. Another point of difference is that usually the inguinal variety can be easily reduced with the patient standing, by making pressure with the hand, while in femoral hernia it is almost always impossible to reduce the tumor until the patient has been placed in the recumbent posture, and even then reduction takes place very slowly. It is well to look with extreme suspicion upon any tumor in the femoral space that is easily reduced while the patient is standing. In the male much valuable information may be obtained by invaginating the thin tissues of the scrotum upon the finger, following the cord up to the external ring and carefully examining the condition of the external ring as well as locating the relative position of the tumor. With the tip of the finger in the external ring it can usually be decided whether the protrusion is to its inside,direct hernia,-or to its outer side and a little lower,-femoral hernia.

Here the author would again caution the examiner against passing the finger up thic canal. Such method of examining the canal is reprehensible and may lead to hernia where none exists.

There is one small round tumor forming in the femoral space that is easily reducible, that has the typical shape of femoral hernia, and that is frequently mistaken by the examiner, viz., a varicose condition of some of the vessels of this vicinity. Varix of some of the larger vessels of this region may prove very perplexing to those of moderate experience (fig. 
$\left.I_{72}\right)$. The points of differential diagnosis are first, and most important, great ease of reduction in varix; second, the fluid feel of the contents and peculiar impulse upon coughing.

FIG. I 72 .

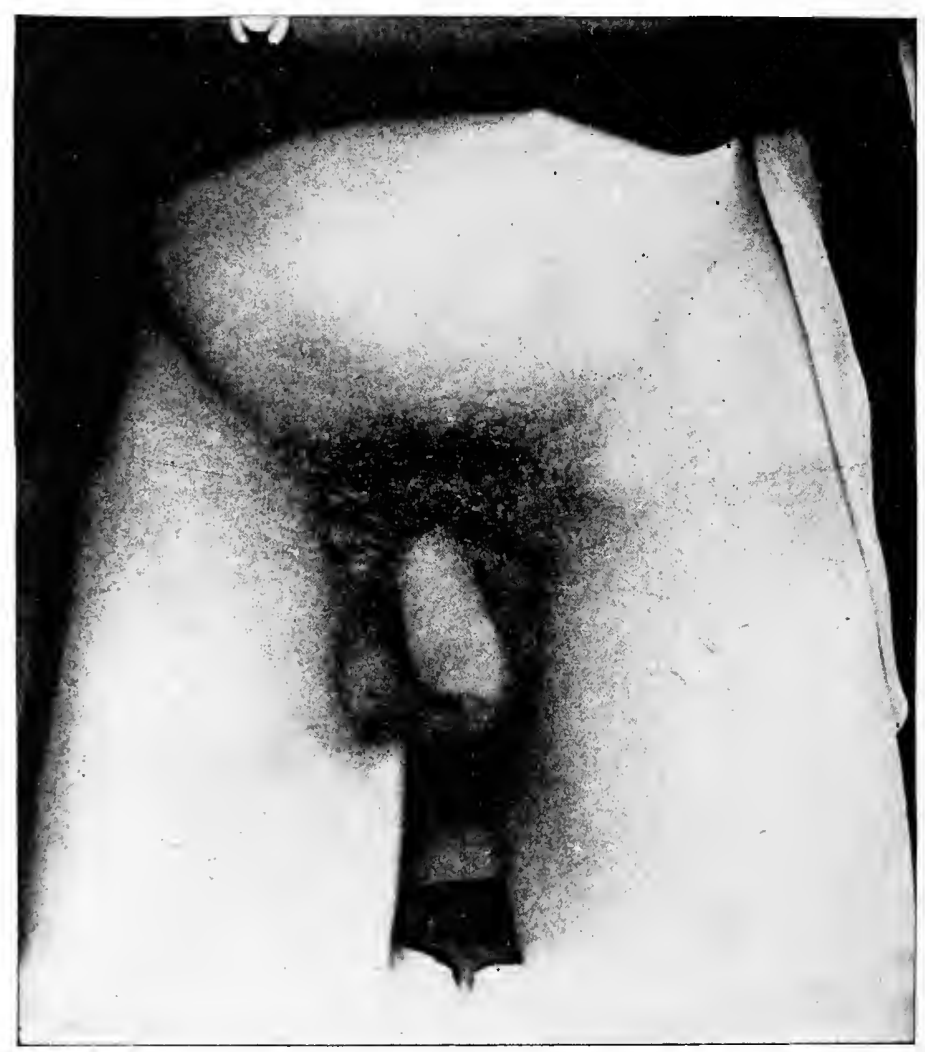

A left varicose saphena vein. (Eccles.)

Femoral hernia, as previously stated, is slow and rather difficult of reduction, and it is almost always a necessity that the paticnt should be recumbent before it can be accomplished. Varix, on the contrary, is soft and readily compressible with the patient in the standling position. By pressure over it with the hand it quickly disappears and as quickly returns when the 
pressure is removed. Furthermore, there is a strong impulse in the varix when the patient conghs which does not exist in femoral hernia. The impulse gives the sensation of "thrill" rather than of expansion. It is the impulse of fluid, and has that characteristic feel under the fingers. A single varix of this type may exist without other vessels of the region, or on

FIG. I 73 .

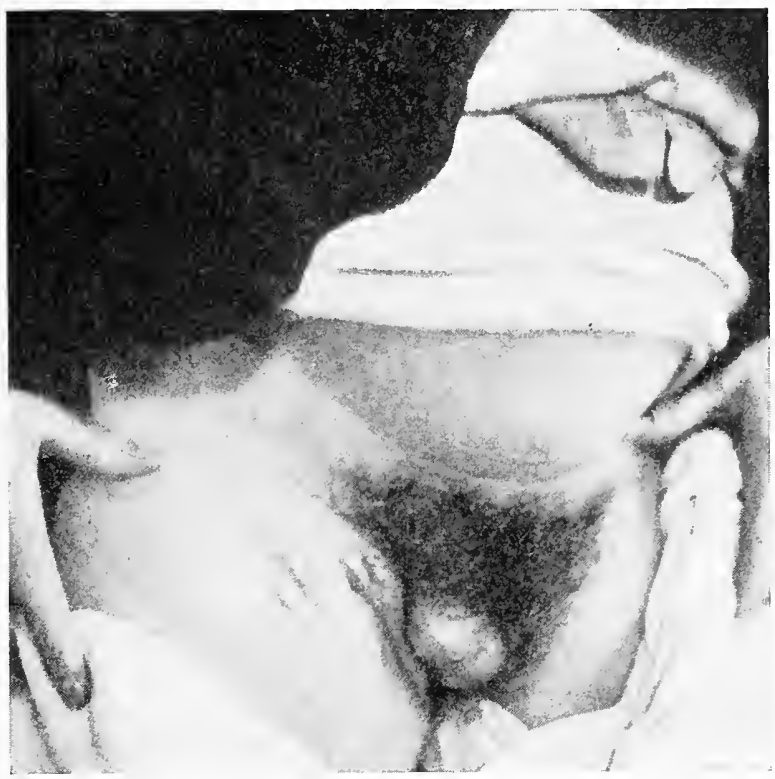

Femoral and labial varix in a woman of 35 years, 6 months pregnant.

the lower leg. being affected. Usually in suspected cases, however, an examination of the leg will reveal varicose bunches about the ankle, calf, or popliteal space.

During pregnancy some women are very liable to a condition of varix that is mainly limited to the labia and femoral region (see fig. I73). In these cases the suspicious appearance of the tumor and a consideration of the general condition usually lead to a correct diagnosis. Varix occurs most fre- 
quently in women of middle age, but I had in my clinic during the past year a young girl of eighteen years where the condition was marked and perplexing. The case was afterward taken into another hospital as one of femoral hernia. The patient's family physician, who was present at the operation, informed me that no hernia was found, but the varix was tied off. I had advised against this operation as not necessary and as attended by considerable danger. The following indicates the possible danger. Dr. William J. Taylor (Annals of Surgcry, July, 1905, p. 127) reports a case of a woman thirty years of age who had been wearing a truss for a supposed femoral hernia. Upon operation he found a varicose condition of the saphenous vein. He ligated the vein below the enlargement and then ligated it again three-quarters of an inch from the femoral vein. Seven days later she went into collapse and died the ninth day after operation. Post-mortem showed heart clot.

Haberern reports (Deutsche Medisinische Wochenschrift, Dec. 20, I906) cutting down on what he believed to be incarcerated femoral hernia and a gush of blood poured out. The saphenous vein was tied off together with the plexus of inflamed varicosities. Patient recovered. He believes that the difficulties of diagnosis between incarcerated femoral hernia and varicosites of this type to be considerable.

A violent inflammation of the superficial glands of the femoral region is seldom mistaken for hernia because of local pain, heat, and usually discoloration of the skin. In the case of glandular trouble it has a feeling of closeness to the surface that is seldom present in hernia. Incarcerated omentum in a femoral sac, from which it is most difficult to distinguish adenitis, is usually accompanied by at least some abdominal discomfort, even though actual pain is not present. The author recently operated upon a case where the diagnosis was very uncertain, as there harl been considerable local pain and there was fluctuation. The tumor had a history of two weeks' duration and gradual increase in size. Nine years previously he had oper- 
ated for femoral hernia upon the opposite side in the same patient and obtained a permanent cure. The present operation revealed a dark-colored femoral sac filled with a coffee-colored fluid, the result of strangulation of a small piece of omentum.

FIG. 174 .

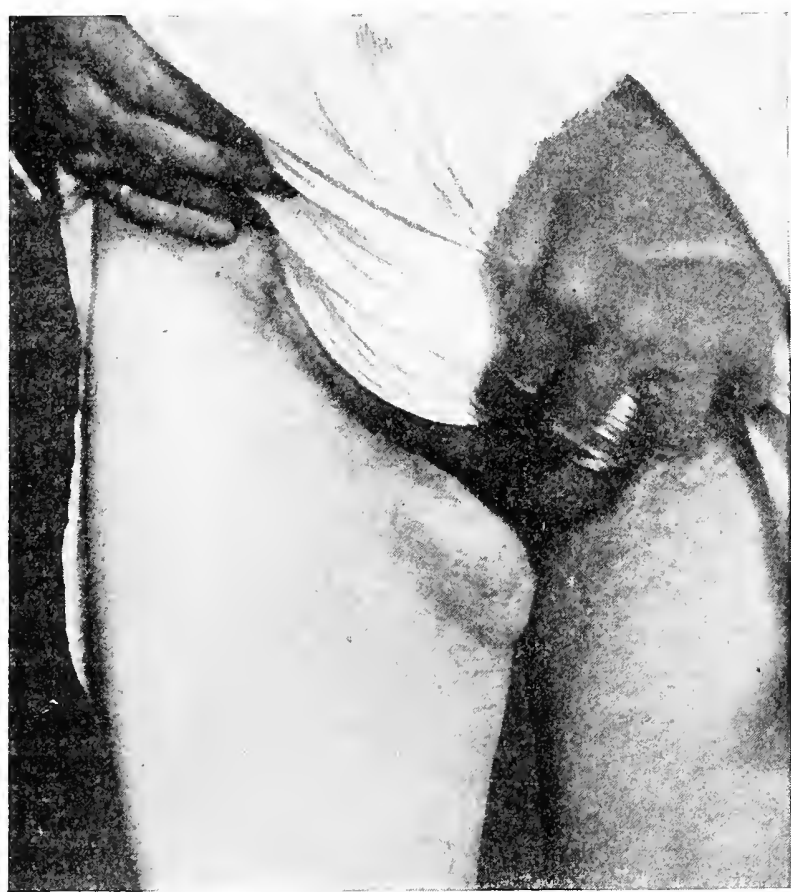

Lipoma simulating femoral hernia. In this case the lipoma is in the subcutaneous fat and does not protrude through the femoral ring.

The woman had suffered no abdominal symptoms, and had discomfort, rather than pain, locally.

Hydrocele of a sac, the neck of which has been obliterated by truss pressure, may present symptoms almost identical with those just narrated, but without the local discomfort. Its elasticity and the smoothness of the surface of the tumor are the usual guides. Between fluid tumors resulting from hydro- 
cele of the sac, or from strangulated omentum, it may be impossible to make an exact diagnosis previous to operation, as in the case just cited. The author has seen two cases of lympho-sarcoma that were at first examination quite perplexing, but fortunately these are of rare occurrence.

Psoas abscess is very rarely mistaken for femoral hernia, as it occurs most frequently in young persons suffering from Pott's disease of the spine, and is therefore easily recognized.

Subperitoneal fat, or lipoma (fig. I74), not only frequently precedes femoral hernia, but is easily mistaken for it. As its treatment should be the same as for hernia the obscurity surrounding the diagnosis is of no serious importance. If it can be reduced through the femoral opening it may be retained by a truss, and if irreducible it should be removed by operation.

Irreducibility.-Femoral hernia becomes irreducible more than ten times as often as inguinal, and the contents are most frequently omentum. Owing to the smallness of the neck of the sac and the inelasticity of its surroundings, it is improbable that bowel would be irreducible without being attended with the usual violent symptoms of strangulated hernia. Very rarely, however, in extremely large and old hernix this may occur. 


\section{CHAPTER XVI.}

\section{MECHANICAL TREATMENT OF FEMORAL HERNIA.}

In no form of abdominal hernia is prompt and efficient treatment more important than in that variety known as femoral, nor is there any form where more difficulty is experienced in carrying treatment into effect. This refers more especially to its palliative or sruss treatment, and is due to the extreme difficulty of producing sufficient pressure over the deep-seated femoral opening to prevent a protrusion through it without making intolerable pressure on adjoining important nerves and blood vessels. Furthermore, even if the exact compression of the canal has been obtained, nothing but the most careful and accurate fitting of the truss spring will maintain the location of the pad. It is easily displaced by the motions of the leg, upon the muscles of which its lower edge necessarily rests, or by the folding over of the abdominal wall against its top.

For these reasons, and for the additional reason that femoral hernia is never cured by truss-wearing, no matter how young the patient nor how recent and small the hernia, this form should always have the benefit of present-day surgery and be cured, unless there is some other physical condition which is contraindicative. This statement is not intended to convey the impression that there are not hundreds who go through life and escape accident, but it means that they are much more liable to accident and experience greater inconvenience from truss-wearing than those who are afflicted with inguinal hernia.

What has been said of truss-fitting in general, the taking of a diagram of the pelvis, which is especially important in these cases, and the shaping of truss springs need not be repeated here. 
Group of Trusses for Femioral Hernia.

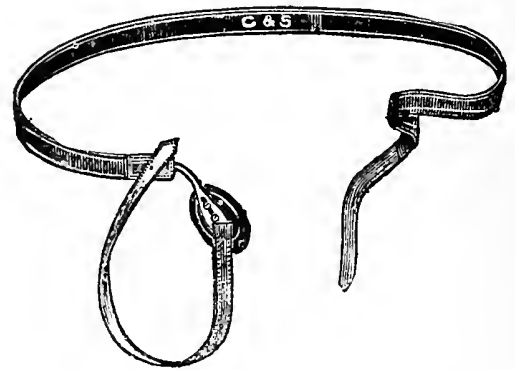

I. Chase femoral-hernia truss, hard rubber.

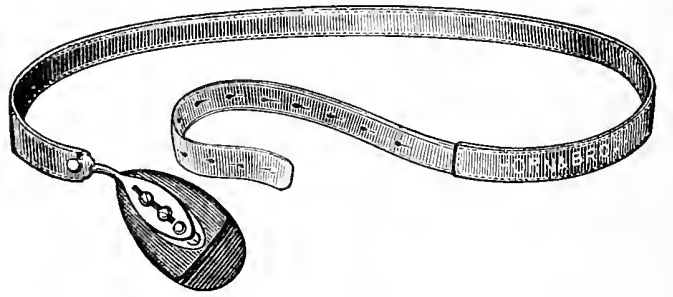

2. Chase femoral-hernia truss, cedar pad, leather cover.

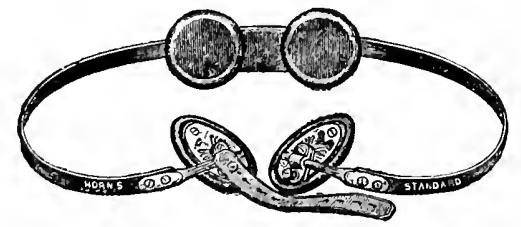

3. Double, hard-rubber, extension-neck truss.

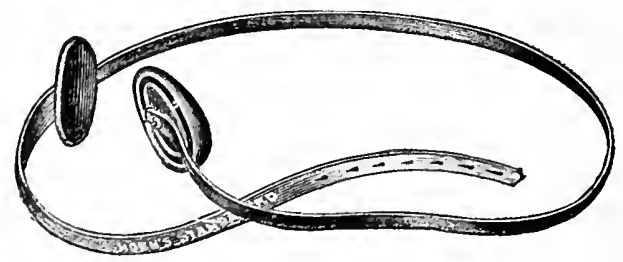

4. Ilard-rubber cross-body truss. 
Group of Trusses for Femoral Hernia (Contimued).

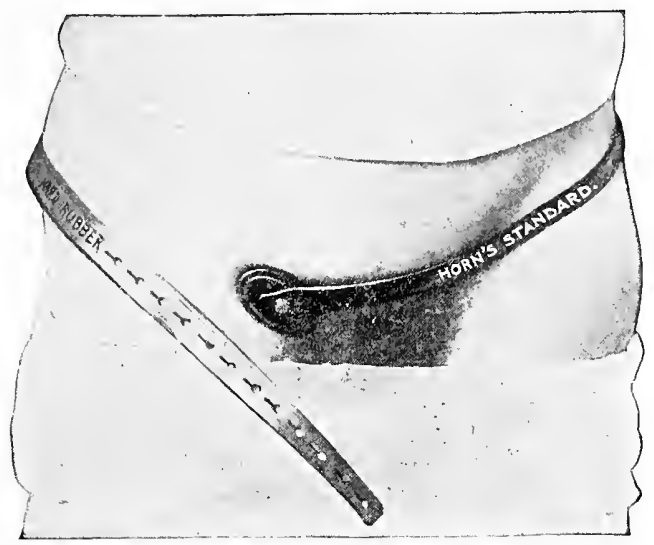

5. Cross-body truss applied for femoral hernia.

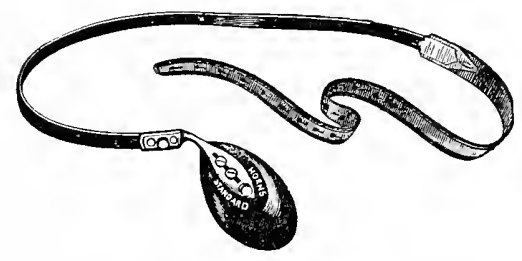

6. Chase hard-rubber truss.

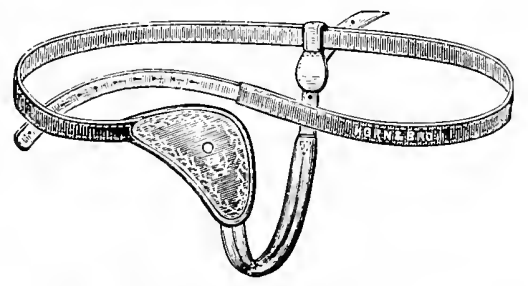

7. French truss with perineal strap.

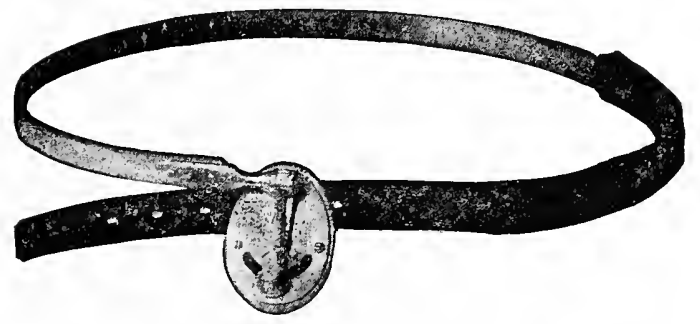

8. Foster ratchet truss, hard rubber. 
Grovp of Trusses for Femoral Hernia (Continued).

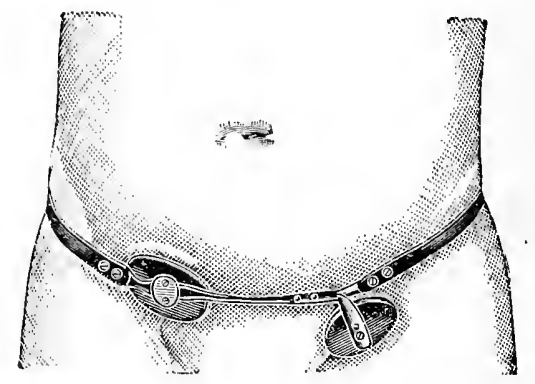

9. Hood truss modified for femoral hernia on left side.

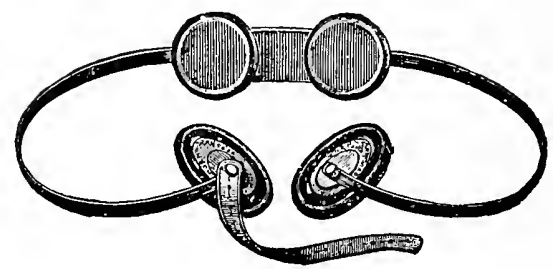

Io. Double hard-rubber truss. Made for inguinal hernia; good form for femoral.

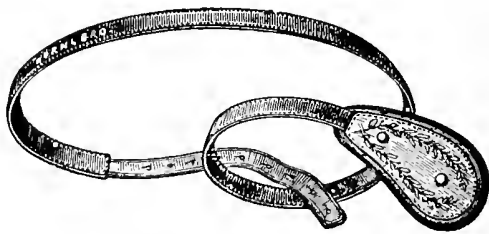

II. French truss, leather and hard rubber.

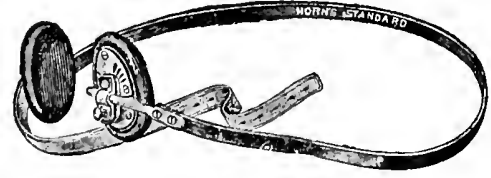

12. Extension neck hard-rubber cross-body truss. 
MECHANICAL TREATMENT: FEMORAL. 321

Group of Trusses for Femoral Hernia (Continued).

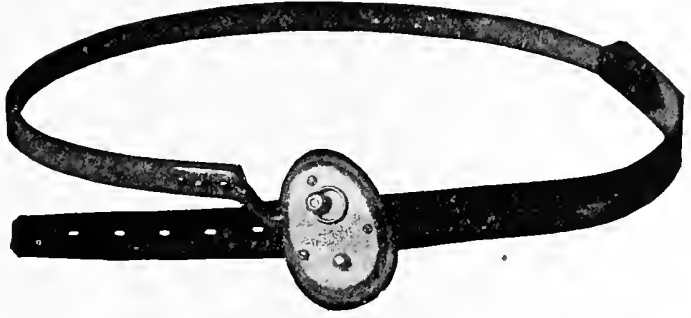

13. Turn-pad hard-rubber truss

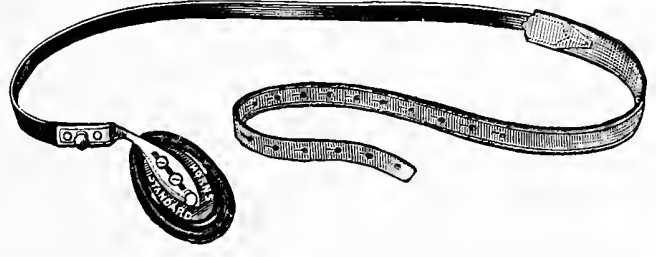

I4. Chase hard-rubber femoral truss.

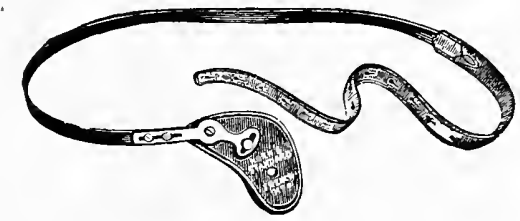

I5. Adjustable French truss, hard rubber.

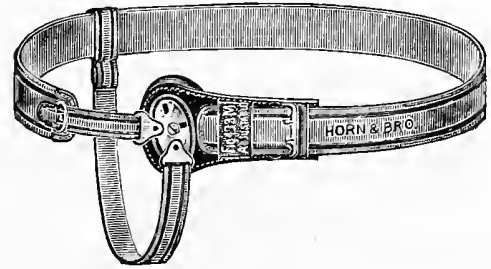

16. Elastic truss. 
One of the most important points in selecting a truss for femoral hernia is that its retaining pad shall be small enough to fit into the deep femoral space without impinging upon the spine of the pubes at the imner side or upon the femoral vessels at the outer side of the hernial opening. The pad should be

FIG. 175 .

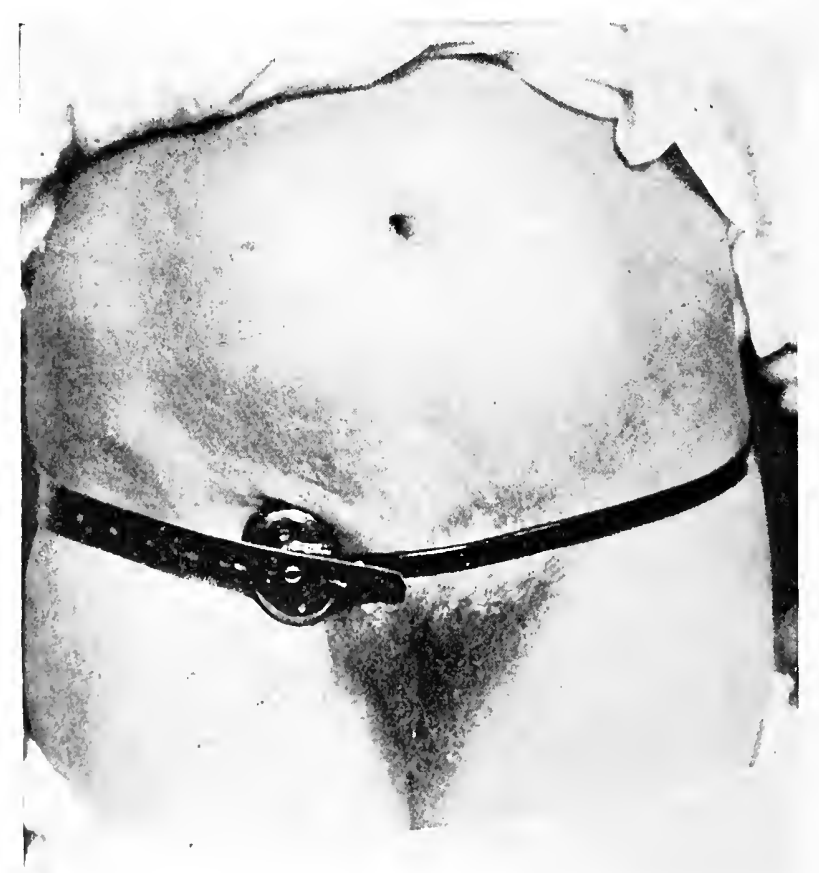

Cross-body hard-rubber truss with water pad. Applied to right femoral hernia.

narrow, not too long, and (leep enough to sink well into the fenoral space inmediatcly beneath Poupart's ligament; having a pressure hackwards towards the thigh, and slightly upwards. The parl shown in no. 4. well answers this purpose, in a thin person, and has leen much used on them by the author. If, however, the patient is at all fat it is not sufficiently deep. 
A small-sized, but prominent, water pad (fig. I 75) is very comfortable in these cases, but should be used only on those who can be relied upon to report for inspection with regularity,

Fig. 176 .

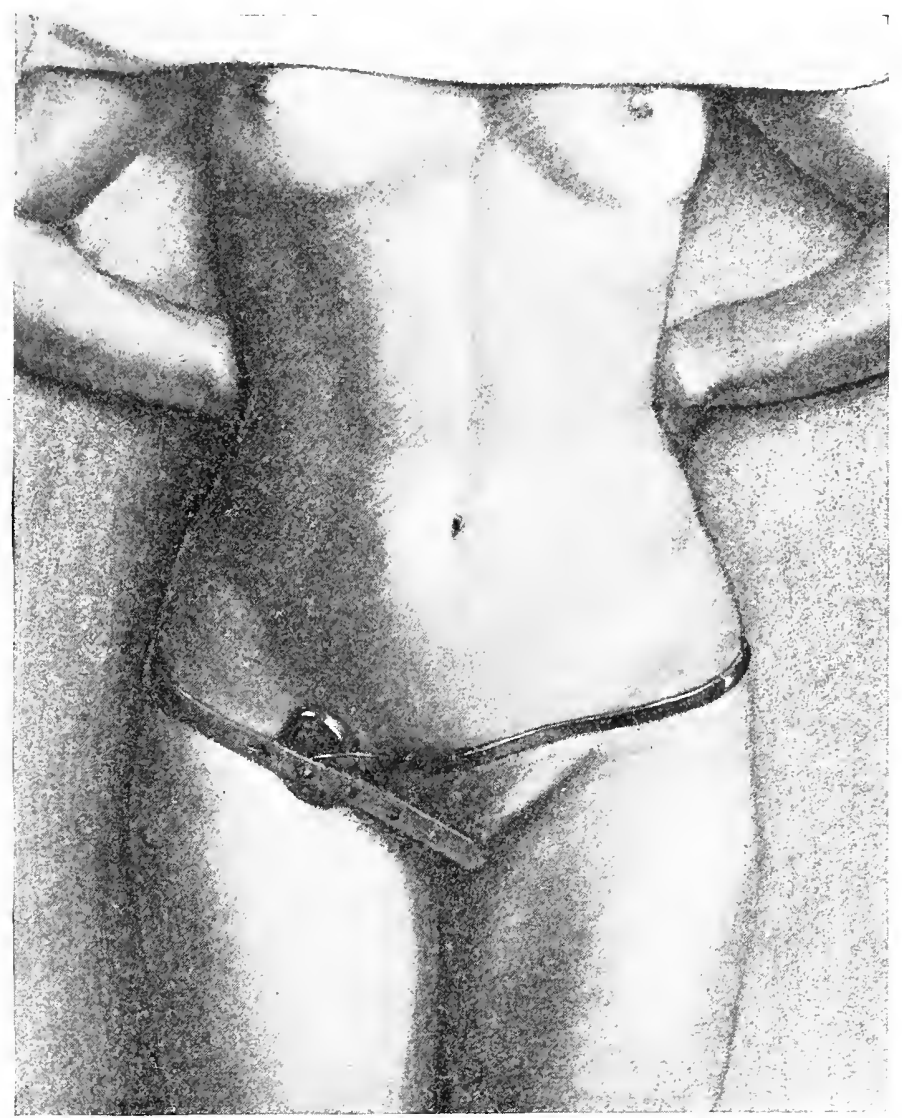

Cross-body hard-rubber truss with deep pad, for femoral hernia.

owing to its perishable character. These pads are liable to flatten out in two or three months' wear and must be renewed. This change of shape is particularly dangerous in femoral hernia, as the pressure then comes upon Poupart's ligament or 
the spine of the pubes and is held away from the hernial opening.

Fig. I 75 shows applied, a form of truss known in the trade as a hard-rubber cross-body spring, with water pad, and the combination forms a most excellent truss for femoral hernia. The small hard-rubber pad is usually preferred. This truss, when properly fitted, can be worn without the perineal strap always so objectionable. This is especially referred to as it is kept in stock by nearly all truss dealers and is therefore readily within the reach of most practitioners. As found in the market, it is designed and shaped for use in cases of inguinal hernia, but the modifications necessary to transform it into a femoral hernia truss are easily made: In selecting a spring, secure one with the lightest obtainable pressure, as femoral hernia never requires as strong pressure for its retention as inguinal. Then select the smaller pad, as the one ordinarily used on the spring is too large. Such a pad will usually be found in the stock of the clealer on the youth's size of inguinal truss, and can be easily transferred to the spring selected. The essential changes now to be made are as follows :

(I) Changing the uniform curve across the front of the spring so that the pad will rest flat against the femoral space. In making this change warm thoroughly the rubber that covers the spring by passing it through the flame of a spirit lamp, or by putting in boiling water, and then bend the spring with the hands or pliers, being careful not to bend it too near the screw hole.

(2) In the shaping of the spring it must be lengthened in front so that it reaches over against the thigh. In the general fitting, the method of making a diagram clescribed under the article on inguinal hernia must be followed closely, as in this way. better than any other, can accurate adjustment be secured.

The cross-borly spring passes around the hip opposite the affecterl sicle (as shown in fig. I 76 ), just above the trochanter 
major, in very nearly the position occupied by the inguinal hernia truss; the pad is placed about one inch lower, its lower edge resting upon the top of the thigh. The spring must be about half an inch longer in front than when used for inguinal hernia. Some of the trusses known as the French (no. 7 of group) or German style (fig. I 77 ) are very good for femoral hernia. The two styles named are alike except that the "French" are lighter in construction and therefore better

FIG. I 77 .

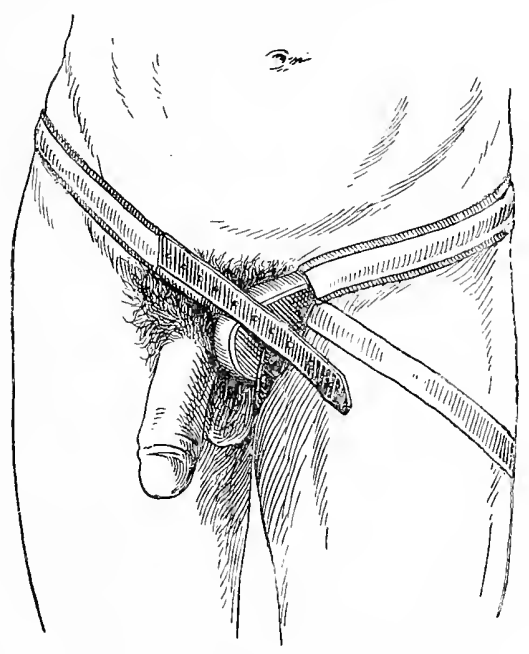

German femoral truss applied. Note that direction of pressure of the pad is towards the spine of the pubes instead of into the femoral space and towards the thigh. Good illustration of poor truss-fitting.

adapted for femoral hernia. If this truss is carefully shaped by the diagram method it can usually be successfully worn without the perineal strap, but if it fails to maintain an exact position, it must be worn even though irksome. The spring in this truss does not pass across the abdomen, but goes around the hip of the affected side. The English form of this type is even better on account of its having a smaller pad (fig. I 78 ).

Springs that go on from the sicle of the hernia, like the German, French, and English type, are better suited to femoral 
than to inguinal hernia, as the curve of the spring brings the direction of pressure toward the thigh. The truss known as the " Chase " is of this type and is very good. Some inguinal trusses with adjustable pads known as "Common Sense" and "Excelsior" trusses,-very similar in design,-are also good for femoral hernia if the spring pressure is sufficiently diminished. One is shown in fig. I79.

FIG. 178 .

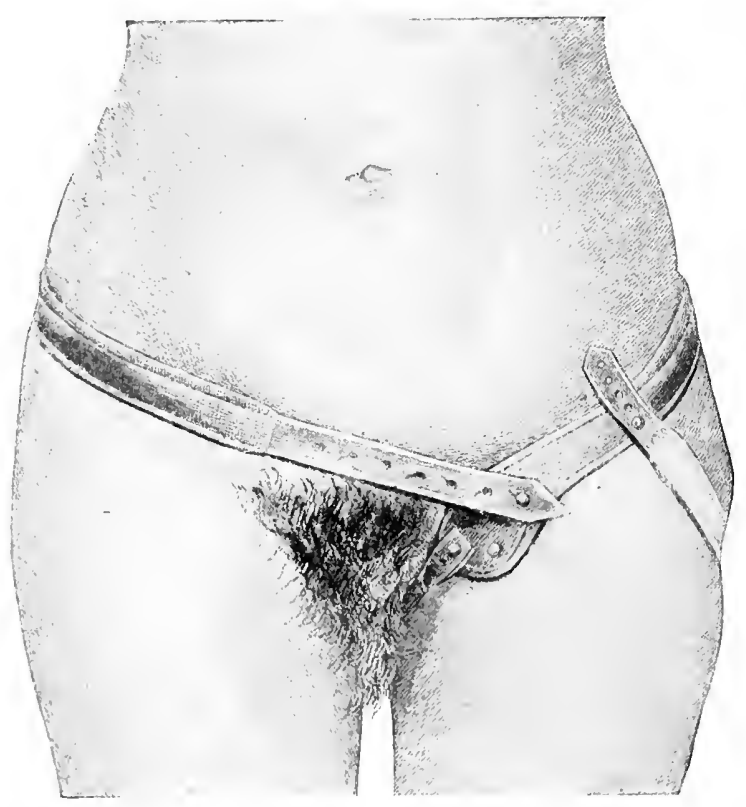

Light form of femoral truss, English form. (Macready.) 1llustration of good-fitting truss.

Several manufacturers have modified the "Hood" truss for use in femoral hernia, but in my own hands it has not proven as satisfactory as those of the cross-body type. A truss of the latter type, designed by me many years ago, is shown in fig. 18o. This has a light cross-body spring, the parl being supported on an arm, which is in turn attached to the spring by a ratchet. There was also a slot in the face- 
plate of the pad that allowed of adjustment. Between the ratchet and the slot very accurate adjustment of the pad could be obtained.

FIG. I 79 .

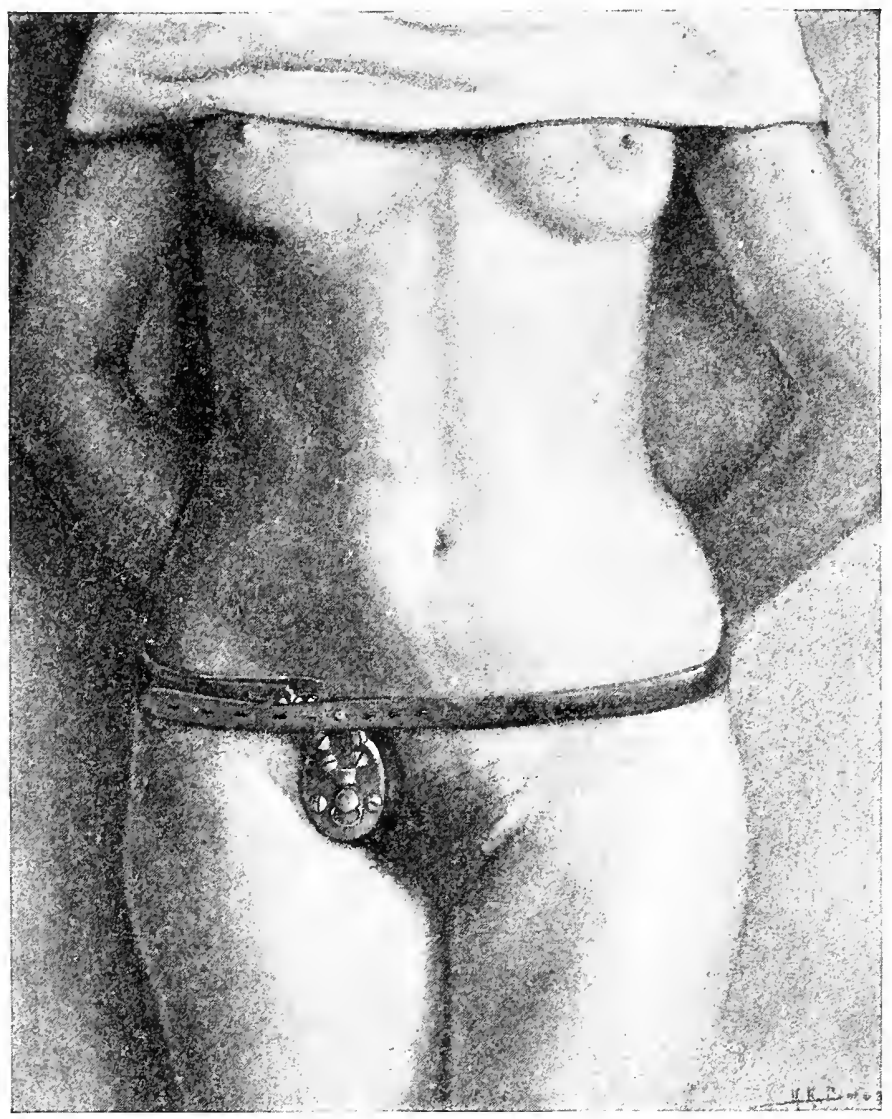

Adjustable truss made for inguinal, also suitable for femoral hernia.

The Elastic Truss is strongly advised against as being both unreliable and unconfortable. The author has seen cases which have become strangulated, and many others which have increased in severity under its use. Nor has the author ever 
seen any form of bandage that he considered in the least degree safe. While waiting for a suitable truss, if one is not at hand, a tight bandage with a moderately hard compress in the

FIG. I 80 .

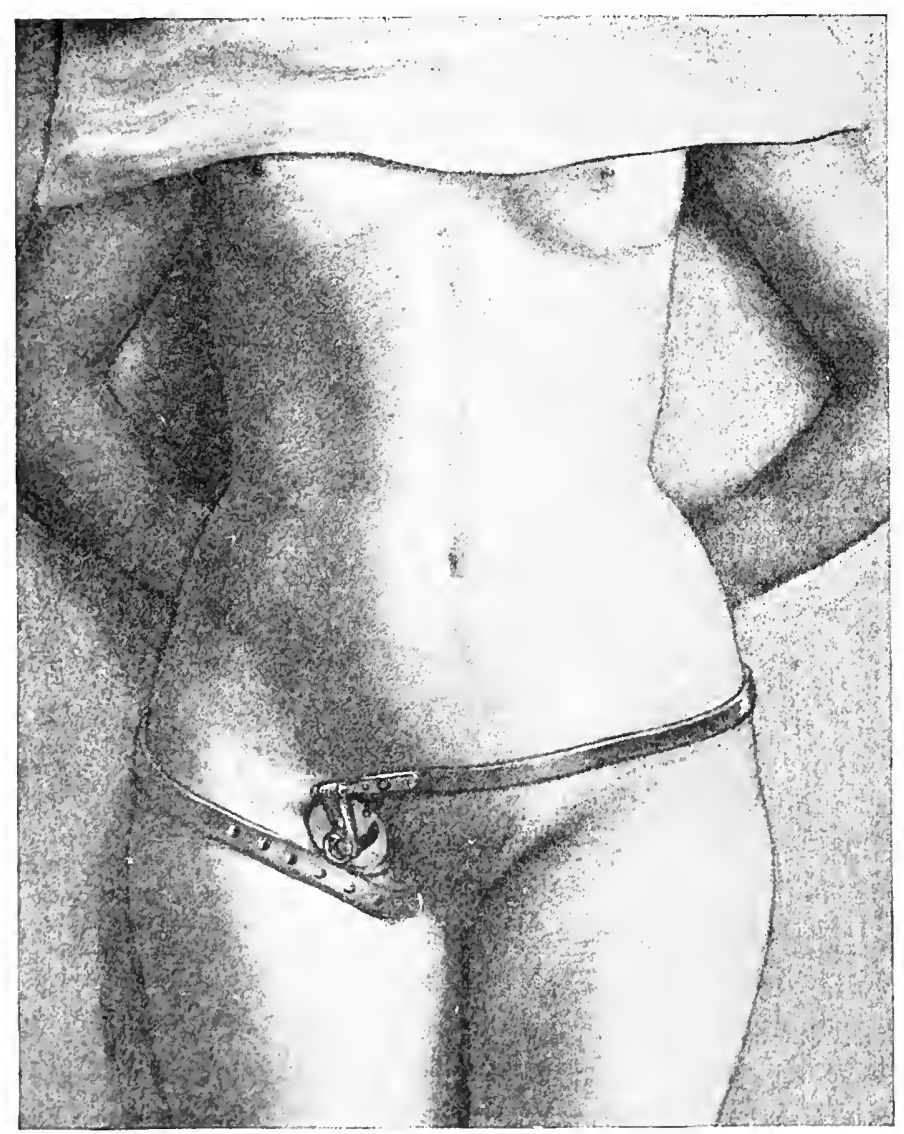

De Garmo femoral truss.

femoral space is somewhat protective and should be used only until something better can be done.

In clouble femoral hernia the choice of truss should be between the "Double French" and the double hard-rubber 
truss shown in no. Io of group, the latter with small pads being very much preferred. This truss is made for inguinal hernia and the curve at the end of the spring must be modified in order to have the pads rest flat upon the thigh. It seldom requires the thigh strap to keep it in position. Fig. I8I shows a truss of this type applied to an inguinal hernia on the left side and femoral on the right. This combination has proven thor-

FIG. I 8 I.

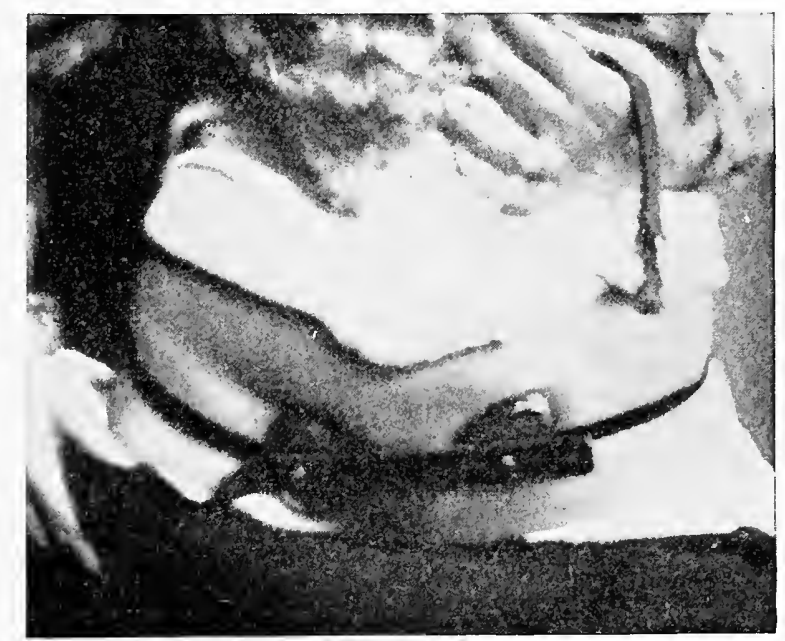

Woman of 45 years with right femoral and left inguinal hernia retained by double hardrubber truss and water pads. The latter are for temporary use only, the hard-rubber pads being better.

oughly satisfactory in many cases. A smaller pad than the one shown in this photograph was put on later.

Irreducible femoral hernia should rarely be treated mechanically, as the only safety in these cases is in operation; in some cases, however, the attending circumstances are such that operative means cannot be carried out and some substitute must be used. If the hernia is small a concave pad may be used, preferably on the cross-body spring. Usually in such cases the perineal or thigh strap is necessary to keep the 
pad in place. The contents of irreducible femoral hernia is almost uniformly omentum, and in a few instances the author has seen its absorption occur under the pressure of a concave pad, and has then changed to the convex pad generally used in femoral hernia. The idea advanced in some of the older works upon this subject that it is dangerous to make truss pressure upon irreducible omentum has been too often disproven in the experience of the author to allow of anything but the most emphatic denial. 


\section{CHAPTER XVII.}

\section{SURGICAL CURE OF FEMIORAL HERNIA.}

The history of the operative cure of femoral hernia is peculiar, in that works upon the subject have repeatedly stated that owing to the formation of the so-called "ring" its cure is uncertain and improbable. At the same time the experience of the individual operator has constantly shown, in this country at least, that if he be careful to clear the femoral opening of sac and all foreign tissue and to close it by almost any method, a cure is more than likely to result. The truth, it would then seem, is that the objections to its surgical cure have been theoretical rather than practical, yet this has led to the suggestion of many different methods by many different operators.

So far as to him known, the report of the author before the Surgical Section of the New York Academy of Medicine (Annals of Surgery, August, I905, p. 209) of I IO cases operated upon by one method and by the same operator, is the largest that has been made. This method is given in this work to the exclusion of others, not because it was original with the author, but because of its simplicity, ease of execution, and the permanence of its results. It was first done by him, on March 4, I890, and has been taught to his classes at the New York Post-Graduate Medical School and Hospital since that date.

The cases recorded in the report referred to were met with in operating upon a series of 1,250 abdominal hernix. The I Io femoral hernixe were in 99 patients, 83 of whom were females and I6 males. Eighty-eight patients had single and I I had double femoral hernia. Of the single hernix, 59 were on the right side and 29 on the left. One patient had double femoral hernia and left inguinal. Three had double inguinal 
and single femoral hernia. Two had single femoral and inguinal hernia on the same side, making 5 cases who had femoral and inguinal hernia on the same side. Six had femoral hernia on one and inguinal on the opposite side.

In 28 patients, strangulation of the hernia existed at the time of the operation, and 82 were operated upon for the cure of the hernia. The ages were, 4 moder ten years; 6 between ten and twenty years; 18 between twenty and thirty years; 34 between thirty and forty years; I 5 between forty and fifty years; I I between fifty and sixty years; 5 between sixty and seventy years; 5 between seventy and eighty years; I over eighty years. The youngest patient was eight years of age and the oldest eighty-one years. The latter was operated upon in a private house, in the middle of the night, for femoral hernia of enormous size that had existed for thirty years and which had been strangulated for six hours. She lived nine years after the operation, during which time she wore no truss and had no recurrence.

Mortality.-In the entire number only one death has occurred, and that was an old woman of seventy years, who had suffered from strangulated hernia for three days, during which time she had been subjected to the most violent attempts at reduction. Perforation of the bowel was found, and, owing to the moribund condition of the patient, the intestine was fastened in the wound and freely opened. She died of exhaustion twenty-four hours later.

Recurrences.-In one case there was recurrence three weeks after the operation from violent vomiting due to acute indigestion. This case was re-operated upon eight months afterwards, and has remained cured three years. One patient, a man, who had double inguinal and right femoral hernia, was supposed to have a recurrence of the femoral hernia. Upon re-operating, the protrusion was found to be subperitoneal fat that had slipped through under Poupart's ligament, but no hernial sac had formed. In one other case, believed to be identical with the one just narrated, a woman of thirty-five 
years had a small swelling in the femoral region nine months after operation. A light truss was applied and worn one year, and she has now been five years without support and no protrusion. It is believed that this also was a small protrusion of subperitoneal fat, and absorption was produced by truss pressure. I have never seen a femoral hernia cured by truss pressure, no matter how young the patient nor how small the protrusion.

One woman of seventy-five years of age had a recurrence within eight months of the operation, and, so far as I know, this is the only actual recurrence. By far the greater number of these cases have been traced and the permanence of the cure ascertained. Three cases operated upon were recurrent following some previous operation, the character of which is unknown. All of these recurrent cases have remained cured for more than four years. Two cases had by mistake been operated upon for inguinal hernia, when, in reality, femoral hernia existed, and it is a rather remarkable fact that both were done by operators noted in other lines of surgical work.

Contents.-Contents of the herniæ were in most instances intestine or omentum, or both. In one instance a small and unhealthy ovary was found in the sac. In two cases of strangulation with quite acute symptoms, appendices epiploicæ were found strangulated. In these cases the bowel itself was held firmly against the femoral opening, but the lumen of the intestine was not constricted. Cysts in or around the sac were found in 4 cases.

In one case, the daughter of a well-known physician, strangulation was coincident with the first protrusion of the hernia. In stepping from a railroad-car, the step being much higher than she had estimated, a hernia was forced through the femoral canal, and urgent symptoms at once presented.

The safety and comfort of the patient demand that every case of femoral hernia, whether reducible or irreducible, shall be cured unless there is something in the condition of the patient that positively contraindicates an operation. Its peculiar 
anatomical surroundings make femoral hernia an unsually dangerous condition, and rencler its stuccessful treatment by mechanical means uncertain as well as attended by discomfort. On the other hand, viewed from the surgical side it is considered the safest of all herniae for operative cure; the operation is easier of execution and it is fully as permanent in its results as that done on any other form of hernia. The danger in the

FIG. IS2.

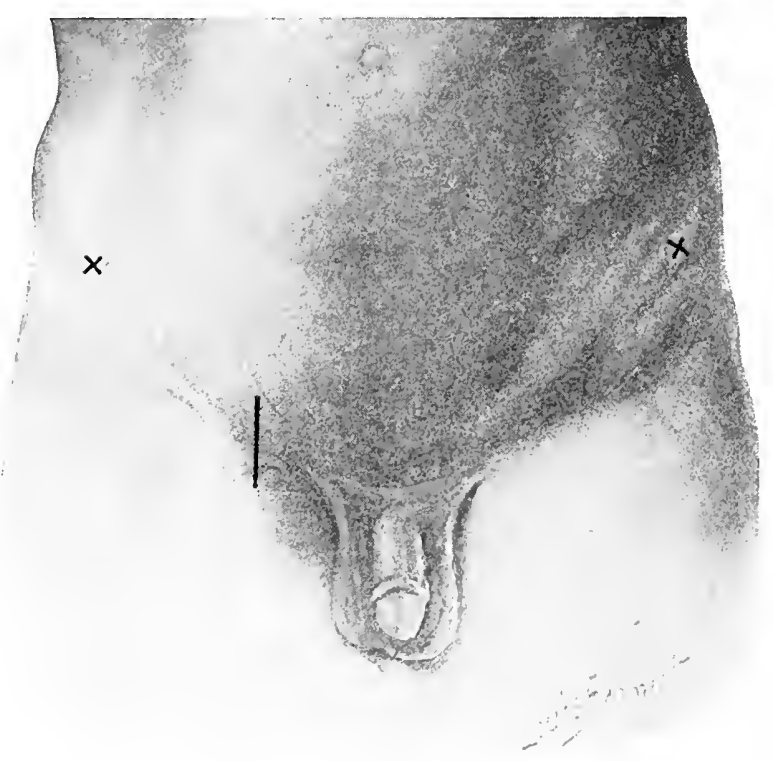

Showing location and direction of incision for femoral heruia operation.

hands of a cualified surgeon in an uncomplicated case would actually seem to be limited to whatever attends the giving of the ansesthetic. The preparation of the patient, and for the operation, should be as carefully made as for any other abchominal operation.

Operation.-The incision for the operation here described should be between two and three inches long, parallel with and to the inner sirle of the femoral vessels (fig. I82). The 
upper angle of the wound should be well up over Poupart's ligament and extend down over the saphenous opening. Many times the line of separation between the superficial fascia and the deep transversalis fascia, that has been pusherl down in front of the peritoneum by the hernia, will be so distinctly seen as to lead the operator to feel that he has the true sac. On

\section{FIG. $8_{3}$.}

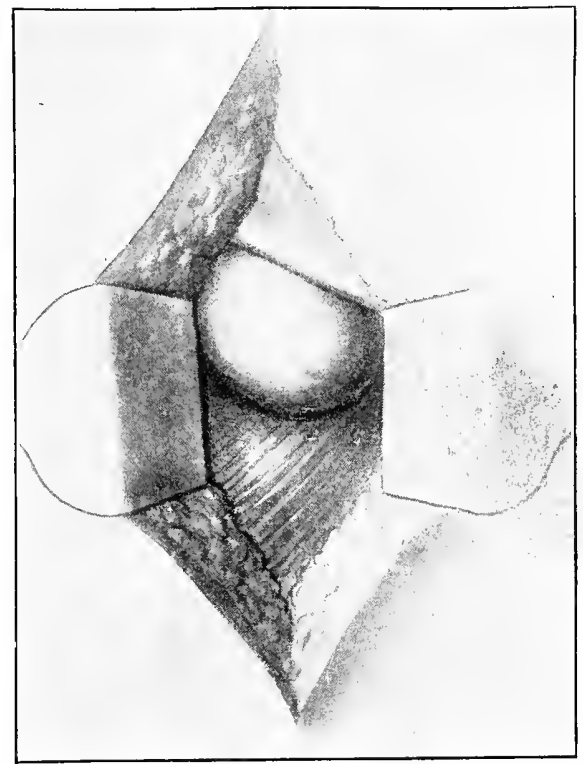

Showing protrusion under Poupart's ligament. The femoral vessels are to the outer side of the sac. The sac should have been shown with smaller neck.

cutting through this, however, he will come upon the subperitoneal fat (sometimes mistaken for adherent omentum) and then reach the bluish-white, true hernial sac. As in other locations when the true sac is opened, there is almost uniformly found evidence of the normal abdominal fluid and the shiny surface characteristic of peritoneum.

When the skin and the superficial fascia are incised, usually the sac and its subperitoneal fat will come into the 
wound, appearing like an encysted lipoma (fig. I 83), and, before separating the sac, it is best that this entire mass should be lifted out of its bed by thumb forceps and blunt dissection, so that its neck where it passes under Poupart's ligament shall be entirely free from its surroundings. By traction on the sac (fig. I $S_{+}$) and its superimposed fat, this neck may not only be freed, but it will be materially lengthened, so that when it is

FIG. I 84 .

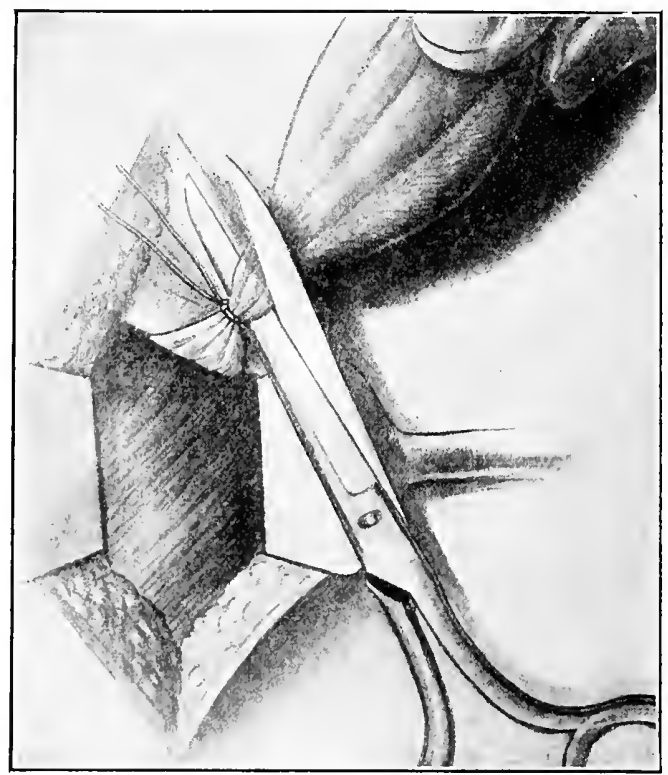

Sac forcibly drawn down while being ligated and cut away. This after examining its interior to see that no adhesions exist.

finally ligated and cut off it will retract within the abdominal cavity, leaving the femoral canal free of foreign tissue. This is absolutely essential to the subsequent permanent cure of the case. It must be borne in mind that extreme traction might easily bring into the operative field either an angle of the bladder wall to the inner sicle, or the deep cpigastric vessels upon the upper surface of the sac. Both of these have been seen in this operation. 
The sac should be opened, and where omentum is found adherent it should be carefully ligated, cut away, and its stump reduced to the abdominal cavity. Adherent intestine will rarely be found, but where it is, the adhesions must either be broken ıp, or, if too firm, the adherent part may be cut out of the sac and left attached to the bowel. When in doubt, the latter method is by far the safer.

FIG. 185 .

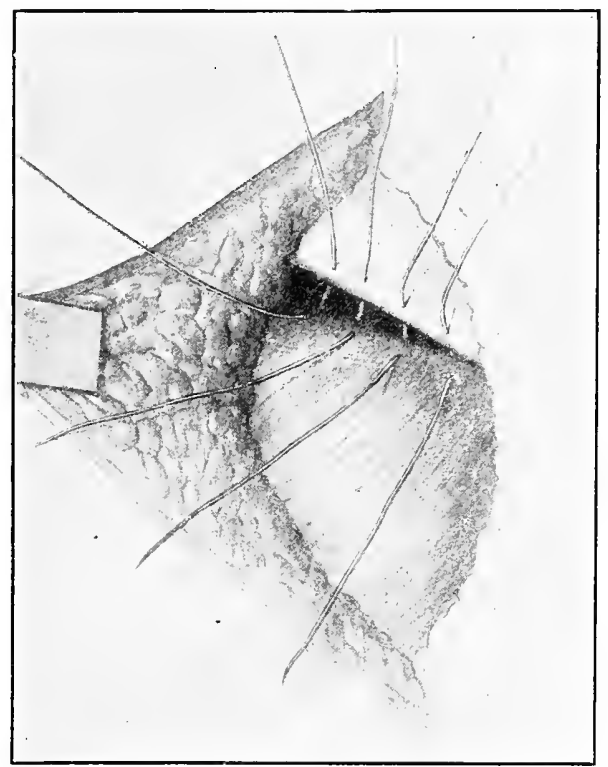

Showing sutures of kangarootendon passing through Poupart's ligament above and through all tissues to periosteum of ramus of pubes below.

The sac, having been entirely freed of its contents, is tied off as high up as possible while it is being forcibly drawn down by an assistant. Great care must be used to insure the perfect freedom of the neck of the sac from protruding bowel or omentum while the ligature is being placed. After tying with strong catgut (a double strand of no. 2 plain is preferred), pass the needle, which has been previously threaded with it, through the neck of the sac and tie again. This gives a double 
ligature, anchored by perforation between the two, which prerents slipping off.

When the sac is cut away the stump should be examined to be sure that no bleeding vessels remain, and not until then should the encls of the ligature be cut, after which the stump usually retracts within the abdomen. If this is prevented by

FIG. I 86.

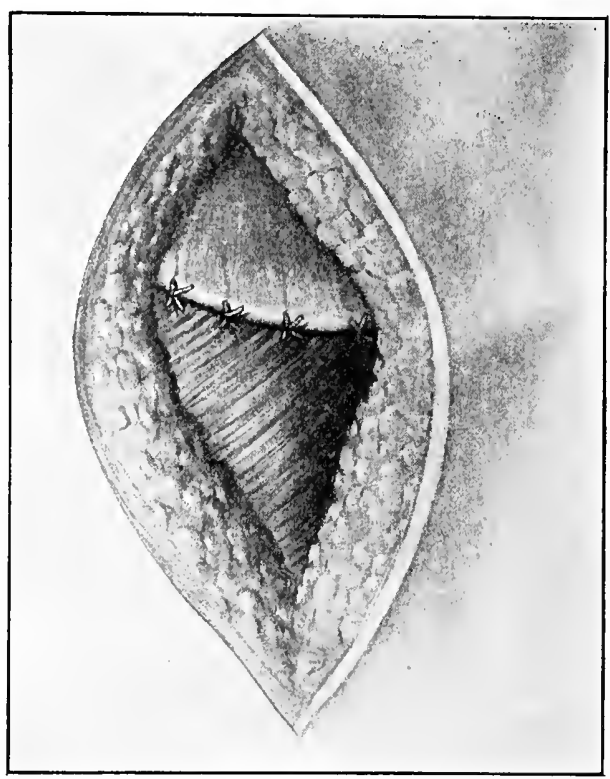

Showing depression of Poupart's ligament to ramus of pubes by sutures tied down.

connective tissue which has not been broken, it should be carefully pushed back, leaving the femoral opening absolutely free.

This opening is closed in the following manner by good sized kangaroo tendon threaded in a strong blunt needle (fig. I $\left.8_{5}\right)$. Press the end of the finger firmly into the femoral opening under Poupart's ligament, and pass the needle through the ligament upon the finger-point. This perforation should be well towards the outer sicle of the canal and close to the femoral vein. The operator should assure himself, by pressure 
of the finger against the ramus of the pubes, that the vessels are out of the way, and then pass the point of the needle fully down to the periosteum of the pubic bone, taking up all tissues over

FIG. I 87 .

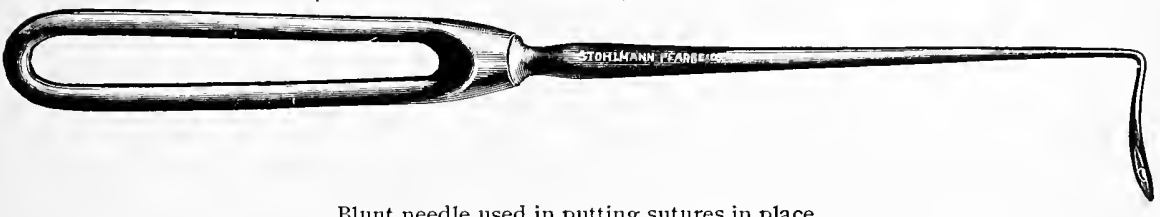

Blunt needle used in putting sutures in place.

it. This constitutes the first stitch, but should not be tied until the others are in place. Sutures should then be placed in

FIG. I 88 .

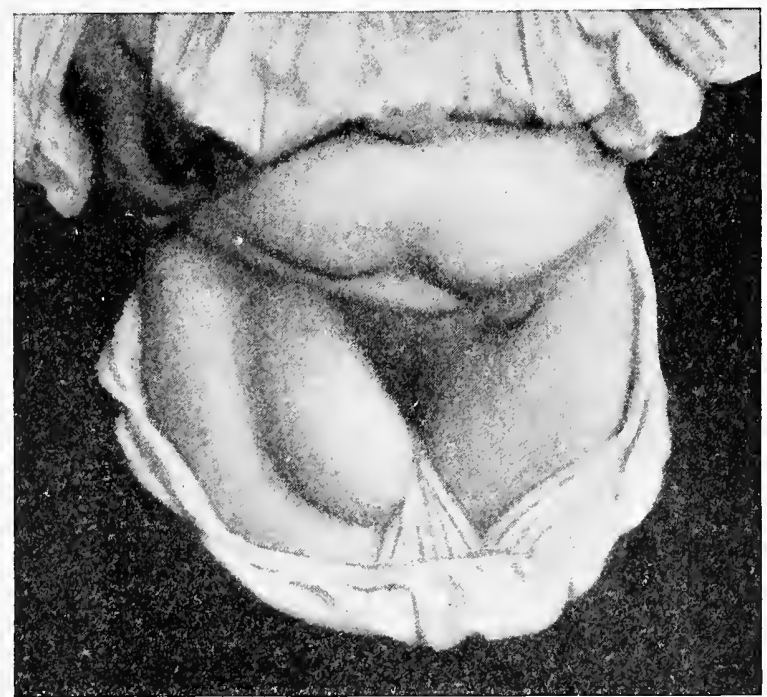

Double reducible femoral hernia in a woman of 60 years. Left side does not show, but was nearly the size of a hen's egg. Swelling above pubes is due to subcutaneous fat.

the same manner every quarter of an inch apart until near the spine of the pubes (fig. 186). Usually three or four will completely close the femoral opening. 
When tied down and the ends cut moderately close the fascia should be closed in by plain catgut, to avoid a pocket in the tissues that otherwise may result, and the skin may then be closed by buried sutures of the same. I have usually covered the wound by collodion and a compress of sterilized gauze held in place by a figure-of-eight bandage. In ten days the dressings are changed and a bandage for tem-

FIG. I 89 .

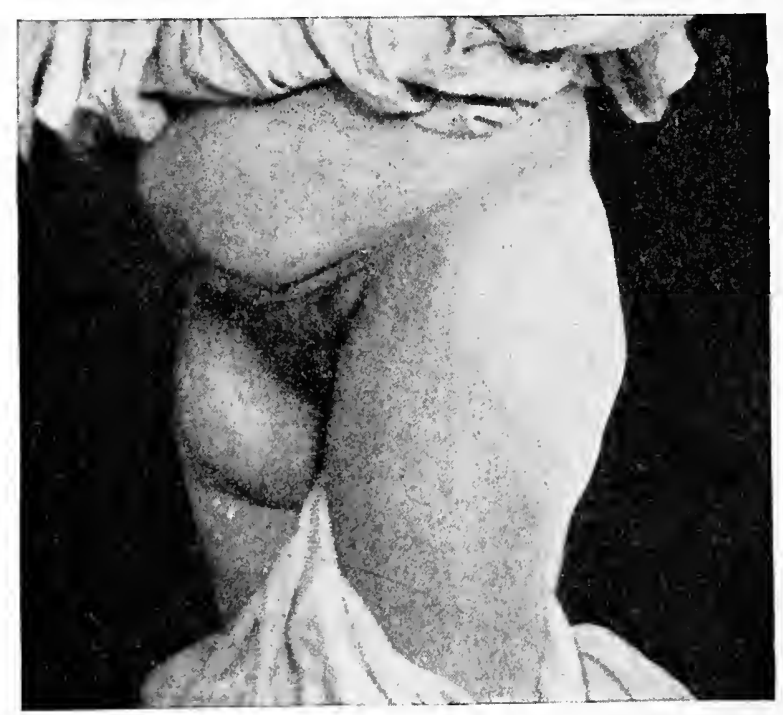

Same case as shown in fig. I88; side view.

porary support is applied. If healing has been complete, the patient is allowed to sit up on the tenth and leave the house on the fourteenth day after the operation.

The bandlage used after the first dressing consists of a pelvic belt, of three thicknesses of canton flannel, with a compress of gauze over the former site of the hernia, and a perineal strap to prevent its slipping up. This is to be worn for four weeks. Neither truss or other permanent support should be wrorn. 
As it is not an uncommon occurrence to have both inguinal and femoral hernia on the same side, it is deemed best to say a few words regarding the combined operations: A single incision will answer every purpose, but it should be a little longer, beginning over the centre of the inguinal canal and curving downward, passing to the inner side and parallel with the femoral vessels to the saphenous opening; this gives easy access to both canals. The femoral sac should be removed, the

FIG. Igo.

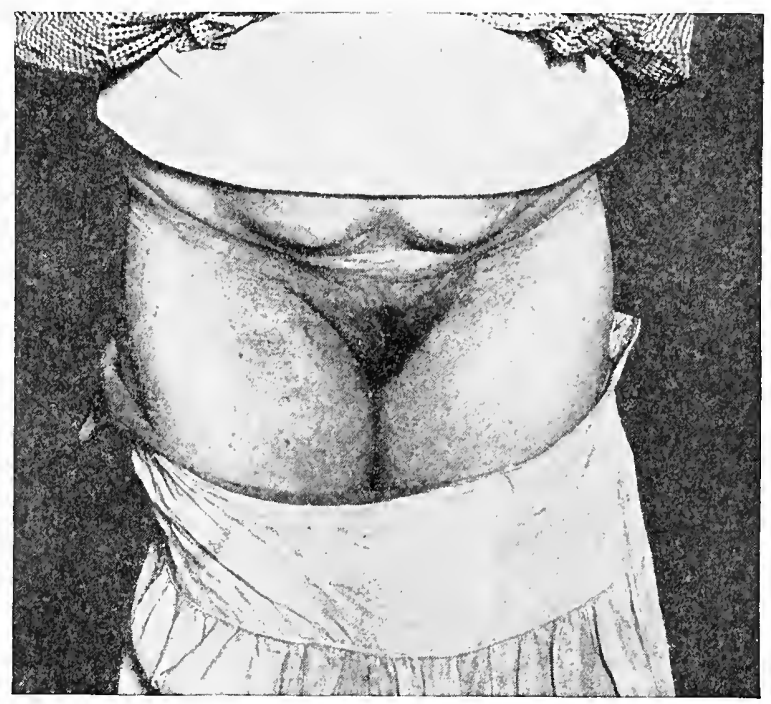

Same case as shown in fig. 188 , six weeks after double operation.

canal closed as already described, then the inguinal hernia should be operated upon as though no complication existed.

Suture.-The idea that the suture in the operation described would make too much pressure on the femoral vein is a very natural one, but it is believed that with ordinary care this will never occur. At least in the cases presented 110 indication of undue pressure has ever been observed. There has been no change in the technique of this operation during the fifteen years of its use by me, except in the suture material. 
In the first 22 operations, No. Io braided silk was used for closing the femoral opening. In 3 , silkworm gut was employed: but in the last 85 cases, i.c., since November 20 , I896, kangaroo tendon has been used exclusively. There can be no question at the present day, it would seem to me, that the last-named substance approaches more nearly than any other

Fig. I9I.

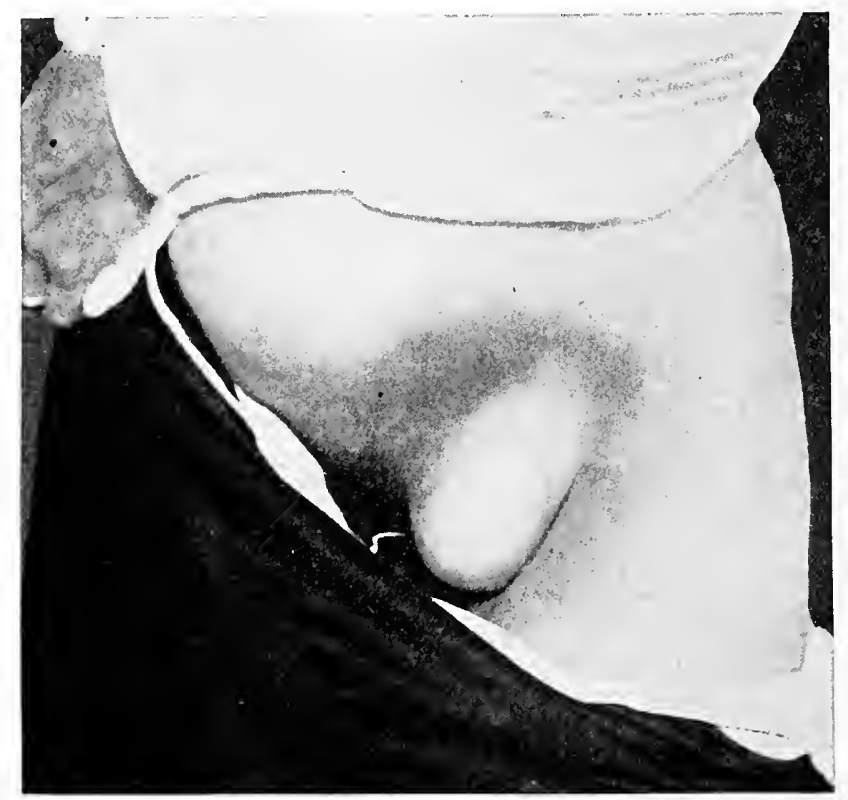

Femoral hernia of unusual type, due to cutting of Poupart's ligament in operation for strangulated hernia. Notice broad base of tumor. Woman 38 years old, 4 months pregnant.

the ideal suture for this purpose. The deep sutures have been placed by a blunt needle with handle (fig. I 87 ). (It is believer that the use of a sharp needle, or even a blunt needle in a holder, is attended by considerable danger of injury to the femoral vein.)

This operation answers equally well in femoral hernix of either large or small size, and has frequently been used, as in 
figs. I88, I 89 and I90, for enormous protrusions on one side and a small hernia on the other on the same patient. Following operations for strangulated femoral hernia it can be used almost as quickly as the parts can be closed without any curative method, and it insures the patient against subsequent disaster. In only one patient have I been unable to make this closure, and this was the case in which it was necessary to leave the bowel open. In giving this method and excluding the many others that have been suggested I fully realize that I open wide the door of criticism, but it has been my effort throughout this work to simplify, as far as possible, the operative procedures advised.

I am confident that the circular suture, used by some good and experienced operators, which includes Poupart's ligament above and the tissues covering the ramus of the pubes below, will accomplish the same end. The essential element of success is in clearing the femoral opening of sac and all other foreign tissues. On the contrary, I have no confidence whatever in the so-called plastic operations which rely for success upon the transplanting of muscle or tendon. Nor have I confidence in those which attempt to close the saphenous opening in the fascia of the thigh. The origin of the hernia is at the peritoneal surface of the abdominal wall and here its remedy should be applied. 


\section{CHAPTER XVIII.}

\section{UMBILICAL HERNIA.}

For reasons stated later, I have not classified hernia into the umbilical cord, which is really a malformation, with umbilical hernia, as done by most authors.

Umbilical hernia is a protrusion of any of the abdominal contents through the umbilical ring. The cases may be divided into those of infancy and those of adult life. By "infancy" it is intended to refer to children under five years of age. Even this division is not based upon any anatomical or pathological consideration, but is used because in infancy these herniæe can be cured by purely mechanical means, while in the adult a cure never results without surgical interference. The division is therefore merely one of convenience.

Umbilical hernia constitutes about $8 \mathrm{r} / 2$ per cent. of all hernix. Up to the tenth year of age it is about equally divided between the sexes, but in later life it is more frequent in the female in the proportion of 2.7 to $\mathrm{I}$.

In adult life the umbilicus is three-quarters of an inch above the highest point of the iliac crest, and opposite the disk between the third and fourth lumbar vertebra. At two years of age it occupies the exact middle point of the body measured from head to foot. Earlier it is below and later it is above this point. In the foetus it is close to the pubes. It is the final point of closure of the lateral abdominal walls and surrounds the vessels of the cord which become obliterated at birth. These obliterated vessels form four strong bands of fibrous tissue which pass through the umbilical ring and end in the umbilical scar. The umbilical vein descends from above, the two umbilical arteries and urachus from below; the urachus holding the umbilicus firmly against the lower margin of the ring. 
The umbilical ring is the opening in the linea alba for the transmission of the nutrient vessels before birth, and if normal should surround closely the remains of the cord when that slonghs off. This ring is occupied by the obliterated vessels and a small amount of fat. The peritoneum in this region is thin and firmly adherent, especially above the umbilicus. The transversalis fascia, fortified by additional fibres known as the umbilical fascia, is beneath the linea alba. Defects in closure of this ring may be anywhere from a large cleft in the muscular wall, constituting so-called congenital umbilical hernia, to a mere weakness. These defects are most frequently found above the navel.

The parts within the abdomen most frequently involved in umbilical hernia are the omentum, the large and small intestine. The greater omentum starts from the lower border of the stomach, descending over the small intestine, forming a louble fold of peritonetm. It passes downward a variable distance, folds upon itself, and returns to envelop the transverse colon, back of which it is continued and attached to the posterior abdominal wall, forming the mesocolon. According to Dr. Zabe (Thirtccntl Intcmational Congress, Paris, I900), in umbilical protrusions, traction upon the omentum impedes the motions of the stomach, and depresses the arch of the colon, resulting in an enteroptosis. Eventually the stomach has a tendency to become vertical and the prolapsed intestine becomes stenosed, causing what he aptly calls "hernial dyspepsia."

Cause.-It does not require deep research to discover the predisposing cause of hernia in this region. The opening in the linea alba, for the transmission of the prenatal vessels of nutrition, is filled in after birth by the remains of those vessels and fibrous or cicatricial tissue. Undoubtedly this is the weakest point in the whole abdominal wall, but from its location it is not subjected to as great a strain as those parts lower down. In conditions of marked obesity, or when intra-abdominal growths exist, this umbilical ring is subjected to great and con- 
stant strain, and it then stretches or even occasionally tears, allowing the contained viscera to protrude.

Frequent pregnancies, by repeated over-distention of the muscular wall, lead to the common occurrence of umbilical hernia in women between forty and fifty years of age. Women who at this period of life have put on an inordinate amount of fat are especially liable to it. In fact, the latter is believed to be a far more potent cause than pregnancy. With the great increase of fat there is frequently a corresponding degeneration of the muscular tissue, so that with increased intra-abdominal pressure there is decreased power of retention. Further than this, such women are very liable to fatty degeneration of the heart muscle and are therefore dangerous patients upon whom to do extensive surgical work.

Umbilical hernia not uncommonly results from, or may form a complication of, acites, the fluid forming in the abdominal cavity being forced through the umbilical ring as well as, in some cases, through both inguinal canals.

In infancy umbilical hernia is believed to result almost uniformly from defective closure, in the median line, of the lateral walls of the abclomen. This predisposing cause is also thought to account for many cases occurring in thin people whose abdomens have not been subjected to abnormal distention. The defective muscular and tendinous structures about the navels of many children is quite apparent; in some instances children reach adult life safely only to develop hernia later, and in others the tissues so harden and develop as to gain for them complete immunity. Given this defective structure, it is usually easy to find in crying, coughing, or constipation an immediate cause. The last named is thought to be most common. I cannot enclorse the view of some authors that there are in these cases, as in the congenital type of inguinal hernia, a preformed sac that has persisted during the years preceding the occurrence of hernia.

Size and Form.-In the infant umbilical hernia usually prescnts as a circular swelling at the umbilical ring, varying in 
size from the end of the little finger to that of the fist. The photograph shown in fig. I92 represents a common size as met with in practice.

The form of the tumor in infants is almost always circular and projects from the body with little tendency to dissect up the adjoining skin, as in the cases occurring in the adult, even though the hernia attains unusual size. Umbilical hernia in infancy is rarely irreducible. In the adult this form of hernia

FIG. 192.

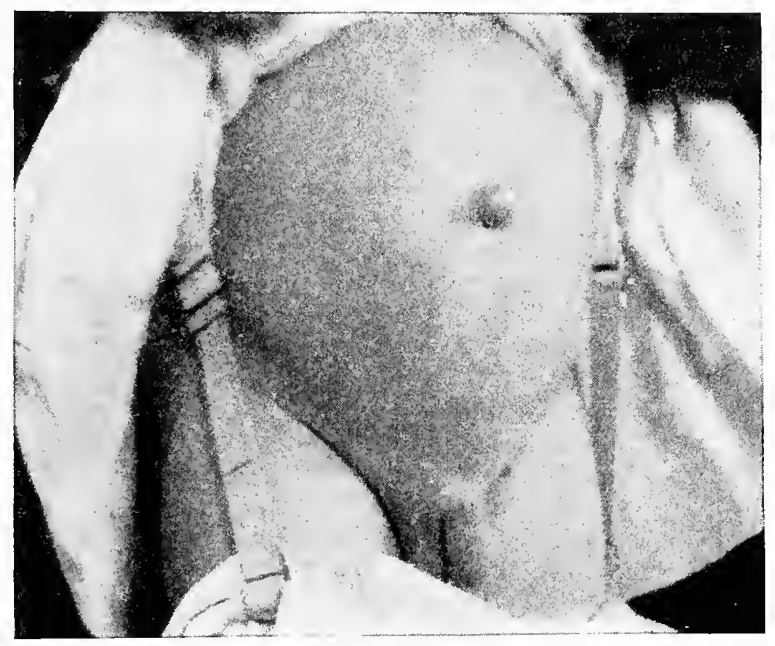

Small umbilical hernia in a child 3 years old. Present since early infancy; truss constantly worn, with little indication of cure.

may become a tumor of enormous proportions. I saw in consultation several years since a case where the largest part of the tumor measured forty-two inches in circumference and thirtyfour inches at its neck. The woman, who was about sixty-five years old, carried this protrusion in a bag arrangement made of canvas supported by suspenders over the shoulders.

The form of the hernia in the adult is very irregular and influenced by the contents of the sac and by resisting bands encountered while passing through the umbilical ring. The 
tough bands, the remains of the umbilical vessels, may divide the tumor into several parts, giving it a lobulated appearance (figs. 193. I94 and 195). These tumors become pendulous and in some instances hang well down over the pubes towards the knees.

FIG. I93.

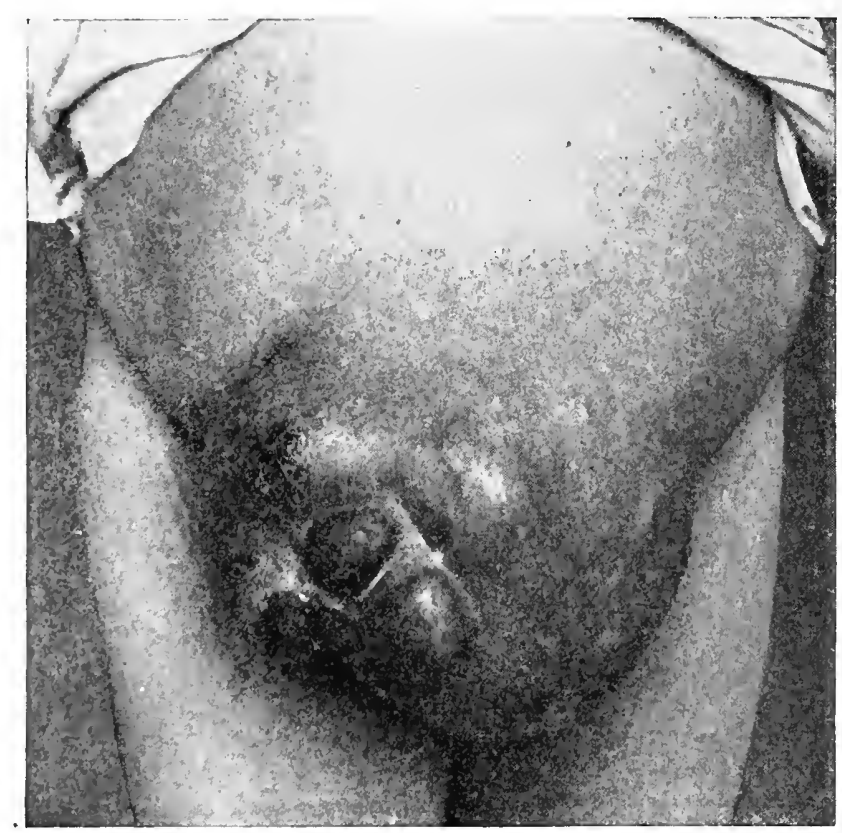

Enormous irreducible umbilical hernia, containing whole of transverse colon and large amount of small intestine, all adherent. Very little omentum present. Dark spots to right of tumor are ulcerations through coverings, allowing leakage of abdominal fuid.

The coverings of umbilical hernia are the skin, superficial, (leep, and subserous fascia, and the peritoneum. The layers of fascire are so thin as to be indistinguishable, and an incision through the overlying skin will almost always open the peritoneum, owing to the intimate union between the outer and inner coverings. When it is considered that intestine is commonly adherent to the peritoneal surface, the importance of 
making the incision well to one side of the tumor will be realized.

Contents of Sac.-In infancy the hernial sac seldom contains anything but omentum or intestine, but in the adult it may, like inguinal hernia, contain any of the abdominal or

FIG. 194 .

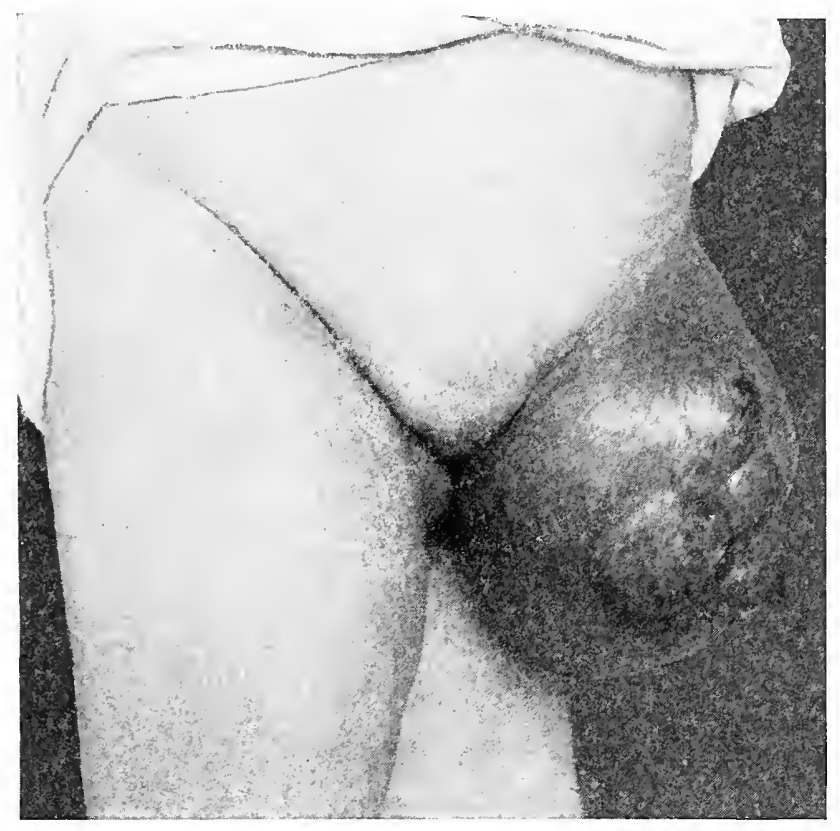

Side view of previous case.

pelvic contents. The omentum or colon are most frequently present. In one case I found the fimbriated extremity of the fallopian tube adherent outsicle of the umbilical ring.

Condition.--Omentum, ordinarily the first to protrude in umbilical hernia, readily becomes adherent. In this position it increases in bulk, both by additions from inside the abdomen and by growth of that part which is retained outside. This hypertrophy is doubtless due to pressure at the hernial opening 
FIG. I 95 .

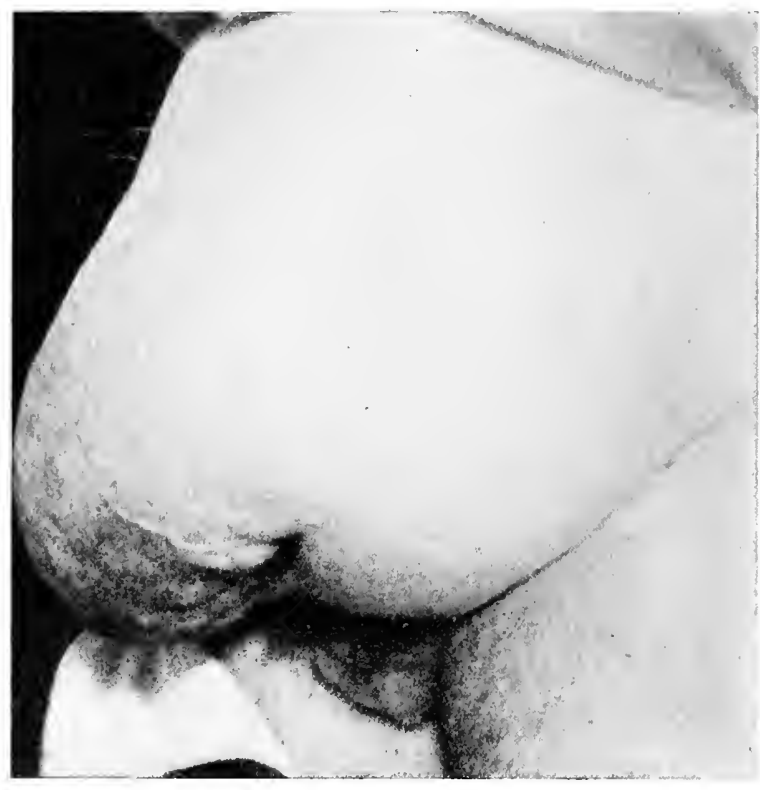

Man of 45 years. Enormous umbilical hernia. Partially reducible.

FIG. ${ }_{96} 6$.

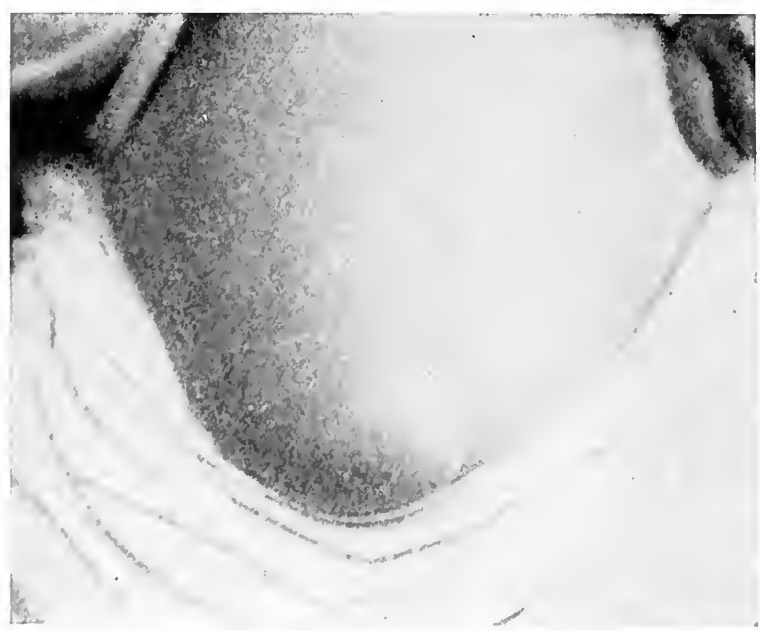

Imbilical heruia (reduchte) in woman weighing about $200 \mathrm{lb}$. 
FIG. 197 .

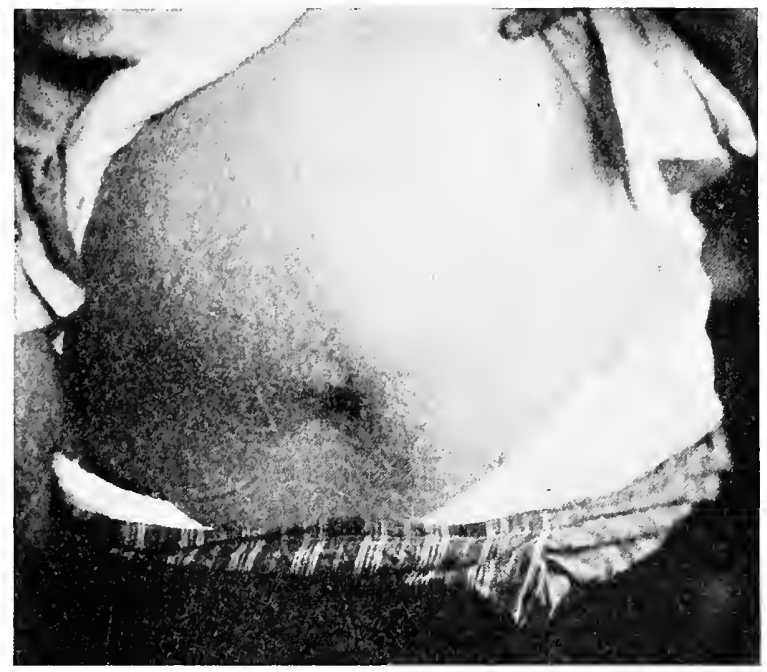

Irreducible umbilical hernia in a woman weighing $229 \mathrm{lb}$. Hernia is larger than the fist, but does not show on account of the thickness of overlying fat. The protrusion is almost entirely to the right of the umbilicus.

FIG. 198.

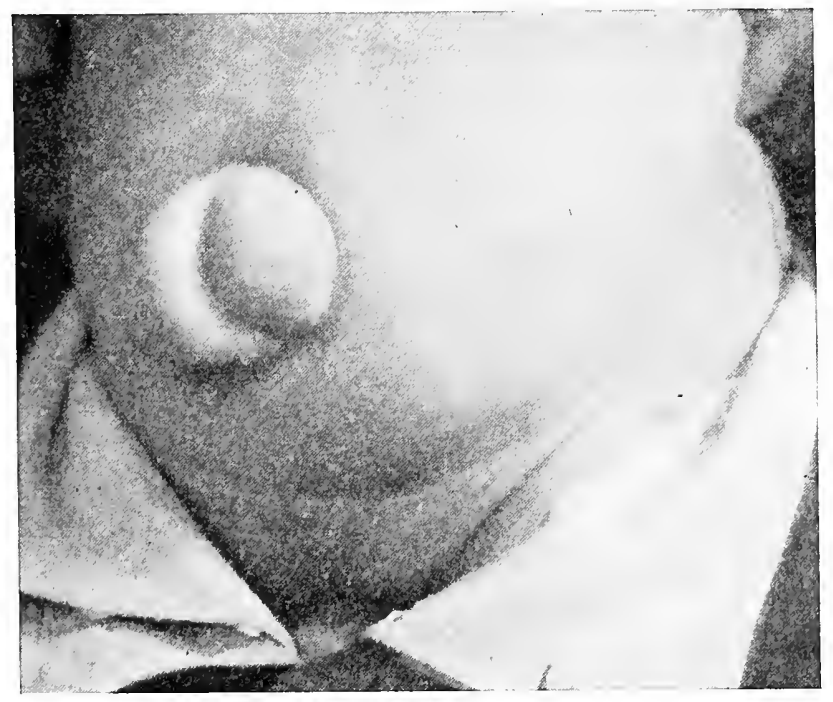

Man 45 years of age. Umbilical hernia irreducible for six years. 
upon the neck of the protrusion, thereby impeding venous circulation, while the arteries continue to pump blood into the parts.

FIG. I99.

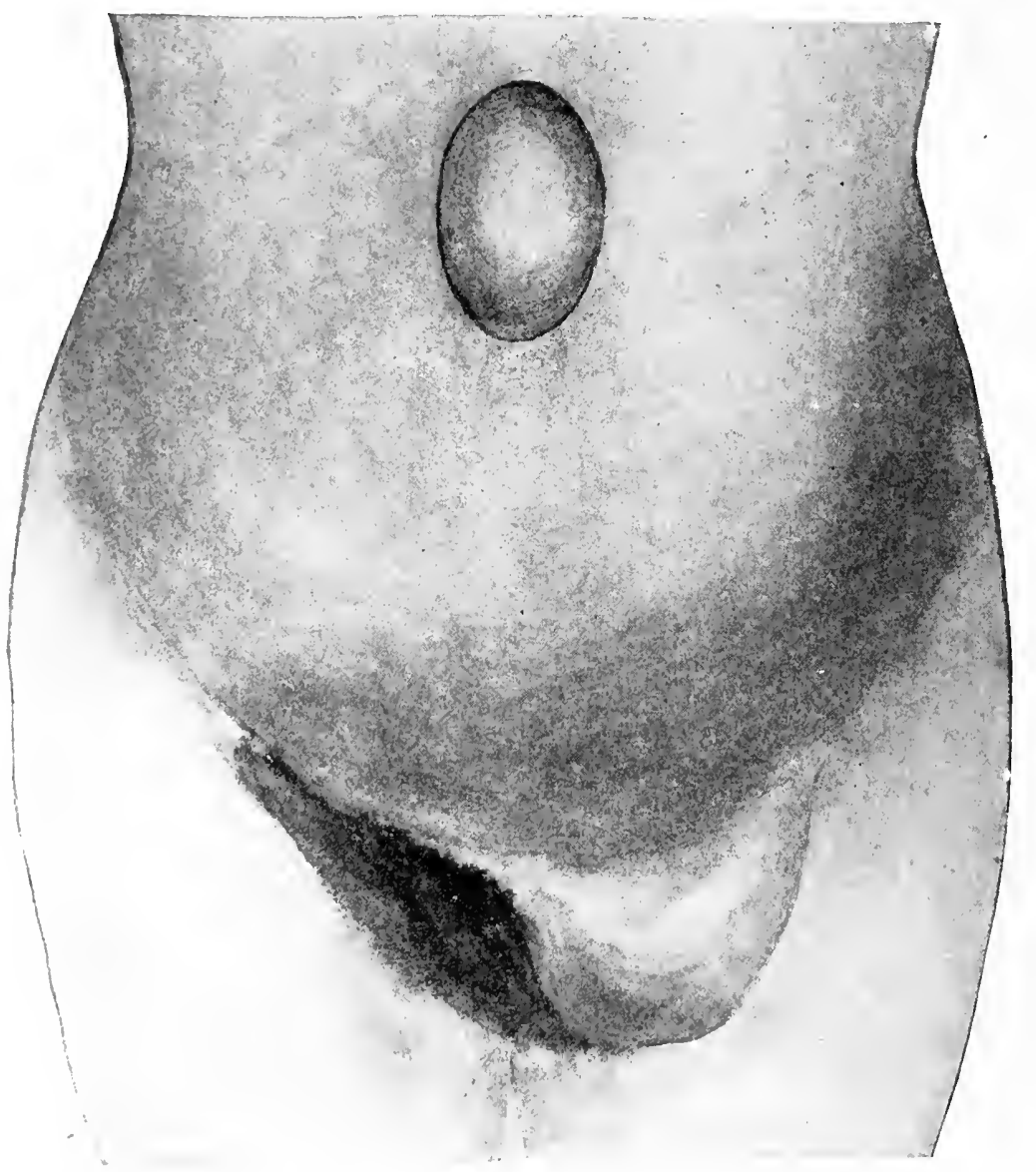

Before operation. Drawing showing the femoral and umbilical hernix, with practically no indication of the presence of the other hernize. (Bainbridge.)

Following the omental protrusion the transverse colon is guite likely to descend and a few loops of small intestine are pretty sure to be present in hernia of large size. The sacs of 
FIG. 200.

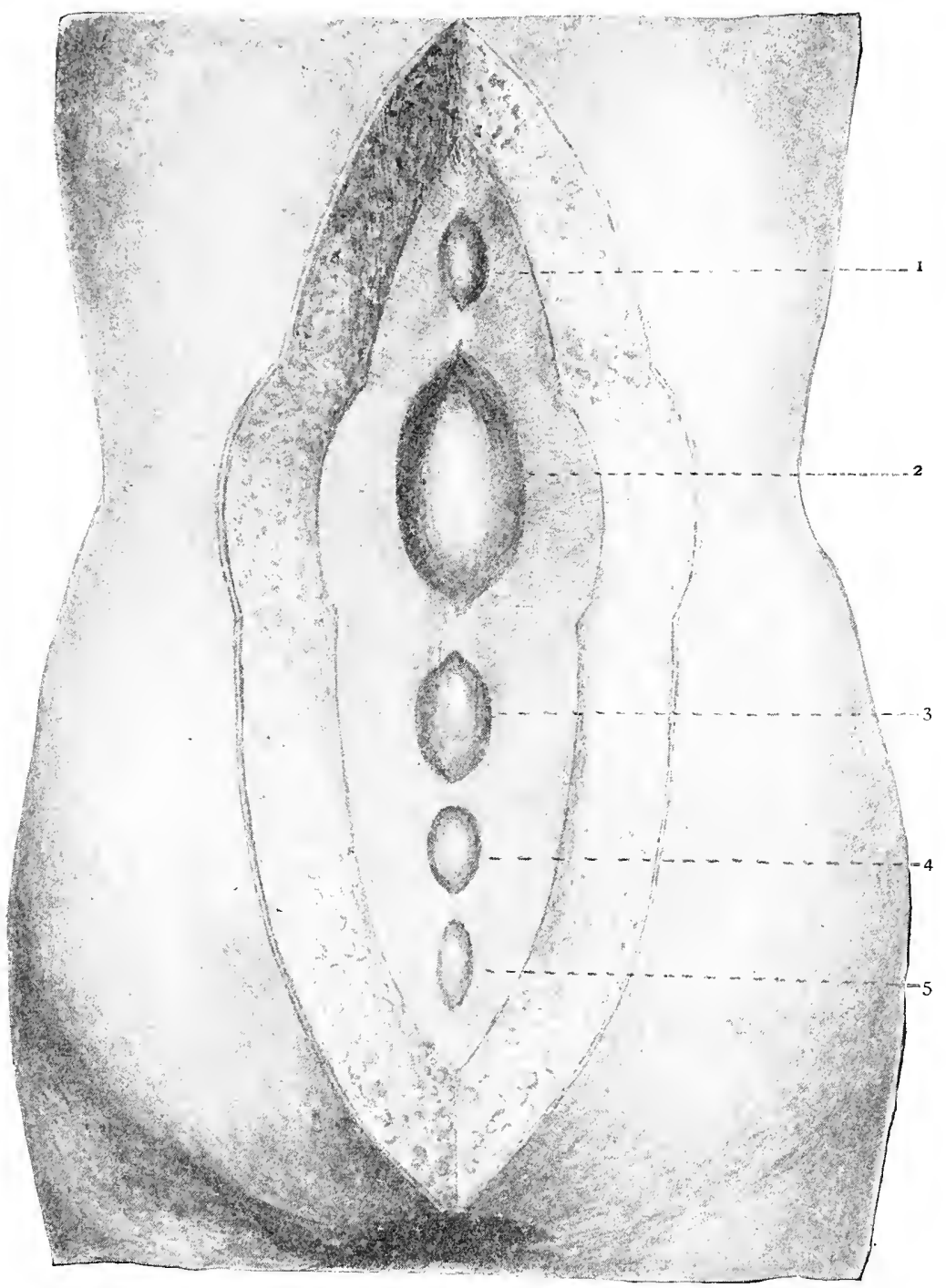

Drawing showing conditions found in central line of abdomen at time of operation. I. Hernia epigastrica. 2. Umbilical entero-epiplocele. 3. Para-umbilical epiplocele. 4. Paraumbilical entero-epiplocele. 5. Lipoma with small epiplocele underneath. (Bainbridge.) 
large umbilical hernia seem especially liable to localized inflammation; this extending to the contents results in a matting together and soon causes the hernia to become irreducible.

Symptoms.-There is little trouble in making a diagnosis of umbilical hernia. In the infant a reducible tumor in the umbilical region may be accepted as an umbilical hernia. An error that is sometimes made is in mistaking an unusually long and prominent navel for an umbilical hernia. Several instances of this kind have been seen by the anthor; one in a girl of fourteen, bronght to him from a distant state. She had worn a truss since one year old for supposed umbilical hernia. No hernia was found, and from the history elicited it was confidently believed that none had ever been present. The navel, about the size of an adult little finger, projected fully threequarters of an inch from the abdominal surface. There was no protrusion inside of this loose skin. Sereral similar cases have been seen in the hernia clinic at the Post-Graduate Hospital.

Aside from the tumor there are few symptoms attending an umbilical hernia in infancy or early chilchood. In the adult, however, it is frequently accompanied by considerable local pain, and gastro-intestinal symptoms are marked. Nausea and even vomiting may be produced when there is no strangulation, and this is believed to be due to traction upon the stomach.

In the larger hernice obstinate constipation, eventually terminating in intestinal obstruction and death, results from the crippled condition of the bowel. Common types of this form of hernia as seen in the adtult are shown in figs. I96, I97 and I98. The case of Bainbridge (The Post-Graduate, February, J905) is of special interest in showing the defective closure throughout the merlian line of the abdominal wall. In this case there cxisterl five hernice in the median line besides a femoral hernia (figs. I99 and 200). 


\section{CHAPTER XIX.}

\section{MECHANICAL TREATMENT OF UMBILICAL HERNIA.}

The discussion of the treatment of umbilical hernia naturally divides itself into a consideration of methods which are palliative or mechanical, and methods which are curative or surgical; this again divides the cases into those occurring in infancy and very early childhood, which are readily cured by palliative means, and those occurring in adult life, when a cure is never obtained except by surgery.

Treatment of Umbilical Hernia in Infancy.-The cure of sniall umbilical protrusions in early infancy is, in some instances, accomplished independently of the family doctor. The grandmother has, on discovering the condition, made a compress of a half dollar, if particularly wealthy, but more frequently of a button-mold, and has held it in place by a bellyband for two or three months, and the hernia has been cured without the doctor ever having known that it existed. This illustrates by what simple methods umbilical hernia may be cured in the new-born child, and makes it seem strange that in the adult its cure can never be obtained, except by a surgical operation.

It has been my habit for many years, both in my clinical and private work, to put no trusses on babies under one year of age, or, in other words, before they begin to walk. They have, with rare exceptions, been treated by a compress over the navel, held in place by one or more strips of zinc oxide plaster. The compress has sometimes, and preferably, been a har,d-rubber umbilical pad, such as is found on infant trusses; sometimes it has been a wooden button-mold, and many times a roll of gauze wrapped about by plaster with the sticky side out so that it would stay just where placed. Fine cork with its edges 
beveled forms an excellent compress. The hernia should be reduced, the compress placed over the navel, and inch-wide strips of zinc oxide plaster placed across it at different angles, the ends extending about two-thirds of the distance around the body. Two strips are usually sufficient and many times one will answer every purpose. It is not well to have the plaster meet in the back, as there is then no allowance for abdominal distension. I have seen a child who had inguinal hernia produced by plaster applied in this manner and the consequent

FIG. 201.

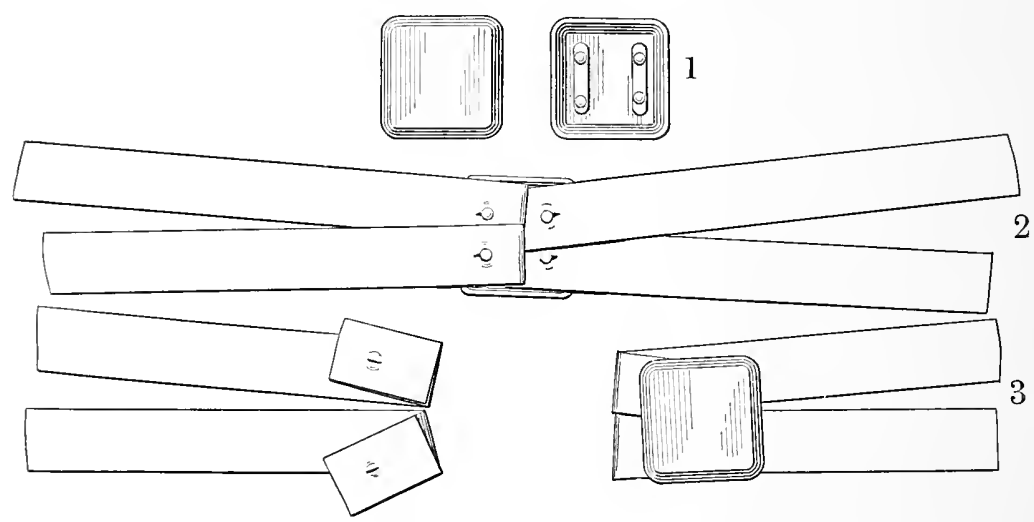

Dr. S. W. Kelley's combination of hard-rubber plate and plaster. I. Hard rubber plate. 2. Plate with plaster attached. 3. Plaster unbuttoned and plate turned back.

forcing of the abdominal contents into the lower abdomen. This dressing should be removed once a week and renewed after the parts have been bathed.

The suggestion of Dr. Samuel IV. Kelley is an excellent one (Ohio State Medical Jour., November 18, I905) of having a hard-rubber pad with buttons on it, folding the front ends of the plaster back upon itself and cutting a button-hole in the plaster (figs. 2OI and 202). The mother can remove the umbilical plate every day for the purpose of washing the skin without disturbing the plaster, and she can easily renew any part of the plaster that may become loosened. In Germany the method 
of using the skin and subcutaneous tissues in the vicinity of the navel as a compress has been adopted and seems to have met with success. The skin and loose tissue on either side of the umbilicus are grasped with thumb and fingers and infolded, the plaster being then placed over. When I first read of the method it impressed me as being particularly good, and I at once adopted it in my clinic; but we found that there was great liability to ulceration of the skin surfaces that were folded together, and soon went back to the use of the compress and plaster, as already described.

FIG. 202.

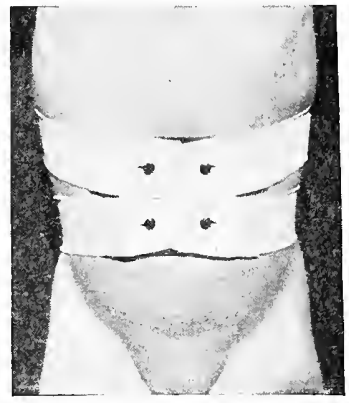

Dr. S. W. Kelley's method of combining plaster with hard-rubber plate. Plaster is foided back upon itself, and has button holes cut in it.

The length of time required to cure an infant of umbilical hernia seems to depend somewhat upon its age. At three months of age it can ordinarily be cured in three months, but at six months of age it will frequently require six months, while a full year of mechanical support is usually required after the child walks. When it is a year old I seldom resort to the plaster and compress, experience having proven that a light spring controls the hernia with greater certainty. In the group of umbilical trusses for infants will be seen several good forms. My preference with very small children is for the single-spring trusses covered with either hard rubber or celluloid so that they can be kept perfectly clean and worn in the bath. In children 


\section{Grolp of Infant Libilical Trusses.}

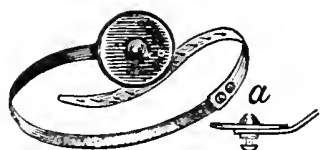

I. Infant's hard-rubber single-spring umbilical truss. Sizes, Io to 21 inches.

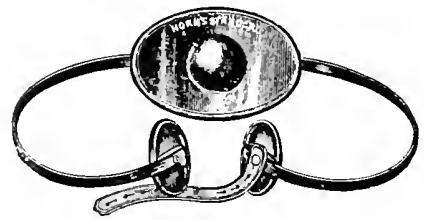

3. Youth's double-spring hard-rubber umbilical truss.

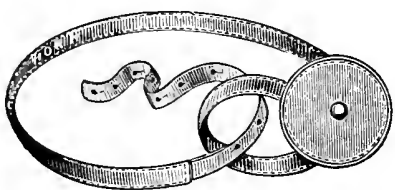

5. Fine French kid or cedar pad. umbilical truss.

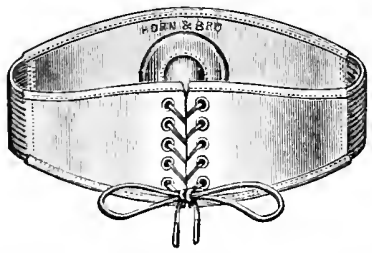

7. Umbilical belt truss, sateen band, elastic sides to lace in back. Kid or liardrubber pad.

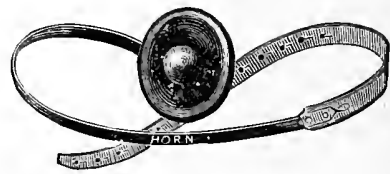

2. Infant's hard-rubber single-spring umbilical truss. (Youth's sizes, 22 to 29 inches.)

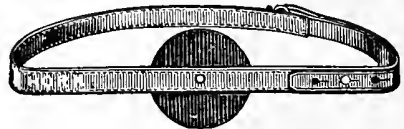

4. Bow spring (leather covered) cedar pad umbilical truss.

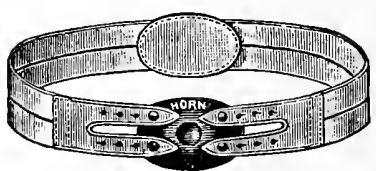

6. Double band elastic hard-rubber plate umbilical truss.

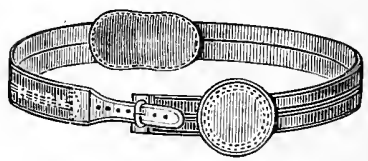

8. Elastic umbilical truss, hard-rubber cedar, or kid pad.

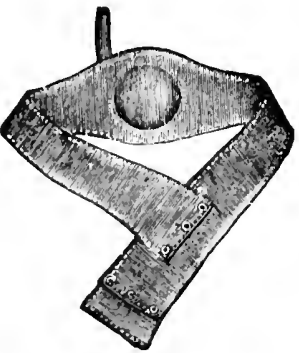

9. Soft-rubber belt with inflated air pad. 
of three years and over I an quite partial to light double springs with the fastening in the back, as shown in fig. 203.

The selection of the pad or button that presses into the umbilicus is a matter of importance. If the child is very fat the centre projection on the umbilical pad must be quite prominent, in order to reach down to the abdominal wall; if very thin such a prominent centre would do actual harm by wedging

FIG. 203.

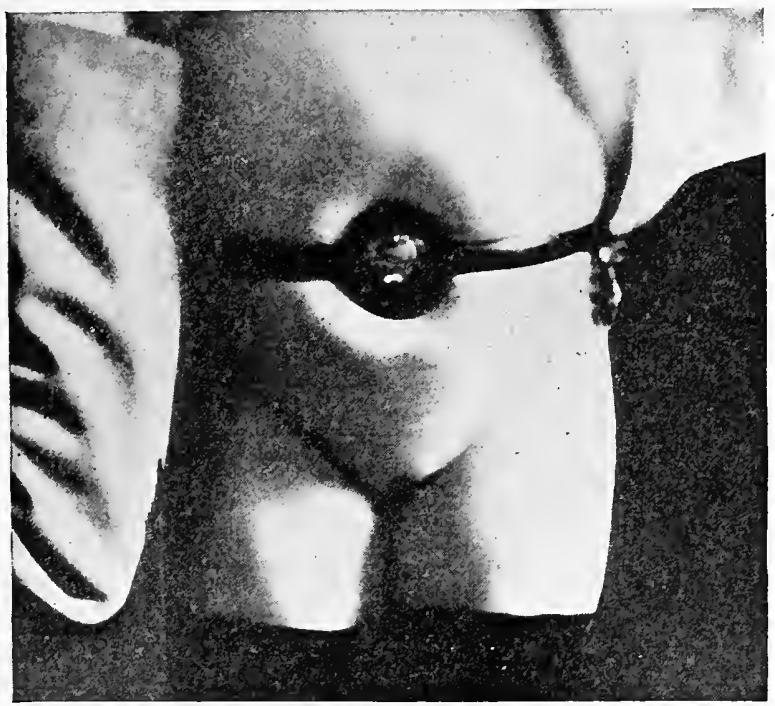

Umbilical hernia (in a child 3 years old) retained by a hard-rubber truss, the spring going around the body on both sides.

itself into the umbilical ring and preventing closure. Some-times in very thin children the use of a perfectly flat surface is attended by better results. As in inguinal hernia, these cases must be kept under the frequent observation of the physician in order to obtain good results. The children are growing rapidly, and this growth must be provided for. Here, also, as in inguinal hernia, infants are good truss wearers if the skin is kept perfectly ciean and dry. 
Children under five years of age are almost always cured by the means suggested if they are kept under care; the curability of umbilical hernia by mechanical support, however, diminishes rapidly after passing the third year, and, while in recent cases a few cures may be obtained, even in children ten or twelve years old, this fortunate result has been very rare in my experience. It has been my practice to recommend operative cure in all children who have passed the tenth year, and in some mich earlier, where there appeared little prospect of curing by means of the truss. Where springs are used their accurate adjustment will be greatly enhanced by resort to the lead-tape diagram method, which has been described under the mechanical treatment of inguinal hernia.

Mechanical Treatment of Umbilical Hernia in the Adult. - In the adult umbilical hernia is unquestionably the most difficult of all hernize to treat either mechanically or surgically, and for this reason its occurrence should always be looked upon as a serious matter even though the hernia be insignificant in size and giving no immediate discomfort. In fact, the most prompt and persistent treatment should be insisted upon from its very inception in order to protect the patient against the many ills and clangers that are sure to follow its neglect.

While there is not such a large variety of trusses made for this form of hernia, there are several good ones from which to select in order to meet the special indications of the case. In small hernia upon a person of medium weight, the single spring trusses are lighter, more convenient and consequently better. Little dependence can be placed upon trusses made of elastic bands, and cantion in their use is therefore advised. In those cases where it seems advisable to use a truss at night they answer the purpose admirably, but under their use during the lay most cases grow worse.

In selecting springs preference shotild always be given to those covered by hard rubber or celluloid. In shaping them it must be borne in mind that those covered with hard rubber must be warmed before bending. What has been said about 
Group of Adult Umbilical Trusses.

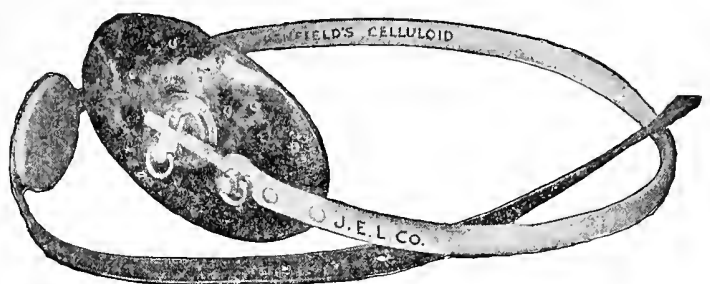

I. Celluloid single-spring umbilical truss.

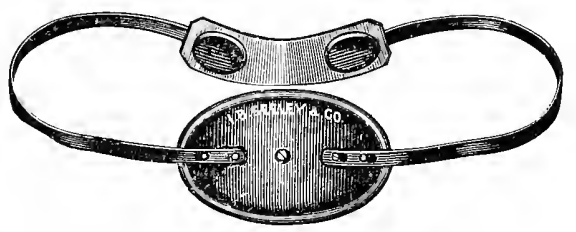

2. Double-spring hard-rubber umbilical truss.

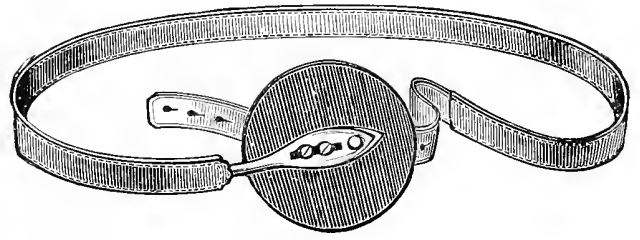

3. Chase umbilical truss. Leather cover, cedar pad. 
Group of Adult Uabilical Trusses (Continued).

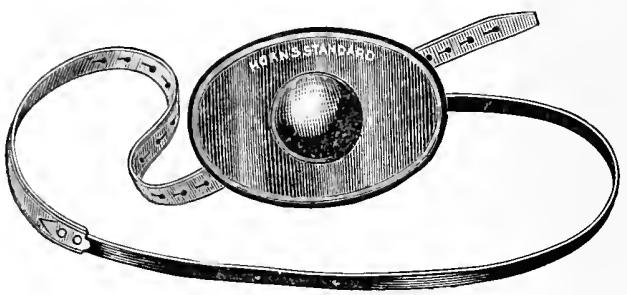

4. Single-spring hard-rubber umbilical truss.

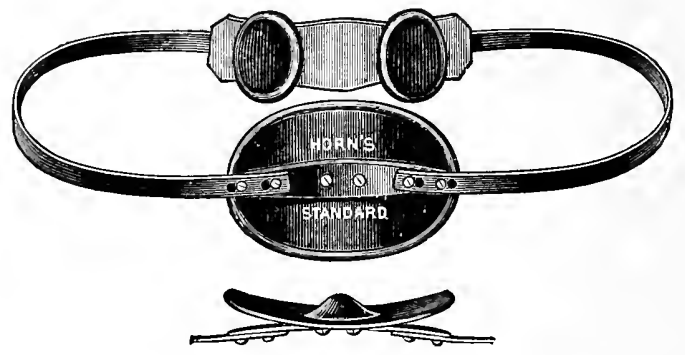

5. Elliptic double-spring hard-rubber umbilical truss.

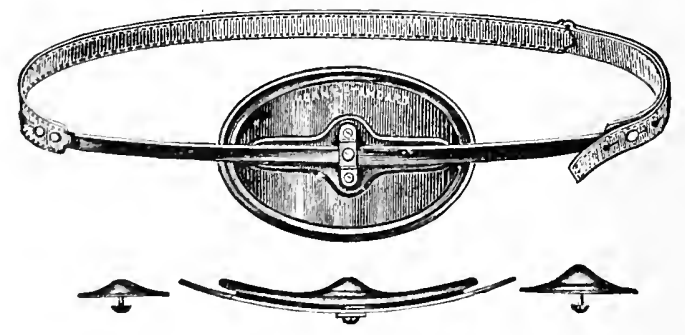

6. Bow-spring hard-rubber umbilical truss. 
MECHANICAL TREATMEN'T: UMBILICAL. 363

Group of Adult Umbilical Trusses (Continued).

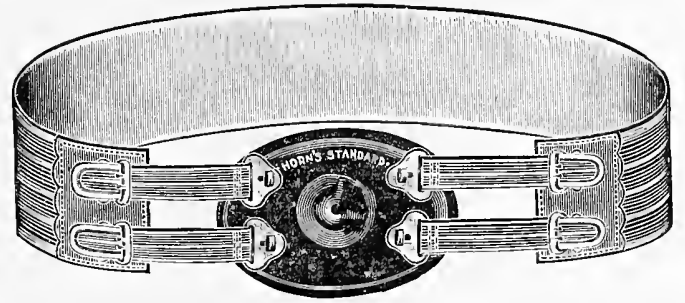

7. Elastic umbilical truss.

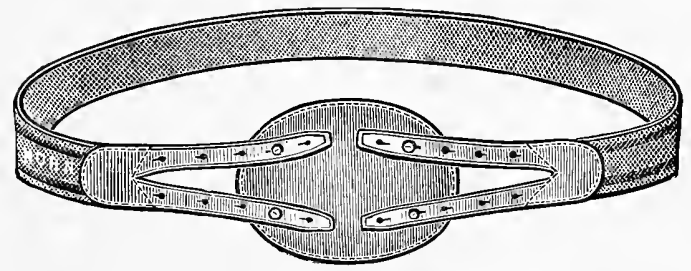

8. Narrow band elastic umbilical truss.

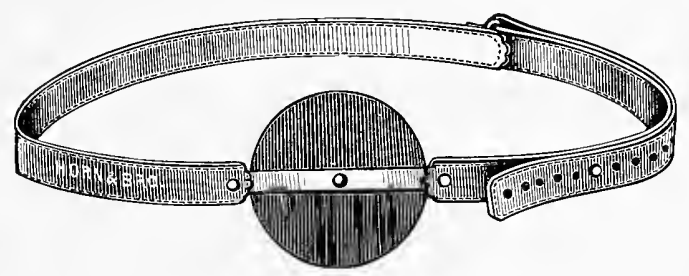

9. Bow-spring leather umbilical truss:

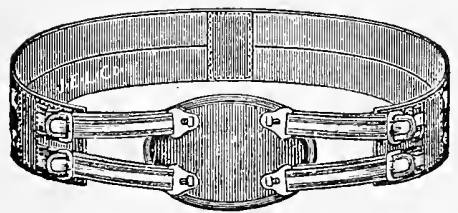

Io. Elastic umbilical truss, celluloid plate.

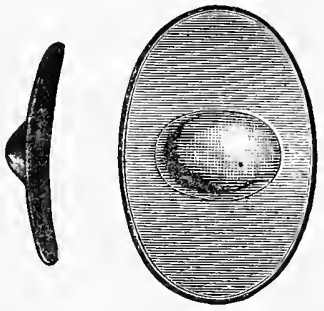

Concave hard-rubber pads. 

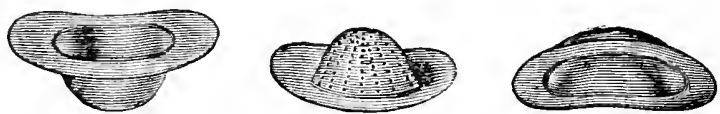

Concave hard-rubber pads for use inside of abdominal belts in irreducible umbilical hernia.

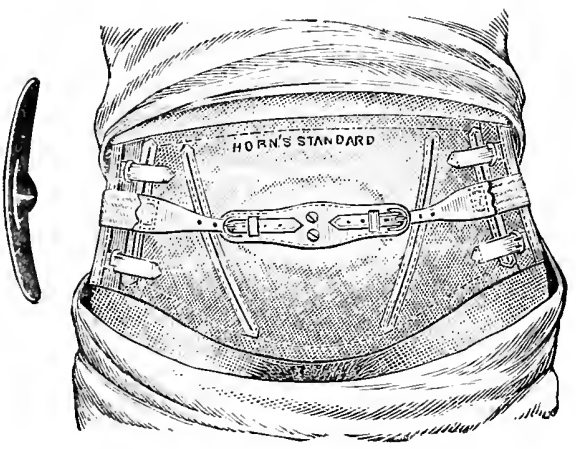

11. Combination belt and umbilical pad.

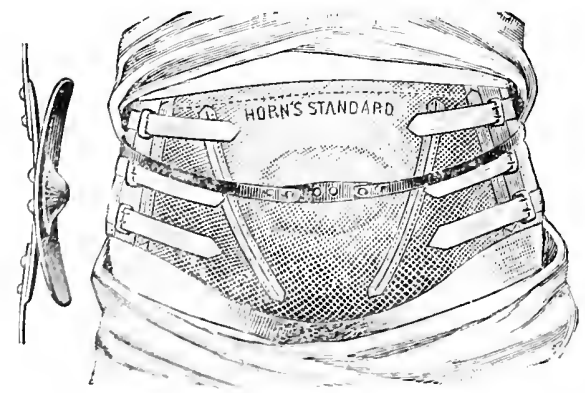

12. Combination belt and umbilical pad with springs outsicle of belt. 
MECHANICAL TREATMENT: UMBILICAL. 365

\section{Group of Adult Umbilical Trusses (Continued).}

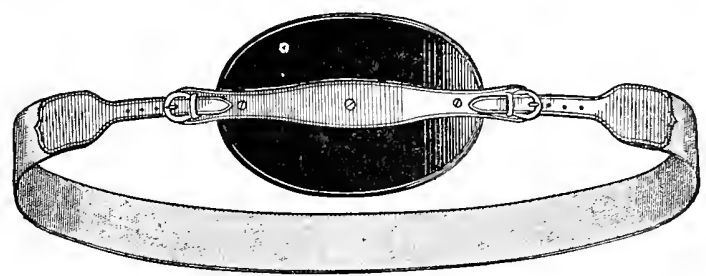

13. Extra hard-rubber pad, elliptic spring, and band to be used in combination with abdominal belt.

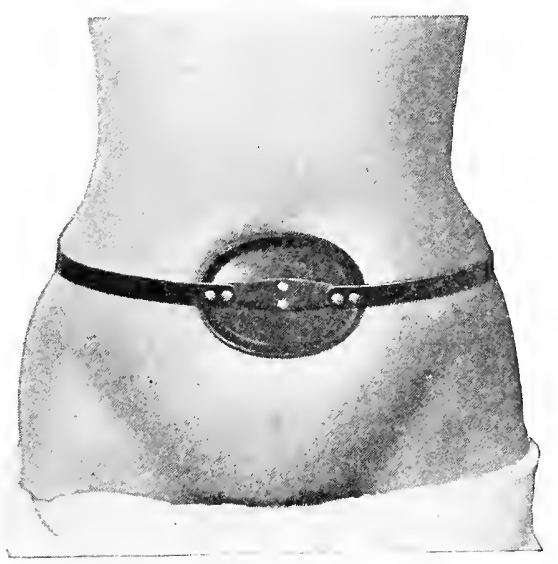

14. Hard-rubber elliptic-spring umbilical truss applied.

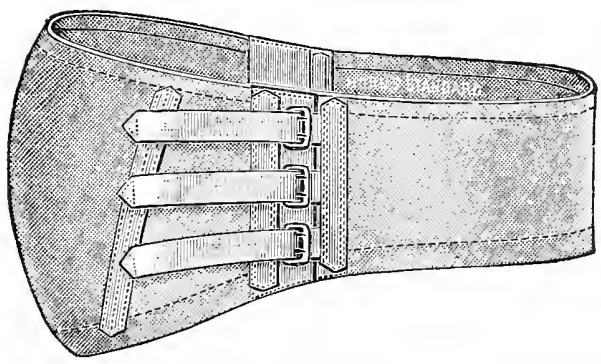

I5. Belt for general abdominal support, to which may be added umbilical pad and extra retaining band. Woven silk or cotton-covered thread. 
Ortumes of Umillical Plates and Centres.

Showing Actual Sizes.

FIG. 20.4.

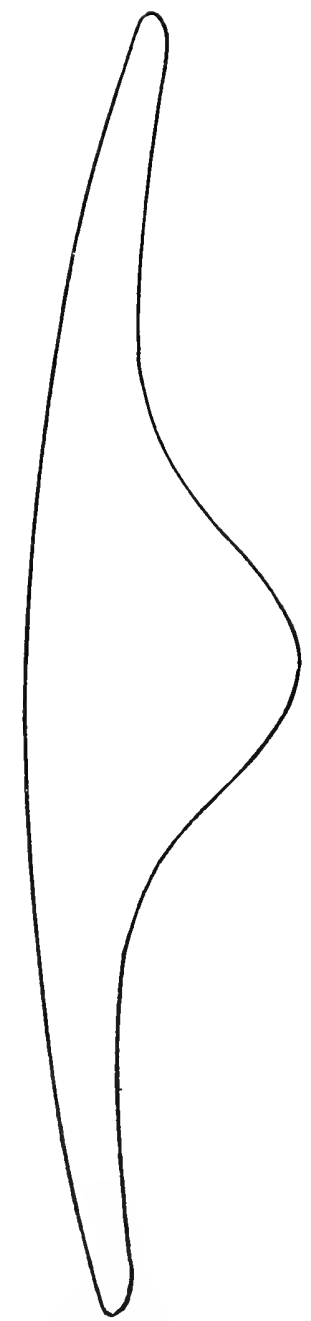

Solid cedar pad, $5 \%$ inches.
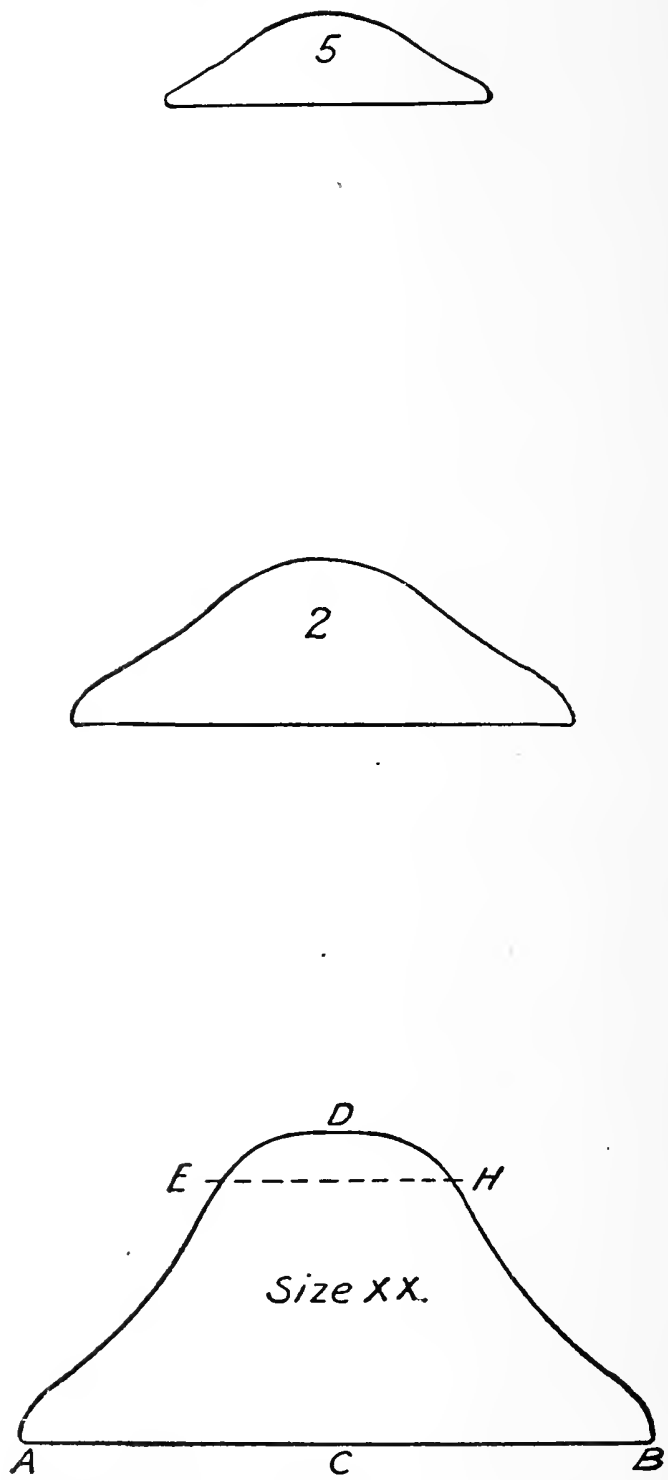

Ilard-rubber (hollow) navel centres for the umbilical pad. 
MECHANICAL 'TREATMEN'T: UMBILICAL. 367

selecting a centre-piece for the plate suitable for the individual child applies with even greater force in the case of the adult. Fat people must have a deep centre and thin persons one that

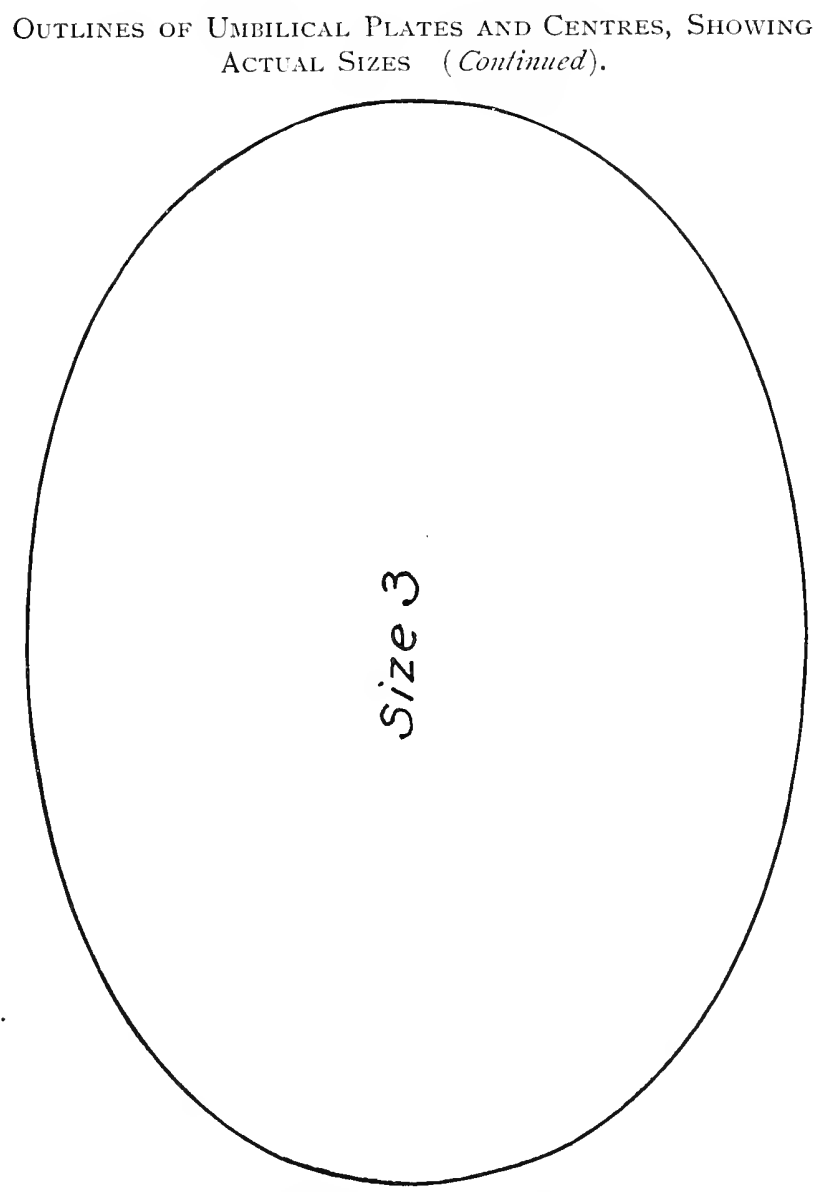

Hard-rubber plates. Size 3 is $4 \frac{3}{3} \times 3^{\mathrm{T}} / 8$ inches; size 2 is $6 \times 4 / 4$ inches, and size $\mathrm{I}$ is $7 / 4 \times 5$ inches, on which are used seven sizes of detachable centre pads, of which size $\mathrm{xx}$ is the largest and size 5 the smallest.

is not so deep. (See fig. 204 for an illustration of the size of plates and centre-pieces as ordinarily manufactured by the truss makers.) 
For fat and heary patients the double-spring truss will afford a firmer and more secure support. In these trusses the springs fasten on stud-head screws that are solidly fixed in the plate, but where very strong pressure is desired the one with an elliptic spring is better. This spring is attached in its

F1G. 205.

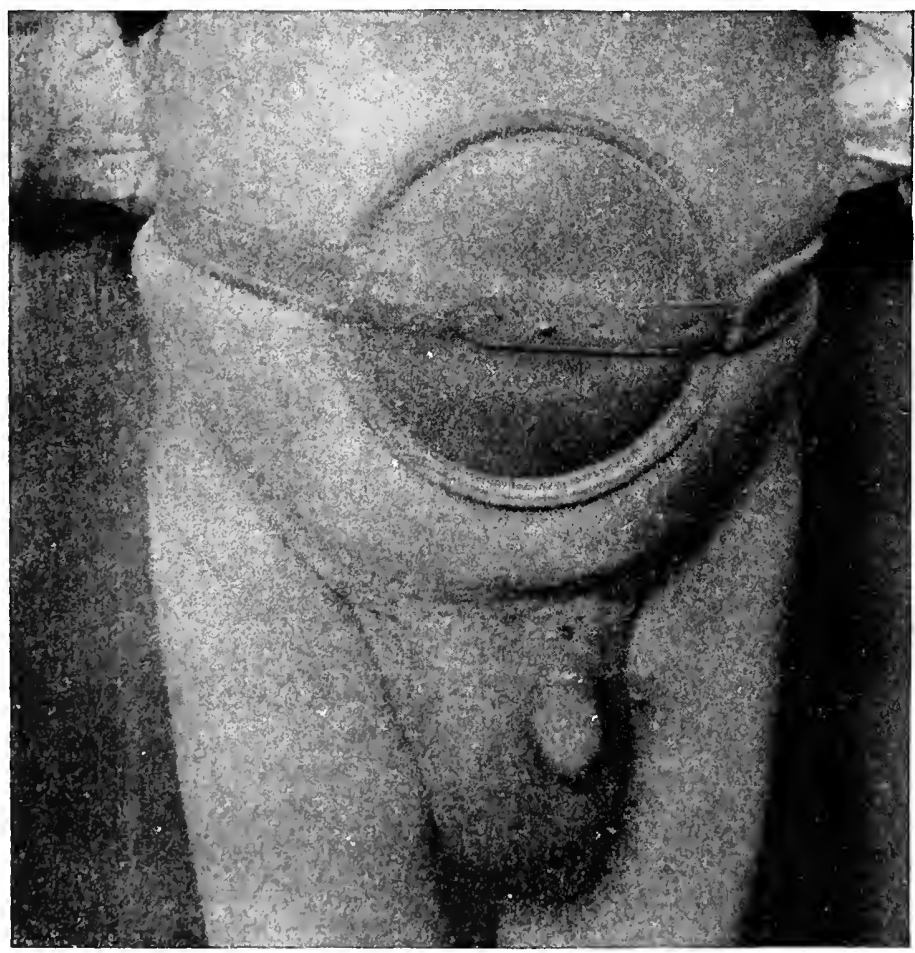

An English umbilical truss, adjusted. (Eccles.)

miclle and the encls arch out away from the plate. When the burly springs are fastened upon the stud-head screws in the ends of the elliptic spring its action is to throw the plate farther in, griving greatly increased pressure. In extrenely large umbilical plate (fig. 205), with a prominent centre, is used in Englanrl. The whole truss is thickly covered with leather, and 
compared with those made in this country is a cumbersome affair.

Where the whole abdomen is pendulous, it is frequently desirable to combine general abdominal support with retention of the hernia, as shown in the illustrations. This is an

\section{FIG. 206 .}

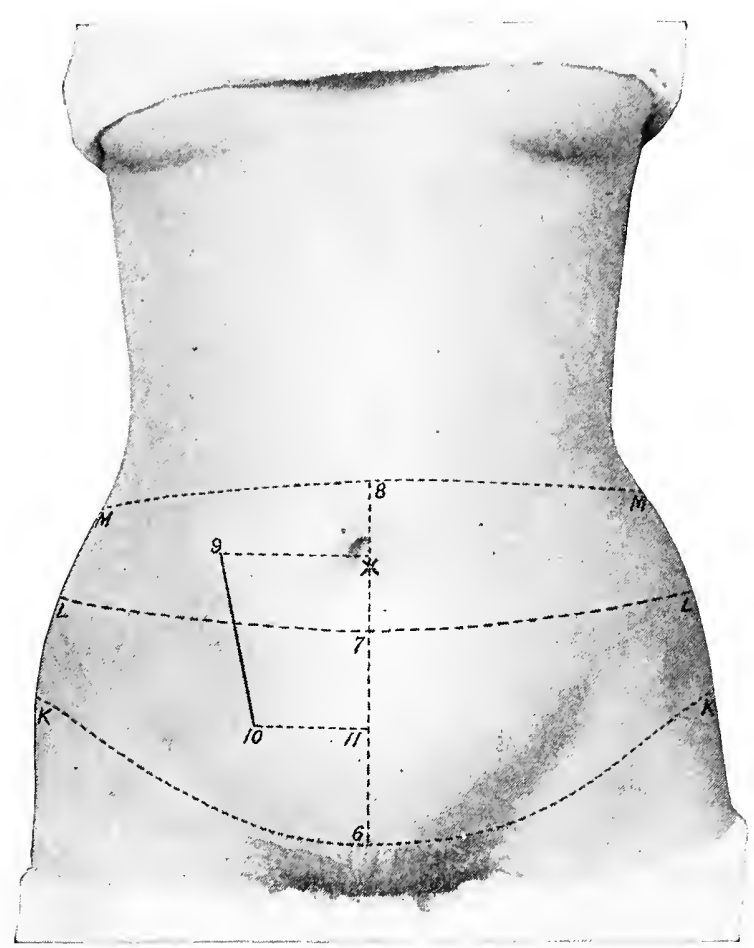

Diagram for abdominal belt and umbilical truss measure.- - . Umbilical hernia: Circumference on line with navel and shape by lead tape (see Truss-fitting, Inguinal hernia) on same line. 2. Abdominal belts: Circumference at $\mathrm{K}, \mathrm{L}, \mathrm{M}$; length from 6 to 8 . If an umbilical plate is to be added, give distance from 6 to navel. 3. Ventral hernia belts: Circumference M, L, K, 6 to 8. Distance of top of pad from navel to 9, of bottom of pad, Io to II.

admirable combination for corpulent people suffering from large reducible or irreducible umbilical hernia. The belt not only gives valuable general support, but prevents the truss 
springs becoming imbedded in the fat. The umbilical plate, being inside the belt, is also held more securely in place. Where a belt is ordered as a part of the support, careful measures of the abdomen should be forwarded to the manufacturer. The diagran shown in fig. 206 will aid the physician in taking these measures, and the letters which designate the points of circumference are understood by all reputable manufacturers.

Hernize containing large omental protrusions are very liable to inflammatory conditions, which may be mistaken for true strangulation. This inflammation is ordinarily due to pro-

FIG. 207.

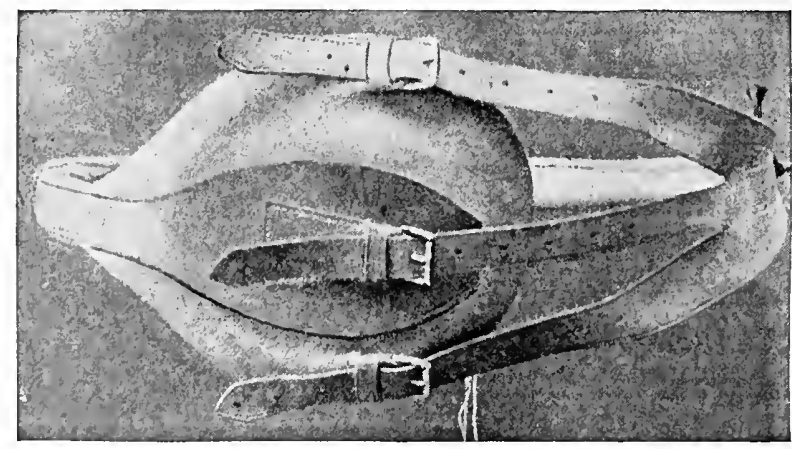

English rim-plate concave-pad truss for irreducible umbilical hernia. (Eccles.)

trusion of a new mass of omentum and its constriction at the hernial aperture. If allowed to follow their own course, sloughing is liable to occur. Long continued hot applications I feel sure favors this result. I have found the use of ice more satisfactory, and have kept it on for several days at a time, with the most happy results. Let it be distinctly understood that I (i) not approve of any such delay if there is the least indication of intestinal obstruction. By the application of ice and daily grentle manipulations, the hernia can frequently be restored to its former condition.

Irreducible Umbilical Hernia.-This leads to the mechanical treatment of those cases which are only partially reducible. 
$\Lambda$ few of these can, by confinement to bed and repeated gentle taxis, be converted into reducible herniæ. At least an attempt should be made to reduce all that is possible; then a concave pad, fitting exactly the remaining protrusion, and attached to one of the springs already described, should be applied. In large people, the combination of belt and spring with concave pad of suitable size, is especially desirable. After wearing one of these concave pads over an irreducible hernia, the protrusion may so diminish in size that a smaller pad will be required. The English use a form of truss shown in fig. 207, which, it would seem, might be useful in the cases under consideration.

The treatment of irreducible umbilical hernia by mechanical means is not attended by an amount of success that is encouraging. In fact the history of such cases is one of constant increase in size, discomfort, and danger. Unless there is some special contra-indication it will be best to advise such patients to submit to surgical treatment. 


\section{CHAPTER XX.}

\section{SURGICAL CURE OF UMBILICAL HERNIA.}

Those having little practical experience will find the literature of this subject confusing, and in many instances misleading, inasmuch as authors differ so widely in their statements. We are assured by some, who should speak with authority, that the operations for the cure of umbilical hernia are particularly dangerous and usually followed by failure to obtain a cure. Then again men of such ripe experience as Dr. George Ben Johnson of Richmond tells us (Medical Register, August I 5, 1897) that most authors are too conservative. Dr. Johnson says, "I am astonished at the cautious manner in which some of our best authors advise the procedure." Those of us who know his work know that Dr. Johnson is himself a conservative man; we also know that some of these umbilical cases are particularly dangerous cases for operative treatment. The truth is that we cannot speak positively upon the subject as a whole; we must attempt to separate and recognize those cases that are safe for operation, and when we are forced to operate upon those that are dangerous we should do so with a full understanding of the responsibilities involved.

As previously stated, most umbilical herniæ of early life need never come to the operating table, if given proper mechanical support for a suitable length of time. In some instances, however, the muscular defect is too great to be overcome and the question will arise as to the advisability of operative relief. These cases may all be classed as safe for operation, and a permanent cure is quite certain to follow. I have no knowlerlge of a fatality or a failure following an operation on young patients.

Operations upon adults of middle and advanced life must be consirlered more carefully, and examination of Dr. Johnson's 
" excluded" list will at once show the reason of his positive and favorable opinion. Here are those who, according to his opinion, should not be operated upon: "The old and feeble, where there is extensive separation of the recti muscles below the umbilicus, where there is so much hypertrophied omentum that its removal would prove dangerous to life, where there is extreme atrophy of the surrounding muscles." To this I would add the excessively fat, who are liable to degeneration of the heart as well as other muscular structures. We at once recognize the fact that a large percentage of umbilical herniae as seen in the adult come within the limits of this excluded list, and should therefore be considered as extra-hazardous as regards danger to life and the permanence of cure.

My personal views have changed materially within the past ten years. Formerly I was inclined to avoid the surgical treatment of umbilical hernia in adults. I have, however, had the mortification of seeing some of those same cases, through neglect of the patient or my own inability to so adjust support as to control the hernia, grow gradually but surely worse. When first seen they might have been operated upon with a small amount of danger, but later that risk has increased many times. For this reason I have come to feel that all cases of umbilical hernia should, if possible, be operated upon while the protrusion is small. The larger these hernia become the greater are the dangers attending their cure, and the poorer are the structures which are used in effecting closure of the hernial ring.

Operation.-The preparation of the patient should be made with all the extreme care that would be exercised in any other laparotomy. Even greater attention must be given to the sterilization of the skin, which is frequently in bad condition either from ulceration due to over-distention, or from the effects of truss-wearing.

Many authors direct that a vertical incision shall be made over the most prominent part of the tumor. This, I am convinced, is fratght with great danger. The incision should 
rarely, if ever, be made in the median line, as at this point the skin and sac are usually so intimately united as to make their separate division impossible; furthermore, the intestine may be adherent to the interior of the sac and opened at the first sweep of the knife. The incision should be elliptical in form, beginning well above and spreading out on each side of the narel so as to avoid to a great extent the risk of opening into the sac. The dissection of the subcutaneous fat can be rapidly carried down to the aponeurosis and then carefully upon this surface towards the neck of the sac. When the neck of sac thus apprcached is freed on all sides it can be lifted up and an opening made through it to examine its contents, all reducible parts having been previously returned to the abdomen. If the sac is free it can at once be cut away at its neck, leaving the adherent fundus attached to the removed ellipse of skin. If on opening the side of the sac a mass of adherent omentum is found, it is best to draw it out through this opening, ligate it by multiple ligatures, cut it away and reduce the stump. Then cut away the sac, which will be removed with attached omentum, skin, and umbilicus. This method saves much valuable time that is lost when opening directly into the top of the sac: If it is found that intestine is adherent then it is better to carefully extend the side incision into the sac, laying it wide open. Patches of sac firmly adherent to the bowel should be cut out and left upon the intestinal surface rather than incur risk of serious laceration by attempting to strip them loose. In cutting away the sac it should be far enough from the abdominal surface to leave plenty of material for closure of the peritoneum.

The method of closing the umbilical ring should depend largely upon the conditions found. If the surrounding structures are of normal thickness, the stripping away of the peritoneum from the edges of the ring, its closure by plain catgut, and then the splitting of the aponenrotic structures into two layers and their separate closure, preferably by kangaroo tendron, and finally the closure of the skin, will result in an effective repair of the distended ring. 
If the case is one where intra-abdominal pressure will be great and liable to tear the sutures through the tissues, put in before closing the tendinous layers, three or four relaxation sutures of silkworm gut. These are put in by a long needle between the layers, if the wall has been split, or just outside of the peritoneum if not, and extended well back from the wound on either side. They are not tied until the other steps in the operation are complete.

The wound is now closed in the following manner: Peritoneum by continuous suture of catgut. Each layer of abdominal wall separately, by heavy strands of chromicized kangaroo tendon, and skin by subcutaneous catgut. The relaxation sutures are then drawn tight and tied. These are cut and removed about the tenth or twelfth day. The usual dressings under a firm binder are applied. This is not disturbed for ten days, unless there is pain or elevation of temperature.

Overlapping of Abdominal Wall.-At the meeting of the Medical Society of the State of New York at Albany, February I, I899, in a paper on the treatment of umbilical hernia, I made the following statement (Transactions $M c d$. Soc., State of New York, I899): "In several cases where the abdominal wall was thin, and intra-abdominal pressure not great, I have succeeded in overlapping the cut edges, so that, when complete, it gave two layers of tendinous structure in the median line. I am quite partial to this method, but in some cases it cannot be used on account of the extent to which the abdominal cavity is thereby diminished."

I had previous to I899 operated upon 6 cases by this method, my first having been on March 9, I896, at the New York Post-Graduate Hospital. This statement is made definitely because of the fact that American writers have recently been accused (P. T. Diaknow, M.D., Annals of Surgcry, July, I906) of not giving credit to Russian authors whose first writings were according to Dr. Diaknow, by himself in I898, and Dr. Sapiejhko (Annals of Russian Surgery) 
in 1900. The truth is that every operator of good surgical common sense would be very likely sooner or later to see his opportunity of making a closure of this type, and without feeling that he had made any great discovery. Dr. Joseph A. Blake of New lork was perhaps the first to demonstrate the method properly by drawings showing its technique (Mcdical

FIG. 20S.

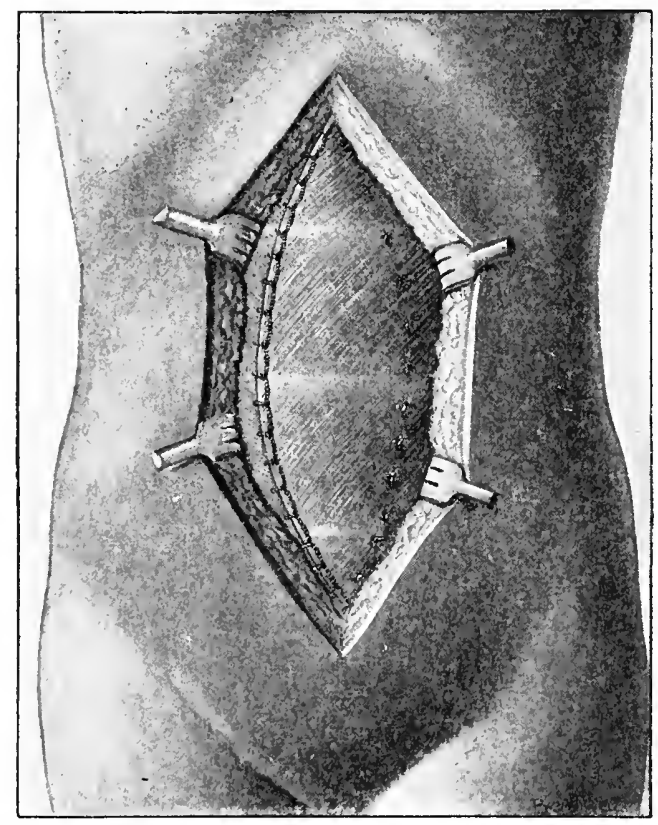

Vertical overlapping of abdominal wall in umbilical and ventral hernia. (Size of incision exaggerated.)

Record, May 25, I901). His claims for it were as follows: "( (2) The breaking of the lines of suture. (3) The broad surfaces for union. (4) The obliteration of the separation of the recti, and the reduction in the size of the abdomen."

After several years' experience with this operation $I$ feel that the claims made for it are fully justified. The method is 
executed as follows: After the removal of the sac, the linea alba, with the peritoneum, is divided for one and a half or two inches above and below the umbilical ring. The peritoneum is separated from the abdominal wall on both sides to whatever distance the overlapping can be done, and it is then closed in the median line by a running suture of catgut. The denuded aponeurosis of the right side is slipped under that of the left and fastened there by interrupted sutures. Kangaroo tendon is preferred for this purpose. The other edge of the flap is now united to the right side by a running suture of the same material (fig. 208). Blake used the interrupted suture for the closure of both lines. The skin is closed in the usual way. This gives a broad surface of union and has proven protective in cases that seemed almost hopeless so far as permanence of cure was concerned. In some instances the intra-abdominal pressure is so great as to make it almost impossible to get the material with which to do this overlapping. These cases should, if possible, be recognized before they come to the table and the pressure relieved by light diet for some days before the operation, and the complete freeing of the intestinal tract by cathartics.

Dr. William J. Mayo of Rochester, Minn., prefers to do the overlapping in a transverse direction and his operation has been largely followed in the West. My own experience with it would not lead me to adopt it as a routine method. $\mathrm{He}$ gives the steps of his operation of transverse overlapping of the abdominal wall as follows (Jour. A.M.A., July 25, I903): "( I) Transverse elliptical incisions are made surrounding the umbilicus and hernia; this is deepened to the base of the hernial protrusion.

(2) The surfaces of the aponeurotic structures are carefully cleared two and a half to three inches in all directions from the neck of the sac (fig. 209).

(3) The fibrous and peritoneal coverings of the hernia are divided in a circular manner at the neck, exposing its contents. If intestinal viscera are present, the adhesions are separated 
and restitution made. The contained omentum is ligated and removed with the entire sac of the hernia, and without tedious dissection of the adherent omentum.

(4) An incision is made through the aponeurotic and peritoneal structures of the ring, extending one inch or less

\section{FIG . 209 .}

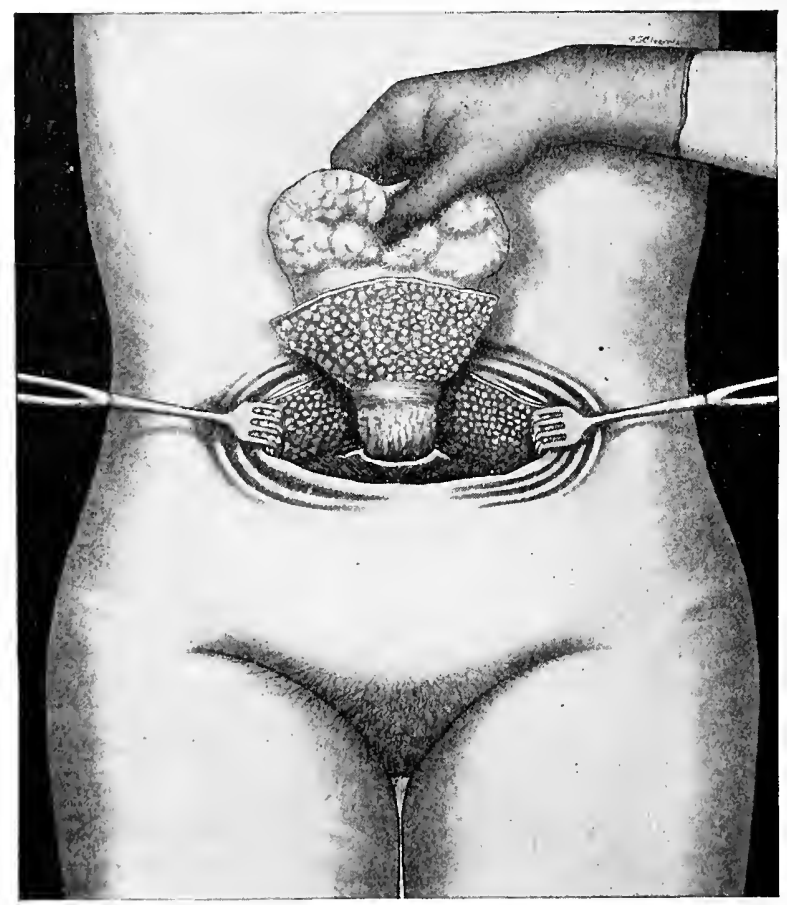

Transverse elliptic incision to aponeurosis, and circular division of sac neck. (Mayo.)

transversely to each side, and the peritoneum is separated from the under surface of the upper of the two flaps thus formed.

(5) Beginning from two to two and one-half inches from the margin of the upper flap, three to four mattress sutures of silk or other permanent material are introduced, the loop firmly grasping the upper margin of the lower flap; sufficient traction is marle on these sutures to enable peritoneal approximation 
with running suture of catgut. The mattress sutures are then drawn into position, sliding the entire lower flap into the pocket previously formed between the aponeurosis and the peritoneum above (fig. 2IO).

FIG. 2 IO.

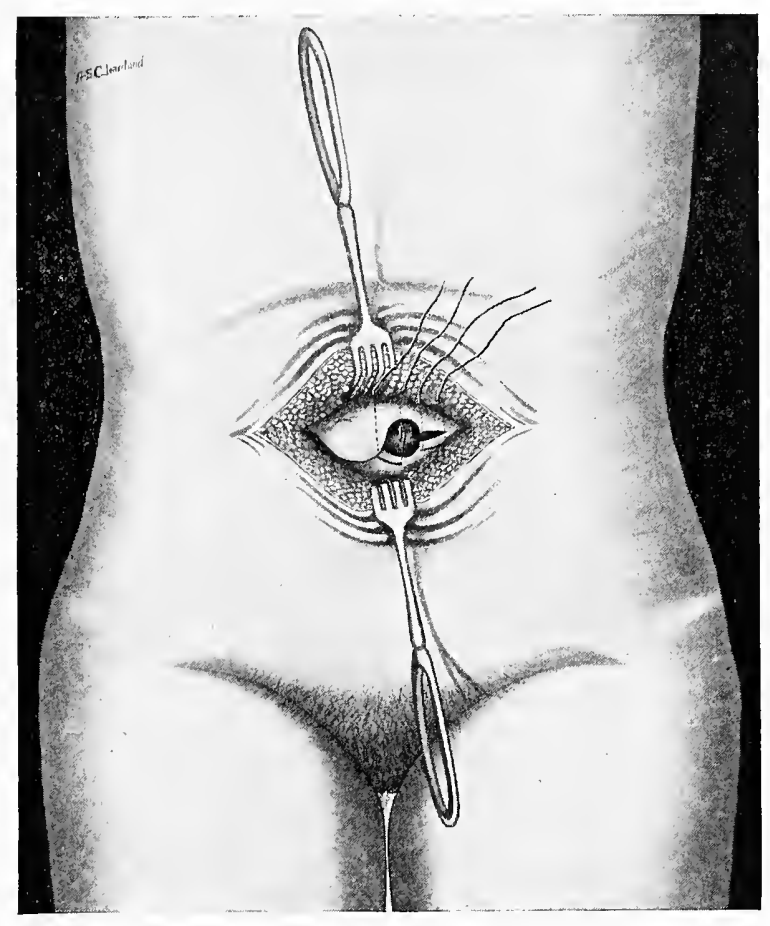

Lower flap slipped into pocket between peritoneum and aponeurosis. Mattress sutures ready to tie. (Mayo.)

(6) The free margin of the upper flap is fixed by catgut sutures to the surface of the aponeurosis below, and the superficial incision closed in the usual manner. In the larger hernix the incisions through the fibrous coverings of the sac may be made somewhat above the base, thereby increasing the amount of tissue available for use in the overlapping process" (fig. $2 \mathrm{II}$ ). 
As to whether the overlapping shall be in a vertical or transverse line must, it seems to me, be decided by the operator after the parts are open and it can be seen in which direction there is the least resistance. In making this a basis upon which the incision rests it has appeared to me that in the

FIG. 2II.

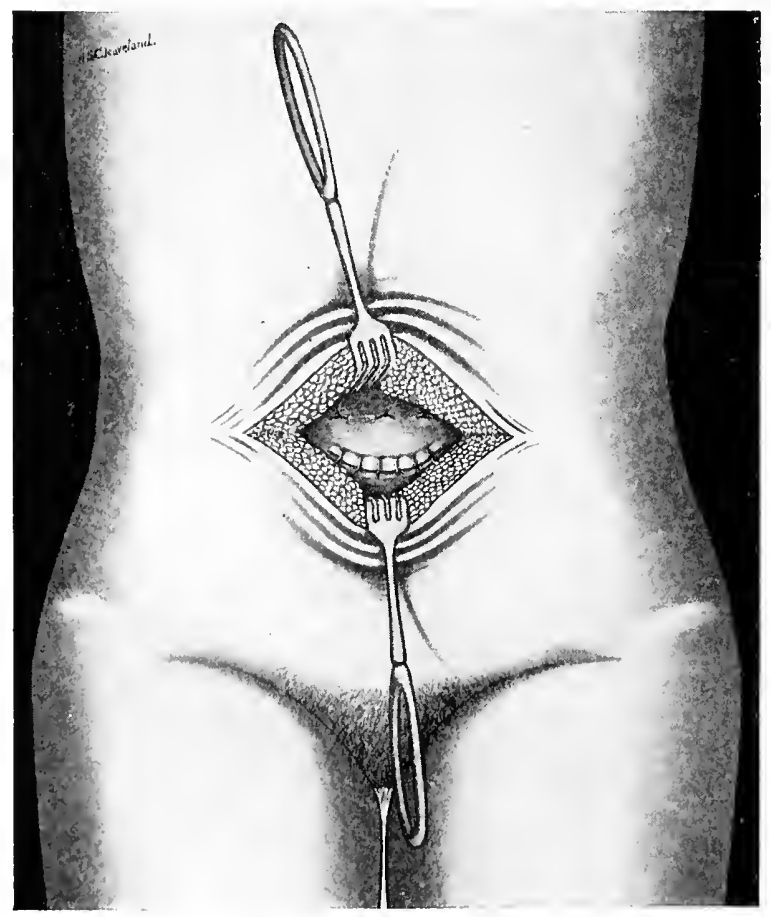

Ypper flap closed over lower and stitched to aponeurosis. (Mayo.)

greater number of cases it would be easier to overlap the aponeurotic structures vertically than transversely. Furthermore, this, to my mind, more nearly restores the normal relative position of the recti muscles, which are so widely separated in the large hernire in which one or the other of these operations seems so well calculated to cure.

The excision of the umbilicus, which I have for years fol- 
lowed in nearly every case, is also believed to be an important step toward a permanent cure. The umbilical ring is a weak spot in the abdominal wall because of its surrounding a mass of tissue that is never completely obliterated. When operating, by removing entirely this mass, we materially aid in the absolute closure of that ring. The suggestion that the sheath of the recti be cut and their fibres united in the median line is not in many cases practicable, especially in large hernix where there is special difficulty in obtaining a cure, because these muscles are so widely separated and so thin that it is not possible to bring them together.

The after treatment of cases operated upon for umbilical hernia should differ somewhat from that given in the ingtinal and femoral types. The patient should be confined to bed for at least two weeks and in very large herniæ even longer. A good snug binder with a compress over the former site of the hernia should be worn for from two to three months. If the hernia has been large and the structures poor it is far better to have a good woven elastic belt worn for a year or even permanently than to take the risk of losing the benefits of the operation. It is believed the best policy to tell patients who have been operated upon for this form of hernia that the risk of recurrence is much greater than in other forms, and that much depends upon them and the care they take of themselves.

\section{CONGENITAL UMBILICAL HERNIA.}

It scarcely comes within the intent of this work to speak of congenital umbilical hernia, which in reality is not hernia, but rather a malformation or lack of development. It is not a protrusion of abdominal contents through the umbilical ring, but a portion of the viscera which has never been closed within the abdominal cavity. The umbilical ring has really never formed, and, as in spina bifida, the lateral halves of the body wall have failed to unite. The subject is briefly discussed here, however, to correct a rather common error among physicians that most umbilical hernix in infancy are of the congenital type. 
This condition is found at the birth of the child, the abdominal wall having failed completely to enclose its normal contents. There is an unclosed cleft in the wall and the protrucling viscera are covered only by the attenuated coverings of the umbilical cord. Lindfors (Centralblatt fïr Gynäk, p. 255, I $88_{4}$ ) gives the number of cases of this malformation in $2 \mathrm{I}, 000$ confinements, at the Munich Lying-in Hospital, as I in every 5, $\mathrm{I} 8_{4}$ births. In size it may be anywhere from that of a walnut to complete eventration containing in addition to the intestinal tract the stomach, liver, spleen, and even the heart. ${ }^{1}$

Diagnosis.-The sac is translucent, and its contents are usually intestine, but may be a part or the whole of any of the abdominal viscera. Diagnosis should not be difficult, but doubt may be caused by possible confusion with hydrocele of the umbilical cord. The treatment of these cases is far from satisfactory, but all authors agree that in immediate operation there is greater safety.

Dr. Willis Macdonald (Am. Jour. Abst., p. 7, I890) gives a history of 12 cases treated by compress and bandage, of which 9 died, while of 19 others subjected to early laparotomy only 2 died. The tumor may be partially or fully reduced, and retained by dry aseptic gauze dressings held in place by strips of zinc oxide plaster. The tumor must be handled with extreme caution and this dressing merely employed while preparing for an operation.

The operation consists in closure of abdominal wall ovèr the stump of the sac, after that has been tied off and cut away. A belly-band with compress should be worn for several months afterwards.

${ }^{1}$ Those who wish to follow the subject more fully are advised to consult the very valuable articie upon the subject by Dr. Charles Green Cumston, of Boston (Mcdical Record, September 23, 1905). 


\section{CHAPTER XXI.}

\section{VENTRAL HERNIA.}

A protrusion of the abdominal contents at any part of the abdominal wall other than the umbilical, ingtinal, or femoral region may be correctly termed a ventral hernia. In orcler to constitute a hernia the tumor must have a neck and a lining of peritoneum. Those cases of deficiency at birth of the muscular wall, with consequent bulging of one side or a part of one side of the abdomen, are looked upon as congenital malformations rather than ventral herniæ.

The two principal causes of ventral hernia are defective points in the tendinous closure of the abdominal wall, and traumatism. The latter in itself forms an important division of the subject. Eccles (Hermia, p. I9o) uses the terms "spontaneous" and "traumatic" ventral hernia. As these terms are concise and expressive they will be used in discussion.

Spontaneous Ventral Hernia.-Macready, in collecting statistics on this subject, found that this form was nearly four times more frequent in the male than in the female, and that it occurred most frequently in middle life, although it may be found at any age. In 2I,8 I 2 herniæe examined, 38 ventral herniæ, or I in every 574 cases, were found.

Its relation to other hernix is shown by the following table from his work:

\begin{tabular}{|c|c|c|c|c|c|}
\hline Sex. & Inguinal. & Femoral. & Umbilical. & $\begin{array}{c}\text { Vent in Linea } \\
\text { Alba. }\end{array}$ & $\begin{aligned} & \text { Ventral } \\
& \text { + Inguinal. }\end{aligned}$ \\
\hline $\begin{array}{l}\text { Male ....... } \\
\text { Female.... }\end{array}$ & $\begin{array}{r}\mathrm{I} 7,538 \\
\mathrm{I}, \mathrm{SO} 3\end{array}$ & $\begin{array}{r}46 \mathrm{I} \\
\mathrm{I}, \mathrm{I} 97\end{array}$ & $\begin{array}{l}209 \\
566\end{array}$ & $\begin{array}{r}\text { I5 } \\
6\end{array}$ & $\begin{array}{r}\text { I5 } \\
2\end{array}$ \\
\hline
\end{tabular}

Spontaneous ventral hernia never protrudes through muscular tissue, but always through some of the tendinous structure of the abdominal wall. It is therefore most frequently found in the median line coming through some 
defective spot in the linea alba, but it may come through at either edge of the recti muscles, the linea transversa, or linea semilunaris. Various names have been applied to it according to the location of the hernia (as, epigastric ventral hernia to all of those protrucling above the navel), but here, as in other parts of this work, multiplicity of names will be avoided as leading only to confusion.

The linea alba being weaker in structure above than below the navel, we find, as might be expected, a greater number of ventral protrusions above this point. Protrusions of subperitoneal fat (commonly called lipoma) through these openings may simulate ventral hernia so closely as to defy diagnosis before operation. This, however, is unimportant, as they are not only frequently the forerunners of true hernia, but by traction upon the peritoneum they are equally painful. Some authors have called these tumors fatty herniæ, but it is hardly necessary to give them any title other than ventral hernia, diagnosis seldom being made before operation. These remarks do not apply to true lipomata that form in the cellular tissue outside of the abdominal wall and which can usually be readily lifted up from the muscular surface. In the latter cases there is ordinarily no pain or discomfort and the patient is concerned in the growth merely as something abnormal.

Spontaneous ventral hernia is usually found as a small round tumor varying in size from the end of the little finger to that of an English walnut. Cases have, rarely, been recorded where the tumor has become large and pendulous, but such have not come within my own experience. The contents of these hernix are most frequently omentum, but may be intestine or both. The sac is frequently small and at the base of the subperitoneal fat which has protruded first, dragging the peritoneum through the aperture after it. These small tumors may, in many instances, be demonstrated by having the patient lie prone on a hard surface and then attempt to raise his hearl and shoulders. In other cases they are better found by having the patient stoop forward and cough while standing. 
Symptoms.-The amount of pain and discomfort that frequently attend this form of hernia is entirely out of proportion to the size of the swelling. The diagnosis of obscure abdominal trouble would many times be made clear by the discovery of this apparently insignificant "lump." The patient may know that it is there and still not associate his abdominal trouble with it. I have seen several cases where the overlooking of a small ventral hernia has led to the patient's great discomfort and unnecessary expense. One man upon whom I operated had been treated by various physicians for " dyspepsia," "renal colic," "gall-stones," " ulcer of the stomach," and was finally told that his distress was due to some malignant growth. He had called the attention of one or two to this little swelling about two and a half inches above the navel and a little to the left of the median line, but was told that it was of no account. In this case, as in some others I have seen, there was no local pain. These little protrusions may cause dragging abdominal pains and gastro-intestinal disturbances quite remote from the seat of the trouble. "Cramps" and vomiting were both present in the case above referred to, and yet at operation nothing was found in the sac but a very small piece of adherent omentum. The cure was absolute both as to the distressing symptoms and the hernial protrusion. Notwithstanding these symptoms actual strangulation seldom occurs in this form of hernia. Lockwood ("Hunterian Lectures on the Morbid Anatomy, Pathology, and Treatment of Hernia," p. I37) found one of the appendices epiploicæ of the colon protruding through a small hole in the linea semilunaris at the level of the anterior superior spine of the ilium and incarcerated. It can be readily seen what an amount of disturbance would result from such a condition without producing symptoms of intestinal obstruction.

Treatment.-The treatment of spontaneous ventral hernia by mechanical means is not usually attended by any great degree of success. They are seldom fully reducible on account of the protruding fat outside of the sac, and they are at points 
on the body where the accurate adjustment of a truss is extremely difficult. I have usually had greater success with a light double-spring umbilical truss than any other. The pad may be much smaller than that used in umbilical hernia and its inner surface nearly flat. The plates ordinarily used on the youth's umbilical trusses answer this purpose very well. If the protrusion is near or below the navel the plate may be held in place by an elastic belt.

Operative Treatment.-If there is considerable pain -and these cases seldom seek relief unless there is-it is believed that they should at once submit to the operative cure of the condition. They are unquestionably the most satisfactory of all hernix under surgical treatment. The operation consists in an incision just large enough to enucleate the tumor which is almost always present, freeing its neck so that fresh peritoneum is drawn into the hernial opening, then opening the sac and liberating whatever is found. The neck of the sac is ligated with plain catgut, and if it has previously been freed from the edges of the aperture its stump will drop back into the abdominal cavity, and the opening, which is usually very small, can be readily closed by kangaroo tendon or chromic gut.

Oskar Witzel (Samml. Klin. Vortr. Volkmam, i890, No. Io, p. 45), noticing that these openings in the linea alba were usually wider from side to side, advised their closure in a transverse line. The direction of closure should be in the line of least resistance and the edges should be overlapped if possible. "The abdomen should be entered in every case in order to free adhesions that may exist." Personally I have not found that necessary, but certainly would not hesitate to do so if in the least doubt about having fully liberated any adhesions present.

Traumatic Ventral Hernia.-When we speak of traumatic ventral hernia we have reference to hernia following some form of injury to the abdominal wall. The injury may have been in the form of an abscess or a blow, but it comes far more fre- 
quently from some incisive wound which has divided the muscular fibre.

In the author's early experience, shortly after the Civil War, several cases of this type were seen which resulted from stab wounds usually inflicted by the bayonet. These cases were, so far as seen, all small protrusions which were easily controlled by simple mechanical supports. The experience of

Fig. 212.

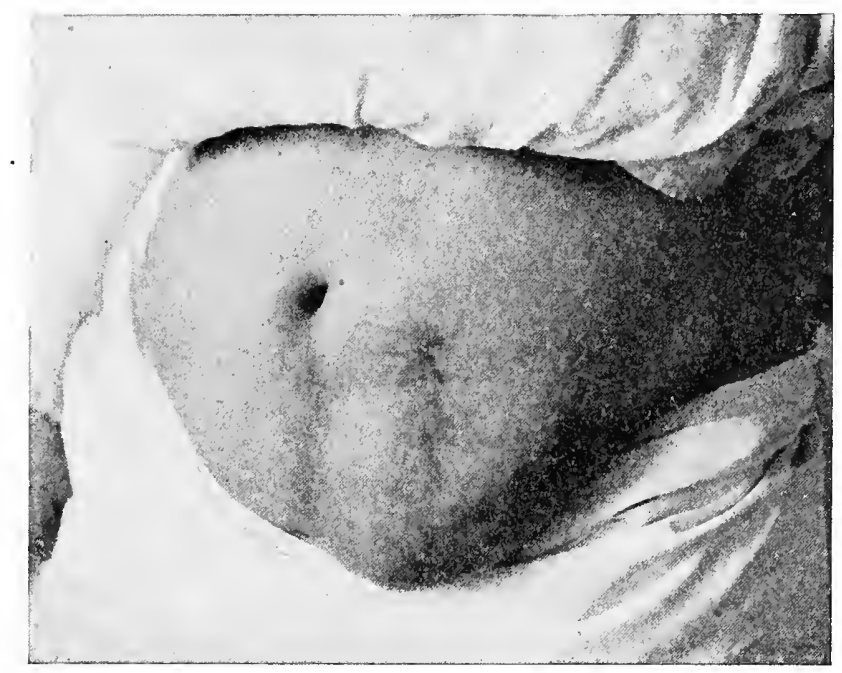

Post-operative ventral hernia, after six operations for cure. Woman 34 years old. Original operation was ovariotomy.

recent years, however, has been with ventral herniæ of quite a different type, enormous in size and extremely difficult to control. They have followed the line of the surgeon's knife in incisions of the abdominal wall. A very common form of this hernia is shown in fig. 2I2. The woman there shown was at the time of this photograph only thirty-four years old. Her original operation was for an ovariotomy, the reason for which could never be ascertained. Following apparent recovery an abdominal abscess formed and required evacuation. On recov- 
ery from this the woman became enormously fat and the hernia, which had been present from the first, increased to a size equal to that of her head. Four successive attempts to cure the hernia failed owing to lack of material with which to close the enormous opening present.

Unfortunately hernia due to stretching of an abdominal cicatrix is quite common, its frequency, as a rule, depending on

FIG. $2 \mathrm{I} 3$.

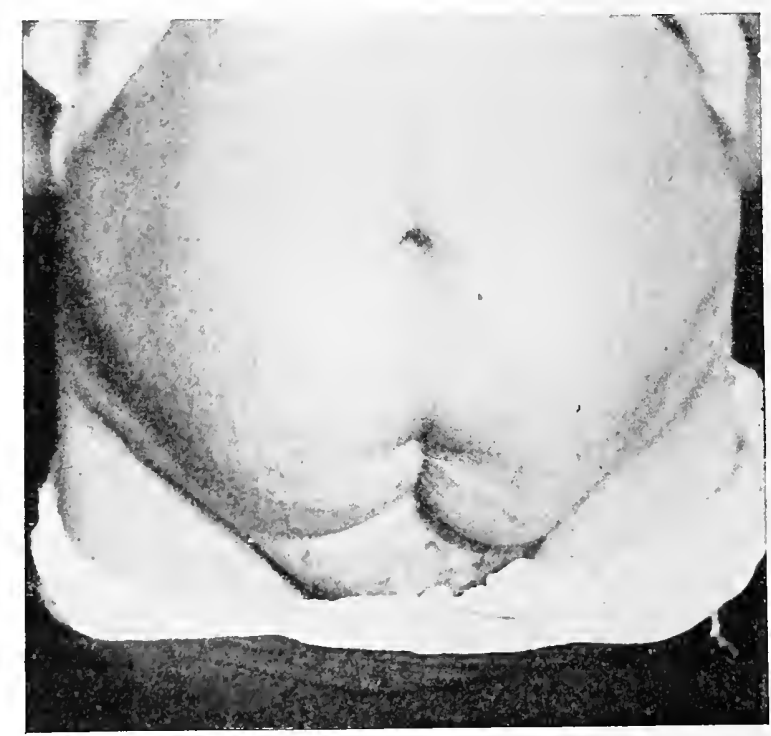

Irregular deposit of fat over right inguinal region, but no hernia. Case operated upon five years previously for ventral hernia, size of large cocoa-nut.

the manner in which the wound of a laparotomy is closed and want of care in providing the patient with a proper and wellfitting abdominal bandage either for a time or constantly, as the case may demand. Even in these days of asepsis, from lack of care at times, suppuration cloes take place with the resulting granulation instead of primary union. Diagnosis usually is easily macle, but in some instances deposit of fat may be misleadling as shown in fig. 2 I 3 . 
A very common type of traumatic ventral hernia are those following operations for appendicitis. Trammatic ventral hernixe following all abdominal operations are fortunately much less frequent than they were ten years ago from the fact that surgeons fully appreciate its serious liability and are using every possible precaution to prevent its occurrence.

\section{FIG. 2 I 4 .}

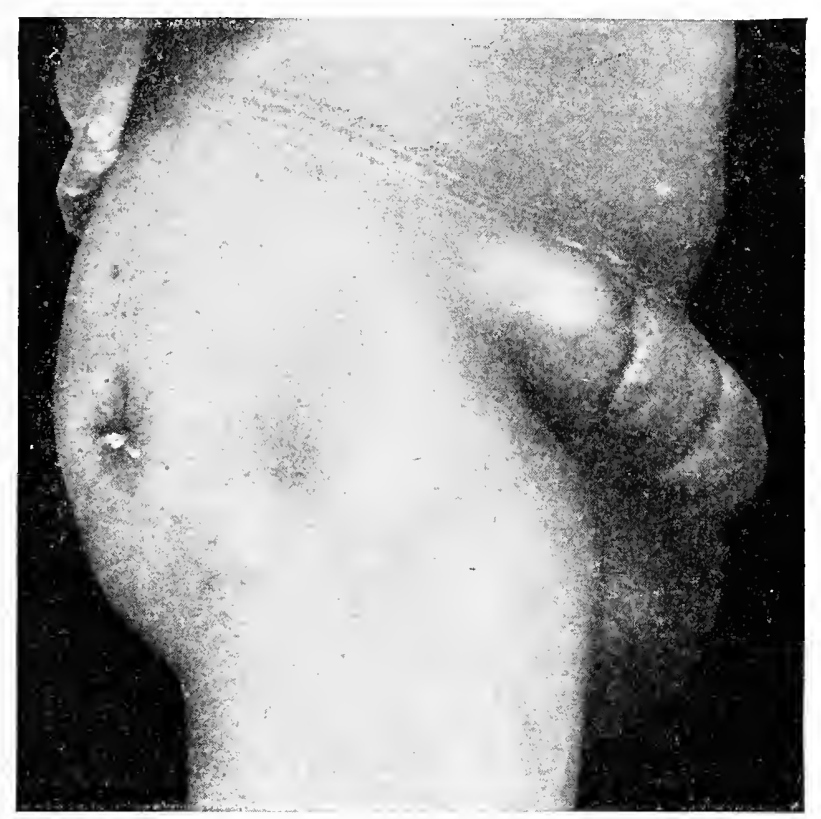

Post-operative ventral hernia. Perforating gun-shot wound. Entrance of ball gluteal, exit inguinal region. Hernia the result of operative attempts to close suppurating sinus.

A few years ago the operation for the removal of the appendix, even where no abscess existed, involved the cutting directly across, with consequent destruction of, the internal oblique muscle, and all the nerves and blood vessels with which this region is so richly endowed. Complete restoration of the parts was practically impossible and hernia resulted in a large proportion of cases. This in some abscess cases may, even 
now, be a necessity as a life-saving measure, but most surgeons, in this country at least, have learned that quite extensive work can be done in this region by splitting each muscle in the direction of its fibres and holding them apart by retractors. Where the abdomen is entered by this method and deep drainage is not required ventral hernia very seldom results. The case shown

FIG. 2I 5.

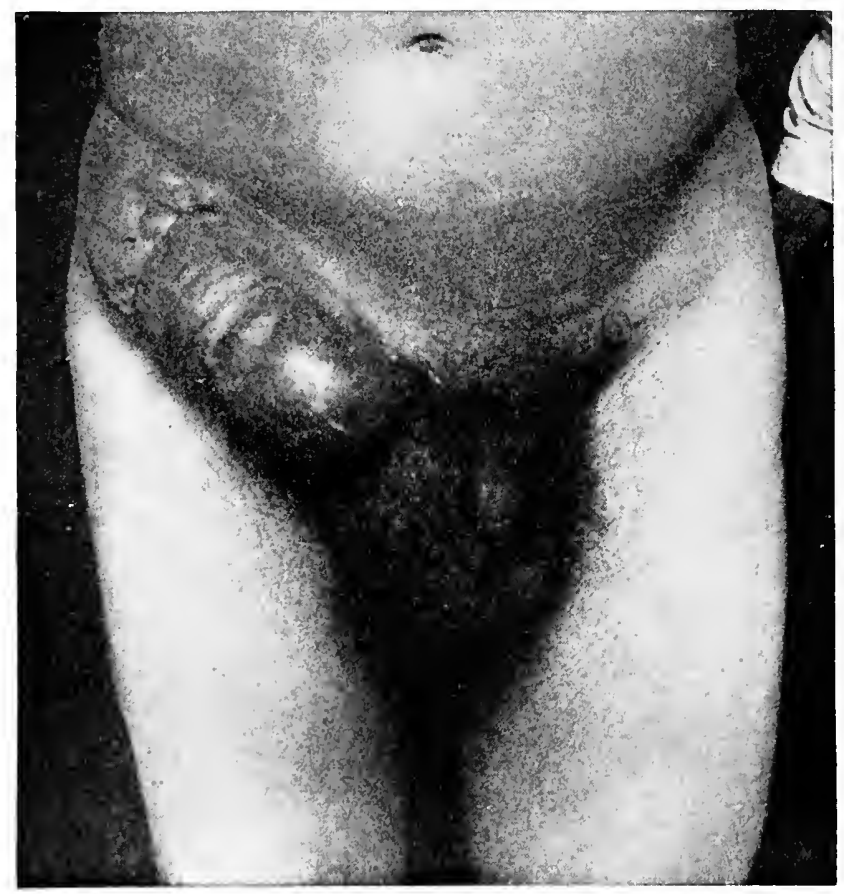

Front view of previous case.

in fig. 2 I 4 and 2 I 5 is of rather unusual form, and resulted from the indiscriminate cutting away of the muscles of the lower abclomen in an attempt to cure a sinus following a gunshot wound. The sinus was of very little importance when it is consirlered in connection with the hernia that was the direct result of the operative work, which also failed in its original purpose. 
Traumatic ventral herniæ are as varied in size and shape as the patients are numerous. When the protrusion once starts it increases rapidly unless checked by suitable and prompt treatment. In its passage through the wall there are usually some fibrous bands which retard a portion of the tumor and cause it to become irregular in shape; it may come out on both sides

FIG. 2 I 6 .

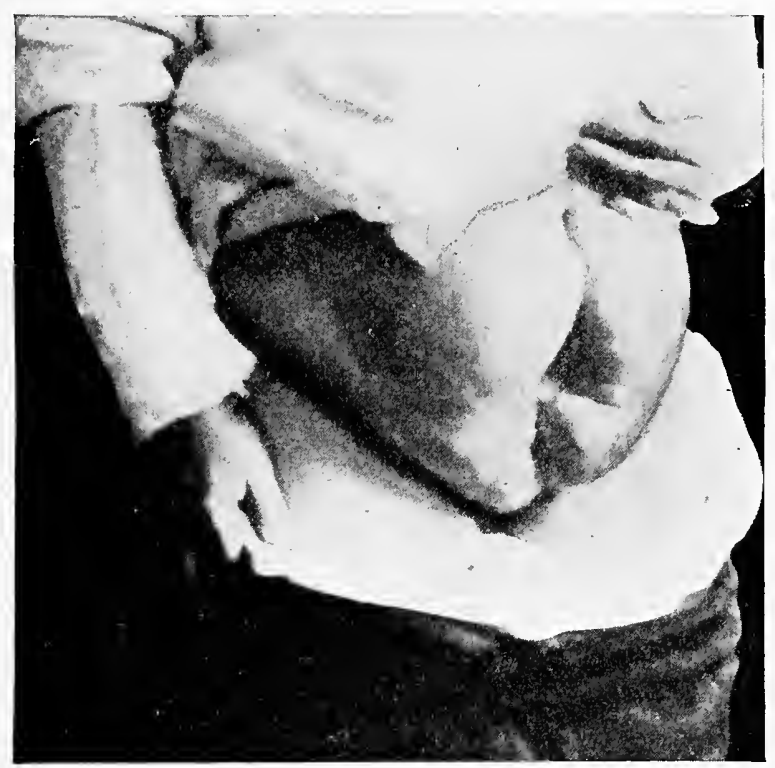

Bilateral post-operative ventral hernia, in woman aged 44 years. Hysterectomy and ovariotomy seven years previously.

of the median line, as shown in fig. $2 \mathrm{I} 6$, or it may protrude at several different places along the line of incision. The contents of the hernix under consideration may be any of the abdominal or pelvic viscera, as in other forms of hernia. (Dr. Howard Kelly (Gynecology, vol. ii, p. 466) shows a photograph of a woman upon whom previous celiotomy had been done, with pregnant uterus protruding through a traumatic ventral hernia. She had a normal labor at term and 
was delivered of a living child.) Omentum is usually the first to protrude and as the opening enlarges intestine soon follows. Adhesions quickly form between the omentum and sides of the sac and not uncommonly between one of these and the intestine. In its earliest stage it is freely reducible, but if allowed to go untreated it very shortly becomes only partially so. It is usually not very painful at first, but as it increases in size is not only more painful but is accompanied by a sense of weakness that borders closely upon prostration. Strangulation of these large traumatic ventral hernia is rarely reported, as the neck of the tumor is usually so large as to allow free circulation through the bowel. Gradual loss of peristaltic action in the bowel, eventually terminating in intestinal obstruction, is far more likely to occur.

MECHANICAL TREATMENT OF TRAUMATIC VENTRAL HERNIA.

Many cases of traumatic ventral hernia seen early will show very satisfactory results under mechanical treatment. They must be carefully watched, for, like cases of umbilical hernia, there is in them a strong tendency to grow worse if not kept under perfect control. Contrary to the rule in umbilical hernia, however, they not infrequently improve very materially under the use of mechanical support, and I have seen a few that have been cured without surgical interference.

If the surgeon doubts the stability of his abdominal closure he should give the parts support either by a truss spring to which has been arljusted a suitable pad, or with a general abdominal belt with a special compress arranged over the line of incision. The spring is always best where the wound has been on either sirle of the median line and, in fact, usually preferable in cases of median incision. The effect of the belt is especially good in some cases where there is great tendency to rapirl increase of fat, as it undoubterlly in a measure checks this. Good, firm support must, however, be afforded by a surface that is not so conical in shape as to force itself directly into the cicatrix. 
The hard-rubber cross-body spring made for the treatment of inguinal hernia has frequently been used with satisfaction. It is shaped by the lead tape diagram method suggested in the chapter on the mechanical treatment of inguinal hernia. Such a truss is shown in fig. 217, applied to a woman sixtyfive years old, following an operation for an abscess case of

\section{FIG. 217 .}

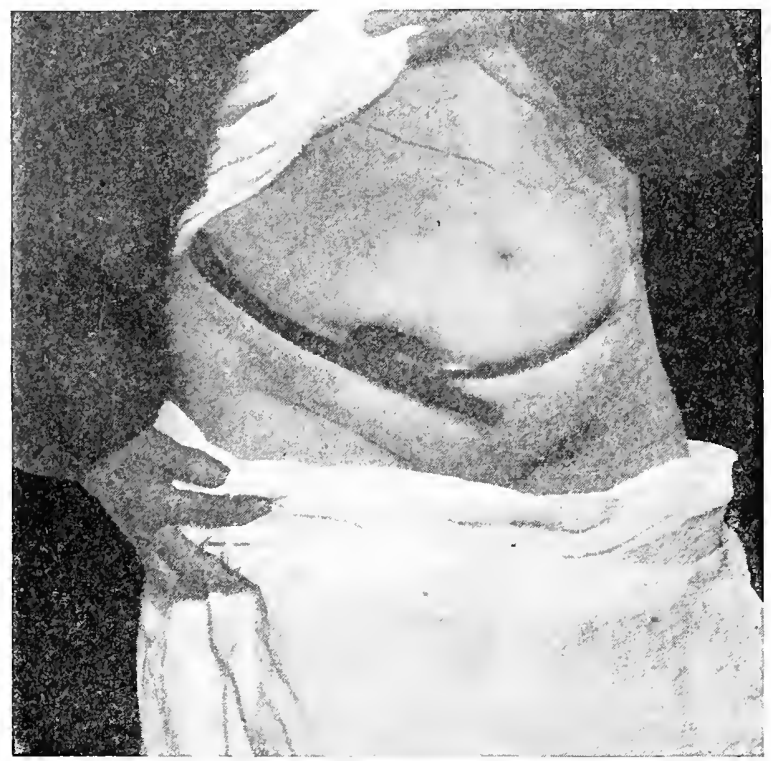

Woman 65 years old, with ventral hernia following operation for appendicitis, retained by hard-rubber cross-body truss, with ordinary inguinal pad.

a!pendicitis, where drainage was necessary for many weeks. Inguinal springs used for this purpose should be reduced in pressure considerably, as the amount required is much less than for other forms of hernia. In a large number of patients the pad used in inguinal hernia can also be used here, as shown. In fact, where the patient is rather fat it is better than the perfectly flat pads made by most truss-makers. The large flat pad rests upon the skin and subcutaneous fat, but does not 
Group of Trusses for Ventral Hernia.

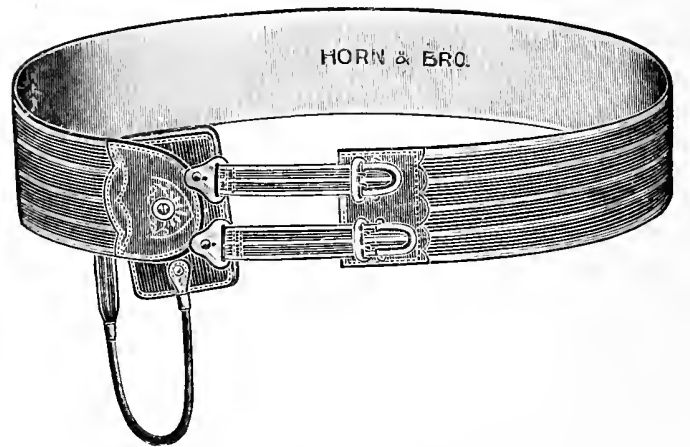

1. Elastic ventral hernia truss. Soft- or hard-rubber pad.

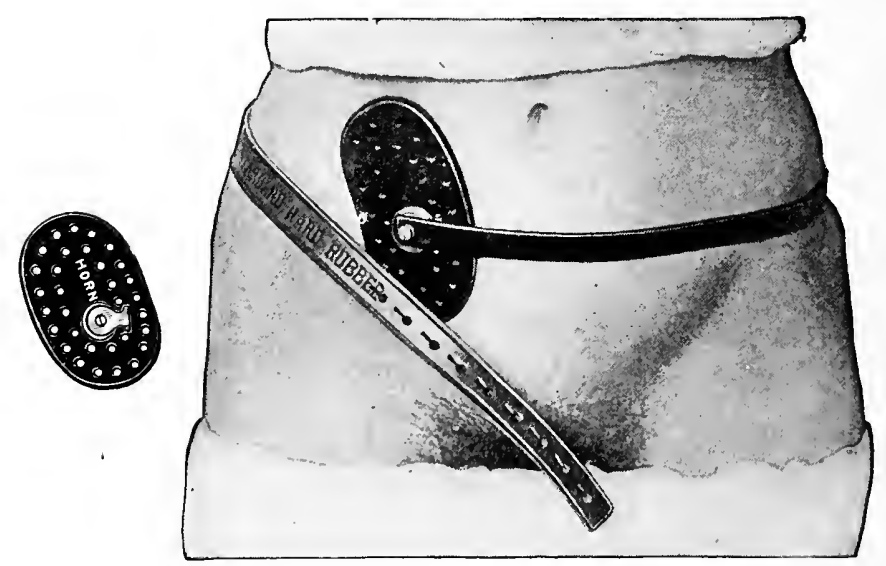

2. Hard-rubber cross-body spring with perforated pad for ventral hernia.

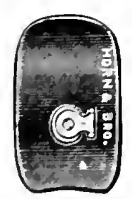

A

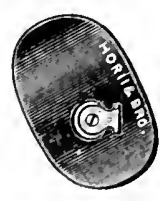

I)

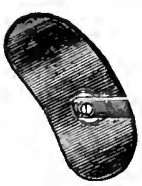

I)

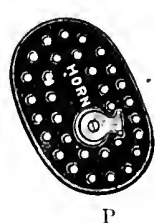

$\mathrm{P}$

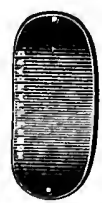

E

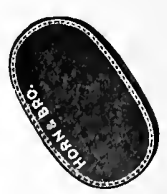

$\mathrm{S}$

3. A variety of pads for ventral hernia. 
Group of Trusses for Ventral Hernia (Continued).

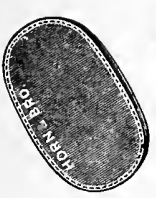

Soft

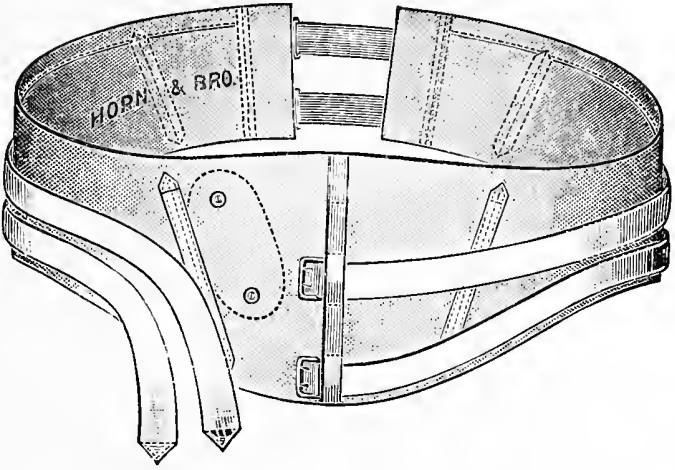

4. Laparotomy belt, with hard-rubber or soft compress.
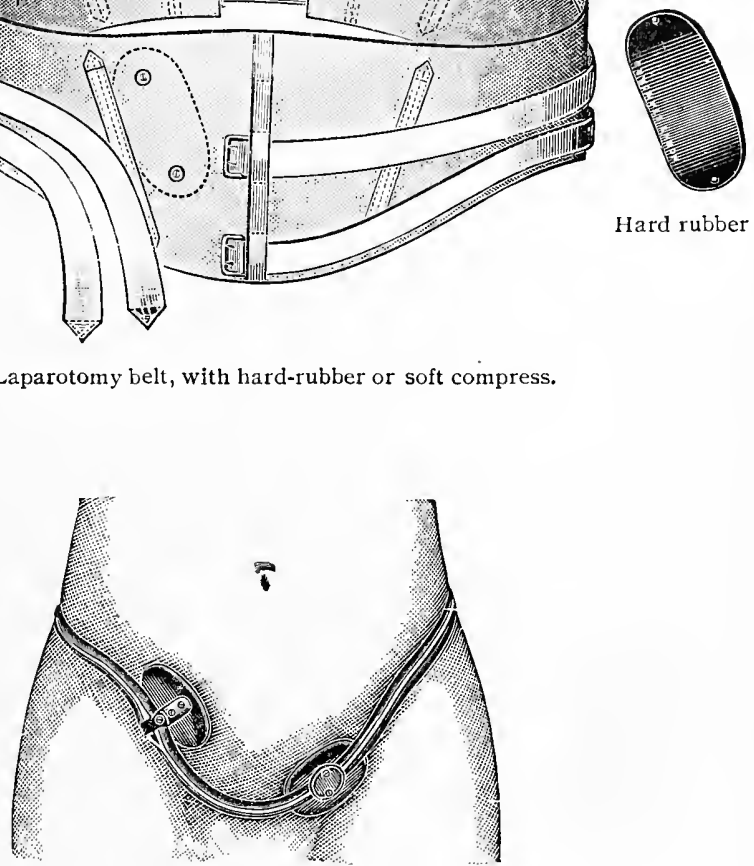

5. Hood truss. Modified for ventral hernia on right side, and inguinal on left.

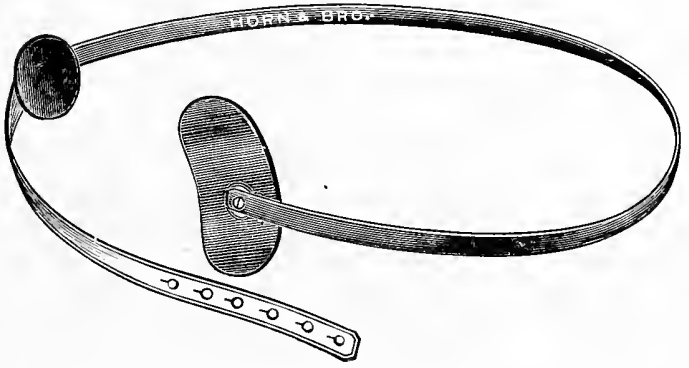

6. Cross-body hard-rubber ventral hernia truss to which any desired form of pad may be attached. 
reach down to the muscular wall where the difficulty exists. In young and thin subjects the flat pads are admirably well adapted for the purpose.

In enormous protrusions, such as shown in the accompanying illustrations, nothing short of a well-made and accurately-fitted, strong canvas belt will give the general abdominal stupport that is demanded. In these extreme cases it amounts to the supplying of an artificial wall, and one who has not had experience cannot fully appreciate the difficulties involved. Patience, perseverance, and many refittings will, however, accomplish a great deal, and when we consider that it is in just this class of cases that surgical relief is almost hopeless, the importance of the service of a good belt maker will be more fully realized. A belt used after an abdominal operation should be of stout, unstretching material-not elastic, although this is often inserted to save trouble in fitting. It is to be worn next the skin and straps should not be needed under the thighs to hold it down or make it comfortable. This will ensure the patient using it as directed.

\section{SURGICAL TREATMENT OF TRAUMATIC VENTRAL HERNIA.}

No hard and fast rules for operating upon traumatic ventral hernia can be formulated, nor can we speak of its surgical cure with the same degree of certainty that is justifiable to use in connection with other forms of hernia. Many small protrusions, where the muscles, vessels, and nerves have not been ruthlessly cut, can be permanently cured by a secondary operation.

Cases following operations for appendicitis are, as a rule, (quite amenable to successful treatment, especially if the incision has been pretty well out toward the crest of the ilitm. When the internal oblique muscle has heen divided, its outer stump retracts toward Poupart's ligament and rapidly atrophies so that a few months later it has become worthless to use in closing the hernial opening. In this conclition the only hope of making an efficient closure is in getting out freely that part 
of the muscle which lies toward the median line. This usually retains a fairly normal condition and if freely stripped away from the aponeurosis of the external oblique can be brought over against Poupart's ligament and sutured there somewhat as is clone in the Bassini operation for the cure of inguinal hernia. If this is clone and the aponeurosis of the external oblique is closed tightly over it a cure will almost certainly result.

The success of these operations depends upon freeing the muscular and tendinous layers of the abdominal wall from adhesions, and then, after excising as much as possible of the cicatrical tissue, bringing them together in their normal relations. Every layer should be sutured independently. The suture material used by the author has been no. 2 plain catgut for the peritoneum, kangaroo tendon for muscular and tendinous structures, and where possible the skin has been closed subcutaneously by the same material as is used for the peritoneum.

The overlapping of the fascial layers may sometimes be used to advantage in these cases. The bringing together of the opening edge to edge is of little use, and sutures that require considerable tension to tie will soon cut through. Dr. Carl Beck of New York (Mcdical Nca's, October 27, I900) reports a case that seemed hopeless by ordinary means, where he obtained a cure by turning out a flap cut from the rectus muscle to fill in the gap that could not otherwise have been closed. 


\section{CHAPTER XXII.}

\section{RARE FORMS OF HERNIA.}

Lumbar Hernia.-There has been considerable discussion as to the exact point at which lumbar hernia leaves the abdomen, but this is unimportant. It is at best a rare condition, and it is not in accord with the intent of this work to enter into the discussion of fine points that are of no practical value to the general practitioner.

Whether it most frequently protrudes at Petit's triangle or Braun's space is immaterial to its proper mechanical or surgical treatment. Its occurrence is very rare, only 4 cases having been seen at the London Truss Society up to I893, the total number of reported cases up to that date being 26 , according to Macready.

Lumbar hernia forms a tumor in the lumbar region which may vary in size from a walnut to that of a child's head, and presents all of the peculiar characteristics of abdominal hernia in other locations. It is usually reducible, and if containing intestine is resonant on percussion with the peculiar gurgling sound found elsewhere. It may be spontaneous or traumatic, but Borchardt (Berliner klinische Wochenschrift, December 9, I9OI) divides these hernix into four groups according to the etiologic factors causing them; those of traumatic origin, those following abscesses, those arising spontaneously without any known cause, and the congenital lumbar hernia. Out of 43 lumbar hernix, he has collected I9 following injuries. The number following psoas or pelvic abscesses which break in this region is comparatively small, and no doubt the pointing of the abscess is in some part caused by the natural weakness of the abdominal wall. The form which is commonly spoken of as spontaneous also tends to occur in the region known as Petit's triangle. Symptoms of strangulation 
are of comparatively frequent occurrence. Ten cases of this kind have been reported. In 5 of these the strangulation was of a transient character. The case shown in fig. 2 I 8 was due to congenital defect and the protrusion occurred on both sides, the right being much the larger.

The treatment mechanically of lumbar hernia is attended by considerable trouble on account of its peculiar location

FIG. 2 I 8.

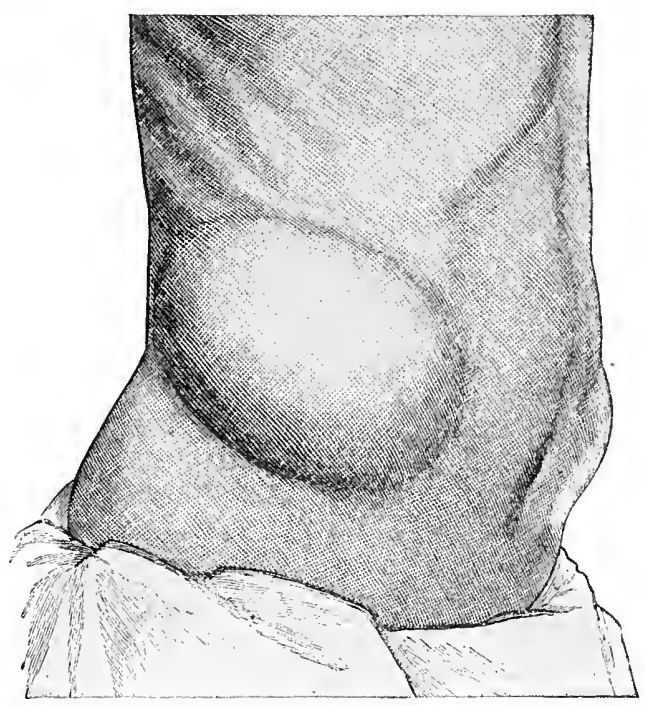

Spontaneous double lumbar hernia due to defective muscular structure. (Macready.)

and the difficulty in securing counter-pressure for the trussspring. Abdominal belts with a compress over the hernia may be useful, but a spring designed by Mr. Kingdon of the London Truss Society is the best appliance so far noted in any publication (figs. 2 I 8 and 219). The base of action is in the cushioned, covered plate, held in position, midway between the trochanter and the crest of the ilium, by a perineal strap. The spring attached to the front of this plate, by ball and socket, passes obliquely across the front of 
the abdomen around the right side, and holds the retaining pad also by ball-and-socket attachment. From this point a strap passes to the back of the curved plate upon which it buttons. Lumbar hernixe, as a rule, show considerable improvement under efficient mechanical support, and some cases have so far improved as to allow of the abandonment of the appliance.

The operative treatment of lumbar hernia should be carried out upon the same general principles that govern the

FIG. 2 I 9.

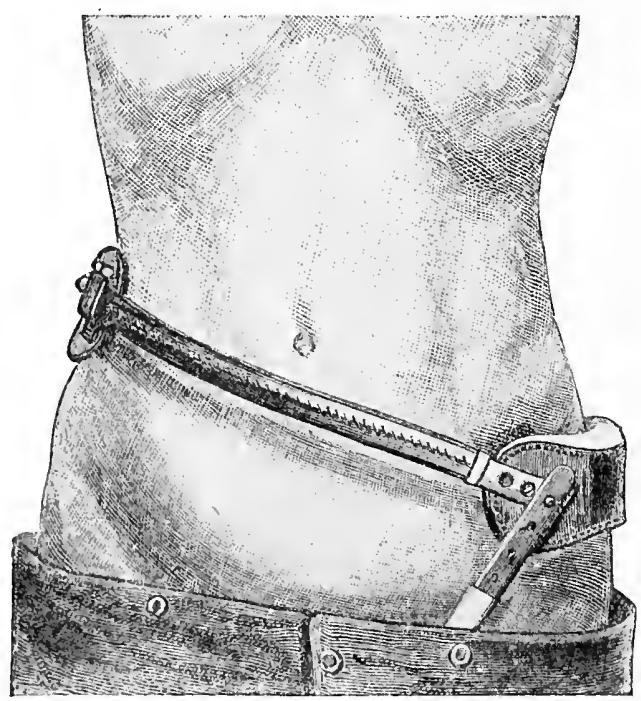

Truss for right lumbar heruia designed by Mr. Kingdon. (MFacready.)

surgical treatment of ventral hernia in other locations. This consists in the enucleation and opening of the sac, the reduction of its contents, the ligation of the neck, and finally the closure of the hernial aperture. The overlapping of the structures to secure union by a broader surface will usually be found feasible and effective in this region.

Dr. Chas. N. Dowd of New York has recently elaborated an operation (Amnals of Surgery, February, I907, p. 245) for 
this form of hernia that seems commendable, and with his permission I reproduce his cuts which illustrate his method more clearly than words could.

The child shown in photograph fig. 220 was three and a half years old and had worn a belt without improvement for *

FIG. 220.

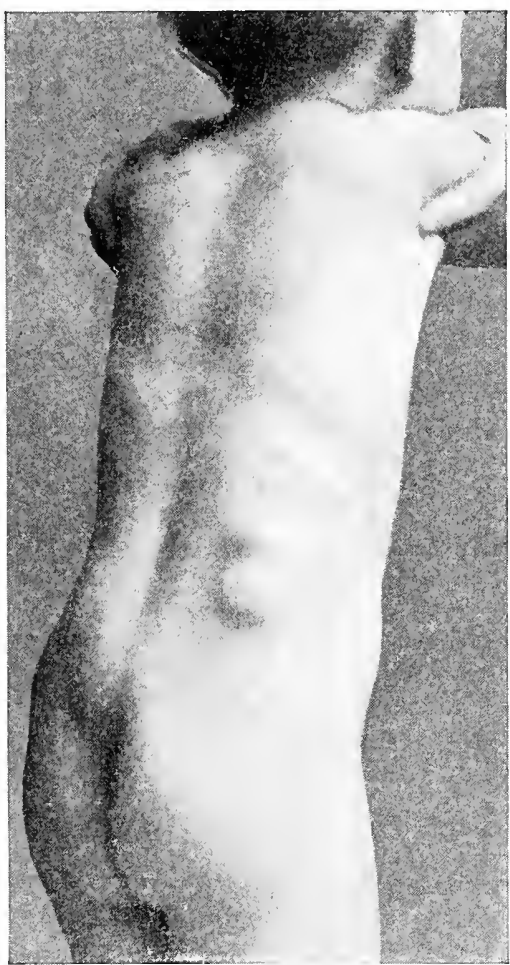

Congenital lumbar hernia, presenting through an enlarged triangle of Petit. (Dowd.)

two years. The protrusion was the size of a goose egg, and at operation was found to come through the triangle of Petit. The hernial sac was distinct but without a narrow neck. At the lower end of the protrusion the appendix vermiformis was found and removed: "A portion of the sac was exsected, and the tissues were then brought together from the sides; the 
margins of the external oblique and the latissimus dorsi being drawn together as far as possible. After this was done, there was, however, a triangular defect above the crest of the illium. An effort was made to close this in with an aponeurotic flap turned up from below. The fascia lata and the aponeurotic

FIG. 22 I.

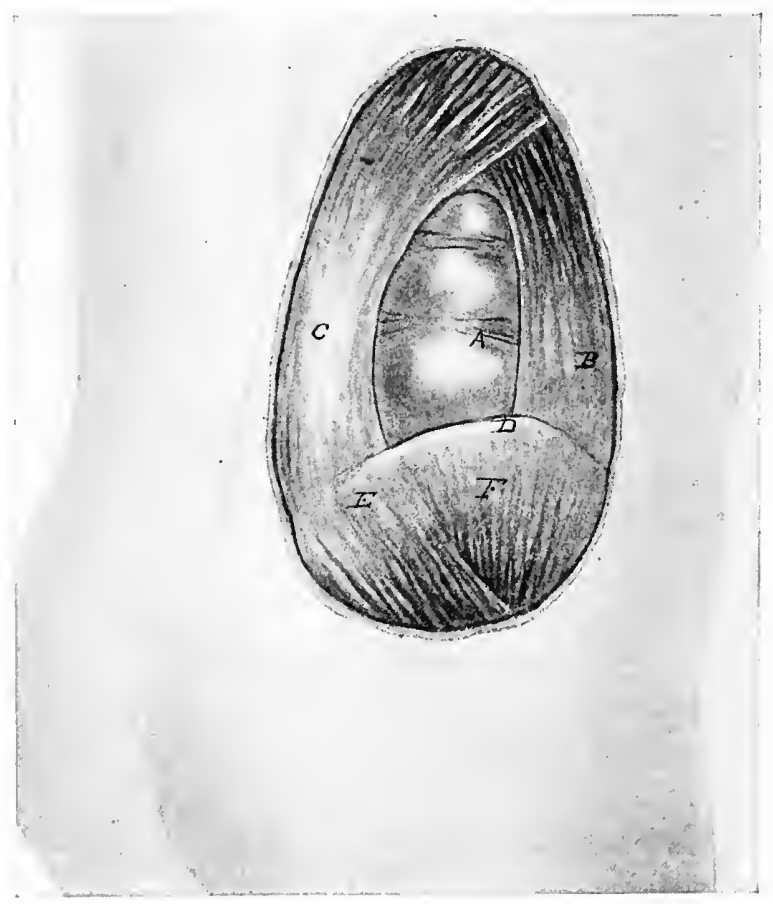

Congenital lumbar hernia (Dowd.) $A$, transversalis fascia ; $B$, external oblique muscle; $C$, latissimus dorsi muscle; $D$, crest of ilium; $E$, gluteus maximus muscle; $F$, gluteus medius muscle.

tissue about the insertion of the gluteus maximus and medius formed a fibrous layer which could be used as a flap, and which was turned up, having the attachment at the crest for the illium as a linge. This was stitched in place with chromic gut, some sutures passing through the previously mentioned transverse band, some through the edge of the latissimus dorsi, and 
others through the edge of the external oblique muscle. There was, however, still a triangular defect above the flap, and this, together with the repaired area, was covered by turning forward a flap cut from the aponeurosis of the latissimus dorsi. This was stitched to the external oblique" (see figs. 22I, 222, and 223).

FIG. 222 .

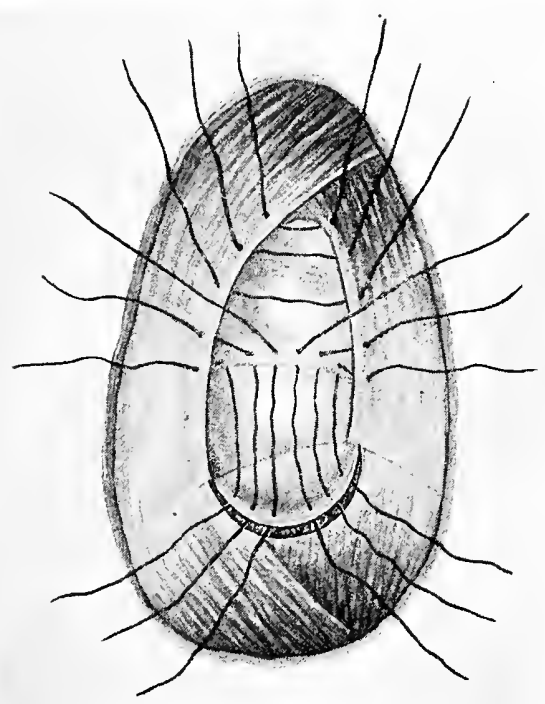

Operation for the cure of congential lumbar hernia (Dowd).-Flap composed of fascia lata and aponeurotic part of gluteus maximus and medius. Stitches placed for suturing this flap to the lumbar-fascia, to the external oblique muscle and to the latissimus dorsi muscle and for drawing the upper parts of the latissimus dorsi and external oblique together.

Obturator Hernia.-Obturator hernia presents as an obscure, deep-seated swelling upon the thigh, below Scarpa's triangle. It is rarely recognized until strangulation has occurred or until after death has taken place. Berger, in 
examining I0,000 cases of hernia at the Paris Central Bureau, found one case of obturator hernia. It leaves the pelvis through the obturator canal with the nerve and vessels of the same name. It most commonly occurs in women who lave passed the middle age of life, and it may be double. The contents of the sac are most frequently intestine, but

FIG. 223 .

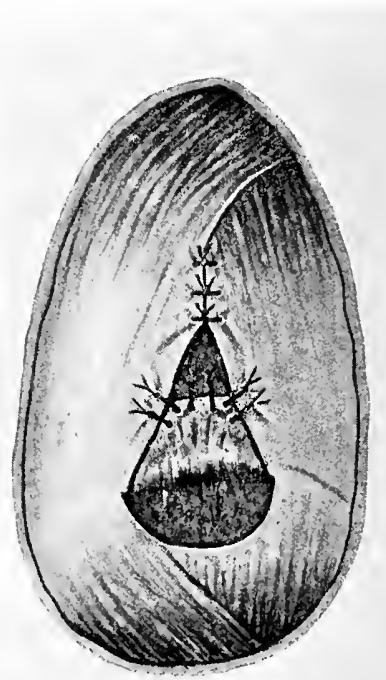

Stitches tied, leaving a triangular defect above the llap. (Dowd.)

the bladkler and uterine appendages have also been found in it.

Franz Schopf (Iricher Klinische Wochonschrift, February 19, 1903) reported, including his own case, 5 cases of protrusion of the tube and ovary in obturator hernia. In one the uterus was inclurled. The same writer states that during twenty years in a Vienna hospital in 393 cases of strangulated hernia 3 were for obturator hernia. 
Diagnosis is seldom made until strangulation occurs or after an autopsy is made. The tumor is more easily felt than seen. Macready thinks it is very liable to be overlooked on account of faulty methods of examination. He says, "It is most easily approached from the inner side of the thigh. The thigh must be flexed, rotated outwards, and carried inwards to relax the adductor muscle, and the finger placed against the descending ramus of pubes behind the adductor longus. The finger may also explore the inner opening of the obturator canal from the vagina or rectum."

The treatment of this form of hernia has not been attended by success and 84.4 per cent. of those operated upon for strangulation have died. If recognized it is not probable that any form of appliance could be so adjusted as to give the patient any degree of comfort and safety. It is beyond question that the safer plan for the patient to adopt would be an attempt to effect a cure by operative means and the approach to the hernia should be through the abdomen.

Sciatic Hernia.-This form of hernia is so extremely rare that it is mentioned only to call attention to its possible occurrence. Its name indicates its location. It comes through the great sacro-sciatic foramen and its contents may be omentum, intestine, ovary, or bladder. Regarding treatment there has been little suggested that would be of real service to the average surgeon.

Perineal, ischio-rectal, and vaginal hernia all pass down through the pelvic outlet in protruding and require only brief consideration here. All herniæe that protrude through the pelvic floor properly are perineal hernix, but they have been given different names according to their coverings. If they push the vaginal wall down between the labia, or if the rectal wall is carried down, they are accordingly called vaginal or ischiorectal. If they descend in the wall between the bladder and the rectum, pushing the skin before them, they are called perineal hernia. 
These hernix have their origin in Douglas's cul-de-sac and may be caused by defective development of the muscular structure of the pelvic floor, or by accidental injury; rarely where no such cause is ascertainable, they have been called congenital. They occur most frequently in the female. In Macready's collection of 40 cases only 6 were in the male. The contents may be those found in hernia elsewhere, but are be-

FIG. 224 .

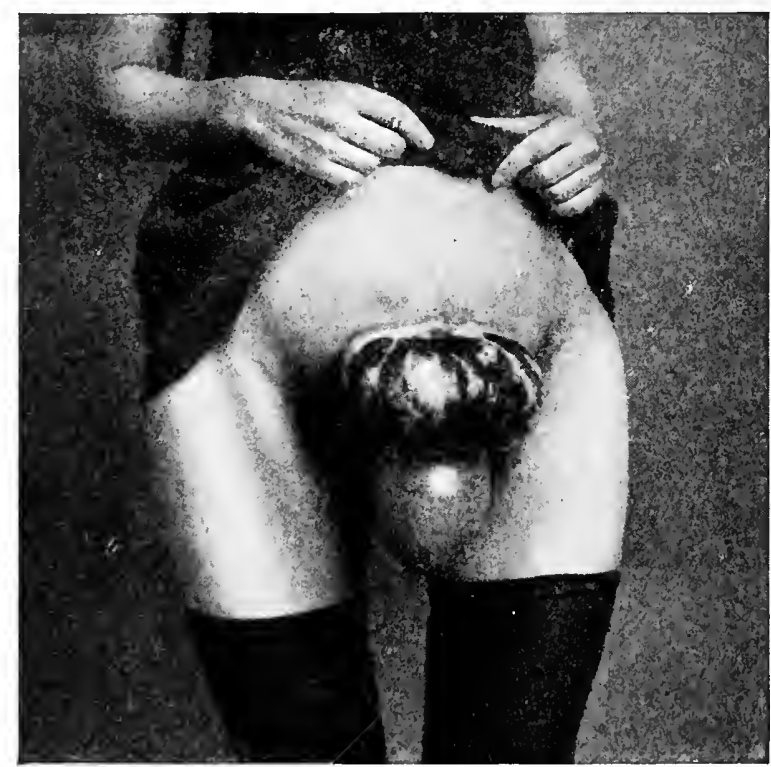

Woman aged 30 years. Exstrophy of bladder and vaginal hernia.

lieved to be most frequently small intestine. The sigmoid flexure, the bladder, and the ovary have been found. In the female the hernia may come down at the side of the vagina and enter the labia, but more frequently the vaginal wall is carried down as its external covering, and is liable to be mistaken for cystocele or labial abscess. They may also protrude either at the posterior or anterior part of the vaginal entrance, and in some instances become enormous in size if left unattended. 
The case shown in the photograph (fig. 224) came to me as a private patient many years ago. In addition to the enormous protrusion there shown, which contained the uterus, ovaries, and a large mass of intestine, the woman suffered from extrophy of the bladder, the anterior wall of that organ being entirely absent. The appliance designed for her relief is shown, applied, in fig. 225. It consisted of a steel frame

FIG. 225 .

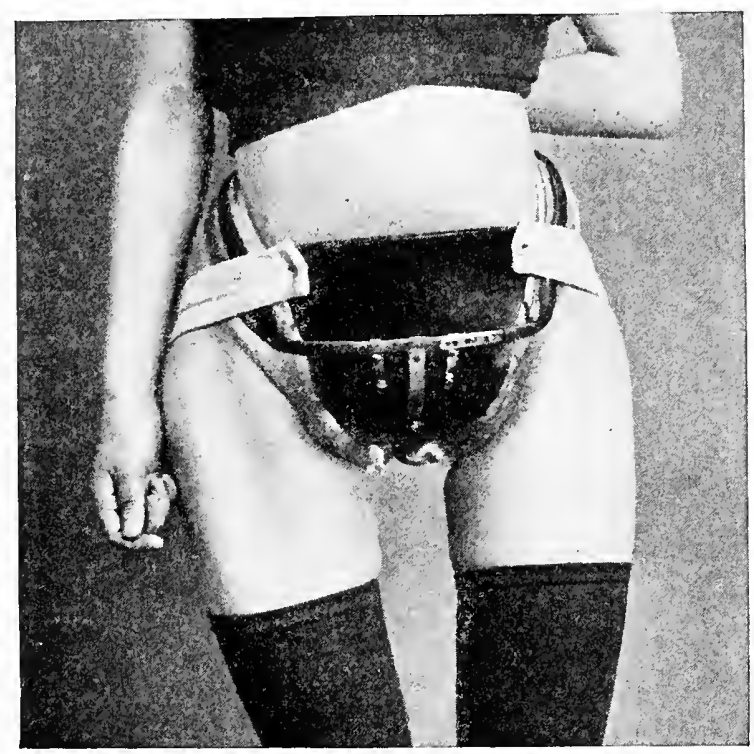

Same case, with supporting appliances on.

arching over each hip and resting firmly upon the crest of the ilium of either side. This frame held a large hard-rubber plate so shaped as to pass over the pubic bone and support the entire perineum. This served the double purpose of retaining, perfectly and comfortably, the pelvic and abdominal contents as well as protecting the patient against the constant wetting with urine that dribbled from the ureters. The urine was carried off through a flexible tube, from the bottom of the plate to a 
rubber bag strapped to the leg. She wore this appliance at night, as well as during the day, and assured me that for the first time in her life she had been enabled to sleep without being wet from her neck to her feet.

As shown by the foregoing case, perineal hernia, even of enormous proportions, can be controlled by properly designed supports. Ordinarily the one shown in fig. 226 answers the purpose very well. This consists of a metal base held in place over the sacrum by a leather belt about two inches wide. To this base is attached, by means of a screw, two light hard-

FIG. 226.

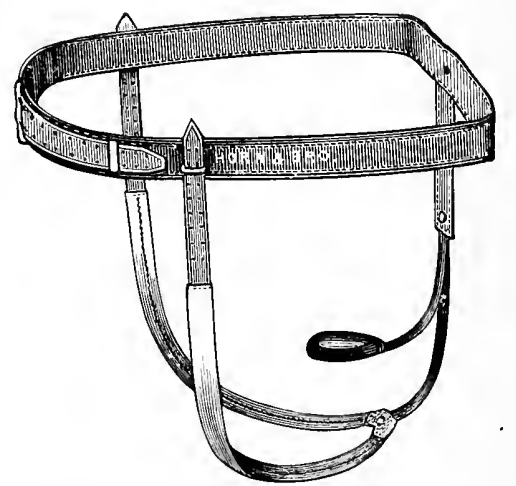

Appliance for perineal ischio-rectal, or vaginal hernia. The spring comes from the back and may have attached a pad of any desired size or shape.

rubber covered steel springs. The inner one of these springs has a short curve and should terminate at about the centre of the intended field of support. To this spring any form of pad desired may be attached. The outer and longer spring passes down over the inner one, and curving forward has attached to it the perineal straps that come up in front and are buckled to the belt. By this combination of the two springs the amount of pressure to be used can be very accurately adjusted.

A few cases of raginal hernia, if retecterl early, may be controlled by the introduction of a suitable pessary, but such relief is very likely to be only temporary. Little of value has been written upon the surgical treatment of vaginal hernia. 


\section{CONTRA-INDICATIONS TO THE SURGICAL CURE OF ABDOMINAL HERNIA.}

There are certain indications which should cause us to hesitate in advising the operative cure of hernia; the added danger in the individual case may be greater than is incurred by the continuance of the disease.

Excessive fat is one of the most common contra-indications in people of middle life. It is particularly bad if its accumulation has been aided by heavy beer drinking, which not only leads to the production of excessive fat, but causes a corresponding degeneration of muscular tissue. This degenerative change is quite noticeable in the abdominal wall and is equally present in the heart muscle. When such conditions exist operative treatment is considered dangerous and there is more liability to a recurrence of the hernia. Still it may be advisable that they should be operated upon, owing to rapidly approaching complete disability and the increasing dangers of their condition; either they or their friends should be told of the danger of operation and the probable necessity of wearing good support for a year or longer.

Ascites.-In no case, except as a life-saving measure, should an attempt be made to cure hernia by operation in any patient who has an excess of fluid in the abdominal cavity, no matter whether this be temporary and trifling or serious and permanent. Such patients should be assured that no danger attends the presence of the fluid in the hernial sac and that no operation is advisable while it exists.

Enormous hernia with intestinal adhesions are especially dangerous for operation, owing to the amount of handling of the bowel and the intra-abdominal pressure to which it is subjected after the reduction in large quantities. Usually these 
cases can be recognized before operation by percussion, after all that is reducible has been returned to the abdomen. If the resonant note usually found over the bowel is obtained it will be known that adherent intestine must be dealt with.

Tubercular patients, unles's in an advanced stage, stand the operation perfectly well, and it has, in the author's experience, in several instances seemed advisable to operate upon them, and he has had no occasion to regret having done so.

Age.-The condition of the patient has been found more important as a contra-indication than advanced age. Old people, if organic lesions are not actually present, stand the operation well and are not particularly liable to recurrence.

In all of these cases that would, in insurance phraseology, be termed extra-hazardous, much can be done to diminish the danger by having every preparation complete for doing the work as rapidly as possible. Assistants and nurses should be selected and coached so that they will thoroughly understand and perform their various duties promptly. The anæsthetist should always, in such a case, be a person of admitted judgment and experience.

ACCIDENTS FOLLOWING OPERATIVE CURE OF HERNIA.

Thrombosis of Femoral Vein.-This, fortunately, is an accident that very rarely follows operation for the cure of abdominal hernia, yet it must be considered as one of the possible accidents. Goldner, reporting the results in 800 Bassini operations in Prof. Albert's clinic, Vienna (Arch. für Klinische Chirurgic, Bond lxviii, Heft I, I902), states that 3 patients died, one during narcosis and 2 from embolism. "Both of the latter had large varices on the legs and this condition should impose caution in doing a radical operation."

In my own experience, in I,400 operations for abdominal hernia, I have had 3 cases of thrombosis of the femoral vein. one terminating fatally by pulmonary embolism. The latter, a man about forty, having the appearance of being poorly 
fed and overworked but otherwise apparently in good health, was operated on for an ordinary complete inguinal hernia on the left side. The time required was about twenty-five minutes, and nothing occurred to which the subsequent trouble could be traced. Everything appeared favorable up to the seventh day, at which time he began to complain of extreme pain in the left leg, and evidence of thrombosis was plainly visible. Five days later pulmonary symptoms developed. The healing of the wound was primary and there was no varicose condition in his case.

The two other cases were women of about thirty-five years. One a frail woman upon whom an operation for right femoral hernia had been done about six days before, developed unnistakable evidence of thrombus in the left leg. The operation (on the opposite side) had been a very simple one and the wound healed completely by first intention. The patient was lame for three months and then recovered perfect use of the limb. The third case was a large, healthy woman, operated on for supposed irreducible inguinal hernia, which proved to be a lipoma protruding from the canal. This was removed without difficulty and the parts healed by the tenth day without infection. About the sixth day pain developed suddenly and the left leg. began to swell. Troublesome lameness continued for several months.

It is not possible to discuss the cause of these accidents here as in reality it is unknown. They are liable to occur in all abdominal and pelvic operations. If the reader wishes to pursue the subject he is advised to consult the excellent article on Embolism by Eugene Boise (Surgery, Gyn. and Obstr., July, I906), who believes that it never occurs in a normal condition of the blood.

Secondary Hemorrhage is an accident seldom known in these days of careful ligation of vessels. Its most serious occurrence is in connection with the ligation of omentum. These vessels have no surrounding muscular tissue to aid in their contraction, and if the ligature is inefficient, or slips off, 
the bleeding will be continuous and probably fatal. All liability to such accident can be guarded against by careful ligation of individual ressels unaccompanied by fat.

Peritonitis is, with aseptic surgery, practically unknown in operations for the cure of abdominal hernia. Undue tratmatism should most certainly be carefully avoided.

Sepsis.-General sepsis resulting from operations for the cure of hernia is now extremely rare, but the occasional operator should realize that its danger is always present, and that for its occurrence he is personally responsible.

\section{INTERNAL HERNIA.}

It is not within the intent of this work to consider so-called internal hernize, such as diaphragmatic, hernia of the foramen of Vinslow, of the duodeno-jejunal recess, of the inter-sigmoid recess, or retro-resical hernia While in one sense these belong to the subject of abdominal hernia, in another they belong to that of intestinal surgery. The diagnosis of these conditions is very rarely made before operation, frequently not until the autopsy. If they reach the surgeon they come as cases of intestinal obstruction, with little evirlence that the bowel may be incarcerated at one of the points named. 


\section{CHAPTER XXIV.}

\section{STRANGULATED INGUINAL HERNIA.}

Few things in the practice of medicine are more alarming, not only to the patient and his family but to the family physician, than the occurrence of strangulated hernia. A tumor which was formerly reducible is suddenly found hard, tender, painful, and irreducible. If the strangulation is acute and the obstruction complete, the agony of the patient is beyond description. That which was a few minutes before considered the threatenings of a mild colic, has rapidly advanced to a degree of suffering too great for human endurance. A strong man will cry out like a child in the intensity of his suffering. Shortly vomiting intervenes to add to his distress, he breaks out into a cold sweat, and if aid is not promptly afforded he goes into a collapse and death is a welcome relief.

Cause.-Volumes have been written discussing various theories as to the cause of strangulation, and those who wish to pursue their investigation along theoretical lines should consult such writings, since this work deals, as far as possible, with facts. Theory is of little interest to the man who suddenly finds himself violently sick, nor does it aid the attending physician in this emergency. The patient, as a rule, has had a hernia for several years and has known how to replace it, but now finds this impossible. The hernia has probably become impacted at the smallest part of the neck, and under the pressure of this impaction the normal action of the bowel has been checked. Furthermore, we know full well that while the circulation in the bowel wall may not have been completely shut off this will promptly take place, with consequent destruction of all tissues under constriction.

In cases where the symptoms gradually reach the most distressing stage, it is probable that enough of the protruding 
contents is forced into the most constricted portion of the sac to impede venous circulation. The arteries, whose resistance is greater, continue to pump blood down into the tumor, but the return of venous blood is prevented by the constriction. Swelling and effusion of fluid then take place, increasing the pressure until finally the whole mass is as completely shut off from the cavity of the abdomen as it would be by strong ligature.

Location of Stricture.-Many times it cannot be told before operating where the constriction will be found, nor is this extremely important. In hernia of the congenital variety it will in many instances be found che to fibrous rings. These rings are, perhaps, the remains of nature's attempts to carry out her original design of obliterating this tubular neck between the cavity of the abdomen and cavity of the tunica vaginalis. Whatever may have been their origin, they are tough, inelastic, and usually situated in the neck of the sac which they completely surround. Sometimes, however, they are just above the testicle and may at this point be the cause of constriction.

In the greater number of cases of strangulated inguinal hernia, the constriction is at or near the external ring and is caused by the dense and strong tissues outside of the sac at this point, but it occasionally happens that the cause of strangulation is within the sac and from some band of connective tissue. This is usual in cases of old hernix of large size. Rarely, it will have its origin in the twisting of the bowel upon itself within the sac, or more frequently, from slipping through a hole in the omentum which forms a part of the hernia.

Irreducibility is in itself no evidence of strangulated hernia, as it many times happens that those who have large hernice cannot for the time reduce them, either from the quantity that has protruded or from the position in which the contents have come down; temporary incarceration results, but no accompanying symptoms of strangulation. No immediate concern need be felt about such cases, but they must be watched with strictest care, as they are on the borderland of strangulated hernia, and may become such at any moment. 
Such tumors are, in many instances, not tense or hard, and the handling of them is unattended by pain. As soon as they begin to get hard or tender, they should be subjected to immediate operation. It is very seldom that a hernia becomes irreducible from its outset and it rarely happens that strangulation takes place in one just formed. The author has seen several exceptions to this, notably one just operated upon. A young woman, in stepping from a car'where the step was much higher than she had anticipated, felt an inmediate pain in the left groin. Shortly afterward; acute abdominal pain and vomiting came on, and it was discovered that she was suffering from a femoral hernia in a state of strangulation.

Physical Signs.-One of the physical signs of inguinal hernia, recently strangulated, is a tumor of variable size anywhere in the inguinal region or the scrotum. It feels hard, especially toward the point where its neck enters the abdomen. If the symptoms are acute it may have the elastic feel of fluid, as effusion rapidly occurs under tight constriction. Discoloration of the surface seldom takes place until very late, and is then more frequently the result of violent handling than from other causes.

It must be borne in mind that the appearance of the surface is no indication whatever of the condition of the parts within. There is likely to be heat after the strangulation has lasted for some hours, but not in the earlier stages. It should also be remembered that an inflamed hydrocele, either in the scrotum or encysted in the inguinal canal, may be deceptive, but neither will be accompanied by general symptoms of strangulation. (This also applies to inflamed hydrocele in the canal of Nuck in the female. The latter is more likely to be confusing since many physicians are not aware that hydrocele occurs in the female.) A case was seen by the author in consultation, where all the physical signs of strangulated hernia were present-that is, a small tumor in the inguinal region, extremely sensitive, and with a history of hernia and trusswearing. To add to the obscurity of the case the patient had 
felt that he might vomit at any moment, although he had not done so. He was sick only when the tumor was handled. It proved to be a case of orchitis in a testicle which had never reached its destination in the scrotum.

\section{SYMPTOMS.}

Pain.-It almost invariably happens that the very first pain experienced from strangulated inguinal hernia is not, as would be expected, at the point of stricture, nor even in the region of hernia, but in the vicinity of the umbilicus-an ill-defined, colicky pain that is likely to lead the patient to believe that he has eaten something which is disagreeing with him. This may increase to an extreme pain without local symptoms to lead the patient to examine his hernia, but in most instances there occurs more or less discomfort in the inguinal region, and the true condition of the swelling is discovered. The pain increases in intensity with more or less rapidity, according to the acuteness of stricture, and it is paroxysmal, appearing at times to have left the patient entirely and then returning with renewed force. This undoubtedly corresponds with the peristaltic action of the bowel which makes endeavors with increasing violence to free itself. If at this point the physician steps in and relieves the pain by a hypodermic injection of morphine, the patient's mind is also entirely relieved, but the pathological changes go on uninterruptedly and the man is rapidly advancing to his death. The pain is described by the patient as a most terrible distress rather than a pain, and it usually extends over the entire abdomen.

The following cases illustrate in a striking manner how little dependence can be placed upon the location of pain in strangulated hemia. A wonnan, seventy-five years of age, with valvular heart trouble and otherwise not in good general condition, developerl extreme abdominal distress, which was soon followerl by vomiting and complete intestinal obstruction. The family physician saw the case on the first clay and discovered that she harl small hernice in both the umbilical and femoral 
regions, but the patient assured him that there was no noticeable change in these swellings. Both had existed for several years and neither had been reducible for a long time. Local symptoms were entirely lacking. Hypodermics made a doubtful case still more obscure, as temporarily they relieved the pain. On the third day the case was seen by the author in consultation. It was at once decided that one of the herniæ was in trouble, and an immediate operation was strongly urged. There was no guide as to which hernia should be operated upon, except that femoral hernia is more liable to strangulation than the umbilical variety. Ether was given and a loop of small bowel was found caught under Poupart's ligament in the femoral canal. The bowel was in bad condition, but after applying hot cloths for half an hour, it was returned to the abdomen. The patient made a perfectly good recovery.

In hernix that have been long strangulated, the bowel after a time becomes paralyzed or gangrenous and the pain in a measure subsides. Distention ensues and the patient is very likely to die, even if an operation is performed. This remark must not be taken as intimating that the operation should not be done even in the most desperate case. Death is absolutely certain without it, and cases apparently moribund are not infrequently saved by it.

Vomiting.-The vomiting in strangulated hernia is doubtless reflex in character and may begin within a few moments of the strangulation or may be delayed for several hours. It is perhaps the most important symptom. If a portion of the bowel high up in the intestinal tract is involved, it is very likely to come on early, but this also depends somewhat upon the tightness of the constriction. It is quite certain that in cases involving the large bowel vomiting is very likely to be delayed. The contents of the stomach are first vomited and later the contents of the small intestine, when the characteristic fecal odor is presented. In former years it was considered good practice not to operate for strangulated hernia until stercoraceous or fecal vomiting had occurred. It is not surprising 
that few recovered after operation, and to-day such waiting would be plainly criminal.

Pain and vomiting usually indicate the severity of the case and the urgent need of haste. If the symptoms are violent prompt relief must be afforded or the patient will die. It happens rarely that complete clestruction of the bowel coats have taken place without vomiting having been present. The cessation of romiting in a given case, after having existed for some time, is a most grave symptom, and usually denotes complete collapse, or paralysis of the bowel, either of which is pretty certain to lead to a fatal termination.

Constipation is usually complete. In a fairly good proportion of the cases there will be one movement of the bowels after strangulation has taken place, the lower bowel merely emptying itself. There may be tenesmus and frequent desire and the feeling that a movement is about to occur. Cathartics, by increasing peristalsis and vomiting, merely add to the sufferings of the patient, and should never be given. Eructations and hiccough are frequently early and persistent symptoms.

There is one exception to constipation as a symptom, and that is in a case of partial enterocœle, which will be referred to $z$ little later.

Thirst is intense, and if indulged the fluid is at once rejected by the stomach.

The Pulse is many times an important aid in deciding as to the severity of a given case of strangulated hernia, and impending collapse is first indicated by changes in it. The grave character of a case may be indicated by it where other symptoms are not very prominent.

Temperature.-It is surprising how many physicians make the mistake of supposing that so long as there has been no elevation of temperature the case is not in great danger. The truth is that there is more frequently subnormal temperature than elevation. A neglected case which has developed general peritonitis may have elevation of temperature, but more die in 
collapse without ever reaching that stage. Ordinarily there is little change of temperature throughout the case, and it is important only when subnormal, which indicates the need for haste in the preparations for relief.

Respiration is almost always hurried, until the patient is passing into a condition of stupor, when it may become slow and stertorous.

Collapse may come on almost immediately after strangulation has taken place, or may be delayel for several hours. It is at any time a very grave symptom, and has been considered by some inevitably fatal.

That it is not universally fatal, the author has had several illustrations. In one case, that of a man nearly eighty years of age, who was apparently in complete collapse and about to die, with strangulation for about three days, the operation was done without an anæsthetic, as he was in a stupor. The bowel was found in bad condition, but, under the application of hot towels, showed evidence of recovery and was returned to the abdomen. The patient's condition improved before the operation was completed, and he made a slow but complete recovery. It has been noticed in several instances that the pulse, respiration, and general condition begin to improve shortly after the constriction is cut. It is also believed that the hot-water applications which have been so frequently used in these severe cases, acts as a stimulant to the patient. In one patient recently operated upon, collapse came on again after there had been general improvement during the operation, and the woman came very near dying on the table.

An otherwise healthy woman, sixty-eight years of age, suffering from an inguinal hernia on the right side, placed herself in the hands of one of the quack firms that promised to cure hernia by hypodermic injection. The first two injections, an interval of one week between, were fairly painful, but were tolerated without much complaint; the third, however, produced the most excruciating pain and prevented her leaving the bed on the following day. She grew rapidly worse and 
began to vomit, and her family physician came in and administered opiates. On the morning of the second day consultation was called, and the case pronounced strangulated hernia, and was sent to the author for operation. When she arrived at the private hospital she was in partial collapse, with the whole body bathed in cold perspiration, and suffering from the most agonizing pain. Preparations were quickly made and the operation begun. Her daughter was informed that death might occur before the operation was completed.

Upon opening the parts, it was found that the injection for the so-called "cure" of hernia had been through the neck of the sac into the coat of the bowel, and the violent inflammation following had resulted in a complete stricture of the bowel. The bowel was adherent in the canal, but it could not be decided positively whether this adhesion was due to the injection, or had previously existed. After the constriction had been cut and hot applications made to the bowel, the patient's condition improved rapidly, but just as she was about to be transferred to the bed it was discovered that she had again gone into a state of collapse so complete that at one time she was thought to be dead. After an hour's work with the use of oxygen, stimulants, and hot saline solutions under the skin and per rectum, she was restored and made a complete and rapid recovery.

This case has been given somewhat at length, as containing several illustrative points.

Ist. - That the injection of irritants about the canal may cause deatl..

2nd.-That strangulation may be secondary to an earlier inflammation.

3rd.-That patients may suffer and even die, from the recurrence of the symptoms of collapsc.

4th. - That it is not best to abandon too early efforts at restoration of a patient in collapse.

Collapse may come on so early and violently in cases of strangulater hernia, as to cause the death of the patient before 
it is possible to afford relief. The amount of injury to the bowel cannot be estinated by the degree of collapse in which the patient is found. In some cases, when death has occurred early, the bowel has not been found badly damaged. Collapse and death must, in these cases, come wholly from reflex action. In cases long neglected, where fecal vomiting has existecl for some time, poisoning may occur from this source and death result either before or after the operation. It is in cases of just this character that general anæsthesia should, if possible, be avoided, as it adds to the depression which already exists, and there is also liability of the patient draving into the lungs, by inhalation, the poisonous fluids which are being constantly vomited. Convulsions are not uncommon as an early symptom in young children, and they may occur, though very rarely, in the adult.

Peritonitis, Local and General.-In almost every instance of strangulated hernia, some indications of localized peritonitis will be found, even though the case may have been of only a few hours' duration. This may consist merely of the exudation of a little plastic lymph, which glues the protruding contents to the side of the sac, or a quite active peritoneal inflammation, extending in every direction from the point of the constriction. It is believed that death more frequently results from shock due to pressure on constricted bowel than from general peritonitis. The latter, however, does occur, and when it is present the prognosis must be of the gravest character. Again, it will be urged, however, never to abandon a case no matter how hopeless it may appear. Better give the patient his only chance of life, even though he die on the table.

General peritonitis may develop from the point of constriction, following the peritoneal surface, or it may be communicated from the bowel, which is enormously distended above this point. From the latter cause it may become general, either before, or after the operation. Perforation of the bowel into the peritoneal cavity is usually followed by immediate collapse and early death. 
Where there is extreme abdominal distention, the prognosis is always bad, as it frequently indicates complete paralysis of the bowel above the point of constriction. In such patients one or more normal evacuations may occur after the operation, and then the symptoms of intestinal obstruction are again presented and the patient dies. The operator is quite liable under such conditions to think that he has failed to remove all constrictions of the bowel at the time of the operation, when in reality, the cause of death has been complete paralysis of the over-distended bowel.

Partial Enterocele.-This form of hernia is where one coat of the bowel has been caught and strangulated, but the entire lumen has not been occluded. The fibrous rings which frequently form in hernial sacs have been previously alluded to, and into a ring of this character such a hernia may protrude and become strangulated. This form of hernia has frequently been referred to as Lavaters, Littres, and Richter's hernia. Diagnosis is obscure, from the fact that a very small tumor, if any, can be found, and also that there is not complete obstruction of the bowel. It not infrequently occurs that there is a tendency to diarrhœa. The pain is, however, characteristic and similar to that found in other forms of strangulated hernia; that is, coming in paroxysms with intervals of relief. The distention found in other cases is not ustually present, and the stools are occasionally bloody.

It is seldom that such cases can be relieved except by operation, and the following case indicates their deceptive character: A man, twenty-four years of age, had left inguinal hernia since early childhood, and had never worn a truss. Symptoms of strangulation occurred five days before he was seen by the author, vomiting beginning on the second day. An operation was done on that day by the attending physician, who opened down to the external ring, incising a distencled sac of the congenital variety. From this considerable clark-colored fluicl escaperl, but otherwise it appeared empty. As the finger could be passed freely in either direction, 
he assumed that the hernia had been reduced under ether, and closed the parts. As later, symptoms increased in severity, the author was called to see the case three days after the operation. Marked evidence of intestinal strangulation was present. Extreme pain was masked somewhat by the free hypodermic use of morphia. The man had a dusky skin, was becoming stupid, had a distended abdomen, and a weak heart.

The wound was re-opened and the canal split to the internal ring. A knuckle of small intestine was found imprisoned just at the upper end of the canal, and as the stricture was divided, the gut dropped back into the abdomen. By enlarging the opening somewhat, and making gentle pressure upon the abdominal wall above, it was obtained again and brought outside for inspection. The anterior wall of the bowel had been under sharp constriction, but its mesenteric attachments had not been under pressure, so that the circulation of the posterior surface had been maintained. The bowel was dark and edematous, but under the application of hot water a change of.color was noticeable, and it was returned to the abdomen. The man made a prompt recovery.

Where we have acute symptoms, showing us that the intestine is involved, it is desirable that we should see the bowel in order to decide whether or not is is safe to return it to the abdominal cavity.

Inflamed Glands, occurring in those who have been known to have suffered from hernia, may cause considerable obscurity in the diagnosis. It will at once be seen, by lack of general abdominal symptoms, that the intestine is not involved, but it is not so.easy to decide that a tumor may not be a mass of inflamed omentum. We almost invariably, however, have a few abdominal symptoms where the incarceration involves omentum exclusively.

Orchitis in Retained Testicle or Torsion of Cord may cause some confusion in diagnosis with physicians who do not see many cases of this character. The table of Eccles (" Imperfectly Descended Testis," W. McAdam Eccles, M.S., 
F.R.C.S., Wm. Wood \& Co., I903) is so suggestive that it is thought best to introduce it here.

Differential Diagnoses of Torsion of Spermatic Cord, Strangulated Hernia and Acute Lymphadenitis.

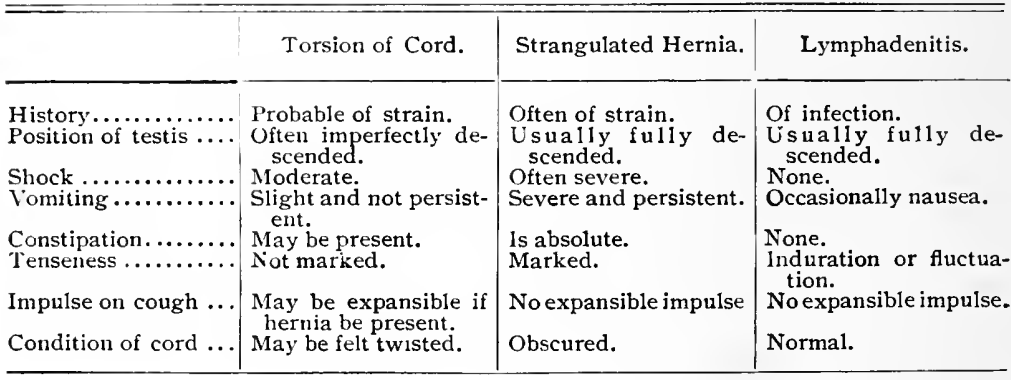

The lodgment of a testicle in the inguinal canal is quite liable to be mistaken for strangulated hernia. Reference has already been made to such a case seen in consultation, where two physicians had failed to recognize the true condition. The error is quite excusable on account of nausea and vomiting frequently present in this condition. Intestinal obstruction is not present, however, and the character of the pain is quite different from that present when the bowel is strangulated. In the latter condition the pain is intermittent, corresponding with the violent peristalsis, while in orchitis there is an intense, dull, sickening pain which is constant. The onset of the pain is usually quite different, that of strangulated bowel being sudden and rather violent in character from the first; in orchitis there is usually a history of traumatism with gradual increase of the symptoms. The absence of the testicle from the scrotum should also cause suspicion as to the true cause of the trouble.

Scrotal Hernia of Enormous Size.-In these cases symptoms of strangulation are seldom of the acute variety. Usually they contain Jarge masses of omentum, and the protrusion of the bowel insicle is somewhat protected from extreme pressure even though it may be strangulated. If the contents of these larger hernix should be exclusively omentum, the symptoms 
will then be more of an inflammatory character, and under rest and cold applications may subside without operation. On the contrary, however, if the constriction is very tight, sloughing of the omental mass may occur, seriously complicating the case.

A case of this character which had been five weeks under treatment was brought to the author from a distant city. During most of this time the man had been in bed and applications of hot olive oil had been made to the tumor. Upon operation it was not only found that the omentum in the scrotum was about to slough, but that the entire omentum, above as wel! as below the point of constriction, was involved. This omentum was inflamed, thickened, and attached to the peritoneal surface, so that it was necessary to leave it in about the condition found, although that portion in the scrotum was removed and the abdomen closed. The wound closed by primary union, but it was found at the end of the second week after the operation that general abdominal trouble was increasing. A hard tumor had formed nearly on a level with the umbilicus and about three inches to the outer side. An incision was made into this, and for nearly three weeks sloughing omentum was discharged, but the man eventually made a good recovery.

Strangulation, occurring in hernize of enormous size, is frequently preceded by a long term of obstinate constipation, finally terminating in complete intestinal obstruction. Such cases, unless operated on very early, are quite sure to prove fatal, as they have their origin in the paralysis of the loop, or loops, of bowel which have formed the contents of the hernia. The bowel, being held by adhesions, has gradually lost its peristaltic power, and when paralysis has restlted symptoms of intestinal obstruction are presented. Such cases do well for a few days after the operation, when intestinal obstruction again comes on and the patient dies. In these large hernix, strangulation may occur by bands of connective tissue which hold the bowel at an acute angle. At the time of the operation, 
such bowel will be found in perfectly normal condition, but doubled upon itself, in such a manner that its lumen is occluded.

Children.-As a general rule, the symptoms of strangulation in young children are not quite so violent and acute as in older persons, nor is the occurrence of strangulation attended by quite the same amount of danger. Many cases of temporary incarceration have been seen in children under treatment by mechanical means, with little indication of pain or intestinal obstruction, yet the hernia was hard and irreducible. Such cases, of course, require close watching, but an early. operation is not necessarily indicated. The author has, in a number of instances, sent the mother home with the child in this condition, with instructions to make gentle pressure over the tumor when the child was asleep; in almost every instance this has proven sufficient to cause its reduction. With careful and proper manipulation cases demanding immediate relief can generally be reduced while the child is under chloroform.

The author has never seen a case of strangulated hernia in infancy that he could not reduce under chloroform by careful manipulation, but he freely admits that such cases may occur. Even more care must be observed than in handling the adult, owing to the greater delicacy of the structures. Force that would be allowable in the adult would be violence if used on an infant. After such herniæ have been reduced it is not uncommon for the child to have one or more bloody stools. 


\section{CHAPTER XXV.}

\section{MEDICAL TREATMENT OF STRANGULATED HERNIA.}

Perhaps the very best way to discuss this branch of the subject is to state at once that there is no medical side to the treatment of strangulated hernia, leaving the subject there. To many this would doubtless appear dogmatic; on the other hand, there is reason to treat of the medical side in order to show its dangers. Certain it is that medication is time-consuming, and therefore dangerous, and should be avoided in almost every case.

The literature of the subject is not lacking in the number of remedies which have been recommended in these cases. Large doses of atropine, opiates, and other drugs, as well as strong decoctions of coffee, have been recommended. Atropine and morphine have been given by physicians, having in mind the theory that strangulation comes from a spasm of muscular tissue, and that when this spasmodic action is overcome reduction of the hernia can be accomplished. It is believed that all operators of large experience will bear out the statement that no such condition is found to exist at the time of operation. Perhaps no drug has been the cause of more deaths in strangulated hernia than opium and its products. The hypodermic injection of morphine, which has been such a blessed relief to the patient, has misled both him and the attendant into the belief that the conditions were improved, while in reality the pathological changes which take place in strangulated intestine have been rapidly advancing, and when the physician has discovered his error it is too late to save the patient. This means of relief to the suffering may and should be given while preparations for a more rational and radical form of relief are being made. 
The use of coffee or cathartics is thoroughly irrational, although they have, in some instances, hastened relief by causing violent peristaltic action of the bowel and thereby aiding in its withdrawal from the point of constriction. This, no doubt, is the only action which strong decoctions of coffee have. The coffee has one advantage over cathartics in that it is a powerful stimulant and delays the period of collapse. The cathartic is sure to leave the patient in much worse condition than before it was taken, and only in the rarest instances does it accomplish the desired result.

Safety is on the side of limiting medication to the hypodermic use of morphine (preferably combined with atropine), and the application of cold to the tumor, to afford the patient partial relief while preparations for the operation are being made; these should be advanced with all possible haste, even though the opiates have temporarily placed the patient in a perfectly comfortable condition.

Taxis.-By the word "taxis," a very indefinite idea is conveyed to the mind of the average physician regarding a certain form of manipulation, which may be more or less violent, according to the peculiarities of the manipulator, and the works on surgery, even of the later day, contain very little precise information regarding its technique.

Taxis comes midway between the medical and the surgical treatment of strangulated hernia, but properly belongs to the surgical side, and the operator should bear in mind that it is a delicate surgical procedure to attempt to reduce strangulated intestine by means of manipulation. He should realize the fact that not only is he liable to fail to accomplish the desired result, but he is apt to add materially to the risks which his patient has already incurred. The length of time this method should be userl can scarcely be stated, as one man will do more harm to the parts than another.

There are perhaps few things in surgery where more actual skill is required and can be displayed than in the reduction of strangulated bowel by manipulation, or so-called taxis. 
The author has, for many years, avoided the use of this word almost entirely, and has taught the reduction of hernia by what he has called traction and compression, which is accomplished in the following manner:

Try at once to assure your patient that you are not going to add to his torture, and confirm this in his mind by handling the tumor with the greatest gentleness. By this you will secure his co-operation instead of unconscious resistance. Place him on a table with the hips well elevated, instead of working over a soft and yielding bed. An ordinary kitchen table, with the legs at one end elevated six or seven inches, answers every purpose, and is obtainable in almost every house. When the patient is in position, first gently crowd the entire abdominal contents away from lower abdomen toward the chest, then work the fingers of one hand around the neck of the tumor where it issues from the abdomen, holding its bulk in the palm of the hand if possible, and, instead of trying to push the tumor back into the abdomen, try to draw it farther down. Now, with the other hand, grasp the canal with its contents (if inguinal hernia) gently but firmly between the thumb and fingers, and, while making traction, and compression with the hand that is holding the tumor, manipulate the canal with a kneading motion. This can be done without adding to any extent to the patient's pain, and will succeed when more rude handling fails.

When you push upwards on strangulated hernia, usually you carry it up over the ring upon the abdominal wall, and accomplish nothing more. In the method suggested, by traction you lengthen out the mass that is blockading the canal, favoring the effect which you afterwards produce by compression, i.e., the partial emptying of engorged blood vessels, and the displacement of gases and fluids. This is further aided by the action of the fingers upon the canal, working the bowel free at the point of constriction. This work should be done only while the patient is conscious, as his endurance of pain should clearly indicate the amount of force that it is desirable to use and beyond which it is never safe to go. 
The amount of time which should be expended upon these cases depend somewhat upon their character. If the hernia is an extremely large one, and its contents chiefly omentum, considerable time may be allowed in this manipulation, carefully executed, without fear of doing harm to the patient. If, on the other hand, the hernia is small, with acute symptoms of strangulated intestine, not more than fifteen minutes should be expended in attempts at reduction. If it cannot be reduced in this time, nothing but harm can come of prolonged handling. So far as possible, not more than one physician should ever attempt to reduce a hernia by manipulation. The second one called will do well to take none of the responsibility of handling the tumor, but should proceed at once to the operation. It should be borne in mind that rude and violent handling of a strangulated bowel is far more dangerous than an operation, even though the latter be done by a man who does not consider himself an expert.

External Applications.-The literature on this branch of the subject is full of delusions and snares. Every sort of poultice, hot and cold, that conld possibly come to the imagination of man, has been applied to strangulated hernia. There is only one external application which should be applied, and only in the earliest stages of the difficulty, and that is cold. Sulphuric ether, allowed to drip slowly over the tumor, has been very strongly recommended, and no doubt has scored some successes. Ethel chloride spray has also been suggested. The only effect that such an application has is the cold produced by rapid eraporation, and if it can be obtained more conveniently in this way than by the application of ice, it is all right. Personally ice is preferred. If delay is necessary in preparing for the operation in a given case, two things should always be resorter to in a pallative way-the hyporlermic injection of morphine in relieve pain, and the immediate application of ice upon the tumor. Again it must be urgerl that this application must he only in the earlier stages before pathological changes have taken place. 
Herniæe of the large type, containing large masses of omentum, and where the symptoms are not very acute, may frequently be reduced after the application of an ice-bag for twenty-four hours or less. This amount of delay must certainly not be tolerated in cases where there is extreme pain and vomiting. Aspiration in order to draw off the fluids of the tumor and thereby aid in its reduction formerly had strong advocates. Not only is there serious risk of injuring the already damaged bowel, but it is believed by the author that the fluid is both a protection to the gut and an aid in its reduction.

Anæsthesia for the Reduction of Hernia.-The opinion of the author on this point has been indicated by the statement made before, that taxis should be used only on conscious patients. It is believed that it seldom happens that a judicious amount of force is used where the patient is uncler the effects of anæsthetics, and that this force applied to strangulated intestine is more dangerous than operation for its relief. An anæsthetic should seldom be given until all preparations for the operation have been completed. This rule does not hold good with infants, in whom reduction is so frequently accomplished.

In some instances, where the patient is very much alarmed regarding the operative part of the work, it is a comfort to him to be assured that an attempt will be made to reduce the hernia after the anæsthetic has been given, and that only in case this fails will the operation be done; manipulation should be even more gently executed than were the attempts at reduction while the patient was conscious. 


\section{CHAPTER XXVI.}

\section{SURGICAL TREATMENT OF STRANGULATED INGUINAL HERNIA.}

As previously intimated, the treatment of strangulated hernia, from first to last, belongs strictly within the domain of surgery. The cases are, however, usually first seen by the practitioner of medicine, who, perhaps, ordinarily does nothing but minor surgery and who has been taught to look upon an operation for this affliction as belonging to the major operations. Unfortunately, also, a surgeon may not be within easy call and hence the liability to delay means death to the patient. In an emergency of this character it is far better that the practitioner subject his patient to the risk attendant upon an unskilful operation rather than to those involved in delay. The life-saving element in this operation is not difficult to carry out if done early in the case before complications, due to disease, have set in. It is purely a mechanical problem and rerquires no such anatomical knowledge as is usually supposed. An unyielding band of some sort surrounds the intestine and must be cut.

There are two operations that every physician should be prepared to perform at the very shortest notice; viz., Tracheotony and Herniotomy. The difficulties of both have been exaggerated by the too careful anatomical consideration of the parts. I do not mean by this to discourage minute and exact anatomical knowledge; but it is not possible that every physician should remain throughout life a perfect anatomist, nor should he be discouraged from executing these life-saving measures because he has forgotten the number and rlistribution of the ressels and nerves of the parts.

The preparations and instruments may, if necessary, be of the simplest character. The author has operated with nothing 
but a knife, clamp, scissors, and needle from a borrowed pocket case, and silk from the work-basket of the patient's wife. It is beyond question that the patient's life was saved in this instance by the immediate release of the imprisoned gut. Where time allows, it is unquestionably better that every preparation be made that usually attends any other abdominal surgery. In any case the parts must be thoroughly cleaned, the water, instruments, and towels used about the wound boiled, and the greatest source of danger, the operator's hands, must be made aseptic by much scrubbing, closely trimmed nails, and such antiseptics as may be at command.

If fecal vomiting has already occurred, it is advisable to wash out the stomach before giving an anæsthetic. The presence of this poisonous matter in the stomach certainly leads to toxemia and adds to existing shock and prostration. Furthermore, vomiting, which is quite sure to occur during anæsthesia, may result in carrying this matter into the lungs by inspiration, thus causing pneumonia. The question as to the advisability of giving a general anæsthetic, or resorting to local anæsthesia, must be seriously considered. If the case has already existed for some time, with shock and fecal vomiting present, sensation is considerably blunted, and by the aid of local anæsthesia the operation may be done without unbearable pain. The local anæsthetic recommended is cocaine by the Schleick method, always being careful not to exceed the physiological dose of the drug.

The Incision.-If the hernia is large, this should be a little longer than that made in the operation for the radical cure, but on the same lines. It should extend from above the internal ring to a point over the upper part of the pubic bone, following the direction of the cord in the male, and opening up the entire canal.

In making this incision the superficial pubic vessels will be cut at the lower angle of the wound and the superficial epigastric vessels at its upper angle. Both are between the skin and the external oblique muscle, and, while they will probably 
require clamping, are unimportant. If they are large and bleed freely it is better to tie them at once with small-sized catgut; if small, merely allow the clamps to remain on while other work is being done; they will then frequently be found permanently closed. It is essential, however, that no oozing points remain when the parts are closed.

The opcning of the canal should now be accomplished by splitting the aponeurosis of the external oblique from a little above the internal ring to the external ring. Where the external ring is sufficiently free to allow of it, this is best done by slipping in a grooved director and cutting upon it, but in strangulated hernia it is seldom possible owing to the constricting bands about the ring. In this case a small opening may be made in the aponeurosis directly over the internal ring and the cutting clone by knife or blunt pointed scissors, following down in the direction of its fibres to the external ring. In many instances when the canal has been freely opened and the constricting bands at the external ring cut, the cause of the strangulation will have been removed and the hernia can be reduced; it is highly important, however, that this should not be done until the contents of the sac have been carefully examined. On the contrary, it may be that the constriction is in the neck of the sac, or by transverse bands within the sac itself, or a loop of bowel may be strangulated through a hole in the omentum. In any case the sac must be opened. The sac, having now been well exposed, should be carefully stripped loose from its adliesions and lifted, with the cord still attached, out of the canal.

This loosening of the sac is done by gently pressing the fingers around it in every direction, and it is easier while the sac is full than when it is emptied. Even when very large and in the scrotum, it may, in this way, be delivered through the high incision. The cord may sometimes be easily separated before opening the sac, but unless the fatal band of constriction has already been cut, very little time should be lost in this work, but proceed at once to the opcning of the sac. 
The discussion as to whether or not the sac should be opened belongs to a past age; it should always be opened.

Much care must be exercised at this stage of the operation, as it not uncommonly happens that the bowel has become adherent to the sac, and to cut carelessly one would be quite certain to open into the intestine. In almost every instance of strangulated bowel there is rapid effusion of fluid, and the opening into the sac should be at a point where this is felt to be present. Usually this will be at or near the bottom of the sac. This fluid may be a clear, colorless serum, or it may be of dark coffee color and of the most offensive odor. If of the latter variety it is full of septic matter and indicates a very grave state of affairs.

The sac having been freely opened, the constriction should be searched for and cut. The bowel should now be drawn down so that the part which has been subjected to the greatest pressure can be inspected. This usually is the point of greatest danger. The bowel may present any degree of injury, from a mere congestion of its surface vessels to gangrene and perforation, according to the length of time strangulation has existed, the tightness of the stricture, and the amount of rough handling it has been subjected to by those trying to reduce it. It is not uncommon to find it a dark claret color, and if there are no gangrenous spots, it may recover fully from this degree of injury under proper treatment. When the bowel has been drawn well down and all constriction removed, it is believed that there is nothing so beneficial as the application of towels wrung out of hot sterilized water. It is perfectly justifiable to spend half an hour or more, if necessary, in restoring the bowel in this way. If a change in color to a lighter shade is effected, it may be assumed that the bowel will live. If, however, there are spots of a dull, ashen-gray color, which have lost the lustre natural to the bowel, it may be known that these are liable to perforation if returned to the abdomen. If not too large, they may be folded in and the healthy edges of the bowel united by Lembert sutures of fine silk. 
If perforation has actually occurred, and the bowel is so far damaged as to preclude its repair by the turning in of its torn edges, then resection, or the formation of an artificial anus, should be considered. In this desperate condition, unless the operator is quite familiar with intestinal surgery, it will be a life-saring measure to anchor the bowel in the wound by a few stitches, covering it by moist, warm dressings, to be frequently changed. Should the patient survive, resection may be done as a secondary operation under more farorable conditions. Gibson in a valuable article on "Gangrenous Hernia " (Annals of Surgory, vol. xxxii. p. 486) gives the mortality in these cases, in the three most common forms of hernia, as follows: Inguinal, 26 per cent.; femoral, 37 per cent.; umbilical, 67 per cent. If the operator has at hand the Murphy button, primary resection may be quickly done, but even the handling of the bowel necessary for that method adds to the profound shock from which the patient usually suffers in the extreme cases under consideration.

Strangulated omentum found within the sac should always be removed, as returning it to the abdomen adds materially to the patient's danger. Omentum which has been under pressure and is inflamed may slough if returned to the abdominal cavity. Its ligation should be carefully done, as accidents from secondary hemorrhages have occurred, even in the hands of noted operators. It is well understood now, however, that accidents come from ligating large masses of omentum together and the subsequent slipping of the ligature. The omentum should be spread out thin and every vessel that can be seen tied, with as little fat as possible included. The fat should then be tied separately. This subject has been fully dealt with uncler the surgical cure of hernia.

An oc'ary or testicle in the canal must be treated according to its conclition. If unhealthy, it is very easy to remove it; if normal. it should be restored to its natural position. A normal testicle with a cord so short that it will not reach the scrotum should be treated in the manner described under the 
heading, "Complications in the Operative Cure of Inguinal Hernia." The reader is also referred to this section for details regarding the closure of the wound, as it should, with rare exceptions, be so closed as to effect a permanent cure of the trouble which has proven such a serious menace to the life of the patient.

The aftcr-trcatmont of the case should be conducted upon general principles to meet conditions present. Shock should be as promptly overcome as possible by hypodermic and rectal stimulation, and by surrounding the body with artificial heat. One of our most valuable means of combating shock is transfusion, the introduction of normal saline solution into the circulation. A strong decoction of coffee for rectal enema has proven efficacious. It is not deemed good practice to give cathartics early after strangulated hernia, especially if the bowel has been badly compressed. Pressure paralysis is liable to result, even though the coats of the gut have suffered little visible damage. It is better to give the bowel complete rest for a few days unless there are other symptoms contraindicating this course. It is also better to empty the lower bowel thoroughly by enema before giving laxatives. It must be remembered that perforation has occurred as late as the tenth day after doubtful bowel has been returned to the abdomen. Fluid food should therefore be continued for this length of time in suspicious cases.

Hemorrhage from the bowel, of greater or lesser degree. may occur after the return of the damaged gut, coming on from the first to the third day after the operation. Children are especially liable to this, and it has been seen where strangulation existed for only a few hours, and where the hernia had been carefully reduced without operation. In no instance has a fatal or very serious result been seen by the author. If this hemorrhage is accompanied by persistent diarrhœe it becomes a more serious matter, and repeated small doses of opium are recommended. The Subgallate of Bismuth, in doses of from 5 to to grains every hour, has, in the experience of the author, 
acted very nicely and saved the giving of opium, which is usually contra-indicated by the condition of the patient. A few loose movements produced by cathartics, unwisely given before the operation, must not be mistaken for the condition here named.

In almost every instance of strangulated hernia evidence is found of localized peritonitis, which, in a few cases, becomes general after the operation and must be treated accordingly. First among remedies is believed to be the ice coil, but this must not be applied while the patient is still in a condition of shock from the effects of strangulation. All of these complications result from delay, and will not be seen where prompt operative relief has been afforded. The operation itself is not one of danger. 


\section{CHAPTER XXVII.}

\section{STRANGULATED FEMORAL HERNIA.}

Symptoms.-The symptoms of strangulated femoral hernia do not differ materially from those attending stranguation at other points, except that the attack is likely to be more violent, the prostration (shock) is more profound, and disastrous results more quickly supervene if relief is not promptly afforded. The reasons for this especial violence in femoral hernia have been entered into in the anatomical considerations of the subject and need not be repeated here; but the urgent necessity for the earliest action possible cannot too often be impressed upon the physician, hor must he allow the temporary relief afforded by opiates to mislead or delay him for a moment. From the beginning of an attack to an early fatal issue the destructive pathological changes advance rapidly.

When the intestine forms the contents of the hernia there may be some premonitory abdominal discomfort, but it is more common for the pains to become severe at once, followed rapidly by all of the grave symptoms of acute intestinal obstruction; general abdominal pain, vomiting, prostration, and collapse. Old and feeble people may die from this collapse or shock within a very brief period of time, and before pathological changes have taken place to a sufficient degree to cause death.

Absence of local pain has undoubtedly misled the physician in very many instances, and hastened a fatal issue. It is not uncommon that the abdominal distress is so great that the patient's attention is not called to the site of the hernia at all; he may not even know that hernia exists. Contrary to experience in inguinal hernia, the author has seen several cases of femoral where it was quite evident that strangulation had taken place with the very first protrusion. Where decided abdominal 
disturbances exist all locations where hernia commonly occurs should be carefully examined and any abnormal condition found should be looked upon with decided suspicion. In the femoral region, especially, a small kernel no larger than the end of the little finger, the existence of which is wholly mnknown to the patient, may be an imprisoned knuckle of bowel which will certainly result in death if not recognized and relieved.

Where omentum only is strangulated, the symptoms may be of the mildest type, amounting to discomfort rather than pain in the abdomen, with a burning, or dragging sensation, the latter usually in the umbilical region. While these cases demand early surgical attention, there is not the urgent need for haste as when the bowel is imprisoned. In fact, this accident occurs many times without the true condition being realized by either the physician or the patient. When the temporary congestion of the strangulated omentum stubsides or its fat is entirely destroyed by cutting off its circulation, it leaves the patient with a permanently irreducible hernia. These hernize are very likely to increase in size, they are more dangerous to the patient than the reducible type, and should therefore be cured by operative means at the earliest convenient moment.

Reduction by Taxis.-All that has been said regarding the reduction of inguinal hernia by so-called "Taxis," or manipulation, applies here, with two exceptions: (I) The length of time that taxis is used, and the degree of force employed, should be decidedly modified. The tightness and knife-edge cluaracter of the constriction in strangulated femoral hernia make the chances of damaging the bowel by handling much greater and the probabilities of success less. Therefore, the greatest caution and gentleness in handling should be exercised. (2) The direction of pressure should be downward and toward the centre of the thigh. Any pressure upivard only draws the intestine up or'or Poupart's ligament and cloes not airl in the least in its reduction. 
Medical Treatment.-Properly there is no medical treatment for strangulated femoral hernia. If the hernia is large and the symptoms not very acute, indicating that the mass may contain considerable omentum, the ice bag should be applied while preparing for operation. This is not advisable if the attack has been of long enough duration to allow of destructive pathological changes, and if shock is present. It will not be of much service if the hernia is small and its contents exclusively intestine. Morphine combined with atropine should be used for the immediate relief of the patient while preparations are being made to operate, but with no other idea than that of temporary comfort. Do not be delayed one moment in prompt action, by the great relief that it affords.

\section{OPERATION FOR STRANGULATED FEMORAL HERNIA.}

All preparations for the operation for strangulated femoral hernia should be attended by the same strict regard for asepsis that is carried out in every important surgical procedure. The incision is the same as in the operation for the cure of femoral hernia. The sac should be loosened from its bed and brought out through the skin incision and its neck fully freed in the canal before opening. The constriction in femoral hernia is almost invariably beneath Poupart's ligament, and may be either in the neck of the sac itself or by its surrounding ligamentous structures. After raising the sac and its contents out of its bed, and the neck has been well freed, it is then carefully opened. It may be split up to and through its neck with little danger of injury to the bowel. This almost uniformly divides the constricting band. In cutting the stricture in strangulated femoral hernia, keep constantly in view the fact that the parts are to be so closed afterwards as to protect the patient against recurrence of the trouble. This cannot be done if Poupart's ligament is divided.

On opening the sac it is very common to find it filled with a coffee-colored fluid which is a product of strangulation and does not necessarily indicate that the case is particularly 
serious. If this fluid has a strong fecal odor, every precaution should be used to protect the wound, as it is quite sure to contain septic matter. The adhesions ordinarily found between bowel and sac are of recent origin and are usually easily separated. If, however, they are so firm as to endanger the tearing of the intestine, it is better surgery to cut out this adherent patch of sac and leave it attached to the bowel. No harm will result from this method of procedure.

All omentum that has been under constriction should be ligated and cut away in the manner described in the chapter on inguinal hernia. Intestine that has been constricted must be carefully examined for damaged places and especially must it be drawn down until normal bowel is seen on both ends of the loop in order to inspect the line that has formed at the point of greatest pressure.

Hot towels, as already suggested (see Inguinal Hernia), will obviate the necessity for resection in many instances. The completion of the operation should be in accordance with the suggestions for operative cure. It seldom happens that the case is so extreme that the proper closure of the parts to secure a permanent cure cannot be carried out without additional risk to the patient. 


\section{CHAPTER XXVIII.}

\section{STRANGULATED UMBILICAL HERNIA.}

In no cases of strangulated hernia is the prognosis more grave than in those occurring at the umbilicus. This is for the two-fold reason that strangulation usually occurs in hernixe of enormous size, where adhesions are numerous, and in very fat patients whose resistance is poor. It can seldom be reducerl by taxis, as the abdominal wall is so flexible as to furnish very little resistance, and, when the hernia is pushed upon, the whole wall is carried back and nothing is accomplished. The symptoms are in every respect similar to those of intestinal strangulation elsewhere. The use of the ice-bag here has, in the author's experience, been attended with good results, owing, doubtless, to the fact that in many instances umbilical hernia contains a large amount of omentum.

The operation must be conducted on the same general principles which govern that for other cases of umbilical hernia. Great caution is necessary in making the initial incision, as distended bowel may lie in such close contact with the inner surface of the sac that the cutting of one is pretty sure to accidentally open into the other. Here, as in other cases of strangulated hernia, fluid is quite sure to collect in some part of the sac, and usually it can be safely opened by taking up a small piece of the latter between anatomical forceps. In many cases the knuckle of bowel that is in trouble may be inside of a layer of omentum, and on opening the sac it is a great error to try to reduce the whole mass without examination. The bowel that is pinched must be found and its condition carefully considered. That which has been previously said regarding the treatment of strangulated intestine applies here as well. In one of my own cases, on opening a large sac a mass of omentum and a loop of intestine were found, both apparently normal, except 
that the former was hypertrophied and adherent in several places. The intestine was easily reduced through a perfectly patent umbilical ring, whereupon it was discovered that the strangulation of the bowel was through a hole in the omentum just within the abdominal wall. In another case the bowel was found incarcerated uncler a tough fibrous band, the remains of the obliterated unbilical vessels; it was flexed upon itself at such an acute angle as to entirely close the lumen of the bowel.

Umbilical sacs of large size are much more liable to have connecting fibrous bands running across them in various ways than large sacs in other forms of hernia, and in these bands intestine is quite liable to become entangled. In these, as in all other large hernix, all parts of the bowel that are reducible should be returned to the cavity of the abdomen as quickly as possible and with the smallest amount of handling. Such parts as cannot be immediately replaced should be covered with moist, hot, sterile towels, changed sufficiently often to maintain a temperature of about roo degrees.

The treatment of strangulation in the rarer forms, ventral, lumbar, obturator, and perineal hernia, needs no special consideration. When there is intestinal obstruction and hernia exists at any point, time will usually be saved by operating first at the site of this hernia, wherever located, even though there are no local symptoms. 


\title{
SUMMARY OF I,4II PERSONAL OPERATIONS FOR ABDOMINAL HERNIA.
}

\author{
(Tabulated January, I907.)
}

Males, 757. Females, 274. Patients, r,o3r.

Ages under Io years (27 under 5 years; youngest 5 months). . I66

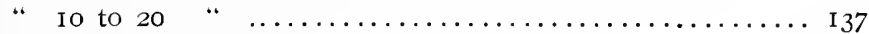

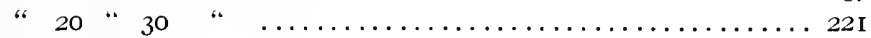

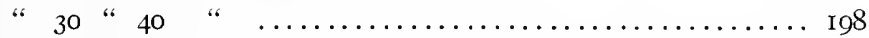

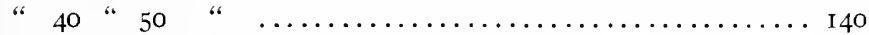

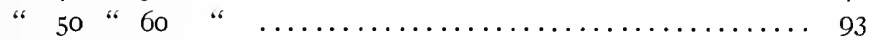

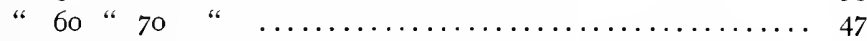

" 70 " 80 "

" over 80 "

Under I4 years, 239. Over I4 years, 792.

Inguinal Hernia: Right, 7I3. Left, 524. (Double, 253)....1237 (I205 were operated upon by Bassini method)

Femoral Hernia: Right, 75. Left, 44. (Double, I2)..... I I9 (II9 operated upon by De Garmo method)

Umbilical Hernia

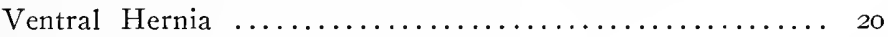

I4I I

5 had Inguinal Hernia on one side and Femoral on the other.

6 " Double Inguinal and Femoral.

2 " Inguinal and Femoral on same side.

I “ Double Inguinal and Umbilical.

I “ Inguinal and Umbilical.

I " Femoral and Umbilical.

Triple operations were done upon the same patient in 7 instances. The Bladder was recognized but not opened........... I7

The Bladder not recognized and opened for sac.......... I

(A11 in inguinal hernia)

Ovary in canal (Youngest in girl of 7 years)

Fallopian tube adherent outside Umbilical ring........... I

The Appendix was found involved and removed through inguinal

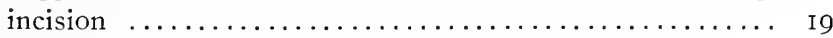

Retained Testes: Right, 39. Left, 33. (Double, 8)...... 72 
Varicocele sufficient to require operation............. 32

(Operation through inguinal incision in $3 \mathrm{r}$ )

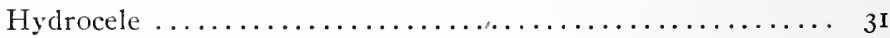

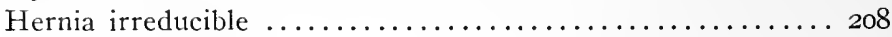

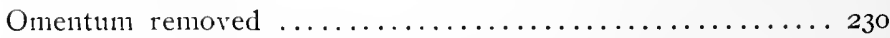

Hernia strangulated (Inguinal 21, Femoral I6, Umbilical 8) . 45

Recurrences, 19. Reoperated upon, $9 \ldots \ldots \ldots \ldots \ldots \ldots \ldots$ ro

\section{Mortality.}

43 Operations for Strangulated Hernia: deaths........ 9

1257 " " Cure of Hernia: deaths.......... 8

The operations for cure of hernia were not all operations of choice.

While some were considered "extra hazardous," it was believed safer to operate than to leave the patient to the risks of his condition. My-

Ist death (Case No. 429) in the latter class was a man of 75 years, completely disabled by the size of his hernia. He stood the operation perfectly, and his condition was good until on the third night, when he got out of bed and went to the toilet. He was missed from bed on return to ward of the night nurse and was found in the toilet room in a state of collapse, from which he never recovered.

2d death (Case No. 467). Boy 8 years old. Syphilitic meningitis 4 days after operation. Autopsy by Professor Brooks, Pathologist of Post-Graduate Hospital, showed wound and abdominal cavity in normal condition. One brother of this boy had died of meningitis after a four hours' illness, and another brother has partial paralysis. These facts were not known to author before operating. 3 death (Case No. 583). Man 48 years old. On 7 th day Sepsis. $4^{\text {th }}$ death (Case No. 628). Man 49 years old, very fat. On 6th day Volvulus, following operation for sigmoid hernia.

5th death (Case No. 73I). Man 55 years. Left Complete Inguinal Hernia. I Ith day Pulmonary Embolism following thrombus of left femoral vein.

6th death (Case No. 965). Man 42, enormously fat. Hypertrophied mesentery. Inoperable case. Shock.

7 th death (Case No. I280). Man 56. Weight 300 lbs. Very large right Scrotal hernia. Infection through drainage tube in scrotum. Sepsis on 5th day.

8th death (Case No r364). Man 43. Double inguinal hernia of small size. No unfavorable symptoms. Death on 3rd day. Apoplexy or pulmonary embolism. 


\section{INDEX}

\section{A}

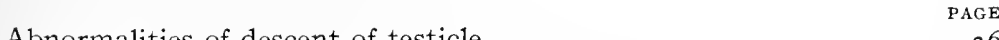

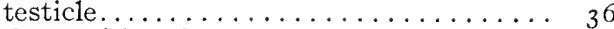

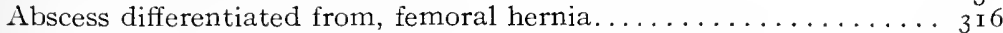

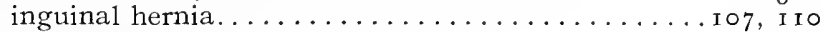

Accidents of hernia operation. .................... 4 Io

Acquired hernia..........................

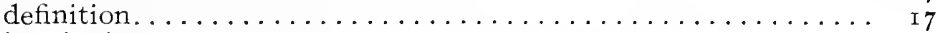

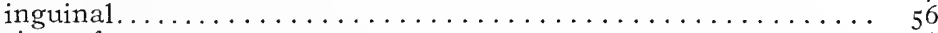

time of appearance......................... 8

Adhesions of contents to sac in inguinal hernia as complication, to

operating.............................. 6 r

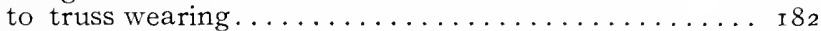

Age, as contra-indication to hernia operation. ............ 4 I0

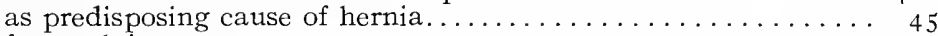

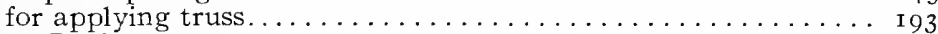

Albert, Professor.......................... 4 I0

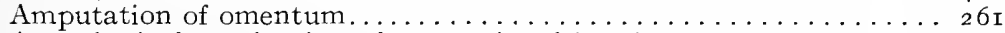

Anæesthesia for reduction of strangulated hernia. . . . . . . . . . 43 I

Anatomical defects as predisposing cause of hernia........... 46

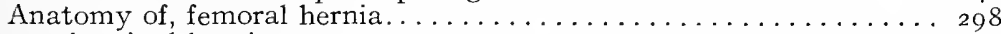

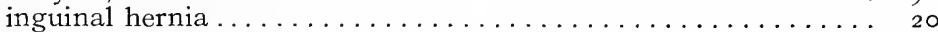

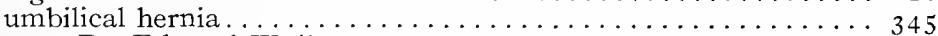

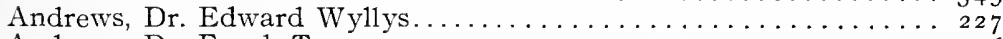

Andrews, Dr. Frank T........................ 256

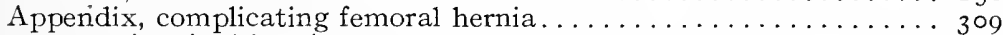

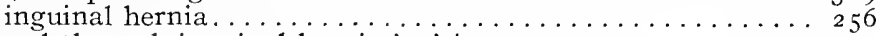

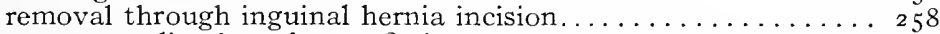

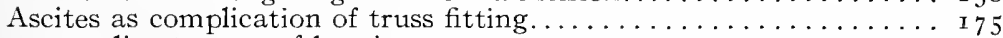

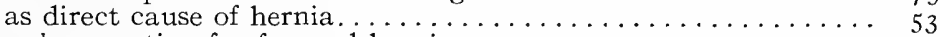

Author's operation for femoral hernia. . . . . . . . . . . . . 445

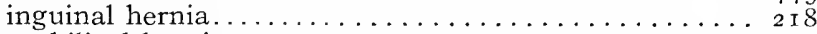

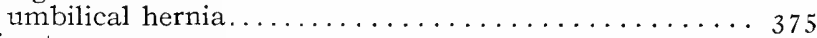

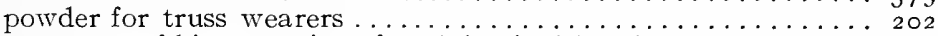

summary of his operations for abdominal hernia. . . . . . . . 445

Baldwin, Dr. J. F.......................... 286

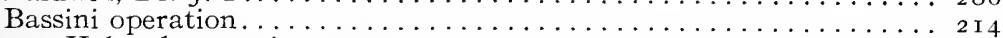

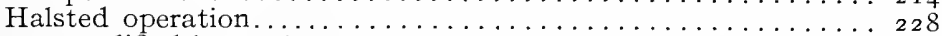

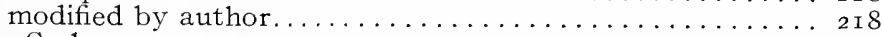

Beck, Dr. Carl. . . . . . . . . . . . . . . . . . . . . . . 397

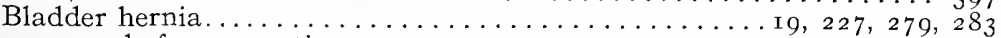

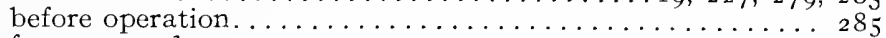

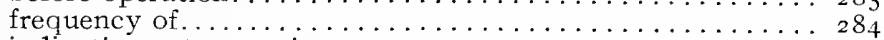

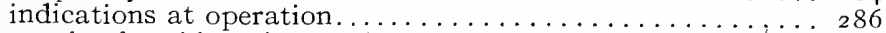

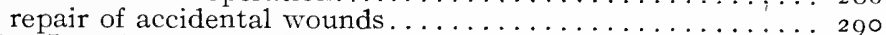

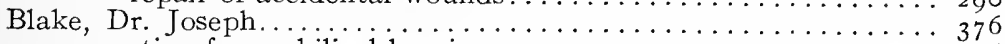

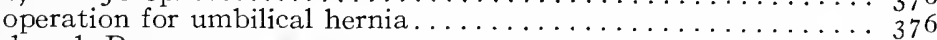

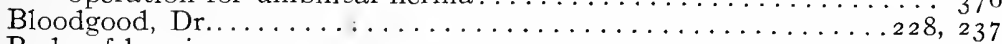

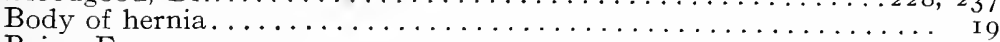

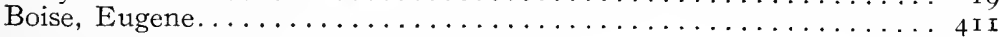




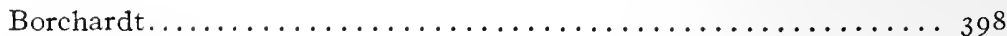

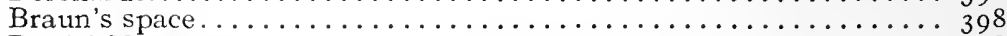

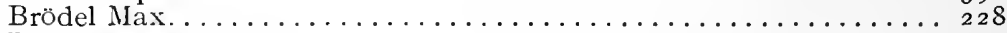

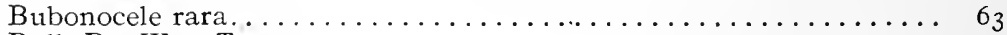

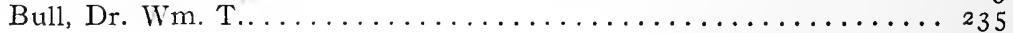

C

Cæcal hernia....................... 19,63, 277, 279

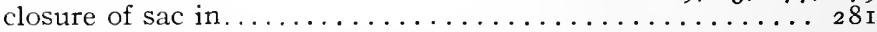

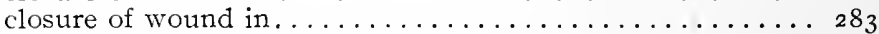

differentiated from other inguinal hernia........... 98

Canal of Nuck............................. 42

Care of skin under truss......................

Causes of, difficulty in operating direct hernia............ 227

femoral hernia.............................. 304

inguinal hernia....................... 44

direct.............................. 50

predisposing $\ldots \ldots \ldots \ldots \ldots \ldots \ldots \ldots \ldots \ldots \ldots \ldots \ldots \ldots \ldots, 44$

strangulated inguinal hernia................ 4 r 3

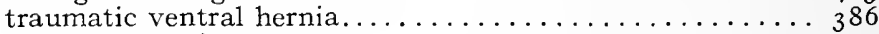

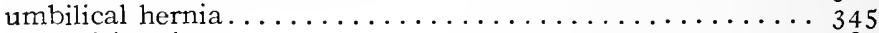

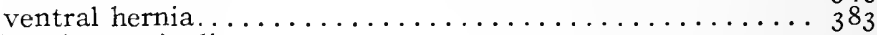

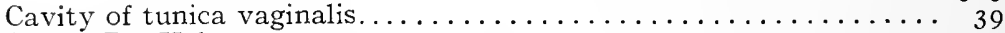

Chase, Dr. Heber......................... I 35

truss for femoral hernia ........................ 326

inguinal hernia.................. I 29, I35

Children with strangulated inguinal hernia............. 426

Classification of trusses. . . . . . . . . . . . . $8 \ldots \ldots \ldots \ldots$ I 8

Closure, of sac in cæcal hernia.................. 28 I

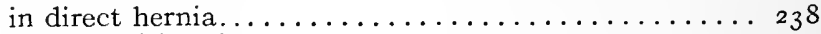

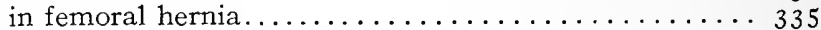

in sigmoid hernia................ 28 I

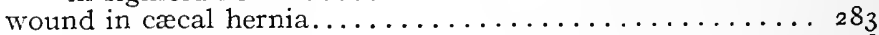

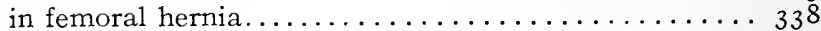

in inguinal hernia.................... 223

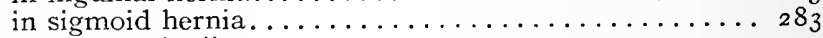

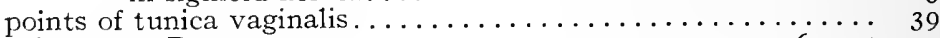

Coley, Dr. Wm. B........................ 225, 235

Combination of hernias. . . . . . . 8

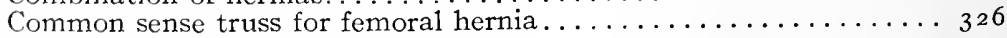

Complete hernia........................... 55

Complications and their treatment in operation for inguinal hernia.. 239

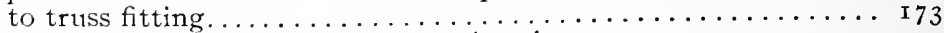

Comnression and traction in reducing hernia ............. 429

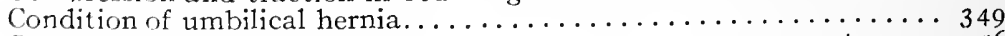

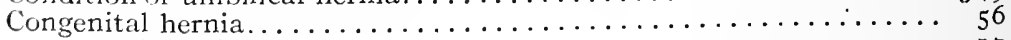

definition......................... 7

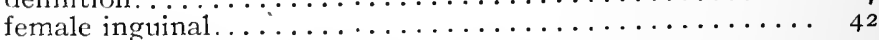

male inguinal....................... 40

differentiated from icquired inguinal hernia....... 92

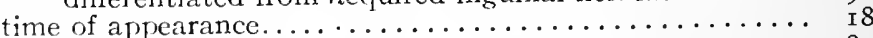

umbilical hernia ..........................

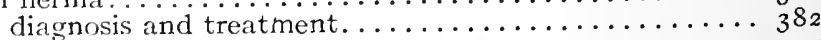

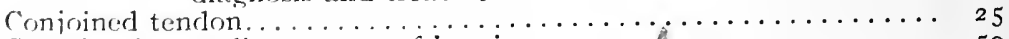

Constipation as direct cause of hernia................... 50

constituents of hernia...................... I9

Construction of trusses. . . . . . . . . . . 


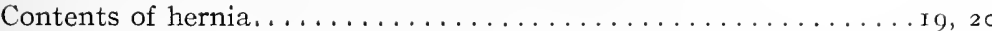

femoral hernia......................... 309,333

inguinal hernia..................... 79

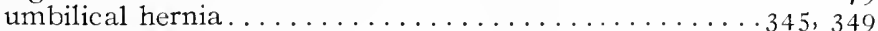

Continuous truss wearing. . . . . . . . . . . . . . 202

Contra-indications to surgical cure of abdominal hernia........ 409

Cooper, Sir Astley........................... 44

Cough as direct cause of hernia ........................

Coverings of inguinal hernia........................ 77

umbilical hernia.............................. 348

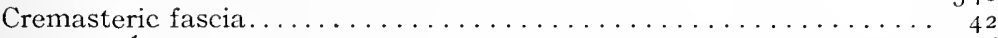

muscle.......................

Cross body truss............................ I29, I 69

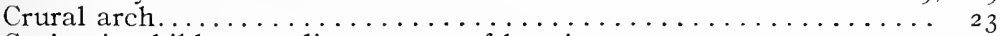

Crying in children as direct cause of hernia.............. 53

Curative treatment of inguinal hernia................ I 3

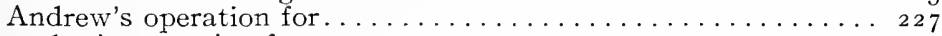

author's operation for ................. 2 I 8

Bassini's operation for .................... 2 I 6

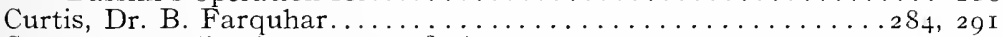

Cysts as complication to truss fitting.............. I 73

differentiated from inguinal hernia................ I Io

of tunica vaginalis....................... 40

D

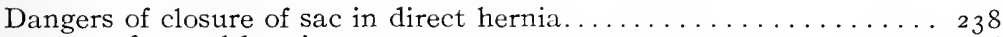

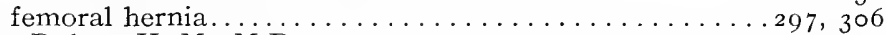

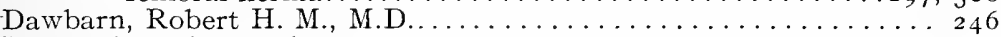

Deep epigastric vessels......................... 27, 29

Definition of hernia........................ I 7

acquired hernia.................... I 7

congenital hernia................ I 7 , I

femoral hernia ................. I 7

inguinal hernia.................. I 7

umbilical hernia................... $7,3+4$

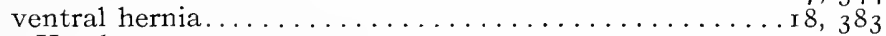

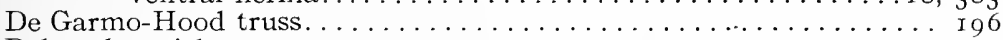

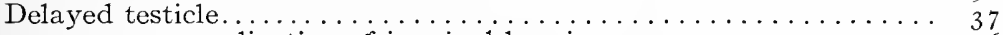

as complication of inguinal hernia.............. I 76

complication of inguinal hernia operation......... 239

predisposing cause of inguinal hernia........... 46

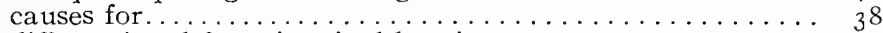

differentiated from inguinal hernia............

function of $\ldots \ldots \ldots \ldots \ldots \ldots \ldots \ldots \ldots \ldots \ldots \ldots \ldots \ldots \ldots \ldots$

Descent of ovary as complication to truss fitting........... I 80

testicle............................ 34

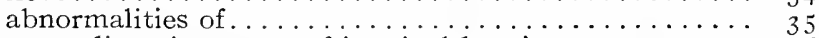

as predisposing cause of inguinal hernia.......... 46

tunica vaginalis as predisposing cause of inguinal hernia... 46

Diagnosis of congenital umbilical hernia ................. $3^{2}$

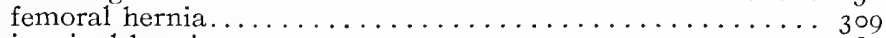

inguinal hernia. . . . . . . . . .

oblique inguinal hernia from other conditions........ I02

traumatic ventral hernia.................. 388

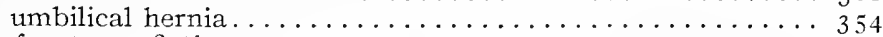

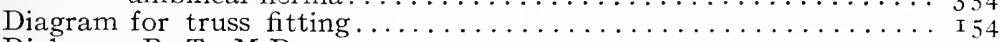

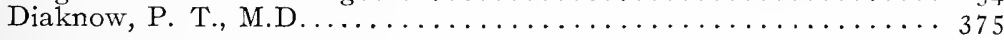


Diaphragmatic hernia. . . . . . . . . . . . . . . . . . 42

Differential diagnosis, between femoral and inguinal hernia....... I I I

femoral hernia...........................

strangulated inguinal hernia................. 423

types of inguinal hernia.................... 9I

Difficulties of mechanical treatment of femoral hernia........ 3 I 7

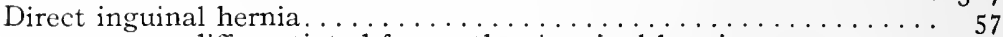

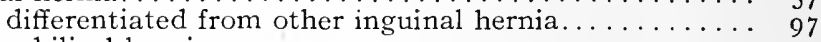

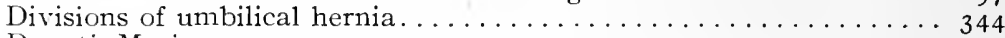

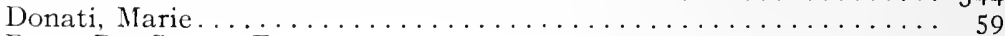

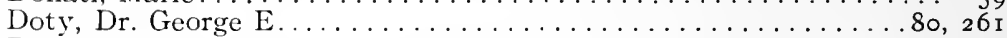

Double truss .............................. I 47

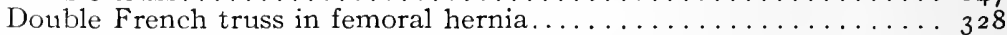

Dowd. Dr. Chas. N........................... . 400

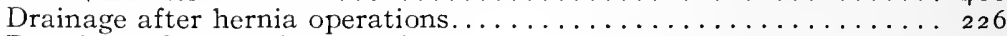

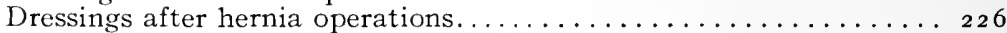

Dummy pad on truss ........................ I 4 I

Duodeno-jejunal recess....................... 4 I 2

Eccles, W. McAdam, M.S................. 35, 36, 383, 423

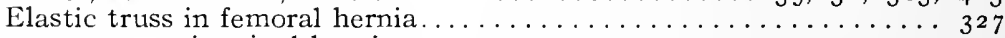

inguinal hernia............................... I 2 I

English truss........................... I 2 I

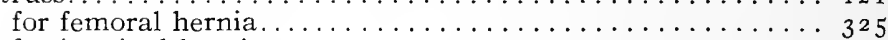

for inguinal hernia. ...................... I 24

for umbilical hernia. . . . . . . . . . . . . . . 369

Enlarged veins as complication to operation for inguinal hernia.... 239

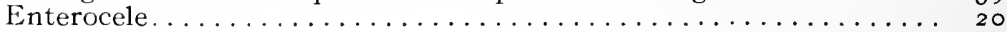

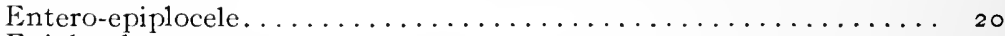

Epiplocele............................... 20

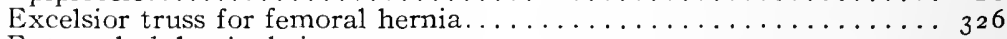

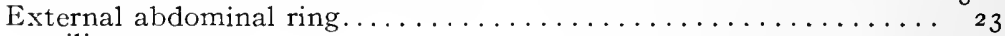

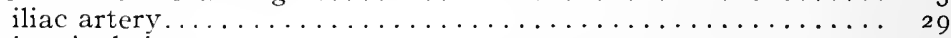

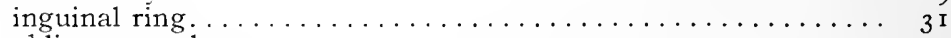

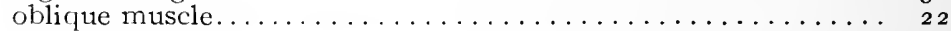

pudic vessels. . . . . . . . . . . . . . . . . . . 22

F

Fat as, complication of operation for oblique ingtuinal hernia. . . . . 239 contra-indication of operation of abdominal hernia...... . . 409 predisposing cause of hernia.................. 49

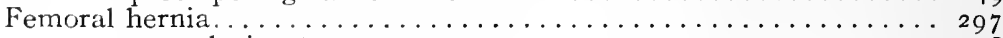

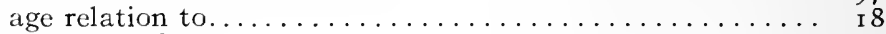

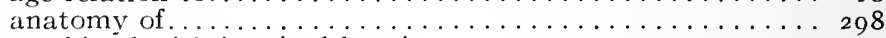

combined with inguinal hernia.............. 340

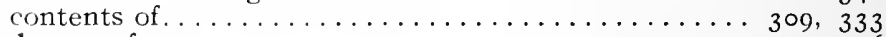

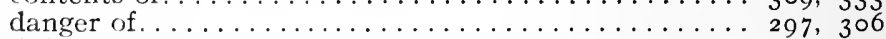

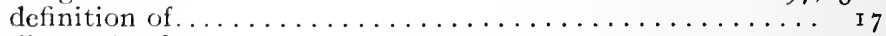

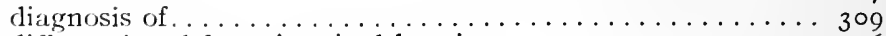

differentiated from inguinal hernia.............. I I , 3 I 6

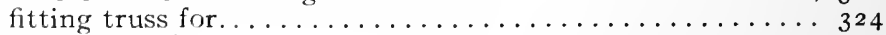

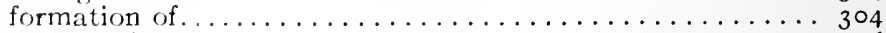

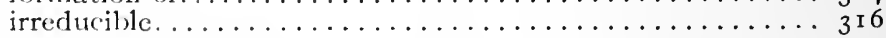

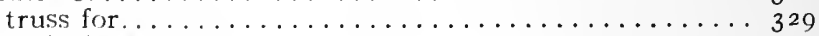

mechanical treatment............................ $3^{\text {I }} 7$

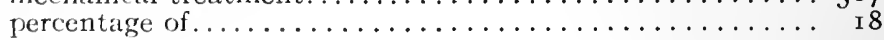


PAgE

Femoral hernia, post-operative treatment of $\ldots \ldots \ldots \ldots \ldots \ldots$

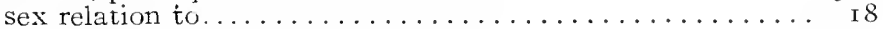

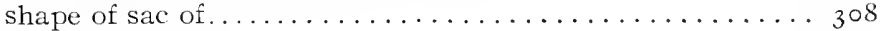

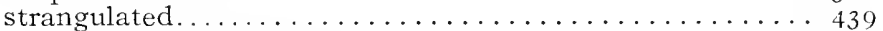

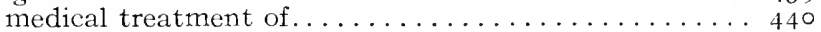

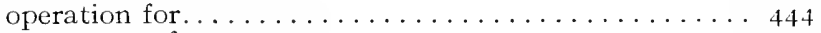

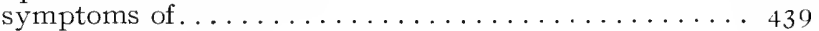

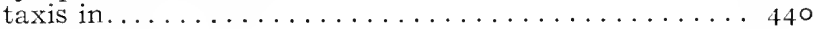

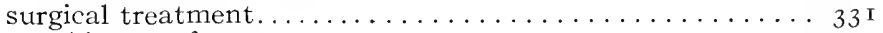

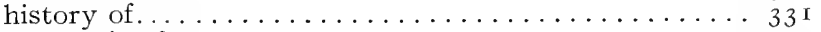

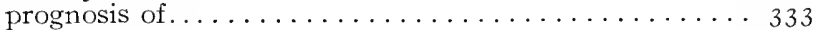

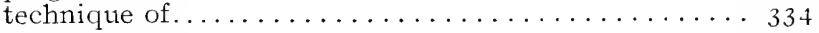

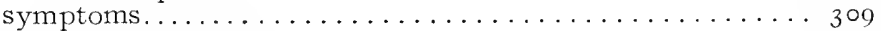

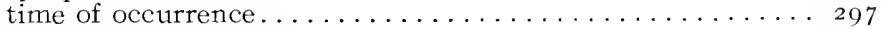

Femoral ring. . . . . . . . . . . . . . . . . . . . . . 299

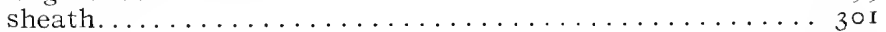

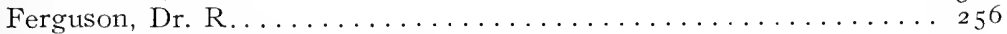

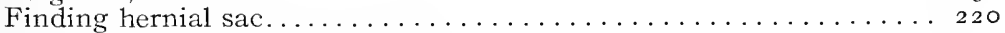

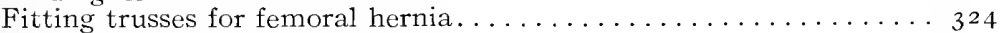

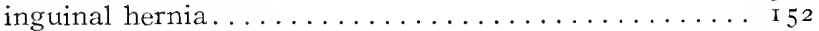

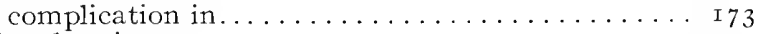

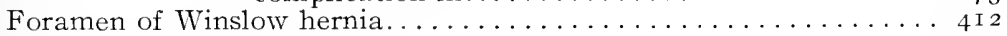

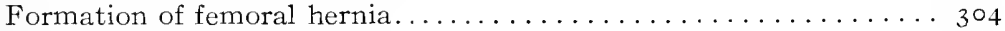

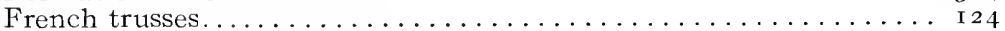

Function of delayed testicle..................... 39

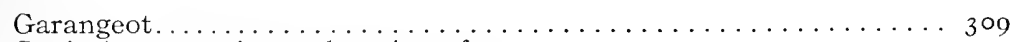

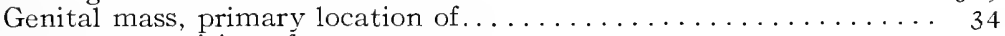

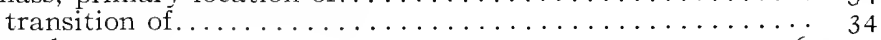

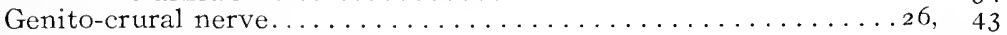

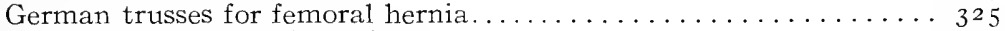

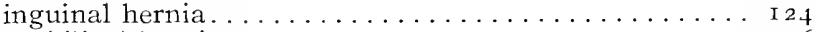

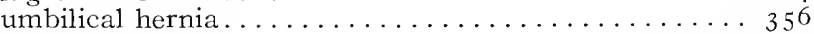

Gibson, Dr. C. L...................... 284, 290, 436

Gimbernat's ligament. . . . . . . . . . . . . . . . . . . 23, 299

Goldner............................... 20, 410

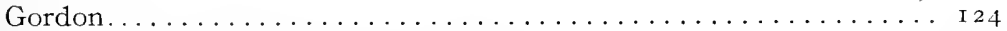

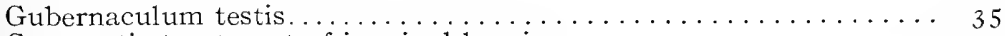

Gymnastic treatment of inguinal hernia............... 204

Seaver's views on ....................... 206

\section{$\mathrm{H}$}

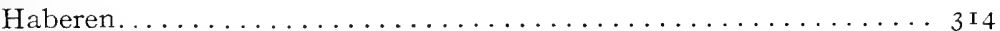

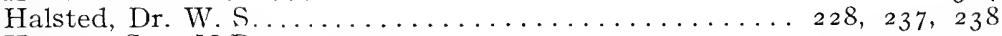

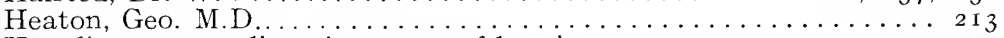

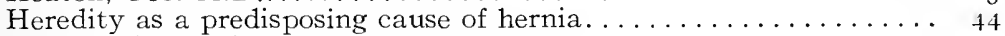

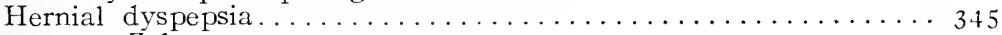

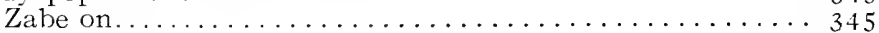

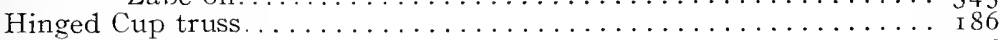

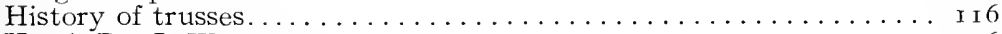

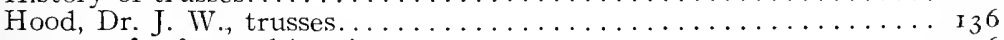

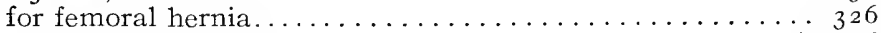

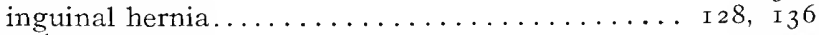

their advantages......................... I 42

Horwitz, Dr. Orville ....................... 29 I 
Hydrocele differentiated from, femoral hernia............. I $^{\mathrm{I}} 5$

inguinal hernia .................... Io8

congenital differentiated from inguinal hernia........... I04

Hypogastric fossæ........................... $3^{\mathrm{r}}$

I

Ilio-hypogastric nerve. $\ldots \ldots \ldots \ldots \ldots \ldots \ldots \ldots \ldots \ldots \ldots \ldots, 30$

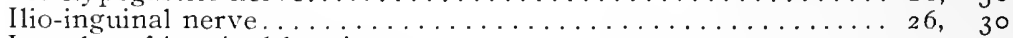

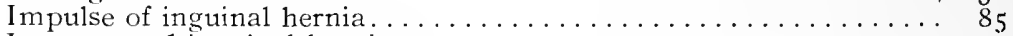

Incarcerated inguinal hernia........................

Incision for combined inguinal and femoral hernia........... 340

Incomplete hernia......................... 54

Infantile hernia............................... 75

Infant umbilical hernia treatment.................... 355

Inflamed inguinal hernia..................... 70

Inflamed glands differentiated from strangulated inguinal hernia.. . 423

Inf undibuliform fascia ................... $27,4^{2}$

Inguinal adenitis differentiated from oblique inguinal hernia....... I I

Inguinal canal. ........................... 27

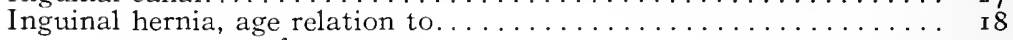

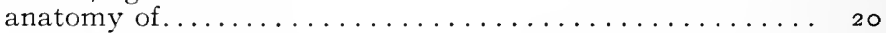

Andrews' operation for ..................... 227

author's operation for ............... 2 I 8

Bassini's operation for ................... 2 I 6

combined with femoral hernia................. 340

curative treatment for $\ldots \ldots \ldots \ldots \ldots \ldots \ldots \ldots \ldots \ldots \ldots \ldots \ldots \ldots$ I 3

definition $\ldots \ldots \ldots \ldots \ldots \ldots \ldots \ldots \ldots \ldots \ldots \ldots \ldots \ldots \ldots \ldots \ldots \ldots$

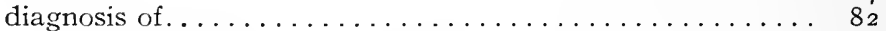

differential...................... 102

differentiated from femoral hernia.............. I I I

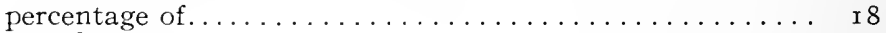

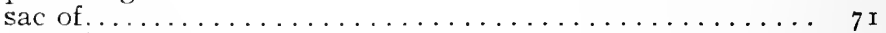

sex relation to....................... I 8

strangulated....................... $44^{\mathrm{r}} 3$

differential diagnosis of................... 423

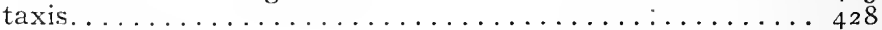

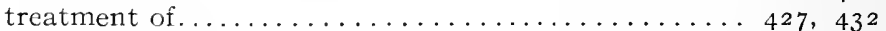

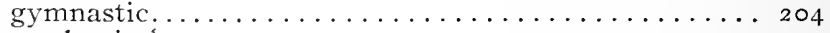

mechanical.................... I I 4

surgical..................... $2 \mathrm{I}_{4}$

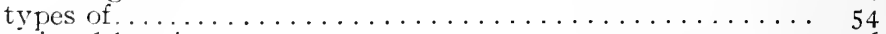

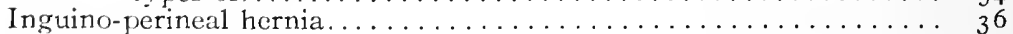

Injection of hernia.................... 2 I 2

Instructions to truss wearers. . . . . . . . . . .

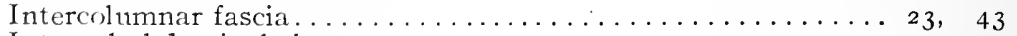

Internal abdominal ring. .................... 27

hernia....................

inguinal hernia........................ 3 I

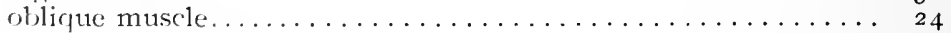

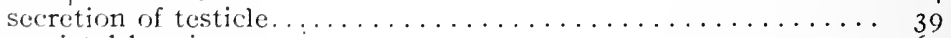

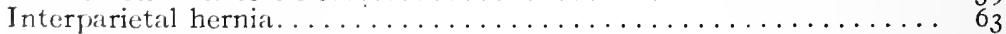

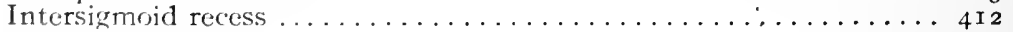

Interstitial hernia.......................... $6_{3}$

crmplicating truss fitting.............. r 80

Invagination of scrotal tissue in diagnosis of inguinal hernia.... 86

Irreducible hernia.......................... I0, 20

femoral........................ 
PAGE

Irreducible femoral hernia, truss for $\ldots \ldots \ldots \ldots \ldots \ldots \ldots \ldots \ldots \ldots \ldots \ldots$
inguinal. $\ldots \ldots \ldots \ldots \ldots \ldots \ldots \ldots \ldots$

strangulated....................... $4^{14}$

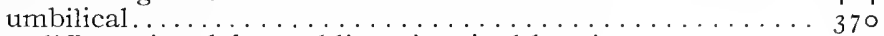

tumor differentiated from oblique inguinal hernia......... I 88

$\mathrm{J}$

Johnson, Dr. George Ben., views on operation for umbilical hernia. . $37^{2}$

$\mathrm{K}$

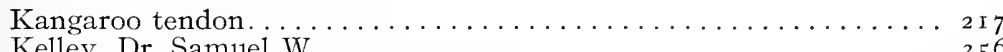

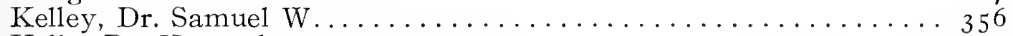

Kelly, Dr. Howard. . . . . . . . . . . . . . . . . . . 39 I

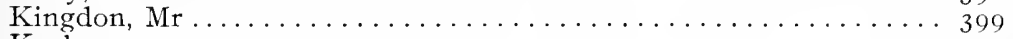

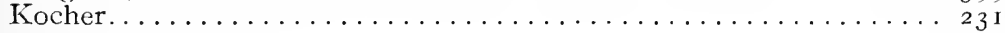

L

Labial hernia............................ 55

varix differentiated from oblique inguinal hernia........... I05

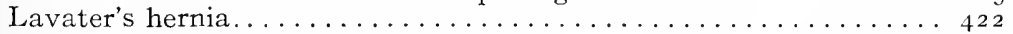

Lead tape method of measuring for truss.............. I 54

Lifting as direct cause of hernia............... 5 I

Ligation of blood vessels in inguinal hernia............. 219

hernial sac........................ 222

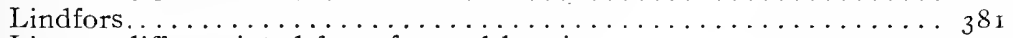

Lipoma differentiated from femoral hernia............. 3 I 2

oblique inguinal hernia ................ I

Littres hernia........................... 422

Location of spontaneous ventral hernia.............. 384

stricture in strangulated inguinal hernia........... 414

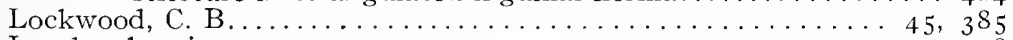

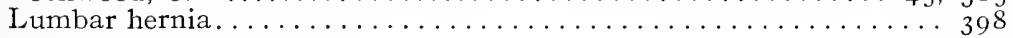

\section{M}

Macdonald, Dr. Willis...................... 382

Macfadden, Bernard ....................... 2 I I

Macready, Jonathan, F. C. H. . I 24, 186, 297, 309, 383, 398, 405, 406

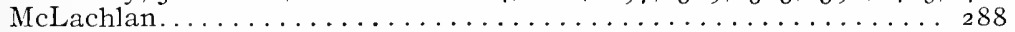

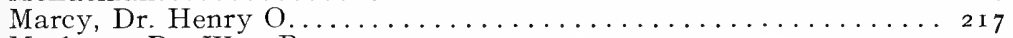

Mathews, Dr. Wm. P....................... 255

Mayo, Dr. Wm. J., operations for umbilical hernia.......... 377

Measuring for truss...................... 152

Mechanical treatment for, femoral hernia.............. 3 7

inguinal hernia. .................. I I 4

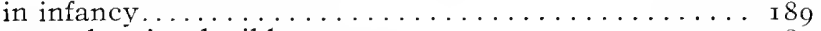

when irreducible............... I 83

lumbar hernia...................... 399

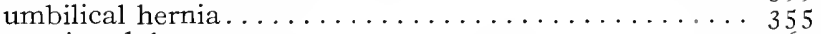

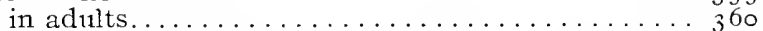

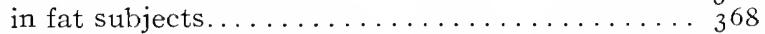

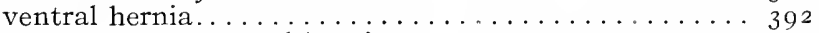

Medical treatment for strangulated hernia.............. 427

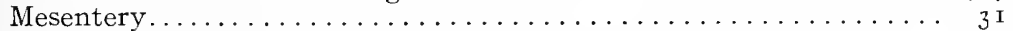

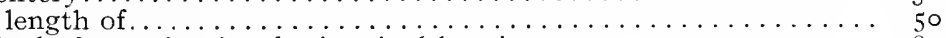

Method of examination for inguinal hernia............. 84 
PAGE

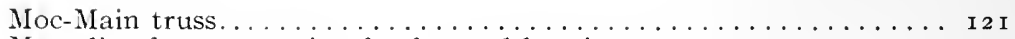

Mortality from operation for femoral hernia. . . . . . . . . . $33^{2}$

Mouth of hernia........................... I9

\section{$\mathbf{N}$}

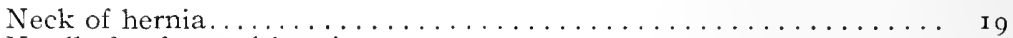

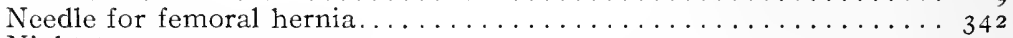

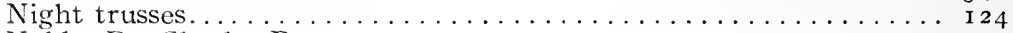

Noble, Dr. Charles P....................... 292

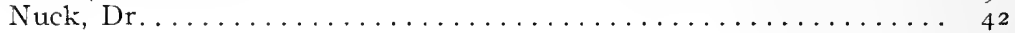

$\mathbf{O}$

Oblique inguinal hernia....................... 54 acquired differentiated from other types of inguinal hernia. . . 94

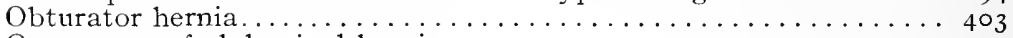

Occurrence of abdominal hernia.................. I

Ody, Salmon and. ........................... I 29

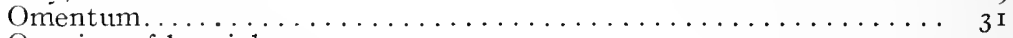

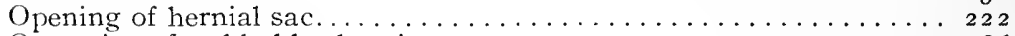

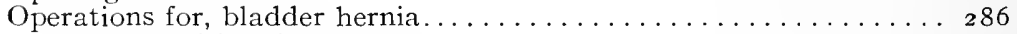

cæcal hernia........................ 2 So

delayed testicle with inguinal hernia............ 247

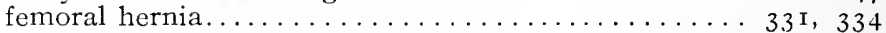

combined with inguinal hernia.................... 340

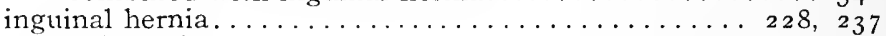

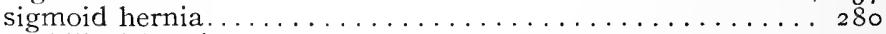

umbilical hernia........................ 373

Orchitis differentiated from strangulated inguinal hernia....... 423

Overlapping abdominal wall in umbilical hernia........... 375

\section{$\mathbf{P}$}

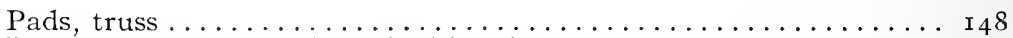

Palliative treatment of inguinal hernia................ I I 3

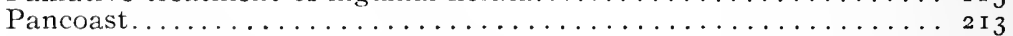

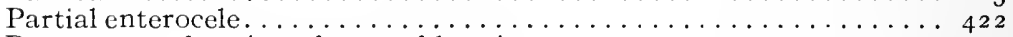

Percentage of various forms of hernia $\ldots \ldots \ldots \ldots \ldots \ldots \ldots$ r 8

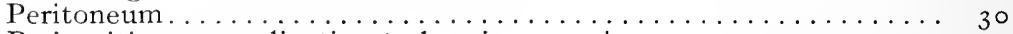

Peritonitis as complication to hernia operation............ 4 r 2

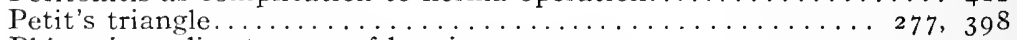

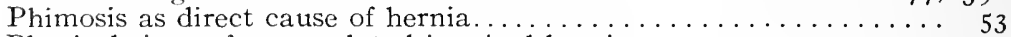

Physical signs of strangulated inguinal hernia............... 4 I 5

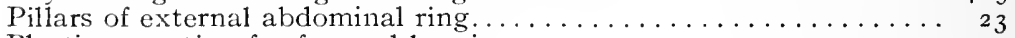

Plastic operation for femoral hernia................. 343

Plummer, Dr. S. C........................ 286

Post-operative treatment for femoral hernia.............. 340

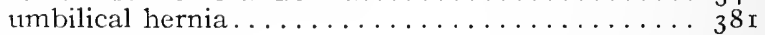

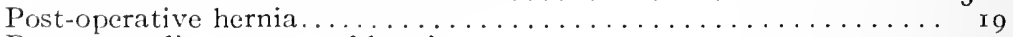

Posture as direct cause of hernia................. 5 I

Poupart's ligament......................... 23, 298

Powder for truss wearers....................... 202

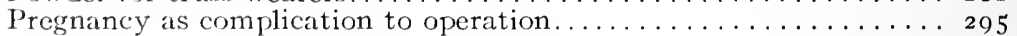

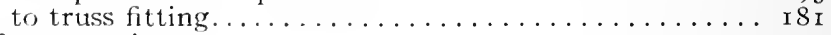

Preparation for operation. ..........................

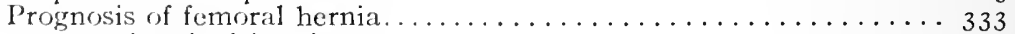
inguinal hernia......................... I 4 obturator hernia...................... 405 
Proge

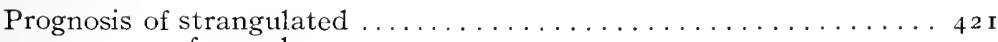

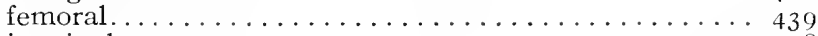

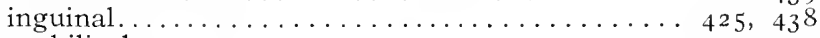

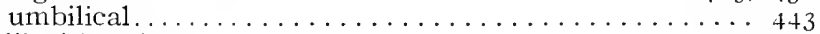

umbilical hernia . . . . . . . . . . . . . . . . 368

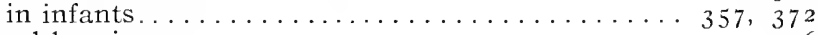

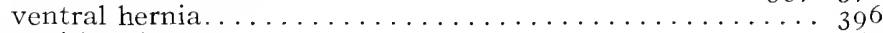

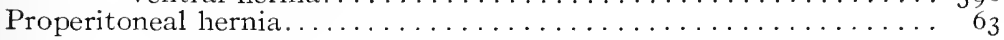

Q

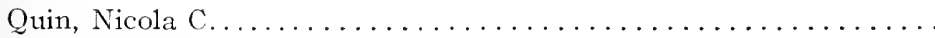

$\mathrm{R}$

Radical cure truss $\ldots \ldots \ldots \ldots \ldots \ldots \ldots \ldots \ldots \ldots \ldots \ldots$

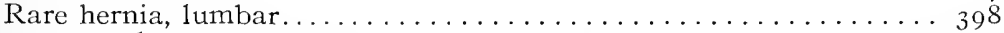

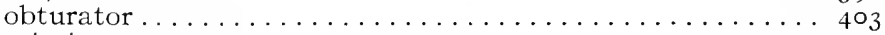

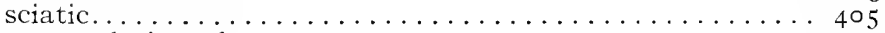

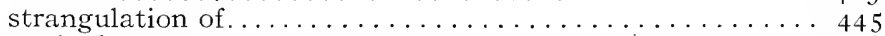

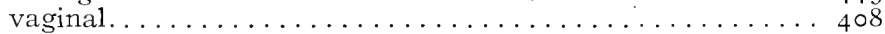

Rat-tail truss ............................ I

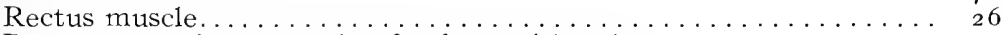

Recurrences after operation for femoral hernia........... 332

Reducible hydrocele as complication to fitting truss . . . . . . . . . . . . I 75

Reducibility of non-strangulated inguinal hernia. . . . . . . . 68 , 82

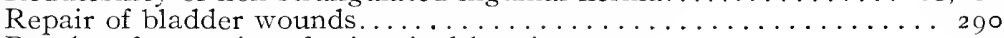

Results of operations for inguinal hernia............... 235

failure of obliteration of tunica vaginalis............. 39

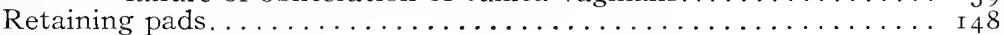

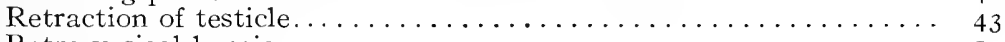

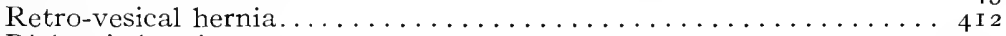

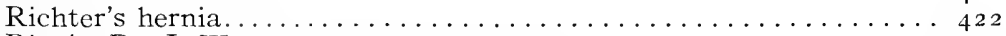

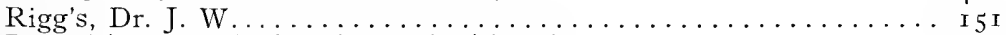

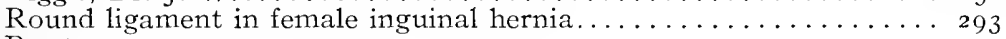

Rupture............................. I 7

$\mathbf{S}$

Salmon and $\mathrm{Ody} \ldots \ldots \ldots \ldots \ldots \ldots \ldots \ldots \ldots \ldots \ldots \ldots$

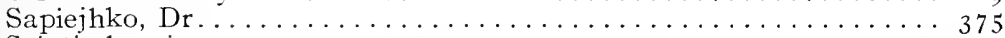

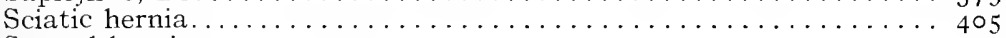

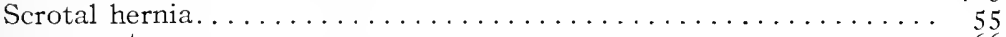

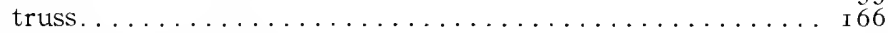

Seaver, Jay W., A.M., M.D . . . . . . . . . . . . . . . . 204

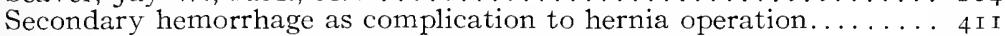

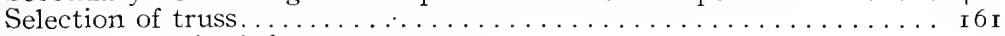

for infants......................... I94

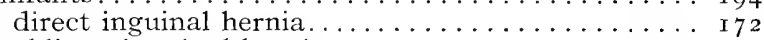

oblique inguinal hernia ....................... 6 r

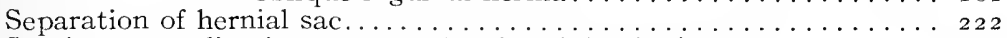

Sepsis as complication to operation for abdominal hernia....... 4 I 2

Sex as predisposing cause of hernia. ................ 45

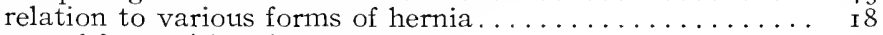

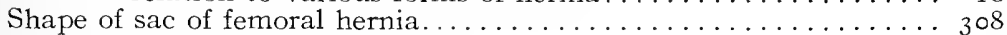

Shaping truss for femoral hernia. . . . . . . . . . . . . . . . $33^{\text {I } 7}$

inguinal hernia......................... I 52 , 5 I 57

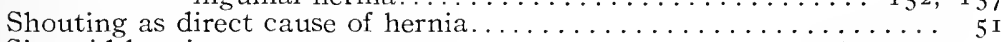

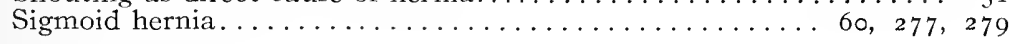


PAGE

Sigmoid hernia differentiated from other inguinal hernia........ $9^{8}$

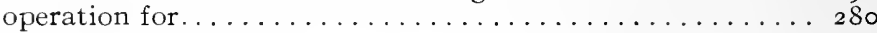

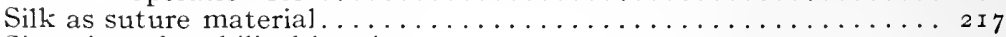

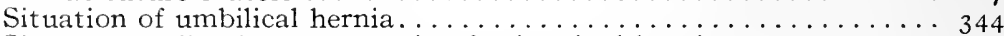

Size as complication to operation for inguinal hernia.......... 265

contra-indication to operation for abdominal hernia..... 409

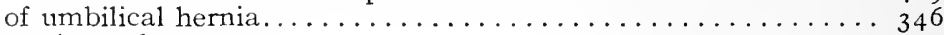

Spermatic cord............................ 29

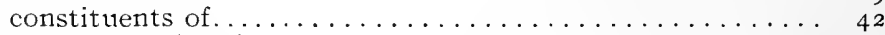

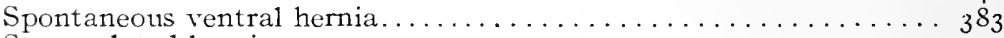

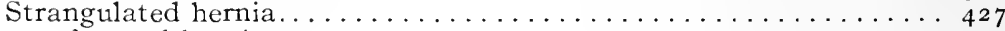

femoral hernia.......................... 439

inguinal hernia..................... $70,4 \mathbf{1} 3$

rarer hernia.......................... 444

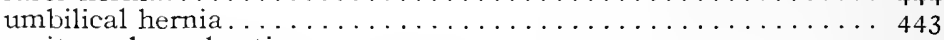

Sub-peritoneal areolar tissue....................... 27

fat as complication to truss fitting............... I 74

Summary of author's operations for abdominal hernia........ 445

Superficial epigastric vessels..................... 22

fascia ...................... 2 I

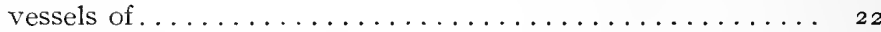

Surgical anatomy of inguinal region. . . . . .

Surgical treatment of, femoral hernia.............. $33^{\mathrm{I}}$

inguinal hernia................. 2 I 4

Andrews' operation for ................ 227

author's operation for ............... 2 I 8

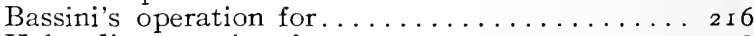

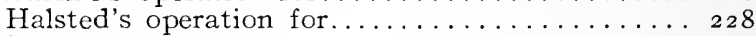

in female..................... 292

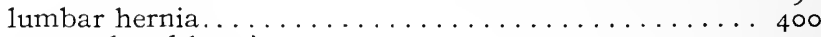

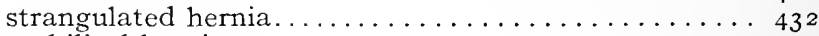

umbilical hernia................... 372

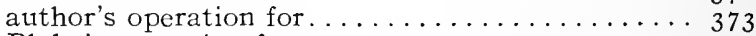

Blake's operation for.............. 375

Dr. Johnson's views on .............. 372

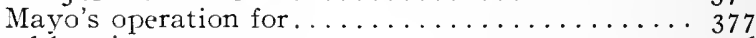

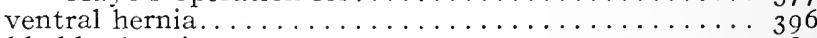

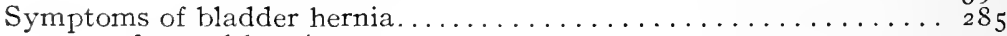

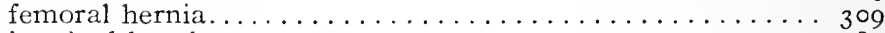

inguinal hernia .................... $88_{2}$

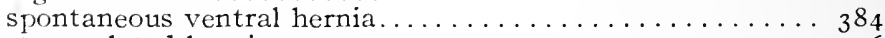

strangulated hernia...................... 4 I 6

umbilical hernia ....................... 354

$\mathrm{T}$

Taylor, Dr. Gen. H.......................... 2 I I

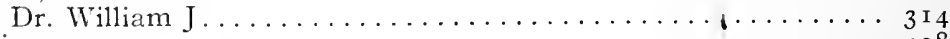

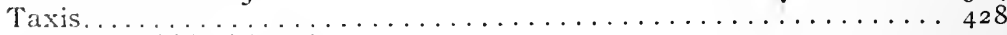

on umbilical hernia........................ 444

Testicle................................... 34

Theory of operation for inguinal hernia.............. $2 \mathbf{I}_{4}$

Thrombosis of femoral vein as accident to hernia operations..... 4 I0

Time of occurrence of femoral hernia.................. 297

Time of obliteration of tunica vaginalis . . . . . . . . . . . . 39

Trel, Dr............................. I 47

Traction in reduction of hernia.................... 429

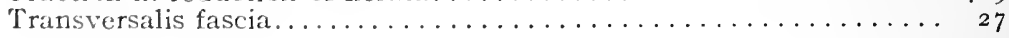


P.AGE

Transversalis muscle in inguinal hernia................ 25

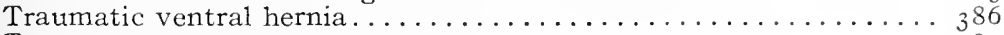

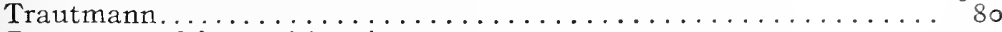

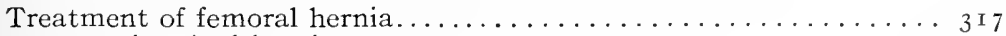

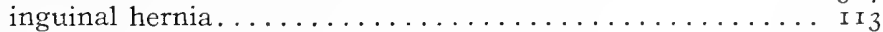

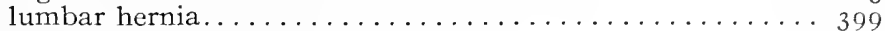

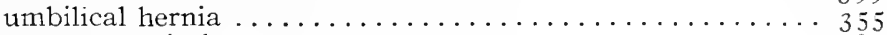

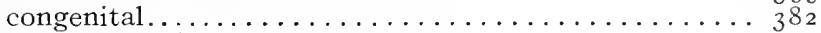

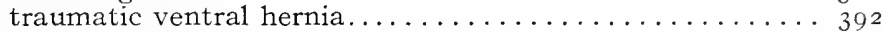

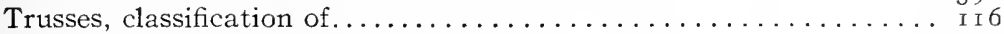

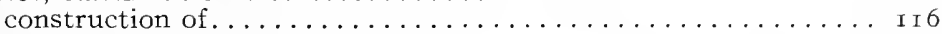

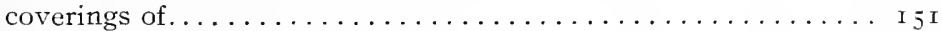

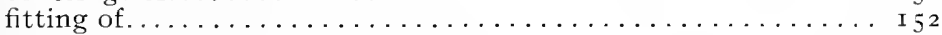

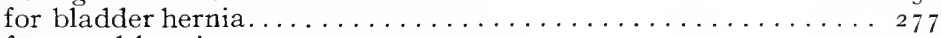

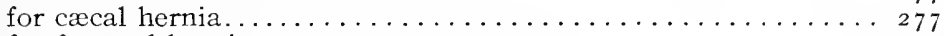

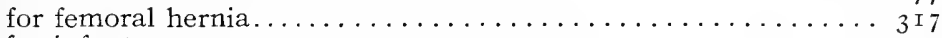

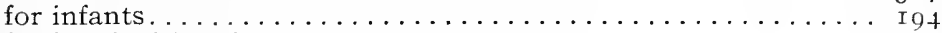

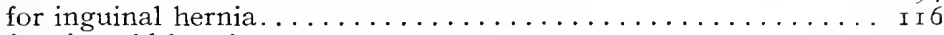

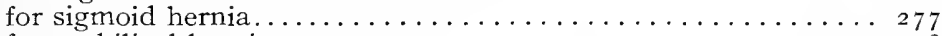

for umbilical hernia. . . . . . . . . . . . . . . . 358

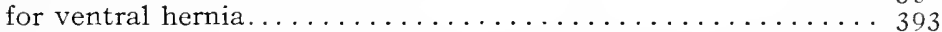

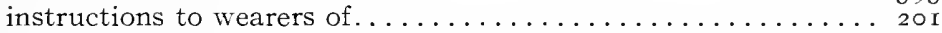

varieties of........................ I 2 I

Tuberculosis as contra-indication to operating abdominal hernia . . 4 io

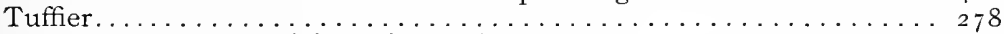

Tumors differentiated from femoral hernia............. 3 I 6

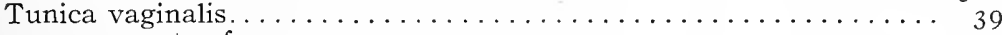

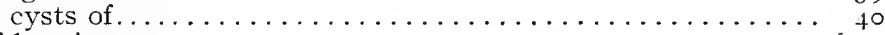

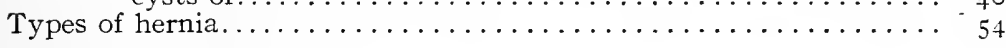

$\mathbf{U}$

Umbilical hernia . . . . . . . . . . . . . . . . . . . . $3+4$

age relation to...................

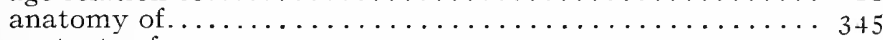

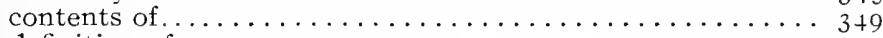

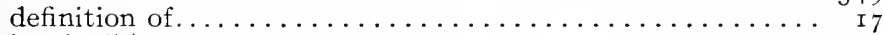

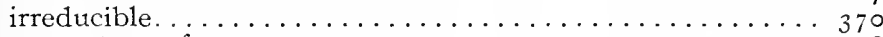

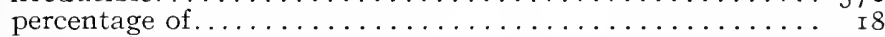

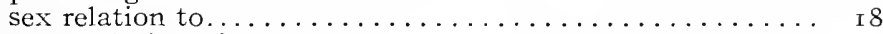

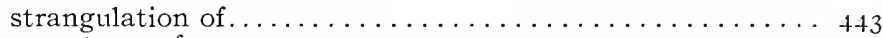

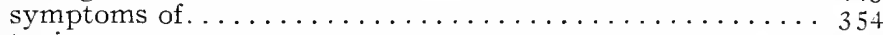

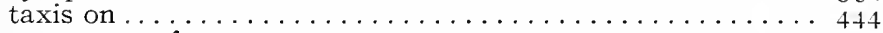

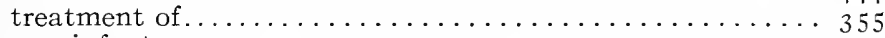

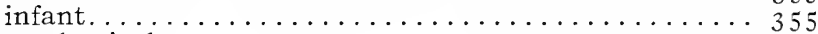

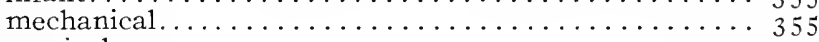

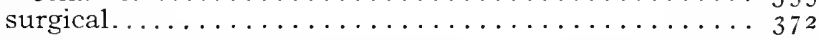

Unclassified trusses............................ I 47

Undescended ovary differentiated from oblique inguinal hernia...... I Io

Undescended testicle, see delayed testicle . . . . . . . . . . 37

Urinary obstruction as direct cause of hernia . . . . . . . . . . $5^{2}$

Vaginal hernia............................. 408

Varicocele as complication to operation for inguinal hernia....... 239

complication to truss fitting.................. 76

differentiated from femoral hernia . . . . . . . . . . . . . . 3 I I

inguinal hernia.................... I O $4_{4}$ 


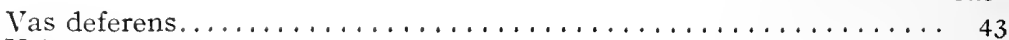

Velpean............................ $2 \mathrm{I}_{3}$

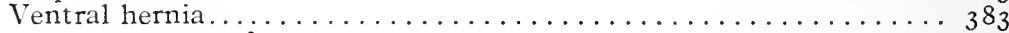

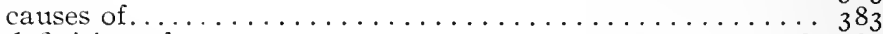

definition of $\ldots \ldots \ldots \ldots \ldots \ldots \ldots \ldots \ldots \ldots \ldots \ldots \ldots \ldots \ldots \ldots \ldots \ldots$

percentage of .................. r 8

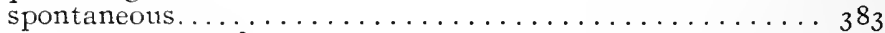

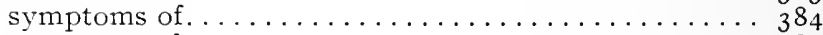

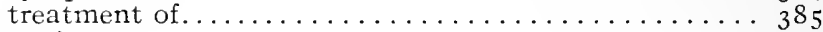

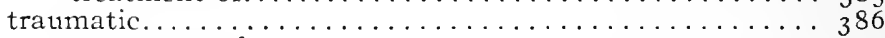

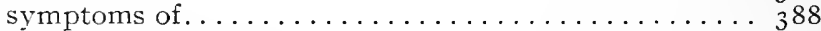

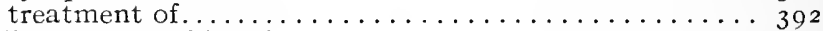

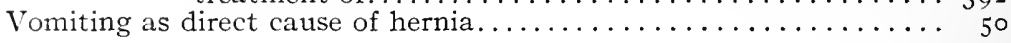

W

Winsboro, Dr. Rudolph........................ 288

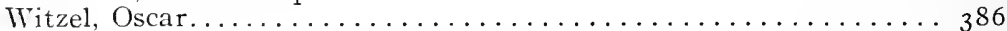

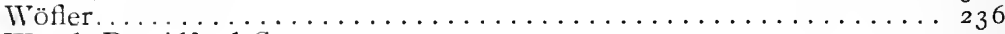

Wood, Dr. Alfred C......................................... 309

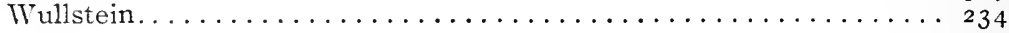

Zabe, Dr., Hernial dyspepsia ...................... 345 
. 

(1)

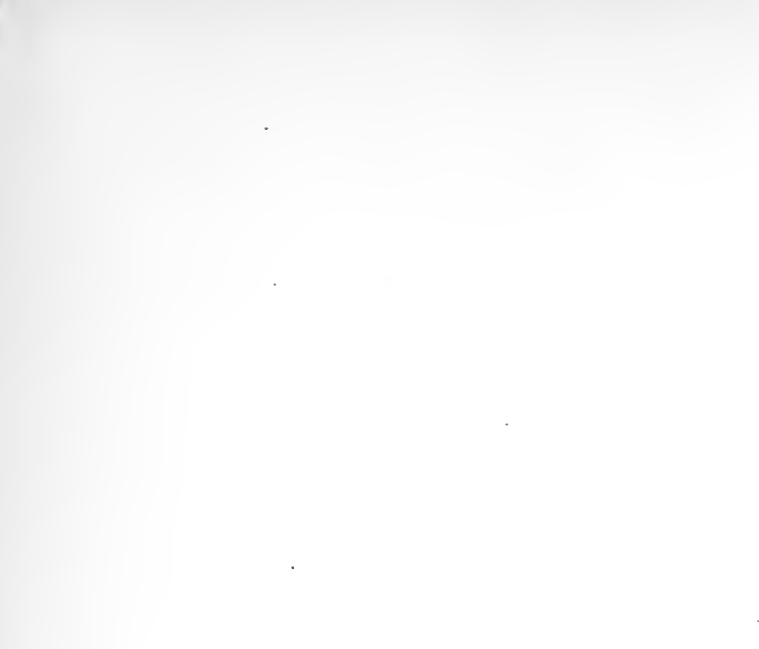



RD 621 D 361907 C.1 LRIES (hSI. StX

Abd 1907 C.1

Hum hernia its dragnosis and trea

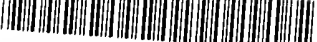

2002098296 


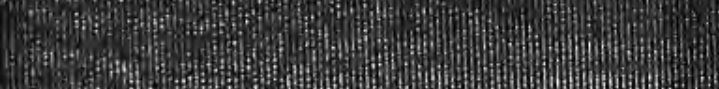

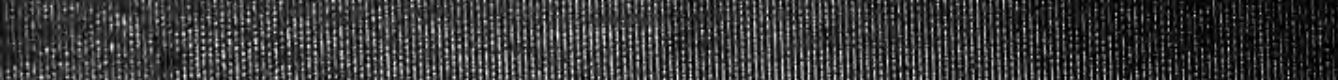

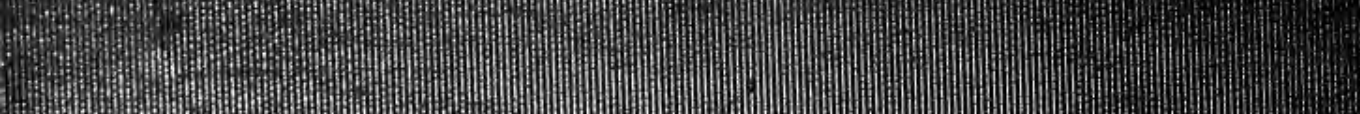

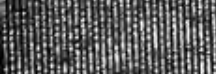

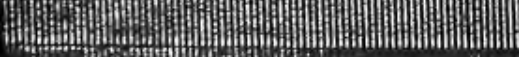
(3) 